\title{
PESTICIDE, NUTRIENT, WATER-DISCHARGE AND PHYSICAL- PROPERTY DATA FOR THE MISSISSIPPI RIVER AND SOME OF ITS TRIBUTARIES, APRIL 1991-SEPTEMBER 1992
}

by R.H. Coupe, D.A. Goolsby, J.L. Iverson, D.J. Markovchick, and S.D. Zaugg

U.S. GEOLOGICAL SURVEY

Open-File Report 93-657 


\section{U.S. DEPARTMENT OF THE INTERIOR \\ BRUCE BABBITT, Secretary}

U.S. GEOLOGICAL SURVEY

Gordon P. Eaton, Director

The use of trade, product, industry, or firm names is for descriptive purposes only and does not imply endorsement by the U.S. Government.

For additional information write to:

Chief, Branch of Regional Research

U.S. Geological Survey

Box 25046, MS 418

Denver Federal Center

Denver, CO 80225
Copies of this report can be purchased from:

U.S. Geological Survey

Earth Science Information Center

Open-File Reports Section

Box 25286, MS 517

Denver Federal Center

Denver, CO 80225 


\section{CONTENTS}

Abstract-1- 1

Introduction -.- 1

Purpose and scope-1-_o 2

Acknowledgments- 2

Data collection --_o 4

Sampling sites-_._._. 4

Upper Mississippi River Basin-_-_ 4

Missouri River Basin -

Ohio River Basin -

Lower Mississippi River Basin-_- 5

Sampling schedule-- 5

Sample-collection and processing procedures - 6

Water discharge and physical properties-ar 7

Analytical procedures and results -1

Pesticides -

Solid-phase extraction 100 milliliters-

Solid-phase extraction 1 liter

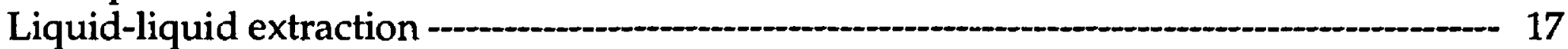

Nutrients - 17

Water discharge-- 20

Physical properties - 20

Quality-assurance procedures and results 30

Field quality-assurance procedures - 30

Laboratory quality-assurance procedures - 33

References cited -_o 36

Data section - 39

\section{FIGURES}

Figure 1. Map showing location of sampling sites -

2. Diagram showing sampling schedule -

3-7. Graphs showing:

3. Atrazine concentrations at three Mississippi River sampling sites

4. Alachlor concentrations at three Mississippi River sampling sites -.......... 11

5. Cyanazine concentrations at three Mississippi River sampling sites -ב-_- 12

6. Metolachlor concentrations at three Mississippi River sampling sites -_-____-_-_ 13

7. Nitrate concentrations at three Mississippi River sampling sites --._. 19

8-15. Water-discharge hydrograph for the:

8. Mississippi River at Clinton, Iowa--_... 21

9. Mississippi River at Thebes, Ill. -.._. 22

10. Mississippi River at Tarbert Landing, La., and for the Mississippi River diversions into the Atchafalaya River -ב-2 23

11. White River at Petersburg, Ind.-_-_- 24

12. Ohio River at Metropolis, Ill.-___- 25

13. Illinois River at Valley City, Ill. - _ 26 
14. Platte River at Louisville, Nebr. --_-27

15. Water-discharge hydrograph for the Missouri River at Hermann, Mo. -.-_-_--28

16. Specific conductance at three Mississippi River sampling sites -..-29

17. Box plots showing percent recoveries of laboratory spikes of alachlor, atrazine, cyanazine, desethylatrazine, metolachlor, metribuzin, prometon, and simazine for solid-phase extraction 100-milliliter method --_n

18. Box plots showing percent recoveries of laboratory spikes of alachlor, atrazine, carbofuran, cyanazine, diazinon, EPTC, metolachlor, metribuzin, prometon, simazine, and trifluralin for solid-phase extraction 1-liter method

\section{TABLES}

Table 1. Analytical reporting limits for selected herbicides

2. Precision and accuracy data from seven determinations of analytes by the solidphase extraction 100-milliliter method in surface water spiked at concentrations of 0.2 and 2.0 micrograms per liter

3. Precision and accuracy data from seven determinations of analytes by the solidphase extraction 1-liter method in surface water spiked at concentrations of 0.1 and 1.0 microgram per liter---

4. Precision and accuracy data for measurements of physical properties and nutrients, April 1991-September 1992 -...-18

5. Results of split samples analyzed by the two solid-phase extraction methods and the liquid-liquid extraction method

6. Results of blind spike samples analyzed by solid-phase extraction 100-milliliter method--.-

7. Pesticide results from solid-phase extraction 100 -milliliter method-______-_-_4

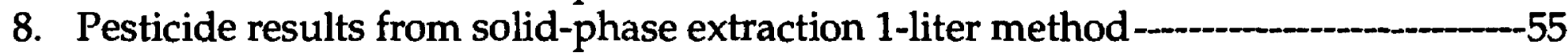

9. Water-discharge, physical-property, nutrient, and suspended-sediment data---- 101 


\section{CONVERSION FACTORS AND ABBREVIATED WATER-QUALITY UNITS}

Multiply

cubic meter per second $\left(\mathrm{m}^{3} / \mathrm{s}\right)$

meter $(\mathrm{m})$

liter (L)

kilometer $(\mathrm{km})$

square kilometer $\left(\mathrm{km}^{2}\right)$
By

35.31

3.281

0.2642

0.6214

0.3861
To obtain

cubic foot per second

foot

gallon

mile

square mile

Abbreviated water-quality units used in this report:

microsiemens per centimeter at 25 degrees Celsius $(\mu \mathrm{S} / \mathrm{cm})$

milligram per liter (mg/L)

microgram per liter $(\mu \mathrm{g} / \mathrm{L})$

milliliter (mL)

microliter $(\mu \mathrm{L})$

millimeter $(\mathrm{mm})$

micrometer $(\mu \mathrm{m})$

To convert degrees Celsius $\left({ }^{\circ} \mathrm{C}\right)$ to degree Fahrenheit $\left({ }^{\circ} \mathrm{F}\right)$, use the following formula:

$$
{ }^{\circ} \mathrm{F}=9 / 5\left({ }^{\circ} \mathrm{C}\right)+32
$$




\title{
PESTICIDE, NUTRIENT, WATER-DISCHARGE, AND PHYSICAL-PROPERTY DATA FOR THE MISSISSIPPI RIVER AND SOME OF ITS TRIBUTARIES, APRIL 1991-SEPTEMBER 1992
}

\author{
by R.H. Coupe, D.A. Goolsby, J.L. Iverson, D.J. Markovchick, \\ and S.D. Zaugg
}

\begin{abstract}
This report presents pesticide, nutrient, water-discharge, and physical-property data for the Mississippi River and some of its tributaries collected during April 1991 through September 1992. Surface-water samples were collected at eight sites. Three sites were on the Mississippi River and one each on the Ohio, Illinois, Missouri, Platte (tributary to the Missouri River), and White (tributary to the Ohio River) Rivers. The samples were collected to determine the temporal and spatial distribution and mass transport of selected pesticides and nutrients, especially those associated with the production of row crops.

Procedures for the collection of representative surface-water samples are described as well as the analytical methods used to determine the pesticide and nutrient concentrations. Field and laboratory quality-assurance procedures are described, and the results are presented.
\end{abstract}

\section{INTRODUCTION}

The Mississippi River drains an area of intensive row-crop production. Millions of pounds of herbicides, insecticides, and nitrogen fertilizers are applied annually to improve crop yields. Recent studies have shown that many of these compounds are transported into the Mississippi River and eventually are discharged into the Gulf of Mexico.

Studies conducted by the U.S. Geological Survey (USGS) in 1989 and 1990 indicate that herbicides are flushed from cropland each spring and summer and are transported into tributary streams to the Mississippi River (Goolsby and others, 1991b; Thurman and others, 1991, 1992). During May and June 1989, maximum concentrations of four herbicides (alachlor, atrazine, cyanazine, and metolachlor) ranged from 10 to more than $100 \mu \mathrm{g} / \mathrm{L}$ in streams draining several hundred to several thousand square kilometers. Similar concentrations were measured in these streams again in 1990 (Goolsby and others, 1991b).

Water samples collected at many points on the Mississippi River and its tributaries as part of a study of sediment-related transport of organic contaminants in the Mississippi River have shown that major rivers such as the Missouri, Ohio, and Mississippi are affected by the discharge of herbicides from tributary streams (Meade and Stevens, 1990; Moody and Meade, 1992). Pereira and Rostad (1990) reported concentrations of atrazine and alachlor as large as about $1 \mu \mathrm{g} / \mathrm{L}$ in samples collected between St. Louis, Mo., and New Orleans, La., during May and June 1988. During mid-June 1990, atrazine concentrations in this same reach of the Mississippi River ranged from 1.5 to $3.0 \mu \mathrm{g} / \mathrm{L}$ (Moody and Goolsby, 1993). 
In order to better understand the occurrence, temporal and spatial distribution, and mass transport of selected pesticides and nutrients in the Mississippi River, the USGS conducted an intensive study of the river and several large tributaries during 1991 and 1992. Surface-water samples were collected at eight sites (fig. 1). Three were on the Mississippi River and one each on the Ohio, Illinois, Missouri, Platte (tributary to the Missouri River), and White (tributary to the Ohio River) Rivers. Additional information describing the study can be obtained from Goolsby and others (1991a).

\section{Purpose and Scope}

The purpose of this report is to describe the data-collection and analytical methods and the field and laboratory quality-assurance procedures, and to present the pesticide, nutrient, water-discharge, and physical-property data from surface-water samples collected as part of this study. These data are derived from almost 600 samples collected at eight sites from April 1991 through September 1992.

\section{Acknowledgments}

Samples for this study were collected by USGS employees from the Illinois, Indiana, Kentucky, Iowa, Missouri, Nebraska, and Louisiana District offices and analyzed by the USGS National Water Quality Laboratory (NWQL) in Arvada, Colo. This study would not have been possible without the hard work and dedication displayed by these men and women. 


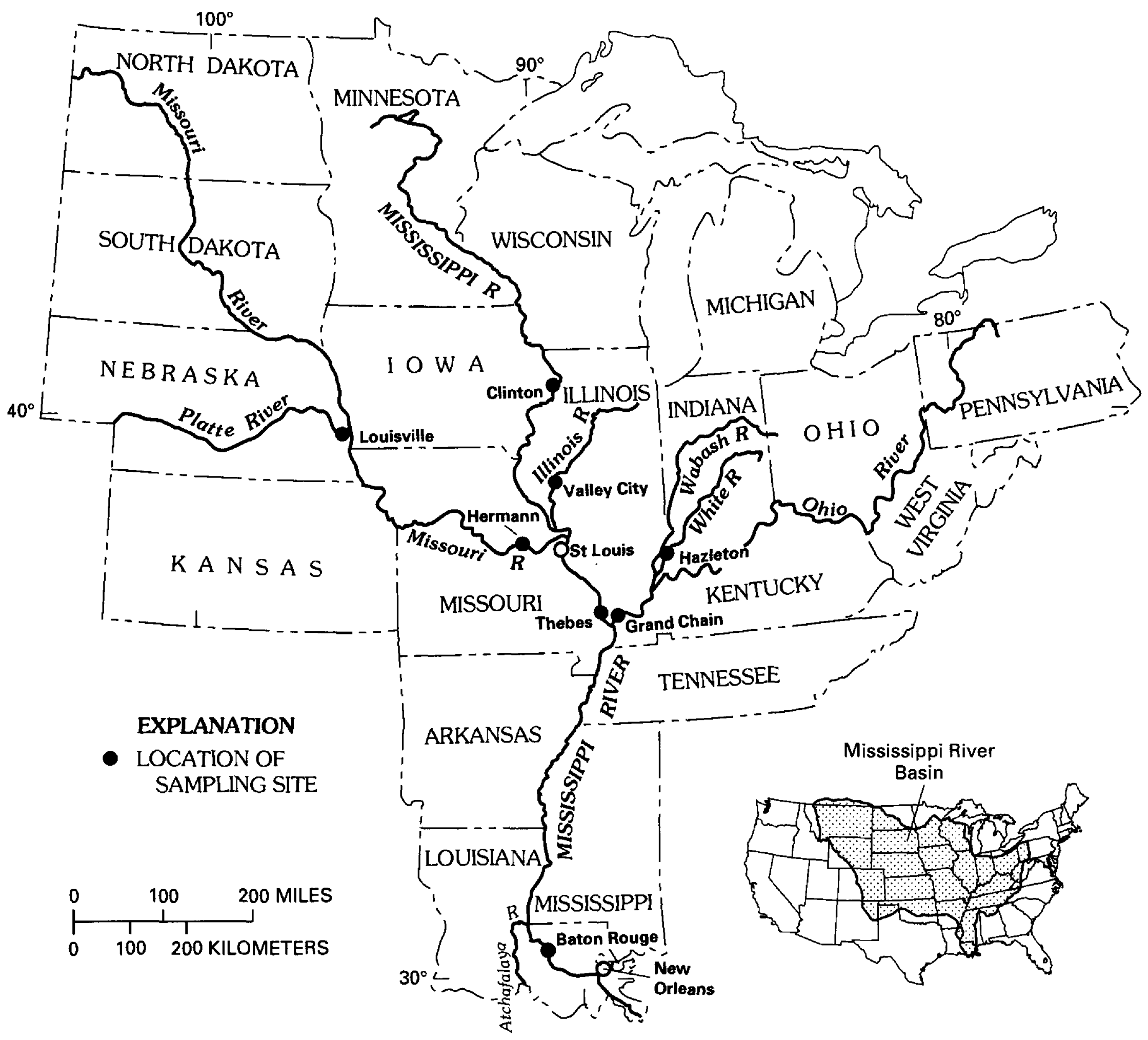

Figure 1.-Location of sampling sites. 


\section{DATA COLLECTION}

This section provides a description of the sampling sites, the rationale for their selection, the sampling schedule, documentation of sample-collection and processing procedures, and measurements of water discharge and physical properties.

\section{Sampling Sites}

Sampling sites were selected at three USGS water-discharge gaging stations on the Mississippi River (fig. 1). Sampling sites also were selected at the water-discharge gaging stations closest to the mouths of the Ohio, Illinois, and Missouri Rivers, and on one stream that is a secondary tributary to the Ohio River and one that is tributary to the Missouri River. The following is a description, by river basin, of each of the eight sampling sites (the 8-digit number following the name of each site is the USGS station number). Site locations are shown in figure 1.

\section{Upper Mlssissippl River Basin}

1. Mississippi River at Clinton, Iowa, station 05420500 (drainage area $221,700 \mathrm{~km}^{2}$ ): This site is the northernmost sampling site on the Mississippi River. Samples from this site provide a measure of the agricultural chemical inputs from the upper basin States of northeastern Iowa, Minnesota, and Wisconsin.

2. Illinois River at Valley City, Ill., station 05586100 (drainage area $69,260 \mathrm{~km}^{2}$ ): Samples from this site provide a measure of the inputs from the Illinois River, a tributary to the Upper Mississippi River, that drains an area of intensive row-crop agriculture.

3. Mississippi River at Thebes, Ill., station 07022000 (drainage area $1,847,200 \mathrm{~km}^{2}$ ): Samples from this site provide a measure of agricultural chemicals discharged from the Upper Mississippi and Missouri River Basins and represent essentially all of the Mississippi River discharge upstream from the Ohio River.

\section{Missourl River Basin}

4. Platte River at Louisville, Nebr., station 06805500 (drainage area $222,200 \mathrm{~km}^{2}$ ): Samples from this site measure the inputs from a major tributary to the Missouri River. The Platte River drains an area of intensely irrigated agriculture in Nebraska.

5. Missouri River at Hermann, Mo., station 06934500 (drainage area 1,357,000 $\mathrm{km}^{2}$ ): This site is relatively near the mouth of the Missouri River, and samples provide an estimate of agricultural chemical input to the Mississippi River from the entire Missouri River Basin.

\section{Ohio River Basin}

6. White River near Hazleton, Ind., station 03374100 (drainage area $29,300 \mathrm{~km}^{2}$ ): This relatively small basin drains an area of intensive agriculture in central and western Indiana. The White River discharges to the Wabash River, which in turn discharges to the Ohio River. 
7. Ohio River at Grand Chain, Ill., station 03612500 (drainage area $526,000 \mathrm{~km}^{2}$ ): Samples from this site provide a measure of inputs from the Ohio River Basin to the Mississippi River.

\section{Lower Mississlppl Rlver Basin}

8. Mississippi River at Baton Rouge, La., station 07373420 (drainage area $2,914,000 \mathrm{~km}^{2}$ ): Samples from this site and estimates of the Mississippi River diversions into the Atchafalaya River provide a measure of the total agricultural chemical discharge from the Mississippi River Basin to the Gulf of Mexico.

\section{Sampling Schedule}

Sample collection for this study began in April 1991 and continued through September 1992 (fig. 2). Samples were collected about once per week, but collection was more frequent during late spring and summer, when the concentrations of agricultural chemicals are expected to be highest, and less frequent in the winter, when concentrations of these chemicals are expected to be lowest. The sampling schedule was as follows:

April 1991: One sample per week.

May 6-July 15, 1991: Two samples per week (except one sample per week for the Ohio River at Dam 53 near Grand Chain, Ill.).

July 15-October 30, 1991: One sample per week (sample collection at White River near Hazleton, Ind., continued at two samples per week through August).

November 1991-February 1992: One sample every 2 weeks (except for Mississippi River at Baton Rouge, La., where sampling frequency remained at one sample per week).

March 1992-July 1992: One sample per week (sample collection was discontinued at Illinois River at Valley City, Ill., after March 1992. Sample-processing procedures were changed for the Platte River at Louisville, Nebr., after May 7, 1992, and data are not included in this report. Also, because of changes in sample-processing procedures, few data are included for the White River near Hazleton, Ind., after April 29, 1992). Sample collection was discontinued at all remaining sites except the White River near Hazleton, Ind., and the Mississippi River at Baton Rouge, La., at the end of July 1992.

August 26, 1992: Sample collection was discontinued at the White River at Hazleton, Ind.

September 23, 1992: Sample collection was discontinued at the Mississippi River at Baton Rouge, La.

The increased sampling frequency during May, June, and July provided more information on the concentrations and transport of agricultural chemicals during the first-flush events following pesticide application. Special efforts were made to distribute these samples over the discharge hydrograph to obtain the best estimates of mass transport of agricultural chemicals. 


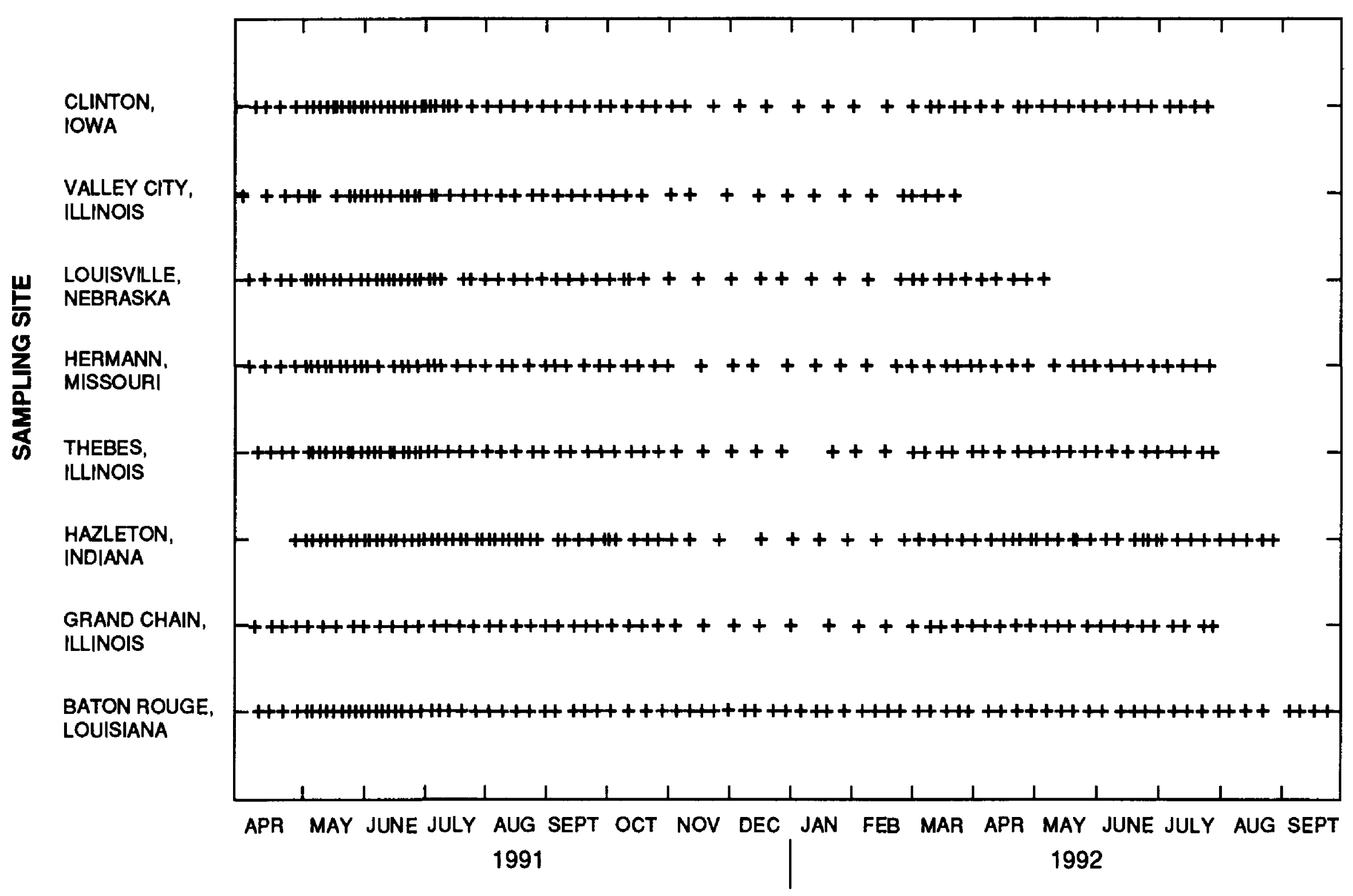

Figure 2.--Sampling schedule.

\section{Sample-Collection and Processing Procedures}

Samples were collected by equal-discharge-increment or equal-width-increment procedures (Edwards and Glysson, 1988) at all sites except Mississippi River at Baton Rouge, La. At each sampling site, water samples were collected in glass containers at five or more locations across the river by using depth-integrating samplers and were composited in large glass or stainless-steel containers. A Teflon cone splitter was then used to divide the composite sample into subsamples to be analyzed for the concentrations of dissolved herbicides and insecticides; dissolved nitrate, nitrite, and ammonianitrogen; dissolved orthophosphate; total organic plus ammonia-nitrogen; total phosphorus; and suspended sediment. This procedure provided a sample that was representative of the entire cross section of the river.

Previous work has indicated that dissolved solutes in the Mississippi River at Baton Rouge, La., are well mixed vertically and laterally (C.R. Demas, U.S. Geological Survey, Baton Rouge, La., oral commun., 1991). Therefore, to minimize sample-collection costs, samples at Baton Rouge were collected from the upper $6 \mathrm{~m}$ of the water column at the end of a pier that extends about $45 \mathrm{~m}$ from shore. As a quality-assurance measure, samples were collected once at several points across the river channel at Baton Rouge, La., to verify that the river water was well mixed. In addition, samples were collected concurrently from the Mississippi River near St. Francisville, La., station 07373420, on about a monthly basis, using the depth-integrating and compositing procedures outlined in this report. This station is about $56 \mathrm{~km}$ upstream from the pier from which the routine samples were collected. These data are 
listed later in this report. Samples for total organic plus ammonia-nitrogen, total phosphorus, and suspended sediment were not collected at this site.

In this study, samples for herbicide and insecticide analysis were filtered through a $142-\mathrm{mm}$-diameter glass-fiber filter with a nominal pore size of $0.7 \mu \mathrm{m}$ using aluminum or stainless-steel filter holders. Filtration was accomplished using either compressed nitrogen gas or pumps with ceramic and (or) Teflon pump mechanisms. The filtrate was collected in precleaned glass bottles. Samples for dissolved nutrients (nitrogen and phosphorus compounds) were filtered through a $0.45-\mu \mathrm{m}$ membrane filter. Total and dissolved nutrients were preserved with mercuric chloride. All herbicide, insecticide, and nutrient samples were chilled immediately after collection and were shipped to the NWQL in Arvada, Colo., for analysis.

Sample processing was completed onsite immediately after collection except for the Ohio River and the Platte River sites. These sites were located close to USGS offices; therefore, samples from these sites were taken to the office for processing. In most cases, this increased the time between sample collection and processing by less than 1 hour.

\section{Water Discharge and Physical Properties}

The accuracy of water-discharge measurements depends on the stability of stage-discharge relations. Discharge data in this report are estimated to be "good," meaning that 95 percent of the data are within 10 percent of the true value, except for a few estimated discharges that are within 15 percent of the true value.

Onsite measurements for specific conductance and $\mathrm{pH}$ were made on the composite mixture for each sample except for those from the White River near Hazleton, Ind., where in-situ measurements from the center of flow were made. Stream temperature was measured in-situ. Except for Mississippi River at Baton Rouge, La., measurements of water discharge were obtained from stage-discharge relations at stations operated by the USGS. Water-discharge data for the Baton Rouge site were provided by the U.S. Army Corps of Engineers (COE), New Orleans District Office (Cecil W. Soileau, U.S. Army Corps of Engineers, New Orleans, La., written commun., 1992). 


\section{ANALYTICAL PROCEDURES AND RESULTS}

All water samples were analyzed at the NWQL in Arvada, Colo., for herbicides, insecticides, nitrogen, and phosphorus compounds. Analytical procedures used to analyze for herbicides, insecticides, and nutrients are briefly described below. Suspended-sediment concentrations were determined by USGS laboratories in Iowa City, Iowa, Rolla, Mo., or Louisville, Ky.

\section{Pesticides}

Three analytical procedures were used at the NWQL to analyze for the pesticides of interest in this study - two solid-phase extraction (SPE) procedures and a liquid-liquid extraction (LLE) procedure that uses methylene chloride. The primary procedures used to obtain the data presented in this report are the two SPE methods. One of the SPE procedures (SPE100 $\mathrm{mL}$ ) uses $100 \mathrm{~mL}$ of water and has reporting limits of $0.05-0.2 \mu \mathrm{g} / \mathrm{L}$. It was used to analyze for 10 herbicides and 2 atrazine metabolites. The other SPE procedure (SPE1L) uses 1 liter of water and has reporting limits of 0.002-0.01 $\mu \mathrm{g} / \mathrm{L}$. SPE1L was used to analyze for 28 herbicides, 16 insecticides, and 2 fungicides. The reporting limits for these analytical procedures for five representative herbicides (alachlor, atrazine, cyanazine, metolachlor, and simazine) are shown in table 1.

Table 1.-Analytical reporting limits for selected herbicides

[SPE100mL, solid-phase 100-milliliter extraction; SPE1L, solidphase 1-liter extraction; LLE, liquid-liquid extraction; $\mu \mathrm{g} / \mathrm{L}$, microgram per liter]

\begin{tabular}{lllc}
\hline Herbicide & $\begin{array}{c}\text { SPE100mL } \\
(\mu \mathbf{g} / \mathbf{L})\end{array}$ & $\begin{array}{c}\text { SPE1L } \\
(\mu \mathbf{g} / \mathbf{L})\end{array}$ & $\begin{array}{c}\text { LLE } \\
(\mu \mathbf{g} / \mathbf{L})\end{array}$ \\
\hline Alachlor & 0.05 & $0.015 / 0.003^{*}$ & 0.2 \\
Atrazine & 0.05 & $0.010 / 0.002$ & 0.1 \\
Cyanazine & $0.2 / 0.05^{*}$ & $0.050 / 0.005$ & 0.2 \\
Metolachlor & 0.05 & $0.005 / 0.002$ & 0.2 \\
Simazine & 0.05 & $0.015 / 0.010$ & 0.1 \\
\hline
\end{tabular}

* Reporting limit changed to this value about January 1992.

\section{Solld-Phase Extraction 100 Millliters}

This procedure is used for the isolation and analysis of triazine and other nitrogen-containing herbicides. The procedure is described in detail by Sandstrom and others (1991) and is a modification of the procedure described by Thurman and others (1990). Approximately $100 \mathrm{~mL}$ of sample is pumped through a disposable C-18 solid-phase extraction cartridge. Prior to extraction, a surrogate standard (terbuthylazine) is added to the sample to aid in determining the extraction efficiency and in interpreting the analytical results. After extraction, the cartridges are dried with nitrogen gas and eluted with $1.8 \mathrm{~mL}$ of hexane-isopropanol (3:1) to remove the extracted compounds. The eluent is evaporated to about $100 \mu \mathrm{L}$ and herbicides are analyzed on a gas chromatograph equipped with a capillary column. 
Herbicides are identified and quantified with a mass spectrometer detector based on selected ion monitoring of the parent compound and two characteristic ions for each herbicide. Precision and accuracy data for this method are shown in table 2.

Tabie 2.-Precision and accuracy data from seven determinations of analytes by the soildphase extraction 100-miiliiiter method in surface water spiked at concentrations of 0.2 and 2.0 micrograms per liter

$[\mu \mathrm{g} / \mathrm{L}$, microgram per liter; $\%$, percent]

\begin{tabular}{|c|c|c|c|c|c|c|}
\hline Compound & $\begin{array}{c}\text { Mean } \\
\text { concen- } \\
\text { tration } \\
(\mu g / L)\end{array}$ & $\begin{array}{l}\text { Precision } \\
\text { (standard } \\
\text { deviation, } \\
\text { in } \mu g / L \text { ) }\end{array}$ & $\begin{array}{c}\text { Accuracy } \\
\text { (mean of } \\
\text { spiked } \\
\text { concen- } \\
\text { tration) } \\
(\%)\end{array}$ & $\begin{array}{c}\text { Mean } \\
\text { concen- } \\
\text { tration } \\
(\mu \mathrm{g} / L)\end{array}$ & $\begin{array}{c}\text { Precision } \\
\text { (standard } \\
\text { deviation, } \\
\text { in } \mu g / L \text { ) }\end{array}$ & $\begin{array}{c}\text { Accuracy } \\
\text { (mean of } \\
\text { spiked } \\
\text { concen- } \\
\text { tration) } \\
(\%)\end{array}$ \\
\hline & \multicolumn{3}{|c|}{$0.2 \mu g / L$ spike } & \multicolumn{3}{|c|}{$2.0 \mu \mathrm{g} / \mathrm{L}$ spike } \\
\hline Alachlor & 0.215 & 0.011 & 107 & 1.433 & 0.059 & 72 \\
\hline Ametryn & 0.174 & 0.009 & 87 & 1.361 & 0.077 & 68 \\
\hline Atrazine & 0.151 & 0.008 & 75 & 1.217 & 0.072 & 61 \\
\hline Cyanazine & 0.282 & 0.022 & 141 & 1.579 & 0.059 & 79 \\
\hline $\begin{array}{l}\text { Desethyl- } \\
\text { atrazine }\end{array}$ & 0.214 & 0.052 & 107 & 1.365 & 0.104 & 68 \\
\hline $\begin{array}{l}\text { Desisopropyl- } \\
\text { atrazine }\end{array}$ & 0.144 & 0.055 & 72 & 1.365 & 0.104 & 68 \\
\hline Metolachlor & 0.244 & 0.025 & 122 & 1.569 & 0.066 & 78 \\
\hline Metribuzin & 0.201 & 0.010 & 101 & 1.602 & 0.042 & 80 \\
\hline Prometryn & 0.177 & 0.017 & 88 & 1.333 & 0.080 & 67 \\
\hline Prometon & 0.235 & 0.011 & 118 & 1.552 & 0.081 & 78 \\
\hline Propazine & 0.147 & 0.008 & 74 & 1.207 & 0.077 & 60 \\
\hline Simazine & 0.177 & 0.009 & 88 & 1.253 & 0.069 & 63 \\
\hline
\end{tabular}

The results from this procedure are shown in table 7 (at end of report). Time-series plots of atrazine, alachlor, cyanazine, and metolachlor for the sampling sites on the Mississippi River at Clinton, Iowa, Thebes, Ill., and Baton Rouge, La., are shown in figures 3-6. The maximum contaminant levels (MCL) or health advisory levels (HA) for drinking water established by the U.S. Environmental Protection Agency (EPA, 1992) also are shown in figures 3-6. MCL's and HA's are based on average annual concentrations of contaminants and not on concentrations in individual samples. 

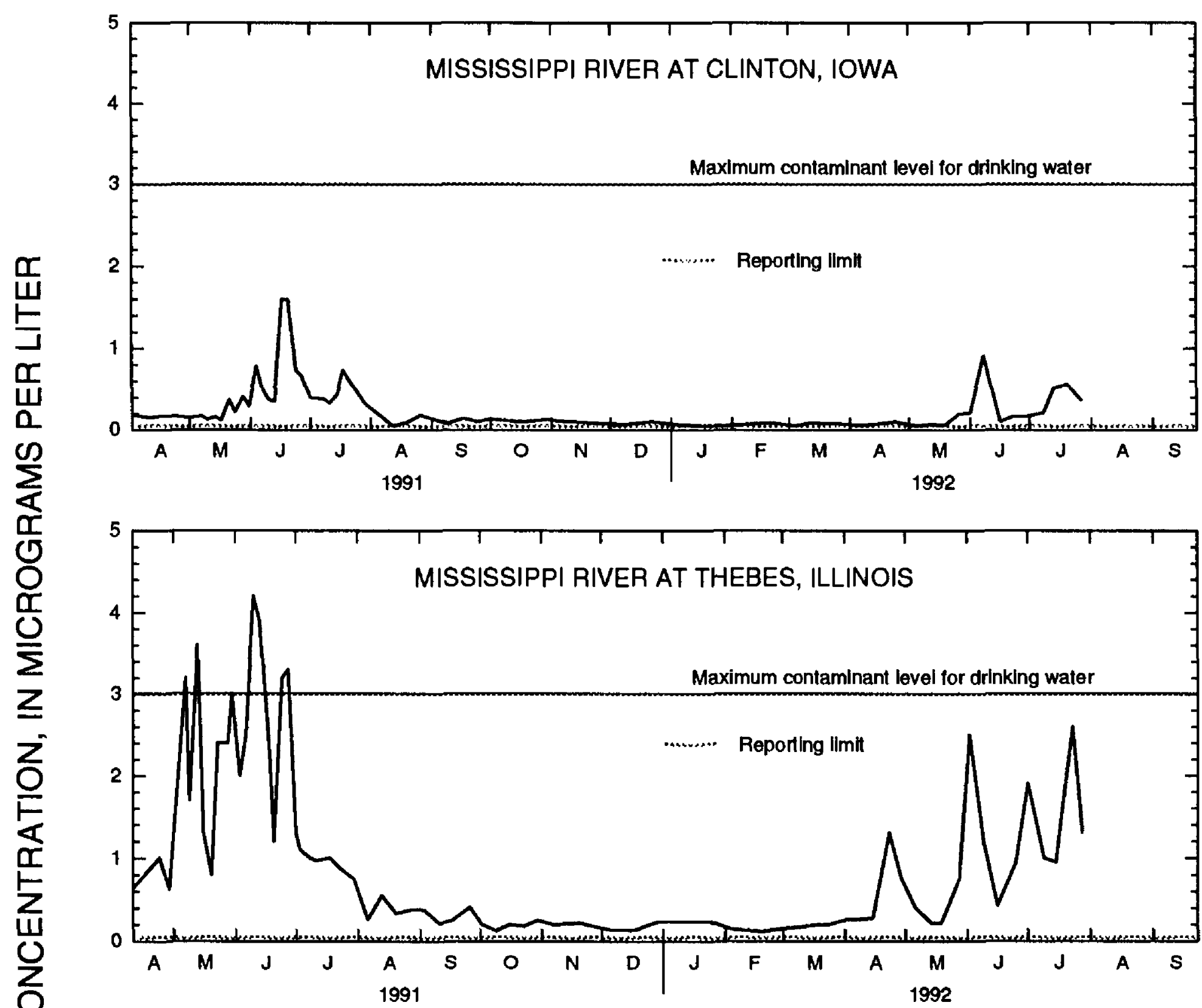

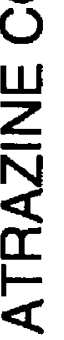

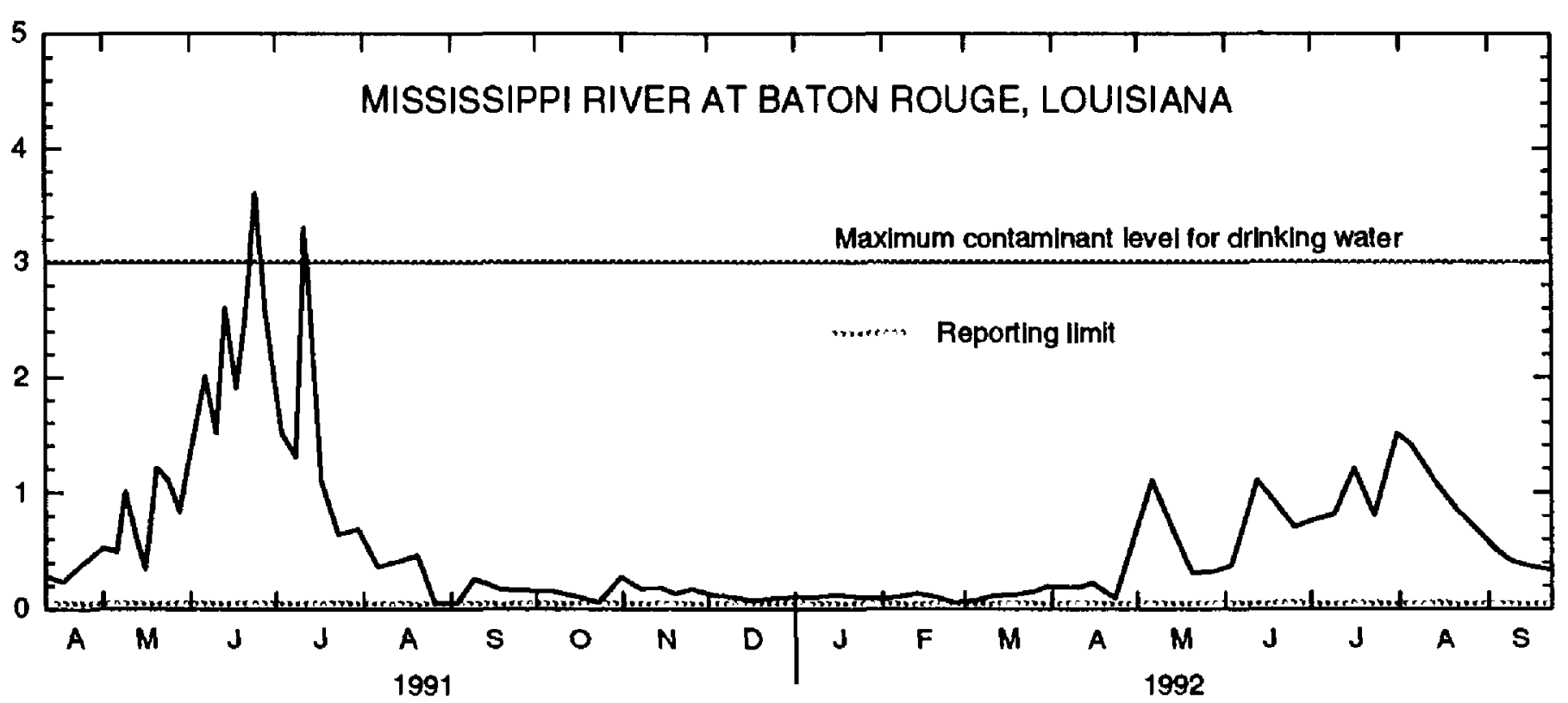

Figure 3.--Atrazine concentrations at three Mississippi River sampling sites. 

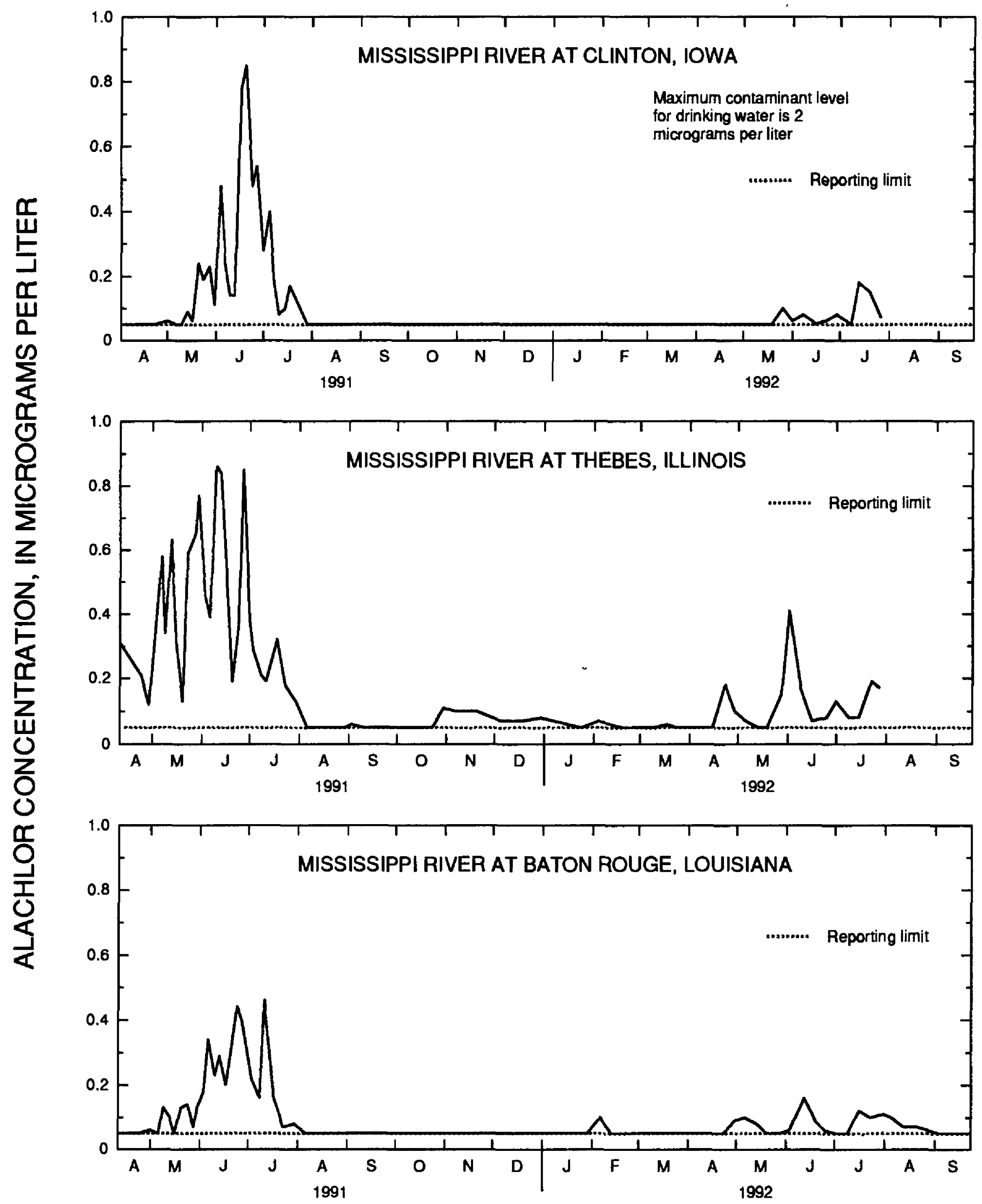

Figure 4.--Alachlor concentrations at three Mississippi River sampling sites. 


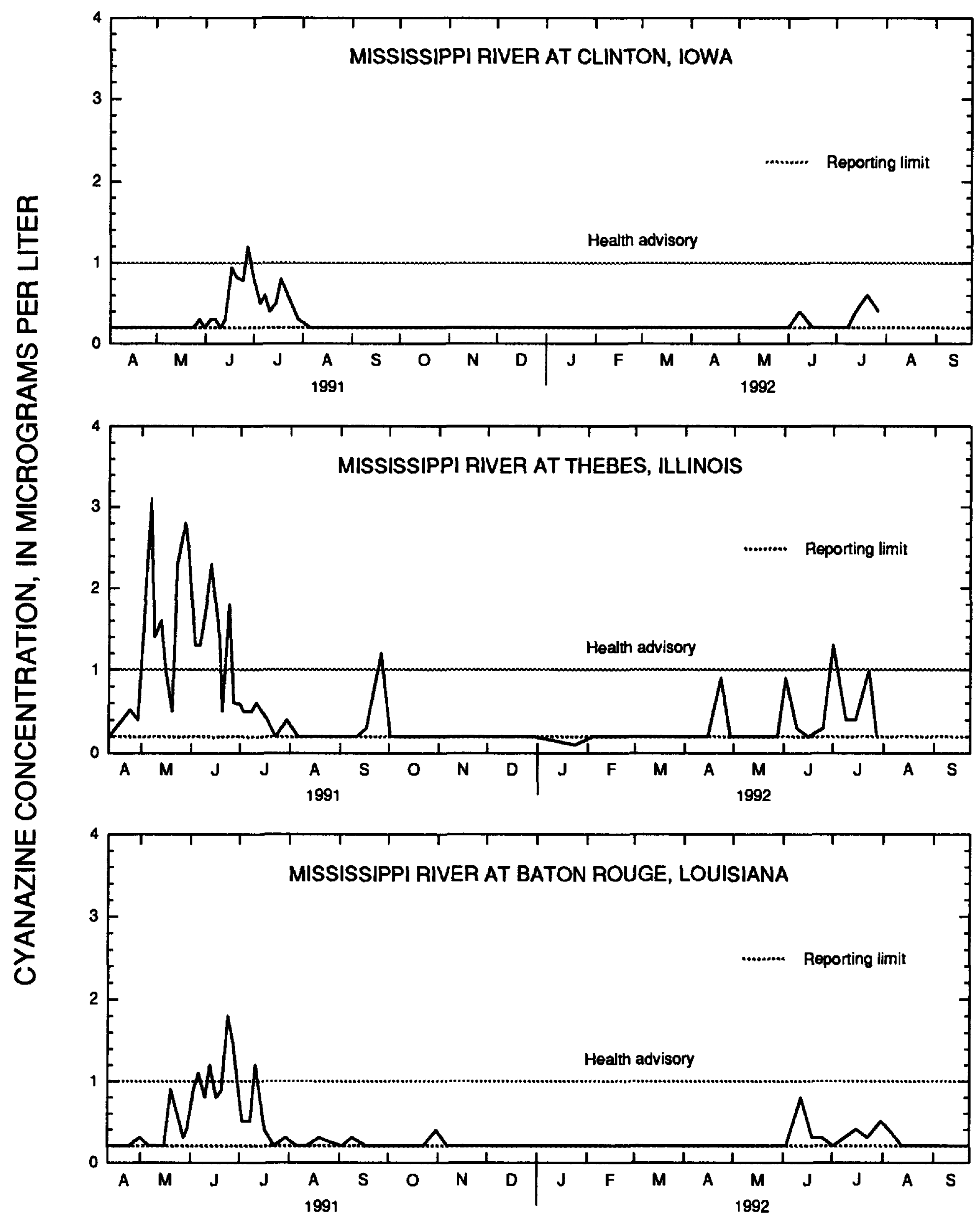

Figure 5.--Cyanazine concentrations at three Mississippi River sampling sites. 


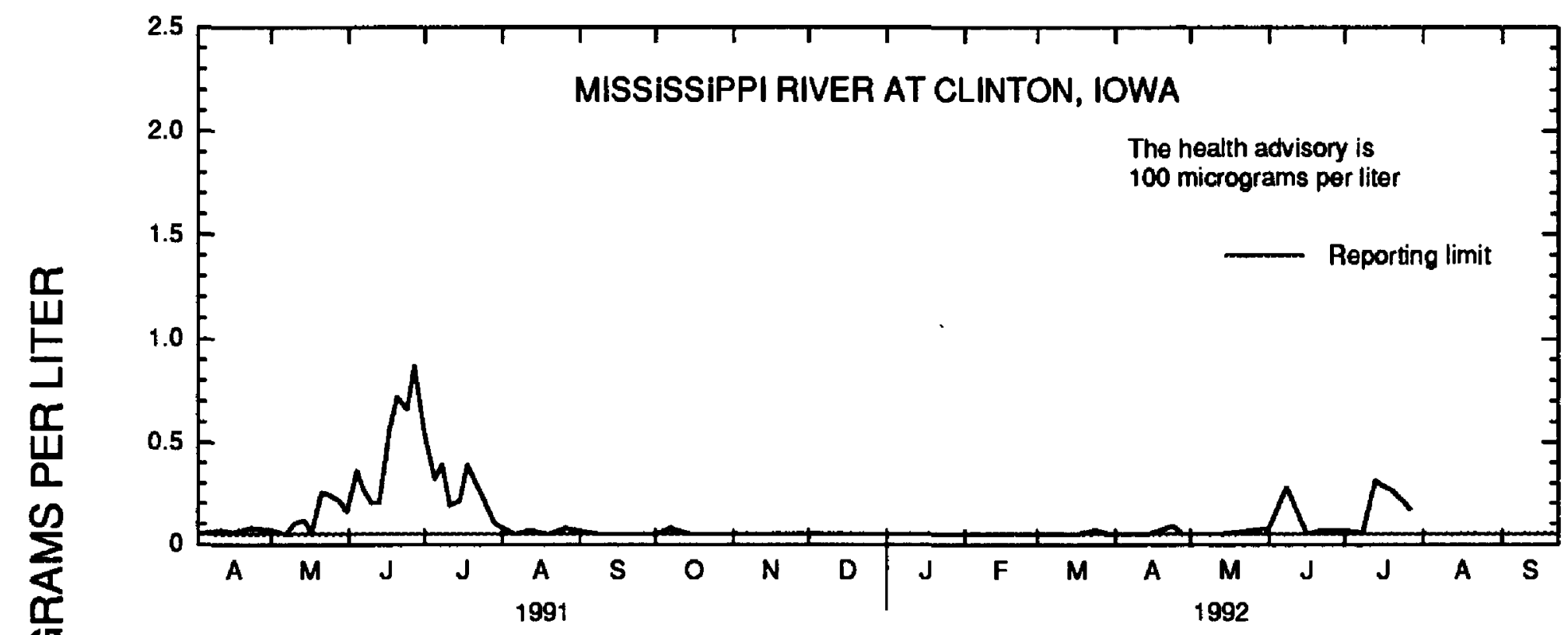

音
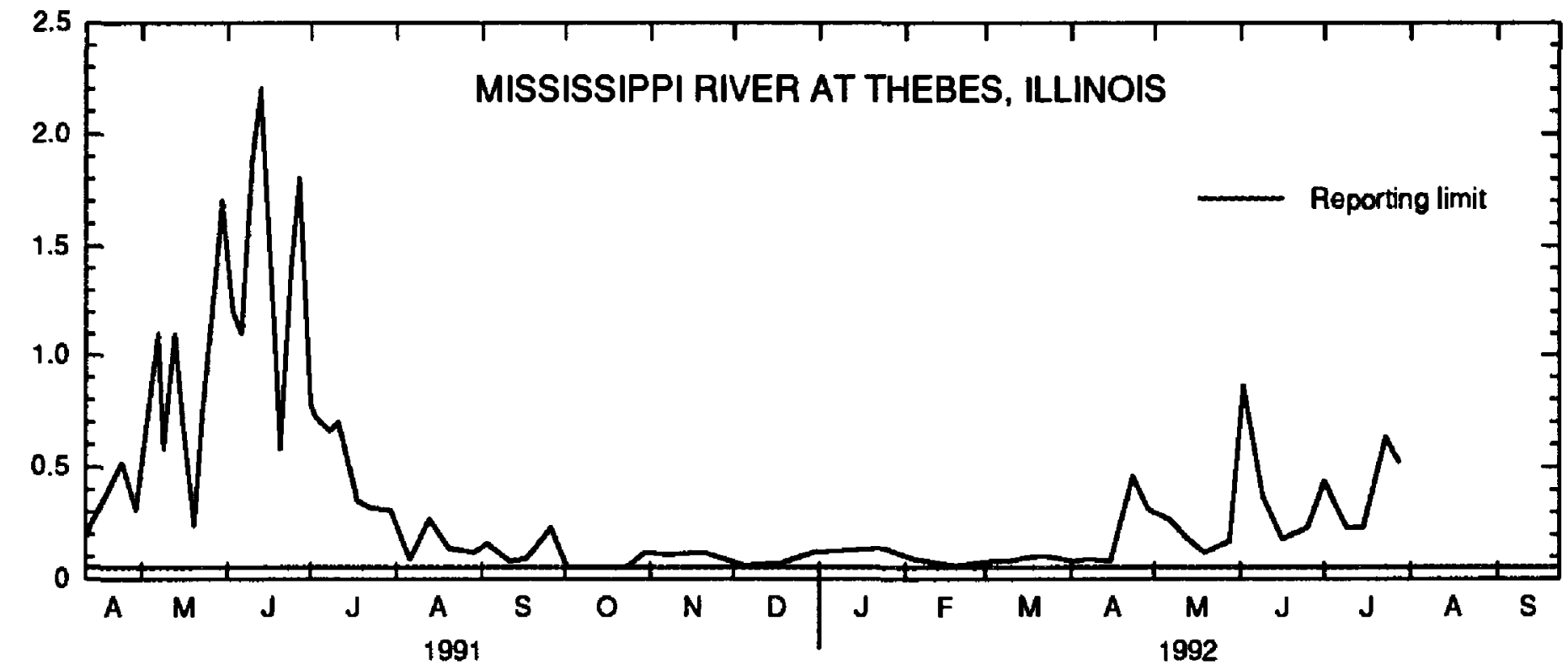

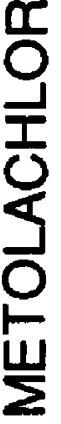

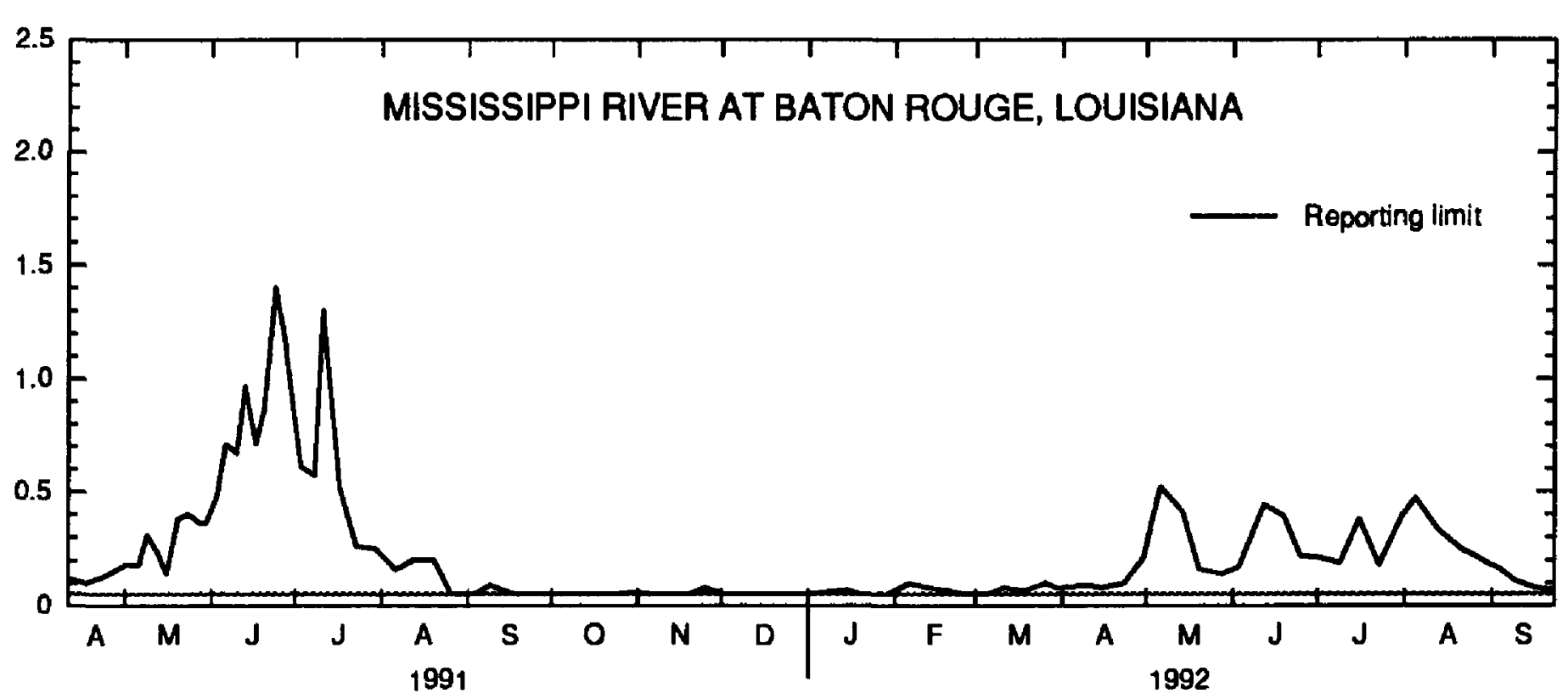

Figure 6.--Metolachlor concentrations at three Mississippi River sampling sites. 
The solid-phase extraction 1-liter (SPE1L) method was used to analyze for both herbicides and insecticides (S.D. Zaugg, U.S. Geological Survey, written commun., 1993). It is more sensitive than the SPE100mL method and more suitable for detecting low concentrations of pesticides. One-liter water samples are extracted in the laboratory on disposable $\mathrm{C}-18$ solid-phase extraction cartridges. Prior to extraction, three surrogate standards $\left(\mathrm{D}_{6}\right.$-Gamma- $\mathrm{HCH}$, terbuthylazine, and $\mathrm{D}_{10}$-Diazinon) are added to the sample to aid in determining the extraction efficiency and in interpreting the analytical results. The cartridges are then eluted with $2.5 \mathrm{~mL}$ of hexane-isopropanol (3:1). Then, $0.100 \mu \mathrm{L}$ of a toluene solvent containing three deuterated polyaromatic hydrocarbons internal standards are added to the eluent prior to nitrogen evaporation to $0.1 \mathrm{~mL}$. Two microliters of the final extract are then injected onto a gas chromatograph equipped with a capillary column to analyze for pesticides. Pesticides are identified and quantified with a mass spectrometer detector based on selected ion monitoring of the parent compound and two characteristic ions for each pesticide. Multipoint calibration curves are constructed for each pesticide using analytical reference standards representing a range in concentration of $0.004-2.0 \mu \mathrm{g} / \mathrm{L}$ as reported for the actual samples. Initially, SPE1L was used to determine 30 compounds but, by the end of the study, 46 compounds were identified. No samples from the Mississippi River at Baton Rouge, La., were analyzed using SPE1L. Precision and accuracy data for this method are shown in table 3 . Results from this procedure are shown in table 8 (at end of report). 
Table 3.-Precision and accuracy data from seven determinations of analytes by the solidphase extraction 1-liter method in surface water spiked at concentrations of 0.1 and 1.0 microgram per liter

[ $\mu \mathrm{g} / \mathrm{L}$, microgram per liter; $\%$, percent]

\begin{tabular}{|c|c|c|c|c|c|c|}
\hline Compound & $\begin{array}{c}\text { Mean } \\
\text { concen- } \\
\text { tration } \\
(\mu g / L)\end{array}$ & $\begin{array}{c}\text { Precision } \\
\text { (standard } \\
\text { deviation, } \\
\text { in } \mu g / L \text { ) }\end{array}$ & $\begin{array}{c}\text { Accuracy } \\
\text { (mean of } \\
\text { spiked } \\
\text { concen- } \\
\text { tration) } \\
(\%)\end{array}$ & $\begin{array}{c}\text { Mean } \\
\text { concen- } \\
\text { tration } \\
(\mu g / L)\end{array}$ & $\begin{array}{c}\text { Precision } \\
\text { (standard } \\
\text { deviatlon, } \\
\text { in } \mu g / L \text { ) }\end{array}$ & $\begin{array}{c}\text { Accuracy } \\
\text { (mean of } \\
\text { spiked } \\
\text { concen- } \\
\text { tration) } \\
(\%)\end{array}$ \\
\hline & \multicolumn{3}{|c|}{$0.1 \mu \mathrm{g} / \mathrm{L}$ spike } & \multicolumn{3}{|c|}{$1.0 \mu \mathrm{g} / \mathrm{L}$ spike } \\
\hline $\begin{array}{r}\text { Alachlor } \\
\text { (Lasso) }\end{array}$ & 0.095 & 0.006 & 95 & 0.827 & 0.036 & 83 \\
\hline Alpha-HCH & 0.077 & 0.005 & 77 & 0.654 & 0.016 & 65 \\
\hline Atrazine & 0.103 & 0.007 & 103 & 0.812 & 0.028 & 81 \\
\hline $\begin{array}{l}\text { Azinphos- } \\
\text { methyl }\end{array}$ & 0.042 & 0.006 & 42 & 0.233 & 0.024 & 23 \\
\hline Benfluralin & 0.060 & 0.006 & 60 & 0.619 & 0.058 & 62 \\
\hline Butylate & 0.085 & 0.010 & 85 & 0.853 & 0.023 & 85 \\
\hline Carbaryl & 0.190 & 0.032 & 190 & 0.927 & 0.039 & 93 \\
\hline Carbofuran & 0.119 & 0.006 & 119 & 0.925 & 0.031 & 93 \\
\hline $\begin{array}{l}\text { Chlorpyrifos } \\
\text { (Dursban) }\end{array}$ & 0.080 & 0.008 & 80 & 0.671 & 0.040 & 67 \\
\hline Cyanazine & 0.066 & 0.003 & 66 & 0.629 & 0.034 & 63 \\
\hline Dacthal & 0.087 & 0.007 & 87 & 0.821 & 0.035 & 82 \\
\hline DDE & 0.045 & 0.007 & 45 & 0.397 & 0.051 & 40 \\
\hline Diazinon & 0.130 & 0.009 & 130 & 0.825 & 0.027 & 83 \\
\hline Dieldrin & 0.062 & 0.005 & 62 & 0.577 & 0.045 & 58 \\
\hline $\begin{array}{l}\text { 2,6-Diethylana- } \\
\text { line }\end{array}$ & 0.067 & 0.006 & 67 & 0.738 & 0.018 & 74 \\
\hline Dimethoate & 0.034 & 0.006 & 34 & 0.066 & 0.009 & 7 \\
\hline Disulfoton & 0.141 & 0.005 & 141 & 0.746 & 0.020 & 75 \\
\hline $\begin{array}{c}\text { EPTAM } \\
\text { (EPTC) }\end{array}$ & 0.083 & 0.004 & 83 & 0.861 & 0.022 & 86 \\
\hline Ethalfluralin & 0.068 & 0.006 & 68 & 0.645 & 0.046 & 65 \\
\hline Ethoprop & 0.096 & 0.011 & 96 & 0.835 & 0.028 & 84 \\
\hline $\begin{array}{l}\text { Fonofos } \\
\text { (Dyfonate) }\end{array}$ & 0.073 & 0.005 & 73 & 0.738 & 0.019 & 74 \\
\hline Lindane & 0.101 & 0.005 & 101 & 0.785 & 0.022 & 79 \\
\hline Linuron & 0.037 & 0.002 & 37 & 0.257 & 0.023 & 26 \\
\hline
\end{tabular}


Table 3.--Precision and accuracy data from seven determinations of analytes by the solidphase extraction 1-liter method in surface water spiked at concentrations of 0.1 and 1.0 microgram per liter-Continued

\begin{tabular}{|c|c|c|c|c|c|c|}
\hline Compound & $\begin{array}{l}\text { Mean } \\
\text { concen- } \\
\text { tration } \\
(\mu g / L)\end{array}$ & $\begin{array}{c}\text { Precision } \\
\text { (standard } \\
\text { deviation, } \\
\text { in } \mu g / L \text { ) }\end{array}$ & $\begin{array}{c}\text { Accuracy } \\
\text { (mean of } \\
\text { spiked } \\
\text { concen- } \\
\text { tration) } \\
(\%)\end{array}$ & $\begin{array}{c}\text { Mean } \\
\text { concen- } \\
\text { tration } \\
(\mu \mathrm{g} / \mathrm{L})\end{array}$ & $\begin{array}{l}\text { Precision } \\
\text { (standard } \\
\text { deviation, } \\
\text { in } \mu g / L \text { ) }\end{array}$ & $\begin{array}{c}\text { Accuracy } \\
\text { (mean of } \\
\text { spiked } \\
\text { concen- } \\
\text { tration) } \\
(\%)\end{array}$ \\
\hline & \multicolumn{3}{|c|}{$0.1 \mu g / L$ spike } & \multicolumn{3}{|c|}{$1.0 \mu \mathrm{g} / \mathrm{L}$ spike } \\
\hline Malathion & 0.085 & 0.006 & 85 & 0.761 & 0.037 & 76 \\
\hline $\begin{array}{l}\text { Methyl- } \\
\text { parathion }\end{array}$ & 0.071 & 0.006 & 71 & 0.619 & 0.030 & 62 \\
\hline Metolachlor & 0.087 & 0.004 & 87 & 0.880 & 0.033 & 88 \\
\hline Metribuzin & 0.056 & 0.004 & 56 & 0.430 & 0.017 & 43 \\
\hline Molinate & 0.081 & 0.004 & 81 & 0.845 & 0.024 & 85 \\
\hline Napropamide & 0.079 & 0.004 & 79 & 0.803 & 0.010 & 80 \\
\hline Parathion & 0.068 & 0.006 & 68 & 0.680 & 0.032 & 68 \\
\hline Pebulate & 0.081 & 0.004 & 81 & 0.864 & 0.023 & 86 \\
\hline Pendimethalin & 0.064 & 0.004 & 64 & 0.647 & 0.054 & 65 \\
\hline $\begin{array}{r}\text { Permethrin } \\
\text { (Ambush) }\end{array}$ & 0.039 & 0.006 & 39 & 0.316 & 0.047 & 32 \\
\hline Phorate & 0.105 & 0.005 & 105 & 0.742 & 0.018 & 74 \\
\hline Prometon & 0.098 & 0.011 & 98 & 0.670 & 0.061 & 67 \\
\hline Pronamide & 0.120 & 0.010 & 120 & 1.221 & 0.040 & 122 \\
\hline Propachlor & 0.082 & 0.006 & 82 & 0.816 & 0.035 & 82 \\
\hline Propanil & 0.083 & 0.008 & 83 & 0.770 & 0.031 & 77 \\
\hline $\begin{array}{c}\text { Propargite } \\
\text { (Omite) }\end{array}$ & 0.056 & 0.005 & 56 & 0.566 & 0.067 & 57 \\
\hline Simazine & 0.080 & 0.005 & 80 & 0.679 & 0.028 & 68 \\
\hline Tebuthiuron & 0.090 & 0.009 & 90 & 0.773 & 0.060 & 77 \\
\hline Terbacil & 0.125 & 0.010 & 125 & 0.833 & 0.027 & 83 \\
\hline Terbufos & 0.105 & 0.004 & 105 & 0.755 & 0.025 & 76 \\
\hline Thiobencarb & 0.076 & 0.006 & 76 & 0.761 & 0.029 & 76 \\
\hline Triallate & 0.071 & 0.005 & 71 & 0.703 & 0.022 & 70 \\
\hline Trifluralin & 0.063 & 0.004 & 63 & 0.635 & 0.057 & 64 \\
\hline
\end{tabular}




\section{Liquld-Liquld Extraction}

Liquid-liquid extraction is a long-established procedure for the analysis of triazine and other nitrogen-containing compounds (Wershaw and others, 1987). The procedure is based on extraction of a 1-L sample with methylene chloride. Extracts are analyzed on a gas chromatograph equipped with dual nitrogen-phosphorus detectors. This procedure is slightly less sensitive than the two solid-phase extraction procedures but has a long and well-documented history of use. It was used in this study only for quality-control purposes in conjunction with the two SPE procedures described previously.

\section{Nutrients}

Dissolved nitrite plus nitrate, nitrite, ammonia, and orthophosphate were determined by an automated colorimetric procedure (Fishman and Friedman, 1989). Dissolved nitrate was calculated as the difference between determinations for nitrite plus nitrate and nitrite. Total organic plus ammonia nitrogen and total phosphorus were determined on unfiltered water samples following a digestion procedure in which organic and particulate forms of nitrogen and phosphorus were converted to dissolved inorganic forms. After digestion, the samples were analyzed by an automated colorimetric procedure (Fishman and Friedman, 1989). Precision and accuracy data for this method are shown in table 4.

Analytical results for nutrients are listed in table 9 (at back of report). A time-series plot of nitrate concentrations for the sampling sites on the Mississippi River at Clinton, Iowa, Thebes, Ill., and Baton Rouge, La., is shown in figure 7. 
Table 4.-Precision and accuracy data for measurements of physical propertles and nutrients, April 1991-September 1992

[MPV, most probable value based on analyses by multiple laboratories; $N$, number of measurements; $\mu \mathrm{S} / \mathrm{cm}$, microsiemens per centimeter at 25 degrees Celsius; diss, dissolved; $\mathrm{mg} / \mathrm{L}$, milligram per liter; --, no data]

\begin{tabular}{|c|c|c|c|c|c|c|c|c|}
\hline $\begin{array}{l}\text { Property or } \\
\text { nutrient }\end{array}$ & MPV & $\mathbf{N}$ & $\begin{array}{l}\text { Precision } \\
\text { (standard } \\
\text { deviation) }\end{array}$ & $\begin{array}{l}\text { Accuracy } \\
\text { (mean of } \\
\text { measured } \\
\text { concen- } \\
\text { tration) }\end{array}$ & MPV & $\mathbf{N}$ & $\begin{array}{l}\text { Precision } \\
\text { (standard } \\
\text { deviation) }\end{array}$ & $\begin{array}{l}\text { Accuracy } \\
\text { (mean of } \\
\text { measured } \\
\text { concen- } \\
\text { tration) }\end{array}$ \\
\hline & \multicolumn{4}{|c|}{ Sample 1} & \multicolumn{4}{|c|}{ Sample 2} \\
\hline $\begin{array}{l}\text { Specific conduc- } \\
\text { tance }^{1}, \text { in } \\
\mu S / \mathrm{cm}\end{array}$ & -- & 73 & 6.57 & 472.3 & -- & 67 & 14.6 & 807.2 \\
\hline $\mathrm{pH}^{1}$, in units & -- & 70 & 0.03 & 7.29 & - & 80 & 0.04 & 7.90 \\
\hline $\begin{array}{l}\text { Ammonia } \\
\text { nitrogen, diss, } \\
\text { in } \mathrm{mg} / \mathrm{L}\end{array}$ & 0.05 & 96 & 0.01 & 0.05 & 0.21 & 17 & 0.03 & 0.22 \\
\hline $\begin{array}{l}\text { Nitrite plus } \\
\text { nitrate nitro- } \\
\text { gen, diss, } \\
\text { in } \mathrm{mg} / \mathrm{L}\end{array}$ & 0.36 & 22 & 0.03 & 0.33 & 1.50 & 16 & 0.08 & 1.42 \\
\hline $\begin{array}{l}\text { Organic plus } \\
\text { ammonia nitro- } \\
\text { gen, total, } \\
\text { in } \mathrm{mg} / \mathrm{L}\end{array}$ & 0.79 & 85 & 0.19 & 0.83 & 1.39 & 78 & 0.10 & 1.40 \\
\hline $\begin{array}{l}\text { Orthophosphate, } \\
\text { diss, in } \mathrm{mg} / \mathrm{L}\end{array}$ & 0.09 & 96 & 0.01 & 0.09 & 0.88 & 73 & 0.02 & 0.90 \\
\hline $\begin{array}{l}\text { Total phospho- } \\
\text { rus, in } \mathrm{mg} / \mathrm{L}\end{array}$ & 0.19 & 22 & 0.02 & 0.17 & 1.60 & 71 & 0.12 & 1.58 \\
\hline
\end{tabular}

${ }^{1}$ From U.S. Geological Survey field proficiency, quality-assurance program (D.L. Stanley, written commun., May 14, 1992). 

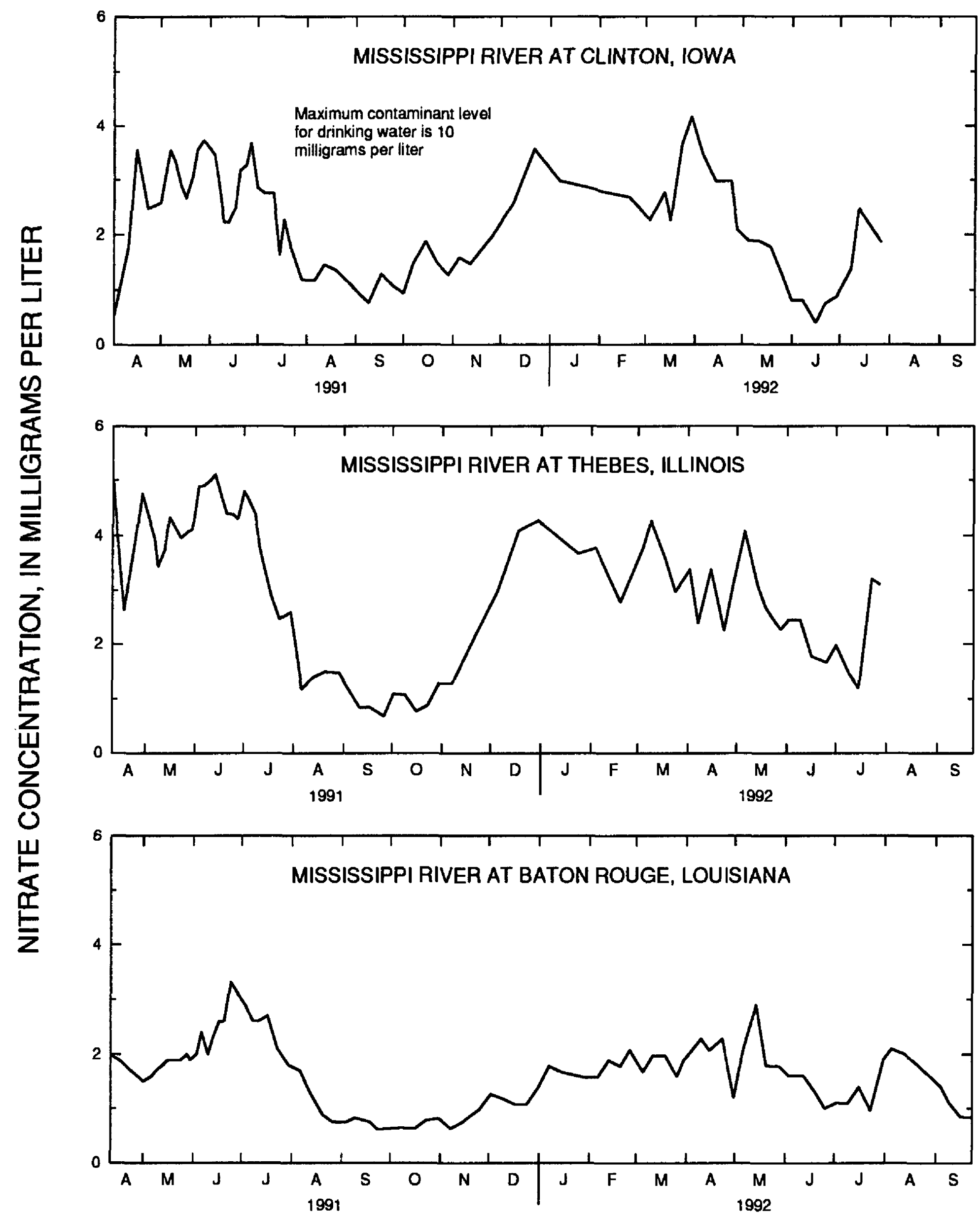

Figure 7.--Nitrate concentrations at three Mississippi River sampling sites. 


\section{Water Dlscharge}

At all sites except Baton Rouge, where water-discharge data are provided by the COE, stage was recorded continuously by the USGS. Stage measurements were calibrated by comparison to permanent reference marks. Water discharge was calculated from stage using stage-discharge relations (Kennedy, 1983, p. 30-32). From recordings of stage, daily mean values of water discharge were calculated and permanently stored in the USGS National Water Data Storage and Retrieval System (WATSTORE). Water discharge normally was measured with current meters using techniques described by Buchanan and Somers (1969). Water-discharge measurements were used to confirm or adjust a rating for converting stage to water discharge. Daily mean water-discharge values for the day that samples were collected are shown in table 9 (at back of report). Water-discharge hydrographs for the Mississippi River at Clinton, Iowa, Thebes, Ill., and Baton Rouge, La., are shown in figures 8-10. Water-discharge hydrographs at the five sites on tributaries are shown in figures 11-15.

Water-discharge data provided by the COE for Baton Rouge are for Tarbert Landing, La., about $129 \mathrm{~km}$ upstream from Baton Rouge. The COE also provided water-discharge data for Mississippi River water that is diverted into the Atchafalaya River upstream from Tarbert Landing (fig. 1). The sum of the water discharge at Tarbert Landing and the Atchafalaya diversion closely represents the total water discharge from the Mississippi River Basin upstream from Baton Rouge.

Water-discharge data for White River at Hazleton, Ind., are from White River at Petersburg, Ind., station 03374000 . This site is approximately $21.4 \mathrm{~km}$ upstream from Hazleton. Comparison of simultaneous water-discharge measurements between the two sites indicates that there is a negligible increase in water discharge of the White River between Petersburg and Hazleton (J.D. Martin, U.S. Geological Survey, Indianapolis, Ind., oral commun., 1992).

Water-discharge data for the Ohio River at Dam 53 near Grand Chain, Ill., are from Ohio River at Metropolis, Ill., station 03611500. This site is located $29 \mathrm{~km}$ upstream from Grand Chain, Ill. Discharges at both sites are nearly equivalent as tributary inflow is negligible.

\section{Physical Properties}

Specific conductance, $\mathrm{pH}$, and water temperature were measured at the time samples were collected for analysis.

Specific conductance and $\mathrm{pH}$ were measured using either a single or a multiple-parameter meter. These meters were calibrated before each use with standard conductance-reference and $\mathrm{pH}$-buffer solutions. Temperature values were obtained using a mercury thermometer. The temperature measurements are estimated to be accurate within \pm 0.5 degree Celsius. These data are shown in table 9 (at back of report). A time-series plot of specific conductance in the Mississippi River at Clinton, Iowa, Thebes Ill., and Baton Rouge, La., is shown in figure 16.

Suspended-sediment concentrations were determined at USGS laboratories in Rolla, Mo., Iowa City, Iowa, or Louisville, Ky., using standard filtration and evaporation methods (Guy, 1969; Matthes and others, 1992). Based on sediment reference samples, the mean accuracy of suspended-sediment analyses is estimated to be about 92 percent of known values at low concentrations (less than 15 milligrams of sediment in sample) and about 96 percent of known values at intermediate concentrations (150-300 milligrams sediment in sample). However, estimates of sediment-concentration sampling error are not available. Suspended-sediment data are listed in table 9 (at back of report). 


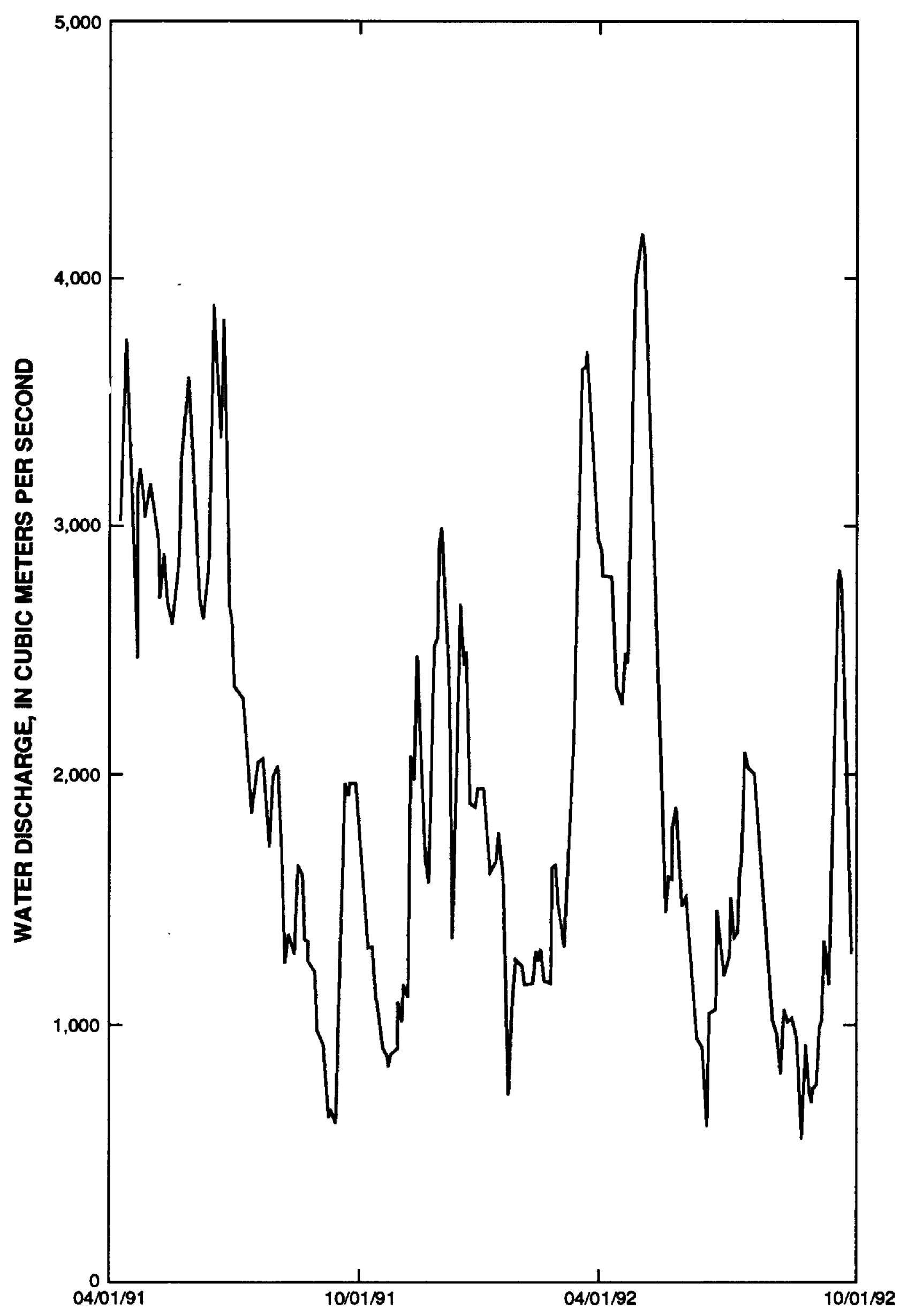

Figure 8.--Water-discharge hydrograph for the Mississippi River at Clinton, lowa. 


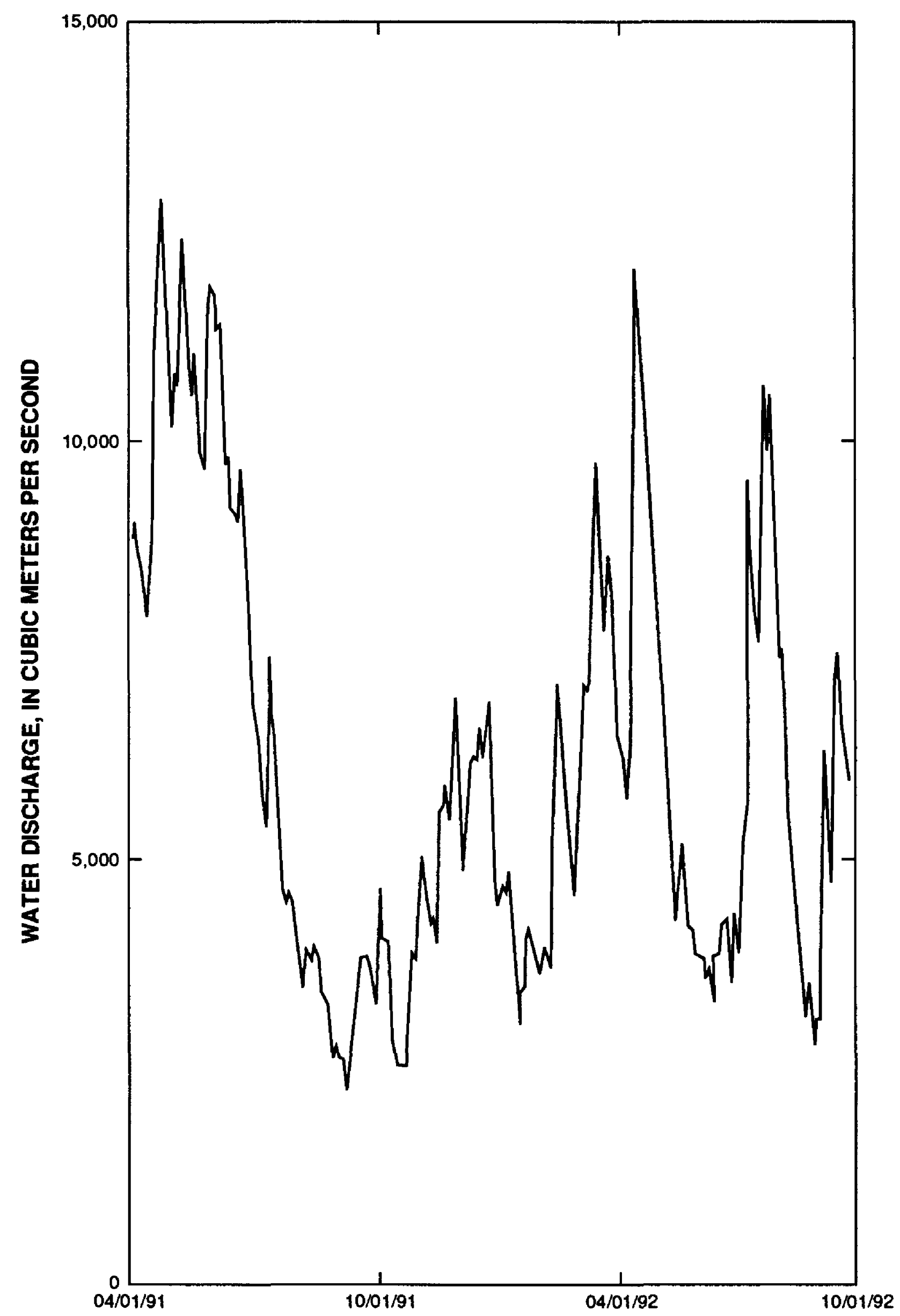

Figure 9.--Water-discharge hydrograph for the Mississippi River at Thebes, Ill. 


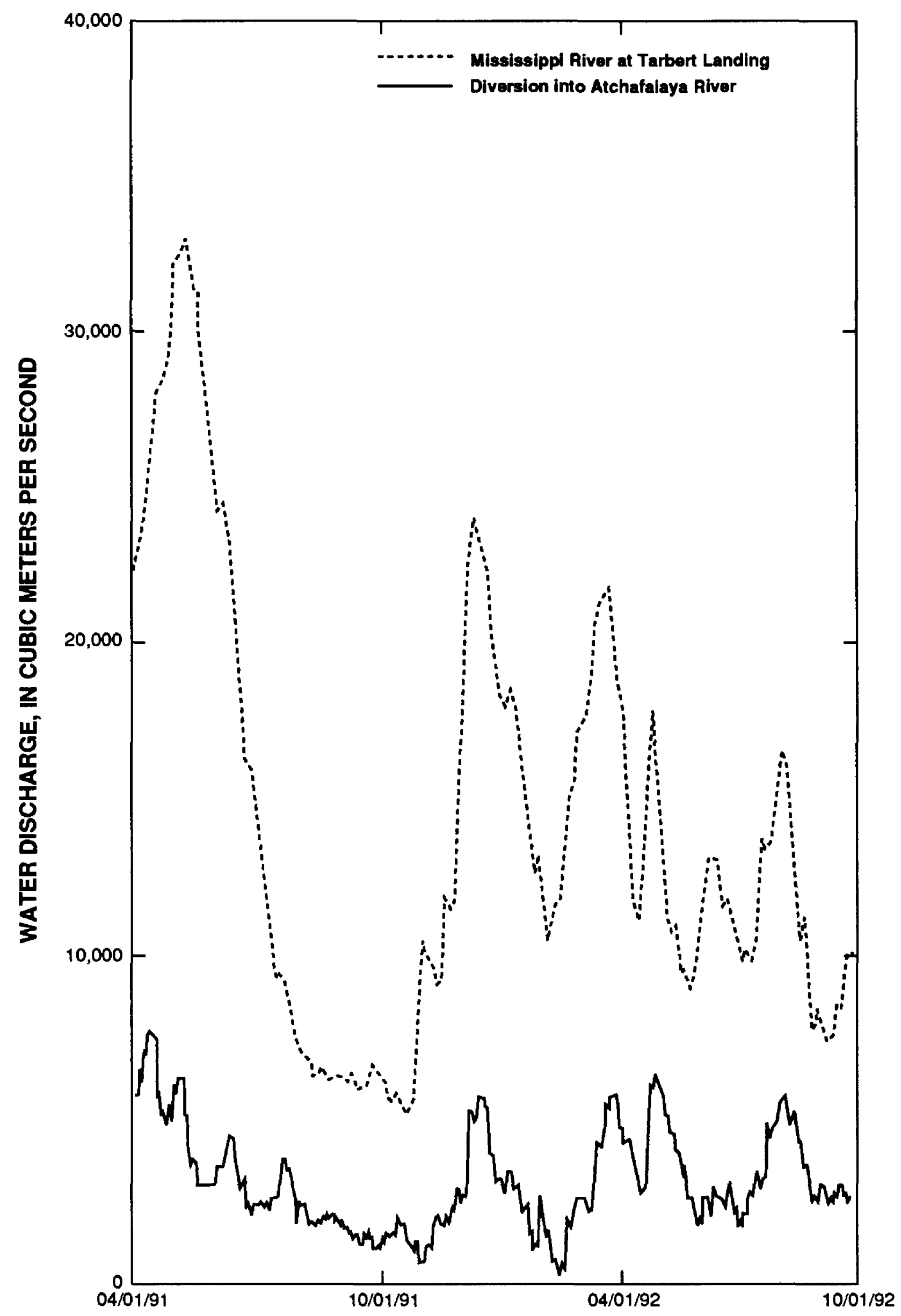

Figure 10.-Water-discharge hydrograph for the Mississippi River at Tarbert Landing, La., and for the Mississippi River diversions into the Atchafalaya River. 


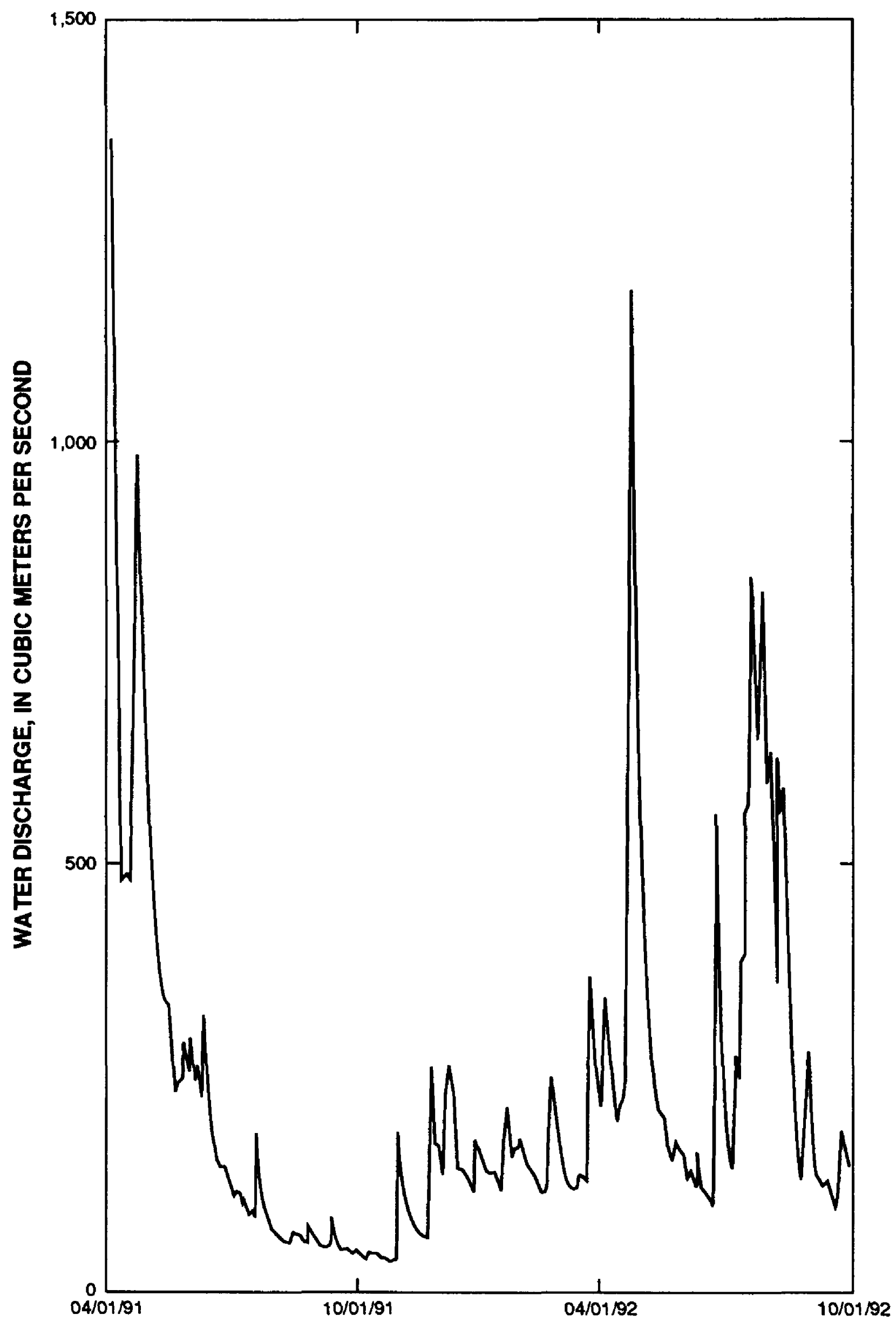

Figure 11.--Water-discharge hydrograph for the White River at Petersburg, Ind. 


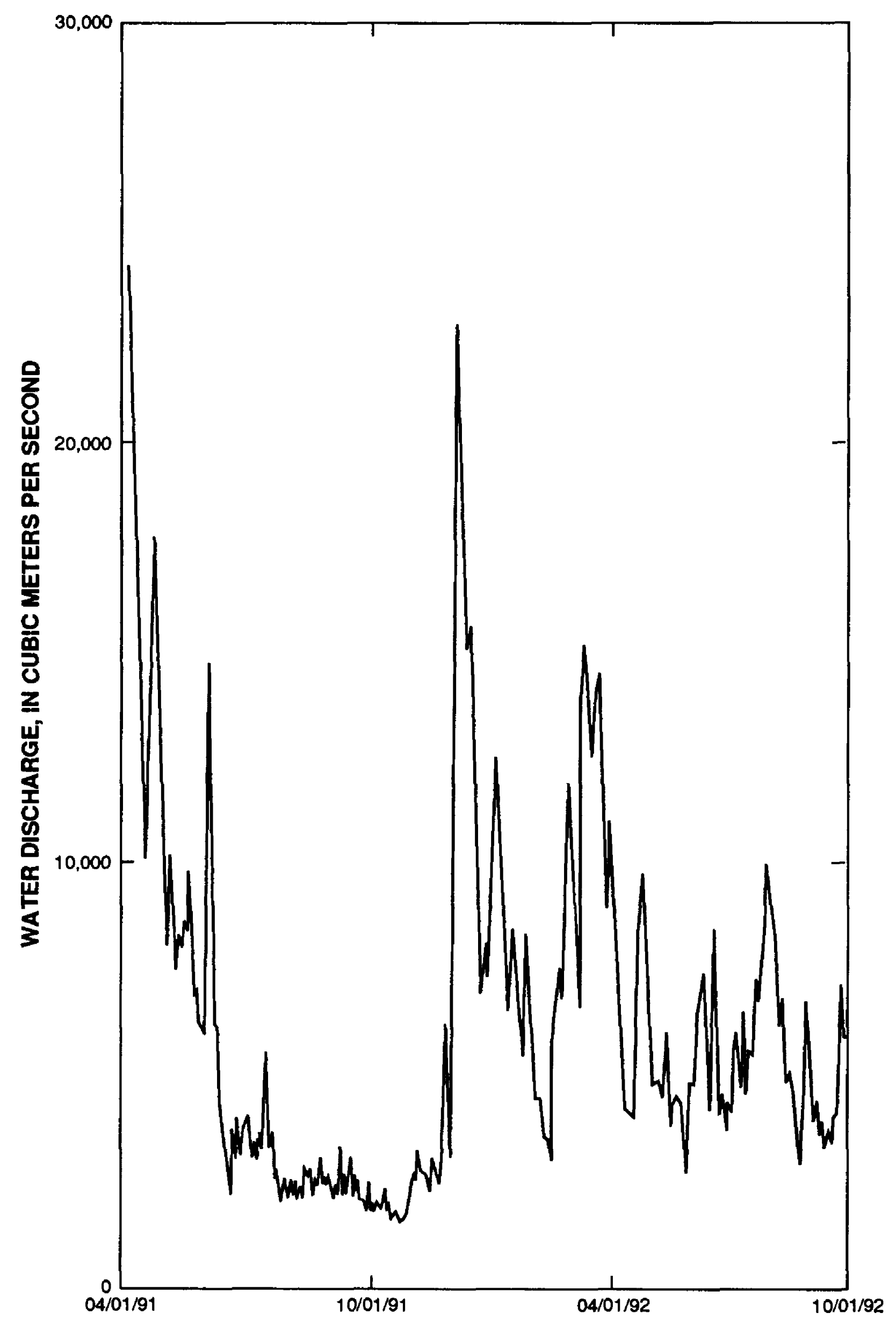

Figure 12.-Water-discharge hydrograph for the Ohio River at Metropolis, Ill. 


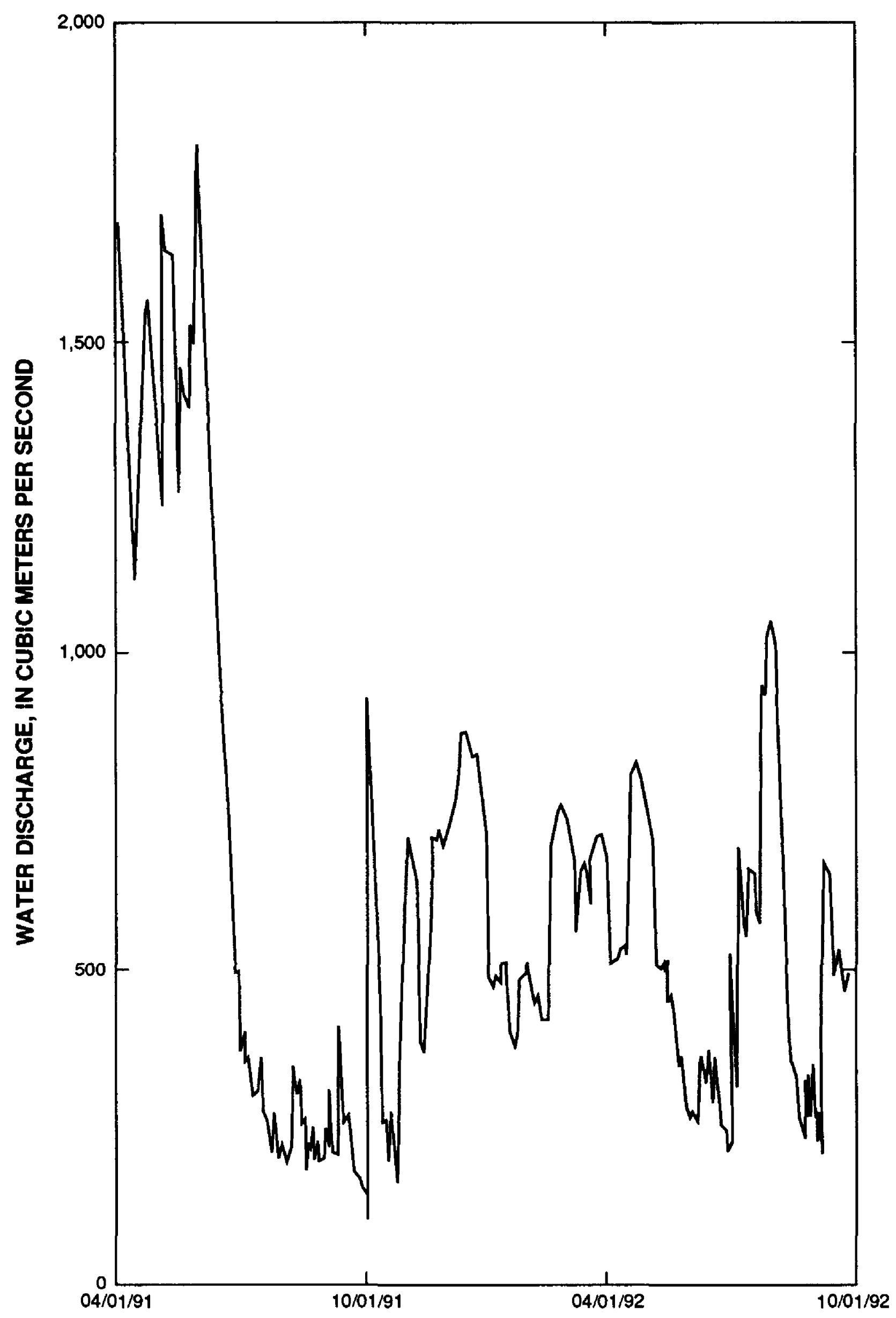

Figure 13.--Water-discharge hydrograph for the Illinois River at Valley City, Ill. 


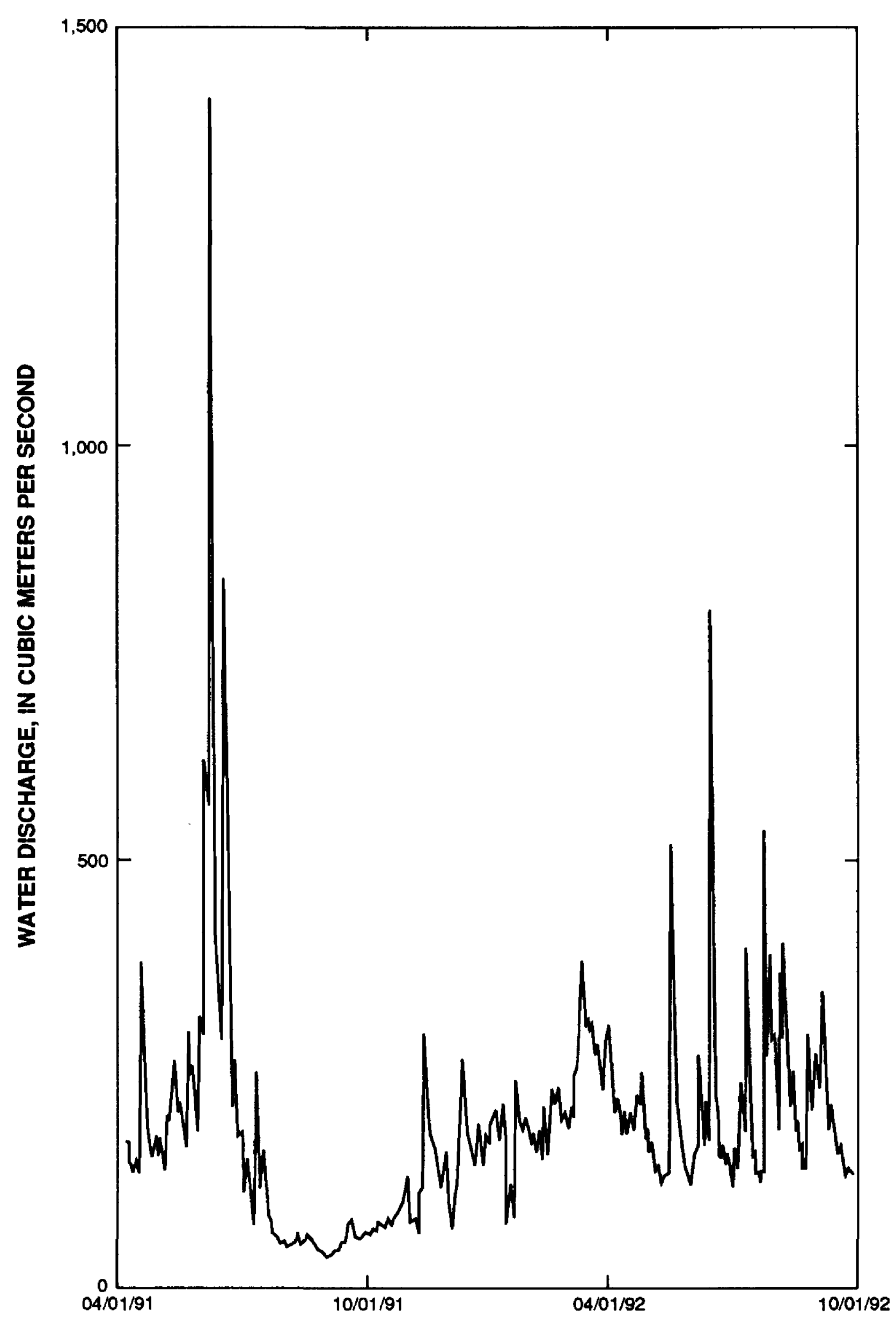

Figure 14.--Water-discharge hydrograph for the Platte River at Louisville, Nebr. 


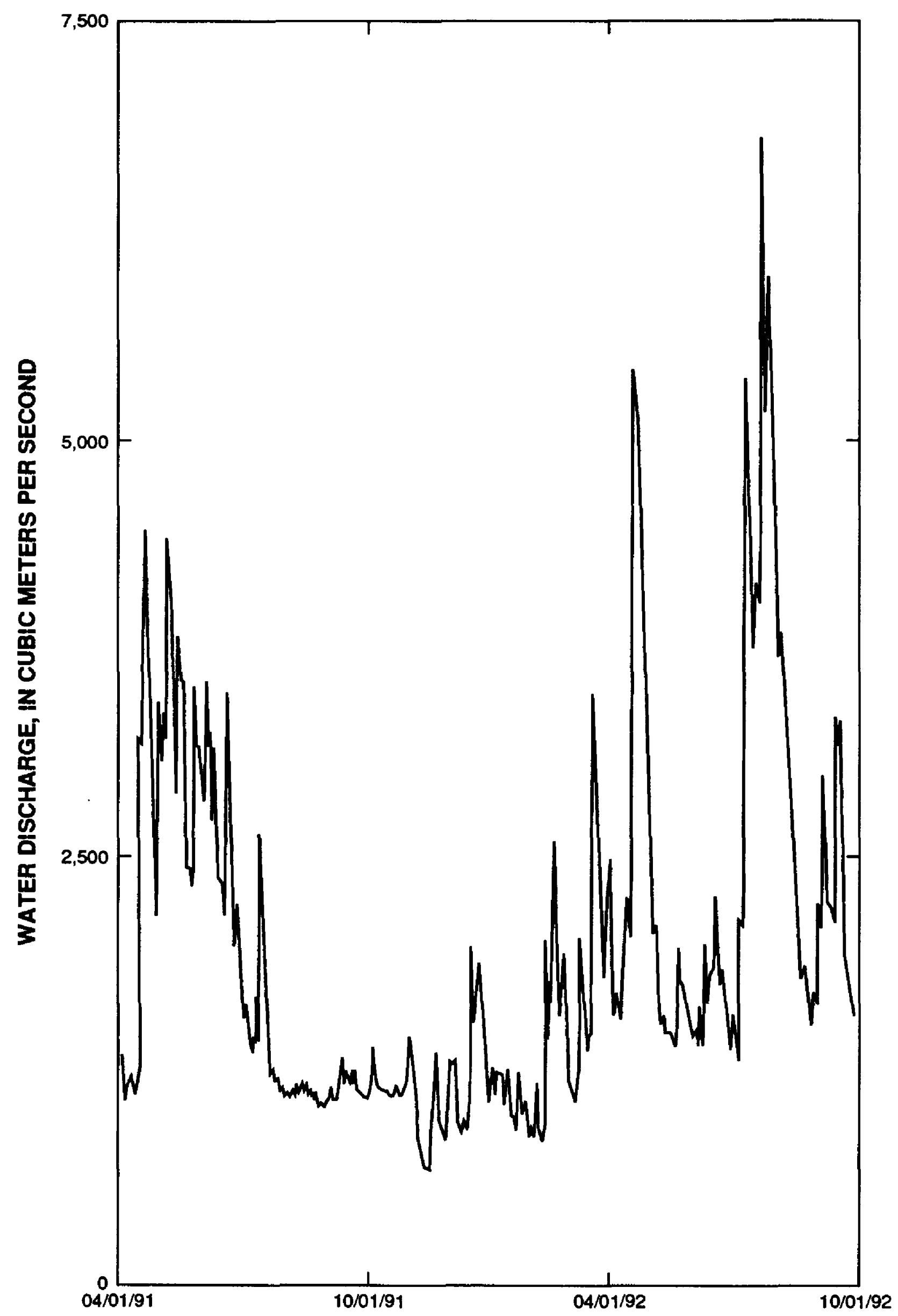

Figure 15.--Water-discharge hydrograph for the Missouri River at Hermann, Mo. 

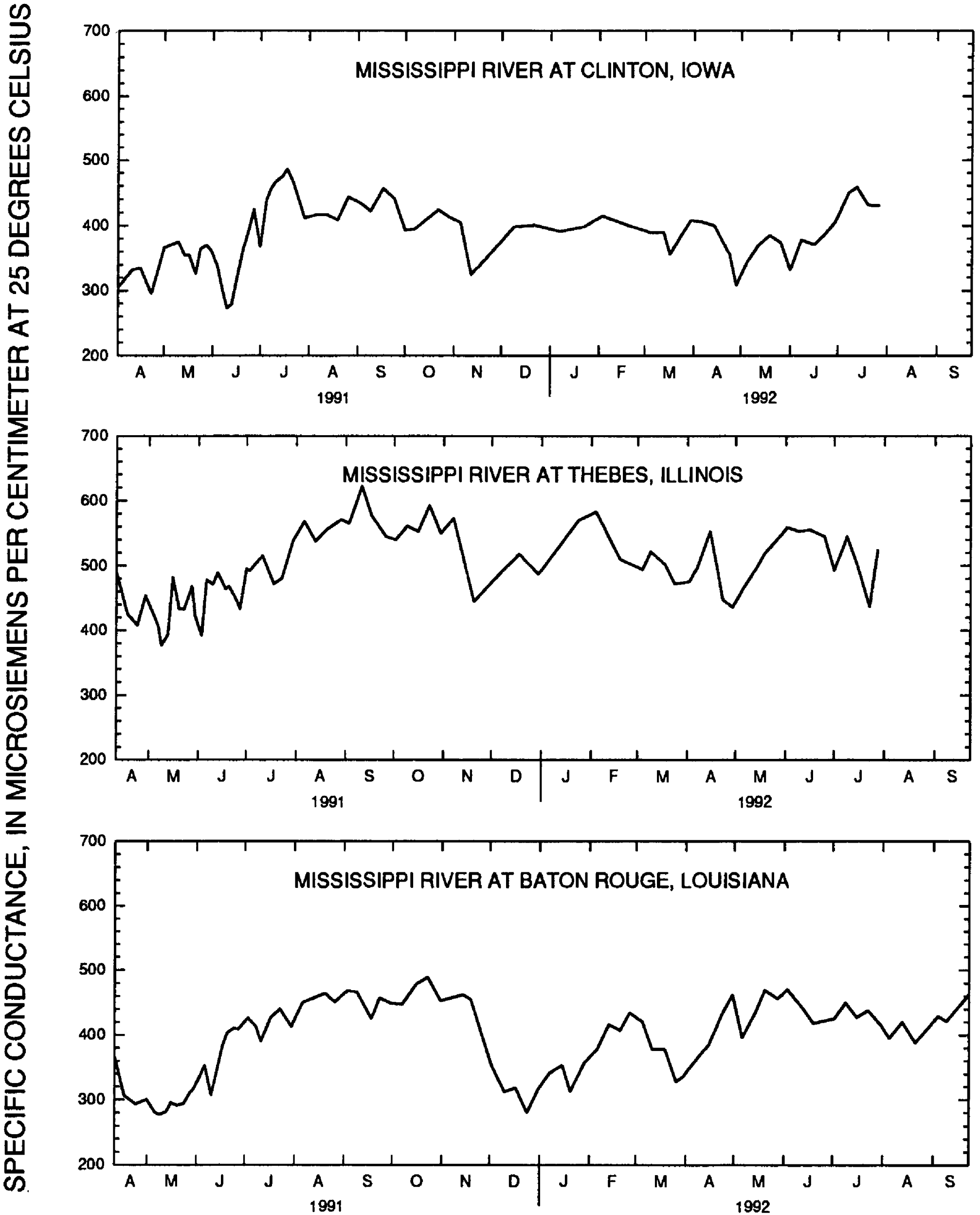

Figure 16.--Specific conductance at three Mississippi River sampling sites. 


\section{QUALITY-ASSURANCE PROCEDURES AND RESULTS}

Quality-assurance procedures for this study had two components: field quality assurance implemented by study personnel and the NWQL's internal quality-assurance program. Field quality-assurance procedures included the submission of field-equipment blanks, blind spikes, and split samples to be analyzed for the constituents of interest. Laboratory quality-assurance procedures included the determination of surrogate compound recoveries in each sample, laboratory reagent-water blank, laboratory reagent-water spikes, and an assessment of the instrument system. A summary of the quality-assurance procedures, blanks, spikes, and splits for April, May, and June 1991 is given by Goolsby and others (1991a).

\section{Field Quality-Assurance Procedures}

Before collection of each sample, all sampling equipment was washed with a phosphate-free laboratory detergent, rinsed with tap water and distilled or deionized water, rinsed with a small amount of methanol, and allowed to air dry. At the sampling site, all equipment (collection container, compositing container, cone splitter, pump mechanism, and filter) was rinsed with water from the stream. Glass-fiber filters and glass sample containers were baked at about $350^{\circ} \mathrm{C}$ to remove organic material.

A field-equipment blank for pesticides was obtained with about every tenth sample. The blank consists of organic-free water that was processed through all of the sampling and filtration equipment. The blank is analyzed for all pesticides of interest. There were no pesticides detected above the reporting limits in any of the 40 field-equipment blanks analyzed by SPE100mL. Of the 28 field-equipment blanks analyzed by SPE1L, only one had a concentration above the reporting limit. This blank was processed on June 18, 1991, and had an atrazine concentration of $0.013 \mu \mathrm{g} / \mathrm{L}$ and a metolachlor concentration of $0.007 \mu \mathrm{g} / \mathrm{L}$. In this instance, the concentrations probably were caused by laboratory processing of the blank following the processing of an ambient sample with high pesticide concentrations (M.W. Sandstrom, U.S. Geological Survey, National Water Quality Laboratory, Arvada, Colo., oral commun., 1992). Sample-collection and processing procedures used in the field were periodically reviewed for conformance with protocols established for the study.

Nine samples were analyzed by both SPE methods and by the LLE method for comparison purposes. The results are summarized in table 5.

A total of 12 blind-spike samples (spiked-reagent water sent to the NWQL disguised as a routine sample) were sent to the NWQL during May, June, and July 1991 to be analyzed by SPE100mL. The spiked concentration, the concentration determined by the NWQL, and the percent recovery [(NWQL concentration/spiked concentration) $\times 100$ ] are shown in table 6 for atrazine, alachlor, cyanazine, metolachlor, metribuzin, simazine, and desethylatrazine.

For quality-assurance purposes, monthly samples were collected from the Mississippi River near St. Francisville, La., using the depth-integrating and compositing procedures outlined earlier in this report.

These samples can be compared with those collected from the Mississippi River at Baton Rouge, where the samples are collected at the end of a 46-m pier extending out into the Mississippi River. St. Francisville is about $56 \mathrm{~km}$ upstream from the Baton Rouge site. These data are shown in table 7.

On July 23, 1991, five samples were collected at equally spaced intervals across the Mississippi River at Baton Rouge (starting at 1316 hours near the left bank and ending at 1320 hours near the right bank) concurrently with the sample collected from the end of the pier (1315 hours). These samples were collected to verify that the river water is well mixed both laterally and vertically at this site. These data are shown in table 7. 
Tabie 5.-Resuits of spiit samples anaiyzed by the two soild-phase extraction methods and the liquid-liquid extraction method

[All results in micrograms per liter; SPE100mL, solid-phase extraction 100 milliliter; SPE1L, solid-phase extraction 1 liter; LLE, liquid-liquid extraction; <, less than]

\begin{tabular}{|c|c|c|c|c|c|c|}
\hline Date & Method & Alachior & Atrazine & Cyanazine & Metolachlor & Simazine \\
\hline \multicolumn{7}{|c|}{ Iliinois River at Vailey City, iliinois } \\
\hline \multirow[t]{3}{*}{$5 / 22 / 91$} & SPE100mL ${ }^{1}$ & 0.92 & 3.4 & 2.9 & 2.1 & 0.05 \\
\hline & SPE1L & 1.9 & 8.7 & 6.6 & 4.6 & 0.13 \\
\hline & LLE $^{2}$ & 1.3 & 7.0 & 6.0 & 3.0 & 0.1 \\
\hline \multirow[t]{3}{*}{$6 / 14 / 91$} & SPE100mL & 0.54 & 3.8 & 2.0 & 1.4 & 0.12 \\
\hline & SPE1L & 0.54 & 4.1 & 2.3 & 1.5 & 0.12 \\
\hline & LLE & 0.6 & 4.3 & 2.6 & 1.5 & 0.16 \\
\hline \multirow[t]{3}{*}{$6 / 24 / 91$} & SPE100mL & 0.17 & 2.4 & 1.4 & 0.63 & 0.09 \\
\hline & SPE1L & 0.21 & 2.7 & 1.2 & 0.78 & 0.09 \\
\hline & LLE $^{2}$ & 0.18 & 2.8 & 2.0 & 0.63 & 0.10 \\
\hline \multirow[t]{3}{*}{$7 / 11 / 91$} & SPE100mL & 0.05 & 0.70 & 0.3 & 0.12 & 0.06 \\
\hline & SPE1L & 0.05 & 0.75 & 0.24 & 0.17 & 0.07 \\
\hline & $\operatorname{LLE}^{1,2}$ & $<0.1$ & 0.34 & 0.2 & 0.1 & 0.02 \\
\hline \multicolumn{7}{|c|}{ Piatte River at Louisviilie, Nebraska } \\
\hline \multirow[t]{3}{*}{$5 / 21 / 91$} & SPE100 $\mathrm{mL}^{1}$ & 3.6 & 8.3 & 6.8 & 3.1 & $<0.05$ \\
\hline & SPE1L & 6.5 & 16.9 & 9.0 & 5.2 & 0.08 \\
\hline & LLE $^{2}$ & 3.3 & 12.8 & 7.6 & 3.3 & 0.06 \\
\hline \multirow[t]{3}{*}{$5 / 29 / 91$} & SPE100mL & 1.4 & 6.5 & 1.7 & 2.2 & 0.07 \\
\hline & SPE1L & 0.77 & 4.2 & 0.79 & 1.4 & 0.04 \\
\hline & LLE $^{2}$ & 1.1 & 8.1 & 1.2 & 1.6 & 0.07 \\
\hline \multirow[t]{3}{*}{$6 / 4 / 91$} & SPE100mL & 1.7 & 5.7 & 3.7 & 1.9 & 0.06 \\
\hline & SPE1L & 2.1 & 11.0 & 13.0 & 2.6 & 0.10 \\
\hline & LLE $^{2}$ & 1.6 & 9.0 & 8.7 & 1.5 & 0.09 \\
\hline \multirow[t]{3}{*}{$6 / 7 / 91$} & SPE100mL & 3.2 & 10.0 & 7.3 & 2.0 & 0.06 \\
\hline & SPEIL & 3.7 & 13.9 & 20.3 & 2.4 & 0.10 \\
\hline & LLE & 3.9 & 13.2 & 10.9 & 1.8 & 0.1 \\
\hline \multirow[t]{3}{*}{$7 / 8 / 91$} & SPE100mL & $<0.05$ & 0.77 & 0.4 & 0.08 & $<0.05$ \\
\hline & SPE1L & 0.04 & 1.2 & 0.47 & 0.16 & 0.02 \\
\hline & LLE $^{2}$ & 0.03 & 0.74 & 0.6 & 0.12 & 0.02 \\
\hline
\end{tabular}

${ }^{1}$ Sample had a low surrogate recovery.

${ }^{2}$ Values below reporting limits given in table 1 are estimates. 


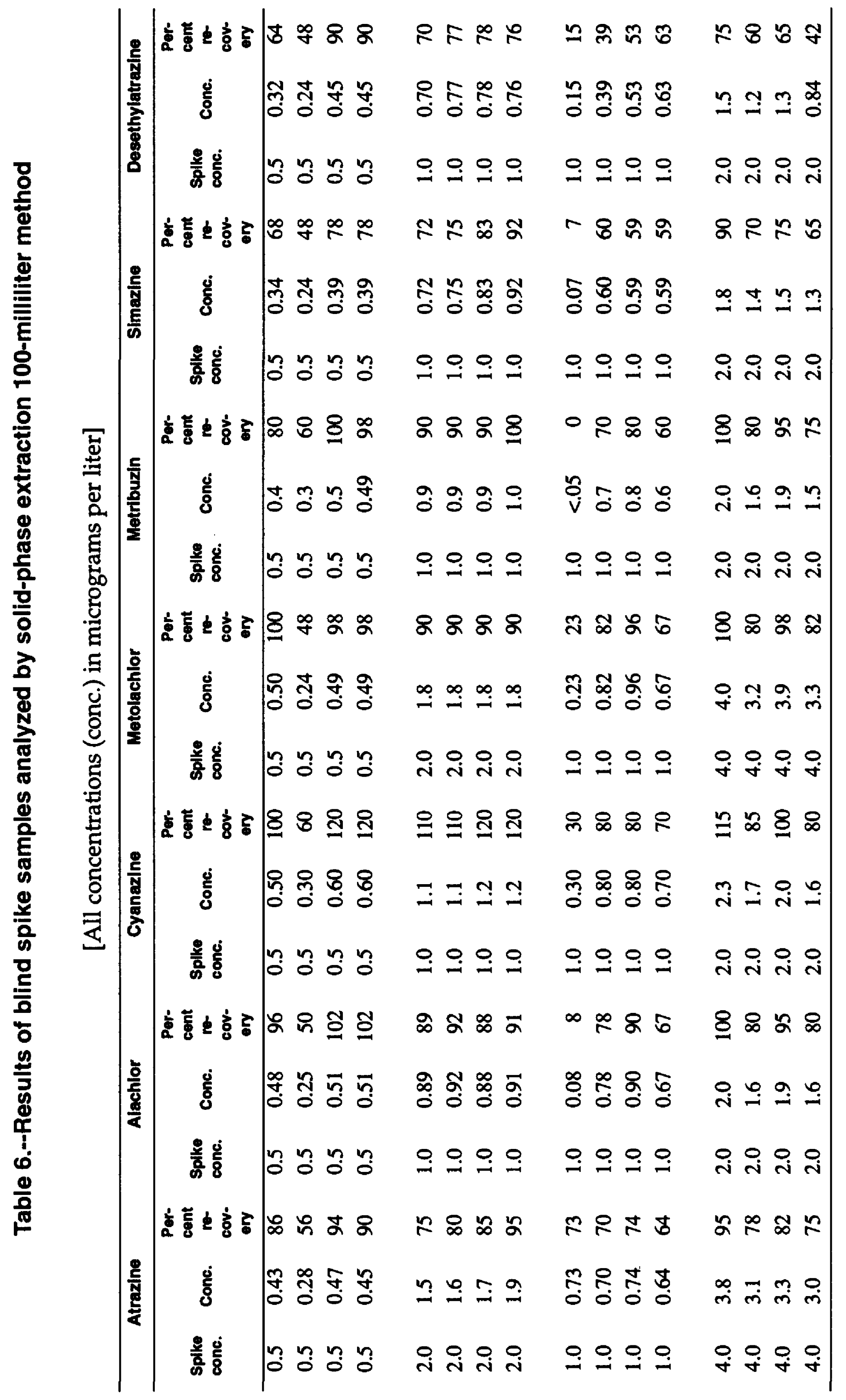




\section{Laboratory Quality-Assurance Procedures}

The routine quality-assurance procedures used in the NWQL included the analysis of laboratory reagent blanks (LRB), determination of surrogate compound recoveries in each sample, blank and laboratory-spiked samples, and the assessment of the instrument system. These procedures are discussed below.

Before processing any samples, the analyst determined if there were any glassware or reagent interferences. Each time a set of samples was extracted or the reagents were changed, an LRB was analyzed. If the LRB produced a peak that would prevent the determination of a compound, the source of contamination was determined and eliminated.

A surrogate was added to each sample to monitor for extraction efficiency. When the surrogate recovery from a sample or blank was determined to be low according to accepted statistical methods of establishing control limits, the following were checked: (1) Calculations to locate possible errors, (2) spiking solutions for degradation, (3) possibility of contamination, and (4) instrument performance. If those checks did not reveal the cause of the contamination, the extract was reanalyzed. If a blank extract reanalysis failed the control-limit criteria, the problem was corrected before continuing. If sample extract reanalysis met the surrogate recovery criteria, the data were reported. If the sample extract continued to fail the recovery criteria, project personnel were notified.

At least one laboratory spiked sample, per sample set, was analyzed for the pesticides of interest (all samples extracted within a 24-hour period). Laboratory-spike recovery data for the principal pesticides detected in this study are shown in figures 17 and 18 . The distribution of percent recovery values are shown in box plots.

If the recovery value of any compound fell outside the control-limit criteria, the source of the problem was identified and resolved before continuing analyses. Initially, because the method was new, the control-limit criteria for the SPE100mL method were set at 30-percent recovery. The current control limits were established after 1991 and are shown in figure 17. These criteria represent the minimum and maximum percent recoveries acceptable for a given analysis. Control-limit criteria for the SPE1L method had not been established at the date of this report (January 1993).

Instrument performance was checked daily. First, a calibration check was done on the mass spectrometer to ensure there were no air leaks in the system and to ensure that the system was tuned to the correct ion mass range. Prior to the analysis of each sample set and every 10 samples thereafter, a standard solution (or solutions) was analyzed containing the target compounds and evaluated to ensure system performance complied with established criteria. 


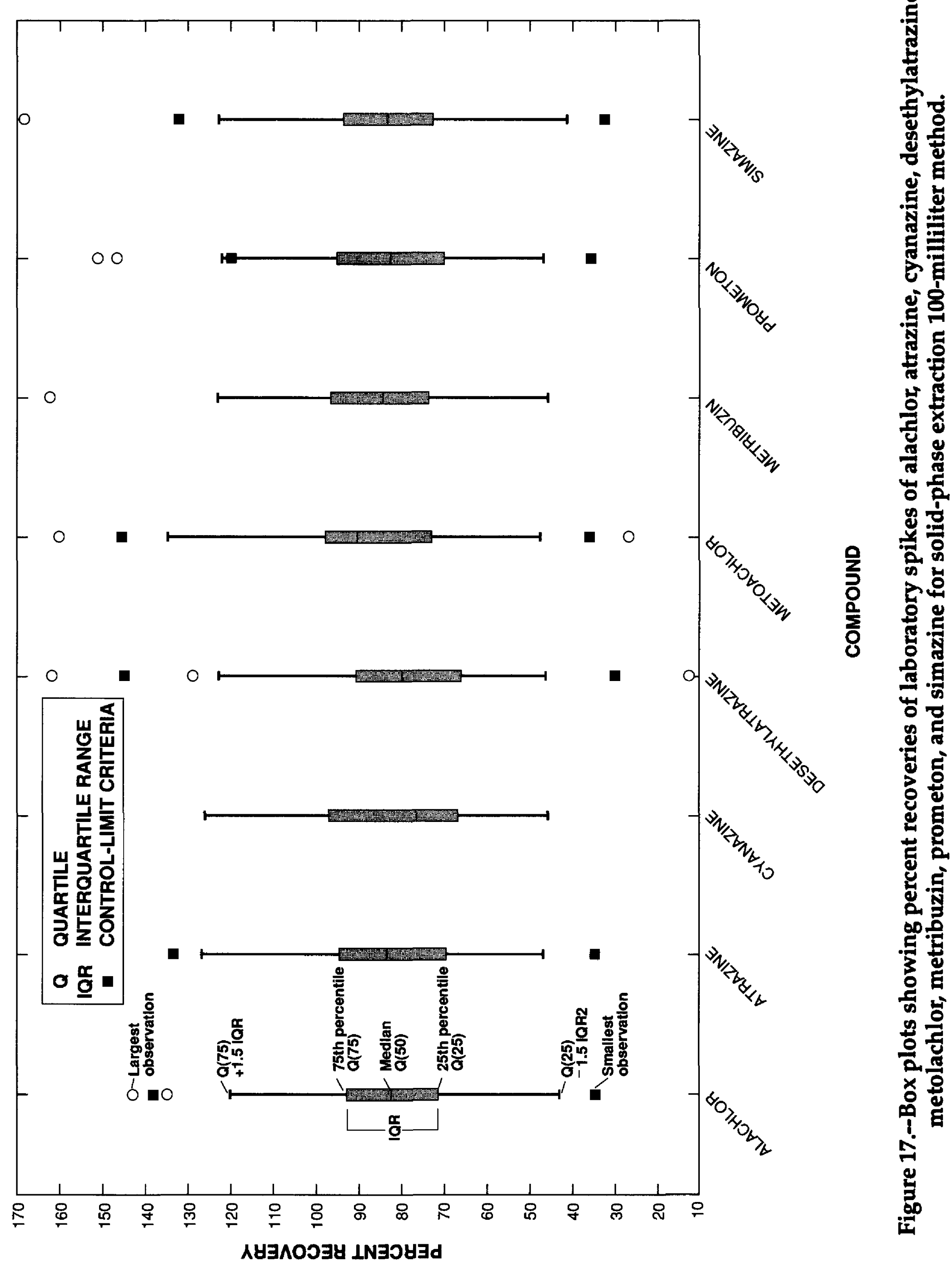




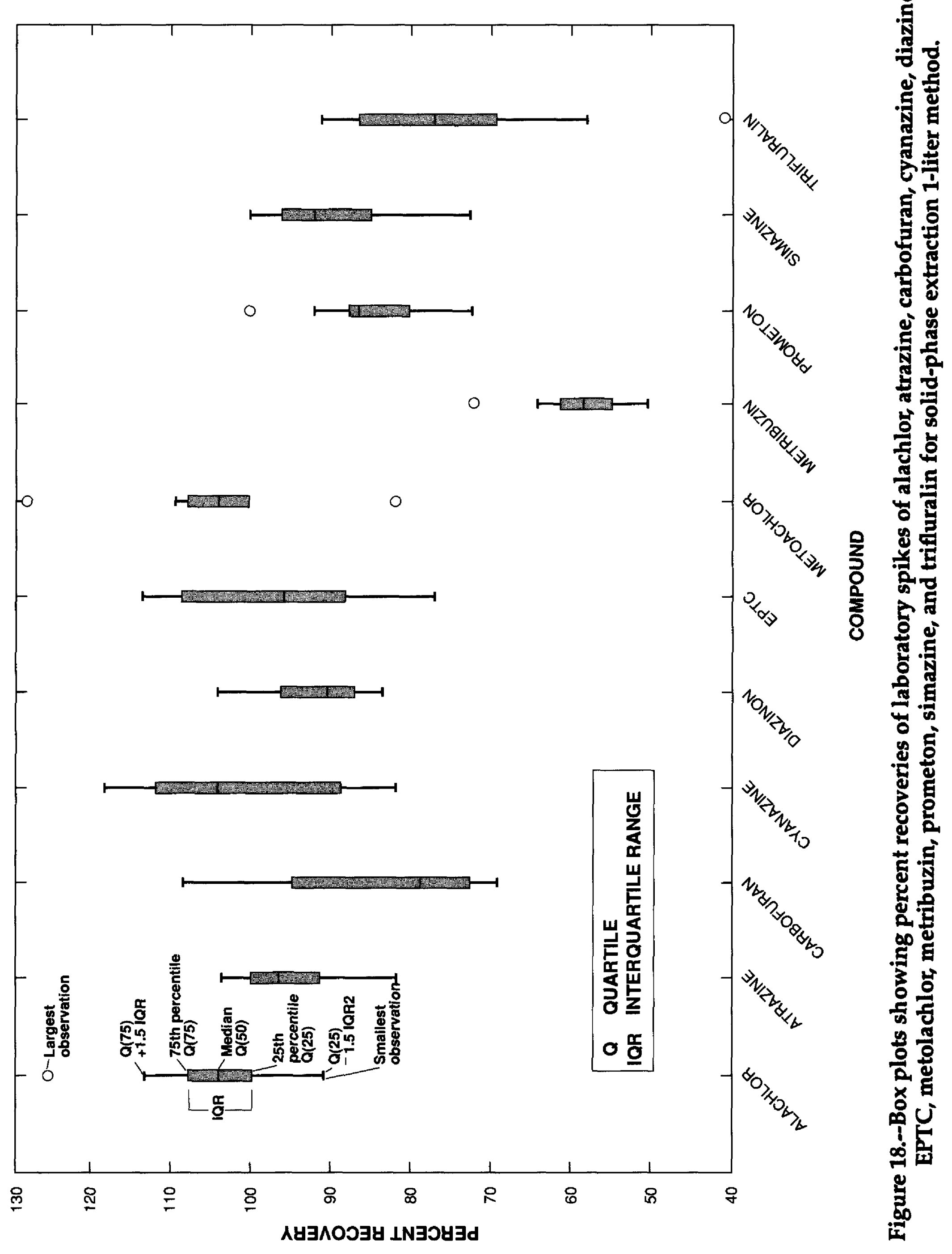




\section{REFERENCES CITED}

Buchanan, T.J., and Somers, W.P., 1969, Discharge measurements at gaging stations: U.S. Geological Survey Techniques of Water-Resources Investigations, book 3, chap. A8, 65 p.

Edwards, T.K., and Glysson, G.D., 1988, Field methods for measurement of fluvial sediment: U.S. Geological Survey Open-File Report 86-53, 118 p.

Fishman, M.J., and Friedman, L.C., 1989, Methods for the determination of inorganic substances in water and fluvial sediments: U.S. Geological Survey Techniques of Water-Resources Investigations, book 5 , chap. A1, $545 \mathrm{p}$.

Goolsby, D.A., Coupe, R.H., and Markovchick, D.J., 1991a, Distribution of selected herbicides and nitrate in the Mississippi River and its major tributaries: U.S. Geological Survey WaterResources Investigations Report 91-4163, 35 p.

Goolsby, D.A., Thurman, E.M., and Kolpin, D.W., 1991b, Herbicides in streams-Midwestern United States, in Irrigation and Drainage, Proceedings of the 1991 American Society of Civil Engineers Conference: Honolulu, Hawaii, July 22-26, 1991, p. 17-23.

Guy, H.P., 1969, Laboratory theory and methods for sediment analysis: U.S. Geological Survey Techniques of Water-Resources Investigations, book 5, chap. C1, $58 \mathrm{p}$.

Kennedy, D.J., 1983, Computation of continuous records of streamflow: U.S. Geological Survey Techniques of Water-Resources Investigations, book 3, chap. A13, 53 p.

Matthes, W.J., Sholar, C.J., and George, J.R., 1992, Quality-assurance plan for the analyses of fluvial sediment by laboratories of the U.S. Geological Survey: U.S. Geological Survey OpenFile Report 91-467, $31 \mathrm{p}$.

Meade, R.H., and Stevens, H.H., Jr., 1990, Strategies and equipment for sampling suspended sediment and associated toxic chemicals in large rivers-with emphasis on the Mississippi River: Science of the Total Environment, v. 97/98, p. 125-135.

Moody, J.A., and Goolsby, D.A., 1993, Spatial variability of triazine herbicides in the Lower Mississippi River: Environmental Science and Technology, v. 27, p. 2120-2126.

Moody, J.A., and Meade, R.H., 1992, Hydrologic and sedimentologic data collected during three cruises at low water on the Mississippi River and some of its tributaries, July 1987-June 1988: U.S. Geological Survey Open-File Report 91-485, 143 p.

Pereira, W.E., and Rostad, C.E., 1990, Occurrence, distributions, and transport of herbicides and their degradation products in the Lower Mississippi River and its tributaries: Environmental Science and Technology, v. 24, p. 1400-1406.

Sandstrom, M.W., Wydoski, D.S., Schroeder, M.P., Zamboni, J.L., and Foreman, W.T., 1991, Methods of analysis by the U.S. Geological Survey National Water Quality Laboratory; determination of organonitrogen herbicides in water by solid-phase extraction and capillary column gas chromatography/mass spectrometry with selected ion monitoring: U.S. Geological Survey Open-File Report 91-519, 26 p. 
Thurman, E.M., Goolsby, D.A., Meyer, M.T., and Kolpin, D.W., 1991, Herbicides in surface water of the midwestern United States-The effect of the spring flush: Environmental Science and Technology, v. 25 , no. 10 , p. $1794-1796$.

Thurman, E.M., Goolsby, D.A., Meyer, M.T., Mills, M.S., Pomes, M.L., and Kolpin, D.W., 1992, A reconnaissance study of herbicides and their metabolites in surface water of the midwestern United States using immunoassay and gas chromatography/mass spectrometry: Environmental Science and Technology, v. 26, p. 2440-2447.

Thurman, E.M., Meyer, M.T., Pomes, M.L., Perry, C.E., and Schwab, A.P., 1990, Enzyme-linked immunosorbent assay compared with GC/MS for the determination of herbicides in water: Analytical Chemistry, v. 62, p. 2043-2048.

U.S. Environmental Protection Agency, 1992, Drinking water regulations and health advisories: Office of Water, Washington, D.C., 13 p.

Wershaw, R.L., Fishman, M.J., Grabbe, R.R., and Lowe, L.E., eds., 1987, Method for the determination of organic substances in water and fluvial sediments: U.S. Geological Survey Techniques of Water Resources-Investigations, book 5, chap. A3, $80 \mathrm{p}$. 


\section{DATA SECTION}


$[\mu \mathrm{g} / \mathrm{L}$, micrograms per liter; <, less than; diss, dissolved; --, no data]

\begin{tabular}{|c|c|c|c|c|c|c|c|c|c|c|c|c|c|}
\hline Date & Time & $\begin{array}{l}\text { Ala- } \\
\text { chlor } \\
\text { dlss } \\
(\mu g /)\end{array}$ & $\begin{array}{c}\text { Ame- } \\
\text { tynn } \\
\text { disa } \\
(\mu g / L)\end{array}$ & $\begin{array}{l}\text { Atra- } \\
\text { zina } \\
\text { diss } \\
(\mu g / L)\end{array}$ & $\begin{array}{l}\text { Cyana- } \\
\text { zine } \\
\text { diss } \\
(\mu g / L)\end{array}$ & $\begin{array}{l}\text { Desethyl- } \\
\text { strazine } \\
\text { diss } \\
(\mu g /)\end{array}$ & $\begin{array}{c}\text { Desiso- } \\
\text { propyt- } \\
\text { atrazine } \\
\text { dlss } \\
(\mu \mathrm{g} / \mathrm{L})\end{array}$ & $\begin{array}{c}\text { Metole- } \\
\text { chlor } \\
\text { diss } \\
(\mu g / L)\end{array}$ & $\begin{array}{l}\text { Metrl- } \\
\text { buzin } \\
\text { diss } \\
(\mu g / L)\end{array}$ & $\begin{array}{c}\text { Pro- } \\
\text { motryn } \\
\text { diss } \\
(\mu g / L)\end{array}$ & $\begin{array}{c}\text { Pro- } \\
\text { meton } \\
\text { diss } \\
(\mu g / L)\end{array}$ & $\begin{array}{l}\text { Prope- } \\
\text { zine } \\
\text { diss } \\
(\mu g /)\end{array}$ & $\begin{array}{c}\text { Sima- } \\
\text { zine } \\
\text { diss } \\
(\mu g / 2)\end{array}$ \\
\hline
\end{tabular}

APRIL 1991

\begin{tabular}{|c|c|c|c|c|c|c|c|c|c|c|c|c|c|}
\hline 02 & 1040 & $<0.05$ & $<0.05$ & 0.18 & $<0.20$ & 0.17 & $<0.05$ & $<0.05$ & $<0.05$ & $<0.05$ & $<0.05$ & $<0.05$ & $<0.05$ \\
\hline 11 & 1100 & $<0.05$ & $<0.05$ & 0.15 & $<0.20$ & 0.14 & $<0.05$ & 0.07 & $<0.05$ & $<0.05$ & $<0.05$ & $<0.05$ & $<0.05$ \\
\hline 16 & 1150 & $<0.05$ & $<0.05$ & 0.17 & $<0.20$ & 0.11 & $<0.05$ & 0.06 & $<0.05$ & $<0.05$ & $<0.05$ & $<0.05$ & $<0.05$ \\
\hline 23 & 1030 & $<0.05$ & $<0.05$ & 0.17 & $<0.20$ & 0.18 & $<0.05$ & 0.08 & $<0.05$ & $<0.05$ & $<0.05$ & $<0.05$ & $<0.05$ \\
\hline \multicolumn{14}{|l|}{ MAY } \\
\hline 01 & 1150 & 0.06 & $<0.05$ & 0.15 & $<0.20$ & 0.06 & $<0.05$ & 0.07 & $<0.05$ & $<0.05$ & $<0.05$ & $<0.05$ & $<0.05$ \\
\hline 07 & 1115 & $<0.05$ & $<0.05$ & 0.17 & $<0.20$ & 0.15 & $<0.05$ & 0.05 & $<0.05$ & $<0.05$ & 0.17 & $<0.05$ & $<0.05$ \\
\hline 10 & 1030 & $<0.05$ & $<0.05$ & 0.13 & $<0.20$ & 0.14 & $<0.05$ & 0.10 & $<0.05$ & $<0.05$ & $<0.05$ & $<0.05$ & $<0.05$ \\
\hline 14 & 1120 & 0.09 & $<0.05$ & 0.16 & $<0.20$ & 0.17 & $<0.05$ & 0.12 & $<0.05$ & $<0.05$ & $<0.05$ & $<0.05$ & $<0.05$ \\
\hline 17 & 1045 & 0.06 & $<0.05$ & 0.12 & $<0.20$ & 0.05 & $<0.05$ & 0.06 & $<0.05$ & $<0.05$ & $<0.05$ & $<0.05$ & $<0.05$ \\
\hline 21 & 1140 & 0.24 & $<0.05$ & 0.37 & $<0.20$ & 0.14 & $<0.05$ & 0.25 & $<0.05$ & $<0.05$ & 0.09 & $<0.05$ & 0.06 \\
\hline 24 & 1205 & 0.19 & $<0.05$ & 0.22 & 0.20 & 0.09 & $<0.05$ & 0.24 & $<0.05$ & $<0.05$ & $<0.05$ & $<0.05$ & $<0.05$ \\
\hline 28 & 1210 & 0.23 & $<0.05$ & 0.41 & 0.30 & 0.08 & $<0.05$ & 0.21 & $<0.05$ & $<0.05$ & $<0.05$ & $<0.05$ & $<0.05$ \\
\hline 31 & 1015 & 0.11 & $<0.05$ & 0.29 & $<0.20$ & 0.08 & $<0.05$ & 0.16 & $<0.05$ & $<0.05$ & $<0.05$ & $<0.05$ & 0.05 \\
\hline \multicolumn{14}{|l|}{ JUNE } \\
\hline 04 & 1300 & 0.48 & $<0.05$ & 0.78 & 0.30 & 0.09 & $<0.05$ & 0.36 & $<0.05$ & $<0.05$ & $<0.05$ & $<0.05$ & 0.11 \\
\hline 07 & 1100 & 0.24 & $<0.05$ & 0.50 & 0.30 & 0.08 & $<0.05$ & 0.26 & $<0.05$ & $<0.05$ & $<0.05$ & $<0.05$ & $<0.05$ \\
\hline 10 & 1130 & 0.14 & $<0.05$ & 0.37 & 0.20 & 0.05 & $<0.05$ & 0.20 & $<0.05$ & $<0.05$ & $<0.05$ & $<0.05$ & $<0.05$ \\
\hline 13 & 1410 & 0.14 & $<0.05$ & 0.35 & 0.30 & 0.07 & $<0.05$ & 0.20 & $<0.05$ & $<0.05$ & $<0.05$ & $<0.05$ & $<0.05$ \\
\hline 17 & 1040 & 0.78 & $<0.05$ & 1.6 & 0.94 & 0.12 & $<0.05$ & 0.56 & $<0.05$ & $<0.05$ & $<0.05$ & $<0.05$ & 0.06 \\
\hline 20 & 1345 & 0.85 & $<0.05$ & 1.6 & 0.82 & 0.23 & $<0.05$ & 0.72 & $<0.05$ & $<0.05$ & 0.05 & $<0.05$ & 0.06 \\
\hline 24 & 1055 & 0.48 & $<0.05$ & 0.73 & 0.78 & 0.14 & $<0.05$ & 0.66 & $<0.05$ & $<0.05$ & $<0.05$ & $<0.05$ & 0.05 \\
\hline 27 & 0915 & 0.54 & $<0.05$ & 0.66 & 1.2 & 0.11 & $<0.05$ & 0.87 & $<0.05$ & $<0.05$ & $<0.05$ & $<0.05$ & $<0.05$ \\
\hline \multicolumn{14}{|l|}{ JULY } \\
\hline 01 & 1025 & 0.28 & $<0.05$ & 0.40 & 0.80 & 0.11 & $<0.05$ & 0.54 & $<0.05$ & $<0.05$ & $<0.05$ & $<0.05$ & $<0.05$ \\
\hline 05 & 1205 & 0.40 & $<0.05$ & 0.39 & 0.50 & 0.10 & $<0.05$ & 0.32 & $<0.05$ & $<0.05$ & $<0.05$ & $<0.05$ & $<0.05$ \\
\hline 08 & 1130 & 0.18 & $<0.05$ & 0.38 & 0.60 & 0.11 & $<0.05$ & 0.39 & $<0.05$ & $<0.05$ & $<0.05$ & $<0.05$ & $<0.05$ \\
\hline 11 & 1300 & 0.08 & $<0.05$ & 0.33 & 0.40 & 0.06 & $<0.05$ & 0.19 & $<0.05$ & $<0.05$ & $<0.05$ & $<0.05$ & $<0.05$ \\
\hline 15 & 1025 & 0.10 & $<0.05$ & 0.44 & 0.50 & 0.12 & 0.10 & 0.21 & 0.11 & $<0.05$ & 0.13 & $<0.05$ & $<0.05$ \\
\hline 18 & 1200 & 0.17 & $<0.05$ & 0.73 & 0.80 & 0.15 & $<0.05$ & 0.39 & $<0.05$ & $<0.05$ & $<0.05$ & $<0.05$ & $<0.05$ \\
\hline 22 & 1050 & -- & - & -- & -- & -- & -- & -. & -- & -- & -- & -- & - \\
\hline $\begin{array}{r}29 \\
\text { AUGGS }\end{array}$ & 1230 & $<0,05$ & $<0.05$ & 0.32 & 0.30 & 0.08 & $<0.05$ & 0.10 & $<0.05$ & $<0.05$ & $<0.05$ & $<0.05$ & $<0.05$ \\
\hline \multicolumn{14}{|l|}{ AUGUS } \\
\hline $\begin{array}{l}06 \\
12\end{array}$ & 1240 & $<0.05$ & $\begin{array}{l}<0.05 \\
<0.05\end{array}$ & 0.17 & $<0.20$ & $<0.05$ & $<0.05$ & $<0.05$ & $<0.05$ & $<0.05$ & $<0.05$ & $<0.05$ & $<0.05$ \\
\hline $\begin{array}{l}12 \\
19\end{array}$ & 1125 & $<0.05$ & $<0.05$ & $<0.05$ & $<0.20$ & $<0,05$ & $<0.05$ & 0.07 & $<0.05$ & $<0.05$ & $<0.05$ & $<0.05$ & $<0.05$ \\
\hline $\begin{array}{l}19 \\
26\end{array}$ & 1145 & $<0.05$ & $<0.05$ & 0.09 & $<0.20$ & $<0.05$ & $<0.05$ & $<0.05$ & $<0.05$ & $<0.05$ & $<0.05$ & $<0.05$ & $<0.05$ \\
\hline $\begin{array}{r}26 \\
\text { SEPTEM }\end{array}$ & 1205 & $<0.05$ & $<0.05$ & 0.18 & $<0.20$ & 0.10 & $<0.05$ & 0.08 & 0.06 & $<0.05$ & $<0.05$ & $<0.05$ & $<0.05$ \\
\hline \multicolumn{14}{|c|}{ SEPTEMBER } \\
\hline 03 & 1200 & -- & -- & -- & -- & - & -- & -- & - & -- & - & -. & - \\
\hline 09 & 1500 & $<0.05$ & $<0.05$ & 0.08 & $<0.20$ & $<0.05$ & $<0.05$ & $<0.05$ & $<0.05$ & $<0.05$ & $<0.05$ & $<0.05$ & $<0.05$ \\
\hline 17 & 1215 & $<0.05$ & $<0.05$ & 0.14 & $<0.20$ & 0.08 & $<0.05$ & $<0.05$ & $<0.05$ & $<0.05$ & $<0.05$ & $<0.05$ & $<0.05$ \\
\hline 24 & 1215 & $<0.05$ & $<0.05$ & 0.10 & $<0.20$ & 0.06 & $<0.10$ & 0.05 & $<0.05$ & $<0.05$ & $<0.05$ & $<0.05$ & $<0.05$ \\
\hline \multicolumn{14}{|c|}{ OCTOBER 1991} \\
\hline 01 & 1200 & $<0.05$ & $<0.05$ & 0.13 & $<0.20$ & 0.09 & 0.10 & $<0.05$ & $<0.05$ & $<0.05$ & $<0.05$ & $<0.05$ & $<0.05$ \\
\hline 07 & 1230 & $<0.05$ & $<0.05$ & 0.12 & $<0.20$ & 0.09 & $<0.10$ & 0.08 & $<0.05$ & $<0.05$ & $<0.05$ & $<0.05$ & $<0.05$ \\
\hline
\end{tabular}




\begin{tabular}{|c|c|c|c|c|c|c|c|c|c|c|c|c|c|}
\hline Date & Time & $\begin{array}{l}\text { Ala- } \\
\text { chlor } \\
\text { diss } \\
(\mu g / L)\end{array}$ & $\begin{array}{l}\text { Ame- } \\
\text { tryn } \\
\text { diss } \\
(\mu g / L)\end{array}$ & $\begin{array}{l}\text { Atra- } \\
\text { zine } \\
\text { diss } \\
(\mu g / L)\end{array}$ & $\begin{array}{l}\text { Cyana- } \\
\text { zine } \\
\text { dlss } \\
\text { ( } \mu \mathrm{g} / \mathrm{L})\end{array}$ & $\begin{array}{l}\text { Desethyl- } \\
\text { atrazine } \\
\text { diss } \\
(\mu g / L)\end{array}$ & $\begin{array}{l}\text { Desiso- } \\
\text { propyl- } \\
\text { atrazine } \\
\text { diss } \\
(\mu \mathrm{g} / \mathrm{L})\end{array}$ & $\begin{array}{l}\text { Metola- } \\
\text { chior } \\
\text { diss } \\
(\mu g / L)\end{array}$ & $\begin{array}{l}\text { Metrl- } \\
\text { buzin } \\
\text { dlss } \\
(\mu g / L)\end{array}$ & $\begin{array}{l}\text { Pro- } \\
\text { metryn } \\
\text { dlss } \\
(\mu g / L)\end{array}$ & $\begin{array}{l}\text { Pro- } \\
\text { meton } \\
\text { dlss } \\
(\mu \mathrm{g} / \mathrm{L})\end{array}$ & $\begin{array}{l}\text { Propa- } \\
\text { zine } \\
\text { diss } \\
(\mu \mathrm{g} / \mathrm{L})\end{array}$ & $\begin{array}{c}\text { Sima- } \\
\text { zine } \\
\text { dlss } \\
(\mu \mathrm{g} / \mathrm{L})\end{array}$ \\
\hline
\end{tabular}

OCTOBER 1991 (cont.)

$\begin{array}{clllllllllllll}15 & 1300 & <0.05 & <0.05 & 0.10 & <0.20 & 0.10 & <0.05 & <0.05 & <0.05 & <0.05 & <0.05 & <0.05 & <0.05 \\ 22 & 1230 & <0.05 & <0.05 & 0.11 & <0.20 & 0.10 & <0.05 & 0.05 & <0.05 & <0.05 & <0.05 & <0.05 & <0.05 \\ 29 & 1330 & <0.05 & <0.05 & 0.13 & <0.20 & 0.10 & <0.05 & 0.05 & <0.05 & <0.05 & <0.05 & <0.05 & <0.05 \\ \text { O5EMBER } & & & & & & & & & & & & & \\ 12 & 1245 & <0.05 & <0.05 & 0.11 & <0.20 & 0.08 & <0.05 & <0.05 & <0.05 & <0.05 & <0.05 & <0.05 & <0.05 \\ & 1255 & <0.05 & <0.05 & 0.10 & <0.20 & 0.08 & <0.05 & <0.05 & <0.05 & <0.05 & <0.05 & <0.05 & <0.05\end{array}$

$\begin{array}{llllllllllll}26 & 1320 & <0.05 & <0.05 & 0.08 & <0.20 & 0.08 & <0.05 & <0.05 & <0.05 & <0.05<0.05<0.05\end{array}$

DECEMBER

\begin{tabular}{|c|c|c|c|c|c|c|c|c|c|c|c|c|c|}
\hline 09 & 1200 & $<0.05$ & $<0.05$ & 0.07 & $<0.20$ & 0.06 & $<0.05$ & $<0.05$ & $<0.05$ & $<0.05$ & $<0.05$ & $<0.05$ & $<0.05$ \\
\hline 22 & 1330 & $<0.05$ & $<0.05$ & 0.10 & $<0.20$ & 0.11 & $<0.05$ & 0.05 & $<0.05$ & $<0.05$ & $<0.05$ & $<0.05$ & $<0.05$ \\
\hline \multicolumn{14}{|c|}{ ANUARY 1992} \\
\hline 07 & 1210 & $<0.05$ & $<0.05$ & 0.06 & $<0.20$ & 0.06 & $<0.05$ & $<0.05$ & $<0.05$ & $<0.05$ & $<0.05$ & $<0.05$ & $<0.05$ \\
\hline 22 & 1340 & $<0.05$ & $<0.05$ & $<0.05$ & $<0.20$ & $<0.05$ & $<0.05$ & $<0.05$ & $<0.05$ & $<0.05$ & $<0.05$ & $<0.05$ & $<0.05$ \\
\hline \multicolumn{14}{|c|}{ BRUARY } \\
\hline 03 & 1245 & $<0.05$ & $<0.05$ & 0.06 & $<0.20$ & 0.06 & $<0.05$ & $<0.05$ & $<0.05$ & $<0.05$ & $<0.05$ & $<0.05$ & 0. \\
\hline
\end{tabular}

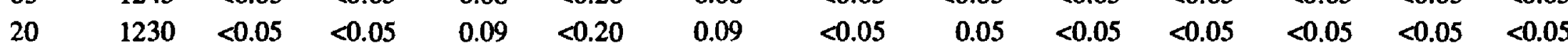

MARCH

$04 \quad 1100<0.05<0.05$

$13 \quad 1200<0.05<0.05$

0.05

$0.05<0.20<0.05$

$\begin{array}{lll}17 & 1400 & <0.05 \\ 24 & 1125 & <0.05\end{array}$

$<0.05$

$0.09<0.20$

$<0.05$

$<0.05<0.05<0.05<0.05$

$<0.05$

$0.08<0.20$

0.10

$<0.05$

0.05

0.09

$<0.05$

0.05

$<0.05$

$<0.05$

$<0.05$

$<0.05<0.05$

$30 \quad 1155<0.05<0.05$

$0.08<0.20$

$0.07<0.05$

0.07

$<0.05<0.05$

$0.07<0.20$

$0.07<0.10$

0.05

$<0.05<0.05$

$<0.05$

$<0.05<0.05<0.05$

APRIL

06

$1215<0.05$

$<0.05$

$0.06<0.20$

0.06

$<0.05$

$<0.05$

$<0.05$

$<0.05$

$<0.05$

$<0.05$

$<0.05<0.05$

$14 \quad 1130<0.05$

$0.07<0.20$

0.06

$<0.05$

$<0.05$

$<0.05$

$<0.05$

$<0.05$

$<0.05$

$<0.05$

$<0.05$

$0.08<0.20 \quad 0.07$

$<0.05$

$<0.05<0.05<0.05$

$<0.05$

$<0.05$

$<0.05$

$<0.05<0.05$

$0.05<0.20$

0.05

$<0.05<0.05<0.05<0.05$

$<0.05<0.05<0.05$

$0.06<0.05 \quad 0.06<0.05<0.05$

$0.06<0.20$

0.07

0.05

$\begin{array}{lll}0.07 & 0.05 & 0.05\end{array}$

0.05

$<0.05<0.05$

$<0.05<0.05$

JUNE

$01 \quad 1200 \quad 0.06<0.05$

$0.21<0.20$

$0.09<0.05$

$0.08<0.05<0.05$

$<0.05$

$<0.05$

$\begin{array}{lll}08 & 1235 & 0.08\end{array}$

$<0.05$

$0.91 \quad 0.40$

0.13

$<0.05$

0.28

0.06

$<0.05$

$<0.05$

$<0.05$

$22 \quad 1230 \quad 0.06$

$<0.05$

$0.16<0.20$

$0.07<0.05$

$<0.05<0.05<0.05$

$<0.05$

$<0.05<0.05$

$\begin{array}{llll}29 & 1300 & 0.08 & <0.05\end{array}$

$0.16<0.20$

$0.07<0.05$

$0.07<0.05<0.05$

$<0.05$

$<0.05<0.05$

$<0.05<0.05<0.05$

JULY 1992

$\begin{array}{rrrr}08 & 0815 & <0.05 & <0.05 \\ 13 & 1225 & 0.18 & <0.05 \\ 20 & 1145 & 0.15 & <0.05 \\ 27 & 1215 & 0.07 & <0.05\end{array}$

$\begin{array}{ll}0.21 & 0.20\end{array}$

0.06

$\begin{array}{rlll}<0.10 & 0.06 & <0.05 & <0.05 \\ 0.13 & 0.31 & <0.05 & <0.05 \\ 0.15 & 0.26 & <0.05 & <0.05 \\ 0.08 & 0.17 & <0.05 & <0.05\end{array}$

$<0.05<0.05<0.05$

$<0.05<0.05<0.05$

$\begin{array}{ll}0.51 & 0.40 \\ 0.56 & 0.60\end{array}$

0.13

$<0.05$

$<0.05<0.05$

$\begin{array}{cccc}0.40 & 0.11 & 0.08 & 0.17 \\ 05586100 & \text { - Illinois } & \text { River at Valley } & \text { City }\end{array}$

$<0.05<0.05<0.05$

APRIL 1991

\begin{tabular}{|c|c|c|c|c|c|c|c|c|c|c|c|c|c|}
\hline 05 & 1230 & $<0.05$ & $<0.05$ & 0.18 & $<0.20$ & 0.14 & $<0.05$ & 0.15 & $<0.05$ & $<0.05$ & 0.10 & $<0.05$ & 0.06 \\
\hline 17 & 1330 & 0.62 & $<0.05$ & 2.4 & 1.3 & 0.16 & $<0.05$ & 1.5 & 0.06 & $<0.05$ & $<0.05$ & $<0.05$ & $<0.05$ \\
\hline 26 & 1000 & 0.14 & $<0.05$ & 0.96 & 0.69 & 0.15 & $<0.05$ & 0.92 & $<0.05$ & $<0.05$ & $<0.05$ & $<0.05$ & $<0.05$ \\
\hline
\end{tabular}


Table 7.-Pesticide results from solid-phase extraction 100-milliliter method-Continued

\begin{tabular}{|c|c|c|c|c|c|c|c|c|c|c|c|c|c|}
\hline Date & Time & $\begin{array}{l}\text { Ala- } \\
\text { chlor } \\
\text { diss } \\
(\mu g /)\end{array}$ & $\begin{array}{c}\text { Ame- } \\
\text { tryn } \\
\text { diss } \\
(\mu g / L)\end{array}$ & $\begin{array}{l}\text { Atra- } \\
\text { zine } \\
\text { diss } \\
(\mu \mathrm{g} / \mathrm{L})\end{array}$ & $\begin{array}{l}\text { Cyana- } \\
\text { zine } \\
\text { diss } \\
(\mu \mathrm{g} / \mathrm{L})\end{array}$ & $\begin{array}{l}\text { Desethyl- } \\
\text { atrazine } \\
\text { diss } \\
(\mu g / L)\end{array}$ & 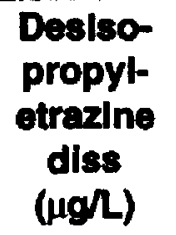 & $\begin{array}{l}\text { Metols- } \\
\text { chlor } \\
\text { diss } \\
\text { ( } \mu \mathrm{g} / \mathrm{L})\end{array}$ & 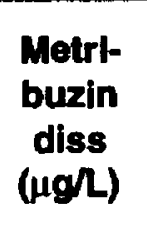 & $\begin{array}{l}\text { Pro- } \\
\text { metryn } \\
\text { diss } \\
(\mu g /)\end{array}$ & $\begin{array}{l}\text { Pro- } \\
\text { meton } \\
\text { diss } \\
(\mu g \Omega)\end{array}$ & $\begin{array}{l}\text { Propa- } \\
\text { zine } \\
\text { diss } \\
(\mu g / \text { ) }\end{array}$ & $\begin{array}{l}\text { Sime- } \\
\text { zine } \\
\text { diss } \\
(\mu g / L)\end{array}$ \\
\hline \multicolumn{14}{|c|}{05586100 - Illinois River at Valley City-Continned } \\
\hline \multicolumn{14}{|l|}{ MAY } \\
\hline 03 & 1545 & 0.28 & $<0.05$ & 0.95 & 1.1 & 0.13 & $<0.05$ & 0.86 & $<0.05$ & $<0.05$ & $<0.05^{\prime}$ & $<0.05$ & $<0.05$ \\
\hline 08 & 1245 & 2.0 & $<0.05$ & 5.0 & 5.6 & 0.37 & $<0.05$ & 2.6 & 0.11 & $<0.05$ & $<0.05$ & $<0.05$ & 0.06 \\
\hline 10 & 1100 & 3.0 & $<0.05$ & 6.3 & 2.9 & 0.54 & $<0.05$ & 4.4 & 0.20 & $<0.05$ & 0.09 & $<0.05$ & 0.14 \\
\hline 17 & 1145 & 0.84 & $<0.05$ & 2.5 & 2.5 & 0.20 & $<0.05$ & 2.0 & $<0.05$ & $<0.05$ & $<0.05$ & $<0.05$ & $<0.05$ \\
\hline 19 & 1115 & 1.6 & $<0.05$ & 5.5 & 6.6 & 0.67 & $<0.05$ & 1.6 & 0.12 & $<0.05$ & $<0.05$ & $<0.05$ & 0.07 \\
\hline 22 & 1715 & 0.92 & $<0.05$ & 3.4 & 2.9 & 0.33 & $<0.05$ & 2.1 & 0.08 & $<0.05$ & $<0.05$ & $<0.05$ & 0.05 \\
\hline 29 & 1130 & -- & -- & -- & -. & -- & -. & - & $\ldots$ & -- & -- & - & -- \\
\hline 31 & 1045 & 0.97 & $<0.05$ & 4.9 & 2.5 & 0.60 & $<0.05$ & 2.0 & 0.09 & $<0.05$ & $<0.05$ & $<0.05$ & 0.07 \\
\hline \multicolumn{14}{|l|}{ JUNE } \\
\hline 04 & 1215 & 0.85 & $<0.05$ & 4.6 & 2.7 & 0.48 & $<0.05$ & 2.1 & 0.08 & $<0.05$ & $<0.05$ & $<0.05$ & 0.09 \\
\hline 06 & 1200 & 1.0 & $<0.05$ & 5.2 & 2.0 & 0.36 & $<0.05$ & 2.3 & 0.08 & $<0.05$ & $<0.05$ & $<0.05$ & 0.09 \\
\hline 11 & 1345 & 0.84 & $<0.05$ & 4.7 & 1.8 & 0.60 & $<0.05$ & 2.0 & $<0.05$ & $<0.05$ & 0.06 & $<0.05$ & 0.14 \\
\hline 14 & 1200 & 0.54 & $<0.05$ & 3.8 & 2.0 & 0.41 & $<0.05$ & 1.4 & $<0.05$ & $<0.05$ & $<0.05$ & $<0.05$ & 0.12 \\
\hline 18 & 1130 & 0.36 & $<0.05$ & 2.1 & 0.90 & 0.21 & $<0.05$ & 0.78 & $<0.05$ & $<0.05$ & $<0.05$ & $<0.05$ & 0.07 \\
\hline 20 & 1130 & 0.34 & $<0.05$ & 2.8 & 1.4 & 0.35 & $<0.05$ & 0.83 & $<0.05$ & $<0.05$ & 0.05 & $<0.05$ & 0.12 \\
\hline 24 & 1130 & 0.17 & $<0.05$ & 2.4 & 1.4 & 0.31 & $<0.05$ & 0.63 & $<0.05$ & $<0.05$ & $<0.05$ & $<0.05$ & 0.09 \\
\hline 27 & 1100 & 0.21 & $<0.05$ & 1.9 & 0.80 & 0.25 & $<0.05$ & 0.55 & $<0.05$ & $<0.05$ & 0.05 & $<0.05$ & 0.07 \\
\hline \multicolumn{14}{|l|}{ JULY } \\
\hline 01 & 0930 & 0.08 & $<0.05$ & 0.70 & 0.20 & 0.13 & $<0.05$ & 0.22 & $<0.05$ & $<0.05$ & $<0.05$ & $<0.05$ & 0.05 \\
\hline 03 & 1100 & 0.64 & $<0.05$ & 0.52 & 0.60 & 0.39 & $<0.05$ & 0.71 & 0.44 & $<0.05$ & $<0.05$ & 0.05 & 0.48 \\
\hline 09 & 1000 & $<0.05$ & $<0.05$ & 0.55 & 0.20 & 0.08 & $<0.05$ & 0.11 & $<0.05$ & $<0.05$ & 0.05 & $<0.05$ & $<0.05$ \\
\hline 11 & 1045 & $<0.05$ & $<0.05$ & 0.70 & 0.30 & 0.10 & $<0.05$ & 0.12 & $<0.05$ & $<0.05$ & 0.06 & $<0.05$ & 0.06 \\
\hline 18 & 1115 & $<0.05$ & $<0.05$ & 0.46 & 0.20 & 0.11 & $<0.05$ & 0.11 & $<0.05$ & $<0.05$ & $<0.05$ & $<0.05$ & 0.05 \\
\hline 25 & 1340 & $<0.05$ & $<0.05$ & 0.18 & $<0.20$ & 0.06 & $<0.05$ & $<0.05$ & $<0.05$ & $<0.05$ & 0.06 & $<0.05$ & $<0.05$ \\
\hline 31 & 1100 & $<0.05$ & $<0.05$ & 0.31 & $<0.20$ & 0.08 & $<0.05$ & $<0.05$ & $<0.05$ & $<0.05$ & 0.12 & $<0.05$ & $<0.05$ \\
\hline \multicolumn{14}{|l|}{ AUGUST } \\
\hline 06 & 1015 & $<0.05$ & $<0.05$ & 0.29 & $<0.20$ & 0.10 & $<0.05$ & 0.07 & $<0.05$ & $<0.05$ & 0.08 & $<0.05$ & 0.05 \\
\hline 13 & 1200 & $<0.05$ & $<0.05$ & 0.35 & $<0.20$ & 0.15 & $<0.05$ & 0.12 & $<0.05$ & $<0.05$ & 0.11 & $<0.05$ & 0.05 \\
\hline 21 & 1230 & $<0.05$ & $<0.05$ & 0.10 & $<0.20$ & $<0.05$ & $<0.05$ & $<0.05$ & $<0.05$ & $<0.05$ & $<0.05$ & $<0.05$ & $<0.05$ \\
\hline 28 & 1015 & $<0.05$ & $<0.05$ & 0.10 & $<0.20$ & 0.05 & $<0.05$ & $<0.05$ & $<0.05$ & $<0.05$ & 0.05 & $<0.05$ & $<0.05$ \\
\hline \multicolumn{14}{|c|}{ SEPTEMBER 1991} \\
\hline 03 & 1050 & $<0.05$ & $<0.05$ & 0.07 & $<0.20$ & $<0.05$ & $<0.05$ & $<0.05$ & $<0.05$ & $<0.05$ & 0.10 & $<0.05$ & $<0.05$ \\
\hline 10 & 1200 & $<0.05$ & $<0.05$ & 0.16 & $<0.20$ & 0.06 & $<0.10$ & $<0.05$ & $<0.05$ & $<0.05$ & 0.08 & $<0.05$ & $<0.05$ \\
\hline 17 & 1100 & $<0.05$ & $<0.05$ & 0.15 & $<0.20$ & 0.07 & $<0.10$ & $<0.05$ & $<0.05$ & $<0.05$ & 0.07 & $<0.05$ & 0.05 \\
\hline 24 & 1100 & $<0.05$ & $<0.05$ & 0.10 & $<0.20$ & 0.05 & $<0.10$ & $<0.05$ & $<0.05$ & $<0.05$ & 0.06 & $<0.05$ & $<0.05$ \\
\hline \multicolumn{14}{|c|}{ OCTOBER } \\
\hline 01 & 1130 & $<0.05$ & $<0.05$ & 0.13 & 0.07 & 0.08 & 0.10 & $<0.05$ & $<0.05$ & $<0.05$ & 0.19 & $<0.05$ & 0.05 \\
\hline 08 & 1330 & $<0.05$ & $<0.05$ & 0.30 & $<0.20$ & 0.12 & $<0.10$ & 0.27 & $<0.05$ & $<0.05$ & 0.05 & $<0.05$ & 0.05 \\
\hline 15 & 1215 & $<0.05$ & $<0.05$ & 0.11 & $<0.20$ & $<0.05$ & $<0.05$ & 0.05 & $<0.05$ & $<0.05$ & $<0.05$ & $<0.05$ & $<0.05$ \\
\hline 22 & 1130 & $<0.05$ & $<0.05$ & 0.09 & $<0.20$ & 0.06 & $<0.10$ & $<0.05$ & $<0.05$ & $<0.05$ & 0.07 & $<0.05$ & 0.05 \\
\hline \multicolumn{14}{|c|}{ NOVEMBER } \\
\hline 05 & 1140 & 0.08 & $<0.05$ & 0.22 & $<0.20$ & 0.11 & $<0.05$ & 0.14 & $<0.05$ & $<0.05$ & 0.10 & $<0.05$ & $<0.05$ \\
\hline 15 & 1130 & $<0.05$ & $<0.05$ & 0.20 & $<0.20$ & 0.09 & $<0.05$ & 0.15 & $<0.05$ & $<0.05$ & 0.06 & $<0.05$ & $<0.05$ \\
\hline \multicolumn{14}{|c|}{ DECEMBER } \\
\hline 03 & 1030 & $<0.05$ & $<0.05$ & 0.26 & $<0.20$ & 0.13 & $<0.05$ & 0.19 & $<0.05$ & $<0.05$ & $<0.05$ & $<0.05$ & $<0.05$ \\
\hline 18 & 1200 & $<0.05$ & $<0.05$ & 0.15 & $<0.20$ & 0.09 & $<0.05$ & 0.12 & $<0.05$ & $<0.05$ & $<0.05$ & $<0.05$ & $<0.05$ \\
\hline
\end{tabular}


Table 7.--Pesticide results from solid-phase extraction 100-milliliter method-Continued

\begin{tabular}{|c|c|c|c|c|c|c|c|c|c|c|c|c|c|}
\hline Date & Time & $\begin{array}{l}\text { Ala- } \\
\text { chior } \\
\text { diss } \\
(\mu g / L)\end{array}$ & $\begin{array}{l}\text { Ame- } \\
\text { tryn } \\
\text { diss } \\
(\mu g / L)\end{array}$ & $\begin{array}{l}\text { Atra- } \\
\text { zine } \\
\text { diss } \\
(\mu g / L)\end{array}$ & $\begin{array}{l}\text { Cyana- } \\
\text { zine } \\
\text { diss } \\
(\mu g h)\end{array}$ & $\begin{array}{l}\text { Desethyl- } \\
\text { atrazine } \\
\text { diss } \\
(\mu g / L)\end{array}$ & $\begin{array}{c}\text { Desiso- } \\
\text { propyl- } \\
\text { atrazine } \\
\text { diss } \\
(\mu \mathrm{g} / \mathrm{L})\end{array}$ & $\begin{array}{l}\text { Metoia- } \\
\text { chlor } \\
\text { diss } \\
(\mu g / L)\end{array}$ & $\begin{array}{c}\text { Metri- } \\
\text { buzin } \\
\text { dlss } \\
(\mu g / L)\end{array}$ & $\begin{array}{c}\text { Pro- } \\
\text { metryn } \\
\text { diss } \\
(\mu g / L)\end{array}$ & $\begin{array}{c}\text { Pro- } \\
\text { meton } \\
\text { diss } \\
(\mu g / L)\end{array}$ & $\begin{array}{l}\text { Propa- } \\
\text { zine } \\
\text { diss } \\
(\mu g / L)\end{array}$ & $\begin{array}{l}\text { Sima- } \\
\text { zine } \\
\text { diss } \\
(\mu g / L)\end{array}$ \\
\hline
\end{tabular}

JANUARY 1992

\begin{tabular}{|c|c|c|c|c|c|c|c|c|c|c|c|c|c|}
\hline 02 & 1330 & $<0.10$ & $<0.10$ & 0.18 & $<0.20$ & 0.12 & $<0.10$ & 0.13 & $<0.10$ & $<0.10$ & $<0.10$ & $<0.10$ & $<0.10$ \\
\hline 15 & 1645 & $<0.05$ & $<0.05$ & 0.16 & $<0.20$ & 0.08 & 0.05 & 0.13 & $<0.05$ & $<0.05$ & $<0.05$ & $<0.05$ & $<0.05$ \\
\hline 30 & 1345 & $<0.05$ & $<0.05$ & 0.17 & $<0.20$ & $<0.05$ & $<0.05$ & 0.14 & $<0.05$ & $<0.05$ & $<0.05$ & $<0.05$ & $<0.05$ \\
\hline \multicolumn{14}{|c|}{ EBRUARY } \\
\hline 12 & 1200 & $<0.05$ & $<0.05$ & 0.13 & $<0.20$ & 0.09 & $<0.05$ & 0.11 & $<0.05$ & $<0.05$ & $<0.05$ & $<0.05$ & $<0.05$ \\
\hline 28 & 1245 & $<0.05$ & $<0.05$ & 0.15 & $<0.20$ & 0.09 & $<0.05$ & 0.12 & $<0.05$ & $<0.05$ & $<0.05$ & $<0.05$ & $<0.05$ \\
\hline \multicolumn{14}{|c|}{ IARCH } \\
\hline 03 & 1100 & $<0.05$ & $<0.05$ & 0.14 & $<0.20$ & 0.09 & $<0.05$ & 0.09 & $<0.05$ & $<0.05$ & $<0.05$ & $<0.05$ & $<0.05$ \\
\hline 10 & 1015 & $<0.05$ & $<0.05$ & 0.12 & $<0.20$ & 0.09 & $<0.05$ & 0.09 & $<0.05$ & $<0.05$ & $<0.05$ & $<0.05$ & $<0.05$ \\
\hline 17 & 1050 & $<0.05$ & $<0.05$ & 0.16 & $<0.20$ & 0.09 & $<0.05$ & 0.14 & $<0.05$ & $<0.05$ & 0.08 & $<0.05$ & $<0.05$ \\
\hline 25 & 1045 & $<0.05$ & $<0.05$ & 0.22 & $<0.20$ & 0.09 & $<0.05$ & 0.16 & $<0.05$ & $<0.05$ & $<0.05$ & $<0.05$ & $<0.05$ \\
\hline
\end{tabular}

APRIL 1991

\begin{tabular}{|c|c|c|c|c|c|c|c|c|c|c|c|c|c|}
\hline 09 & 1250 & 0.10 & $<0.05$ & 0.25 & $<0.20$ & 0.12 & $<0.05$ & 0.10 & 0.19 & $<0.05$ & $<0.05$ & $<0.05$ & $<0.05$ \\
\hline 16 & 1030 & $<0.05$ & $<0.05$ & 0.37 & $<0.20$ & 0.07 & 0.05 & 0.11 & $<0.05$ & $<0.05$ & $<0.05$ & $<0.05$ & $<0.05$ \\
\hline 24 & 1030 & $<0.05$ & $<0.05$ & 0.06 & $<0.20$ & $<0.05$ & $<0.05$ & $<0.05$ & $<0.05$ & $<0.05$ & $<0.05$ & $<0.05$ & $<0.05$ \\
\hline 29 & 1000 & 0.14 & $<0.05$ & 0.66 & $<0.20$ & 0.06 & $<0.05$ & 0.30 & $<0.05$ & $<0.05$ & $<0.05$ & $<0.05$ & $<0.05$ \\
\hline \multicolumn{14}{|l|}{ IAY } \\
\hline 06 & 1115 & 0.27 & $<0.05$ & 1.3 & 0.38 & 0.18 & 0.15 & 0.47 & $<0.05$ & $<0.05$ & 0.11 & $<0.05$ & $<0.05$ \\
\hline 09 & 0940 & 0.14 & $<0.05$ & 0.81 & 0.20 & 0.06 & $<0.05$ & 0.21 & $<0.05$ & $<0.05$ & $<0.05$ & $<0.05$ & 0.09 \\
\hline 13 & 1110 & 0.20 & $<0.05$ & 0.87 & $<0.20$ & 0.17 & $<0.05$ & 0.21 & $<0.05$ & $<0.05$ & $<0.05$ & $<0.05$ & $<0.05$ \\
\hline 16 & 1130 & 0.17 & $<0.05$ & 0.35 & 0.40 & 0.05 & $<0.05$ & 0.09 & $<0.05$ & $<0.05$ & $<0.05$ & $<0.05$ & $<0.05$ \\
\hline 21 & 0945 & 3.6 & $<0.05$ & 8.3 & 6.8 & 0.29 & 0.16 & 3.1 & $<0.05$ & $<0.05$ & $<0.05$ & $<0.05$ & $<0.05$ \\
\hline 24 & 0740 & 0.51 & $<0.05$ & 2.4 & 1.4 & 0.12 & $<0.05$ & 0.70 & $<0.05$ & $<0.05$ & $<0.05$ & $<0.05$ & $<0.05$ \\
\hline 29 & 1015 & 1.4 & $<0.05$ & 6.5 & 1.7 & 0.56 & 0.21 & 2.2 & $<0.05$ & $<0.05$ & $<0.05$ & $<0.05$ & 0.07 \\
\hline 31 & 0735 & 2.1 & $<0.05$ & 6.8 & 7.0 & 0.48 & $<0.05$ & 2.6 & 0.09 & $<0.05$ & $<0.05$ & $<0.05$ & 0.06 \\
\hline \multicolumn{14}{|c|}{ UNE 1991} \\
\hline 04 & 0800 & 1.7 & $<0.05$ & 5.7 & 3.7 & 0.44 & 0.21 & 1.9 & $<0.05$ & $<0.05$ & 0.06 & $<0.05$ & 0.06 \\
\hline 07 & 1415 & 3.2 & $<0.05$ & 10 & 7.3 & 0.48 & 0.26 & 2.0 & 0.12 & $<0.05$ & $<0.05$ & 0.08 & 0.06 \\
\hline 11 & 1045 & 0.80 & $<0.05$ & 5.4 & 2.0 & 0.29 & $<0.05$ & 1.3 & 0.10 & $<0.05$ & $<0.05$ & $<0.05$ & $<0.05$ \\
\hline 14 & 0750 & 0.99 & $<0.05$ & 4.7 & 1.9 & 0.49 & 0.18 & 1.4 & 0.28 & $<0.05$ & $<0.05$ & $<0.05$ & 0.05 \\
\hline 18 & 0935 & 0.59 & $<0.05$ & 3.4 & 3.2 & 0.34 & $<0.05$ & 0.80 & 0.08 & $<0.05$ & $<0.05$ & $<0.05$ & $<0.05$ \\
\hline 21 & 1020 & 0.15 & $<0.05$ & 1.9 & 1.3 & 0.23 & $<0.05$ & 0.34 & $<0.05$ & $<0.05$ & $<0.05$ & $<0.05$ & $<0.05$ \\
\hline 24 & 1110 & 0.23 & $<0.05$ & 1.8 & 0.70 & 0.19 & $<0.05$ & 0.33 & $<0.05$ & $<0.05$ & $<0.05$ & $<0.05$ & $<0.05$ \\
\hline 27 & 1020 & 0.10 & $<0.05$ & 1.4 & 0.90 & 0.19 & $<0.05$ & 0.20 & $<0.05$ & $<0.05$ & $<0.05$ & $<0.05$ & $<0.05$ \\
\hline \multicolumn{14}{|l|}{ ULY } \\
\hline 01 & 0950 & 0.06 & $<0.05$ & 0.63 & 0.30 & 0.13 & $<0.05$ & 0.11 & 0.13 & $<0.05$ & 0.05 & $<0.05$ & $<0.05$ \\
\hline 03 & 0950 & 0.09 & $<0.05$ & 0.95 & 0.73 & 0.16 & 0.14 & 0.22 & $<0.05$ & $<0.05$ & $<0.05$ & $<0.05$ & $<0.05$ \\
\hline 08 & 1100 & $<0.05$ & $<0.05$ & 0.77 & 0.40 & 0.10 & $<0.05$ & 0.08 & $<0.05$ & $<0.05$ & $<0.05$ & $<0.05$ & $<0.05$ \\
\hline 11 & 1020 & 0.25 & $<0.05$ & 2.6 & 0.40 & 0.24 & $<0.05$ & 0.43 & $<0.05$ & $<0.05$ & $<0.05$ & $<0.05$ & $<0.05$ \\
\hline 15 & 0950 & $<0.05$ & $<0.05$ & 1.2 & 0.20 & 0.18 & $<0.05$ & 0.19 & $<0.05$ & $<0.05$ & $<0.05$ & $<0.05$ & $<0.05$ \\
\hline 25 & 1000 & $<0.05$ & $<0.05$ & 0.42 & $<0.20$ & 0.09 & $<0.05$ & $<0.05$ & $<0.05$ & $<0.05$ & $<0.05$ & $<0.05$ & $<0.05$ \\
\hline 29 & 0940 & $<0.05$ & $<0.05$ & 0.44 & $<0.20$ & 0.11 & $<0.05$ & $<0.05$ & $<0.05$ & $<0.05$ & $<0.05$ & $<0.05$ & $<0.05$ \\
\hline \multicolumn{14}{|c|}{ UGUST } \\
\hline 06 & 1130 & $<0.05$ & $<0.05$ & 0.22 & $<0.20$ & 0.08 & $<0.05$ & $<0.05$ & $<0.05$ & $<0.05$ & $<0.05$ & $<0.05$ & $<0.05$ \\
\hline 12 & 1115 & $<0.05$ & $<0.05$ & 0.30 & $<0.20$ & 0.09 & $<0.05$ & $<0.05$ & $<0.05$ & $<0.05$ & $<0.05$ & $<0.05$ & $<0.05$ \\
\hline 20 & 1000 & $<0.05$ & $<0.05$ & $<0.05$ & $<0.20$ & $<0.05$ & $<0.05$ & $<0.05$ & $<0.05$ & $<0.05$ & $<0.05$ & $<0.05$ & $<0.05$ \\
\hline 27 & 0940 & $<0.05$ & 0.09 & 0.34 & $<0.20$ & 0.11 & 0.08 & $<0.05$ & $<0.05$ & $<0.05$ & $<0.05$ & $<0.05$ & $<0.05$ \\
\hline
\end{tabular}


Tabie 7.--Pesticide results from soiid-phase extraction 100-miliiliter method--Continued

\begin{tabular}{|c|c|c|c|c|c|c|c|c|c|c|c|c|c|}
\hline Date & Time & $\begin{array}{c}\text { Ala- } \\
\text { chlor } \\
\text { diss } \\
(\mu g / L)\end{array}$ & $\begin{array}{l}\text { Ame- } \\
\text { tryn } \\
\text { dlss } \\
(\mu g / L)\end{array}$ & $\begin{array}{c}\text { Atra- } \\
\text { zine } \\
\text { dlss } \\
(\mu g / L)\end{array}$ & $\begin{array}{l}\text { Cyana- } \\
\text { zine } \\
\text { diss } \\
(\mu g / L)\end{array}$ & $\begin{array}{c}\text { Desethyl- } \\
\text { atrazine } \\
\text { diss } \\
(\mu g / L)\end{array}$ & $\begin{array}{c}\text { Desiso- } \\
\text { propyl- } \\
\text { atrazine } \\
\text { dlss } \\
(\mathrm{ua} / \mathrm{L})\end{array}$ & $\begin{array}{l}\text { Metola- } \\
\text { chlor } \\
\text { diss } \\
(\mu g / L)\end{array}$ & $\begin{array}{c}\text { Metrl- } \\
\text { buzin } \\
\text { diss } \\
(\mu g / L)\end{array}$ & $\begin{array}{c}\text { Pro- } \\
\text { metryn } \\
\text { diss } \\
(\mu g / L)\end{array}$ & $\begin{array}{c}\text { Pro- } \\
\text { meton } \\
\text { diss } \\
(\mu g / L)\end{array}$ & $\begin{array}{l}\text { Propa- } \\
\text { zine } \\
\text { diss } \\
(\mu g / L)\end{array}$ & $\begin{array}{c}\text { Sima- } \\
\text { zine } \\
\text { diss } \\
(\mu g / L)\end{array}$ \\
\hline
\end{tabular}

SEPTEMBER

\begin{tabular}{|c|c|c|c|c|c|c|c|c|c|c|c|c|c|}
\hline 03 & 0950 & $<0.05$ & $<0.05$ & 0.17 & $<0.20$ & $<0.05$ & $<0.05$ & $<0.05$ & $<0.05$ & $<0.05$ & $<0.05$ & $<0.05$ & $<0.05$ \\
\hline 10 & 1030 & $<0.05$ & $<0.05$ & 0.21 & $<0.20$ & 0.07 & $<0.05$ & $<0.05$ & $<0.05$ & $<0.05$ & $<0.05$ & $<0.05$ & $<0.05$ \\
\hline 17 & 1115 & $<0.05$ & $<0.05$ & 0.19 & $<0.20$ & 0.07 & $<0.10$ & $<0.05$ & $<0.05$ & $<0.05$ & $<0.05$ & $<0.05$ & $<0.05$ \\
\hline 24 & 1135 & $<0.05$ & $<0.05$ & 0.14 & $<0.20$ & $<0.05$ & $<0.05$ & $<0.05$ & $<0.05$ & $<0.05$ & $<0.05$ & $<0.05$ & $<0.05$ \\
\hline \multicolumn{14}{|c|}{ OCTOBER } \\
\hline 01 & 1115 & 0.05 & 0.11 & 0.22 & 0.10 & 0.10 & 0.06 & 0.06 & 0.11 & 0.11 & 0.15 & $<0.05$ & 0.06 \\
\hline 08 & 1030 & $<0.05$ & $<0.05$ & 0.15 & $<0.20$ & 0.06 & $<0.05$ & $<0.05$ & $<0.05$ & $<0.05$ & $<0.05$ & $<0.05$ & $<0.05$ \\
\hline 15 & 1130 & $<0.05$ & $<0.05$ & 0.14 & $<0.20$ & 0.07 & $<0.05$ & $<0.05$ & $<0.05$ & $<0.05$ & $<0.05$ & $<0.05$ & $<0.05$ \\
\hline 24 & 1200 & $<0.05$ & $<0.05$ & 0.34 & 0.50 & 0.09 & $<0.05$ & $<0.05$ & $<0.05$ & $<0.05$ & $<0.05$ & $<0.05$ & 0.06 \\
\hline \multicolumn{14}{|c|}{ NOVEMBER } \\
\hline 05 & 1200 & $<0.05$ & $<0.05$ & 0.25 & $<0.20$ & 0.07 & $<0.05$ & 0.06 & $<0.05$ & $<0.05$ & $<0.05$ & $<0.05$ & $<0.05$ \\
\hline 19 & 1140 & $<0.05$ & $<0.05$ & 0.62 & 1.2 & 0.05 & $<0.05$ & $<0.05$ & $<0.05$ & $<0.05$ & $<0.05$ & $<0.05$ & $<0.05$ \\
\hline \multicolumn{14}{|c|}{ DECEMBER } \\
\hline 05 & 1130 & -- & -- & -- & -. & $\cdots$ & - & -. & -. & -- & -- & -. & -. \\
\hline 20 & 1000 & $<0.05$ & $<0.05$ & 0.14 & $<0.20$ & 0.10 & $<0.05$ & 0.05 & $<0.05$ & $<0.05$ & $<0.05$ & $<0.05$ & $<0.05$ \\
\hline 30 & 1020 & $<0.10$ & $<0.10$ & $<0.10$ & $<0.20$ & $<0.10$ & $<0.05$ & $<0.10$ & $<0.10$ & $<0.10$ & $<0.10$ & $<0.10$ & $<0.10$ \\
\hline \multicolumn{14}{|c|}{ JANUARY 1992} \\
\hline 14 & 1000 & $<0.05$ & $<0.05$ & 0.10 & $<0.20$ & 0.07 & $<0.05$ & $<0.05$ & $<0.05$ & $<0.05$ & $<0.05$ & $<0.05$ & $<0.05$ \\
\hline 28 & 1000 & $<0.05$ & $<0.05$ & 0.11 & $<0.20$ & 0.09 & $<0.05$ & $<0.05$ & $<0.05$ & $<0.05$ & $<0.05$ & $<0.05$ & $<0.05$ \\
\hline \multicolumn{14}{|c|}{ FEBRUARY 1992} \\
\hline 11 & 1030 & $<0.05$ & $<0.05$ & 0.10 & $<0.20$ & 0.09 & $<0.05$ & $<0.05$ & $<0.05$ & $<0.05$ & $<0.05$ & $<0.05$ & $<0.05$ \\
\hline 27 & 1000 & $<0.05$ & $<0.05$ & 0.08 & $<0.20$ & 0.08 & $<0.05$ & $<0.05$ & $<0.05$ & $<0.05$ & $<0.05$ & $<0.05$ & $<0.05$ \\
\hline \multicolumn{14}{|l|}{ MARCH } \\
\hline 05 & 1010 & $<0.05$ & $<0.05$ & 0.15 & $<0.20$ & 0.08 & $<0.05$ & $<0.05$ & $<0.05$ & $<0.05$ & $<0.05$ & $<0.05$ & $<0.05$ \\
\hline 09 & 0945 & $<0.05$ & $<0.05$ & 0.11 & $<0.20$ & 0.09 & $<0.05$ & $<0.05$ & $<0.05$ & $<0.05$ & $<0.05$ & $<0.05$ & $<0.05$ \\
\hline 17 & 1145 & $<0.05$ & $<0.05$ & 0.13 & $<0.20$ & 0.08 & $<0.05$ & $<0.05$ & $<0.05$ & $<0.05$ & $<0.05$ & $<0.05$ & $<0.05$ \\
\hline 23 & 1040 & $<0.05$ & $<0.05$ & 0.10 & $<0.20$ & 0.08 & $<0.05$ & $<0.05$ & $<0.05$ & $<0.05$ & $<0.05$ & $<0.05$ & $<0.05$ \\
\hline 31 & 1110 & $<0.05$ & $<0.05$ & 0.10 & $<0.20$ & 0.08 & $<0.05$ & $<0.05$ & $<0.05$ & $<0.05$ & $<0.05$ & $<0.05$ & $<0.05$ \\
\hline 07 & & & & & & & & & & & & Cone & \\
\hline 14 & 1010 & $<0.05$ & $<0.05$ & 0.12 & $<0.20$ & 0.06 & $<0.05$ & 0.06 & $<0.05$ & $<0.05$ & $<0.05$ & $<0.05$ & $<0.05$ \\
\hline 23 & 1020 & 0.05 & $<0.05$ & 0.14 & $<0.20$ & 0.07 & $<0.05$ & 0.07 & $<0.05$ & $<0.05$ & $<0.05$ & $<0.05$ & $<0.05$ \\
\hline 29 & 1030 & $<0.05$ & $<0.05$ & 0.12 & $<0.20$ & 0.06 & $<0.05$ & 0.06 & $<0.05$ & $<0.05$ & $<0.05$ & $<0.05$ & $<0.05$ \\
\hline \multicolumn{14}{|l|}{ MAY } \\
\hline 07 & 1100 & 0.08 & $<0.05$ & 0.22 & $<0.20$ & $<0.05$ & $<0.05$ & 0.08 & $<0.05$ & $<0.05$ & $<0.05$ & $<0.05$ & 0.19 \\
\hline \multirow{2}{*}{\multicolumn{14}{|c|}{6934500 - Missouri River at Hermann, Missouri }} \\
\hline PRIL 1 & & & & & & & & & & & & & \\
\hline 09 & 1331 & 0.07 & $<0.05$ & 0.37 & $<0.20$ & 0.15 & 0.11 & 0.15 & $<0.05$ & $<0.05$ & 0.08 & $<0.05$ & $<0.05$ \\
\hline 16 & 1030 & 0.21 & $<0.05$ & 1.2 & 0.60 & $<0.05$ & $<0.05$ & 0.38 & $<0.05$ & $<0.05$ & $<0.05$ & $<0.05$ & $<0.05$ \\
\hline 24 & 1200 & 0.11 & $<0.05$ & 1.1 & 0.45 & 0.06 & $<0.05$ & 0.53 & $<0.05$ & $<0.05$ & $<0.05$ & $<0.05$ & $<0.05$ \\
\hline \multicolumn{14}{|l|}{ MAY } \\
\hline 01 & 1130 & 0.26 & $<0.05$ & 1.6 & 0.70 & 0.11 & 0.08 & 0.79 & $<0.05$ & $<0.05$ & $<0.05$ & $<0.05$ & $<0.05$ \\
\hline 06 & 1100 & 0.33 & $<0.05$ & 2.6 & 1.5 & 0.22 & 0.10 & 1.0 & $<0.05$ & $<0.05$ & $<0.05$ & $<0.05$ & $<0.05$ \\
\hline 09 & 0930 & 0.47 & $<0.05$ & 3.2 & 0.80 & 0.27 & 0.20 & 0.77 & 0.18 & $<0.05$ & $<0.05$ & $<0.05$ & 0.09 \\
\hline 13 & 1000 & 0.18 & $<0.05$ & 1.9 & 0.60 & 0.23 & 0.18 & 0.55 & $<0.05$ & $<0.05$ & $<0.05$ & $<0.05$ & 0.07 \\
\hline 16 & 1020 & 0.17 & $<0.05$ & 1.8 & 0.50 & 0.18 & $<0.05$ & 0.43 & $<0.05$ & $<0.05$ & $<0.05$ & $<0.05$ & $<0.05$ \\
\hline 20 & 1000 & 0.19 & $<0.05$ & 1.7 & 0.70 & 0.13 & $<0.05$ & 0.48 & $<0.05$ & $<0.05$ & $<0.05$ & $<0.05$ & $<0.05$ \\
\hline 24 & 1000 & 0.68 & $<0.05$ & 2.7 & 2.1 & 0.10 & $<0.05$ & 1.4 & $<0.05$ & $<0.05$ & $<0.05$ & $<0.05$ & $<0.05$ \\
\hline
\end{tabular}




\begin{tabular}{|c|c|c|c|c|c|c|c|c|c|c|c|c|c|}
\hline Date & Time & $\begin{array}{l}\text { Ala- } \\
\text { chior } \\
\text { diss } \\
(\mu g / L)\end{array}$ & $\begin{array}{l}\text { Ame- } \\
\text { tryn } \\
\text { diss } \\
(\mu g / L)\end{array}$ & $\begin{array}{c}\text { Atra- } \\
\text { zine } \\
\text { diss } \\
(\mu g / L)\end{array}$ & $\begin{array}{l}\text { Cyana- } \\
\text { zine } \\
\text { diss } \\
(\mu g / L)\end{array}$ & $\begin{array}{c}\text { Desethyl- } \\
\text { atrazina } \\
\text { diss } \\
(\mu g / L)\end{array}$ & $\begin{array}{l}\text { Desiao- } \\
\text { propyl- } \\
\text { atrazine } \\
\text { diss } \\
(\mu \mathrm{g} /)\end{array}$ & $\begin{array}{l}\text { Metola- } \\
\text { chlor } \\
\text { diss } \\
(\mu \mathrm{g} / \mathrm{L})\end{array}$ & $\begin{array}{l}\text { Motri- } \\
\text { buzin } \\
\text { diss } \\
(\mu g / L)\end{array}$ & $\begin{array}{c}\text { Pro- } \\
\text { motryn } \\
\text { diss } \\
(\mu g / L)\end{array}$ & $\begin{array}{l}\text { Pro- } \\
\text { moton } \\
\text { diss } \\
(\mu g / L)\end{array}$ & $\begin{array}{l}\text { Propa- } \\
\text { zine } \\
\text { diss } \\
(\mu g / L)\end{array}$ & $\begin{array}{l}\text { Sima- } \\
\text { zine } \\
\text { diss } \\
(\mu g / L)\end{array}$ \\
\hline
\end{tabular}

MAY (cont.)

\begin{tabular}{|c|c|c|c|c|c|c|c|c|c|c|c|c|c|}
\hline 28 & 1030 & 0.57 & $<0.05$ & 2.9 & 1.6 & 0.24 & $<0.05$ & 1.3 & 0.09 & $<0.05$ & $<0.05$ & $<0.05$ & $<0.05$ \\
\hline 31 & 1040 & 0.42 & $<0.05$ & 3.2 & 2.0 & 0.17 & $<0.05$ & 1.1 & 0.08 & $<0.05$ & $<0.05$ & $<0.05$ & $<0.05$ \\
\hline \multicolumn{14}{|l|}{ JUNE } \\
\hline 03 & 1000 & 0.27 & $<0.05$ & 3.1 & 1.6 & 0.29 & $<0.05$ & 0.80 & $<0.05$ & $<0.05$ & $<0.05$ & $<0.05$ & $<0.05$ \\
\hline 06 & 1000 & 0.57 & $<0.05$ & 5.4 & 2.0 & 0.24 & 0.14 & 1.7 & 0.09 & $<0.05$ & $<0.05$ & $<0.05$ & $<0.05$ \\
\hline 11 & 1000 & 0.41 & $<0.05$ & 5.7 & 4.3 & 0.48 & 0.25 & 1.4 & 0.17 & $<0.05$ & 0.06 & 0.06 & 0.08 \\
\hline 13 & 1000 & 0.92 & $<0.05$ & 5.7 & 4.7 & 0.52 & 0.16 & 1.7 & 0.12 & $<0.05$ & $<0.05$ & $<0.05$ & 0.06 \\
\hline 21 & 1000 & 0.43 & $<0.05$ & 5.5 & 1.8 & 0.35 & 0.18 & 2.0 & 0.13 & $<0.05$ & $<0.05$ & $<0.05$ & $<0.05$ \\
\hline 25 & 1130 & 0.23 & $<0.05$ & 3.9 & 2.1 & 0.39 & $<0.05$ & 1.7 & 0.12 & $<0.05$ & $<0.05$ & $<0.05$ & $<0.05$ \\
\hline 27 & 1045 & 0.29 & $<0.05$ & 3.5 & 0.60 & 0.29 & 0.28 & 0.93 & 0.19 & $<0.05$ & 0.09 & 0.05 & 0.07 \\
\hline \multicolumn{14}{|c|}{ JULY 1992} \\
\hline 01 & 1100 & 0.14 & $<0.05$ & 1.2 & 0.32 & 0.16 & 0.13 & 0.35 & 0.13 & $<0.05$ & 0.05 & $<0.05$ & $<0.05$ \\
\hline 03 & 1000 & 0.08 & $<0.05$ & 1.1 & 0.30 & 0.14 & 0.10 & 0.30 & $<0.05$ & $<0.05$ & $<0.05$ & $<0.05$ & $<0.05$ \\
\hline 08 & 1030 & 0.05 & $<0.05$ & 0.74 & 0.20 & 0.12 & 0.09 & 0.18 & $<0.05$ & $<0.05$ & $<0.05$ & $<0.05$ & $<0.05$ \\
\hline 11 & 1000 & $<0.05$ & $<0.05$ & 0.59 & $<0.20$ & 0.05 & $<0.05$ & 0.13 & $<0.05$ & $<0.05$ & $<0.05$ & $<0.05$ & $<0.05$ \\
\hline 15 & 1030 & $<0.05$ & $<0.05$ & 1.3 & $<0.20$ & 0.11 & $<0.05$ & 0.27 & 0.13 & $<0.05$ & $<0.05$ & $<0.05$ & $<0.05$ \\
\hline 22 & 1015 & $<0.05$ & $<0.05$ & 1.0 & 0.30 & 0.19 & 0.11 & 0.19 & 0.12 & $<0.05$ & 0.14 & $<0.05$ & $<0.05$ \\
\hline 29 & 1100 & $<0.05$ & $<0.05$ & 0.52 & $<0.20$ & 0.10 & $<0.05$ & 0.10 & $<0.05$ & $<0.05$ & $<0.05$ & $<0.05$ & $<0.05$ \\
\hline \multicolumn{14}{|c|}{ AUGUST } \\
\hline 05 & 1000 & $<0.05$ & $<0.05$ & 0.23 & $<0.20$ & $<0.05$ & $<0.05$ & 0.08 & $<0.05$ & $<0.05$ & $<0.05$ & $<0.05$ & $<0.05$ \\
\hline 13 & 1100 & $<0.05$ & $<0.05$ & 0.14 & $<0.20$ & $<0.05$ & $<0.05$ & 0.05 & $<0.05$ & $<0.05$ & $<0.05$ & $<0.05$ & $<0.05$ \\
\hline 19 & 1000 & $<0.05$ & $<0.05$ & 0.22 & $<0.20$ & $<0.05$ & $<0.05$ & 0.09 & $<0.05$ & $<0.05$ & $<0.05$ & $<0.05$ & $<0.05$ \\
\hline 27 & 1100 & $<0.05$ & $<0.05$ & 0.30 & $<0.20$ & 0.12 & 0.09 & 0.12 & $<0.05$ & 0.07 & 0.14 & $<0.05$ & $<0.05$ \\
\hline \multicolumn{14}{|c|}{ SEPTEMBER } \\
\hline 05 & 1030 & $<0.05$ & $<0.05$ & 0.16 & $<0.20$ & $<0.05$ & $<0.05$ & 0.10 & $<0.05$ & $<0.05$ & $<0.05$ & $<0.05$ & $<0.05$ \\
\hline 09 & 1000 & $<0.05$ & $<0.05$ & 0.09 & $<0.20$ & $<0.05$ & $<0.05$ & $<0.05$ & $<0.05$ & $<0.05$ & $<0.05$ & $<0.05$ & $<0.05$ \\
\hline 16 & 1000 & 0.05 & $<0.05$ & 0.14 & $<0.20$ & $<0.05$ & $<0.05$ & 0.08 & $<0.05$ & $<0.05$ & $<0.05$ & $<0.05$ & $<0.05$ \\
\hline 23 & 0945 & $<0.05$ & $<0.05$ & 0.14 & $<0.20$ & $<0.05$ & $<0.10$ & 0.06 & $<0.05$ & $<0.05$ & $<0.05$ & $<0.05$ & $<0.05$ \\
\hline \multicolumn{14}{|c|}{ OCTOBER } \\
\hline 01 & 1030 & $<0.05$ & $<0.05$ & 0.18 & $<0.20$ & 0.06 & $<0.05$ & 0.05 & $<0.05$ & $<0.05$ & $<0.05$ & $<0.05$ & $<0.05$ \\
\hline 07 & 1000 & $<0.05$ & $<0.05$ & 0.15 & $<0.20$ & 0.07 & $<0.05$ & 0.05 & $<0.05$ & $<0.05$ & $<0.05$ & $<0.05$ & $<0.05$ \\
\hline 15 & 1100 & $<0.05$ & $<0.05$ & 0.14 & $<0.20$ & 0.06 & $<0.10$ & $<0.05$ & $<0.05$ & $<0.05$ & $<0.05$ & $<0.05$ & $<0.05$ \\
\hline 21 & 1000 & $<0.05$ & $<0.05$ & 0.42 & $<0.20$ & 0.08 & $<0.10$ & 0.29 & $<0.05$ & $<0.05$ & $<0.05$ & $<0.05$ & $<0.05$ \\
\hline 29 & 1000 & $<0.05$ & $<0.05$ & 0.18 & $<0.20$ & 0.09 & $<0.05$ & 0.09 & $<0.05$ & $<0.05$ & $<0.05$ & $<0.05$ & $<0.05$ \\
\hline \multicolumn{14}{|c|}{ NOVEMBER } \\
\hline 04 & 1030 & $<0.05$ & $<0.05$ & 0.16 & $<0.20$ & 0.05 & $<0.05$ & 0.07 & $<0.05$ & $<0.05$ & $<0.05$ & $<0.05$ & $<0.05$ \\
\hline 21 & 1030 & $<0.05$ & $<0.05$ & 0.18 & $<0.20$ & 0.05 & $<0.05$ & 0.18 & $<0.05$ & $<0.05$ & $<0.05$ & $<0.05$ & $<0.05$ \\
\hline \multicolumn{14}{|c|}{ DECEMBER } \\
\hline 06 & 1240 & $<0.05$ & $<0.05$ & 0.13 & $<0.20$ & $<0.05$ & $<0.05$ & 0.07 & $<0.05$ & $<0.05$ & $<0.05$ & $<0.05$ & $<0.05$ \\
\hline 16 & 1130 & $<0.05$ & $<0.05$ & 0.21 & $<0.20$ & 0.05 & $<0.05$ & 0.30 & $<0.05$ & $<0.05$ & $<0.05$ & $<0.05$ & $<0.05$ \\
\hline \multicolumn{14}{|c|}{ JANUARY 1992} \\
\hline 02 & 1030 & $<0.05$ & $<0.05$ & 0.14 & $<0.20$ & 0.06 & $<0.05$ & 0.08 & $<0.05$ & $<0.05$ & $<0.05$ & $<0.05$ & $<0.05$ \\
\hline 16 & 1200 & $<0.10$ & $<0.10$ & 0.30 & $<0.20$ & $<0.10$ & $<0.10$ & 0.40 & $<0.10$ & $<0.10$ & $<0.10$ & $<0.10$ & $<0.10$ \\
\hline 28 & 1300 & $<0.05$ & $<0.05$ & 0.21 & $<0.20$ & 0.06 & $<0.05$ & 0.33 & $<0.05$ & $<0.05$ & $<0.05$ & $<0.05$ & $<0.05$ \\
\hline \multicolumn{14}{|c|}{ FEBRUARY } \\
\hline 10 & 1000 & $<0.05$ & $<0.05$ & 0.12 & $<0.20$ & 0.07 & $<0.05$ & 0.12 & $<0.05$ & $<0.05$ & $<0.05$ & $<0.05$ & $<0.05$ \\
\hline 24 & 1200 & $<0.05$ & $<0.05$ & 0.18 & $<0.20$ & 0.09 & $<0.05$ & 0.12 & $<0.05$ & $<0.05$ & $<0.05$ & $<0.05$ & $<0.05$ \\
\hline
\end{tabular}


Tabie 7.-Pesticide results from solid-phase extraction 100-milliliter method--Continued

\begin{tabular}{|c|c|c|c|c|c|c|c|c|c|c|c|c|c|}
\hline Date & TIme & $\begin{array}{c}\text { Ala- } \\
\text { chlor } \\
\text { diss } \\
(\mu g / L)\end{array}$ & $\begin{array}{c}\text { Ame- } \\
\text { tryn } \\
\text { diss } \\
(\mu g / L)\end{array}$ & $\begin{array}{l}\text { Atra- } \\
\text { zlne } \\
\text { diss } \\
(\mu g / L)\end{array}$ & $\begin{array}{l}\text { Cyana- } \\
\text { zlne } \\
\text { dlss } \\
(\mu g / 2)\end{array}$ & $\begin{array}{l}\text { Desethyl- } \\
\text { atrazine } \\
\text { diss } \\
(\mu g / L)\end{array}$ & $\begin{array}{c}\text { Deslso- } \\
\text { propyl- } \\
\text { atrazine } \\
\text { dlss } \\
(\mu \mathrm{g} / \mathrm{L})\end{array}$ & $\begin{array}{l}\text { Metola- } \\
\text { chlor } \\
\text { dlss } \\
(\mu g / 2)\end{array}$ & $\begin{array}{c}\text { Metri- } \\
\text { buzln } \\
\text { diss } \\
(\mu g / L)\end{array}$ & $\begin{array}{c}\text { Pro- } \\
\text { metryn } \\
\text { diss } \\
(\mu g / L)\end{array}$ & $\begin{array}{l}\text { Pro- } \\
\text { meton } \\
\text { diss } \\
(\mu g / L)\end{array}$ & $\begin{array}{l}\text { Propa- } \\
\text { zlne } \\
\text { dlss } \\
(\mu g / L)\end{array}$ & $\begin{array}{l}\text { Sima- } \\
\text { zine } \\
\text { diss } \\
(\mu g / L)\end{array}$ \\
\hline \multicolumn{14}{|c|}{ 6934500 - Missouri River at Hermann, Missouri-Continued } \\
\hline \multicolumn{14}{|l|}{ MARCH } \\
\hline 03 & 1100 & $<0.05$ & $<0.05$ & 0.21 & $<0.20$ & 0.09 & $<0.05$ & 0.11 & $<0.05$ & $<0.05$ & $<0.05$ & $<0.05$ & $<0.05$ \\
\hline 12 & 1200 & $<0.05$ & $<0.05$ & 0.29 & $<0.20$ & 0.11 & $<0.05$ & 0.15 & $<0.05$ & $<0.05$ & $<0.05$ & $<0.05$ & $<0.05$ \\
\hline 20 & 0830 & $<0.05$ & $<0.05$ & 0.23 & $<0.20$ & 0.07 & $<0.05$ & 0.13 & $<0.05$ & $<0.05$ & $<0.05$ & $<0.05$ & $<0.05$ \\
\hline 26 & 1130 & $<0.05$ & $<0.05$ & 0.34 & $<0.20$ & 0.10 & $<0.05$ & 0.12 & $<0.05$ & $<0.05$ & $<0.05$ & $<0.05$ & $<0.05$ \\
\hline \multicolumn{14}{|c|}{ APRIL 1992} \\
\hline 01 & 1000 & $<0.05$ & $<0.05$ & 0.22 & $<0.20$ & 0.06 & $<0.10$ & 0.08 & $<0.05$ & $<0.05$ & $<0.05$ & $<0.05$ & $<0.05$ \\
\hline 06 & 1230 & $<0.05$ & $<0.05$ & 0.26 & $<0.20$ & 0.07 & $<0.05$ & 0.12 & $<0.05$ & $<0.05$ & $<0.05$ & $<0.05$ & $<0.05$ \\
\hline 14 & 1030 & 0.07 & $<0.05$ & 0.54 & 0.20 & $<0.05$ & $<0.05$ & 0.28 & $<0.05$ & $<0.05$ & $<0.05$ & $<0.05$ & $<0.05$ \\
\hline 21 & 1300 & 0.07 & $<0.05$ & 0.94 & 0.50 & 0.08 & $<0.05$ & 0.40 & $<0.05$ & $<0.05$ & $<0.05$ & $<0.05$ & $<0.05$ \\
\hline 29 & 1015 & 0.14 & $<0.05$ & 1.4 & 0.70 & 0.13 & $<0.05$ & 0.60 & $<0.05$ & $<0.05$ & $<0.05$ & $<0.05$ & $<0.05$ \\
\hline \multicolumn{14}{|l|}{ MAY } \\
\hline 12 & 1000 & $<0.05$ & $<0.05$ & 0.29 & $<0.20$ & 0.06 & $<0.05$ & 0.27 & $<0.05$ & $<0.05$ & $<0.05$ & $<0.05$ & $<0.05$ \\
\hline 21 & 1200 & $<0.05$ & $<0.05$ & 0.33 & 0.20 & 0.06 & 0.07 & 0.20 & $<0.05$ & $<0.05$ & 05 & $<0.05$ & $<0.05$ \\
\hline 26 & 1130 & 0.98 & $<0.05$ & 5.6 & 5.2 & 0.31 & 0.30 & 2.6 & 0.08 & $<0.05$ & $<0.05$ & 0.05 & 0.11 \\
\hline \multicolumn{14}{|l|}{ JUNE } \\
\hline 01 & 1330 & 0.37 & $<0.05$ & 3.1 & 0.80 & 0.25 & 0.15 & 1.2 & 0.06 & $<0.05$ & $<0.05$ & $<0.05$ & 0.10 \\
\hline 09 & 1030 & $<0.05$ & $<0.05$ & 0.17 & $<0.20$ & 0.09 & $<0.05$ & 0.05 & $<0.05$ & $<0.05$ & $<0.05$ & $<0.05$ & $<0.05$ \\
\hline 15 & 1230 & 0.19 & $<0.05$ & 1.2 & 0.40 & 0.17 & 0.12 & 0.45 & 0.09 & $<0.05$ & $<0.05$ & $<0.05$ & $<0.05$ \\
\hline 22 & 0945 & 0.13 & $<0.05$ & 1.4 & 0.40 & 0.25 & 0.15 & 0.38 & 0.08 & $<0.05$ & $<0.05$ & $<0.05$ & 0.05 \\
\hline 30 & 1130 & 0.10 & $<0.05$ & 1.7 & $<0.20$ & 0.23 & 0.17 & 0.53 & 0.11 & $<0.05$ & $<0.05$ & $<0.05$ & $<0.05$ \\
\hline \multicolumn{14}{|l|}{ JULY 1992} \\
\hline 07 & 0930 & $<0.05$ & $<0.05$ & 0.90 & 0.20 & 0.17 & 0.10 & 0.18 & 0.07 & $<0.05$ & $<0.05$ & $<0.05$ & $<0.05$ \\
\hline 14 & 1200 & 0.16 & $<0.05$ & 1.8 & 0.50 & 0.31 & 0.16 & 0.43 & 0.06 & $<0.05$ & $<0.05$ & $<0.05$ & 0.05 \\
\hline 21 & 1030 & 0.24 & $<0.05$ & 3.4 & 0.70 & 0.73 & 0.34 & 0.79 & 0.08 & $<0.05$ & $<0.05$ & $<0.05$ & 0.06 \\
\hline 28 & 1230 & 0.07 & $<0.05$ & 1.3 & $<0.20$ & 0.40 & 0.19 & 0.30 & 0.06 & $<0.05$ & $<0.05$ & $<0.05$ & $<0.05$ \\
\hline \multicolumn{14}{|c|}{07022000 - Mississippi River at Thebes, $\mathrm{Ill}$. } \\
\hline \multicolumn{14}{|c|}{ APRIL 1991} \\
\hline 11 & 1500 & 0.31 & $<0.05$ & 0.64 & $<0.20$ & 0.37 & $<0.05$ & 0.20 & $<0.05$ & $<0.05$ & $<0.05$ & $<0.05$ & $<0.05$ \\
\hline 18 & 1130 & -- & -- & -- & -- & -- & -- & -- & -- & -- & -- & -- & -- \\
\hline 24 & 0930 & 0.21 & $<0.05$ & 1.0 & 0.52 & 0.10 & $<0.05$ & 0.52 & $<0.05$ & 0.05 & $<0.05$ & 0.05 & 0.05 \\
\hline 29 & 1230 & 0.12 & $<0.05$ & 0.63 & 0.40 & 0.11 & $<0.05$ & 0.31 & $<0.05$ & $<0.05$ & $<0.05$ & $<0.05$ & $<0.05$ \\
\hline \multicolumn{14}{|l|}{ MAY } \\
\hline 07 & 1345 & 0.58 & $<0.05$ & 3.2 & 3.1 & 0.28 & $<0.05$ & & 0.09 & $<0.05$ & $<0.05$ & $<0.05$ & $<0.05$ \\
\hline 09 & 0900 & 0.34 & $<0.05$ & 1.7 & 1.4 & 0.17 & $<0.05$ & 0.58 & 0.09 & $<0.05$ & $<0.05$ & $<0.05$ & $<0.05$ \\
\hline 13 & 1330 & 0.63 & $<0.05$ & 3.6 & 1.6 & 0.34 & 0.18 & 1.1 & 0.17 & $<0.05$ & $<0.05$ & $<0.05$ & 0.10 \\
\hline 16 & 1200 & 0.31 & $<0.05$ & 1.3 & 1.0 & 0.15 & $<0.05$ & 0.69 & $<0.05$ & $<0.05$ & $<0.05$ & $<0.05$ & $<0.05$ \\
\hline 20 & 1430 & 0.13 & $<0.05$ & 0.80 & 0.50 & 0.11 & $<0.05$ & 0.24 & $<0.05$ & $<0.05$ & $<0.05$ & $<0.05$ & $<0.05$ \\
\hline 23 & 0916 & 0.59 & $<0.05$ & 2.4 & 2.3 & 0.30 & $<0.05$ & 0.75 & 0.08 & $<0.05$ & $<0.05$ & $<0.05$ & $<0.05$ \\
\hline 28 & 1300 & 0.65 & $<0.05$ & 2.4 & 2.8 & 0.26 & $<0.05$ & 1.4 & $<0.05$ & $<0.05$ & $<0.05$ & $<0.05$ & $<0.05$ \\
\hline 30 & 1330 & 0.77 & $<0.05$ & 3.0 & 2.5 & 0.39 & $<0.05$ & 1.7 & 0.08 & $<0.05$ & $<0.05$ & $<0.05$ & $<0.05$ \\
\hline \multicolumn{14}{|l|}{ JUNE 1991} \\
\hline 03 & 1345 & 0.46 & $<0.05$ & 2.0 & 1.3 & 0.14 & $<0.05$ & 1.2 & $<0.05$ & $<0.05$ & $<0.05$ & $<0.05$ & $<0.05$ \\
\hline 06 & 1045 & 0.39 & $<0.05$ & 2.5 & 1.3 & 0.15 & $<0.05$ & 1.1 & $<0.05$ & $<0.05$ & $<0.05$ & $<0.05$ & $<0.05$ \\
\hline 10 & 1400 & 0.86 & $<0.05$ & 4.2 & 1.8 & 0.40 & $<0.05$ & 1.9 & $<0.05$ & $<0.05$ & $<0.05$ & $<0.05$ & $<0.05$ \\
\hline 13 & 1500 & 0.84 & $<0.05$ & 3.9 & 2.3 & 0.39 & $<0.05$ & 2.2 & $<0.05$ & $<0.05$ & $<0.05$ & $<0.05$ & $<0.05$ \\
\hline 18 & 1430 & 0.39 & $<0.05$ & 2.2 & 1.4 & 0.15 & $<0.05$ & 1.1 & $<0.05$ & $<0.05$ & $<0.05$ & $<0.05$ & $<0.05$ \\
\hline 20 & 1100 & 0.19 & $<0.05$ & 1.2 & 0.50 & 0.08 & $<0.05$ & 0.58 & $<0.05$ & $<0.05$ & $<0.05$ & $<0.05$ & $<0.05$ \\
\hline
\end{tabular}


Table 7.--Pesticide resuits from solid-phase extraction 100-miiiiiiter method--Continued

\begin{tabular}{|c|c|c|c|c|c|c|c|c|c|c|c|c|c|}
\hline Date & Time & $\begin{array}{l}\text { Ala- } \\
\text { chlor } \\
\text { diss } \\
(\mu g / \mathrm{L})\end{array}$ & $\begin{array}{l}\text { Ame- } \\
\text { tryn } \\
\text { diss } \\
(\mu g h)\end{array}$ & 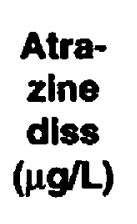 & $\begin{array}{l}\text { Cyana- } \\
\text { zlne } \\
\text { dlss } \\
(\mu g / \text { ) }\end{array}$ & $\begin{array}{c}\text { Desethyl- } \\
\text { atraztne } \\
\text { dlss } \\
(\mu g /)\end{array}$ & $\begin{array}{l}\text { Desiso- } \\
\text { propyl- } \\
\text { atrazine } \\
\text { diss } \\
\text { ( } \mathrm{g} / \mathrm{l} \text { ) }\end{array}$ & $\begin{array}{l}\text { Metola- } \\
\text { chlor } \\
\text { diss } \\
(\mu g / \mathrm{L})\end{array}$ & $\begin{array}{l}\text { Metrl- } \\
\text { buzin } \\
\text { dlss } \\
\text { (ugn) }\end{array}$ & $\begin{array}{l}\text { Pro- } \\
\text { metryn } \\
\text { diss } \\
(\mu g / 4)\end{array}$ & $\begin{array}{l}\text { Pro- } \\
\text { meton } \\
\text { diss } \\
(\mu g / L)\end{array}$ & $\begin{array}{l}\text { Prope- } \\
\text { zine } \\
\text { diss } \\
(\mu g / \mathrm{L})\end{array}$ & 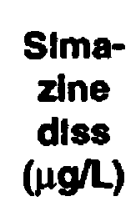 \\
\hline
\end{tabular}

JUNE 1991 (cont.)

07022000 - Mississippi River at Thebes, Ill.-Continued

\begin{tabular}{|c|c|c|c|c|c|c|c|c|c|c|c|c|c|}
\hline 24 & 1300 & 0.36 & $<0.05$ & 3.2 & 1.8 & 0.29 & $<0.05$ & 1.4 & 0.08 & $<0.05$ & $<0.05$ & $<0.05$ & 0.05 \\
\hline 27 & 1400 & 0.85 & $<0.05$ & 3.3 & 0.60 & 0.37 & 0.34 & 1.8 & 0.18 & $<0.05$ & 0.09 & 0.05 & 0.08 \\
\hline \multicolumn{14}{|l|}{ JULY } \\
\hline 01 & 1320 & 0.38 & $<0.05$ & 1.3 & 0.59 & 0.20 & $<0.05$ & 0.78 & 0.16 & $<0.05$ & $<0.05$ & $<0.05$ & 0.05 \\
\hline 03 & 0950 & 0.29 & $<0.05$ & 1.1 & 0.50 & 0.18 & $<0.05$ & 0.72 & 0.1 & $<0.05$ & $<0.05$ & $<0.05$ & $<0.05$ \\
\hline 08 & 1330 & 0.21 & $<0.05$ & 1.0 & 0.50 & 0.16 & $<0.05$ & 0.66 & $<0.05$ & $<0.05$ & $<0.05$ & $<0.05$ & 0.05 \\
\hline 11 & 1400 & 0.19 & $<0.05$ & 0.97 & 0.60 & 0.10 & $<0.05$ & 0.70 & $<0.05$ & $<0.05$ & $<0.05$ & $<0.05$ & $<0.05$ \\
\hline 18 & 0930 & 0.32 & $<0.05$ & 1.0 & 0.40 & 0.19 & 0.16 & 0.35 & 0.1 & $<0.05$ & 0.13 & $<0.05$ & 0.05 \\
\hline 23 & 1300 & 0.18 & $<0.05$ & 0.88 & $<0.20$ & $<0.05$ & $<0.05$ & 0.32 & $<0.05$ & $<0.05$ & $<0.05$ & $<0.05$ & $<0.05$ \\
\hline 30 & 1000 & 0.13 & $<0.05$ & 0.75 & 0.40 & 0.15 & $<0.05$ & 0.31 & $<0.05$ & $<0.05$ & $<0.05$ & $<0.05$ & $<0.05$ \\
\hline \multicolumn{14}{|c|}{ AUGUST } \\
\hline 06 & 1300 & $<0.05$ & $<0.05$ & 0.26 & $<0.20$ & 0.06 & $<0.05$ & 0.09 & $<0.05$ & $<0.05$ & $<0.05$ & $<0.05$ & $<0.05$ \\
\hline 13 & 0930 & 0.05 & $<0.05$ & 0.55 & $<0.20$ & $<0.05$ & $<0.05$ & 0.27 & $<0.05$ & $<0.05$ & $<0.05$ & $<0.05$ & $<0.05$ \\
\hline 20 & 1330 & $<0.05$ & $<0.05$ & 0.34 & 0.20 & 0.11 & $<0.05$ & 0.14 & $<0.05$ & $<0.05$ & $<0.05$ & $<0.05$ & $<0.05$ \\
\hline 29 & 1100 & 0.05 & $<0.05$ & 0.39 & $<0.20$ & 0.13 & 0.12 & 0.12 & $<0.05$ & $<0.05$ & 0.14 & $<0.05$ & $<0.05$ \\
\hline \multicolumn{14}{|c|}{ SEPTEMBER } \\
\hline 03 & 1500 & 0.06 & $<0.05$ & 0.38 & $<0.20$ & 0.14 & $<0.05$ & 0.16 & $<0.05$ & $<0.05$ & $<0.05$ & $<0.05$ & $<0.05$ \\
\hline 11 & 1000 & $<0,05$ & $<0.05$ & 0.21 & $<0.20$ & 0.06 & $<0.05$ & 0.08 & $<0.05$ & $<0.05$ & $<0.05$ & $<0.05$ & $<0.05$ \\
\hline 17 & 1400 & $<0.05$ & $<0.05$ & 0.25 & 0.30 & 0.09 & $<0.10$ & 0.09 & $<0.05$ & $<0.05$ & $<0.05$ & $<0.05$ & $<0.05$ \\
\hline 26 & 0930 & $<0.05$ & $<0.05$ & 0.41 & 1.2 & 0.08 & $<0.10$ & 0.23 & $<0.05$ & $<0.05$ & $<0.05$ & $<0.05$ & $<0.05$ \\
\hline \multicolumn{14}{|c|}{ OCTOBER } \\
\hline 02 & 1300 & 0.05 & $<0.05$ & 0.20 & $<0.20$ & $<0.05$ & $<0.05$ & 0.05 & $<0.05$ & $<0.05$ & $<0.05$ & $<0.05$ & 0.03 \\
\hline 09 & 1030 & $<0.05$ & $<0.05$ & 0.13 & $<0.20$ & 0.05 & $<0.10$ & $<0.05$ & $<0.05$ & $<0.05$ & $<0.05$ & $<0.05$ & $<0.05$ \\
\hline 16 & 1430 & $<0.05$ & $<0.05$ & 0.20 & $<0.20$ & 0.09 & $<0.10$ & 0.05 & $<0.05$ & $<0.05$ & $<0.05$ & $<0.05$ & $<0.05$ \\
\hline 23 & 0945 & 0.05 & $<0.05$ & 0.18 & $<0.20$ & 0.08 & $<0.10$ & $<0.05$ & $<0.05$ & $<0.05$ & $<0.05$ & $<0.05$ & $<0.05$ \\
\hline 30 & 1400 & 0.11 & $<0.05$ & 0.25 & $<0.20$ & 0.10 & $<0.05$ & 0.12 & $<0.05$ & 0.07 & $<0.05$ & $<0.05$ & $<0.05$ \\
\hline NOVEM & & & & , & & & & & & & & & \\
\hline 07 & 0800 & 0.10 & $<0.05$ & 0.20 & $<0.20$ & 0.09 & $<0.05$ & 0.11 & $<0.05$ & $<0.05$ & 0.06 & $<0.05$ & 0.05 \\
\hline 20 & 1030 & 0.10 & $<0.05$ & 0.22 & $<0.20$ & 0.10 & $<0.05$ & 0.12 & $<0.05$ & $<0.05$ & $<0.05$ & $<0.05$ & $<0.05$ \\
\hline \multicolumn{14}{|c|}{ DECEMBER } \\
\hline 05 & 1045 & 0.07 & $<0.05$ & 0.14 & $<0.20$ & 0.07 & $<0.10$ & 0.06 & $<0.05$ & $<0.05$ & $<0.05$ & $<0.05$ & $<0.05$ \\
\hline 18 & 1100 & 0.07 & $<0.05$ & 0.14 & $<0.20$ & 0.08 & $<0.05$ & 0.07 & $<0.05$ & $<0.05$ & $<0.05$ & $<0.05$ & $<0.05$ \\
\hline 30 & 1400 & 0.08 & $<0.05$ & 0.24 & $<0.20$ & 0.13 & $<0.10$ & 0.12 & $<0.05$ & $<0.05$ & $<0.05$ & $<0.05$ & $<0.05$ \\
\hline \multicolumn{14}{|c|}{ JANUARY 1992} \\
\hline 24 & 0930 & $<0.05$ & $<0.05$ & 0.24 & $<0.20$ & 0.09 & $<0.05$ & 0.14 & $<0.05$ & $<0.05$ & $<0.05$ & $<0.05$ & $<0.05$ \\
\hline \multicolumn{14}{|c|}{ FEBRUARY } \\
\hline 04 & 1030 & 0.07 & $<0.05$ & 0.15 & $<0.20$ & 0.08 & $<0.05$ & 0.09 & $<0.05$ & $<0.05$ & $<0.05$ & $<0.05$ & $<0.05$ \\
\hline 19 & 0930 & $<0.05$ & $<0.05$ & 0.12 & $<0.20$ & 0.09 & $<0.05$ & 0,06 & $<0.05$ & $<0.05$ & $<0.05$ & $<0.05$ & $<0.05$ \\
\hline \multicolumn{14}{|l|}{ MARCH } \\
\hline 04 & 0945 & $<0.05$ & $<0.05$ & 0.16 & $<0.20$ & 0.09 & $<0.05$ & 0.08 & $<0.05$ & $<0.05$ & $<0.05$ & $<0.05$ & $<0.05$ \\
\hline 09 & 1300 & $<0.05$ & $<0.05$ & 0.17 & $<0.20$ & 0.08 & $<0.05$ & 0.08 & $<0.05$ & $<0.05$ & $<0.05$ & $<0.05$ & $<0.05$ \\
\hline 18 & 0945 & 0.06 & $<0.05$ & 0.20 & $<0.20$ & 0.11 & $<0.05$ & 0.10 & $<0.05$ & $<0.05$ & $<0.05$ & $<0.05$ & $<0.05$ \\
\hline 24 & 1330 & $<0.05$ & $<0.05$ & 0.20 & $<0.20$ & 0.07 & $<0.05$ & 0.10 & $<0.05$ & $<0.05$ & $<0.05$ & $<0.05$ & $<0.05$ \\
\hline \multicolumn{14}{|l|}{ APRIL } \\
\hline 02 & 0930 & $<0.05$ & $<0.05$ & 0.26 & $<0.20$ & 0.09 & $<0.05$ & 0.08 & $<0.05$ & $<0.05$ & $<0.05$ & $<0.05$ & $<0.05$ \\
\hline 07 & 1330 & $<0.05$ & $<0.05$ & 0.26 & $<0.20$ & 0.09 & $<0.05$ & 0.09 & $<0.05$ & $<0.05$ & $<0.05$ & $<0.05$ & $<0.05$ \\
\hline 15 & 1030 & $<0.05$ & $<0.05$ & 0.28 & $<0.20$ & 0.05 & $<0.05$ & 0.08 & $<0.05$ & $<0.05$ & $<0.05$ & $<0.05$ & $<0.05$ \\
\hline
\end{tabular}


Table 7.-Pesticide results from solid-phase extraction 100-miliiliter method-Continued

\begin{tabular}{|c|c|c|c|c|c|c|c|c|c|c|c|c|c|}
\hline Date & TIme & $\begin{array}{l}\text { Ala- } \\
\text { chior } \\
\text { diss } \\
(\mu g / L)\end{array}$ & $\begin{array}{l}\text { Ame- } \\
\text { tryn } \\
\text { diss } \\
(\mu g / L)\end{array}$ & $\begin{array}{l}\text { Atra- } \\
\text { zine } \\
\text { diss } \\
(\mu g / L)\end{array}$ & $\begin{array}{l}\text { Cyana- } \\
\text { zine } \\
\text { diss } \\
(\mu g / L)\end{array}$ & $\begin{array}{c}\text { Desethyl- } \\
\text { atrazine } \\
\text { diss } \\
(\mu g / L)\end{array}$ & 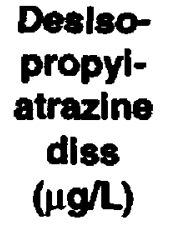 & $\begin{array}{l}\text { Metola- } \\
\text { chior } \\
\text { diss } \\
(\mu g /)\end{array}$ & $\begin{array}{l}\text { Metri- } \\
\text { buzin } \\
\text { diss } \\
(\mu g / L)\end{array}$ & $\begin{array}{l}\text { Pro- } \\
\text { metryn } \\
\text { diss } \\
(\mu g / L)\end{array}$ & $\begin{array}{l}\text { Pro- } \\
\text { moton } \\
\text { diss } \\
(\mu g /)\end{array}$ & $\begin{array}{l}\text { Propa- } \\
\text { zine } \\
\text { diss } \\
(\mu g / L)\end{array}$ & $\begin{array}{l}\text { Sima- } \\
\text { zine } \\
\text { diss } \\
(\mu g / L)\end{array}$ \\
\hline \multicolumn{14}{|c|}{07022000 - Mississippi River at Thebes, Ill.-Continued } \\
\hline \multicolumn{14}{|c|}{ APRIL (cont.) } \\
\hline 23 & 1330 & 0.18 & $<0.05$ & 1.3 & 0.90 & 0.11 & $<0.05$ & 0.46 & 0.08 & $<0.05$ & $<0.05$ & $<0.05$ & 0.07 \\
\hline 29 & 1100 & 0.10 & $<0.05$ & 0.76 & $<0.20$ & 0.09 & $<0.05$ & 0.31 & $<0.05$ & $<0.05$ & $<0.05$ & $<0.05$ & $<0.05$ \\
\hline \multicolumn{14}{|l|}{ MAY } \\
\hline 06 & 1300 & 0.07 & $<0.05$ & 0.41 & 0.20 & 0.07 & $<0.05$ & 0.27 & $<0.05$ & $<0.05$ & $<0.05$ & $<0.05$ & $<0.05$ \\
\hline 14 & 0930 & 0.05 & $<0.05$ & 0.22 & $<0.20$ & 0.08 & $<0.05$ & 0.17 & $<0.05$ & $<0.05$ & $<0.05$ & $<0.05$ & $<0.05$ \\
\hline 19 & 1300 & 0.05 & $<0.05$ & 0.22 & $<0.20$ & 0.06 & $<0.05$ & 0.12 & $<0.05$ & $<0.05$ & $<0.05$ & $<0.05$ & $<0.05$ \\
\hline 28 & 0900 & 0.15 & $<0.05$ & 0.75 & 0.20 & 0.12 & $<0.10$ & 0.17 & $<0.05$ & $<0.05$ & $<0.05$ & $<0.05$ & 0.13 \\
\hline \multicolumn{14}{|l|}{ JUNE } \\
\hline 02 & 1300 & 0.41 & $<0.05$ & 2.5 & 0.90 & 0.25 & 0.12 & 0.86 & $<0.05$ & $<0.05$ & $<0.05$ & $<0.05$ & 0.11 \\
\hline 09 & 0930 & 0.17 & $<0.05$ & 1.2 & 0.30 & 0.17 & 0.10 & 0.37 & $<0.05$ & $<0.05$ & $<0.05$ & $<0.05$ & 0.08 \\
\hline 16 & 1330 & 0.07 & $<0.05$ & 0.43 & 0.20 & 0.10 & $<0.05$ & 0.18 & $<0.05$ & $<0.05$ & $<0.05$ & $<0.05$ & 0.06 \\
\hline 25 & 1000 & 0.08 & $<0.05$ & 0.94 & 0.30 & 0.17 & 0.13 & 0.23 & 0.09 & $<0.05$ & $<0.05$ & $<0.05$ & $<0.05$ \\
\hline \multicolumn{14}{|l|}{ JULY } \\
\hline 01 & 1300 & 0.13 & $<0.05$ & 1.9 & 1.3 & 0.30 & 0.21 & 0.44 & 0.1 & $<0.05$ & $<0.05$ & $<0.05$ & $<0.05$ \\
\hline 09 & 0915 & 0.08 & $<0.05$ & 1.0 & 0.40 & 0.23 & 0.16 & 0.23 & $<0.05$ & $<0.05$ & $<0.05$ & $<0.05$ & 0.07 \\
\hline 15 & 1230 & 0.08 & $<0.05$ & 0.95 & 0.40 & 0.21 & 0.14 & 0.23 & $<0.05$ & $<0.05$ & $<0.05$ & $<0.05$ & 0.06 \\
\hline 23 & 1000 & 0.19 & $<0.05$ & 2.6 & 1.0 & 0.60 & 0.34 & 0.63 & 0.07 & $<0.05$ & $<0.05$ & $<0.05$ & 0.07 \\
\hline 28 & 1300 & 0.17 & $<0.05$ & 1.3 & $<0.20$ & 0.38 & 0.25 & 0.52 & 0.18 & $<0.05$ & $<0.05$ & $<0.05$ & 0.05 \\
\hline \multicolumn{14}{|c|}{03374100 - White River at Hazleton, Ind. } \\
\hline \multicolumn{14}{|l|}{ MAY 1991} \\
\hline 01 & 1000 & $<0.05$ & $<0.05$ & 0.26 & $<0.20$ & 0.06 & $<0.05$ & 0.19 & $<0.05$ & $<0.05$ & $<0.05$ & $<0.05$ & $<0.05$ \\
\hline 06 & 1115 & $<0.05$ & $<0.05$ & 0.35 & $<0.20$ & 0.06 & $<0.05$ & 0.16 & $<0.05$ & $<0.05$ & $<0.05$ & $<0.05$ & 0.07 \\
\hline 09 & 1215 & $<0.05$ & $<0.05$ & 0.42 & 0.20 & 0.07 & $<0.05$ & 0.20 & $<0.05$ & $<0.05$ & $<0.05$ & $<0.05$ & 0.06 \\
\hline 13 & 1300 & 0.68 & $<0.05$ & 3.7 & 0.70 & 0.30 & $<0.05$ & 1.1 & 0.18 & $<0.05$ & 0.10 & $<0.05$ & 0.33 \\
\hline 16 & 1130 & 0.08 & $<0.05$ & 0.91 & 0.60 & 0.10 & $<0.05$ & 0.35 & $<0.05$ & $<0.05$ & $<0.05$ & $<0.05$ & 0.12 \\
\hline 20 & 1045 & 0.25 & $<0.05$ & 1.4 & 0.70 & 0.13 & $<0.05$ & 0.27 & $<0.05$ & $<0.05$ & $<0.05$ & $<0.05$ & 0.14 \\
\hline 23 & 1100 & 1.3 & $<0.05$ & 3.4 & 1.4 & 0.37 & $<0.05$ & 1.5 & 0.11 & $<0.05$ & 0.12 & $<0.05$ & 0.30 \\
\hline 28 & 1100 & 3.2 & $<0.05$ & 8.6 & 4.4 & 0.84 & $<0.05$ & 2.2 & 0.27 & $<0.05$ & 0.14 & 0.06 & 0.51 \\
\hline 30 & 1200 & 2.1 & $<0.05$ & 9.2 & 2.8 & 1.0 & $<0.05$ & 3.3 & 0.25 & $<0.05$ & 0.07 & $<0.05$ & 0.72 \\
\hline \multicolumn{14}{|l|}{ JUNE } \\
\hline 03 & 1200 & 0.88 & $<0.05$ & 8.2 & 2.1 & 0.94 & $<0.05$ & 2.8 & 0.16 & $<0.05$ & $<0.05$ & $<0.05$ & 0.58 \\
\hline 06 & 1145 & 1.3 & $<0.05$ & 5.1 & 1.3 & 0.51 & $<0.05$ & 2.0 & 0.16 & $<0.05$ & 0.06 & $<0.05$ & 0.38 \\
\hline 10 & 1200 & 1.2 & $<0.05$ & 5.5 & 2.0 & 0.78 & $<0.05$ & 2.2 & 0.20 & $<0.05$ & $<0.05$ & $<0.05$ & 0.70 \\
\hline 13 & 1100 & 0.96 & $<0.05$ & 5.8 & 1.6 & 0.80 & $<0.05$ & 1.9 & 0.11 & $<0.05$ & $<0.05$ & $<0.05$ & 0.45 \\
\hline 18 & 1200 & 0.30 & $<0.05$ & 3.2 & 0.87 & 0.44 & $<0.05$ & 0.89 & 0.14 & $<0.05$ & 0.06 & $<0.05$ & 0.17 \\
\hline 20 & 1130 & 0.19 & $<0.05$ & 2.6 & 0.50 & 0.25 & $<0.05$ & 0.85 & $<0.05$ & $<0.05$ & 0.12 & $<0.05$ & 0.10 \\
\hline 24 & 1215 & 0.27 & $<0.05$ & 2.7 & 0.60 & 0.29 & $<0.05$ & 0.98 & $<0.05$ & $<0.05$ & 0.13 & $<0.05$ & 0.16 \\
\hline 27 & 1130 & 0.08 & $<0.05$ & 2.0 & 0.50 & 0.31 & $<0.05$ & 0.54 & $<0.05$ & $<0.05$ & 0.06 & $<0.05$ & 0.12 \\
\hline \multicolumn{14}{|l|}{ JULY } \\
\hline 01 & 1145 & $<0.05$ & $<0.05$ & 1.3 & 0.30 & 0.18 & $<0.05$ & 0.29 & $<0.05$ & $<0.05$ & 0.07 & $<0.05$ & 0.07 \\
\hline 03 & 1100 & 0.08 & $<0.05$ & 1.3 & 0.30 & 0.24 & $<0.05$ & 0.40 & $<0.05$ & $<0.05$ & 0.06 & $<0.05$ & 0.10 \\
\hline 08 & 1200 & 0,05 & $<0.05$ & 0.99 & 0.20 & 0.20 & $<0.05$ & 0.30 & $<0.05$ & $<0.05$ & 0.07 & $<0.05$ & 0.08 \\
\hline 11 & 1130 & $<0.05$ & $<0.05$ & 1.4 & 0.30 & 0.24 & $<0.05$ & 0.33 & $<0.05$ & $<0.05$ & $<0.05$ & $<0.05$ & 0.68 \\
\hline 15 & 1200 & 0.08 & $<0.05$ & 1.8 & 0.20 & 0.29 & 0.25 & 0.83 & 0.11 & $<0.05$ & $<0.05$ & $<0.05$ & 0.16 \\
\hline 18 & 1300 & 0.06 & $<0.05$ & 1.2 & 0.20 & 0.26 & 0.23 & 0.41 & 0.12 & $<0.05$ & 0.20 & $<0.05$ & 0.10 \\
\hline 22 & 1300 & $<0.05$ & $<0.05$ & 0.92 & 0.20 & 0.17 & $<0.05$ & 0.22 & $<0.05$ & $<0.05$ & 0.07 & $<0.05$ & 0.06 \\
\hline 25 & 1200 & $<0.05$ & $<0.05$ & 0.58 & $<0.20$ & 0.10 & $<0.05$ & 0.13 & $<0.05$ & $<0.05$ & $<0.05$ & $<0.05$ & $<0.05$ \\
\hline 30 & 1145 & 0.05 & $<0.05$ & 0.61 & $<0.20$ & 0.16 & $<0.05$ & 0.17 & 0.07 & $<0.05$ & 0.08 & $<0.05$ & $<0.05$ \\
\hline
\end{tabular}


Table 7.-Pesticide resuits from solid-phase extraction 100-milliliter method-Continued

\begin{tabular}{|c|c|c|c|c|c|c|c|c|c|c|c|c|c|}
\hline Date & Time & $\begin{array}{l}\text { Aia- } \\
\text { chior } \\
\text { diss } \\
(\mu g / L)\end{array}$ & $\begin{array}{l}\text { Ame- } \\
\text { tryn } \\
\text { diss } \\
(\mu g / L)\end{array}$ & $\begin{array}{l}\text { Atra- } \\
\text { zine } \\
\text { diss } \\
(\mu g / L)\end{array}$ & $\begin{array}{l}\text { Cyana- } \\
\text { zine } \\
\text { diss } \\
(\mu g / L)\end{array}$ & $\begin{array}{l}\text { Desethyi- } \\
\text { atrazine } \\
\text { diss } \\
(\mu g / L)\end{array}$ & $\begin{array}{l}\text { Deslao- } \\
\text { propyi- } \\
\text { atrazine } \\
\text { diss } \\
\text { (ug/L) }\end{array}$ & $\begin{array}{l}\text { Metola- } \\
\text { chior } \\
\text { diss } \\
(\mu \mathrm{g} / \mathrm{L})\end{array}$ & $\begin{array}{l}\text { Metri- } \\
\text { buzin } \\
\text { diss } \\
(\mu g /)\end{array}$ & $\begin{array}{l}\text { Pro- } \\
\text { motryn } \\
\text { diss } \\
(\mu g /)\end{array}$ & $\begin{array}{l}\text { Pro- } \\
\text { moton } \\
\text { diss } \\
(\mu g /)\end{array}$ & $\begin{array}{l}\text { Propa- } \\
\text { zine } \\
\text { diss } \\
(\mu g /)\end{array}$ & $\begin{array}{c}\text { Sima- } \\
\text { zine } \\
\text { diss } \\
(\mu g / L)\end{array}$ \\
\hline
\end{tabular}

AUGUST

\begin{tabular}{|c|c|c|c|c|c|c|c|c|c|c|c|c|c|}
\hline 01 & 1145 & $<0.05$ & $<0.05$ & 0.66 & 0.20 & 0.18 & $<0.05$ & 0.12 & $<0.05$ & $<0.05$ & 0.06 & $<0.05$ & 0.06 \\
\hline 06 & 1200 & $<0.05$ & $<0.05$ & 0.26 & $<0.20$ & 0.08 & $<0.05$ & $<0.05$ & $<0.05$ & $<0.05$ & $<0.05$ & $<0.05$ & $<0.05$ \\
\hline 08 & 1300 & $<0.05$ & $<0.05$ & 0.22 & $<0.20$ & $<0.05$ & $<0.05$ & $<0.05$ & $<0.05$ & $<0.05$ & $<0.05$ & $<0.05$ & $<0.05$ \\
\hline 12 & 1100 & $<0.05$ & $<0.05$ & 0.29 & $<0.20$ & $<0.05$ & $<0.05$ & 0.11 & $<0.05$ & $<0.05$ & $<0.05$ & $<0.05$ & $<0.05$ \\
\hline 15 & 1200 & $<0.05$ & $<0.05$ & 0.39 & $<0.20$ & 0.14 & $<0.05$ & 0.07 & $<0.05$ & $<0.05$ & $<0.05$ & $<0.05$ & 0.05 \\
\hline 19 & 1115 & $<0.05$ & $<0.05$ & 0.29 & $<0.20$ & 0.09 & $<0.05$ & 0.05 & $<0.05$ & $<0.05$ & 0.06 & $<0.05$ & $<0.05$ \\
\hline 22 & 1230 & $<0.05$ & $<0.05$ & 0.38 & $<0.20$ & 0.12 & $<0.05$ & 0.07 & $<0.05$ & $<0.05$ & 0.09 & $<0.05$ & $<0.05$ \\
\hline 26 & 1245 & $<0.05$ & 0.07 & 0.41 & $<0.20$ & 0.14 & 0.11 & 0.07 & $<0.05$ & $<0.05$ & 0.17 & $<0.05$ & 0.05 \\
\hline 29 & 1200 & $<0.05$ & $<0.05$ & 0.36 & $<0.20$ & 0.13 & 0.12 & 0.07 & $<0.05$ & $<0.05$ & 0.19 & $<0.05$ & $<0.05$ \\
\hline \multicolumn{14}{|c|}{ EPTEMBER } \\
\hline 09 & 1100 & $<0.05$ & $<0.05$ & 0.41 & $<0.20$ & 0.16 & 0.10 & 0.13 & $<0.05$ & $<0.05$ & 0.06 & $<0.05$ & $<0.05$ \\
\hline 12 & 1100 & $<0.05$ & $<0.05$ & 0.33 & $<0.20$ & 0.10 & $<0.10$ & 0.09 & $<0.05$ & $<0.05$ & 0.06 & $<0.05$ & $<0.05$ \\
\hline 19 & 1130 & $<0.05$ & $<0.05$ & 0.30 & $<0.20$ & 0.12 & $<0.10$ & 0.08 & $<0.05$ & $<0.05$ & 0.10 & $<0.05$ & 0.06 \\
\hline 26 & 1200 & $<0.05$ & $<0.05$ & 0.30 & $<0.20$ & 0.12 & $<0.10$ & 0.08 & $<0.05$ & $<0.05$ & 0.08 & $<0.05$ & 0.05 \\
\hline \multicolumn{14}{|c|}{ OCTOBER 1991} \\
\hline 03 & 1000 & $<0.05$ & $<0.05$ & 0.35 & $<0.20$ & 0.12 & 0.12 & 0.06 & $<0.05$ & $<0.05$ & 0.16 & $<0.05$ & 0.06 \\
\hline 08 & 1100 & $<0.05$ & $<0.05$ & 0.23 & $<0.20$ & 0.09 & $<0.10$ & 0.06 & $<0.05$ & $<0.05$ & 0.06 & $<0.05$ & 0.05 \\
\hline 17 & 1100 & $<0.05$ & $<0.05$ & 0.24 & $<0.20$ & 0.11 & $<0.10$ & $<0.05$ & $<0.05$ & $<0.05$ & 0.06 & $<0.05$ & 0.06 \\
\hline 24 & 1100 & $<0.05$ & $<0.05$ & 0.22 & $<0.20$ & 0.12 & $<0.05$ & 0.07 & $<0.05$ & $<0.05$ & $<0.05$ & $<0.05$ & $<0.05$ \\
\hline 28 & 1130 & $<0.05$ & $<0.05$ & 0.19 & $<0.20$ & 0.11 & $<0.05$ & 0.05 & $<0.05$ & $<0.05$ & $<0.05$ & $<0.05$ & $<0.05$ \\
\hline 30 & 1130 & 0.05 & $<0.05$ & 0.32 & $<0.20$ & 0.12 & 0.11 & 0.21 & $<0.05$ & $<0.05$ & 0.07 & $<0.05$ & 0.08 \\
\hline \multicolumn{14}{|c|}{ NOVEMBER } \\
\hline 04 & 1130 & $<0.05$ & $<0.05$ & 0.56 & $<0.20$ & 0.16 & $<0.10$ & 0.14 & $<0.05$ & $<0.05$ & $<0.07$ & $<0.05$ & $<0.07$ \\
\hline 13 & 1025 & $<0.05$ & $<0.05$ & 0.29 & $<0.20$ & 0.10 & $<0.05$ & 0.08 & $<0.05$ & $<0.05$ & 0.09 & $<0.05$ & 0.06 \\
\hline 27 & 1200 & $<0.05$ & $<0.05$ & 0.45 & $<0.20$ & 0.17 & $<0.05$ & 0.15 & $<0.05$ & $<0.05$ & 0.08 & $<0.05$ & 0.07 \\
\hline \multicolumn{14}{|c|}{ DECEMBER } \\
\hline 18 & 1200 & $<0.05$ & $<0.05$ & 0.42 & $<0.20$ & 0.20 & 0.09 & 0.10 & $<0.05$ & $<0.05$ & 0.05 & $<0.05$ & 0.06 \\
\hline \multicolumn{14}{|c|}{ JANUARY 1992} \\
\hline 02 & 1200 & $<0.05$ & $<0.05$ & 0.24 & $<0.20$ & 0.13 & $<0.05$ & 0.09 & $<0.05$ & $<0.05$ & 0.07 & $<0.05$ & 0.06 \\
\hline 16 & 1235 & $<0.05$ & $<0.05$ & 0.30 & $<0.20$ & 0.14 & 0.06 & 0.12 & $<0.05$ & $<0.05$ & $<0.05$ & $<0.05$ & $<0.05$ \\
\hline 30 & 1243 & $<0.05$ & $<0.05$ & 0.26 & $<0.20$ & 0.13 & $<0.05$ & 0.08 & $<0.05$ & $<0.05$ & $<0.05$ & $<0.05$ & $<0.05$ \\
\hline \multicolumn{14}{|c|}{ FEBRUARY } \\
\hline 13 & 1240 & $<0.05$ & $<0.05$ & 0.25 & $<0.20$ & 0.14 & 0.06 & 0.08 & $<0.05$ & $<0.05$ & 0.08 & $<0.05$ & 0.06 \\
\hline 27 & 1200 & $<0.05$ & $<0.05$ & 0.25 & $<0.20$ & 0.13 & $<0.05$ & 0.10 & $<0.05$ & $<0.05$ & 0.09 & $<0.05$ & 0.09 \\
\hline \multicolumn{14}{|c|}{ MARCH } \\
\hline 05 & 1130 & $<0.05$ & $<0.05$ & 0.20 & $<0.20$ & 0.10 & $<0.05$ & 0.08 & $<0.05$ & $<0.05$ & $<0.05$ & $<0.05$ & 0.05 \\
\hline 12 & 1115 & $<0.05$ & $<0.05$ & 0.18 & $<0.20$ & 0.11 & $<0.05$ & 0.09 & $<0.05$ & $<0.05$ & $<0.05$ & $<0.05$ & 0.07 \\
\hline 19 & 1133 & $<0.05$ & $<0.05$ & 0.17 & $<0.20$ & 0.11 & 0.06 & 0.07 & $<0.05$ & $<0.05$ & 0.08 & $<0.05$ & 0.11 \\
\hline 26 & 1128 & $<0.05$ & $<0.05$ & 0.18 & $<0.20$ & 0.10 & $<0.05$ & 0.12 & $<0.05$ & $<0.05$ & $<0.05$ & $<0.05$ & 0.09 \\
\hline \multicolumn{14}{|l|}{ APRIL } \\
\hline 02 & 1135 & $<0.05$ & $<0.05$ & 0.36 & $<0.20$ & 0.12 & $<0.05$ & 0.18 & $<0.05$ & $<0.05$ & $<0.05$ & $<0.05$ & 0.07 \\
\hline 09 & 1102 & $<0.05$ & $<0.05$ & 0.26 & $<0.20$ & 0.10 & $<0.05$ & 0.14 & $<0.05$ & $<0.05$ & $<0.05$ & $<0.05$ & 0.12 \\
\hline 16 & 1112 & 0.39 & $<0.05$ & 1.2 & $<0.20$ & 0.13 & 0.05 & 0.26 & $<0.05$ & $<0.05$ & $<0.05$ & $<0.05$ & 0.38 \\
\hline 20 & 1030 & 0.09 & $<0.05$ & 1.0 & 0.30 & 0.17 & 0.05 & 0.57 & $<0.05$ & $<0.05$ & $<0.05$ & $<0.05$ & 0.19 \\
\hline 24 & 1035 & 0.25 & $<0.05$ & 1.4 & 0.40 & 0.20 & $<0.05$ & 1.7 & 0.22 & $<0.05$ & 0.05 & $<0.05$ & 0.17 \\
\hline 29 & 1100 & 0.11 & $<0.05$ & 0.80 & $<0.20$ & 0.16 & $<0.05$ & 0.69 & 0.10 & $<0.05$ & $<0.05$ & $<0.05$ & 0.12 \\
\hline
\end{tabular}


Table 7.--Pesticide results from solid-phase extraction 100-milliliter method-Continued

\begin{tabular}{|c|c|c|c|c|c|c|c|c|c|c|c|c|c|}
\hline Date & Time & $\begin{array}{l}\text { Ala- } \\
\text { chlor } \\
\text { diss } \\
(\mu g / L)\end{array}$ & $\begin{array}{l}\text { Ame- } \\
\text { iryn } \\
\text { diss } \\
(\mu g / L)\end{array}$ & $\begin{array}{l}\text { Atra- } \\
\text { zlne } \\
\text { dlss } \\
(\mu \mathrm{g} / \mathrm{L})\end{array}$ & $\begin{array}{l}\text { Cyana- } \\
\text { zine } \\
\text { diss } \\
(\mu g / L)\end{array}$ & $\begin{array}{c}\text { Desethyl- } \\
\text { atrazlne } \\
\text { diss } \\
(\mu g / L)\end{array}$ & $\begin{array}{l}\text { Deslso- } \\
\text { propyl- } \\
\text { atrazine } \\
\text { dlss } \\
(\mu \mathrm{g} / \mathrm{L})\end{array}$ & $\begin{array}{l}\text { Metola- } \\
\text { chlor } \\
\text { diss } \\
(\mu g / L)\end{array}$ & $\begin{array}{l}\text { Metrl- } \\
\text { buzin } \\
\text { diss } \\
(\mu g / L)\end{array}$ & $\begin{array}{l}\text { Pro- } \\
\text { metryn } \\
\text { dlss } \\
(\mu g /)\end{array}$ & $\begin{array}{l}\text { Pro- } \\
\text { meton } \\
\text { dlss } \\
(\mu g / L)\end{array}$ & $\begin{array}{l}\text { Propa- } \\
\text { zlne } \\
\text { diss } \\
(\mu g / L)\end{array}$ & $\begin{array}{c}\text { Sima- } \\
\text { zine } \\
\text { dlss } \\
(\mu g /)\end{array}$ \\
\hline \multicolumn{14}{|c|}{03374100 - White River at Hazleton, Ind.-Continued } \\
\hline \multicolumn{14}{|l|}{ MAY } \\
\hline 06 & 1115 & 0.08 & $<0.05$ & 0.80 & $<0.20$ & 0.18 & $<0.05$ & 0.66 & 0.10 & $<0.05$ & $<0.05$ & $<0.05$ & 0.12 \\
\hline 12 & 1130 & 0.06 & $<0.05$ & 0.53 & $<0.20$ & 0.12 & $<0.05$ & 0.44 & $<0.05$ & $<0.05$ & 0.05 & $<0.05$ & 0.10 \\
\hline 20 & 1130 & 0.17 & $<0.05$ & 1.4 & $<0.20$ & 0.18 & $<0.05$ & 0.76 & 0.10 & $<0.05$ & 0.06 & $<0.05$ & 0.20 \\
\hline 27 & 1100 & 0.19 & $<0.05$ & 2.2 & 0.40 & 0.27 & 0.12 & 0.46 & $<0.05$ & $<0.05$ & $<0.05$ & $<0.05$ & 0.24 \\
\hline \multicolumn{14}{|l|}{ JUNE 1992} \\
\hline 04 & 1115 & 0.51 & $<0.05$ & 2.4 & 0.60 & 0.34 & $<0.10$ & 1.3 & 0.10 & $<0.05$ & 0.05 & $<0.05$ & 0.22 \\
\hline 10 & 1115 & 0.16 & $<0.05$ & 2.0 & $<0.20$ & 0.36 & 0.20 & 0.66 & $<0.05$ & $<0.05$ & $<0.05$ & $<0.05$ & 0.16 \\
\hline 19 & 1100 & 0.09 & $<0.05$ & 1.3 & 0.40 & 0.25 & $<0.10$ & 0.48 & $<0.05$ & $<0.05$ & $<0.05$ & $<0.05$ & 0.19 \\
\hline 23 & 1130 & 0.63 & $<0.05$ & 2.9 & 0.70 & 0.55 & 0.42 & 2.1 & 0.20 & $<0.05$ & 0.10 & $<0.05$ & 0.16 \\
\hline 25 & 1115 & 1.8 & $<0.05$ & 11 & 3.3 & 1.1 & 0.82 & 4.9 & 0.38 & $<0.05$ & 0.09 & 0.07 & 0.42 \\
\hline 29 & 1115 & 0.90 & $<0.05$ & 6.4 & 1.4 & 0.96 & 0.72 & 2.0 & 0.24 & $<0.05$ & 0.09 & 0.05 & 0.33 \\
\hline \multicolumn{14}{|l|}{ JULY } \\
\hline 02 & 1215 & 0.61 & $<0.05$ & 4.8 & 1.0 & 0.97 & 0.63 & 1.5 & 0.12 & $<0.05$ & 0.12 & 0.05 & 0.24 \\
\hline 10 & 1130 & 0.49 & $<0.05$ & 3.6 & 0.80 & 0.83 & 0.54 & 1.4 & 0.20 & $<0.05$ & 0.20 & $<0.05$ & 0.42 \\
\hline 16 & 1130 & 0.30 & $<0.05$ & 2.1 & 0.40 & 0.62 & 0.45 & 0.86 & 0.08 & $<0.05$ & 0.11 & $<0.05$ & 0.24 \\
\hline 23 & 1145 & 0.28 & $<0.05$ & 2.4 & 0.60 & 0.87 & 0.51 & 0.92 & 0.08 & $<0.05$ & 0.08 & $<0.05$ & 0.20 \\
\hline 31 & 1045 & 0.14 & $<0.05$ & 1.4 & 0.40 & 0.67 & 0.39 & 0.62 & 0.06 & $<0.05$ & 0.06 & $<0.05$ & 0.15 \\
\hline \multicolumn{14}{|l|}{ AUGUST } \\
\hline 06 & 1100 & 0.14 & $<0.05$ & 1.1 & 0.20 & 0.54 & 0.34 & 0.47 & 0.05 & $<0.05$ & 0.07 & $<0.05$ & 0.11 \\
\hline 12 & 1100 & 0.10 & $<0.05$ & 0.72 & $<0.20$ & 0.33 & 0.23 & 0.22 & $<0.05$ & $<0.05$ & $<0.05$ & $<0.05$ & 0.11 \\
\hline 20 & 1145 & 0.06 & $<0.05$ & 0.64 & $<0.20$ & 0.29 & 0.21 & 0.24 & $<0.05$ & $<0.05$ & $<0.05$ & $<0.05$ & 0.12 \\
\hline 26 & 1130 & $<0.05$ & $<0.05$ & 0.63 & $<0.20$ & 0.27 & 0.20 & 0.18 & $<0.05$ & $<0.05$ & $<0.05$ & $<0.05$ & 0.08 \\
\hline
\end{tabular}

APRIL 1991

03612500 - Ohio River at Dam 53 near Grand Chain, Ill.

\begin{tabular}{|c|c|c|c|c|c|c|c|c|c|c|c|c|c|}
\hline 10 & 1200 & $<0.05$ & $<0.05$ & 0.12 & $<0.20$ & 0.10 & $<0.05$ & 0.09 & $<0.05$ & $<0.05$ & $<0.05$ & $<0.05$ & 0.06 \\
\hline 18 & 1045 & $<0.05$ & $<0.05$ & 0.15 & $<0.20$ & $<0.05$ & $<0.05$ & $<0.05$ & $<0.05$ & $<0.05$ & $<0.05$ & $<0.05$ & $<0.05$ \\
\hline 23 & 1110 & $<0.05$ & $<0.05$ & 0.51 & 0.21 & $<0.05$ & $<0.05$ & 0.32 & $<0.05$ & $<0.05$ & $<0.05$ & $<0.05$ & 0.07 \\
\hline \multicolumn{14}{|l|}{ MAY } \\
\hline 01 & 1040 & $<0.05$ & $<0.05$ & 0.18 & $<0.20$ & $<0.05$ & $<0.05$ & 0.08 & $<0.05$ & $<0.05$ & $<0.05$ & $<0.05$ & $<0.05$ \\
\hline 07 & 1022 & $<0.05$ & $<0.05$ & 0.23 & $<0.20$ & 0.10 & $<0.05$ & 0.12 & $<0.05$ & $<0.05$ & 0.09 & $<0.05$ & 0.08 \\
\hline 14 & 1045 & $<0.05$ & $<0.05$ & 0.47 & $<0.20$ & 0.19 & $<0.05$ & 0.10 & $<0.05$ & $<0.05$ & $<0.05$ & $<0.05$ & 0.16 \\
\hline 21 & 1045 & 0.11 & $<0.05$ & 1.7 & 0.40 & 0.17 & $<0.05$ & 0.39 & $<0.05$ & $<0.05$ & $<0.05$ & $<0.05$ & 0.40 \\
\hline 29 & 1030 & 0.05 & $<0.05$ & 0.54 & 0.20 & $<0.05$ & $<0.05$ & 0.10 & $<0.05$ & $<0.05$ & $<0.05$ & $<0.05$ & 0.05 \\
\hline \multicolumn{14}{|l|}{ JUNE } \\
\hline 04 & 1135 & 0.24 & $<0.05$ & 1.6 & 0.50 & 0.16 & $<0.05$ & 0.35 & 0.06 & $<0.05$ & $<0.05$ & $<0.05$ & 0.12 \\
\hline 11 & 1045 & 0.33 & $<0.05$ & 1.9 & 0.50 & 0.11 & $<0.05$ & 0.72 & $<0.05$ & $<0.05$ & $<0.05$ & $<0.05$ & 0.07 \\
\hline 18 & 1040 & 0.40 & $<0.05$ & 2.1 & 0.70 & 0.21 & $<0.05$ & 0.95 & 0.11 & $<0.05$ & $<0.05$ & $<0.05$ & 0.12 \\
\hline 25 & 0946 & 0.13 & $<0.05$ & 1.3 & 0.40 & 0.15 & $<0.05$ & 0.42 & $<0.05$ & $<0.05$ & $<0.05$ & $<0.05$ & 0.11 \\
\hline \multicolumn{14}{|l|}{ JULY } \\
\hline 01 & 0950 & 0.16 & $<0.05$ & 0.83 & $<0.20$ & 0.15 & $<0.05$ & $<0.05$ & $<0.05$ & $<0.05$ & 0.09 & $<0.05$ & 0.09 \\
\hline 09 & 1030 & 0.08 & $<0.05$ & 0.51 & $<0.20$ & 0.10 & $<0.05$ & 0.14 & $<0.05$ & $<0.05$ & $<0.05$ & $<0.05$ & 0.07 \\
\hline 15 & 1100 & $<0.05$ & $<0.05$ & 0.35 & $<0.20$ & $<0.05$ & $<0.05$ & 0.07 & $<0.05$ & $<0.05$ & $<0.05$ & $<0.05$ & $<0.05$ \\
\hline 22 & 1040 & 0.06 & $<0.05$ & 0.78 & 0.20 & 0.15 & 0.09 & 0.19 & $<0.05$ & $<0.05$ & 0.15 & $<0.05$ & 0.10 \\
\hline 29 & 1110 & $<0.05$ & $<0.05$ & 0.22 & $<0.20$ & $<0.05$ & $<0.05$ & 0.05 & $<0.05$ & $<0.05$ & $<0.05$ & $<0.05$ & $<0.05$ \\
\hline \multicolumn{14}{|c|}{ AUGUST 1991} \\
\hline 05 & 1050 & $<0.05$ & $<0.05$ & 0.54 & 0.20 & 0.12 & $<0.05$ & 0.16 & $<0.05$ & $<0.05$ & $<0.05$ & $<0.05$ & 0.05 \\
\hline 12 & 1030 & $<0.05$ & $<0.05$ & 0.14 & $<0.20$ & $0.05^{\circ}$ & $<0.05$ & $<0.05$ & $<0.05$ & $<0.05$ & $<0.05$ & $<0.05$ & 0.05 \\
\hline
\end{tabular}




\begin{tabular}{|c|c|c|c|c|c|c|c|c|c|c|c|c|c|}
\hline Date & TIms & $\begin{array}{l}\text { Ala- } \\
\text { chlor } \\
\text { diss } \\
(\mu g / 4)\end{array}$ & $\begin{array}{l}\text { Amon } \\
\text { tryn } \\
\text { diss } \\
(\mu g /)\end{array}$ & 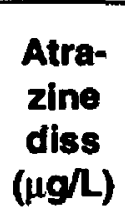 & 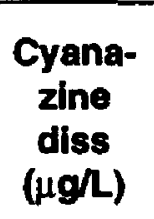 & 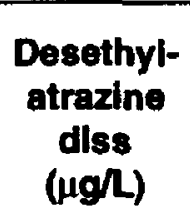 & $\begin{array}{l}\text { Dealso- } \\
\text { propyl- } \\
\text { atrazine } \\
\text { diss } \\
\text { (uch) }\end{array}$ & $\begin{array}{l}\text { Metola- } \\
\text { chlor } \\
\text { dlss } \\
(\mu g / L)\end{array}$ & $\begin{array}{l}\text { Metri- } \\
\text { buzin } \\
\text { dlss } \\
\text { ( } \mu \mathrm{g} / \mathrm{L})\end{array}$ & $\begin{array}{l}\text { Pro- } \\
\text { metryn } \\
\text { diss } \\
(\mu g /)\end{array}$ & 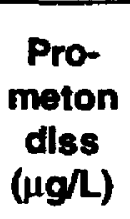 & $\begin{array}{l}\text { Propa- } \\
\text { zlne } \\
\text { diss } \\
\text { (ugh) }\end{array}$ & $\begin{array}{l}\text { Sime- } \\
\text { zine } \\
\text { dlss } \\
\text { (ugn) }\end{array}$ \\
\hline
\end{tabular}

AUGUST 1991 (cont.)

\begin{tabular}{|c|c|c|c|c|c|c|c|c|c|c|c|c|c|}
\hline 20 & 1030 & $<0.05$ & $<0.05$ & 0.11 & $<0.20$ & $<0.05$ & $<0.05$ & $<0.05$ & $<0.05$ & $<0.05$ & $<0.05$ & $<0.05$ & $<0.05$ \\
\hline 27 & 0945 & $<0.05$ & $<0.05$ & $<0.05$ & $<0.20$ & $<0.05$ & $<0.05$ & $<0,05$ & $<0.05$ & $<0.05$ & $<0.05$ & $<0.05$ & $<0.05$ \\
\hline \multicolumn{14}{|c|}{ SEPTEMBER } \\
\hline 03 & 1040 & $<0.05$ & $<0.05$ & $<0.05$ & $<0.20$ & $<0.05$ & $<0.05$ & $<0.05$ & $<0.05$ & $<0.05$ & $<0.05$ & $<0.05$ & $<0.05$ \\
\hline 10 & 1030 & $<0.05$ & $<0.05$ & 0.13 & $<0.20$ & $<0.05$ & $<0.05$ & $<0.05$ & $<0.05$ & $<0.05$ & $<0.05$ & $<0.05$ & $<0.05$ \\
\hline 17 & 0952 & $<0.05$ & $<0.05$ & 0.12 & $<0.20$ & 0.05 & $<0.05$ & $<0.05$ & $<0.05$ & $<0.05$ & $<0.05$ & $<0.05$ & 0.05 \\
\hline 23 & 1040 & $<0.05$ & $<0.05$ & 0.13 & $<0.20$ & 0.50 & $<0.05$ & $<0.05$ & $<0.05$ & $<0.05$ & $<0.05$ & $<0.05$ & $<0.05$ \\
\hline 30 & 1122 & $<0.05$ & $<0.05$ & 0.09 & $<0.20$ & $<0.05$ & $<0.05$ & $<0.05$ & $<0.05$ & $<0.05$ & $<0.05$ & $<0.05$ & $<0.05$ \\
\hline \multicolumn{14}{|c|}{ OCTOBER } \\
\hline 07 & 1125 & $<0.05$ & $<0.05$ & 0.10 & $<0.20$ & 0.06 & $<0.05$ & $<0.05$ & $<0.05$ & $<0.05$ & $<0.05$ & $<0.05$ & $<0.05$ \\
\hline 15 & 0955 & 0.08 & $<0.05$ & 0.13 & $<0.20$ & $<0.05$ & $<0.05$ & 0.10 & $<0.05$ & 0.05 & $<0.05$ & $<0.05$ & $<0.05$ \\
\hline 21 & 1020 & $<0.05$ & $<0.05$ & 0.10 & $<0.20$ & 0.06 & $<0.10$ & $<0.05$ & $<0.05$ & $<0.05$ & $<0.05$ & $<0.05$ & $<0.05$ \\
\hline 29 & 0930 & $<0.05$ & $<0.05$ & 0.23 & $<0.20$ & $<0.05$ & $<0.05$ & 0.07 & $<0.05$ & $<0.05$ & $<0.05$ & $<0.05$ & $<0.05$ \\
\hline \multicolumn{14}{|c|}{ NOVEMBER } \\
\hline 06 & 1040 & $<0.05$ & $<0.05$ & 0.13 & $<0.20$ & 0.05 & $<0.05$ & $<0.05$ & $<0.05$ & $<0.05$ & $<0.05$ & $<0.05$ & $<0.05$ \\
\hline 20 & 0920 & $<0.05$ & $<0.05$ & 0.15 & $<0.20$ & 0.06 & $<0.05$ & 0.06 & $<0.05$ & $<0.05$ & $<0.05$ & $<0.05$ & $<0.05$ \\
\hline \multicolumn{14}{|c|}{ DECEMBER } \\
\hline 05 & 1045 & $<0.05$ & $<0.05$ & 0.12 & $<0.20$ & 0.05 & $<0.05$ & $<0.05$ & $<0.05$ & $<0.05$ & $<0.05$ & $<0.05$ & $<0.05$ \\
\hline 17 & 1010 & $<0.10$ & $<0.10$ & $<0.10$ & $<0.20$ & $<0.05$ & $<0.05$ & $<0.15$ & $<0.05$ & $<0.15$ & $<0.10$ & $<0.05$ & $<0.05$ \\
\hline \multicolumn{14}{|c|}{ JANUARY 1992} \\
\hline 02 & 1005 & $<0.05$ & $<0.05$ & 0.10 & $<0.20$ & 0.08 & $<0.05$ & 0.05 & $<0.05$ & $<0.05$ & $<0.05$ & $<0.05$ & $<0.05$ \\
\hline 21 & 1030 & $<0.05$ & $<0.05$ & 0.08 & $<0.20$ & $<0.05$ & $<0.05$ & 0.05 & $<0.05$ & $<0.05$ & $<0.05$ & $<0.05$ & $<0.05$ \\
\hline \multicolumn{14}{|c|}{ FEBRUARY } \\
\hline 04 & 1125 & $<0.05$ & $<0.05$ & 0.10 & $<0.20$ & 0.30 & 0.12 & $<0.05$ & $<0.05$ & $<0.05$ & $<0.05$ & $<0.05$ & $<0.05$ \\
\hline 18 & 1025 & $<0.05$ & $<0.05$ & 0.08 & $<0.20$ & 0.06 & $<0.05$ & 0.05 & $<0.05$ & $<0.05$ & $<0.05$ & $<0.05$ & $<0.05$ \\
\hline \multicolumn{14}{|l|}{ MARCH } \\
\hline 03 & 1025 & $<0.05$ & $<0.05$ & 0.05 & $<0.20$ & 0.06 & $<0.05$ & $<0.05$ & $<0.05$ & $<0.05$ & $<0.05$ & $<0.05$ & $<0.05$ \\
\hline 11 & 1030 & $<0.05$ & $<0.05$ & $<0.05$ & $<0.20$ & $<0.05$ & $<0.05$ & $<0.05$ & $<0.05$ & $<0.05$ & $<0.05$ & $<0.05$ & $<0.05$ \\
\hline 16 & 1040 & $<0.05$ & $<0.05$ & 0.07 & $<0.20$ & $<0.05$ & $<0.05$ & 0.05 & $<0.05$ & $<0.05$ & $<0.05$ & $<0.05$ & $<0.05$ \\
\hline 24 & 1020 & $<0.05$ & $<0.05$ & 0.08 & $<0.20$ & $<0.05$ & $<0.05$ & 0.05 & $<0.05$ & $<0.05$ & $<0.05$ & $<0.05$ & $<0.05$ \\
\hline 31 & 1030 & $<0.05$ & $<0.05$ & 0.08 & $<0.20$ & $<0.05$ & $<0.05$ & $<0.05$ & $<0.05$ & $<0.05$ & $<0.05$ & $<0.05$ & $<0.05$ \\
\hline \multicolumn{14}{|l|}{ APRIL } \\
\hline 07 & 1015 & $<0.05$ & $<0.05$ & 0.11 & $<0.20$ & 0.05 & $<0.05$ & 0.06 & $<0.05$ & $<0.05$ & $<0.05$ & $<0.05$ & 0.06 \\
\hline 14 & 1035 & $<0.05$ & $<0.05$ & 0.09 & $<0.20$ & 0.05 & $<0.05$ & $<0.05$ & $<0.05$ & $<0.05$ & $<0.05$ & $<0.05$ & $<0.05$ \\
\hline 22 & 1040 & 0.14 & $<0.05$ & 1.3 & 0.30 & 0.08 & $<0.05$ & 0.37 & $<0.05$ & $<0.05$ & $<0.05$ & $<0.05$ & 0.15 \\
\hline 28 & 1104 & 0.14 & $<0.05$ & 0.95 & $<0.20$ & 0.11 & $<0.05$ & 0.51 & 0.07 & $<0.05$ & $<0.05$ & $<0.05$ & 0.14 \\
\hline \multicolumn{14}{|c|}{ MAY 1992} \\
\hline 06 & 0953 & 0.05 & $<0.05$ & 0.42 & 0.20 & 0.07 & $<0.05$ & 0.24 & $<0.05$ & $<0.05$ & $<0.05$ & $<0.05$ & 0.11 \\
\hline 12 & 0955 & $<0.05$ & $<0.05$ & 0.25 & $<0.20$ & 0.05 & $<0.05$ & 0.19 & $<0.05$ & $<0.05$ & $<0.05$ & $<0.05$ & 0.06 \\
\hline 18 & 1025 & $<0.05$ & $<0.05$ & 0.21 & $<0.20$ & 0.05 & $<0.05$ & 0.10 & $<0.05$ & $<0.05$ & $<0.05$ & $<0.05$ & 0.06 \\
\hline 27 & 1030 & 0.19 & $<0.05$ & 1.4 & 0.40 & 0.15 & 0.06 & 0.28 & $<0.05$ & $<0.05$ & $<0.05$ & $<0.05$ & 0.26 \\
\hline \multicolumn{14}{|l|}{ JUNE } \\
\hline 02 & 1045 & 0.28 & $<0.05$ & 3.0 & 0.40 & 0.42 & 0.21 & 0.54 & $<0.05$ & $<0.05$ & $<0.05$ & $<0.05$ & 0.52 \\
\hline 09 & 1105 & 0.06 & $<0.05$ & 0.79 & 0.20 & 0.14 & $<0.05$ & 0.21 & $<0.05$ & $<0.05$ & $<0.05$ & $<0.05$ & 0.18 \\
\hline 16 & 1105 & 0.05 & $<0.05$ & 0.32 & $<0.20$ & 0.08 & $<0.05$ & 0.11 & $<0.05$ & $<0.05$ & $<0.05$ & $<0.05$ & 0.09 \\
\hline 23 & 1023 & 0.05 & $<0.05$ & 0.39 & 0.20 & 0.08 & $<0.05$ & 0.17 & $<0.05$ & $<0.05$ & $<0.05$ & $<0.05$ & 0.12 \\
\hline 29 & 1235 & 0.07 & $<0.05$ & 0.65 & 0.20 & 0.14 & 0.12 & 0.22 & $<0.05$ & $<0.05$ & 0.05 & $<0.05$ & 0.14 \\
\hline
\end{tabular}


Table 7.--Pesticide results from solid-phase extraction 100-milliliter method-Contlnued

\begin{tabular}{|c|c|c|c|c|c|c|c|c|c|c|c|c|c|}
\hline Date & Time & $\begin{array}{l}\text { Ala- } \\
\text { chior } \\
\text { diss } \\
(\mu \mathrm{g} / \mathrm{L})\end{array}$ & $\begin{array}{l}\text { Ame- } \\
\text { tryn } \\
\text { diss } \\
(\mu g / L)\end{array}$ & $\begin{array}{l}\text { Atra- } \\
\text { zine } \\
\text { diss } \\
(\mu g / L)\end{array}$ & $\begin{array}{l}\text { Cyana- } \\
\text { zine } \\
\text { diss } \\
(\mu g / L)\end{array}$ & $\begin{array}{l}\text { Desethyl- } \\
\text { atrazine } \\
\text { diss } \\
(\mu \mathrm{g} / \mathrm{L})\end{array}$ & $\begin{array}{l}\text { Desieo- } \\
\text { propyl- } \\
\text { atrazine } \\
\text { dise } \\
(\mu g / L)\end{array}$ & $\begin{array}{l}\text { Metola- } \\
\text { chior } \\
\text { diss } \\
(\mu \mathrm{g} / \mathrm{L})\end{array}$ & $\begin{array}{l}\text { Motrl- } \\
\text { buzin } \\
\text { diss } \\
(\mu g / L)\end{array}$ & $\begin{array}{l}\text { Pro- } \\
\text { motryn } \\
\text { diss } \\
(\mu g / L)\end{array}$ & $\begin{array}{l}\text { Pro- } \\
\text { meton } \\
\text { diss } \\
(\mu \mathrm{g} / \mathrm{L})\end{array}$ & $\begin{array}{l}\text { Propa- } \\
\text { zine } \\
\text { diss } \\
(\mu g / L)\end{array}$ & $\begin{array}{c}\text { Sima- } \\
\text { zine } \\
\text { dise } \\
(\mu g / L)\end{array}$ \\
\hline \multicolumn{14}{|c|}{03612500 - Ohio River st Dam 53 near Grand Chain, III.-Continued } \\
\hline JULY & & & & & & & & & & & & & \\
\hline 08 & 1012 & 0.19 & $<0.05$ & 0.85 & 0.20 & 0.20 & 0.14 & 0.25 & 0.06 & $<0.05$ & $<0.05$ & $<0.05$ & 0.14 \\
\hline 14 & 1025 & 0.14 & $<0.05$ & 0.91 & 0.30 & 0.22 & 0.15 & 0.31 & $<0.05$ & $<0.05$ & $<0.05$ & $<0.05$ & 0.13 \\
\hline 23 & 1040 & 0.12 & $<0.05$ & 1.1 & 0.30 & 0.29 & 0.19 & 0.47 & 0.06 & $<0.05$ & $<0.05$ & $<0.05$ & 0.14 \\
\hline 28 & 1025 & 0.11 & $<0.05$ & 1.0 & 0.30 & 0.28 & 0.17 & 0.47 & 0.09 & $<0.05$ & 0.06 & $<0.05$ & 0.11 \\
\hline \multicolumn{14}{|c|}{07373420 - Mississippi River near St. Francisville, La. } \\
\hline \multicolumn{14}{|l|}{ MAY 1991} \\
\hline $\begin{array}{l}06 \\
\text { JUNE }\end{array}$ & 1131 & 0.11 & $<0.05$ & 0.70 & $<0.20$ & 0.13 & $<0.05$ & 0.24 & $<0.05$ & $<0.05$ & $<0.05$ & $<0.05$ & 0.10 \\
\hline $\begin{array}{l}17 \\
\text { JULY }\end{array}$ & \multicolumn{12}{|c|}{ JULY } & $<0.05$ \\
\hline \multicolumn{14}{|c|}{ NOVEMBER } \\
\hline $\begin{array}{c}05 \\
\text { DECEMBE }\end{array}$ & 1001 & $<0.05$ & $<0.05$ & 0.17 & $<0.20$ & 0.06 & $<0.05$ & $<0.05$ & $<0.05$ & $<0.05$ & $<0.05$ & $<0.05$ & $<0.05$ \\
\hline $\begin{array}{c}10 \\
\text { JANUARY }\end{array}$ & $\begin{array}{l}1030 \\
92\end{array}$ & $<0.05$ & $<0.05$ & 0.11 & $<0.20$ & 0.05 & $<0.05$ & 0.05 & $<0.05$ & $<0.05$ & $<0.05$ & $<0.05$ & 0.05 \\
\hline \multicolumn{14}{|c|}{ FEBRUARY } \\
\hline \multicolumn{13}{|l|}{ MARCH } & $<0.05$ \\
\hline MAY & 1000 & $<0.05$ & $<0.05$ & 0.15 & $<0.20$ & 0.05 & $<0.05$ & 0.09 & $<0.05$ & $<0.05$ & $<0.05$ & $<0.05$ & 0.07 \\
\hline 14 & 1201 & 0.06 & $<0.05$ & 0.90 & 0.20 & 0.13 & $<0.10$ & 0.35 & $<0.05$ & $<0.05$ & $<0.05$ & $<0.05$ & 0.12 \\
\hline \multicolumn{14}{|l|}{ JUNE } \\
\hline \multicolumn{13}{|l|}{ JULY } & 0.12 \\
\hline $\begin{array}{c}15 \\
\text { AUGUST }\end{array}$ & 1101 & 0.13 & $<0.05$ & 1.2 & 0.40 & 0.23 & 0.14 & 0.39 & $<0.05$ & $<0.05$ & $<0.05$ & $<0.05$ & 0.09 \\
\hline 18 & 1016 & 0.06 & $<0.05$ & 0.88 & 0.20 & 0.30 & 0.15 & 0.24 & 0.08 & $<0.05$ & 0.06 & $<0.05$ & $<0.05$ \\
\hline \multicolumn{14}{|c|}{ SEPTEMBER } \\
\hline \multicolumn{14}{|c|}{07374000 - Mississippi River at Baton Rouge, La. } \\
\hline \multicolumn{14}{|c|}{ APRIL 1991} \\
\hline 11 & 1430 & $<0.05$ & $<0.05$ & 0.28 & $<0.20$ & 0.05 & $<0.05$ & 0.12 & $<0.05$ & $<0.05$ & $<0.05$ & $<0.05$ & $<0.05$ \\
\hline 17 & 1415 & $<0.05$ & $<0.05$ & 0.23 & $<0.20$ & $<0.05$ & $<0.05$ & 0.10 & $<0.05$ & $<0.05$ & $<0.05$ & $<0.05$ & $<0.05$ \\
\hline 24 & 1045 & $<0.05$ & $<0.05$ & 0.39 & $<0.20$ & $<0.05$ & $<0.05$ & 0.13 & $<0.05$ & $<0.05$ & $<0.05$ & $<0.05$ & $<0.05$ \\
\hline \multicolumn{14}{|l|}{ MAY } \\
\hline 01 & 1030 & 0.06 & $<0.05$ & 0.52 & 0.30 & $<0.05$ & $<0.05$ & 0.18 & $<0.05$ & $<0.05$ & $<0.05$ & $<0.05$ & $<0.05$ \\
\hline 06 & 0800 & $<0.05$ & $<0.05$ & 0.49 & 0.20 & 0.16 & $<0.05$ & 0.18 & $<0.05$ & $<0.05$ & 0.09 & $<0.05$ & 0.10 \\
\hline 09 & 1030 & 0.13 & $<0.05$ & 1.0 & 0.20 & 0.16 & $<0.05$ & 0.31 & $<0.05$ & $<0.05$ & 0.09 & $<0.05$ & 0.10 \\
\hline 13 & 0930 & 0.10 & $<0.05$ & 0.59 & $<0.20$ & 0.13 & $<0.05$ & 0.23 & $<0.05$ & $<0.05$ & $<0.05$ & $<0.05$ & 0.08 \\
\hline 16 & 1130 & $<0.05$ & $<0.05$ & 0.34 & 0.20 & $<0.05$ & $<0.05$ & 0.14 & $<0.05$ & $<0.05$ & $<0.05$ & $<0.05$ & $<0.05$ \\
\hline 20 & 1330 & 0.13 & $<0.05$ & 1.2 & 0.90 & 0.11 & $<0.05$ & 0.38 & $<0.05$ & $<0.05$ & $<0.05$ & $<0.05$ & $<0.05$ \\
\hline 24 & 1315 & 0.14 & $<0.05$ & 1.1 & 0.60 & 0.10 & $<0.05$ & 0.40 & $<0.05$ & $<0.05$ & $<0.05$ & $<0.05$ & $<0.05$ \\
\hline 28 & 1255 & 0.07 & $<0.05$ & 0.83 & 0.30 & 0.09 & $<0.05$ & 0.36 & $<0.05$ & $<0.05$ & $<0.05$ & $<0.05$ & $<0.05$ \\
\hline 30 & 1130 & 0.13 & $<0.05$ & 1.1 & 0.40 & 0.11 & $<0.05$ & 0.36 & 0.09 & $<0.05$ & $<0.05$ & $<0.05$ & 0.09 \\
\hline
\end{tabular}




\begin{tabular}{|c|c|c|c|c|c|c|c|c|c|c|c|c|c|}
\hline Date & Time & $\begin{array}{l}\text { Ala- } \\
\text { chior } \\
\text { diss } \\
(\mu g / L)\end{array}$ & $\begin{array}{l}\text { Ame- } \\
\text { tryn } \\
\text { dlss } \\
(\mu g / L)\end{array}$ & $\begin{array}{l}\text { Atra- } \\
\text { zine } \\
\text { diss } \\
(\mu \mathrm{g} / \mathrm{L})\end{array}$ & $\begin{array}{l}\text { Cyana- } \\
\text { zine } \\
\text { dlss } \\
(\mu g / L)\end{array}$ & $\begin{array}{l}\text { Desethyl- } \\
\text { atrazine } \\
\text { dlss } \\
(\mu g / L)\end{array}$ & $\begin{array}{l}\text { Desiso- } \\
\text { propyl- } \\
\text { atrazine } \\
\text { diss } \\
(\mu g / L)\end{array}$ & $\begin{array}{l}\text { Metola- } \\
\text { chlor } \\
\text { diss } \\
(\mu g / L)\end{array}$ & $\begin{array}{c}\text { Metri- } \\
\text { buzin } \\
\text { dlss } \\
(\mu g / L)\end{array}$ & $\begin{array}{c}\text { Pro- } \\
\text { metryn } \\
\text { dlss } \\
(\mu g / L)\end{array}$ & $\begin{array}{l}\text { Pro- } \\
\text { meton } \\
\text { dlas } \\
(\mu g / L)\end{array}$ & $\begin{array}{l}\text { Propa- } \\
\text { zine } \\
\text { dlaa } \\
(\mu g / L)\end{array}$ & $\begin{array}{c}\text { Sima- } \\
\text { zine } \\
\text { dlss } \\
(\mu g / L)\end{array}$ \\
\hline
\end{tabular}

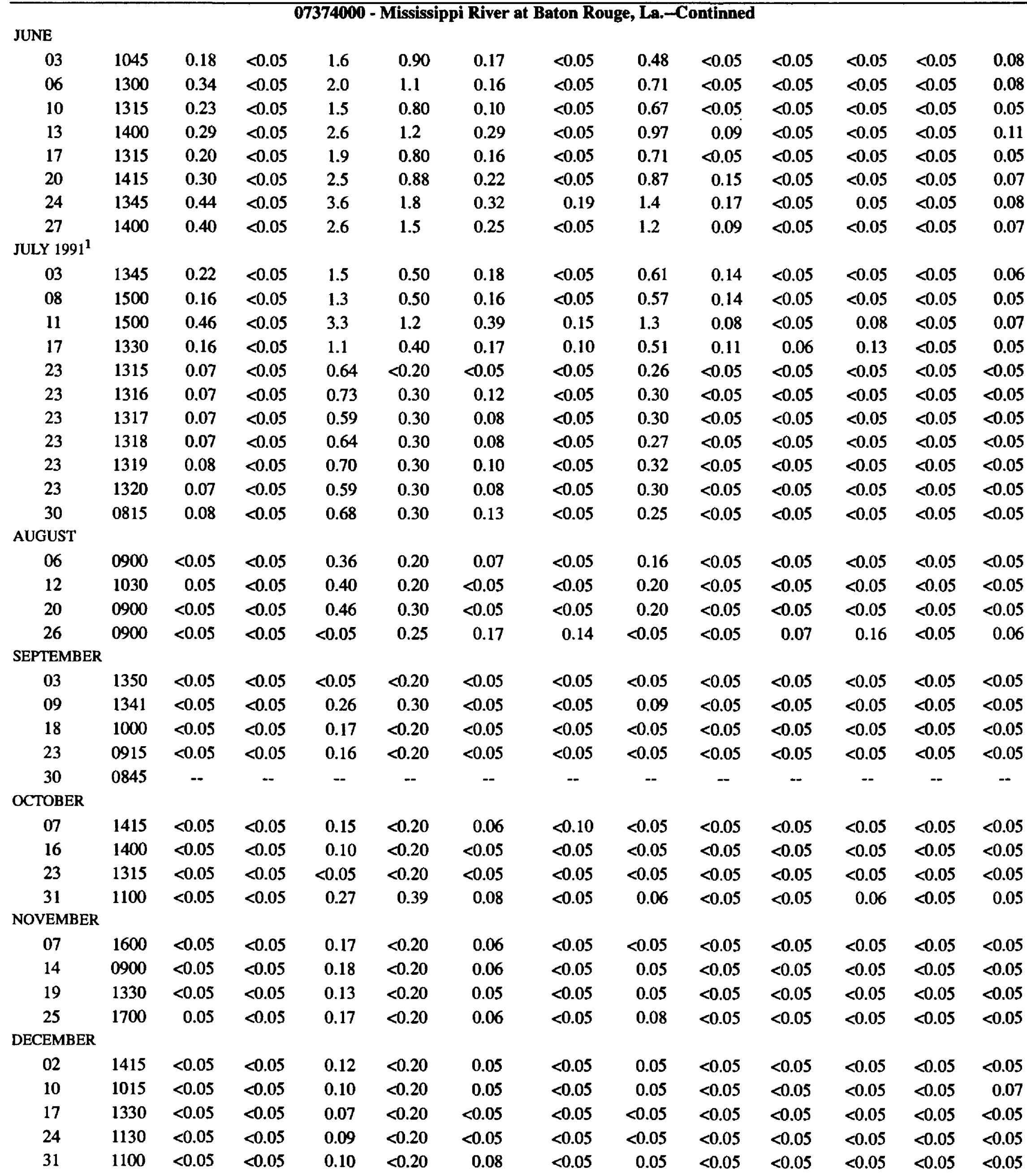


Table 7.-Pesticide results from solid-phase extraction 100-milliliter method-Continued

\begin{tabular}{|c|c|c|c|c|c|c|c|c|c|c|c|c|c|}
\hline Date & Time & $\begin{array}{l}\text { Ala- } \\
\text { chlor } \\
\text { dlss } \\
(\mu g / L)\end{array}$ & 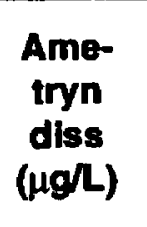 & $\begin{array}{l}\text { Atra- } \\
\text { zine } \\
\text { diss } \\
(\mu g / L)\end{array}$ & $\begin{array}{l}\text { Cyana- } \\
\text { zlne } \\
\text { diss } \\
\text { ( } \mu \mathrm{g} / \mathrm{L})\end{array}$ & $\begin{array}{l}\text { Desethyl- } \\
\text { atrazine } \\
\text { diss } \\
(\mu g / L)\end{array}$ & $\begin{array}{l}\text { Deslso- } \\
\text { propyl- } \\
\text { atrazlne } \\
\text { dlss } \\
\text { ( } \mathrm{gg} / \mathrm{L})\end{array}$ & $\begin{array}{l}\text { Metola- } \\
\text { chlor } \\
\text { diss } \\
(\mu g / \text { ) }\end{array}$ & $\begin{array}{c}\text { Metri- } \\
\text { buzin } \\
\text { dlss } \\
(\mu g / L)\end{array}$ & $\begin{array}{l}\text { Pro- } \\
\text { metryn } \\
\text { diss } \\
(\mu g / L)\end{array}$ & $\begin{array}{l}\text { Pro- } \\
\text { meton } \\
\text { diss } \\
(\mu g / L)\end{array}$ & $\begin{array}{l}\text { Propa- } \\
\text { zine } \\
\text { diss } \\
\text { ( } \mu \mathrm{g} / \mathrm{L} \text { ) }\end{array}$ & $\begin{array}{l}\text { Sima- } \\
\text { zlne } \\
\text { diss } \\
(\mu g / L)\end{array}$ \\
\hline
\end{tabular}

JANUARY 1992

07374000 - Mississippi River st Baton Rouge, La.-Continued

\begin{tabular}{|c|c|c|c|c|c|c|c|c|c|c|c|c|c|}
\hline 07 & 1100 & $<0.05$ & $<0.05$ & 0.10 & $<0.20$ & 0.07 & $<0.05$ & 0.06 & $<0.05$ & $<0.05$ & $<0.05$ & $<0.05$ & $<0.05$ \\
\hline 15 & 1430 & $<0.05$ & $<0.05$ & 0.12 & $<0.20$ & 0.05 & $<0.05$ & 0.07 & $<0.05$ & $<0.05$ & $<0.05$ & $<0.05$ & $<0.05$ \\
\hline 20 & 1645 & $<0.05$ & $<0.05$ & 0.11 & $<0.20$ & 0.05 & $<0.05$ & 0.05 & $<0.05$ & $<0.05$ & $<0.05$ & $<0.05$ & $<0.05$ \\
\hline 29 & 1600 & $<0.05$ & $<0.05$ & 0.09 & $<0.20$ & 0.04 & $<0.05$ & 0.05 & $<0.05$ & $<0.05$ & 0.01 & $<0.05$ & $<0.05$ \\
\hline \multicolumn{14}{|c|}{ FEBRUARY 1992} \\
\hline 06 & 1500 & $<0.10$ & $<0.05$ & $<0.10$ & $<0.20$ & $<0.10$ & $<0.05$ & $<0.10$ & $<0.05$ & $<0.10$ & $<0.10$ & $<0.05$ & $<0.10$ \\
\hline 13 & 1500 & 0.05 & $<0.05$ & 0.13 & $<0.20$ & 0.08 & $<0.05$ & 0.08 & $<0.05$ & $<0.05$ & $<0.05$ & $<0.05$ & 0.06 \\
\hline 20 & 1745 & $<0.05$ & $<0.05$ & 0.10 & $<0.20$ & 0.07 & $<0.05$ & 0.07 & $<0.05$ & $<0.05$ & $<0.05$ & $<0.05$ & $<0.05$ \\
\hline 26 & 1015 & $<0.05$ & $<0.05$ & $<0.05$ & $<0.20$ & $<0.05$ & $<0.05$ & $<0.05$ & $<0.05$ & $<0.05$ & $<0.05$ & $<0.05$ & $<0.05$ \\
\hline \multicolumn{14}{|l|}{ MARCH } \\
\hline 05 & 1100 & $<0.05$ & $<0.05$ & 0.07 & $<0.20$ & $<0.05$ & $<0.05$ & 0.05 & $<0.05$ & $<0.05$ & $<0.05$ & $<0.05$ & $<0.05$ \\
\hline 11 & 1300 & $<0.05$ & $<0.05$ & 0.11 & $<0.20$ & 0.07 & $<0.05$ & 0.08 & $<0.05$ & $<0.05$ & $<0.05$ & $<0.05$ & $<0.05$ \\
\hline 19 & 0900 & $<0.05$ & $<0.05$ & 0.12 & $<0.20$ & 0.05 & $<0.05$ & 0.07 & $<0.05$ & $<0.05$ & $<0.05$ & $<0.05$ & $<0.05$ \\
\hline 26 & 1030 & $<0.05$ & $<0.05$ & 0.15 & $<0.20$ & 0.05 & $<0.05$ & 0.10 & $<0.05$ & $<0.05$ & $<0.05$ & $<0.05$ & 0.05 \\
\hline 30 & 1630 & $<0.05$ & $<0.05$ & 0.19 & $<0.20$ & 0.05 & $<0.05$ & 0.08 & $<0.05$ & $<0.05$ & $<0.05$ & $<0.05$ & $<0.05$ \\
\hline \multicolumn{14}{|l|}{ APRIL } \\
\hline 10 & 1500 & $<0.05$ & $<0.05$ & 0.18 & $<0.20$ & $<0.05$ & $<0.05$ & 0.09 & $<0.05$ & $<0.05$ & $<0.05$ & $<0.05$ & $<0.05$ \\
\hline 15 & 0930 & $<0.05$ & $<0.05$ & 0.22 & $<0.20$ & 0.05 & $<0.05$ & 0.08 & $<0.05$ & $<0.05$ & $<0.05$ & $<0.05$ & $<0.05$ \\
\hline 23 & 1030 & $<0.05$ & $<0.05$ & 0.09 & $<0.20$ & $<0.05$ & $<0.05$ & $<0.10$ & $<0.05$ & $<0.05$ & $<0.05$ & $<0.05$ & $<0.05$ \\
\hline 30 & 0900 & 0.09 & $<0.05$ & 0.64 & $<0.20$ & $<0.05$ & $<0.05$ & 0.21 & $<0.05$ & $<0.05$ & $<0.05$ & $<0.05$ & $<0.05$ \\
\hline \multicolumn{14}{|l|}{ MAY } \\
\hline 06 & 0915 & 0.10 & $<0.05$ & 1.1 & $<0.20$ & 0.11 & $<0.05$ & 0.52 & $<0.05$ & $<0.05$ & $<0.05$ & $<0.05$ & 0.09 \\
\hline 14 & 1330 & 0.08 & $<0.05$ & 0.64 & $<0.20$ & 0.10 & $<0.05$ & 0.41 & $<0.05$ & $<0.05$ & $<0.05$ & $<0.05$ & 0.07 \\
\hline 20 & 1110 & $<0.05$ & $<0.05$ & 0.31 & $<0.20$ & 0.08 & $<0.05$ & 0.16 & $<0.05$ & $<0.05$ & $<0.05$ & $<0.05$ & 0.08 \\
\hline 28 & 1100 & $<0.05$ & $<0.05$ & 0.32 & $<0.20$ & 0.08 & $<0.05$ & 0.14 & $<0.05$ & $<0.05$ & $<0.05$ & $<0.05$ & 0.09 \\
\hline \multicolumn{14}{|l|}{ JUNE } \\
\hline 03 & 1325 & 0.06 & $<0.05$ & 0.37 & $<0.20$ & 0.07 & $<0.05$ & 0.17 & $<0.05$ & $<0.05$ & $<0.05$ & $<0.05$ & 0.08 \\
\hline 12 & 1440 & 0.16 & $<0.05$ & 1.1 & 0.80 & 0.14 & $<0.10$ & 0.44 & 0.10 & $<0.05$ & $<0.05$ & $<0.05$ & 0.14 \\
\hline 19 & 0925 & 0.09 & $<0.05$ & 0.89 & 0.30 & 0.15 & $<0.10$ & 0.39 & 0.11 & $<0.05$ & $<0.05$ & $<0.05$ & 0.14 \\
\hline 25 & 1415 & 0.06 & $<0.05$ & 0.70 & 0.30 & 0.13 & 0.09 & 0.22 & 0.06 & $<0.05$ & $<0.05$ & $<0.05$ & 0.09 \\
\hline \multicolumn{14}{|l|}{ JULY } \\
\hline 02 & 1145 & 0.05 & $<0.05$ & 0.76 & $<0.20$ & 0.10 & 0.09 & 0.21 & 0.06 & $<0.05$ & $<0.05$ & $<0.05$ & 0.08 \\
\hline 09 & 1500 & 0.05 & $<0.05$ & 0.81 & 0.30 & 0.16 & 0.10 & 0.19 & 0.05 & $<0.05$ & $<0.05$ & $<0.05$ & 0.08 \\
\hline 16 & 1050 & 0.12 & $<0.05$ & 1.2 & 0.40 & 0.23 & 0.14 & 0.38 & 0.06 & $<0.05$ & $<0.05$ & $<0.05$ & 0.10 \\
\hline 23 & 1400 & 0.10 & $<0.05$ & 0.80 & 0.30 & 0.18 & 0.12 & 0.18 & $<0.05$ & $<0.05$ & $<0.05$ & $<0.05$ & 0.09 \\
\hline 31 & 1545 & 0.11 & $<0.05$ & 1.5 & 0.50 & 0.33 & 0.19 & 0.39 & 0.05 & $<0.05$ & $<0.05$ & $<0.05$ & 0.10 \\
\hline \multicolumn{14}{|c|}{ AUGUST } \\
\hline 05 & 1200 & 0.10 & $<0.05$ & 1.4 & 0.40 & 0.36 & 0.22 & 0.47 & 0.05 & $<0.05$ & $<0.05$ & $<0.05$ & 0.08 \\
\hline 13 & 0945 & 0.07 & $<0.05$ & 1.1 & 0.20 & 0.34 & 0.17 & 0.33 & 0.08 & $<0.05$ & 0.06 & $<0.05$ & 0.09 \\
\hline 21 & 1415 & 0.07 & $<0.05$ & 0.85 & 0.20 & 0.32 & 0.16 & 0.25 & $<0.05$ & $<0.05$ & 0.06 & $<0.05$ & $<0.05$ \\
\hline \multicolumn{14}{|c|}{ SEPTEMBER } \\
\hline 04 & 1510 & 0.05 & $<0.05$ & 0.50 & 0.20 & 0.23 & 0.12 & 0.16 & $<0.05$ & $<0.05$ & 0.06 & $<0.05$ & $<0.05$ \\
\hline 09 & 0835 & $<0.05$ & $<0.05$ & 0.41 & $<0.20$ & 0.21 & 0.12 & 0.11 & $<0.05$ & $<0.05$ & 0.05 & $<0.05$ & $<0.05$ \\
\hline 16 & 1450 & $<0.05$ & $<0.05$ & 0.36 & $<0.20$ & $<0.05$ & $<0.05$ & 0.08 & $<0.05$ & $<0.05$ & $<0.05$ & $<0.05$ & $<0.05$ \\
\hline 23 & 1300 & $<0.05$ & $<0.05$ & 0.33 & $<0.20$ & $<0.05$ & $<0.05$ & 0.07 & $<0.05$ & $<0.05$ & $<0.05$ & $<0.05$ & $<0.05$ \\
\hline
\end{tabular}

${ }^{1}$ The sample collected at 1315 hours on July 23,1991 , was from the end of the pier. The other samples were collected at equally spaced intervals across the river, starting at 1316 hours near the left bank and ending at 1320 hours near the right bank. 
$[\mu \mathrm{g} / \mathrm{L}$, microgram per liter; <, less than; diss, dissolved; --, no data;

DDE, dichlorodiphenyldichloroethylene; EPTC, S-Ethyl dipropylthiocarbamate;

$\mathrm{HCH}$, Hexachlorocyclohexane]

\begin{tabular}{|c|c|c|c|c|c|c|c|c|c|c|c|c|}
\hline Date & Time & $\begin{array}{c}\text { Alachlor } \\
\text { diss } \\
(\mu g / L)\end{array}$ & $\begin{array}{l}\text { Alpha } \\
\text { HCH } \\
\text { diss } \\
(\mu g / L)\end{array}$ & $\begin{array}{l}\text { Atra- } \\
\text { zine } \\
\text { diss } \\
(\mu g / L)\end{array}$ & $\begin{array}{l}\text { Azin- } \\
\text { phos- } \\
\text { methyl } \\
\text { diss } \\
\text { (ug/L) }\end{array}$ & $\begin{array}{c}\text { Ben- } \\
\text { fluralin } \\
\text { diss } \\
(\mu g / L)\end{array}$ & $\begin{array}{c}\text { Butylate } \\
\text { dlss } \\
(\mu g / L)\end{array}$ & $\begin{array}{c}\text { Carbaryl } \\
\text { diss } \\
(\mu g / L)\end{array}$ & $\begin{array}{l}\text { Carbo- } \\
\text { furan } \\
\text { diss } \\
(\mu g /)\end{array}$ & $\begin{array}{c}\text { Chior- } \\
\text { pyrifos } \\
\text { diss } \\
(\mu g / L)\end{array}$ & $\begin{array}{l}\text { Cyana- } \\
\text { zine } \\
\text { dlss } \\
(\mu g / L)\end{array}$ & $\begin{array}{c}\text { Dachtal } \\
\text { dlss } \\
(\mu g / 4)\end{array}$ \\
\hline
\end{tabular}

\begin{tabular}{|c|c|c|c|c|c|c|c|c|c|c|c|c|}
\hline \multirow{2}{*}{\multicolumn{13}{|c|}{ APRIL 1991}} \\
\hline & \multicolumn{12}{|c|}{ APRIL 1991} \\
\hline 02 & 1040 & -- & -- & -- & - & .. & -. & $\ldots$ & .. & .. & - & - \\
\hline 11 & 1100 & - & -- & -- & - & -- & -- & -- & - & -- & - & - \\
\hline 16 & 1150 & - & -- & -- & -- & -- & -- & -- & - & -- & - & -- \\
\hline 23 & 1030 & -- & -- & -- & - & -- & -- & -- & -- & -- & - & -- \\
\hline \multicolumn{13}{|l|}{ MAY } \\
\hline 01 & 1150 & - & -. & -- & - & -- & -- & -- & -- & -- & - & -- \\
\hline 07 & 1115 & 0.040 & -. & 0.137 & $<0.038$ & -- & $<0.008$ & $<0.046$ & $<0.013$ & $<0.005$ & 0.028 & -- \\
\hline 10 & 1030 & -- & -- & -- & -- & -- & -- & -- & -- & -- & -- & - \\
\hline 14 & 1120 & -- & -- & -. & -. & -- & -. & -- & -- & -- & -- & -- \\
\hline 17 & 1045 & 0.085 & -- & 0.148 & $<0.038$ & -- & $<0.008$ & $<0.046$ & $<0.013$ & $<0.005$ & 0.044 & -- \\
\hline 21 & 1140 & -- & -- & - & $\cdots$ & -- & -- & -- & -- & -- & -- & -- \\
\hline 24 & 1205 & 0.351 & - & 0.390 & $<0.038$ & - & $<0.008$ & $<0.046$ & 0.014 & $<0.005$ & 0.146 & -- \\
\hline 28 & 1210 & 0.335 & -- & 0.620 & $<0.038$ & -- & $<0.008$ & $<0.046$ & 0.008 & 0.009 & 0.294 & -- \\
\hline 31 & 1015 & 0.147 & -- & 0.338 & $<0.038$ & -- & $<0.008$ & $<0.046$ & 0.010 & $<0.005$ & 0.125 & -- \\
\hline \multicolumn{13}{|c|}{ JUNE 1991} \\
\hline 04 & 1300 & 0.478 & -- & 0.897 & $<0.038$ & - & $<0.008$ & $<0.046$ & 0.023 & $<0.005$ & 0.379 & -- \\
\hline 07 & 1100 & 0.192 & - & 0.379 & $<0.038$ & -- & $<0.008$ & $<0.046$ & 0.009 & $<0.005$ & 0.185 & -- \\
\hline 10 & 1130 & 0.182 & -- & 0.447 & $<0.038$ & -- & $<0.008$ & $<0.046$ & 0.008 & $<0.005$ & 0.265 & -- \\
\hline 13 & 1410 & 0.154 & -- & 0.342 & $<0.038$ & -- & $<0.008$ & $<0.046$ & 0.006 & $<0.005$ & 0.190 & - \\
\hline 17 & 1040 & 1.02 & -- & 2.01 & $<0.038$ & -- & $<0.008$ & $<0.046$ & 0.022 & 0.038 & 1.15 & -- \\
\hline 20 & 1345 & 1.04 & -- & 1.79 & $<0.038$ & -- & $<0.008$ & $<0.046$ & 0.037 & $<0.005$ & 0.814 & -- \\
\hline 24 & 1055 & 0.674 & -- & 0.952 & $<0.038$ & - & $<0.008$ & $<0.046$ & $<0.013$ & $<0.005$ & 0.997 & -- \\
\hline 27 & 0915 & 0.648 & -- & 0.782 & $<0.038$ & -- & $<0.008$ & $<0.046$ & 0.016 & $<0.005$ & 1.07 & - \\
\hline \multicolumn{13}{|l|}{ JULY } \\
\hline 01 & 1025 & 0.420 & -- & 0.639 & $<0.038$ & -- & $<0.008$ & $<0.046$ & $<0.013$ & $<0.005$ & 1.12 & -. \\
\hline 05 & 1205 & 0.208 & -- & 0.575 & $<0.038$ & -- & $<0.008$ & $<0.046$ & $<0.013$ & $<0.005$ & 0.751 & -- \\
\hline 08 & 1130 & 0.137 & -. & 0.319 & $<0.038$ & -- & $<0.008$ & $<0.046$ & $<0.013$ & $<0.005$ & 0.474 & -- \\
\hline 11 & 1300 & -- & -- & - & -- & -- & - & - & - & -- & $\cdots$ & -- \\
\hline 15 & 1025 & 0.187 & - & 0.728 & $<0.038$ & -- & $<0.008$ & $<0.046$ & $<0.013$ & $<0.005$ & 0.780 & -- \\
\hline 18 & 1200 & - & - & -- & - & -- & - & -- & -- & -- & -- & -- \\
\hline 22 & 1050 & -- & -- & -- & - & -- & - & - & - & -- & -- & - \\
\hline 29 & 1230 & 0.054 & - & 0.524 & $<0.038$ & -- & $<0.008$ & $<0.046$ & $<0.013$ & $<0.005$ & 0.260 & -- \\
\hline \multicolumn{13}{|c|}{ AUGUST 1991} \\
\hline 06 & 1240 & 0.056 & -- & 0.479 & $<0.038$ & -- & $<0.008$ & $<0.046$ & $<0.013$ & $<0.005$ & 0.193 & -- \\
\hline 12 & 1125 & 0.047 & -- & 0.381 & $<0.038$ & -- & $<0.008$ & $<0.046$ & $<0.013$ & $<0.005$ & 0.160 & - \\
\hline 19 & 1145 & 0.039 &.- & 0.373 & $<0.038$ & -- & $<0.008$ & $<0.046$ & $<0.013$ & $<0.015$ & 0.114 & - \\
\hline 26 & 1205 & 0.020 & -- & 0.269 & $<0.038$ & -- & $<0.008$ & $<0.046$ & $<0.013$ & 0.005 & 0.071 & - \\
\hline \multicolumn{13}{|c|}{ SEPTEMBER } \\
\hline 03 & 1200 & 0.017 & -- & 0.237 & $<0.038$ & -- & $<0.008$ & $<0.046$ & $<0.013$ & $<0.005$ & 0.070 & -- \\
\hline 09 & 1500 & 0.017 & -- & 0.236 & $<0.038$ & -- & $<0.008$ & $<0.046$ & $<0.013$ & $<0.005$ & 0.065 & - \\
\hline 17 & 1215 & 0.020 & -- & 0.254 & $<0.038$ & -- & $<0.008$ & $<0.046$ & $<0.013$ & $<0.005$ & 0.062 & - \\
\hline 24 & 1215 & 0.010 & -- & 0.175 & $<0.038$ & -- & $<0.008$ & $<0.046$ & $<0.013$ & $<0.005$ & 0.039 & -- \\
\hline
\end{tabular}


Table 8.-Pesticide results from solid-phase extraction 1-liter method-Continued

\begin{tabular}{|c|c|c|c|c|c|c|c|c|c|c|c|c|}
\hline Date & Time & $\begin{array}{c}\text { Alachlor } \\
\text { dlss } \\
(\mu g / L)\end{array}$ & $\begin{array}{c}\text { Alpha } \\
\text { HCH } \\
\text { dlss } \\
(\mu g / L)\end{array}$ & $\begin{array}{l}\text { Atra- } \\
\text { zlne } \\
\text { diss } \\
(\mu \mathrm{g} / L)\end{array}$ & $\begin{array}{c}\text { Azin- } \\
\text { phos- } \\
\text { mathyl } \\
\text { dlss } \\
(\mu g / L)\end{array}$ & $\begin{array}{c}\text { Ben- } \\
\text { fluralln } \\
\text { diss } \\
(\mu g / L)\end{array}$ & $\begin{array}{c}\text { Butylate } \\
\text { dlss } \\
(\mu \mathrm{g} / \mathrm{L})\end{array}$ & $\begin{array}{c}\text { Carbaryl } \\
\text { diss } \\
(\mu g / L)\end{array}$ & $\begin{array}{c}\text { Carbo- } \\
\text { furan } \\
\text { diss } \\
(\mu g / L)\end{array}$ & $\begin{array}{c}\text { Chlor- } \\
\text { pyrifoa } \\
\text { disa } \\
(\mu g /)\end{array}$ & $\begin{array}{c}\text { Cyana- } \\
\text { zlne } \\
\text { dlas } \\
(\mu g / L)\end{array}$ & $\begin{array}{c}\text { Dachtal } \\
\text { dlss } \\
(\mu g / L)\end{array}$ \\
\hline \multicolumn{13}{|c|}{05420500 - Mississippi River at Clinton, lowa-Continued } \\
\hline OCTOBER & & & & & & & & & & & & \\
\hline 01 & 1200 & -- & -- & -- & -- & -- & -- & - & -- & -- & -. & -. \\
\hline 07 & 1230 & 0.025 & .. & 0.149 & $<0.038$ & -- & $<0.008$ & $<0.046$ & $<0.013$ & $<0.005$ & 0.028 & -- \\
\hline 15 & 1300 & 0.021 & -- & 0.163 & $<0.038$ & -- & $<0.008$ & $<0.046$ & $<0.013$ & $<0.005$ & 0.026 & -- \\
\hline 22 & 1230 & 0.010 & -- & 0.104 & $<0.038$ & -- & $<0.008$ & $<0.046$ & $<0.013$ & $<0.005$ & 0.018 & - \\
\hline 29 & 1330 & 0.009 & - & 0.100 & $<0.038$ & -- & $<0.008$ & $<0.046$ & $<0.013$ & $<0.005$ & 0.018 & - \\
\hline \multicolumn{13}{|c|}{ NOVEMBER 1991} \\
\hline 05 & 1245 & 0.012 & -- & 0.111 & $<0.038$ & -- & $<0.008$ & $<0.046$ & $<0.013$ & $<0.005$ & 0.019 & - \\
\hline 12 & 1255 & 0.007 & -- & 0.099 & $<0.038$ & - & $<0.008$ & $<0.046$ & $<0.013$ & $<0.005$ & 0.015 & -- \\
\hline 26 & 1320 & $<0.015$ & -- & 0.089 & $<0.038$ & -- & $<0.008$ & $<0.046$ & $<0.013$ & $<0.005$ & 0.026 & -- \\
\hline \multicolumn{13}{|c|}{ DECEMBER } \\
\hline 09 & 1200 & 0.009 & -- & 0.097 & $<0.038$ & -- & $<0.008$ & $<0.046$ & $<0.013$ & $<0.005$ & 0.019 & - \\
\hline 22 & 1330 & 0.010 & -- & 0.074 & $<0.038$ & -- & $<0.008$ & $<0.046$ & $<0.013$ & $<0.005$ & 0.017 & - \\
\hline \multicolumn{13}{|c|}{ JANUARY 1992} \\
\hline 07 & 1210 & 0.006 & $<0.007$ & 0.053 & $<0.038$ & $<0.005$ & $<0.008$ & $<0.046$ & $<0.013$ & $<0.005$ & $<0.013$ & $<0.004$ \\
\hline 22 & 1340 & 0.005 & $<0.007$ & 0.058 & $<0.038$ & $<0.005$ & $<0.008$ & $<0.046$ & $<0.013$ & $<0.005$ & 0.011 & $<0.004$ \\
\hline \multicolumn{13}{|c|}{ FEBRUARY } \\
\hline 03 & 1245 & 0.005 & $<0.007$ & 0.051 & $<0.038$ & $<0.005$ & $<0.008$ & $<0.046$ & $<0.013$ & $<0.005$ & $<0.013$ & $<0.004$ \\
\hline 20 & 1230 & 0.010 & $<0.007$ & 0.074 & $<0.038$ & $<0.005$ & $<0.008$ & $<0.046$ & $<0.013$ & $<0.005$ & 0.019 & $<0.004$ \\
\hline \multicolumn{13}{|c|}{ MARCH 1992} \\
\hline 04 & 1100 & - & -- & -- & -- & -- & -- & -- & - & -- & -- & - \\
\hline 13 & 1200 & 0.013 & 0.002 & 0.075 & $<0.038$ & $<0.013$ & $<0.008$ & $<0.046$ & $<0.013$ & $<0.005$ & 0.015 & $<0.004$ \\
\hline 17 & 1400 & 0.009 & $<0.007$ & 0.068 & $<0.038$ & $<0.013$ & $<0.008$ & $<0.046$ & $<0.013$ & $<0.005$ & 0.013 & $<0.004$ \\
\hline 24 & 1125 & 0.015 & $<0.007$ & 0.061 & $<0.038$ & $<0.013$ & $<0.008$ & $<0.046$ & $<0.013$ & $<0.005$ & $<0.013$ & $<0.004$ \\
\hline 30 & 1155 & 0.012 & $<0.007$ & 0.063 & $<0.038$ & $<0.013$ & $<0.008$ & $<0.046$ & $<0.013$ & $<0.005$ & $<0.013$ & $<0.004$ \\
\hline \multicolumn{13}{|l|}{ APRIL } \\
\hline 06 & 1215 & 0.011 & $<0.007$ & 0.069 & $<0.038$ & $<0.013$ & $<0.008$ & $<0.046$ & $<0.013$ & $<0.005$ & 0.017 & $<0.004$ \\
\hline 14 & 1130 & 0.010 & $<0.007$ & 0.066 & $<0.038$ & $<0.013$ & $<0.008$ & $<0.046$ & $<0.013$ & $<0.005$ & 0.016 & $<0.004$ \\
\hline 24 & 1145 & 0.021 & $<0.007$ & 0.130 & $<0.038$ & $<0.013$ & $<0.008$ & $<0.046$ & $<0.013$ & $<0.005$ & 0.023 & $<0.004$ \\
\hline 28 & 1145 & 0.009 & $<0.007$ & 0.073 & $<0.038$ & $<0.013$ & $<0.008$ & $<0.046$ & $<0.013$ & $<0.005$ & 0.013 & $<0.004$ \\
\hline \multicolumn{13}{|l|}{ MAY } \\
\hline 05 & 1115 & 0.010 & $<0.007$ & 0.058 & $<0.038$ & $<0.013$ & $<0.008$ & $<0.046$ & $<0.013$ & $<0.005$ & 0.015 & $<0.004$ \\
\hline 12 & 1130 & 0.028 & $<0.007$ & 0.058 & $<0.038$ & $<0.013$ & 0.005 & $<0.046$ & $<0.013$ & $<0.005$ & 0.020 & $<0.004$ \\
\hline 19 & 1130 & 0.031 & $<0.007$ & 0.089 & $<0.038$ & $<0.013$ & $<0.008$ & $<0.046$ & $<0.013$ & $<0.005$ & 0.034 & $<0.004$ \\
\hline 26 & 1215 & 0.078 & $<0.007$ & 0.140 & $<0.038$ & $<0.013$ & $<0.008$ & $<0.046$ & $<0.013$ & 0.011 & 0.068 & 0.001 \\
\hline \multicolumn{13}{|c|}{ 05586100 - Illinois River at Valley City } \\
\hline \multicolumn{13}{|c|}{ APRIL 1991} \\
\hline 05 & 1230 & -- & -- & -- & -- & -- & -- & - & -- & -- & -- & -- \\
\hline 17 & 1330 & -- & - & -- & -. & -- & -- & - & -- & -- & -- & -- \\
\hline 26 & 1000 & -- & -- & -- & -- & -- & -- & -- & -- & .. & -- & - \\
\hline \multicolumn{13}{|l|}{ MAY } \\
\hline 03 & 1545 & 0.184 & -- & 0.745 & $<0.038$ & -- & 0.024 & $<0.046$ & $<0.013$ & $<0.005$ & 0.763 & -- \\
\hline 08 & 1245 & 4.19 & -- & 11 & $<0.038$ & -- & 0.088 & $<0.046$ & 0.149 & 0.148 & 7.81 & -- \\
\hline 10 & 1100 & -- & -- & -- & -- & -- & -- & - & -- & -- & -- & -- \\
\hline 17 & 1145 & 0.585 & -- & 2.58 & $<0.038$ & -- & $<0.008$ & $<0.046$ & 0.013 & $<0.005$ & 2.07 & - \\
\hline 19 & 1115 & 1.19 & -- & 4.79 & $<0.038$ & -- & 0.005 & $<0.046$ & 0.038 & 0.063 & 3.28 & -- \\
\hline 22 & 1715 & 1.88 & -- & 8.73 & $<0.038$ & -- & 0.014 & $<0.046$ & 0.159 & $<0.005$ & 6.61 & - \\
\hline 29 & 1130 & 1.96 & - & 11 & $<0.038$ & -- & 0.016 & $<0.046$ & 0.029 & 0.040 & 1.78 & -- \\
\hline 31 & 1045 & 0.931 & -- & 6.76 & $<0.038$ & -- & 0.007 & $<0.046$ & 0.054 & $<0.005$ & 4.75 & -- \\
\hline
\end{tabular}


Table 8.--Pesticide results from solid-phase extraction 1-liter method--Continued

\begin{tabular}{|c|c|c|c|c|c|c|c|c|c|c|c|c|}
\hline Date & Time & $\begin{array}{c}\text { Alachlor } \\
\text { diss } \\
(\mu g / L)\end{array}$ & $\begin{array}{l}\text { Alpha } \\
\text { HCH } \\
\text { diss } \\
(\mu g / L)\end{array}$ & $\begin{array}{l}\text { Atra- } \\
\text { zine } \\
\text { diss } \\
(\mu g / L)\end{array}$ & $\begin{array}{l}\text { Azin- } \\
\text { phos- } \\
\text { methyl } \\
\text { diss }\end{array}$ & $\begin{array}{c}\text { Ben- } \\
\text { fluralln } \\
\text { dlss } \\
(\mu \mathrm{g} / \mathrm{L})\end{array}$ & $\begin{array}{c}\text { Butylate } \\
\text { diss } \\
(\mu g / L)\end{array}$ & $\begin{array}{c}\text { Carbaryl } \\
\text { diss } \\
(\mu g / L)\end{array}$ & $\begin{array}{l}\text { Carbo- } \\
\text { furan } \\
\text { diss } \\
(\mu \mathrm{g} / \mathrm{L})\end{array}$ & $\begin{array}{c}\text { Chlor- } \\
\text { pyrifos } \\
\text { diss } \\
(\mu \mathrm{g} / \mathrm{L})\end{array}$ & $\begin{array}{l}\text { Cyana- } \\
\text { zlne } \\
\text { diss } \\
(\mu g / L)\end{array}$ & $\begin{array}{c}\text { Dachtal } \\
\text { diss } \\
(\mu g / L)\end{array}$ \\
\hline
\end{tabular}

\begin{tabular}{|c|c|c|c|c|c|c|c|c|c|c|c|c|}
\hline \multirow{2}{*}{\multicolumn{13}{|c|}{ JUNE 1991}} \\
\hline & & & & & & & & & & & & \\
\hline \multicolumn{3}{|c|}{$\begin{array}{c}\text { JUNE } 1991 \\
04\end{array}$} & & 6.11 & $<0.038$ & & 0.005 & $<0.046$ & 0.037 & $<0.005$ & 4.26 & -- \\
\hline 06 & 1200 & 0.668 & -- & 4.62 & $<0.038$ & -- & $<0.046$ & $<0.046$ & 0.031 & $<0.005$ & 1.64 & -- \\
\hline 11 & 1345 & 0.691 & -- & 4.78 & $<0.038$ & -- & $<0.046$ & $<0.046$ & 0.024 & $<0.005$ & 3.13 & -- \\
\hline 14 & 1200 & 0.538 & -- & 4.09 & $<0.038$ & -- & $<0.046$ & $<0.046$ & 0.009 & $<0.005$ & 2.32 & -- \\
\hline 18 & 1130 & 0.509 & -- & 3.69 & $<0.038$ & -- & $<0.046$ & $<0.046$ & 0.009 & $<0.005$ & 1.44 & -- \\
\hline 20 & 1130 & 0.396 & -- & 3.39 & $<0.038$ & -- & $<0.046$ & $<0.046$ & $<0.013$ & $<0.005$ & 1.34 & -- \\
\hline 24 & 1130 & 0.206 & -- & 2.74 & $<0.038$ & -- & $<0.046$ & $<0.046$ & $<0.013$ & $<0.005$ & 1.25 & -- \\
\hline 27 & 1100 & 0.214 & -- & 2.06 & $<0.038$ & -- & $<0.046$ & $<0.046$ & 0.033 & $<0.005$ & 0.716 & -- \\
\hline \multicolumn{13}{|l|}{ JULY } \\
\hline 01 & 0930 & 0.109 & -- & 1.77 & $<0.038$ & -- & $<0.046$ & $<0.046$ & 0.015 & $<0.005$ & 0.631 & -- \\
\hline 03 & 1100 & 0.075 & -- & 1.28 & $<0.038$ & -- & $<0.046$ & $<0.046$ & 0.022 & $<0.005$ & 0.456 & -- \\
\hline 09 & 1000 & 0.042 & -- & 0.923 & $<0.038$ & .- & $<0.046$ & $<0.046$ & $<0.013$ & $<0.005$ & 0.271 & .- \\
\hline 11 & 1045 & 0.053 & -- & 0.791 & $<0.038$ & .- & $<0.046$ & $<0.046$ & $<0.013$ & $<0.005$ & 0.240 & -- \\
\hline 18 & 1115 & -- & -- & -- & -- & -- & -- & -- & -- & .- & -- & -- \\
\hline 25 & 1340 & 0.015 & -- & 0.451 & $<0.038$ & -- & $<0.046$ & $<0.046$ & $<0.013$ & $<0.005$ & 0.123 & -- \\
\hline 31 & 1100 & 0.013 & -- & 0.350 & $<0.038$ & -- & $<0.046$ & $<0.046$ & $<0.013$ & $<0.005$ & 0.096 & -- \\
\hline \multicolumn{13}{|l|}{ AUGUST } \\
\hline 06 & 1015 & 0.011 & -- & 0.331 & $<0.038$ & -- & $<0.046$ & $<0.046$ & $<0.013$ & $<0.005$ & 0.087 & - \\
\hline 13 & 1200 & 0.019 & -- & 0.303 & $<0.038$ & - & $<0.046$ & $<0.046$ & $<0.013$ & $<0.005$ & 0.086 & - \\
\hline 21 & 1230 & 0.009 & -- & 0.200 & $<0.038$ & -- & $<0.046$ & $<0.046$ & $<0.013$ & $<0.005$ & 0.050 & -- \\
\hline 28 & 1015 & 0.009 & -- & 0.119 & $<0.038$ & -- & $<0.046$ & $<0.046$ & $<0.013$ & $<0.005$ & 0.037 & -- \\
\hline \multicolumn{13}{|c|}{ SEPTEMBER 1991} \\
\hline 03 & 1050 & 0.009 & -- & 0.141 & $<0.038$ & -- & $<0.046$ & $<0.046$ & $<0.013$ & $<0.005$ & 0.047 & -- \\
\hline 10 & 1200 & 0.021 & -- & 0.219 & $<0.038$ & -- & $<0.046$ & 0.004 & $<0.013$ & $<0.005$ & 0.076 & -- \\
\hline 17 & 1100 & 0.025 & -- & 0.205 & $<0.038$ & -- & $<0.046$ & $<0.046$ & 0.010 & $<0.005$ & 0.089 & -- \\
\hline 24 & 1100 & 0.025 & - & 0.159 & $<0.038$ & -- & $<0.046$ & 0.013 & $<0.013$ & $<0.005$ & 0.046 & -- \\
\hline \multicolumn{13}{|c|}{ OCTOBER } \\
\hline 01 & 1130 & 0.025 & -- & 0.169 & $<0.038$ & -- & $<0.008$ & $<0.046$ & $<0.013$ & $<0.005$ & 0.050 & - \\
\hline 08 & 1330 & 0.064 & -- & 0.463 & $<0.038$ & -- & $<0.008$ & 0.017 & $<0.013$ & $<0.005$ & 0.141 & -- \\
\hline 15 & 1215 & 0.029 & -- & 0.195 & $<0.038$ & -- & $<0.008$ & $<0.046$ & $<0.013$ & $<0.005$ & 0.068 & -- \\
\hline 22 & 1130 & 0.024 & -- & 0.111 & $<0.038$ & -- & $<0.008$ & $<0.046$ & $<0.013$ & $<0.005$ & 0.034 & -- \\
\hline \multicolumn{13}{|c|}{ NOVEMBER } \\
\hline 05 & 1140 & 0.088 & -- & 0.213 & $<0.038$ & -- & $<0.008$ & $<0.046$ & $<0.013$ & $<0.005$ & 0.043 & -- \\
\hline 15 & 1130 & 0.017 & -- & 0.200 & $<0.038$ & -- & $<0.008$ & $<0.046$ & $<0.013$ & $<0.005$ & 0.049 & -- \\
\hline \multicolumn{13}{|c|}{ DECEMBER } \\
\hline 03 & 1030 & 0.032 & -- & 0.234 & $<0.038$ & -- & $<0.008$ & $<0.046$ & $<0.013$ & $<0.005$ & 0.048 & -. \\
\hline 18 & 1200 & 0.026 & .- & 0.211 & $<0.038$ & -. & $<0.008$ & $<0.046$ & $<0.013$ & $<0.005$ & 0.049 & -. \\
\hline \multicolumn{13}{|c|}{ JANUARY 1992} \\
\hline 02 & 1330 & 0.014 & -- & 0.158 & $<0.038$ & -- & $<0.008$ & $<0.046$ & $<0.013$ & $<0.005$ & 0.073 & -- \\
\hline 15 & 1645 & 0.011 & $<0.007$ & 0.140 & $<0.038$ & $<0.013$ & $<0.008$ & $<0.046$ & $<0.013$ & $<0.005$ & 0.043 & $<0.004$ \\
\hline 30 & 1345 & 0.016 & $<0.007$ & 0.150 & $<0.038$ & $<0.013$ & $<0.008$ & $<0.046$ & $<0.013$ & $<0.005$ & 0.054 & $<0.004$ \\
\hline \multicolumn{13}{|c|}{ FEBRUARY 1992} \\
\hline 12 & 1200 & 0.011 & $<0.007$ & 0.130 & $<0.038$ & $<0.013$ & $<0.008$ & $<0.046$ & $<0.013$ & $<0.005$ & 0.130 & $<0.004$ \\
\hline 28 & 1245 & 0.023 & $<0.007$ & 0.190 & $<0.038$ & $<0.013$ & $<0.008$ & $<0.046$ & $<0.013$ & $<0.005$ & 0.068 & $<0.004$ \\
\hline \multicolumn{13}{|l|}{ MARCH } \\
\hline 03 & 1100 & 0.019 & $<0.007$ & 0.160 & $<0.038$ & $<0.013$ & $<0.008$ & $<0.046$ & $<0.013$ & $<0.005$ & 0.061 & $<0.004$ \\
\hline 10 & 1015 & 0.014 & $<0.007$ & 0.180 & $<0.038$ & $<0.013$ & $<0.008$ & $<0.046$ & $<0.013$ & $<0.005$ & 0.062 & $<0.004$ \\
\hline
\end{tabular}


Table 8.-Pesticide results from solid-phase extraction 1-liter method-Continued

\begin{tabular}{|c|c|c|c|c|c|c|c|c|c|c|c|c|}
\hline Dale & Time & $\begin{array}{c}\text { Alachior } \\
\text { diss } \\
(\mu g / L)\end{array}$ & $\begin{array}{c}\text { Alpha } \\
\text { HCH } \\
\text { diss } \\
(\mu g / L)\end{array}$ & $\begin{array}{l}\text { Atra- } \\
\text { zine } \\
\text { diss } \\
(\mu g / L)\end{array}$ & $\begin{array}{c}\text { Azin- } \\
\text { phos- } \\
\text { mothyl } \\
\text { dlss } \\
(\mu \mathrm{g} / \mathrm{L})\end{array}$ & $\begin{array}{l}\text { Ben- } \\
\text { flurailin } \\
\text { diss } \\
(\mu g / L)\end{array}$ & $\begin{array}{c}\text { Butylate } \\
\text { diss } \\
(\mu g / L)\end{array}$ & $\begin{array}{c}\text { Carbaryl } \\
\text { diss } \\
(\mu g / L)\end{array}$ & $\begin{array}{l}\text { Carbo- } \\
\text { furan } \\
\text { diss } \\
(\mu g / L)\end{array}$ & $\begin{array}{c}\text { Chior- } \\
\text { pyrifos } \\
\text { diss } \\
(\mu g / L)\end{array}$ & $\begin{array}{l}\text { Cyana- } \\
\text { zine } \\
\text { diss } \\
(\mu g / L)\end{array}$ & $\begin{array}{c}\text { Dachtal } \\
\text { dlss } \\
(\mu g / 4)\end{array}$ \\
\hline \multicolumn{13}{|c|}{05586100 - Illinois River at Valley City-Continued } \\
\hline \multicolumn{13}{|c|}{ MARCH (cont.) } \\
\hline 17 & 1050 & 0.011 & $<0.007$ & 0.170 & $<0.038$ & $<0.013$ & $<0.008$ & $<0.046$ & $<0.013$ & $<0.005$ & 0.064 & $<0.004$ \\
\hline 25 & 1045 & 0.018 & $<0.007$ & 0.190 & $<0.038$ & $<0.013$ & $<0.008$ & $<0.046$ & $<0.013$ & $<0.005$ & 0.051 & $<0.004$ \\
\hline \multicolumn{13}{|c|}{ 06805500 - Platte River at Louisville, Nebr. } \\
\hline \multicolumn{13}{|c|}{ APRIL 1991} \\
\hline 09 & 1250 & -- & -- & -- & -- & -- & -- & -- & -- & -- & -- & - \\
\hline 16 & 1030 & -- & -- & -- & -- & -- & -- & -- &.- & -- & -- & - \\
\hline 24 & 1030 & -- & -- & -- & -- & -- & -- & -- & -- & - & -- & - \\
\hline 29 & 1000 & 0.139 & -- & 0.590 & $<0.038$ & -- & 0.007 & $<0.046$ & $<0.013$ & $<0.005$ & 0.049 & - \\
\hline \multicolumn{13}{|l|}{ MAY } \\
\hline 06 & 1115 & 0.219 & -. & 0.975 & $<0.038$ & - & 0.005 & $<0.046$ & $<0.013$ & $<0.005$ & 0.312 & - \\
\hline 09 & 0940 & 0.145 & -- & 1.00 & $<0.038$ & -- & 0.007 & $<0.046$ & $<0.013$ & $<0.005$ & 0.182 & - \\
\hline 13 & 1110 & -- & - & -- & -- & -- & -- & -- & -- & -- & -- & -- \\
\hline 16 & 1130 & -- & -- & -- & -- & -- & -- & -- & -- & $\cdots$ & -- & -. \\
\hline 21 & 0945 & 6.51 & -- & 17 & $<0.038$ & -- & 0.021 & $<0.046$ & 0.153 & $<0.005$ & 9.02 & - \\
\hline 24 & 0740 & 0.647 & -- & 3.04 & $<0.038$ & - & $<0.008$ & $<0.046$ & 0.039 & $<0.005$ & 1.75 & - \\
\hline 29 & 1015 & 0.772 & -- & 4.18 & $<0.038$ & -- & $<0.008$ & $<0.046$ & 0.010 & 0.017 & 0.787 & -- \\
\hline 31 & 0735 & 2.50 & -- & 9.52 & $<0.038$ & -- & $<0.008$ & $<0.046$ & 0.028 & $<0.005$ & 7.80 & - \\
\hline \multicolumn{13}{|c|}{ JUNE 1991} \\
\hline 04 & 0800 & 2.09 & - & 11 & $<0.038$ & -- & $<0.008$ & $<0.046$ & 0.053 & $<0.005$ & 13.0 & -- \\
\hline 07 & 1415 & 3.72 & -- & 14 & $<0.038$ & -- & 0.006 & 0.005 & 0.092 & 0.036 & 20.3 & -- \\
\hline 11 & 1045 & 1.05 & - & 7.95 & $<0.038$ & -- & 0.005 & $<0.046$ & 0.093 & $<0.005$ & 4.43 & -- \\
\hline 14 & 0750 & 0.914 & - & 5.26 & $<0.038$ & -- & $<0.008$ & $<0.046$ & 0.078 & 0.062 & 2.32 & -- \\
\hline 18 & 0935 & 0.700 & -- & 4.84 & $<0.038$ & -- & $<0.008$ & 0.004 & 0.119 & $<0.005$ & 3.71 & -- \\
\hline 21 & 1020 & 0.213 & -- & 2.72 & $<0.038$ & -- & $<0.008$ & $<0.046$ & 0.020 & $<0.005$ & 1.52 & -- \\
\hline 24 & 1110 & 0.327 & -- & 2.55 & $<0.038$ & -- & $<0.008$ & 0.006 & 0.191 & $<0.005$ & 0.814 & -- \\
\hline 27 & 1020 & 0.122 & -- & 1.75 & $<0.038$ & - & $<0.008$ & 0.017 & 0.056 & $<0.005$ & 0.746 & - \\
\hline \multicolumn{13}{|l|}{ JULY } \\
\hline 01 & 0950 & 0.036 & - & 1.05 & $<0.038$ & -- & $<0.008$ & 0.022 & $<0.013$ & $<0.005$ & 0.389 & -- \\
\hline 03 & 0950 & 0.092 & -- & 1.58 & $<0.038$ & -- & $<0.008$ & $<0.046$ & 1.27 & $<0.005$ & 1.37 & -- \\
\hline 08 & 1100 & 0.039 & -- & 1.18 & $<0.038$ & -- & $<0.008$ & 0.006 & 0.038 & $<0.005$ & 0.422 & -. \\
\hline 11 & 1020 & 0.177 & -- & 2.03 & $<0.038$ & -- & $<0.008$ & $<0.046$ & 0.295 & $<0.005$ & 0.229 & - \\
\hline 15 & 0950 & 0.058 & - & 1.80 & $<0.038$ & -- & $<0.008$ & $<0.046$ & 0.008 & $<0.005$ & 0.185 & -- \\
\hline 25 & 1000 & 0.016 & - & 0.994 & $<0.038$ & -- & $<0.008$ & $<0.046$ & $<0.013$ & $<0.005$ & 0.161 & -- \\
\hline 29 & 0940 & 0.007 & -- & 0.689 & $<0.038$ & -- & $<0.008$ & $<0.046$ & $<0.013$ & $<0.005$ & 0.090 & - \\
\hline \multicolumn{13}{|c|}{ AUGUST 1991} \\
\hline 06 & 1130 & 0.006 & - & 0.520 & $<0.038$ & -- & $<0.008$ & $<0.046$ & $<0.013$ & $<0.005$ & 0.060 & - \\
\hline 12 & 1115 & 0.005 & - & 0.481 & $<0.038$ & -- & $<0.008$ & $<0.046$ & $<0.013$ & $<0.005$ & 0.068 & - \\
\hline 20 & 1000 & 0.009 & -- & 0.496 & $<0.038$ & - & $<0.008$ & $<0.046$ & $<0.013$ & $<0.005$ & 0.132 & -- \\
\hline 27 & 0940 & 0.005 & -- & 0.409 & $<0.038$ & - & $<0.008$ & $<0.046$ & $<0.013$ & $<0.005$ & 0.047 & -- \\
\hline \multicolumn{13}{|c|}{ SEPTEMBER } \\
\hline 03 & 0950 & $<0.009$ & - & 0.357 & $<0.038$ & -- & $<0.008$ & $<0.046$ & $<0.013$ & $<0.005$ & 0.057 & - \\
\hline 10 & 1030 & 0.007 & -- & 0.281 & $<0.038$ & -- & $<0.008$ & 0.016 & $<0.013$ & $<0.005$ & 0.035 & -- \\
\hline 17 & 1115 & 0.029 & -- & 0.333 & $<0.038$ & -- & $<0.008$ & $<0.046$ & 0.020 & $<0.005$ & 0.087 & -- \\
\hline 24 & 1135 & 0.006 & -- & 0.183 & $<0.038$ & -- & $<0.008$ & $<0.046$ & 0.006 & $<0.005$ & 0.024 & -- \\
\hline \multicolumn{13}{|c|}{ OCTOBER } \\
\hline 01 & 1115 & $<0.009$ & -- & 0.203 & $<0.038$ & -- & $<0.008$ & $<0.046$ & $<0.013$ & $<0.005$ & 0.025 & -- \\
\hline 08 & 1030 & 0.003 & -- & 0.114 & $<0.038$ & -- & $<0.008$ & $<0.046$ & $<0.013$ & $<0.005$ & 0.010 & -- \\
\hline
\end{tabular}


Table 8.--Pesticide results from solid-phase extraction 1-liter method--Continued

\begin{tabular}{|c|c|c|c|c|c|c|c|c|c|c|c|c|}
\hline Date & Time & $\begin{array}{c}\text { Alachlor } \\
\text { diss } \\
(\mu g / L)\end{array}$ & $\begin{array}{l}\text { Alpha } \\
\text { HCH } \\
\text { diss } \\
(\mu g / L)\end{array}$ & $\begin{array}{l}\text { Atra- } \\
\text { zine } \\
\text { diss } \\
(\mu g / L)\end{array}$ & $\begin{array}{c}\text { Azin- } \\
\text { phos- } \\
\text { methyi } \\
\text { diss } \\
(\mu g / L)\end{array}$ & $\begin{array}{c}\text { Ben- } \\
\text { fluralin } \\
\text { diss } \\
(\mu \mathrm{g} / \mathrm{L})\end{array}$ & $\begin{array}{c}\text { Butylate } \\
\text { diss } \\
(\mu g / L)\end{array}$ & $\begin{array}{c}\text { Carbaryi } \\
\text { diss } \\
(\mu g / L)\end{array}$ & $\begin{array}{c}\text { Carbo- } \\
\text { furan } \\
\text { diss } \\
(\mu g / L)\end{array}$ & $\begin{array}{c}\text { Chior- } \\
\text { pyrlios } \\
\text { diss } \\
(\mu \mathrm{g} / \mathrm{L})\end{array}$ & $\begin{array}{l}\text { Cyana- } \\
\text { zine } \\
\text { diss } \\
(\mu g / L)\end{array}$ & $\begin{array}{c}\text { Dachtal } \\
\text { diss } \\
(\mu g / L)\end{array}$ \\
\hline
\end{tabular}

OCTOBER (cont.)

\begin{tabular}{|c|c|c|c|c|c|c|c|c|c|c|c|c|}
\hline 15 & 1130 & $<0.009$ & -- & 0.209 & $<0.038$ & -- & $<0.008$ & $<0.046$ & $<0.013$ & $<0.005$ & 0.016 & -- \\
\hline 24 & 1200 & $<0.009$ & -- & 0.119 & $<0.038$ & -- & $<0.008$ & $<0.046$ & $<0.013$ & $<0.005$ & 0.010 & -- \\
\hline \multicolumn{13}{|c|}{ NOVEMBER } \\
\hline 05 & 1200 & 0.008 & -- & 0.215 & $<0.038$ & -- & $<0.008$ & 0.015 & $<0.013$ & $<0.005$ & 0.074 & -- \\
\hline 19 & 1140 & 0.017 & -- & 0.912 & $<0.038$ & -. & $<0.008$ & $<0.046$ & $<0.013$ & $<0.005$ & 1.86 & - \\
\hline \multicolumn{13}{|c|}{ DECEMBER 1991} \\
\hline 05 & 1130 & -- & -- & -. & - & -- & - & -- & - & -- & - & -- \\
\hline 20 & 1000 & $<0.009$ & $<0.007$ & 0.093 & $<0.038$ & $<0.013$ & $<0.008$ & $<0.046$ & $<0.013$ & $<0.005$ & 0.010 & $<0.004$ \\
\hline 30 & 1020 & 0.010 & $<0.007$ & 0.140 & $<0.038$ & $<0.013$ & $<0.008$ & $<0.046$ & $<0.013$ & $<0.005$ & 0.045 & $<0.004$ \\
\hline \multicolumn{13}{|c|}{ JANUARY 1992} \\
\hline 14 & 1000 & 0.008 & $<0.007$ & 0.083 & $<0.038$ & $<0.013$ & $<0.008$ & $<0.046$ & $<0.013$ & $<0.005$ & 0.020 & $<0.004$ \\
\hline 28 & 1000 & $<0.009$ & $<0.007$ & 0.083 & $<0.038$ & $<0.013$ & $<0.008$ & $<0.046$ & $<0.013$ & $<0.005$ & $<0.013$ & $<0.004$ \\
\hline \multicolumn{13}{|c|}{ FEBRUARY } \\
\hline 11 & 1030 & 0.002 & $<0.007$ & 0.094 & $<0.038$ & $<0.013$ & $<0.008$ & $<0.046$ & $<0.013$ & $<0.005$ & 0.010 & 0.002 \\
\hline 27 & 1000 & 0.005 & $<0.007$ & 0.095 & $<0.038$ & $<0.013$ & $<0.008$ & $<0.046$ & $<0.013$ & $<0.005$ & 0.008 & $<0.004$ \\
\hline \multicolumn{13}{|l|}{ IARCH } \\
\hline 05 & 1010 & 0.005 & 0.007 & 0.170 & $<0.038$ & $<0.013$ & $<0.008$ & $<0.046$ & $<0.013$ & $<0.005$ & $<0.013$ & $<0.004$ \\
\hline 09 & 0945 & 0.008 & $<0.007$ & 0.120 & $<0.038$ & $<0.013$ & $<0.008$ & $<0.046$ & $<0.013$ & $<0.005$ & 0.034 & $<0.004$ \\
\hline 17 & 1145 & 0.008 & $<0.007$ & 0.130 & $<0.038$ & $<0.013$ & $<0.008$ & $<0.046$ & $<0.013$ & $<0.005$ & $<0.013$ & $<0.004$ \\
\hline 23 & 1040 & 0.004 & $<0.007$ & 0.100 & $<0,038$ & $<0.013$ & $<0.008$ & $<0.046$ & $<0.013$ & $<0.005$ & 0.023 & $<0.004$ \\
\hline 31 & 1100 & 0.007 & $<0.007$ & 0.100 & $<0.038$ & $<0.013$ & 0.005 & $<0.046$ & $<0.013$ & $<0.005$ & 0.013 & $<0.004$ \\
\hline \multicolumn{13}{|c|}{ APRIL 1992} \\
\hline 07 & 1100 & 0.005 & $<0.007$ & 0.140 & $<0,038$ & $<0.013$ & $<0.008$ & $<0.046$ & $<0.013$ & $<0.005$ & 0.016 & $<0.004$ \\
\hline 14 & 1010 & 0.004 & $<0.007$ & 0.150 & $<0.038$ & $<0.013$ & $<0.008$ & $<0.046$ & $<0.013$ & $<0.005$ & 0.014 & $<0.004$ \\
\hline 23 & 1020 & 0.038 & $<0.007$ & 0.180 & $<0.038$ & $<0.013$ & $<0.008$ & $<0.046$ & $<0.013$ & $<0.005$ & 0.027 & $<0.004$ \\
\hline 29 & 1030 & 0.017 & $<0.007$ & 0.160 & $<0.038$ & $<0.013$ & $<0.008$ & $<0.046$ & $<0.013$ & $<0.005$ & 0.045 & $<0.004$ \\
\hline \multicolumn{13}{|l|}{ IAY } \\
\hline 07 & 1100 & 0.069 & $<0.007$ & 0.330 & $<0,038$ & $<0.013$ & $<0.008$ & $<0.046$ & $<0.013$ & $<0.005$ & 0.390 & $<0.004$ \\
\hline & & & & & & & & Mo. & & & & \\
\hline
\end{tabular}

\begin{tabular}{|c|c|c|c|c|c|c|c|c|c|c|c|c|}
\hline 09 & 1331 & -- & - & -- & $\cdots$ & - & -- & -- & -- & - & -- & -- \\
\hline 16 & 1030 & -- & -- & -- & -- & -- & -- & -- & -- & -- & -- & -- \\
\hline 24 & 1200 & - & -- & -- & -- & -- & -- & -- & -- & -- & -- & -- \\
\hline \multicolumn{13}{|l|}{ MAY } \\
\hline 01 & 1130 & -- & -- & -- & -- & -- & -. & -- & -- & -- & -- & -- \\
\hline 06 & 1100 & 0.330 & -. & 3.17 & $<0.038$ & -- & 0.024 & $<0.046$ & 0.082 & $<0.005$ & 1.41 & -- \\
\hline 09 & 0930 & -- & -- & -- & -- & -- & -- & .- & -- & .- & -- & -- \\
\hline 13 & 1000 & -- & -- & -- & -- & -- & -- & -- & -- & -- & -- & -- \\
\hline 16 & 1020 & 0.248 & - & 3.03 & $<0.038$ & -- & 0.010 & $<0.046$ & 0.006 & $<0.005$ & 0.476 & -. \\
\hline 20 & 1000 & 0.325 & -. & 2.85 & $<0.038$ & - & $<0.008$ & $<0.046$ & 0.022 & $<0.005$ & 0.830 & -- \\
\hline 24 & 1000 & 1.06 & -. & 6.20 & $<0.038$ & -- & $<0.008$ & $<0.046$ & $<0.013$ & $<0.005$ & 5.13 & -. \\
\hline 28 & 1030 & 0.934 & -- & 6.92 & $<0.038$ & -- & $<0.008$ & $<0.046$ & $<0.013$ & $<0.005$ & 3.00 & - \\
\hline 31 & 1040 & 0.470 & -- & 4.83 & $<0.038$ & -- & $<0.008$ & $<0.046$ & 0.107 & $<0.005$ & 2.68 & -. \\
\hline \multicolumn{13}{|c|}{ JUNE 1991} \\
\hline 03 & 1000 & 0.390 & -- & 4.6 & $<0.038$ &.- & $<0.008$ & 0.006 & 0.029 & $<0.005$ & 2.49 & -- \\
\hline 06 & 1000 & 0.599 & -- & 5.8 & $<0.038$ & -- & $<0.008$ & $<0.046$ & 0.034 & $<0.005$ & 3.49 & -- \\
\hline 11 & 1000 & 0.354 & -- & 4.8 & $<0.038$ & -- & $<0.008$ & $<0.046$ & 0.020 & $<0.005$ & 4.99 & -- \\
\hline 13 & 1000 & 0.866 & -- & 6.2 & $<0.038$ & -- & $<0.008$ & $<0.046$ & 0.030 & $<0.005$ & 7.94 & - \\
\hline
\end{tabular}


Table 8.--Pesticide results from solid-phase extraction 1-liter method-Continued

\begin{tabular}{|c|c|c|c|c|c|c|c|c|c|c|c|c|}
\hline Date & Time & $\begin{array}{c}\text { Alachior } \\
\text { diss } \\
(\mu g / L)\end{array}$ & $\begin{array}{c}\text { Alpha } \\
\text { HCH } \\
\text { diss } \\
(\mu g / L)\end{array}$ & $\begin{array}{l}\text { Atra- } \\
\text { zine } \\
\text { diss } \\
(\mu g / L)\end{array}$ & $\begin{array}{c}\text { Azin- } \\
\text { phos- } \\
\text { methyl } \\
\text { diss } \\
(\mu \mathrm{g} / \mathrm{L})\end{array}$ & $\begin{array}{c}\text { Bon- } \\
\text { fluralin } \\
\text { diss } \\
(\mu \mathrm{g} / \mathrm{L})\end{array}$ & $\begin{array}{c}\text { Butylate } \\
\text { diss } \\
(\mu \mathrm{g} / \mathrm{L})\end{array}$ & $\begin{array}{c}\text { Carbaryl } \\
\text { diss } \\
(\mu g / L)\end{array}$ & $\begin{array}{c}\text { Carbo- } \\
\text { furan } \\
\text { diss } \\
(\mu g / L)\end{array}$ & $\begin{array}{c}\text { Chlor- } \\
\text { Pyrifos } \\
\text { diss } \\
(\mu g / L)\end{array}$ & $\begin{array}{c}\text { Cyana- } \\
\text { zine } \\
\text { diss } \\
(\mu g / L)\end{array}$ & $\begin{array}{c}\text { Dachtal } \\
\text { diss } \\
(\mu g / L)\end{array}$ \\
\hline \multicolumn{13}{|c|}{06934500 - Missouri River at Hermsnn, Mo.--Continned } \\
\hline \multicolumn{13}{|c|}{ JUNE 1991 (cont.) } \\
\hline 21 & 1000 & 0.516 & -- & 8.6 & $<0.038$ & -- & $<0.008$ & $<0.046$ & 0.069 & $<0.005$ & 3.26 & -- \\
\hline 25 & 1130 & 0.458 & -- & 4.1 & $<0.038$ & -- & $<0.008$ & $<0.046$ & 0.021 & $<0.005$ & 1.82 & -- \\
\hline 27 & 1045 & 0.209 & -- & 3.2 & $<0.038$ & -- & $<0.008$ & $<0.046$ & 0.159 & $<0.005$ & 0.883 & -- \\
\hline \multicolumn{13}{|l|}{ ralY } \\
\hline 01 & 1100 & 0.218 & - & 2.75 & $<0.038$ & -- & $<0.008$ & $<0.046$ & 0.020 & $<0.005$ & 0.586 & - \\
\hline 03 & 1000 & 0.075 & -- & 1.28 & $<0.038$ & -. & $<0.008$ & 0.009 & $<0.013$ & $<0.005$ & 0.323 & -- \\
\hline 08 & 1030 & 0.018 & - & 0.57 & $<0.038$ & -- & $<0.008$ & $<0.046$ & $<0.013$ & $<0.005$ & 0.124 & - \\
\hline 11 & 1000 & 0.033 & -- & 0.87 & $<0.038$ & -- & $<0.008$ & $<0.046$ & $<0.013$ & $<0.005$ & 0.168 & -- \\
\hline 15 & 1030 & 0.144 & -- & 1.32 & $<0.038$ & -- & $<0.008$ & $<0.046$ & 0.023 & $<0.005$ & 0.240 & -- \\
\hline 22 & 1015 & 0.029 & -- & 0.93 & $<0.038$ & -- & $<0.008$ & $<0.046$ & $<0.013$ & $<0.005$ & 0.213 & - \\
\hline 29 & 1100 & 0.021 & -- & 0.70 & $<0.038$ & -- & $<0.008$ & $<0.046$ & $<0.013$ & $<0.005$ & 0.094 & - \\
\hline \multicolumn{13}{|c|}{ AUGUST 1991} \\
\hline 05 & 1000 & 0.007 & -- & 0.407 & $<0.038$ & -- & $<0.008$ & $<0.046$ & $<0.013$ & $<0.005$ & 0.096 & -- \\
\hline 13 & 1100 & 0.014 & -- & 0.477 & $<0.038$ & -- & $<0.008$ & $<0.046$ & $<0.013$ & $<0.005$ & 0.089 & -- \\
\hline 19 & 1000 & 0.017 & - & 0.483 & $<0.038$ & -- & $<0.008$ & $<0.046$ & $<0.013$ & $<0.005$ & 0.124 & -. \\
\hline 27 & 1100 & 0.010 & -- & 0.388 & $<0.038$ & -- & $<0.008$ & $<0.046$ & $<0.013$ & $<0.005$ & 0.077 & -- \\
\hline \multicolumn{13}{|c|}{ SEPTEMBER } \\
\hline 05 & 1030 & 0.011 & -- & 0.337 & $<0.038$ & -- & $<0.008$ & $<0.046$ & $<0.013$ & $<0.005$ & 0.030 & - \\
\hline 09 & 1000 & 0.011 & -- & 0.324 & $<0.038$ & -- & $<0.008$ & $<0.046$ & $<0.013$ & $<0.005$ & 0.026 & - \\
\hline 16 & 1000 & 0.011 & -- & 0.241 & $<0.038$ & -- & $<0.008$ & $<0.046$ & $<0.013$ & $<0.005$ & 0.020 & - \\
\hline 23 & 0945 & 0.010 & -- & 0.237 & $<0.038$ & -- & $<0.008$ & $<0.046$ & $<0.013$ & $<0.005$ & 0.017 & -- \\
\hline \multicolumn{13}{|l|}{ OCTOBER } \\
\hline 01 & 1030 & 0.007 & -- & 0.159 & $<0.038$ & -- & $<0.008$ & $<0.046$ & $<0.013$ & $<0.005$ & 0.017 & - \\
\hline 07 & 1000 & $<0.009$ & -- & 0.177 & $<0.038$ & -- & $<0.008$ & $<0.046$ & $<0.013$ & $<0.005$ & 0.015 & - \\
\hline 15 & 1100 & 0.011 & -- & 0.187 & $<0.038$ & -- & $<0.008$ & $<0.046$ & $<0.013$ & $<0.005$ & 0.018 & -- \\
\hline 21 & 1000 & 0.015 & -- & 0.248 & $<0.038$ & -- & $<0.008$ & $<0.046$ & $<0.013$ & $<0.005$ & 0.021 & - \\
\hline 29 & 1000 & 0.006 & - & 0.184 & $<0.038$ & -- & $<0.008$ & $<0.046$ & $<0.013$ & $<0.005$ & 0.011 & - \\
\hline \multicolumn{13}{|c|}{ NOVEMBER 1991} \\
\hline 04 & 1030 & 0.008 & -- & 0.170 & $<0.038$ & -- & $<0.008$ & $<0.046$ & $<0.013$ & $<0.005$ & 0.015 & - \\
\hline 21 & 1030 & 0.017 & -- & 0.273 & $<0.038$ & -- & $<0.008$ & $<0.046$ & $<0.013$ & $<0.005$ & 0.028 & -- \\
\hline \multicolumn{13}{|c|}{ DECEMBER } \\
\hline 06 & 1240 & 0.010 & - & 0.197 & $<0.038$ & -- & $<0.008$ & $<0.046$ & $<0.013$ & $<0.005$ & 0.028 & - \\
\hline 16 & 1130 & 0.011 & - & 0.308 & $<0.038$ & -- & 0.023 & $<0.046$ & $<0.013$ & $<0.005$ & 0.037 & -- \\
\hline \multicolumn{13}{|c|}{ JANUARY 1992} \\
\hline 02 & 1030 & 0.024 & - & 0.296 & $<0.038$ & -- & $<0.008$ & $<0.046$ & $<0.013$ & $<0.005$ & 0.036 & - \\
\hline 16 & 1200 & 0.010 & $<0.007$ & 0.280 & $<0.038$ & $<0.013$ & $<0.008$ & $<0.046$ & $<0.013$ & $<0.005$ & 0.052 & $<0.004$ \\
\hline 28 & 1300 & 0.009 & $<0.007$ & 0.180 & $<0.038$ & $<0.013$ & $<0.008$ & $<0.046$ & $<0.013$ & $<0.005$ & 0.039 & $<0.004$ \\
\hline \multicolumn{13}{|c|}{ FEBRUARY } \\
\hline 10 & 1000 & 0.006 & $<0.007$ & 0.160 & $<0.038$ & $<0.013$ & $<0.008$ & $<0.046$ & $<0.013$ & $<0.005$ & 0.022 & $<0.004$ \\
\hline 24 & 1200 & 0.016 & $<0.007$ & 0.210 & $<0.038$ & $<0.013$ & $<0.008$ & $<0.046$ & $<0.013$ & $<0.005$ & 0.045 & $<0.004$ \\
\hline \multicolumn{13}{|l|}{ MARCH } \\
\hline 03 & 1100 & 0.010 & $<0.007$ & 0.240 & $<0.038$ & $<0.013$ & $<0.008$ & $<0.046$ & $<0.013$ & $<0.005$ & 0.084 & $<0.004$ \\
\hline 12 & 1200 & 0.012 & $<0.007$ & 0.300 & $<0.038$ & $<0.013$ & $<0.008$ & $<0.046$ & $<0.013$ & 0.006 & 0.043 & $<0.004$ \\
\hline 20 & 0830 & 0.010 & $<0.007$ & 0.220 & $<0.038$ & $<0.013$ & 0.023 & $<0.046$ & $<0.013$ & $<0.005$ & 0.044 & 0.004 \\
\hline 26 & 1130 & 0.019 & $<0.007$ & 0.390 & $<0.038$ & $<0.013$ & 0.004 & $<0.046$ & $<0.013$ & $<0.005$ & 0.072 & $<0.004$ \\
\hline \multicolumn{13}{|c|}{ APRIL 1992} \\
\hline 01 & 1000 & 0.010 & $<0.007$ & 0.330 & $<0.038$ & $<0.013$ & 0.013 & $<0.046$ & $<0.013$ & $<0.005$ & 0.072 & $<0.004$ \\
\hline 06 & 1230 & 0.014 & $<0.007$ & 0.320 & $<0.038$ & $<0.013$ & 0.008 & $<0.046$ & $<0.013$ & 0.009 & 0.089 & $<0.004$ \\
\hline
\end{tabular}


Table 8.--Pesticide results from solid-phase extraction 1-liter method--Continued

\begin{tabular}{|c|c|c|c|c|c|c|c|c|c|c|c|c|}
\hline Date & Time & $\begin{array}{c}\text { Alachlor } \\
\text { diss } \\
(\mu g / L)\end{array}$ & $\begin{array}{c}\text { Alpha } \\
\text { HCH } \\
\text { diss } \\
(\mu g / L)\end{array}$ & $\begin{array}{l}\text { Atra- } \\
\text { zine } \\
\text { diss } \\
(\mu g / L)\end{array}$ & $\begin{array}{c}\text { Azin- } \\
\text { phos- } \\
\text { methyl } \\
\text { diss } \\
(\mu g / L)\end{array}$ & $\begin{array}{c}\text { Ben- } \\
\text { fluralin } \\
\text { diss } \\
(\mu g / L)\end{array}$ & $\begin{array}{c}\text { Butylate } \\
\text { diss } \\
\left(\mu g^{\prime} L\right)\end{array}$ & $\begin{array}{c}\text { Carbaryl } \\
\text { diss } \\
\left(\mu g^{\prime} L\right)\end{array}$ & $\begin{array}{c}\text { Carbo- } \\
\text { furan } \\
\text { diss } \\
(\mu g / L)\end{array}$ & $\begin{array}{c}\text { Chlor- } \\
\text { pyrifos } \\
\text { diss } \\
(\mu g / L)\end{array}$ & $\begin{array}{l}\text { Cyana- } \\
\text { zine } \\
\text { diss } \\
(\mu g / L)\end{array}$ & $\begin{array}{c}\text { Dachtal } \\
\text { dlss } \\
(\mu \mathrm{g})\end{array}$ \\
\hline \multicolumn{13}{|c|}{06934500 - Missouri River at Hermann, Mo.-Continued } \\
\hline \multicolumn{13}{|c|}{ APRIL 1992 (cont.) } \\
\hline 14 & 1030 & 0.062 & $<0.007$ & 0.600 & $<0.038$ & $<0.013$ & $<0.008$ & $<0.046$ & $<0.013$ & $<0.005$ & 0.400 & $\theta$ \\
\hline 21 & 1300 & 0.062 & $<0.007$ & 0.880 & $<0.038$ & $<0.013$ & $<0.008$ & $<0.046$ & $<0.013$ & $<0.005$ & 0.430 & \\
\hline 29 & 1015 & 0.160 & $<0.007$ & 2.20 & $<0.038$ & $<0.013$ & 0.009 & $<0.046$ & $<0.013$ & $<0.005$ & 0.920 & $<0$. \\
\hline \multicolumn{13}{|l|}{ MAY } \\
\hline 12 & 1000 & 0.021 & $<0.007$ & 0.510 & $<0.038$ & $<0.013$ & $<0.008$ & $<0.046$ & $<0.013$ & $<0.005$ & 0.160 & 0.4 \\
\hline 21 & 1200 & 0.019 & $<0.007$ & 0.350 & $<0.038$ & $<0.013$ & 0.017 & $<0.046$ & $<0.013$ & 0.007 & 0.140 & \\
\hline 26 & 1130 & 1.00 & $<0.007$ & 4.60 & $<0.038$ & $<0.013$ & 0.008 & $<0.046$ & $<0.013$ & $<0.005$ & 8.90 & \\
\hline \multicolumn{13}{|l|}{ JUNE } \\
\hline 01 & 1330 & 0.430 & $<0.007$ & 3.10 & $<0.038$ & $<0.013$ & $<0.008$ & $<0.046$ & $<0.013$ & $<0.005$ & 2.00 & 0.00 \\
\hline 09 & 1030 & 0.063 & $<0.007$ & 0.690 & $<0.038$ & $<0.013$ & $<0.008$ & $<0.046$ & $<0.013$ & 0.018 & 0.360 & 0.0 \\
\hline 15 & 1230 & 0.190 & $<0.007$ & 1.50 & $<0.038$ & $<0.013$ & $<0.008$ & $<0.046$ & $<0.013$ & $<0.005$ & 0.330 & 0.0 \\
\hline 22 & 0945 & 0.110 & $<0.007$ & 1.80 & $<0.038$ & $<0.013$ & $<0.008$ & $<0.046$ & $<0.013$ & $<0.005$ & 0.390 & 0.0 \\
\hline 30 & 1130 & 0.094 & $<0.007$ & 2.00 & $<0.038$ & $<0.013$ & $<0.008$ & $<0.046$ & $<0.013$ & $<0.005$ & 0.580 & 0.0 \\
\hline \multicolumn{13}{|l|}{ JULY 1992} \\
\hline 07 & 0930 & 0.021 & $<0.007$ & 0.700 & $<0.038$ & $<0.013$ & $<0.008$ & 0.002 & $<0.013$ & $<0.005$ & 0.150 & $<0.0$ \\
\hline 14 & 1200 & 0.130 & $<0.007$ & 1.50 & $<0.038$ & $<0.013$ & $<0.008$ & $<0.046$ & $<0.013$ & $<0.005$ & 0.300 & 0.0 \\
\hline 21 & 1030 & 0.180 & $<0.007$ & 3.00 & $<0.038$ & $<0.013$ & $<0.008$ & $<0.046$ & $<0.013$ & $<0.005$ & 0.520 & 0.0 \\
\hline 28 & 1230 & 0.049 & $<0.007$ & 1.20 & $<0.038$ & $<0.013$ & $<0.008$ & $<0.046$ & $<0.013$ & $<0.005$ & 0.140 & $<0.0$ \\
\hline
\end{tabular}

APRIL 1991

\begin{tabular}{|c|c|c|c|c|c|c|c|c|c|c|c|c|}
\hline 11 & 1500 & -- & -- & -- & -- & -- & -- & -- & -- & -- & -. & -- \\
\hline 18 & 1130 & -- & -- & -- & - & -- & -- & -- & -- & -- & -- & -- \\
\hline 24 & 0930 & -- & -- & -. & -- & -- & -- & -- & -- & -- & -- & -- \\
\hline 29 & 1230 & -- & -- & -- & -- & -- & -- & -- & -- & -- & -- & -- \\
\hline \multicolumn{13}{|l|}{ MAY } \\
\hline 07 & 1345 & 0.497 & -. & 3.63 & $<0.038$ & -- & 0.036 & $<0.046$ & 0.057 & $<0.005$ & 2.76 & - \\
\hline 09 & 0900 & 0.310 & -- & 2.02 & $<0.038$ & -- & 0.017 & 0.005 & 0.013 & $<0.005$ & 1.39 & - \\
\hline 13 & 1330 & -- & -- & -- & -- & -- & - & -- & -- & - & -- & - \\
\hline 16 & 1200 & 0.509 & -- & 2.12 & $<0.038$ & -- & $<0.008$ & $<0.046$ & 0.026 & $<0.005$ & 1.05 & - \\
\hline 20 & 1430 & 0.335 & -- & 2.55 & $<0.038$ & -- & $<0.008$ & $<0.046$ & $<0.013$ & $<0.005$ & 1.06 & - \\
\hline 23 & 0916 & 0.497 & -- & 2.58 & $<0.038$ & -- & $<0.008$ & $<0.046$ & 0.009 & $<0.005$ & 2.25 & -- \\
\hline 28 & 1300 & 0.787 & -- & 2.52 & $<0.038$ & -- & 0.007 & $<0.046$ & 0.012 & $<0.005$ & 0.644 & -- \\
\hline 30 & 1330 & 0.755 & -- & 3.75 & $<0.038$ & -- & 0.004 & 0.005 & 0.047 & $<0.005$ & 2.20 & -- \\
\hline \multicolumn{13}{|c|}{ JUNE 1991} \\
\hline 03 & 1345 & 0.624 & -- & 3.87 & $<0.038$ & -- & $<0.008$ & $<0.046$ & 0.051 & $<0.005$ & 2.45 & -- \\
\hline 06 & 1045 & 0.504 & -- & 3.85 & $<0.038$ & -- & $<0.008$ & $<0.046$ & 0.027 & $<0.005$ & 2.04 & -- \\
\hline 10 & 1400 & 0.625 & -- & 3.67 & $<0.038$ & -- & $<0.008$ & $<0.046$ & 0.025 & $<0.005$ & 2.39 & -- \\
\hline 13 & 1500 & 0.552 & -. & 3.30 & $<0.038$ & -- & $<0.008$ & $<0.046$ & $<0.013$ & $<0.005$ & 1.20 & $\cdots$ \\
\hline 18 & 1430 & 0.701 & -- & 4.16 & $<0.038$ & -- & $<0.008$ & $<0.046$ & 0.018 & $<0.005$ & 2.35 & -- \\
\hline 20 & 1100 & 0.630 & -- & 3.72 & $<0.038$ & -- & $<0.008$ & $<0.046$ & 0.016 & $<0.005$ & 1.56 & -- \\
\hline 24 & 1300 & 0.312 & -- & 5.94 & $<0.038$ & -- & $<0.008$ & 0.018 & 0.076 & $<0.005$ & 2.70 & -- \\
\hline 27 & 1400 & 0.848 & -- & 4.01 & $<0.038$ & -- & $<0.008$ & $<0.046$ & 0.050 & $<0.005$ & 1.40 & -- \\
\hline \multicolumn{13}{|l|}{ JULY } \\
\hline 01 & 1320 & 0.661 & -- & 2.51 & $<0.038$ & -- & $<0.008$ & $<0.046$ & $<0.013$ & $<0.005$ & 0.892 & -- \\
\hline 03 & 0950 & 0.543 & -- & 2.35 & $<0.038$ & -- & $<0.008$ & $<0.046$ & $<0.013$ & 0.026 & 0.861 & -- \\
\hline 08 & 1330 & 0.360 & -- & 1.63 & $<0.038$ & -- & $<0.008$ & 0.005 & $<0.013$ & $<0.005$ & 0.876 & -- \\
\hline 11 & 1400 & 0.333 & -- & 1.62 & $<0.038$ & -- & $<0.008$ & $<0.046$ & $<0.013$ & $<0.005$ & 0.840 & - \\
\hline
\end{tabular}


Table 8.--Pesticide resuits from solid-phase extraction 1-liter method-Continued

\begin{tabular}{|c|c|c|c|c|c|c|c|c|c|c|c|c|}
\hline Data & Tima & $\begin{array}{c}\text { Alachlor } \\
\text { diss } \\
(\mu g h)\end{array}$ & $\begin{array}{l}\text { Alpha } \\
\text { HCH } \\
\text { diss } \\
(\mu \mathrm{g} / L)\end{array}$ & $\begin{array}{l}\text { Atra- } \\
\text { zine } \\
\text { diss } \\
(\mu g / L)\end{array}$ & $\begin{array}{l}\text { Azin- } \\
\text { phos- } \\
\text { mothyl } \\
\text { diss } \\
(\mu g / L)\end{array}$ & $\begin{array}{c}\text { Ban- } \\
\text { fluralin } \\
\text { diss } \\
(\mu g / L)\end{array}$ & $\begin{array}{c}\text { Butyiata } \\
\text { diss } \\
\text { ( } \mu g / L)\end{array}$ & $\begin{array}{c}\text { Carbaryl } \\
\text { diss } \\
(\mu g / L)\end{array}$ & $\begin{array}{l}\text { Carbo- } \\
\text { furan } \\
\text { diss } \\
(\mu g / L)\end{array}$ & $\begin{array}{c}\text { Chior- } \\
\text { pyrifos } \\
\text { diss } \\
(\mu g / 2)\end{array}$ & $\begin{array}{l}\text { Cyana- } \\
\text { zlne } \\
\text { diss } \\
(\mu g / L)\end{array}$ & $\begin{array}{c}\text { Dachtal } \\
\text { diss } \\
(\mu \mathrm{g} / \mathrm{L})\end{array}$ \\
\hline \multicolumn{13}{|c|}{07022000 - Mississippi River at Thebes, Ill,-Continued } \\
\hline \multicolumn{13}{|c|}{ JULY (cont) } \\
\hline 18 & 0930 & -- & -. & -- & -- & -. & .. & -- & -- & -- & -- & - \\
\hline 23 & 1300 & 0.240 & -- & 1.53 & $<0.038$ & .- & $<0.008$ & $<0.046$ & $<0.013$ & $<0.005$ & 0.556 & - \\
\hline 30 & 1000 & 0.154 & - & 1.13 & $<0.038$ & -- & $<0.008$ & $<0.046$ & $<0.013$ & $<0.005$ & 0.535 & - \\
\hline \multicolumn{13}{|c|}{ AUGUST 1991} \\
\hline 06 & 1300 & 0.097 & -. & 0.814 & $<0.038$ & -. & $<0.008$ & $<0.046$ & $<0.013$ & $<0.005$ & 0.363 & - \\
\hline 13 & 0930 & 0.091 & -- & 0.738 & $<0.038$ & -- & $<0.008$ & $<0.046$ & $<0.013$ & $<0.005$ & 0.301 & -- \\
\hline 20 & 1330 & 0.091 & -- & 0.681 & $<0.038$ & -- & $<0.008$ & $<0.046$ & $<0.013$ & $<0.005$ & 0.225 & - \\
\hline 29 & 1100 & 0.057 & -- & 0.498 & $<0.038$ & -- & $<0.008$ & $<0.046$ & $<0.013$ & $<0.005$ & 0.191 & - \\
\hline \multicolumn{13}{|c|}{ SEPTEMBER 1991} \\
\hline 03 & 1500 & 0.049 & -- & 0.452 & $<0.038$ & -- & $<0.008$ & $<0.046$ & $<0.013$ & $<0.005$ & 0.124 & - \\
\hline 11 & 1000 & 0.031 & - & 0.363 & $<0.038$ & -- & $<0.008$ & $<0.046$ & $<0.013$ & $<0.005$ & 0.071 & -- \\
\hline 17 & 1400 & 0.030 & -- & 0.294 & $<0.038$ & -- & $<0.008$ & $<0.046$ & $<0.013$ & $<0.005$ & 0.047 & - \\
\hline 26 & 0930 & 0.062 & -- & 0.280 & $<0.038$ & -- & $<0.008$ & $<0.046$ & $<0.013$ & $<0.005$ & 0.040 & -- \\
\hline \multicolumn{13}{|l|}{ OCTOBER } \\
\hline 02 & 1300 & 0.073 & -- & 0.209 & $<0.038$ & -- & $<0.008$ & $<0.046$ & $<0.013$ & $<0.005$ & 0.035 & - \\
\hline 09 & 1030 & 0.092 & -- & 0.233 & $<0.038$ & -- & $<0.008$ & $<0.046$ & $<0.013$ & $<0.005$ & 0.032 & -- \\
\hline 16 & 1430 & 0.079 & -- & 0.240 & $<0.038$ & -- & $<0.008$ & $<0.046$ & $<0.013$ & $<0.005$ & 0.044 & - \\
\hline 23 & 0945 & 0.084 & -- & 0.263 & $<0.038$ & -- & $<0.008$ & $<0.046$ & $<0.013$ & 0.005 & 0.036 & -- \\
\hline 30 & 1400 & 0.060 & -- & 0.150 & $<0.038$ & -- & $<0.008$ & $<0.046$ & $<0.015$ & 0.005 & $<0.013$ & -- \\
\hline \multicolumn{13}{|c|}{ NOVEMBER } \\
\hline 07 & 0800 & 0.078 & -- & 0.168 & $<0.038$ & -. & $<0.008$ & $<0.046$ & $<0.013$ & $<0.005$ & 0.022 & -- \\
\hline 20 & 1030 & 0.081 & -. & 0.241 & $<0.038$ & -- & $<0.008$ & $<0.046$ & $<0.013$ & $<0.005$ & 0.039 & -- \\
\hline \multicolumn{13}{|c|}{ DECEMBER 1991} \\
\hline 05 & 1045 & 0.116 & -- & 0.198 & $<0.038$ & -- & $<0.008$ & $<0.046$ & $<0.013$ & $<0.005$ & 0.046 & - \\
\hline 18 & 1100 & 0.093 & -- & 0.203 & $<0.038$ & -. & $<0.008$ & $<0.046$ & $<0.013$ & $<0.005$ & 0.040 & -- \\
\hline 30 & 1400 & 0.076 & - & 0.224 & $<0.038$ & -- & $<0.008$ & $<0.046$ & $<0.015$ & $<0.005$ & 0.035 & -- \\
\hline \multicolumn{13}{|c|}{ JANUARY 1992} \\
\hline 24 & 0930 & 0.051 & $<0.007$ & 0.170 & $<0.038$ & $<0.013$ & $<0.008$ & $<0.046$ & $<0.013$ & $<0.005$ & 0.041 & $<0.004$ \\
\hline \multicolumn{13}{|c|}{ FEBRUARY } \\
\hline 04 & 1030 & 0.062 & $<0.007$ & 0.140 & $<0.038$ & $<0.013$ & $<0.008$ & $<0.046$ & $<0.013$ & $<0.005$ & 0.037 & $<0.004$ \\
\hline 19 & 0930 & 0.012 & $<0.007$ & 0.160 & $<0.038$ & $<0.013$ & $<0.008$ & $<0.046$ & $<0.013$ & $<0.005$ & 0.047 & $<0.004$ \\
\hline \multicolumn{13}{|l|}{ MARCH } \\
\hline 04 & 0945 & 0.022 & $<0.007$ & 0.240 & $<0.038$ & $<0.013$ & $<0.008$ & $<0.046$ & $<0.013$ & $<0.005$ & 0.074 & $<0.004$ \\
\hline 09 & 1300 & 0.022 & $<0.007$ & 0.180 & $<0.038$ & $<0.013$ & $<0.008$ & $<0.046$ & $<0.013$ & $<0.005$ & 0.048 & $<0.004$ \\
\hline 18 & 0945 & 0.036 & $<0.007$ & 0.190 & $<0.038$ & $<0.013$ & $<0.008$ & $<0.046$ & $<0.013$ & $<0.005$ & 0.036 & $<0.004$ \\
\hline 24 & 1330 & 0.023 & $<0.007$ & 0.270 & $<0.038$ & $<0.013$ & $<0.008$ & $<0.046$ & $<0.013$ & $<0.005$ & 0.058 & $<0.004$ \\
\hline \multicolumn{13}{|c|}{ APRIL 1992} \\
\hline 02 & 0930 & 0.036 & $<0.007$ & 0.240 & $<0.038$ & $<0.013$ & $<0.008$ & $<0.046$ & $<0.013$ & $<0.005$ & 0.063 & $<0.004$ \\
\hline 07 & 1330 & 0.021 & $<0.007$ & 0.240 & $<0.038$ & $<0.013$ & $<0.008$ & $<0.046$ & $<0.013$ & $<0.005$ & 0.053 & $<0.004$ \\
\hline 15 & 1030 & 0.026 & $<0.007$ & 0.290 & $<0.038$ & $<0.013$ & $<0.008$ & $<0.046$ & $<0.013$ & $<0.005$ & 0.170 & $<0.004$ \\
\hline 23 & 1330 & 0.250 & $<0.007$ & 2.30 & $<0.038$ & $<0.013$ & 0.018 & $<0.046$ & $<0.013$ & $<0.005$ & 1.30 & $<0.004$ \\
\hline 29 & 1100 & 0.200 & $<0.007$ & 2.00 & $<0.038$ & $<0.013$ & $<0.008$ & $<0.046$ & $<0.013$ & $<0.005$ & 0.960 & $<0.004$ \\
\hline \multicolumn{13}{|l|}{ MAY } \\
\hline 06 & 1300 & 0.066 & $<0.007$ & 0.600 & $<0.038$ & $<0.013$ & $<0.008$ & $<0.046$ & $<0.013$ & $<0.005$ & 0.270 & $<0.004$ \\
\hline 14 & 0930 & 0.040 & $<0.007$ & 0.370 & $<0.038$ & $<0.013$ & $<0.008$ & $<0.046$ & $<0.013$ & $<0.005$ & 0.140 & $<0.004$ \\
\hline 19 & 1300 & 0.047 & $<0.007$ & 0.330 & $<0.038$ & $<0.013$ & $<0.008$ & $<0.046$ & $<0.013$ & $<0.005$ & 0.110 & $<0.004$ \\
\hline 28 & 0900 & 0.120 & $<0.007$ & 0.670 & $<0.038$ & $<0.013$ & $<0.008$ & $<0.046$ & $<0.013$ & 0.014 & 0.270 & 0.001 \\
\hline
\end{tabular}


Table 8.--Pesticide results from solid-phase extraction 1-liter method--Continued

\begin{tabular}{|c|c|c|c|c|c|c|c|c|c|c|c|c|}
\hline Date & Time & $\begin{array}{l}\text { Alachlor } \\
\text { diss } \\
(\mu g / L)\end{array}$ & $\begin{array}{l}\text { Alpha } \\
\text { HCH } \\
\text { diss } \\
(\mu g / L)\end{array}$ & $\begin{array}{l}\text { Atra- } \\
\text { zine } \\
\text { diss } \\
(\mu g / L)\end{array}$ & $\begin{array}{c}\text { Azin- } \\
\text { phos- } \\
\text { methyl } \\
\text { diss } \\
(\mu g / L)\end{array}$ & $\begin{array}{c}\text { Ben- } \\
\text { fluralin } \\
\text { diss } \\
(\mu g / L)\end{array}$ & $\begin{array}{c}\text { Butylate } \\
\text { diss } \\
(\mu g / L)\end{array}$ & $\begin{array}{c}\text { Carbaryl } \\
\text { diss } \\
(\mu g / L)\end{array}$ & $\begin{array}{l}\text { Carbo- } \\
\text { luran } \\
\text { diss } \\
(\mu g / L)\end{array}$ & $\begin{array}{c}\text { Chlor- } \\
\text { pyrifos } \\
\text { diss } \\
(\mu g / L)\end{array}$ & $\begin{array}{l}\text { Cyana- } \\
\text { zine } \\
\text { diss } \\
(\mu g / L)\end{array}$ & $\begin{array}{c}\text { Dachtal } \\
\text { dlss } \\
(\mu g / L)\end{array}$ \\
\hline
\end{tabular}

JUNE

$\begin{array}{ccccccccccccc}02 & 1300 & 0.490 & <0.007 & 2.40 & <0.038 & <0.013 & <0.008 & <0.046 & <0.013 & 0.055 & 1.80 & 0.001 \\ 09 & 0930 & 0.170 & <0.007 & 1.10 & <0.038 & <0.013 & <0.008 & <0.046 & <0.013 & 0.029 & 0.500 & 0.001 \\ 16 & 1330 & 0.058 & <0.007 & 0.490 & <0.038 & <0.013 & <0.008 & <0.046 & <0.013 & <0.005 & 0.160 & 0.001 \\ 25 & 1000 & 0.067 & <0.007 & 1.00 & <0.038 & <0.013 & <0.008 & <0.046 & <0.013 & <0.005 & 0.240 & 0.001 \\ \text { JULY 1992 } & & & & & & & & & & & & \\ 01 & 1300 & 0.100 & <0.007 & 1.40 & <0.038 & <0.013 & <0.008 & <0.046 & <0.013 & <0.005 & 0.960 & <0.004 \\ 09 & 0915 & 0.055 & <0.007 & 0.800 & <0.038 & <0.013 & <0.008 & <0.046 & <0.013 & <0.005 & 0.260 & <0.004 \\ 15 & 1230 & 0.051 & <0.007 & 0.700 & <0.038 & <0.013 & <0.008 & <0.046 & <0.013 & <0.005 & 0.280 & <0.004 \\ 23 & 1000 & 0.150 & <0.007 & 2.20 & <0.038 & <0.013 & <0.008 & <0.046 & <0.013 & <0.005 & 0.780 & <0.004 \\ 28 & 1300 & 0.130 & <0.007 & 1.60 & <0.038 & <0.013 & <0.008 & <0.046 & <0.013 & <0.005 & 0.460 & <0.004\end{array}$

MAY 1991

03374100 - White River at Hazleton, Ind.

\begin{tabular}{|c|c|c|c|c|c|c|c|c|c|c|c|c|}
\hline \\
\hline 01 & 1000 & 0.028 & -. & 0.267 & $<0.038$ & -- & 0.028 & $<0.046$ & $<0.013$ & $<0.005$ & 0.067 & -- \\
\hline 06 & 1115 & 0.027 & -- & 0.312 & $<0.038$ & -- & 0.035 & $<0.046$ & $<0.013$ & $<0.005$ & 0.065 & $\cdots$ \\
\hline 09 & 1215 & 0.031 & -- & 0.526 & $<0.038$ & -- & 0.045 & $<0.046$ & $<0.013$ & $<0.005$ & 0.115 & - \\
\hline 13 & 1300 & -- & - & -- & -. & -- & $\cdots$ & -- & $\cdots$ & -. & -- & -- \\
\hline 16 & 1130 & -- & -- & - & -. & -- & - & -- & - & -- & -- & - \\
\hline 20 & 1045 & 0.752 & -- & 4.26 & $<0.038$ & -- & 0.055 & $<0.046$ & 0.022 & $<0.005$ & 1.40 & - \\
\hline 23 & 1100 & 1.40 & -- & 4.74 & $<0.038$ & $\cdots$ & 0.083 & $<0.046$ & 0.018 & $<0.005$ & 1.38 & $\cdots$ \\
\hline 28 & 1100 & 5.25 & -. & 18 & $<0.038$ & -- & 0.018 & $<0.046$ & $<0.013$ & $<0.005$ & 3.44 & - \\
\hline 30 & 1200 & 3.44 & -- & 16 & $<0.038$ & -- & 0.034 & $<0.046$ & 0.091 & $<0.005$ & 3.92 & -- \\
\hline \multicolumn{13}{|l|}{ JUNE } \\
\hline 03 & 1200 & 0.869 & -- & 8.82 & $<0.038$ & -- & 0.017 & $<0.046$ & 0.051 & $<0.005$ & 2.40 & -. \\
\hline 06 & 1145 & 1.19 & -- & 6.02 & $<0.038$ & -- & 0.007 & $<0.046$ & 0.027 & $<0.005$ & 1.27 & -- \\
\hline 10 & 1200 & 1.17 & -- & 6.50 & $<0.038$ & -- & 0.004 & $<0.046$ & 0.033 & $<0.005$ & 2.18 & -- \\
\hline 13 & 1100 & 0.787 & -- & 6.58 & $<0.038$ & -- & $<0.008$ & $<0.046$ & 0.036 & $<0.005$ & 2.07 & -- \\
\hline 17 & 1200 & 0.384 & -- & 3.93 & $<0.038$ & -- & $<0.008$ & $<0.046$ & 0.009 & $<0.005$ & 1.18 & -- \\
\hline 20 & 1130 & 0.276 & -- & 3.79 & $<0.038$ & -- & $<0.008$ & $<0.046$ & $<0.013$ & $<0.005$ & 0.726 & -- \\
\hline 24 & 1215 & 0.381 & -- & 3.95 & $<0.038$ & - & $<0.008$ & $<0.046$ & $<0.013$ & $<0.005$ & 0.798 & - \\
\hline 27 & 1130 & 0.148 & - & 2.97 & $<0.038$ & -- & 0.007 & $<0.046$ & $<0.013$ & $<0.005$ & 0.564 & -- \\
\hline \multicolumn{13}{|l|}{ JULY 1991} \\
\hline 01 & 1145 & 0.075 & -- & 1.84 & $<0.038$ & -- & $<0.008$ & $<0.046$ & $<0.013$ & $<0.005$ & 0.326 & -- \\
\hline 03 & 1100 & 0.063 & -- & 1.96 & $<0.038$ & -- & $<0.008$ & $<0.046$ & $<0.013$ & $<0.005$ & 0.322 & -- \\
\hline 08 & 1200 & 0.034 & - & 1.60 & $<0.038$ & -- & $<0.008$ & $<0.046$ & $<0.013$ & $<0.005$ & 0.235 & -- \\
\hline 11 & 1130 & $<0.009$ & - & 1.32 & $<0.038$ & -- & $<0.008$ & $<0.046$ & $<0.013$ & $<0.005$ & 0.209 & $\cdots$ \\
\hline 15 & 1200 & 0.123 & - & 2.32 & $<0.038$ & -- & $<0.008$ & $<0.046$ & 0.024 & $<0.005$ & 0.239 & -- \\
\hline 18 & 1300 & 0.075 & -- & 2.09 & $<0.038$ & - & $<0.008$ & $<0.046$ & $<0.013$ & $<0.005$ & 0.302 & -- \\
\hline 22 & 1300 & 0.027 & -- & 1.63 & $<0.038$ & -. & $<0.008$ & $<0.046$ & $<0.013$ & $<0.005$ & 0.244 & -- \\
\hline 25 & 1200 & 0.016 & -- & 1.24 & $<0.038$ & -- & $<0.008$ & $<0.046$ & $<0.013$ & $<0.005$ & 0.194 & -- \\
\hline 30 & 1145 & 0.016 & -- & 1.09 & $<0.038$ & -- & $<0.008$ & $<0.046$ & $<0.013$ & $<0.005$ & 0.187 & -- \\
\hline \multicolumn{13}{|l|}{ AUGUST } \\
\hline 01 & 1145 & 0.012 & -- & 0.888 & $<0.038$ & -- & $<0.008$ & $<0.046$ & $<0.013$ & $<0.005$ & 0.132 & -- \\
\hline 06 & 1200 & $<0.009$ & - & 0.765 & $<0.038$ & -- & $<0.008$ & $<0.046$ & $<0.013$ & $<0.005$ & 0.110 & - \\
\hline 08 & 1300 & 0.007 & $-\cdot$ & 0.642 & $<0.038$ & -- & $<0.008$ & $<0.046$ & $<0.013$ & $<0.005$ & 0.101 & -- \\
\hline 12 & 1100 & 0.024 & -- & 0.671 & $<0.038$ & -- & 0.009 & $<0.046$ & $<0.013$ & $<0.005$ & 0.126 & -- \\
\hline 15 & 1200 & 0.010 & -- & 0.619 & $<0.038$ & -- & $<0.008$ & $<0.046$ & $<0.013$ & $<0.005$ & 0.118 & -- \\
\hline 19 & 1115 & 0.011 & -- & 0.621 & $<0.038$ & - & $<0.008$ & $<0.046$ & $<0.013$ & $<0.005$ & 0.086 & -- \\
\hline
\end{tabular}


Table 8.--Pesticide results from solid-phase extraction 1-iiter method--Continued

\begin{tabular}{|c|c|c|c|c|c|c|c|c|c|c|c|c|}
\hline Date & Time & $\begin{array}{c}\text { Alachior } \\
\text { dlss } \\
(\mu g / L)\end{array}$ & $\begin{array}{c}\text { Alpha } \\
\text { HCH } \\
\text { diss } \\
(\mu g / L)\end{array}$ & $\begin{array}{l}\text { Atra- } \\
\text { zine } \\
\text { diss } \\
(\mu g / L)\end{array}$ & $\begin{array}{c}\text { Azin- } \\
\text { phos- } \\
\text { methyl } \\
\text { dlss } \\
(\mu g / L)\end{array}$ & $\begin{array}{c}\text { Ben- } \\
\text { flurailn } \\
\text { diss } \\
(\mu g / L)\end{array}$ & $\begin{array}{c}\text { Butylate } \\
\text { diss } \\
(\mu g / L)\end{array}$ & $\begin{array}{c}\text { Carbaryl } \\
\text { diss } \\
(\mu g / L)\end{array}$ & $\begin{array}{c}\text { Carbo- } \\
\text { furan } \\
\text { dlss } \\
(\mu g / L)\end{array}$ & $\begin{array}{c}\text { Chlor- } \\
\text { pyrifos } \\
\text { diss } \\
(\mu g / L)\end{array}$ & $\begin{array}{c}\text { Cyana- } \\
\text { zine } \\
\text { diss } \\
(\mu g / L)\end{array}$ & $\begin{array}{c}\text { Dachtal } \\
\text { dles } \\
(\mu g / L)\end{array}$ \\
\hline \multicolumn{13}{|c|}{03374100 - White River at Hazleton, Ind.-Continued } \\
\hline \multicolumn{13}{|c|}{ AUGUST (cont.) } \\
\hline 22 & 1230 & 0.008 & -- & 0.530 & $<0.038$ & -- & $<0.008$ & $<0.046$ & $<0.013$ & $<0.005$ & 0.077 & -- \\
\hline 26 & 1245 & 0.009 & -- & 0.538 & $<0.038$ & -. & $<0.008$ & $<0.046$ & $<0.013$ & $<0.005$ & 0.089 & - \\
\hline 29 & 1200 & 0.010 & - & 0.508 & $<0.038$ & -- & $<0.008$ & $<0.046$ & $<0.013$ & $<0.005$ & 0.076 & - \\
\hline \multicolumn{13}{|c|}{ SEPTEMBER 1991} \\
\hline 09 & 1100 & 0.014 & -- & 0.556 & $<0.038$ & -- & $<0.008$ & $<0.046$ & $<0.013$ & $<0.005$ & 0.090 & - \\
\hline 12 & 1100 & 0.017 & -- & 0.492 & $<0.038$ & -- & $<0.008$ & $<0.046$ & $<0.013$ & $<0.005$ & .081 & - \\
\hline 19 & 1130 & 0.022 & -- & 0.521 & $<0.038$ & -- & $<0.008$ & $<0.046$ & $<0.013$ & $<0.005$ & .079 & + \\
\hline 26 & 1200 & 0.019 & -- & 0.553 & $<0.038$ & -- & $<0.008$ & 0.009 & $<0.013$ & $<0.005$ & .087 & -- \\
\hline \multicolumn{13}{|c|}{ OCTOBER } \\
\hline 03 & 1000 & $<0.009$ & -- & 0.381 & $<0.038$ & -- & $<0.008$ & 0.014 & $<0.013$ & $<0.005$ & 0.060 & - \\
\hline 08 & 1100 & 0.007 & -- & 0.327 & $<0.038$ & -- & $<0.008$ & $<0.046$ & $<0.013$ & $<0.005$ & 49 & -- \\
\hline 17 & 1100 & 0.006 & -- & 0.354 & $<0.038$ & -- & $<0.008$ & $<0.046$ & $<0.013$ & $<0.005$ & .068 & -- \\
\hline 24 & 1100 & $<0.009$ & -- & 0.283 & $<0.038$ & -- & $<0.008$ & $<0.046$ & $<0.013$ & $<0.005$ & 0.045 & - \\
\hline 28 & 1130 & 0.007 & -- & 0.261 & $<0.038$ & -- & $<0.008$ & $<0.046$ & $<0.013$ & $<0.005$ & 0.050 & -. \\
\hline 30 & 1130 & 0.046 & -- & 0.390 & $<0.038$ & -- & $<0.008$ & $<0.046$ & 0.017 & $<0.005$ & 0.067 & - \\
\hline \multicolumn{13}{|c|}{ NOVEMBER } \\
\hline 04 & 1130 & $<0.009$ & - & 0.514 & $<0.038$ & -- & $<0.008$ & $<0.046$ & $<0.013$ & $<0.005$ & 0.074 & - \\
\hline 13 & 1025 & 0.017 & -- & 0.289 & $<0.038$ & -- & $<0.008$ & $<0.046$ & 0.013 & $<0.005$ & .049 & -- \\
\hline 27 & 1200 & $<0.009$ & -. & 0.471 & $<0.038$ & -- & 0.002 & $<0.046$ & $<0.013$ & $<0.005$ & 0.070 & - \\
\hline \multicolumn{13}{|c|}{ DECEMBER } \\
\hline 18 & 1200 & 0.036 & -- & 0.427 & $<0.038$ & -- & $<0.008$ & $<0.046$ & $<0.013$ & $<0.005$ & 0.061 & - \\
\hline \multicolumn{13}{|c|}{ JANUARY 1992} \\
\hline 02 & 1200 & 0.014 & -- & 0.228 & $<0.038$ & -- & $<0.008$ & $<0.046$ & $<0.013$ & $<0.005$ & 0.043 & -- \\
\hline 16 & 1235 & 0.032 & $<0.007$ & 0.240 & $<0.038$ & $<0.013$ & $<0.008$ & $<0.046$ & $<0.013$ & $<0.005$ & 0.043 & $<0.004$ \\
\hline 30 & 1243 & 0.024 & $<0.007$ & 0.300 & $<0.038$ & $<0.013$ & $<0.008$ & $<0.046$ & $<0.013$ & $<0.005$ & 0.046 & $<0.004$ \\
\hline \multicolumn{13}{|c|}{ FEBRUARY } \\
\hline 13 & 1240 & 0.013 & $<0.007$ & 0.270 & $<0.038$ & $<0.013$ & $<0.008$ & $<0.046$ & $<0.013$ & $<0.005$ & 0.055 & $<0.004$ \\
\hline 27 & 1200 & 0.028 & $<0.007$ & 0.240 & $<0.038$ & $<0.013$ & $<0.008$ & $<0.046$ & $<0.013$ & $<0.005$ & 0.030 & $<0.004$ \\
\hline \multicolumn{13}{|l|}{ MARCH } \\
\hline 05 & 1130 & 0.016 & $<0.007$ & 0.240 & $<0.038$ & $<0.013$ & $<0.008$ & $<0.046$ & $<0.013$ & $<0.005$ & 0.048 & $<0.004$ \\
\hline 12 & 1115 & 0.011 & $<0.007$ & 0.220 & $<0.038$ & $<0.013$ & $<0.008$ & $<0.046$ & $<0.013$ & $<0.005$ & 0.027 & $<0.004$ \\
\hline 19 & 1133 & 0.012 & $<0.007$ & 0.190 & $<0.038$ & $<0.013$ & $<0.008$ & $<0.046$ & $<0.013$ & $<0.005$ & 0.023 & $<0.004$ \\
\hline 26 & 1128 & 0.021 & $<0.007$ & 0.190 & $<0.038$ & $<0.013$ & $<0.008$ & $<0.046$ & $<0.013$ & $<0.005$ & 0.025 & $<0.004$ \\
\hline \multicolumn{13}{|l|}{ APRIL } \\
\hline 02 & 1135 & 0.020 & $<0.007$ & 0.320 & $<0.038$ & $<0.013$ & 0.003 & $<0.046$ & $<0.013$ & $<0.005$ & 0.053 & $<0.004$ \\
\hline 09 & 1102 & 0.017 & $<0.007$ & 0.280 & $<0.038$ & $<0.013$ & 0.007 & $<0.046$ & $<0.013$ & $<0.005$ & 0.038 & $<0.004$ \\
\hline 16 & 1112 & 0.460 & $<0.007$ & 1.20 & $<0.038$ & $<0.013$ & 0.028 & $<0.046$ & $<0.013$ & $<0.005$ & 0.170 & $<0.004$ \\
\hline 20 & 1030 & 0.094 & $<0.007$ & 1.10 & $<0.038$ & $<0.013$ & 0.013 & $<0.046$ & $<0.013$ & $<0.005$ & 0.300 & $<0.004$ \\
\hline 24 & 1035 & 0.280 & $<0.007$ & 1.90 & $<0.038$ & $<0.013$ & $<0.008$ & $<0.046$ & $<0.013$ & $<0.005$ & 0.550 & $<0.004$ \\
\hline 29 & 1100 & 0.140 & $<0.007$ & 1.30 & $<0.038$ & $<0.013$ & 0.029 & $<0.046$ & $<0.013$ & $<0.005$ & 0.500 & $<0.004$ \\
\hline
\end{tabular}

APRIL 1991

$\begin{array}{ll}10 & 1200 \\ 18 & 1045 \\ 23 & 1110\end{array}$

MAY

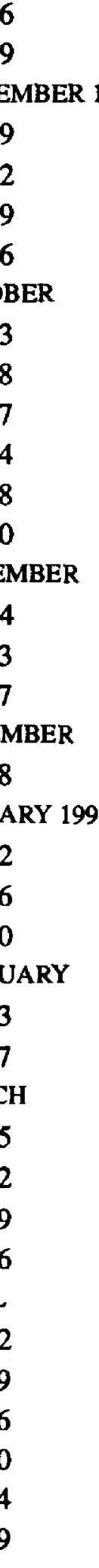

1040

0.020

$-\quad 0.185$

$<0.038$

03612500 - Ohio River at Dam 53 near Grand Chain, Ill.

07

1022

0.016

0.194

$<0.038$

$\begin{array}{lll}-- & 0.015 & <0.046 \\ -- & 0.007 & <0.046\end{array}$

$<0.013$

$<0.005$

0.035

$0.003<0.005 \quad 0.057$ 
Table 8.-Pesticide results from solid-phase extraction 1-liter method-Continued

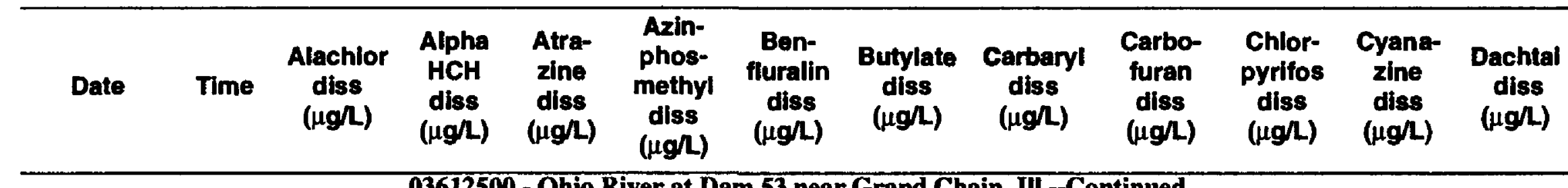

MAY (cont.)

\begin{tabular}{|c|c|c|c|c|c|c|c|c|c|c|c|c|}
\hline 14 & 1045 & -- & -- & - & -- & -- & -- & -- & -- & -- & -- & -- \\
\hline 21 & 1045 & 0.235 & -- & 3.34 & $<0.038$ & $\cdots$ & 0.053 & $<0.046$ & $<0.013$ & $<0.005$ & 0.599 & -- \\
\hline 29 & 1030 & 0.069 & - & 0.658 & $<0.038$ & -- & $<0.008$ & $<0.046$ & 0.016 & $<0.005$ & 0.144 & -- \\
\hline \multicolumn{13}{|l|}{ JUNE } \\
\hline 04 & 1135 & 0.326 & -- & 1.97 & $<0.038$ & -- & 0.020 & $<0.046$ & 0.042 & $<0.005$ & 0.390 & -- \\
\hline 11 & 1045 & 0.267 & -- & 2.13 & $<0.038$ & -- & 0.011 & $<0,046$ & 0.011 & $<0.005$ & 0.370 & - \\
\hline 18 & 1040 & 0.347 & - & 1.83 & $<0.038$ & -- & 0.006 & $<0.046$ & 0.007 & $<0.005$ & 0.641 & -- \\
\hline 25 & 0946 & 0.153 & -- & 1.36 & $<0.038$ & -- & 0.005 & $<0.046$ & $<0.013$ & $<0.005$ & 0.302 & -- \\
\hline \multicolumn{13}{|c|}{ JULY 1991} \\
\hline 01 & 0950 & 0.119 & - & 0.808 & $<0.038$ & -- & 0.007 & $<0.046$ & $<0.013$ & $<0.005$ & 0.113 & -- \\
\hline 09 & 1030 & 0.083 & -- & 0.797 & $<0.038$ & -- & $<0.008$ & $<0.046$ & $<0.013$ & 0.006 & 0.117 & -- \\
\hline 15 & 1100 & 0.054 & -- & 0.699 & $<0.038$ & -- & $<0.008$ & 0.037 & $<0.013$ & $<0.005$ & 0.150 & -- \\
\hline 22 & 1040 & -- & -- & -- & -- & -- & -- & -- & - & -- & -- & -- \\
\hline 29 & 1110 & - & -- & -- & -- & - & -- & $\cdots$ & - & - & -- & -- \\
\hline \multicolumn{13}{|c|}{ AUGUST } \\
\hline 05 & 1050 & - & $\ldots$ & $\ldots$ & -- & -- & -- & - & -- & - & - & - \\
\hline 12 & 1030 & 0.017 & - & 0.388 & $<0.038$ & -- & $<0.008$ & $<0.046$ & $<0.013$ & $<0.005$ & 0.074 & $\cdots$ \\
\hline 20 & 1030 & 0.009 & -- & 0.353 & $<0.038$ & -- & $<0.008$ & $<0.046$ & $<0.013$ & $<0.005$ & 0.055 & -- \\
\hline 27 & 0945 & 0.006 & -- & 0.199 & $<0.038$ & - & $<0.008$ & $<0.046$ & $<0.013$ & $<0.005$ & 0.030 & -- \\
\hline \multicolumn{13}{|c|}{ SEPTEMBER } \\
\hline 03 & 1040 & 0.006 & $\cdots$ & 0.211 & $<0.038$ & -- & $<0.008$ & $<0.046$ & $<0.013$ & $<0.005$ & 0.031 & -- \\
\hline 10 & 1030 & $<0.009$ & - & 0.197 & $<0.038$ & $\cdots$ & $<0.008$ & $<0.046$ & $<0.013$ & $<0.005$ & 0.021 & -- \\
\hline 17 & 0952 & 0.007 & -- & 0.188 & $<0.038$ & -- & $<0.008$ & $<0.046$ & $<0.013$ & $<0.005$ & 0.019 & -- \\
\hline 23 & 1040 & 0.010 & -- & 0.231 & $<0.038$ & -- & $<0.008$ & $<0.046$ & $<0.013$ & $<0.005$ & 0.025 & - \\
\hline 30 & 1122 & $<0.009$ & - & 0.180 & $<0.038$ & -- & $<0.008$ & $<0.046$ & $<0.013$ & $<0.005$ & 0.024 & - \\
\hline \multicolumn{13}{|c|}{ OCTOBER 1991} \\
\hline 07 & 1125 & $<0.009$ & $\cdots$ & 0.104 & $<0.038$ & -- & $<0.008$ & $<0.046$ & $<0.013$ & $<0.005$ & 0.020 & $\cdots$ \\
\hline 15 & 0955 & 0.004 & -- & 0.131 & $<0.038$ & -- & $<0.008$ & $<0.046$ & $<0.013$ & $<0.005$ & 0.020 & -- \\
\hline 21 & 1020 & $<0.009$ & -- & 0.148 & $<0.038$ & -- & $<0.008$ & 0.099 & $<0.013$ & $<0.005$ & 0.024 & -- \\
\hline 29 & 0930 & 0.005 & -- & 0.243 & $<0.038$ & -- & $<0.008$ & $<0.046$ & $<0.013$ & $<0.005$ & 0.054 & -- \\
\hline \multicolumn{13}{|c|}{ NOVEMBER } \\
\hline 06 & 1040 & -- & -- & 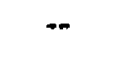 & -- & -- & -- & - & $\cdots$ & 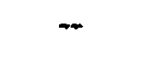 & -- & - \\
\hline 20 & 0920 & 0.009 & -- & 0.173 & $<0.038$ & - & $<0.008$ & $<0.046$ & $<0.013$ & $<0.005$ & 0.034 & $\cdots$ \\
\hline \multicolumn{13}{|c|}{ DECEMBER } \\
\hline 05 & 1045 & 0.009 & -- & 0.144 & $<0.038$ & -- & $<0.008$ & $<0.046$ & $<0.013$ & $<0.005$ & 0.024 & - \\
\hline 17 & 1010 & $<0.009$ & -- & 0.054 & $<0.038$ & - & $<0.008$ & $<0.046$ & $<0.013$ & $<0.005$ & $<0.050$ & $-\cdot$ \\
\hline \multicolumn{13}{|c|}{ JANUARY 1992} \\
\hline 02 & 1005 & $<0.009$ & - & 0.071 & $<0.038$ & -- & $<0.008$ & $<0.046$ & $<0.013$ & $<0.005$ & 0.012 & -- \\
\hline 21 & 1030 & 0.006 & $<0.007$ & 0.061 & $<0.038$ & $<0.013$ & $<0.008$ & 0.006 & $<0.013$ & $<0.005$ & $<0.013$ & $<0.004$ \\
\hline \multicolumn{13}{|c|}{ FEBRUARY } \\
\hline 04 & 1125 & 0.005 & $<0.007$ & 0.047 & $<0.038$ & $<0.013$ & $<0.008$ & $<0.046$ & $<0.013$ & $<0.005$ & 0.014 & $<0.004$ \\
\hline 18 & 1025 & 0.006 & $<0.007$ & 0.062 & $<0.038$ & $<0.013$ & $<0.008$ & $<0.046$ & $<0.013$ & $<0.005$ & 0.024 & $<0.004$ \\
\hline \multicolumn{13}{|c|}{ MARCH 1992} \\
\hline 03 & 1025 & 0.004 & $<0.007$ & 0.038 & $<0.038$ & $<0.008$ & $<0.008$ & 0.007 & $<0.013$ & $<0.005$ & 0.020 & $<0.004$ \\
\hline 11 & 1030 & 0.003 & $<0.007$ & 0.037 & $<0.038$ & $<0.008$ & $<0.008$ & 0.008 & $<0.013$ & $<0.005$ & 0.007 & $<0.004$ \\
\hline 16 & 1040 & 0.004 & $<0.007$ & 0.050 & $<0.038$ & $<0.008$ & $<0.008$ & $<0.046$ & $<0.013$ & $<0.005$ & $<0.013$ & $<0.004$ \\
\hline 24 & 1020 & 0.007 & $<0.007$ & 0.079 & $<0.038$ & $<0.008$ & $<0.008$ & 0.005 & $<0.013$ & $<0.005$ & 0.011 & $<0.004$ \\
\hline 31 & 1030 & 0.007 & $<0.007$ & 0.076 & $<0.038$ & $<0.008$ & $<0.008$ & 0.004 & $<0.013$ & $<0.005$ & 0.012 & $<0.004$ \\
\hline
\end{tabular}


Tabie 8.--Pesticide results from soiid-phase extraction 1-iiter method-Continued

\begin{tabular}{|c|c|c|c|c|c|c|c|c|c|c|c|c|c|}
\hline Date & Time & $\begin{array}{l}\text { Aiachior } \\
\text { diss } \\
(\mu g / L)\end{array}$ & $\begin{array}{c}\text { Aipha } \\
\text { HCH } \\
\text { disa } \\
(\mu g / L)\end{array}$ & $\begin{array}{c}\text { Atra- } \\
\text { zina } \\
\text { diss } \\
(\mu g / L)\end{array}$ & $\begin{array}{c}\text { Azin- } \\
\text { phos- } \\
\text { methyl } \\
\text { diss } \\
(\mu g / L)\end{array}$ & $\begin{array}{c}\text { Ben- } \\
\text { fluralin } \\
\text { diss } \\
(\mu g / L)\end{array}$ & $\begin{array}{c}\text { Butylata } \\
\text { diss } \\
(\mu g / L)\end{array}$ & $\begin{array}{c}\text { Carbary } \\
\text { diss } \\
(\mu g / L)\end{array}$ & & & $\begin{array}{l}\text { Chior- } \\
\text { pyrifos } \\
\text { diss } \\
(\mu g / L)\end{array}$ & $\begin{array}{l}\text { Cyana- } \\
\text { zine } \\
\text { diss } \\
(\mu g / L)\end{array}$ & $\begin{array}{c}\text { Dachtal } \\
\text { diss } \\
(\mu g / L)\end{array}$ \\
\hline \multicolumn{14}{|c|}{03612500 - Ohio River at Dam 53 near Grand Chain, Iil.--Continued } \\
\hline \multicolumn{14}{|l|}{ APRIL } \\
\hline 07 & 1015 & 0.009 & $<0.007$ & 0.110 & $<0.038$ & $<0.008$ & $<0.008$ & 0.005 & \multicolumn{2}{|c|}{$<0.013$} & 0.011 & 0.023 & $<0.004$ \\
\hline 14 & 1035 & 0.007 & $<0.007$ & 0.090 & $<0.038$ & $<0.008$ & $<0.008$ & 0.002 & \multicolumn{2}{|c|}{$<0.013$} & $<0.005$ & 0.016 & $<0.004$ \\
\hline 22 & 1040 & 0.170 & $<0.007$ & 1.60 & $<0.038$ & $<0.008$ & 0.030 & 0.003 & \multicolumn{2}{|c|}{$<0.013$} & $<0.005$ & 0.460 & $<0.004$ \\
\hline 28 & 1104 & 0.180 & $<0.007$ & 1.50 & $<0.038$ & $<0.008$ & 0.010 & $<0.046$ & \multicolumn{2}{|c|}{$<0.013$} & $<0.005$ & 0.570 & $<0.004$ \\
\hline \multicolumn{14}{|l|}{ MAY } \\
\hline 06 & 0953 & 0.039 & $<0.007$ & 0.530 & $<0.038$ & $<0.008$ & 0.021 & 0.003 & \multicolumn{2}{|c|}{$<0.013$} & 0.027 & 0.140 & $<0.004$ \\
\hline 12 & 0955 & 0.024 & $<0.007$ & 0.280 & $<0.038$ & $<0.008$ & 0.017 & \multirow{2}{*}{$\begin{array}{l}<0.046 \\
<0.046\end{array}$} & \multicolumn{2}{|c|}{$<0.013$} & 0.018 & 0.072 & $<0.004$ \\
\hline 18 & 1025 & 0.018 & $<0.007$ & 0.230 & $<0.038$ & $<0.008$ & 0.020 & & $<0$. & 013 & 0.021 & 0.054 & $<0.004$ \\
\hline 27 & 1030 & 0.180 & $<0.007$ & 1.30 & $<0.038$ & $<0.008$ & 0.031 & $<0.046$ & \multicolumn{2}{|c|}{0.024} & 0.027 & 0.460 & 0.001 \\
\hline \multicolumn{14}{|l|}{ JUNE 1992} \\
\hline 02 & 1045 & 0.360 & $<0.007$ & 3.20 & $<0.038$ & $<0.008$ & 0.040 & $<0.046$ & & 074 & 0.038 & 0.550 & 0.001 \\
\hline 09 & 1105 & 0.048 & $<0.007$ & 0.670 & $<0.038$ & $<0.008$ & 0.014 & $<0.046$ & $<0$. & 013 & 0.014 & 0.140 & 0.001 \\
\hline 16 & 1105 & 0.022 & $<0.007$ & 0.320 & $<0.038$ & $<0.008$ & 0.004 & $<0.046$ & $<0$. & 013 & 0.014 & 0.052 & $<0.004$ \\
\hline 23 & 1023 & 0.029 & $<0.007$ & 0.430 & $<0.038$ & $<0.008$ & 0.003 & $<0.046$ & $<0$. & 013 & 0.006 & 0.120 & 0.001 \\
\hline 29 & 1235 & 0.059 & $<0.007$ & 0.760 & $<0.038$ & $<0.008$ & 0.006 & $<0.046$ & $<0$. & 013 & 0.018 & 0.180 & 0.001 \\
\hline JULY & & & & & & & & & & & & & \\
\hline 08 & 1012 & 0.140 & $<0.007$ & 0.600 & $<0.038$ & $<0.008$ & 0.006 & $<0.046$ & $<0$. & 013 & 0.024 & 0.120 & $<0.004$ \\
\hline 14 & 1025 & 0.120 & $<0.007$ & 0.800 & $<0.038$ & $<0.008$ & $<0.008$ & $<0.046$ & $<0$. & 013 & 0.037 & 0.170 & $<0.004$ \\
\hline 23 & 1040 & 0.082 & $<0.007$ & 0.850 & $<0.038$ & $<0.008$ & $<0.008$ & $<0.046$ & $<0$. & 013 & 0.012 & 0.220 & 0.005 \\
\hline 28 & 1025 & 0.100 & $<0.007$ & 1.00 & $<0.038$ & 0.003 & $<0.008$ & $<0.046$ & $<0$. & 013 & 0.038 & 0.230 & 0.002 \\
\hline Dste & Tima & $\begin{array}{c}\text { DDE } \\
\text { diss } \\
(\mu g / L)\end{array}$ & $\begin{array}{l}\text { Dia- } \\
\text { zinon } \\
\text { diss } \\
(\mu g / L)\end{array}$ & $\begin{array}{l}\text { Diei- } \\
\text { drin } \\
\text { diss } \\
(\mu g / L)\end{array}$ & $\begin{array}{c}2,6- \\
\text { Diethyl- } \\
\text { ansiine } \\
\text { diss } \\
(\mu g / L)\end{array}$ & $\begin{array}{l}\text { Dim- } \\
\text { ethoate } \\
\text { diss } \\
(\mu \mathrm{g} / \mathrm{L})\end{array}$ & $\begin{array}{l}\text { Disul- } \\
\text { folon } \\
\text { diss } \\
(\mu g / L)\end{array}$ & $\begin{array}{c}\text { EPTC } \\
\text { diss } \\
(\mu g / L)\end{array}$ & $\begin{array}{l}\text { Ethai- } \\
\text { fiurailin } \\
\text { diss } \\
(\mu g / L)\end{array}$ & $\begin{array}{l}\text { Etho- } \\
\text { prop } \\
\text { diss } \\
(\mu g / L)\end{array}$ & $\begin{array}{c}\text { Fon- } \\
\text { ofos } \\
\text { diss } \\
(\mu g /)\end{array}$ & $\begin{array}{c}\text { Lin- } \\
\text { dane } \\
\text { diss } \\
(\mu g / L)\end{array}$ & $\begin{array}{c}\text { Lin- } \\
\text { uron } \\
\text { diss } \\
(\mu g / L)\end{array}$ \\
\hline & & & & 20500 & - Mississip & pi Rive & inton, Io & owa & & & & & \\
\hline APRIL 1991 & & & & & & & & & & & & & \\
\hline 02 & 1040 & -- & -- & -- & -- & -- & -- & -- & -- & -- & -- & -- & -- \\
\hline 11 & 1100 & -- & -- & -- & -- & -- & -- & - & -- & -- & -- & -- & -- \\
\hline 16 & 1150 & -- & -- & -- & -- & -- & -- & -- & -- & -- & -- & -- & -- \\
\hline 23 & 1030 & -- & -- & -. & $\ldots$ & -- & - & - & -- & -. & -- & - & -- \\
\hline MAY & & & & & & & & & & & & & \\
\hline 01 & 1150 & -- & -- & -- & -- & -- & -- & -- & -- & -- & -- & -- & - \\
\hline 07 & 1115 & $<0.010$ & $<0.008$ & $<0.008$ & -- & -- & $<0.008$ & 0.014 & -- & $<0.012$ & $2<0.008$ & $<0.011$ & $<0.039$ \\
\hline 10 & 1030 & -- & -- & -- & -- & -- & -- & -- & -- & -- & -- & -- & - \\
\hline 14 & 1120 & -- & -- & -- & -- & -- & -- & -- & -- & -- & -- & -- & -- \\
\hline 17 & 1045 & $<0.010$ & $<0.008$ & $<0.008$ & -- & -- & $<0.008$ & 0.009 & -- & $<0.012$ & 0.005 & $<0.011$ & $<0.039$ \\
\hline 21 & 1140 & -- & -- & -- & -- & - & - & - & -- & -- & -- & -- & -- \\
\hline 24 & 1205 & $<0.010$ & $<0.008$ & $<0.008$ & -- & -- & $<0.008$ & 0.070 & -- & $<0.012$ & 0.006 & $<0.011$ & $<0.039$ \\
\hline 28 & 1210 & $<0.010$ & $<0.008$ & $<0.008$ & - & - & $<0.008$ & 0.023 & -. & $<0.012$ & 0.007 & $<0.011$ & $<0.039$ \\
\hline 31 & 1015 & $<0.010$ & $<0.008$ & $<0.008$ & -- & -- & $<0.008$ & 0.012 & -. & $<0.012$ & 0.003 & $<0.011$ & $<0.039$ \\
\hline JUNE 199 & & & & & & & & & & & & & \\
\hline 04 & 1300 & $<0.010$ & $<0.008$ & $<0.008$ & -- & -- & $<0.008$ & 0.011 & -- & $<0.012$ & 0.004 & $<0.011$ & $<0.039$ \\
\hline 07 & 1100 & $<0.010$ & $<0.008$ & $<0.008$ & -- & -- & $<0.008$ & 0.010 & -- & $<0.012$ & 0.003 & $<0.011$ & $<0.039$ \\
\hline 10 & 1130 & $<0.010$ & $<0.008$ & $<0.008$ & -- & - & $<0.008$ & 0.013 & -- & $<0.012$ & $2<0.008$ & $<0.011$ & $<0.039$ \\
\hline 13 & 1410 & $<0.010$ & $<0.008$ & $<0.008$ & -- & -- & $<0.008$ & 0.008 & -- & $<0.012$ & $2<0.008$ & $<0.011$ & $<0.039$ \\
\hline 17 & 1040 & $<0.010$ & $<0.008$ & $<0.008$ & -. & -- & $<0.008$ & 0.006 & -. & $<0.012$ & 0.023 & $<0.011$ & $<0.039$ \\
\hline
\end{tabular}




\begin{tabular}{|c|c|c|c|c|c|c|c|c|c|c|c|c|c|}
\hline Date & Time & $\begin{array}{c}\text { DDE } \\
\text { diss } \\
(\mu g / L)\end{array}$ & $\begin{array}{l}\text { Dia- } \\
\text { zinon } \\
\text { diss } \\
(\mu g / L)\end{array}$ & $\begin{array}{c}\text { Diei- } \\
\text { drin } \\
\text { diss } \\
(\mu g / L)\end{array}$ & $\begin{array}{c}\text { 2,6- } \\
\text { Dethyl- } \\
\text { anailina } \\
\text { diss } \\
(\mu \mathrm{g} / \mathrm{L})\end{array}$ & $\begin{array}{l}\text { Dim- } \\
\text { ethoat } \\
\text { e diss } \\
(\mu g / L)\end{array}$ & $\begin{array}{l}\text { Disul- } \\
\text { foton } \\
\text { diss } \\
(\mu g / L)\end{array}$ & $\begin{array}{c}\text { EPTC } \\
\text { diss } \\
(\mu g /)\end{array}$ & $\begin{array}{l}\text { Ethai- } \\
\text { fluralin } \\
\text { diss } \\
(\mu g / L)\end{array}$ & $\begin{array}{l}\text { Etho- } \\
\text { prop } \\
\text { diss } \\
(\mu g /)\end{array}$ & $\begin{array}{l}\text { Fon- } \\
\text { ofos } \\
\text { diss } \\
(\mu g /)\end{array}$ & $\begin{array}{l}\text { Lin- } \\
\text { dane } \\
\text { diss } \\
(\mu g / L)\end{array}$ & $\begin{array}{l}\text { Lin- } \\
\text { uron } \\
\text { diss } \\
(\mu g / L)\end{array}$ \\
\hline
\end{tabular}

JUNE 1991 (cont.)

\begin{tabular}{|c|c|c|c|c|c|c|c|c|c|c|c|c|c|}
\hline 20 & 1345 & $<0.010$ & $<0.008$ & $<0.008$ & -- & -- & $<0.008$ & 0.008 & -- & $<0.012$ & 0.007 & $<0.011$ & $<0.039$ \\
\hline 24 & 1055 & $<0.010$ & $<0.008$ & $<0.008$ & -- & -- & $<0.008$ & 0.005 & -- & $<0.012$ & 0.004 & $<0.011$ & $<0.039$ \\
\hline 27 & 0915 & $<0.010$ & $<0.008$ & $<0.008$ & -- & -- & $<0.008$ & 0.008 & -- & $<0.012$ & $<0.008$ & $<0.011$ & $<0.039$ \\
\hline \multicolumn{14}{|l|}{ JULY } \\
\hline 01 & 1025 & $<0.010$ & $<0.008$ & $<0.008$ & -- & -- & $<0.008$ & 0.004 & -- & $<0.012$ & $<0.008$ & $<0.011$ & $<0.03$ \\
\hline 05 & 1205 & $<0.010$ & $<0.008$ & $<0.008$ & -- & -- & $<0.008$ & $<0.005$ & -- & $<0.012$ & $<0.008$ & $<0.011$ & $<0.03$ \\
\hline 08 & 1130 & $<0.010$ & $<0.008$ & $<0.008$ & - & - & $<0.008$ & 0.003 & -- & $<0.012$ & $<0.008$ & $<0.011$ & $<0.03$ \\
\hline 11 & 1300 & - & - & - & -- & - & -- & - & -- & -- & -- & -- & - \\
\hline 15 & 1025 & $<0.010$ & $<0.008$ & $<0.008$ & $-\cdot$ & -- & $<0.008$ & $<0.005$ & -- & $<0.012$ & $<0.008$ & $<0.011$ & $<0.03$ \\
\hline 18 & 1200 & - & -- & -- & -- & -. & -- & -- & - & -- & -- & -- & - \\
\hline 22 & 1050 & -- & -- & -- & -- & -- & -- & - & -- & -- & - & -- & - \\
\hline 29 & 1230 & $<0.010$ & $<0.008$ & $<0.008$ & -- & -- & $<0.008$ & $<0.005$ & - & $<0.012$ & $<0.008$ & $<0.011$ & $<0.039$ \\
\hline \multicolumn{14}{|c|}{ AUGUST 1991} \\
\hline 06 & 1240 & $<0.010$ & $<0.008$ & $<0.008$ & -- & -- & $<0.008$ & $<0.005$ & -- & $<0.012$ & $<0.008$ & $<0.011$ & $<0.039$ \\
\hline 12 & 1125 & $<0.010$ & $<0.008$ & $<0.008$ & -- & -- & $<0.008$ & $<0.005$ & -- & $<0.012$ & 0.001 & $<0.011$ & $<0.03$ \\
\hline 19 & 1145 & $<0.010$ & $<0.008$ & $<0.008$ & - & -- & $<0.008$ & $<0.005$ & -- & $<0.012$ & $<0.008$ & $<0.011$ & $<0.039$ \\
\hline 26 & 1205 & $<0.010$ & $<0.008$ & $<0.008$ & -. & -- & $<0.008$ & $<0.005$ & - & $<0.012$ & $<0.008$ & $<0.011$ & $<0.03$ \\
\hline \multicolumn{14}{|c|}{ SEPTEMBER } \\
\hline 03 & 1200 & $<0.010$ & $<0.008$ & $<0.008$ & -- & -- & $<0.008$ & $<0.005$ & -- & $<0.012$ & $<0.008$ & $<0.011$ & $<0.03$ \\
\hline 09 & 1500 & $<0.010$ & $<0.008$ & $<0.008$ & -- & -- & $<0.008$ & $<0.005$ & -- & $<0.012$ & $<0.008$ & $<0.011$ & $<0.03$ \\
\hline 17 & 1215 & $<0.010$ & $<0.008$ & $<0.008$ & -- & -- & $<0.008$ & $<0.005$ & -- & $<0.012$ & $<0.008$ & $<0.011$ & $<0.03$ \\
\hline 24 & 1215 & $<0.010$ & $<0.008$ & $<0.008$ & -- & -- & $<0.008$ & $<0.005$ & -- & $<0.012$ & $<0.008$ & $<0.011$ & $<0.03$ \\
\hline \multicolumn{14}{|c|}{ OCTOBER } \\
\hline 01 & 1200 & -- & - & -- & -- & -- & -- & - & -- & - & - & - & -- \\
\hline 07 & 1230 & $<0.010$ & $<0.008$ & $<0.008$ & -- & -- & $<0.008$ & $<0.005$ & -- & $<0.012$ & $<0.008$ & $<0.011$ & $<0.039$ \\
\hline 15 & 1300 & $<0.010$ & $<0.008$ & $<0.008$ & -- & -- & $<0.008$ & $<0.005$ & -- & $<0.012$ & $<0.008$ & $<0.011$ & $<0.039$ \\
\hline 22 & 1230 & $<0.010$ & $<0.008$ & $<0.008$ & -- & -- & $<0.008$ & $<0.005$ & -- & $<0.012$ & $<0.008$ & $<0.011$ & $<0.039$ \\
\hline 29 & 1330 & $<0.010$ & $<0.008$ & $<0.008$ & -- & -- & $<0.008$ & $<0.005$ & - & $<0.012$ & $<0.008$ & $<0.011$ & $<0.039$ \\
\hline \multicolumn{14}{|c|}{ NOVEMBER 1991} \\
\hline 05 & 1245 & $<0.010$ & $<0.008$ & $<0.008$ & -- & -- & $<0.008$ & $<0.005$ & -- & $<0.012$ & $<0.008$ & $<0.011$ & $<0.039$ \\
\hline 12 & 1255 & $<0.010$ & $<0.008$ & $<0.008$ & -- & -- & $<0.008$ & $<0.005$ & -- & $<0.012$ & $<0.008$ & $<0.011$ & $<0.039$ \\
\hline 26 & 1320 & $<0.010$ & $<0.008$ & $<0.008$ &.- & - & $<0.008$ & $<0.005$ & -- & $<0.012$ & $<0.008$ & $<0.011$ & $<0.039$ \\
\hline \multicolumn{14}{|c|}{ DECEMBER } \\
\hline 09 & 1200 & $<0.010$ & $<0.008$ & $<0.008$ & -- & - & $<0.008$ & $<0.005$ & -- & $<0.012$ & $<0.008$ & $<0.011$ & $<0.039$ \\
\hline 22 & 1330 & $<0.010$ & $<0.008$ & $<0.008$ & - & -- & $<0.008$ & $<0.005$ & -- & $<0.012$ & $<0.008$ & $<0.011$ & $<0.039$ \\
\hline \multicolumn{14}{|c|}{ JANUARY 1992} \\
\hline 07 & 1210 & $<0.010$ & $<0.008$ & $<0.008$ & $<0.006$ & $<0.024$ & $<0.008$ & $<0.005$ & $<0.013$ & $<0.012$ & $<0.008$ & $<0.011$ & $<0.039$ \\
\hline 22 & 1340 & $<0.010$ & $<0.008$ & $<0.008$ & $<0.006$ & $<0.024$ & $<0.008$ & $<0.005$ & $<0.013$ & $<0.012$ & $<0.008$ & $<0.011$ & $<0.039$ \\
\hline \multicolumn{14}{|c|}{ FEBRUARY } \\
\hline 03 & 1245 & $<0.010$ & $<0.008$ & $<0.008$ & $<0.006$ & $<0.024$ & $<0.008$ & $<0.005$ & $<0.013$ & $<0.012$ & $<0.008$ & $<0.011$ & $<0.039$ \\
\hline 20 & 1230 & $<0.010$ & $<0.008$ & $<0.008$ & $<0.006$ & $<0.024$ & $<0.008$ & $<0.005$ & $<0.013$ & $<0.012$ & $<0.008$ & $<0.011$ & $<0.039$ \\
\hline \multicolumn{14}{|l|}{ MARCH } \\
\hline 04 & 1100 & - & -- & -- & -- & - & -- & - & - & - & - & - & -- \\
\hline 13 & 1200 & $<0.010$ & $<0.008$ & $<0.008$ & $<0.006$ & $<0.024$ & $<0.008$ & $<0.005$ & $<0.013$ & $<0.012$ & $<0.008$ & $<0.011$ & $<0.039$ \\
\hline 17 & 1400 & $<0.010$ & $<0.008$ & $<0.008$ & $<0.006$ & $<0.024$ & $<0.008$ & $<0.005$ & $<0.013$ & $<0.012$ & $<0.008$ & $<0.011$ & $<0.039$ \\
\hline 24 & 1125 & $<0.010$ & $<0.008$ & $<0.008$ & $<0.006$ & $<0.024$ & $<0.008$ & $<0.005$ & $<0.013$ & $<0.012$ & $<0.008$ & $<0.011$ & $<0.039$ \\
\hline 30 & 1155 & $<0.010$ & $<0.008$ & $<0.008$ & $<0.006$ & $<0.024$ & $<0.008$ & $<0.005$ & $<0.013$ & $<0.012$ & $<0.008$ & $<0.011$ & $<0.039$ \\
\hline
\end{tabular}


Table 8.-Pesticide resuits from solid-phase extraction 1-liter method-ContInued

\begin{tabular}{|c|c|c|c|c|c|c|c|c|c|c|c|c|c|}
\hline Data & Time & $\begin{array}{c}\text { DDE } \\
\text { diss } \\
(\mu g / L)\end{array}$ & $\begin{array}{c}\text { Dia- } \\
\text { zinon } \\
\text { diss } \\
(\mu g /)\end{array}$ & $\begin{array}{l}\text { Diei- } \\
\text { drin } \\
\text { diss } \\
(\mu g / L)\end{array}$ & $\begin{array}{c}2,6- \\
\text { Dethyl- } \\
\text { analine } \\
\text { diss } \\
(\mu g / L)\end{array}$ & 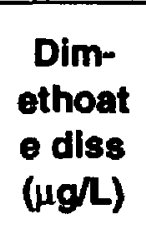 & $\begin{array}{l}\text { Disul- } \\
\text { foton } \\
\text { diss } \\
(\mu g /)\end{array}$ & $\begin{array}{c}\text { EPTC } \\
\text { diss } \\
(\mu g / L)\end{array}$ & $\begin{array}{c}\text { Ethal- } \\
\text { fiuralin } \\
\text { diss } \\
(\mu g / L)\end{array}$ & $\begin{array}{l}\text { Etho- } \\
\text { prop } \\
\text { diss } \\
(\mu g /)\end{array}$ & $\begin{array}{l}\text { Fon- } \\
\text { ofos } \\
\text { diss } \\
(\mu g /)\end{array}$ & $\begin{array}{l}\text { Lin- } \\
\text { dane } \\
\text { diss } \\
(\mu g /))\end{array}$ & 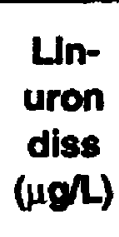 \\
\hline
\end{tabular}

APRIL 1992

\begin{tabular}{|c|c|c|c|c|c|c|c|c|c|c|c|c|c|}
\hline 06 & 1215 & $<0.010$ & $<0.008$ & $<0.008$ & $<0.006$ & $<0.024$ & $<0.008$ & $<0.005$ & $<0.013$ & $<0.012$ & $<0.008$ & $<0.011$ & $<0.039$ \\
\hline 14 & 1130 & $<0.010$ & $<0.008$ & $<0.008$ & $<0.006$ & $<0.024$ & $<0.008$ & $<0.005$ & $<0.013$ & $<0.012$ & $<0.008$ & $<0.011$ & $<0.039$ \\
\hline 24 & 1145 & $<0.010$ & $<0.008$ & $<0.008$ & $<0.006$ & $<0.024$ & $<0.008$ & $<0.005$ & $<0.013$ & $<0.012$ & $<0.008$ & $<0.011$ & $<0.039$ \\
\hline 28 & 1145 & $<0.010$ & $<0.008$ & $<0.008$ & $<0.006$ & $<0.024$ & $<0.008$ & $<0.005$ & $<0.013$ & $<0.012$ & $<0.008$ & $<0.011$ & $<0.039$ \\
\hline \multicolumn{14}{|l|}{ MAY } \\
\hline 05 & 1115 & $<0.010$ & $<0.008$ & $<0.008$ & $<0.006$ & $<0.024$ & $<0.008$ & $<0.005$ & $<0.013$ & $<0.012$ & $<0.008$ & $<0.011$ & $<0.039$ \\
\hline 12 & 1130 & $<0.010$ & $<0.008$ & $<0.008$ & $<0.006$ & $<0.024$ & $<0.008$ & 0.013 & $<0.013$ & $<0.012$ & $<0.008$ & $<0.011$ & $<0.039$ \\
\hline 19 & 1130 & $<0.010$ & $<0.008$ & $<0.008$ & $<0.006$ & $<0.024$ & $<0.008$ & 0.015 & $<0.013$ & $<0.012$ & $<0.008$ & $<0.011$ & $<0.039$ \\
\hline 26 & 1215 & $<0.010$ & $<0.008$ & $<0.008$ & $<0.006$ & $<0.024$ & $<0.008$ & 0.026 & $<0.013$ & $<0.012$ & $<0.008$ & $<0.011$ & $<0.039$ \\
\hline \multicolumn{14}{|c|}{05586100 - Illinois River at Valley City } \\
\hline
\end{tabular}

\begin{tabular}{|c|c|c|c|c|c|c|c|c|c|c|c|c|c|}
\hline 05 & 1230 & -- & -- & -- & -- & -- & -- & -- & -- & -- & -- & -. & -- \\
\hline 17 & 1330 & -- & -- & -- & -. & -- & -- & - & -- & -- & -- & -- & -- \\
\hline 26 & 1000 & - & -- & -- & -- & -- & -- & - & - & -- & -- & -- & -. \\
\hline \multicolumn{14}{|l|}{ MAY } \\
\hline 03 & 1545 & $<0.010$ & $<0.008$ & $<0.008$ & -- & -- & $<0.008$ & 0.057 & -- & $<0.012$ & $<0.008$ & $<0.011$ & $<0.039$ \\
\hline 08 & 1245 & $<0.010$ & $<0.008$ & $<0.008$ & -- & -- & $<0.008$ & 0.203 & - & $<0.012$ & 0.037 & $<0.011$ & $<0.039$ \\
\hline 10 & 1100 & -- & -- & -. & -. & -- & -- & -- & - & -- & -- & -- & -- \\
\hline 17 & 1145 & $<0.010$ & $<0.008$ & $<0.008$ & -- & -- & $<0.008$ & $<0.005$ & -- & $<0.012$ & 0.005 & $<0.011$ & $<0.039$ \\
\hline 19 & 1115 & $<0.010$ & 0.015 & $<0.008$ & -- & -- & $<0.008$ & 0.041 & - & $<0.012$ & 0.020 & $<0.011$ & $<0.039$ \\
\hline 22 & 1715 & $<0.010$ & 0.011 & 0.014 & $-\cdot$ & -- & $<0.008$ & 0.035 & - & $<0.012$ & 0.029 & $<0.011$ & $<0.039$ \\
\hline 29 & 1130 & $<0.010$ & 0.009 & 0.010 & - & -- & $<0.008$ & 0.061 & - & $<0.012$ & 0.033 & $<0.011$ & $<0.039$ \\
\hline 31 & 1045 & $<0.010$ & 0.007 & 0.009 & -- & -- & $<0.008$ & 0.015 & -- & $<0.012$ & 0.021 & $<0.011$ & $<0.039$ \\
\hline \multicolumn{14}{|c|}{ JUNE 1991} \\
\hline 04 & 1215 & $<0.010$ & 0.011 & 0.007 & -- & -- & $<0.008$ & 0.011 & -- & $<0.012$ & 0.014 & $<0.011$ & $<0.039$ \\
\hline 06 & 1200 & $<0.010$ & 0.014 & $<0.008$ & -- & -- & $<0.008$ & 0.008 & - & $<0.012$ & 0.011 & $<0.011$ & $<0.039$ \\
\hline 11 & 1345 & $<0.010$ & 0.016 & 0.004 & - & -- & $<0.008$ & 0.006 & - & $<0.012$ & 0.008 & $<0.011$ & $<0.039$ \\
\hline 14 & 1200 & $<0.010$ & 0.014 & 0.005 & -- & -- & $<0.008$ & 0.003 & -- & $<0.012$ & 0.006 & $<0.011$ & $<0.039$ \\
\hline 18 & 1130 & $<0.010$ & 0.017 & 0.010 & -- & -- & $<0.008$ & $<0.005$ & -- & $<0,012$ & 0.006 & $<0.011$ & $<0.039$ \\
\hline 20 & 1130 & $<0.010$ & 0.021 & $<0.008$ & - & - & $<0.008$ & $<0.005$ & -- & $<0.012$ & 0.007 & $<0.011$ & $<0.039$ \\
\hline 24 & 1130 & $<0.010$ & 0.015 & $<0.008$ & -- & -- & $<0.008$ & $<0.005$ & -- & $<0.012$ & 0.005 & $<0.011$ & $<0.039$ \\
\hline 27 & 1100 & $<0.010$ & 0.012 & $<0.008$ & -- & -- & $<0.008$ & $<0.005$ & - & $<0.012$ & 0.005 & $<0.011$ & $<0.039$ \\
\hline \multicolumn{14}{|l|}{ JULY } \\
\hline 01 & 0930 & $<0.010$ & 0.016 & 0.009 & -- & -- & $<0.008$ & 0.005 & -. & $<0.012$ & $<0.008$ & $<0.011$ & $<0.039$ \\
\hline 03 & 1100 & $<0.010$ & 0.016 & $<0.008$ & -- & -- & $<0.008$ & $<0.005$ & -- & $<0.012$ & $<0.008$ & $<0.011$ & $<0.039$ \\
\hline 09 & 1000 & $<0.010$ & $<0.008$ & $<0.008$ & -- & -- & $<0.008$ & $<0.005$ & -- & $<0.012$ & $<0.008$ & $<0.011$ & $<0.039$ \\
\hline 11 & 1045 & $<0.010$ & 0.013 & $<0.008$ & -- & -. & $<0.008$ & $<0.005$ & -- & $<0.012$ & $<0.008$ & $<0.011$ & $<0.039$ \\
\hline 18 & 1115 & -- & -- & - & $\cdots$ & - & -- & -- & -- & - & -- & -- & -- \\
\hline 25 & 1340 & $<0.010$ & $<0.008$ & $<0.008$ & - & -- & $<0.008$ & $<0.005$ & -. & $<0.012$ & $<0.008$ & $<0.011$ & $<0.039$ \\
\hline 31 & 1100 & $<0.010$ & $<0.008$ & $<0.008$ & -- & -- & $<0.008$ & $<0.005$ & -- & $<0.012$ & $<0.008$ & $<0.011$ & $<0.039$ \\
\hline \multicolumn{14}{|c|}{ AUGUST 1991} \\
\hline 06 & 1015 & $<0.010$ & 0.017 & $<0.008$ & - & -- & $<0.008$ & $<0.005$ & -- & $<0.012$ & $<0.008$ & $<0.011$ & $<0.039$ \\
\hline 13 & 1200 & $<0.010$ & 0.032 & $<0.008$ & -- & -- & $<0.008$ & $<0.005$ & -- & $<0.012$ & $<0.008$ & $<0.011$ & $<0.039$ \\
\hline 21 & 1230 & $<0.010$ & 0.025 & $<0.008$ & -- & -- & $<0.008$ & $<0.005$ & -- & $<0.012$ & $<0.008$ & $<0.011$ & $<0.039$ \\
\hline 28 & 1015 & $<0.010$ & 0.023 & $<0.008$ & -- & -- & $<0.008$ & $<0.005$ & -- & $<0.012$ & $<0.008$ & $<0.011$ & $<0.039$ \\
\hline \multicolumn{14}{|l|}{ SEPTEM } \\
\hline 03 & 1050 & $<0.010$ & 0.035 & $<0.008$ & - & -- & $<0.008$ & $<0.005$ & -- & $<0.012$ & $<0.008$ & $<0.011$ & $<0.039$ \\
\hline 10 & 1200 & $<0.010$ & 0.055 & $<0.008$ & -- & -- & $<0.008$ & $<0.005$ & -- & $<0.012$ & 0.001 & $<0.011$ & $<0.039$ \\
\hline
\end{tabular}


Table 8.-Pesticide results from solid-phase extraction 1-liter method-Continued

\begin{tabular}{|c|c|c|c|c|c|c|c|c|c|c|c|c|c|}
\hline Date & Time & $\begin{array}{c}\text { DDE } \\
\text { diss } \\
(\mu g / L)\end{array}$ & $\begin{array}{l}\text { Dia- } \\
\text { zinon } \\
\text { diss } \\
(\mu g / L)\end{array}$ & $\begin{array}{l}\text { Diel- } \\
\text { drin } \\
\text { diss } \\
(\mu g / L)\end{array}$ & $\begin{array}{c}\text { 2,6- } \\
\text { Dethyl- } \\
\text { analine } \\
\text { diss } \\
(\mu \mathrm{g} / \mathrm{L})\end{array}$ & $\begin{array}{l}\text { Dim- } \\
\text { othoat } \\
\text { e diss } \\
\text { ( } \mu \mathrm{g} / \mathrm{L})\end{array}$ & $\begin{array}{l}\text { Disui- } \\
\text { foton } \\
\text { diss } \\
(\mu g / L)\end{array}$ & $\begin{array}{c}\text { EPTC } \\
\text { dlss } \\
(\mu g / L)\end{array}$ & $\begin{array}{c}\text { Ethal- } \\
\text { furalin } \\
\text { diss } \\
(\mu \mathrm{g} / \mathrm{L})\end{array}$ & $\begin{array}{l}\text { Etho- } \\
\text { prop } \\
\text { diss } \\
(\mu g / L)\end{array}$ & $\begin{array}{l}\text { Fon- } \\
\text { ofos } \\
\text { diss } \\
(\mu \mathrm{g} / \mathrm{L})\end{array}$ & $\begin{array}{c}\text { Lin- } \\
\text { dane } \\
\text { diss } \\
(\mu g / L)\end{array}$ & $\begin{array}{l}\text { Lin- } \\
\text { uron } \\
\text { diss } \\
(\mu g / L)\end{array}$ \\
\hline
\end{tabular}

SEPTEMBER (cont.)

\begin{tabular}{|c|c|c|c|c|c|c|c|c|c|c|c|c|c|}
\hline & & & & & & & & & & & & & \\
\hline 17 & 1100 & $<0.010$ & 0.035 & $<0.008$ & -- & -- & $<0.008$ & $<0.005$ & -- & $<0.012$ & $<0.008$ & $<0.011$ & $<0.039$ \\
\hline 24 & 1100 & $<0.010$ & 0.036 & $<0.008$ & - & -- & $<0.008$ & $<0.005$ & -- & $<0.012$ & $<0.008$ & $<0.011$ & $<0.039$ \\
\hline \multicolumn{14}{|c|}{ OCTOBER } \\
\hline 01 & 1130 & $<0.010$ & 0.050 & $<0.008$ & -- & -- & $<0.008$ & $<0.005$ & -- & $<0.012$ & $<0.008$ & $<0.011$ & $<0.039$ \\
\hline 08 & 1330 & $<0.010$ & 0.082 & $<0.008$ & -- & - & $<0.008$ & $<0.005$ & -- & $<0.012$ & $<0.008$ & $<0.011$ & $<0.039$ \\
\hline 15 & 1215 & $<0.010$ & 0.058 & 0.010 & -- & - & $<0.008$ & $<0.005$ & -- & $<0.012$ & $<0.008$ & $<0,011$ & $<0.039$ \\
\hline 22 & 1130 & $<0.010$ & 0.085 & 0.011 & -- & -- & $<0,008$ & $<0.005$ & - & $<0.012$ & $<0.008$ & $<0.011$ & $<0.039$ \\
\hline \multicolumn{14}{|c|}{ NOVEMBER } \\
\hline 05 & 1140 & $<0.010$ & 0.077 & $<0.008$ & -- & -- & $<0.008$ & $<0.005$ & - & $<0.012$ & $<0.008$ & $<0.011$ & $<0.039$ \\
\hline 15 & 1130 & $<0.010$ & 0.050 & $<0.008$ & -- & -- & $<0.008$ & $<0.005$ & -- & $<0.012$ & $<0.008$ & $<0.011$ & $<0.039$ \\
\hline \multicolumn{14}{|c|}{ DECEMBER 1991} \\
\hline 03 & 1030 & $<0.010$ & 0.019 & $<0.008$ & - & -- & $<0,008$ & $<0.005$ & -- & $<0.012$ & $<0.008$ & $<0.011$ & $<0.039$ \\
\hline 18 & 1200 & $<0.010$ & 0.018 & $<0.008$ & -- & - & $<0.008$ & $<0.005$ & -- & $<0.012$ & $<0.008$ & $<0.011$ & $<0.039$ \\
\hline \multicolumn{14}{|c|}{ JANUARY 1992} \\
\hline 02 & 1330 & $<0.010$ & 0.016 & $<0.008$ & -- & -- & $<0.008$ & $<0.005$ & -- & $<0.012$ & $<0.008$ & $<0.011$ & $<0.039$ \\
\hline 15 & 1645 & $<0.010$ & $<0.008$ & $<0.008$ & $<0.006$ & $<0.024$ & $<0.008$ & $<0.005$ & $<0.013$ & $<0.012$ & $<0.008$ & $<0.011$ & $<0.039$ \\
\hline 30 & 1345 & $<0.010$ & $<0.008$ & $<0.008$ & $<0.006$ & $<0.024$ & $<0.008$ & $<0.005$ & $<0.013$ & $<0.012$ & $<0.008$ & $<0.011$ & $<0.039$ \\
\hline \multicolumn{14}{|c|}{ FEBRUARY } \\
\hline 12 & 1200 & 0.004 & $<0.008$ & $<0.008$ & $<0.006$ & $<0.024$ & $<0.008$ & $<0.005$ & $<0.013$ & $<0.012$ & $<0.008$ & $<0.011$ & $<0.039$ \\
\hline 28 & 1245 & $<0.010$ & $<0.008$ & $<0.008$ & $<0.006$ & $<0.024$ & $<0.008$ & $<0.005$ & $<0.013$ & $<0.012$ & $<0.008$ & $<0.011$ & $<0.039$ \\
\hline \multicolumn{14}{|l|}{ MARCH } \\
\hline 03 & 1100 & $<0.010$ & $<0.008$ & $<0.008$ & $<0.006$ & $<0.024$ & $<0.008$ & $<0.005$ & $<0.013$ & $<0.012$ & $<0.008$ & $<0.011$ & $<0.039$ \\
\hline 10 & 1015 & $<0.010$ & $<0.008$ & $<0.008$ & $<0.006$ & $<0.024$ & $<0.008$ & $<0.005$ & $<0.013$ & $<0.012$ & $<0.008$ & $<0.011$ & $<0.039$ \\
\hline 17 & 1050 & $<0.010$ & $<0.008$ & $<0.008$ & $<0.006$ & $<0.024$ & $<0.008$ & $<0.005$ & $<0.013$ & $<0.012$ & $<0.008$ & $<0.011$ & $<0.039$ \\
\hline 25 & 1045 & $<0.010$ & $<0.008$ & $<0.008$ & $<0.006$ & $<0.024$ & $<0.008$ & $<0.005$ & $<0.013$ & $<0.012$ & $<0.008$ & $<0.011$ & $<0.039$ \\
\hline
\end{tabular}

APRIL 1991

MAY

16

1250

1030

1030

29

1000

MAY

06

\section{9}

13

16

21

24

29

31

JUNE 1991

07

11

14

18

21

$\begin{array}{lccc}1115 & <0.010 & <0.008 & <0.008 \\ 0940 & <0.010 & <0.008 & <0.008 \\ 1110 & -- & -- & -- \\ 1130 & -- & -- & -- \\ 0945 & <0.010 & <0.008 & <0.008 \\ 0740 & <0.010 & <0.008 & <0.008 \\ 1015 & <0.010 & <0.008 & <0.008 \\ 0735 & <0.010 & <0.008 & <0.008 \\ & & & \\ 0800 & <0.010 & <0.008 & <0.008 \\ 1415 & <0.010 & 0.003 & <0.008 \\ 1045 & <0.010 & <0.008 & <0.008 \\ 0750 & <0.010 & 0.006 & <0.008 \\ 0935 & <0.010 & 0.004 & <0.008 \\ 1020 & <0.010 & <0.008 & <0.008\end{array}$

06805500 - Platte River at Louisville, Nebr. 
Table 8.--Pesticide results from solid-phase extraction 1-liter method--Contlnued

\begin{tabular}{|c|c|c|c|c|c|c|c|c|c|c|c|c|c|}
\hline Date & Time & $\begin{array}{c}\text { DDE } \\
\text { diss } \\
(\mu g /)\end{array}$ & $\begin{array}{c}\text { Dia- } \\
\text { zinon } \\
\text { diss } \\
(\mu g / L)\end{array}$ & $\begin{array}{l}\text { Diel- } \\
\text { drin } \\
\text { diss } \\
(\mu g / L)\end{array}$ & $\begin{array}{c}2,6- \\
\text { Dethyi- } \\
\text { ansilne } \\
\text { diss } \\
(\mu g / L)\end{array}$ & $\begin{array}{l}\text { Dim- } \\
\text { ethoat } \\
\text { e diss } \\
(\mu g / L)\end{array}$ & $\begin{array}{l}\text { Disul- } \\
\text { foton } \\
\text { dlss } \\
(\mu g /)\end{array}$ & $\begin{array}{c}\text { EPTC } \\
\text { diss } \\
(\mu g / L)\end{array}$ & $\begin{array}{c}\text { Ethsi- } \\
\text { flurailn } \\
\text { diss } \\
(\mu g / L)\end{array}$ & $\begin{array}{l}\text { Etho- } \\
\text { prop } \\
\text { diss } \\
(\mu g / L)\end{array}$ & $\begin{array}{l}\text { Fon- } \\
\text { ofos } \\
\text { diss } \\
(\mu g / L)\end{array}$ & $\begin{array}{l}\text { Lin- } \\
\text { dane } \\
\text { diss } \\
(\mu g / L)\end{array}$ & $\begin{array}{l}\text { Lin- } \\
\text { uron } \\
\text { diss } \\
(\mu g /)\end{array}$ \\
\hline \multicolumn{14}{|c|}{ O6805500 - Platte River at Louisville, Nebr.-Continued } \\
\hline \multicolumn{14}{|c|}{ JUNE 1991 (cont.) } \\
\hline 24 & 1110 & $<0.010$ & 0.018 & $<0.008$ & -- & -- & $<0.008$ & $<0.005$ & -- & $<0.012$ & 0.005 & $<0.011$ & $<0.039$ \\
\hline 27 & 1020 & $<0.010$ & 0.008 & $<0.008$ & -- & -- & $<0.008$ & $<0.005$ & -- & $<0.012$ & 0.006 & $<0.011$ & $<0.039$ \\
\hline \multicolumn{14}{|l|}{ JULY } \\
\hline 01 & 0950 & $<0.010$ & $<0.008$ & $<0.008$ & -- & -- & $<0.008$ & $<0.005$ & -- & $<0.012$ & $<0.008$ & $<0.011$ & $<0.039$ \\
\hline 03 & 0950 & $<0.010$ & 0.006 & $<0.008$ & -- & -- & $<0.008$ & $<0.005$ & -- & $<0.012$ & 0.042 & $<0.011$ & $<0.039$ \\
\hline 08 & 1100 & $<0.010$ & 0.008 & $<0.008$ & -- & -. & $<0.008$ & $<0.005$ & -- & $<0.012$ & 0.009 & $<0.011$ & $<0.039$ \\
\hline 11 & 1020 & $<0.010$ & 0.044 & $<0.008$ & .- & - & $<0.008$ & $<0.005$ & -- & $<0.012$ & $<0.008$ & $<0.011$ & $<0.039$ \\
\hline 15 & 0950 & $<0.010$ & $<0.008$ & $<0.008$ & -- & -- & $<0.008$ & $<0.005$ & -- & $<0.012$ & $<0.008$ & $<0.011$ & $<0.039$ \\
\hline 25 & 1000 & $<0.010$ & 0.005 & $<0.008$ & -- & -- & $<0.008$ & $<0.005$ & -- & $<0.012$ & $<0.008$ & $<0.011$ & $<0.039$ \\
\hline 29 & 0940 & $<0.010$ & $<0.008$ & $<0.008$ & -- & -- & $<0.008$ & $<0.005$ & -- & $<0.012$ & $<0.008$ & $<0.011$ & $<0.039$ \\
\hline \multicolumn{14}{|c|}{ AUGUST 1991} \\
\hline 06 & 1130 & $<0.010$ & 0.019 & $<0.008$ & -- & -- & $<0.008$ & $<0.005$ & -- & $<0.012$ & $<0.008$ & $<0.011$ & $<0.039$ \\
\hline 12 & 1115 & $<0.010$ & 0.019 & $<0.008$ & -- & -- & $<0.008$ & $<0.005$ & -- & $<0.012$ & $<0.008$ & $<0.011$ & $<0.039$ \\
\hline 20 & 1000 & $<0.010$ & 0.017 & $<0.008$ & -- & -- & $<0.008$ & $<0.005$ & -- & $<0.012$ & $<0.008$ & $<0.011$ & $<0.039$ \\
\hline 27 & 0940 & 0.003 & 0.004 & $<0.008$ & -. & -. & $<0.008$ & $<0.005$ & -- & $<0.012$ & $<0.008$ & $<0.011$ & $<0.039$ \\
\hline \multicolumn{14}{|c|}{ SEPTEMBER } \\
\hline 03 & 0950 & $<0.010$ & 0.006 & $<0.008$ & -- & -- & $<0,008$ & $<0.005$ & -- & $<0.012$ & $<0.008$ & $<0.011$ & $<0.039$ \\
\hline 10 & 1030 & $<0.010$ & 0.023 & $<0.008$ & -- & -- & $<0.008$ & $<0.005$ & -- & $<0.012$ & $<0.008$ & $<0.011$ & $<0.039$ \\
\hline 17 & 1115 & $<0.010$ & 0.007 & $<0.008$ & -- & -- & $<0.008$ & $<0.005$ & -- & $<0.012$ & $<0.008$ & $<0.011$ & $<0.039$ \\
\hline 24 & 1135 & $<0.010$ & $<0.008$ & $<0.008$ & -- & -- & $<0.008$ & $<0.005$ & -. & $<0.012$ & $<0.008$ & $<0.011$ & $<0.039$ \\
\hline \multicolumn{14}{|c|}{ OCTOBER } \\
\hline 01 & 1115 & $<0.010$ & $<0.008$ & $<0.008$ & -- & -- & $<0.008$ & $<0.005$ &.- & $<0.012$ & $<0.008$ & $<0.011$ & $<0.039$ \\
\hline 08 & 1030 & $<0.010$ & $<0.008$ & $<0.008$ & -- & -. & $<0.008$ & $<0.005$ & -. & $<0.012$ & $<0.008$ & $<0.011$ & $<0.039$ \\
\hline 15 & 1130 & $<0.010$ & $<0.008$ & $<0.008$ & -- & -. & $<0.008$ & $<0.005$ & -- & $<0.012$ & $<0.008$ & $<0.011$ & $<0.039$ \\
\hline 24 & 1200 & $<0.010$ & $<0.008$ & $<0.008$ & -- & -- & $<0.008$ & $<0.005$ & -- & $<0.012$ & $<0.008$ & $<0.011$ & $<0.039$ \\
\hline \multicolumn{14}{|c|}{ NOVEMBER } \\
\hline 05 & 1200 & $<0.010$ & $<0.008$ & $<0.008$ & -- & -- & $<0.008$ & $<0.005$ & -- & $<0.012$ & $<0.008$ & $<0.011$ & $<0.039$ \\
\hline 19 & 1140 & $<0.010$ & $<0.008$ & $<0.008$ & -- & -- & $<0.008$ & $<0.005$ & -- & $<0.012$ & $<0.008$ & $<0.011$ & $<0.039$ \\
\hline \multicolumn{14}{|c|}{ DECEMBER 1991} \\
\hline 05 & 1130 & - & -- & -- & -- & -- & -- & -- & -- & -- & -- & -- & -- \\
\hline 20 & 1000 & $<0.010$ & $<0.008$ & $<0.008$ & $<0.006$ & $<0.024$ & $<0.008$ & $<0.005$ & $<0.013$ & $<0.012$ & $<0.008$ & $<0.011$ & $<0.039$ \\
\hline 30 & 1020 & 0.002 & $<0.008$ & $<0.008$ & $<0.006$ & $<0.024$ & $<0.008$ & $<0.005$ & $<0.013$ & $<0.012$ & $<0.008$ & $<0.011$ & $<0.039$ \\
\hline \multicolumn{14}{|c|}{ JANUARY 1992} \\
\hline 14 & 1000 & $<0.010$ & $<0.008$ & $<0.008$ & $<0.006$ & $<0.024$ & $<0.008$ & $<0.005$ & $<0.013$ & $<0.012$ & $<0.008$ & $<0.011$ & $<0.039$ \\
\hline 28 & 1000 & $<0.010$ & $<0.008$ & $<0.008$ & $<0.006$ & $<0.024$ & $<0.008$ & $<0.005$ & $<0.013$ & $<0.012$ & $<0.008$ & $<0.011$ & $<0.039$ \\
\hline \multicolumn{14}{|c|}{ FEBRUARY } \\
\hline 11 & 1030 & $<0.010$ & $<0.008$ & $<0.008$ & $<0.006$ & $<0,024$ & $<0.008$ & $<0.005$ & $<0.013$ & $<0.012$ & $<0.008$ & $<0.011$ & $<0.039$ \\
\hline 27 & 1000 & $<0.010$ & $<0.008$ & $<0.008$ & $<0.006$ & $<0.024$ & $<0.008$ & $<0.005$ & $<0.013$ & $<0.012$ & $<0.008$ & $<0.011$ & $<0.039$ \\
\hline \multicolumn{14}{|l|}{ MARCH } \\
\hline 05 & 1010 & $<0.010$ & $<0.008$ & $<0.008$ & $<0.006$ & $<0.024$ & $<0.008$ & $<0.005$ & $<0.013$ & $<0.012$ & $<0.008$ & $<0.011$ & $<0.039$ \\
\hline 09 & 0945 & $<0.010$ & $<0.008$ & $<0.008$ & $<0.006$ & $<0.024$ & $<0.008$ & $<0.005$ & $<0.013$ & $<0.012$ & $<0.008$ & $<0.011$ & $<0.039$ \\
\hline 17 & 1145 & $<0.010$ & $<0.008$ & $<0.008$ & $<0.006$ & $<0.024$ & $<0.008$ & $<0.005$ & $<0.013$ & $<0.012$ & $<0.008$ & $<0.011$ & $<0.039$ \\
\hline 23 & 1040 & $<0.010$ & $<0.008$ & $<0.008$ & $<0.006$ & $<0.024$ & $<0.008$ & $<0.005$ & $<0.013$ & $<0.012$ & $<0.008$ & $<0.011$ & $<0.039$ \\
\hline 31 & 1100 & $<0.010$ & $<0.008$ & $<0.008$ & $<0.006$ & $<0.024$ & $<0.008$ & $<0.005$ & $<0.013$ & $<0.012$ & $<0.008$ & $<0.011$ & $<0.039$ \\
\hline \multicolumn{14}{|c|}{ APRIL 1992} \\
\hline 07 & 1100 & $<0.010$ & $<0.008$ & $<0.008$ & $<0.006$ & $<0.024$ & $<0.008$ & $<0.005$ & $<0.013$ & $<0.012$ & $<0.008$ & $<0.011$ & $<0.039$ \\
\hline 14 & 1010 & $<0.010$ & $<0.008$ & $<0.008$ & $<0.006$ & $<0.024$ & $<0.008$ & $<0.005$ & $<0.013$ & $<0.012$ & $<0.008$ & $<0.011$ & $<0.039$ \\
\hline
\end{tabular}


Table 8.--Pesticide results from solid-phase extraction 1-liter method--Continued

\begin{tabular}{rlcccccccccccc}
\hline Date & Time & $\begin{array}{c}\text { DDE } \\
\text { diss } \\
(\mu g / L)\end{array}$ & $\begin{array}{c}\text { Dia- } \\
\text { zinon } \\
\text { diss } \\
(\mu g / L)\end{array}$ & $\begin{array}{c}\text { Diel- } \\
\text { drin } \\
\text { diss } \\
(\mu g / L)\end{array}$ & $\begin{array}{c}\text { Dethyl- } \\
\text { analine } \\
\text { diss } \\
(\mu g / L)\end{array}$ & $\begin{array}{c}\text { Dim- } \\
\text { ethoat } \\
\text { ediss } \\
(\mu g / L)\end{array}$ & $\begin{array}{c}\text { Disul- } \\
\text { foton } \\
\text { diss } \\
(\mu g / L)\end{array}$ & $\begin{array}{c}\text { EPTC } \\
\text { disa } \\
(\mu g / L)\end{array}$ & $\begin{array}{c}\text { Ethal- } \\
\text { fluralin } \\
\text { diss } \\
(\mu g / L)\end{array}$ & $\begin{array}{c}\text { Etho- } \\
\text { prop } \\
\text { diss } \\
(\mu g / L)\end{array}$ & $\begin{array}{c}\text { Fon- } \\
\text { ofos } \\
\text { diss } \\
(\mu g / L)\end{array}$ & $\begin{array}{c}\text { Lin- } \\
\text { dane } \\
\text { diss } \\
(\mu g / L)\end{array}$ & $\begin{array}{c}\text { Lin- } \\
\text { uron } \\
\text { diss } \\
(\mu g / L)\end{array}$ \\
\hline
\end{tabular}

APRIL 1992 (cont.)

\begin{tabular}{|c|c|c|c|c|c|c|c|c|c|c|c|c|c|}
\hline 23 & 1020 & $<0.010$ & $<0.008$ & $<0.008$ & $<0.006$ & $<0.024$ & $<0.008$ & $<0.005$ & $<0.013$ & $<0.012$ & $<0.008$ & $<0.011$ & $<0.039$ \\
\hline 29 & 1030 & $<0.010$ & $<0.008$ & $<0.008$ & $<0.006$ & $<0.024$ & $<0.008$ & $<0.005$ & $<0.013$ & $<0.012$ & $<0.008$ & $<0.011$ & $<0.039$ \\
\hline \multicolumn{14}{|l|}{ MAY } \\
\hline 07 & 1100 & $<0.010$ & $<0.008$ & $<0.008$ & $<0.006$ & $<0.024$ & $<0.008$ & $<0.005$ & $<0.013$ & $<0.012$ & $<0.008$ & $<0.011$ & $<0.039$ \\
\hline & & & & & & ra & I。 & nann. & & & & & \\
\hline
\end{tabular}

APRIL 1991

\begin{tabular}{|c|c|c|c|c|c|c|c|c|c|c|c|c|c|}
\hline 09 & 1331 & -- & -- & -- & - & -- & - & -- & -- & -- & -- & -- & - \\
\hline 16 & 1030 & -- & - & -- & -- & -- & - & $\cdots$ & -- & -- & -- & -- & - \\
\hline 24 & 1200 & -. & -- & - & -- & -- & -- & -- & -- & -- & - & -- & -- \\
\hline \multicolumn{14}{|l|}{ MAY } \\
\hline 01 & 1130 & -- & - & -- & $\cdots$ & $\cdots$ & - & -- & -- & -- & -- & - & - \\
\hline 06 & 1100 & $<0.010$ & $<0.008$ & 0.036 & -- &. & $<0.008$ & 0.006 & - & $<0.012$ & $<0.008$ & $<0.011$ & $<0.039$ \\
\hline 09 & 0930 & -- & -- & -- & -- & - & -- & -- & - & -- & -- & -- & $\cdots$ \\
\hline 13 & 1000 & -- & - & -- & -- & - & -- & -- & - & -- & - & - & - \\
\hline 16 & 1020 & $<0.010$ & $<0.008$ & $<0.008$ & -- & - & $<0.008$ & 0.005 & -- & $<0.012$ & 0.006 & $<0.011$ & $<0.039$ \\
\hline 20 & 1000 & $<0.010$ & 0.006 & $<0.008$ & - & -- & $<0.008$ & $<0.005$ & - & $<0.012$ & $<0.008$ & $<0.011$ & $<0.039$ \\
\hline 24 & 1000 & $<0.010$ & $<0.008$ & $<0.008$ & -- & -- & $<0.008$ & 0.030 & - & $<0.012$ & 0.005 & $<0.011$ & $<0.039$ \\
\hline 28 & 1030 & $<0.010$ & $<0.008$ & $<0.008$ & - & -- & $<0.008$ & $<0.005$ & $-\cdot$ & $<0.012$ & $<0.008$ & $<0.011$ & $<0.039$ \\
\hline 31 & 1040 & $<0.010$ & $<0.008$ & $<0.008$ & -- & -- & $<0.008$ & 0.005 & -. & $<0.012$ & 0.003 & $<0.011$ & $<0.039$ \\
\hline \multicolumn{14}{|c|}{ JUNE 1991} \\
\hline 03 & 1000 & $<0.010$ & $<0.008$ & 0.008 & - & -- & $<0.008$ & 0.005 & $\cdots$ & $<0.012$ & $<0.008$ & $<0.011$ & $<0.039$ \\
\hline 06 & 1000 & $<0.010$ & 0.004 & 0.005 & -- & -- & $<0.008$ & $<0.005$ & -- & $<0.012$ & 0.004 & $<0.011$ & $<0.039$ \\
\hline 11 & 1000 & $<0.010$ & 0.004 & $<0.008$ & -- & -- & $<0.008$ & 0.004 & -- & $<0.012$ & 0.003 & $<0.011$ & $<0.039$ \\
\hline 13 & 1000 & $<0.010$ & 0.003 & 0.003 & -- & -- & $<0.008$ & $<0.005$ & -- & $<0.012$ & 0.004 & $<0.011$ & $<0.039$ \\
\hline 21 & 1000 & $<0.010$ & 0.012 & $<0.008$ & -- & - & $<0.008$ & $<0.005$ & -. & $<0.012$ & 0.011 & $<0.011$ & $<0.039$ \\
\hline 25 & 1130 & $<0.010$ & 0.010 & 0.005 & -- & -- & $<0.008$ & $<0.005$ & -- & $<0.012$ & .0 .005 & $<0.011$ & $<0.039$ \\
\hline 27 & 1045 & $<0.010$ & $<0.008$ & 0.007 & -- & -- & $<0.008$ & $<0.005$ & -- & $<0.012$ & $<0.008$ & $<0.011$ & $<0.039$ \\
\hline \multicolumn{14}{|l|}{ JULY } \\
\hline 01 & 1100 & $<0.010$ & 0.006 & $<0.008$ & -- & -- & $<0.008$ & $<0.005$ & -- & $<0.012$ & $<0.008$ & $<0.011$ & $<0.039$ \\
\hline 03 & 1000 & $<0.010$ & $<0.008$ & $<0.008$ & -- & $-\bullet$ & $<0.008$ & $<0.005$ & -- & $<0.012$ & $<0.008$ & $<0.011$ & $<0.039$ \\
\hline 08 & 1030 & $<0.010$ & $<0.008$ & $<0.008$ & - & -- & $<0.008$ & $<0.005$ & - & $<0.012$ & $<0.008$ & $<0.011$ & $<0.039$ \\
\hline 11 & 1000 & $<0.010$ & 0.008 & $<0.008$ & -- & -- & $<0.008$ & $<0.005$ & - & $<0.012$ & $<0.008$ & $<0.011$ & $<0.039$ \\
\hline 15 & 1030 & $<0.010$ & $<0.008$ & $<0.008$ & - & - & $<0.008$ & $<0.005$ & -- & $<0.012$ & $<0.008$ & $<0.011$ & $<0.039$ \\
\hline 22 & 1015 & $<0.010$ & $<0.008$ & $<0.008$ & -- & -- & $<0.008$ & $<0.005$ & - & $<0.012$ & $<0.008$ & $<0.011$ & $<0.039$ \\
\hline 29 & 1100 & $<0.010$ & $<0.008$ & $<0.008$ & - & -- & $<0.008$ & $<0.005$ & - & $<0.012$ & $<0.008$ & $<0.011$ & $<0.039$ \\
\hline \multicolumn{14}{|c|}{ AUGUST 1991} \\
\hline 05 & 1000 & $<0.010$ & $<0.008$ & $<0.008$ & - & - & $<0.008$ & $<0.005$ & -- & $<0.012$ & $<0.008$ & $<0.011$ & $<0.039$ \\
\hline 13 & 1100 & $<0.010$ & $<0.008$ & $<0.008$ & -- & - & $<0.008$ & $<0.005$ & $\cdots$ & $<0.012$ & $<0.008$ & $<0.011$ & $<0.039$ \\
\hline 19 & 1000 & $<0.010$ & $<0.008$ & $<0.008$ & -- & - & $<0.008$ & $<0.005$ & -- & $<0.012$ & $<0.008$ & $<0.011$ & $<0.039$ \\
\hline 27 & 1100 & $<0.010$ & $<0.008$ & $<0.008$ & -- & -- & $<0.008$ & $<0.005$ & -- & $<0.012$ & $<0.008$ & $<0.011$ & $<0.039$ \\
\hline \multicolumn{14}{|c|}{ SEPTEMBER 1991} \\
\hline 05 & 1030 & $<0.010$ & $<0.008$ & $<0.008$ & - & -- & $<0.008$ & $<0.005$ & - & $<0.012$ & $<0.008$ & $<0.011$ & $<0.039$ \\
\hline 09 & 1000 & $<0.010$ & $<0.008$ & $<0.008$ & -- & -- & $<0.008$ & $<0.005$ & -- & $<0.012$ & $<0.008$ & $<0.011$ & $<0.039$ \\
\hline 16 & 1000 & 0.008 & 0.013 & $<0.008$ & -- & -- & $<0.008$ & $<0.005$ & $-\cdots$ & $<0.012$ & $<0.008$ & $<0.011$ & $<0.039$ \\
\hline 23 & 0945 & $<0.010$ & 0.006 & $<0.008$ & -- & -- & $<0.008$ & $<0.005$ & - & $<0.012$ & $<0.008$ & $<0.011$ & $<0.039$ \\
\hline \multicolumn{14}{|c|}{ OCTOBER } \\
\hline 01 & 1030 & $<0.010$ & 0.007 & $<0.008$ & -- & -- & $<0.008$ & $<0.005$ & - & $<0.012$ & $<0.008$ & $<0.011$ & $<0.039$ \\
\hline 07 & 1000 & $<0.010$ & $<0.008$ & $<0.008$ & - & - & $<0.008$ & $<0.005$ & - & $<0.012$ & $<0.008$ & $<0.011$ & $<0.039$ \\
\hline
\end{tabular}


Table 8.-Pesticide results from solid-phase extraction 1-liter method-Continued

\begin{tabular}{|c|c|c|c|c|c|c|c|c|c|c|c|c|c|}
\hline Date & Time & $\begin{array}{c}\text { DDE } \\
\text { diss } \\
(\mu g / L)\end{array}$ & $\begin{array}{l}\text { Dla- } \\
\text { zinon } \\
\text { diss } \\
(\mu \mathrm{g} / \mathrm{L})\end{array}$ & $\begin{array}{l}\text { Diei- } \\
\text { drin } \\
\text { diss } \\
(\mu g / L)\end{array}$ & $\begin{array}{c}2,6- \\
\text { Dethyi- } \\
\text { analine } \\
\text { diss } \\
(\mu g / L)\end{array}$ & $\begin{array}{l}\text { Dim- } \\
\text { ethost } \\
\text { e diss } \\
(\mu g / L)\end{array}$ & $\begin{array}{l}\text { Disul- } \\
\text { foton } \\
\text { diss } \\
(\mu g /)\end{array}$ & $\begin{array}{c}\text { EPTC } \\
\text { diss } \\
(\mu g /)\end{array}$ & $\begin{array}{l}\text { Ethat- } \\
\text { fiuralin } \\
\text { diss } \\
(\mu g / L)\end{array}$ & $\begin{array}{l}\text { Etho- } \\
\text { prop } \\
\text { diss } \\
(\mu g / L)\end{array}$ & $\begin{array}{l}\text { Fon- } \\
\text { ofos } \\
\text { diss } \\
(\mu g /)\end{array}$ & $\begin{array}{l}\text { Lin- } \\
\text { dane } \\
\text { diss } \\
(\mu g / L)\end{array}$ & $\begin{array}{l}\text { Lin- } \\
\text { uron } \\
\text { diss } \\
(\mu g / L)\end{array}$ \\
\hline \multicolumn{14}{|c|}{06934500 - Missouri River at Hermann, Mo.--Continned } \\
\hline \multicolumn{14}{|c|}{ OCTOBER (cont.) } \\
\hline 15 & 1100 & $<0.010$ & $<0.008$ & $<0.008$ & -- & -- & $<0.008$ & $<0.005$ & -- & $<0.012$ & $<0.008$ & $<0.011$ & $<0.039$ \\
\hline 21 & 1000 & $<0.010$ & $<0.008$ & $<0.008$ & -- & -- & $<0.008$ & $<0.005$ & -- & 0.012 & $<0.008$ & $<0.011$ & $<0.039$ \\
\hline 29 & 1000 & $<0.010$ & $<0.008$ & $<0.008$ & -- & -- & $<0.008$ & $<0.005$ & -- & $<0.012$ & $<0.008$ & $<0.011$ & $<0.039$ \\
\hline \multicolumn{14}{|c|}{ NOVEMBER 1991} \\
\hline 04 & 1030 & $<0.010$ & $<0.008$ & $<0.008$ & -. & -- & $<0.008$ & $<0.005$ & -- & $<0.012$ & $<0.008$ & $<0.011$ & $<0.039$ \\
\hline 21 & 1030 & $<0.010$ & $<0.008$ & $<0.008$ & -- & -- & $<0.008$ & $<0.005$ & -- & 012 & $<0.008$ & & $<0.039$ \\
\hline \multicolumn{14}{|c|}{ DECEMBER } \\
\hline 06 & 1240 & 010 & 8 & $<0$ & - & -- & 008 & 005 & -- & 12 & $<0.008$ & & $<0.039$ \\
\hline 16 & 1130 & $<0.010$ & $<$ & $<0.008$ & -- & -- & 8 & 005 & -- & & 08 & & 0.039 \\
\hline \multicolumn{14}{|c|}{ JANUARY 1992} \\
\hline 02 & 1030 & $<0.010$ & 209 & $<0.008$ & -- & -. & $<0.008$ & $<0.005$ & -- & 12 & $<0.008$ & 11 & $\$ 0.039$ \\
\hline 16 & 1200 & 0.002 & $<0.008$ & $<0.008$ & $<0.008$ & $<0.024$ & $<0.008$ & $<0.005$ & $<0.013$ & & 08 & & 0.039 \\
\hline 28 & 1300 & $<0.010$ & $<0.008$ & $<0.008$ & $<0.008$ & $<0.024$ & $<0.008$ & $<0.005$ & $<0.013$ & 12 & $<0.008$ & $<0$. & $<0.039$ \\
\hline \multicolumn{14}{|l|}{ FEBRUAR } \\
\hline 10 & 1000 & 0.003 & $<0.008$ & $<0.008$ & $<0.008$ & $<0.024$ & $<0.008$ & $<0.005$ & $<0.013$ & $<0.012$ & $<0.008$ & $<0.011$ & $<0.039$ \\
\hline 24 & 1200 & 50.010 & $<0.008$ & $<0.008$ & $<0.008$ & $<0.024$ & $<0.008$ & $<0.005$ & $<0.013$ & $<0.012$ & $<0.008$ & $<0.011$ & $<0.039$ \\
\hline \multicolumn{14}{|l|}{ MARCH } \\
\hline 03 & 1100 & $<0.010$ & $<0.008$ & $<0.008$ & $<0.008$ & $<0.024$ & $<0.008$ & $<0.005$ & $<0.013$ & $<0.012$ & $<0.008$ & $<0.011$ & $<0.039$ \\
\hline 12 & 1200 & $<0.010$ & 0.008 & $<0.008$ & $<0.008$ & $<0.024$ & $<0.008$ & $<0.005$ & $<0.013$ & $<0.012$ & $<0.008$ & $<0$ & $<0.039$ \\
\hline 20 & 0830 & 0.003 & 0.008 & $<0.008$ & $<0.008$ & $<0.024$ & $<0.008$ & $<0.005$ & $<0.013$ & 12 & $<0.008$ & 11 & $<0.039$ \\
\hline 26 & 1130 & $<0.010$ & 0.008 & $<0.008$ & 0008 & $<0.024$ & $<0.008$ & $<0.005$ & $<0.013$ & 12 & $<0.008$ & & $<0.039$ \\
\hline \multicolumn{14}{|l|}{ APRIL 19} \\
\hline 01 & 1000 & 010 & 06 & $<0.008$ & $<0.008$ & $<0.024$ & 0.008 & .011 & $<0.013$ & 12 & .008 & & 0.039 \\
\hline 06 & 1230 & 010 & $<0.008$ & $<0.008$ & $<0.008$ & $<0.024$ & $<0.008$ & $<0.005$ & $<0.013$ & & 08 & & $<0.039$ \\
\hline 14 & 1030 & 10 & 8 & $<0$. & $<0$. & 4 & 08 & 05 & $<0.013$ & & & & .039 \\
\hline 21 & & & & & & & & 05 & 13 & & & & .039 \\
\hline 29 & 1015 & $<0$ & $<0$ & $<0$ & $<$ & & 88 & 05 & 13 & & 18 & & 0.039 \\
\hline \multicolumn{14}{|l|}{ MAY } \\
\hline 12 & 1000 & 03 & 0 & $<0$ & $<0$. & $<0.024$ & 08 & $<0.005$ & $<0.013$ & 12 & 8 & & $<0.039$ \\
\hline 21 & 1200 & 0 & 0. & $<0$. & & 4 & 88 & 0.006 & $<0.013$ & 12 & $<0.008$ & & $<0.039$ \\
\hline 26 & 1130 & $<0.010$ & 0.004 & $<0.008$ & 0.001 & $<0.024$ & $<0.008$ & 0.016 & $<0.013$ & $<0.012$ & 0.004 & $<0.011$ & $<0.039$ \\
\hline \multicolumn{14}{|l|}{ JUNE } \\
\hline 01 & 330 & 02 & 002 & $<0.008$ & 002 & $<0.024$ & $<0.008$ & 007 & $<0.013$ & $<0.012$ & $<0.008$ & $<0.011$ & $<0.039$ \\
\hline 09 & 1030 & $<0.010$ & $<0.008$ & $<0.008$ & $<0.008$ & $<0.024$ & $<0.008$ & $<0.005$ & $<0.013$ & 0.012 & $<0.008$ & $<0.011$ & $<0.039$ \\
\hline 15 & 1230 & $<0.010$ & 0.005 & $<0.008$ & $<0.008$ & $<0.024$ & $<0.008$ & $<0.005$ & $<0.013$ & $<0.012$ & $<0.008$ & $<0.011$ & $<0.039$ \\
\hline 22 & 0945 & 0.001 & 0.0 & $<0.008$ & $<0.0$ & $<0.024$ & $<0.008$ & $<0.005$ & $<0.013$ & 12 & $<0.008$ & 11 & $<0.039$ \\
\hline 30 & 1130 & .010 & 0. & $<0.008$ & $<0.00$ & $<0.024$ & $<0.008$ & $<0.005$ & $<0.013$ & $<0.012$ & $<0.008$ & $<0.011$ & $<0.039$ \\
\hline \multicolumn{14}{|c|}{ JULY 1992} \\
\hline 07 & 0930 & $<0.010$ & $<0.008$ & $<0.008$ & $<0.008$ & $<0.024$ & $<0.008$ & 0.007 & $<0.013$ & $<0.012$ & $<0.008$ & $<0.011$ & $<0.039$ \\
\hline 14 & 1200 & $<0.010$ & 0.008 & $<0.008$ & 0.006 & $<0.024$ & $<0.008$ & $<0.005$ & $<0.013$ & $<0.012$ & $<0.008$ & $<0.011$ & $<0.039$ \\
\hline 21 & 1030 & $<0.010$ & $<0.008$ & $<0.008$ & $<0.008$ & $<0.024$ & $<0.008$ & $<0.005$ & $<0.013$ & $<0.012$ & $<0.008$ & $<0.011$ & $<0.039$ \\
\hline 28 & 1230 & $<0.010$ & $<0.008$ & $<0.008$ & $<0.008$ & $<0.024$ & $<0.008$ & $<0.005$ & $<0.013$ & $<0.012$ & $<0.008$ & $<0.011$ & $<0.039$ \\
\hline
\end{tabular}

APRIL 1991

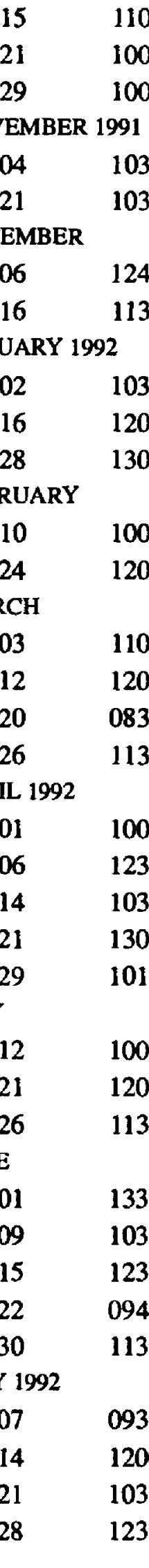

$18 \quad 1130$

$24 \quad 0930$

$29 \quad 1230$

07022000 - Mississippi River at Thebes, Ill. 
Table 8.-Pesticide results from solid-phase extraction 1-liter method--Continued

\begin{tabular}{|c|c|c|c|c|c|c|c|c|c|c|c|c|c|}
\hline Date & Time & $\begin{array}{c}\text { DDE } \\
\text { diss } \\
(\mu g / L)\end{array}$ & $\begin{array}{l}\text { Dia- } \\
\text { zinon } \\
\text { dlss } \\
(\mu g / L)\end{array}$ & $\begin{array}{l}\text { Diel- } \\
\text { drin } \\
\text { dlss } \\
(\mu g / L)\end{array}$ & $\begin{array}{c}\text { 2,6- } \\
\text { Dethyl- } \\
\text { analine } \\
\text { diss } \\
(\mu g / L)\end{array}$ & $\begin{array}{l}\text { Dim- } \\
\text { ethost } \\
\text { e dlss } \\
(\mu \mathrm{g} / \mathrm{L})\end{array}$ & $\begin{array}{l}\text { Disul- } \\
\text { foton } \\
\text { diss } \\
(\mu g / L)\end{array}$ & $\begin{array}{c}\text { EPTC } \\
\text { diss } \\
(\mu g / L)\end{array}$ & $\begin{array}{c}\text { Ethai- } \\
\text { fluralin } \\
\text { diss } \\
(\mu \mathrm{g} / \mathrm{L})\end{array}$ & $\begin{array}{l}\text { Etho- } \\
\text { prop } \\
\text { dlss } \\
(\mu g / L)\end{array}$ & $\begin{array}{l}\text { Fon- } \\
\text { ofos } \\
\text { dlss } \\
(\mu g / L)\end{array}$ & $\begin{array}{c}\text { Lin- } \\
\text { dane } \\
\text { dlss } \\
(\mu g / L)\end{array}$ & $\begin{array}{c}\text { Lin- } \\
\text { uron } \\
\text { dlss } \\
(\mu g / L)\end{array}$ \\
\hline
\end{tabular}

MAY

07

09

$1345<0.010<0.008$

\section{0} $<0.010<0.008$

$<0.008$

\section{0}

1200

1430

$<0.010$

$<0.008<0.008$

16

20

23

28

30

JUNE 1991

$0916<0.010<0.008$

$1300<0.010 \quad 0.005$

$1330<0.010<0.008$

03

$1345<0.010<0.008$

$$
134
$$

$$
1400
$$

1500

1430

1100

1300

1400

JULY

$01<1320<0.010<0.008$

03

\section{0}$$
0950
$$

08

11

18

23

30 AUGUST 1991$$
1400
$$

0930

1300

1000

$06 \quad 1300$

13

1300

0930

$20 \quad 1330$

29

SEPTEMBER

$\begin{array}{cc}03 & 1500 \\ 11 & 1000 \\ 17 & 1400 \\ 26 & 0930 \\ \text { OCTOBER } & \end{array}$

02

$02 \quad 1300$

$09 \quad 1030$

$16 \quad 1430$

$23 \quad 0945$

$30 \quad 1400$

NOVEMBER 1991

$100<0.010$

0.010

$<0.010$

$<0.008$
0.015

$<0.008$

$<0.01$

$<0.010<0$

$<0.008$

$<0.008$

$<0.008$

$<0.008$

0.005

0.005

0.007

$\begin{array}{lll}-- & - & <0.008\end{array}$

$\begin{array}{ccc}-- & - & <0.008 \\ -- & -- & <0.008 \\ -- & -- & --\end{array}$

-. $\quad--$

$\begin{array}{ll}-- & -- \\ -- & --\end{array}$

$<0.008$

\section{$<0.008$}

$<0.008$

$\begin{array}{llll}-. & - & - & <0.008\end{array}$

$\begin{array}{lll}-. & -- & <0.008 \\ -. & -- & <0.008\end{array}$

0.018

$\begin{array}{lll}-- & <0.012 \quad 0.010<0 .\end{array}$

0.013

0.021

$<0.005$

$<0.005$

0.015

0.016

$\quad<0.008$

$-\quad<0.008$

0.012

- $\quad<0.008$

$\begin{array}{ll}-- & <0.008 \\ -- & <0.008\end{array}$

-
$-\quad 0008$

- $\quad<0.008$

- $\quad<0.008$

0.004

$<0.005$

$<0.005$

$<0.005$

$<0.005$

0.004

$--\quad<0.008$

$-\quad<0.008$

$--\quad<0.008$

$-\quad<0.008$

$<0.005$

$<0.005$

$<0.005$

0.009

$--$

$<0.010$

$<.008$

$<0.008$

$-\quad<0.008$

$<0.005$

-.

- $\quad<0.008$

$--\quad<0.008$

-- $\quad<0.008$

- $\quad<0.008$

$<0.005$

$-$

$-$

$-$

$-$

-.

-.

--

$-$

$-$

$--$

$-$

$-$

$--$

$--$

--
--
--
--
--
--
--

$<0.012<0.008$

$<0.012<0.008$

$<0.012<0.008$

$<0.012$

$<0.008$

$<0.012<0.008$

$<0.012<0.008$

$<0.005$

$-$

$<0.012<0.008$

$<0.012<0.008$

$<0.012<0.008$

$<0.012<0.008$

$<0.005$

- $\quad<0.008<0.005$

- $\quad<0.008<0.005$

-- $<0.008<0.005$

-- $\quad<0.008$

$--\quad<0.008$

$-\quad<0.008$

$-\quad<0.008$

$-\quad<0.008$

.- $\quad<0.008$

$<0.005$

$<0.005$

$<0.005$

$<0.010<0.008$

$<0.008$

$<0.008$

$<0.010<0.008$

$<0.008$

20

0800

$<0.010 \quad 0.012$

$<0.008$

$<0.008<0.005$

$-\quad<0.008<0.005$

$--\quad<0.008<0.005$

$-\quad<0.008<0.005$

$--\quad<0.008<0.005$

$-$

$\begin{array}{llllllllllllll}05 & 1045 & <0.010 & 0.007 & <0.008 & - & - & <0.008 & <0.005 & -- & <0.012 & <0.008 & <0.011 & <0.039 \\ 18 & 1100 & <0.010 & <0.008 & <0.008 & -- & -- & <0.008 & <0.005 & -- & <0.012 & <0.008 & <0.011 & <0.039 \\ 30 & 1400 & <0.010 & <0.008 & <0.008 & - & - & <0.008 & <0.005 & -- & <0.012 & <0.008 & <0.011 & <0.039\end{array}$

$\begin{array}{lllll}- & <0.012 & <0.008 & <0.011 & <0.039 \\ .- & <0.012 & <0.008 & <0.011 & <0.039 \\ .- & <0.012 & <0.008 & <0.011 & <0.039 \\ - & <0.012 & <0.008 & <0.011 & <0.039 \\ & & & & \\ - & <0.012 & <0.008 & <0.011 & <0.039 \\ .- & <0.012 & <0.008 & <0.011 & <0.039 \\ -. & <0.012 & <0.008 & <0.011 & <0.039 \\ .- & <0.012 & <0.008 & <0.011 & <0.039 \\ .- & <0.012 & <0.008 & <0.011 & <0.039 \\ & & & & \\ - & <0.012 & <0.008 & <0.011 & <0.039 \\ - & <0.012 & <0.008 & <0.011 & <0.039 \\ & & & & \\ - & <0.012 & <0.008 & <0.011 & <0.039 \\ - & <0.012 & <0.008 & <0.011 & <0.039 \\ - & <0.012 & <0.008 & <0.011 & <0.039\end{array}$


Table 8.--Pesticide results from solid-phase extraction 1-liter method-Continued

\begin{tabular}{|c|c|c|c|c|c|c|c|c|c|c|c|c|c|}
\hline Date & Time & $\begin{array}{c}\text { DDE } \\
\text { diss } \\
(\mu g / L)\end{array}$ & $\begin{array}{l}\text { Dia- } \\
\text { zinon } \\
\text { diss } \\
(\mu \mathrm{g} / \mathrm{L})\end{array}$ & $\begin{array}{l}\text { Diol- } \\
\text { drin } \\
\text { diss } \\
(\mu g / L)\end{array}$ & $\begin{array}{c}2,6- \\
\text { Dethyi- } \\
\text { anailne } \\
\text { diss } \\
(\mu \mathrm{g} / \mathrm{L})\end{array}$ & $\begin{array}{l}\text { Dim- } \\
\text { ethoat } \\
\text { e diss } \\
(\mu g / L)\end{array}$ & $\begin{array}{c}\text { Disul- } \\
\text { foton } \\
\text { diss } \\
(\mu g / L)\end{array}$ & $\begin{array}{c}\text { EPTC } \\
\text { diss } \\
(\mu g / L)\end{array}$ & $\begin{array}{c}\text { Ethal- } \\
\text { fluralin } \\
\text { diss } \\
(\mu g / L)\end{array}$ & $\begin{array}{l}\text { Etho- } \\
\text { prop } \\
\text { diss } \\
(\mu g / L)\end{array}$ & $\begin{array}{l}\text { Fon- } \\
\text { ofos } \\
\text { diss } \\
(\mu g / L)\end{array}$ & $\begin{array}{c}\text { Lln- } \\
\text { dane } \\
\text { diss } \\
(\mu g / L)\end{array}$ & $\begin{array}{c}\text { Lin- } \\
\text { uron } \\
\text { diss } \\
(\mu g / L)\end{array}$ \\
\hline \multicolumn{14}{|c|}{07022000 - Mississippi River at Thebes, Ill.-Continued } \\
\hline \multicolumn{14}{|c|}{ JANUARY 1992} \\
\hline 24 & 0930 & $<0.010$ & $<0.008$ & $<0.008$ & $<0.006$ & $<0.024$ & $<0.008$ & $<0.005$ & $<0.013$ & $<0.012$ & $<0.008$ & $<0.011$ & $<0.039$ \\
\hline \multicolumn{14}{|c|}{ FEBRUARY } \\
\hline 04 & 1030 & 0.003 & $<0.008$ & $<0.008$ & 0.004 & $<0.024$ & $<0.008$ & $<0.005$ & $<0.013$ & $<0.012$ & $<0.008$ & $<0.011$ & $<0.039$ \\
\hline 19 & 0930 & $<0.010$ & $<0.008$ & $<0.008$ & 0.003 & $<0.024$ & $<0.008$ & $<0.005$ & $<0.013$ & $<0.012$ & $<0.008$ & $<0.011$ & $<0.039$ \\
\hline \multicolumn{14}{|l|}{ MARCH } \\
\hline 04 & 0945 & $<0.010$ & $<0.008$ & $<0.008$ & 0.002 & $<0.024$ & $<0.008$ & $<0.005$ & $<0.013$ & $<0.012$ & $<0.008$ & $<0.011$ & $<0.039$ \\
\hline 09 & 1300 & $<0.010$ & $<0.008$ & $<0.008$ & 0.002 & $<0.024$ & $<0.008$ & $<0.005$ & $<0.013$ & $<0.012$ & $<0.008$ & $<0.011$ & $<0.039$ \\
\hline 18 & 0945 & $<0.010$ & $<0.008$ & $<0.008$ & 0.002 & $<0.024$ & $<0.008$ & $<0.005$ & $<0.013$ & $<0.012$ & $<0.008$ & $<0.011$ & $<0.039$ \\
\hline 24 & 1330 & $<0.010$ & $<0.008$ & $<0.008$ & $<0.006$ & $<0.024$ & $<0.008$ & $<0.005$ & $<0.013$ & $<0.012$ & $<0.008$ & $<0.011$ & $<0.039$ \\
\hline \multicolumn{14}{|c|}{ APRIL 1992} \\
\hline 02 & 0930 & $<0.010$ & 0.010 & $<0.008$ & 0.002 & $<0.024$ & $<0.008$ & $<0.005$ & $<0.013$ & $<0.012$ & $<0.008$ & $<0.011$ & $<0.039$ \\
\hline 07 & 1330 & $<0.010$ & $<0.008$ & $<0.008$ & 0.003 & $<0.024$ & $<0.008$ & $<0.005$ & $<0.013$ & $<0.012$ & $<0.008$ & $<0.011$ & $<0.039$ \\
\hline 15 & 1030 & $<0.010$ & $<0.008$ & $<0.008$ & 0.002 & $<0.024$ & $<0.008$ & $<0.005$ & $<0.013$ & $<0.012$ & $<0.008$ & $<0.011$ & $<0.039$ \\
\hline 23 & 1330 & $<0.010$ & 0.010 & $<0.008$ & $<0.006$ & $<0.024$ & $<0.008$ & $<0.005$ & $<0.013$ & $<0.012$ & $<0.008$ & $<0.011$ & $<0.039$ \\
\hline 29 & 1100 & $<0.010$ & 0.010 & $<0.008$ & $<0.006$ & $<0.024$ & $<0.008$ & $<0.005$ & $<0.013$ & $<0.012$ & $<0.008$ & $<0.011$ & $<0.039$ \\
\hline \multicolumn{14}{|l|}{ MAY } \\
\hline 06 & 1300 & $<0.010$ & $<0.008$ & $<0.008$ & $<0.006$ & $<0.024$ & $<0.008$ & $<0.005$ & $<0.013$ & $<0.012$ & $<0.008$ & $<0.011$ & $<0.039$ \\
\hline 14 & 0930 & $<0.010$ & $<0.008$ & $<0.008$ & $<0.006$ & $<0.024$ & $<0.008$ & 0.008 & $<0.013$ & $<0.012$ & $<0.008$ & $<0.011$ & $<0.039$ \\
\hline 19 & 1300 & $<0.010$ & $<0.008$ & $<0.008$ & 0.003 & $<0.024$ & $<0.008$ & $<0.005$ & $<0.013$ & $<0.012$ & $<0.008$ & $<0.011$ & $<0.039$ \\
\hline 28 & 0900 & $<0.010$ & 0.006 & $<0.008$ & 0.001 & $<0.024$ & $<0.008$ & 0.006 & $<0.013$ & $<0.012$ & $<0.008$ & $<0.011$ & $<0.039$ \\
\hline \multicolumn{14}{|l|}{ ЛUNE } \\
\hline 02 & 1300 & $<0.010$ & 0.005 & $<0.008$ & 0.002 & $<0.024$ & $<0.008$ & 0.011 & $<0.013$ & $<0.012$ & $<0.008$ & $<0.011$ & $<0.039$ \\
\hline 09 & 0930 & $<0.010$ & 0.012 & $<0.008$ & 0.006 & $<0.024$ & $<0.008$ & 0.007 & $<0.013$ & $<0.012$ & $<0.008$ & $<0.011$ & $<0.039$ \\
\hline 16 & 1330 & $<0.010$ & $<0.008$ & $<0.008$ & 0.001 & $<0.024$ & $<0.008$ & 0.003 & $<0.013$ & $<0.012$ & $<0.008$ & $<0.011$ & $<0.039$ \\
\hline 25 & 1000 & $<0.010$ & $<0.008$ & $<0.008$ & 0.001 & $<0.024$ & $<0.008$ & 0.004 & $<0.013$ & $<0.012$ & $<0.008$ & $<0.011$ & $<0.039$ \\
\hline \multicolumn{14}{|c|}{ JULY 1992} \\
\hline 01 & 1300 & $<0.010$ & 0.004 & $<0.008$ & 0.001 & $<0.024$ & $<0.008$ & $<0.005$ & $<0.013$ & $<0.012$ & $<0.008$ & $<0.011$ & $<0.039$ \\
\hline 09 & 0915 & $<0.010$ & $<0,008$ & $<0.008$ & 0.003 & $<0.024$ & $<0.008$ & $<0.005$ & $<0.013$ & $<0.012$ & $<0.008$ & $<0.011$ & $<0.039$ \\
\hline 15 & 1230 & $<0.010$ & $<0.008$ & $<0.008$ & $<0.006$ & $<0.024$ & $<0.008$ & $<0.005$ & $<0.013$ & $<0.012$ & $<0.008$ & $<0.011$ & $<0.039$ \\
\hline 23 & 1000 & $<0.010$ & 0.008 & 0.002 & 0.003 & $<0.024$ & $<0.008$ & $<0.005$ & $<0.013$ & $<0.012$ & $<0.008$ & $<0.011$ & $<0.039$ \\
\hline 28 & 1300 & 0.003 & $<0.008$ & $<0.008$ & 0.003 & $<0.024$ & $<0.008$ & $<0.005$ & $<0.013$ & $<0.012$ & $<0.008$ & $<0.011$ & $<0.039$ \\
\hline \multicolumn{14}{|c|}{03374100 - White River at Hazleton, Ind. } \\
\hline \multicolumn{14}{|l|}{ MAY 1991} \\
\hline 01 & 1000 & $<0.010$ & $<0.008$ & $<0.008$ & -- & .. & $<0.008$ & $<0.005$ & .- & $<0.012$ & $<0.008$ & $<0.011$ & $<0.039$ \\
\hline 06 & 1115 & $<0.010$ & 0.006 & $<0.008$ & -- & -. & $<0.008$ & 0.006 & -- & $<0.012$ & $<0.008$ & $<0.011$ & $<0.039$ \\
\hline 09 & 1215 & $<0.010$ & $<0.008$ & $<0.008$ & -- & -- & $<0.008$ & 0.007 & -- & $<0.012$ & $<0.008$ & $<0.011$ & $<0.039$ \\
\hline 13 & 1300 & -- & -- & -- & .. & -- & -- & -. & -- & -- & -- & -- & -- \\
\hline 16 & 1130 & -- & -- & -- & -- & -. & -. & -- & -- & -. & -- & -- & -- \\
\hline 20 & 1045 & $<0.010$ & 0.010 & $<0.008$ & - & -. & $<0.008$ & 0.022 & -- & $<0.012$ & 0.010 & $<0.011$ & $<0.039$ \\
\hline 23 & 1100 & $<0.010$ & 0.016 & $<0.008$ & -- & .. & $<0.008$ & 0.027 & -. & $<0.012$ & 0.006 & $<0.011$ & $<0.039$ \\
\hline 28 & 1100 & $<0.010$ & 0.050 & $<0.008$ & -- & -- & $<0.008$ & $<0.005$ & -- & $<0.012$ & 0.032 & $<0.011$ & $<0.039$ \\
\hline 30 & 1200 & $<0.010$ & 0.012 & 0.008 & -- & .. & $<0.008$ & 0.010 & -. & $<0.012$ & 0.030 & $<0.011$ & $<0.039$ \\
\hline \multicolumn{14}{|l|}{ JUNE } \\
\hline 03 & 1200 & $<0.010$ & $<0.008$ & $<0.008$ & -. & -- & $<0.008$ & 0.004 & .. & $<0.012$ & 0.014 & $<0.011$ & $<0.039$ \\
\hline 06 & 1145 & $<0.010$ & 0.007 & $<0.008$ & -- & -- & $<0.008$ & $<0.005$ & -. & $<0.012$ & 0.004 & $<0.011$ & $<0.039$ \\
\hline 10 & 1200 & $<0.010$ & 0.004 & $<0.008$ & -- & $=$ & $<0.008$ & $<0.005$ & -- & $<0.012$ & $<0.008$ & $<0.011$ & $<0.039$ \\
\hline 13 & 1100 & $<0.010$ & $<0.008$ & $<0.008$ & - & .- & $<0.008$ & $<0.005$ & -- & $<0.012$ & $<0.008$ & $<0.011$ & 0.123 \\
\hline
\end{tabular}


Table 8.--Pesticide results from solid-phase extraction 1-liter method--Continued

\begin{tabular}{|c|c|c|c|c|c|c|c|c|c|c|c|c|c|}
\hline Date & Time & $\begin{array}{c}\text { DDE } \\
\text { disa } \\
(\mu g / L)\end{array}$ & $\begin{array}{c}\text { Dia- } \\
\text { zinon } \\
\text { diss } \\
(\mu g / L)\end{array}$ & $\begin{array}{l}\text { Diei- } \\
\text { drin } \\
\text { dias } \\
(\mu g / L)\end{array}$ & $\begin{array}{c}2,6- \\
\text { Dethyi- } \\
\text { anailine } \\
\text { diss } \\
(\mu g / L)\end{array}$ & $\begin{array}{l}\text { Dim- } \\
\text { ethoat } \\
\text { e diss } \\
(\mu g / L)\end{array}$ & $\begin{array}{c}\text { Diaul- } \\
\text { foton } \\
\text { diss } \\
(\mu g / L)\end{array}$ & $\begin{array}{c}\text { EPTC } \\
\text { diss } \\
(\mu g / L)\end{array}$ & $\begin{array}{l}\text { Ethai- } \\
\text { fiuraiin } \\
\text { diss } \\
(\mu g / L)\end{array}$ & $\begin{array}{l}\text { Etho- } \\
\text { prop } \\
\text { diss } \\
(\mu g / L)\end{array}$ & $\begin{array}{l}\text { Fon- } \\
\text { ofoa } \\
\text { diss } \\
(\mu g / L)\end{array}$ & $\begin{array}{l}\text { Lin- } \\
\text { dane } \\
\text { diss } \\
(\mu g / L)\end{array}$ & $\begin{array}{c}\text { Lin- } \\
\text { uron } \\
\text { disa } \\
(\mu g /))\end{array}$ \\
\hline \multicolumn{14}{|c|}{03374100 - White River at Hazleton, Ind.-Continued } \\
\hline \multicolumn{14}{|c|}{ JUNE (cont.) } \\
\hline 17 & 1200 & $<0.010$ & 0.005 & $<0.008$ & - & - & $<0.008$ & $<0.005$ & -- & $<0.012$ & $<0.008$ & $<0.011$ & $<0.039$ \\
\hline 20 & 1130 & $<0.010$ & 0.009 & $<0.008$ & -- & -- & $<0.008$ & $<0.005$ & -- & $<0.012$ & $<0.008$ & $<0.011$ & $<0.039$ \\
\hline 24 & 1215 & $<0.010$ & 0.011 & $<0.008$ & -- & -- & $<0.008$ & $<0.005$ & -. & $<0.012$ & $<0.008$ & $<0.011$ & $<0.039$ \\
\hline 27 & 1130 & $<0.010$ & 0.010 & $<0.008$ & -- & -- & $<0.008$ & $<0.005$ & - & $<0.012$ & $<0.008$ & $<0.011$ & $<0.039$ \\
\hline \multicolumn{14}{|c|}{ JULY 1991} \\
\hline 01 & 1145 & $<0.010$ & 0.009 & $<0.008$ & -- & -- & $<0.008$ & $<0.005$ & -- & $<0.012$ & $<0.008$ & $<0.011$ & $<0.039$ \\
\hline 03 & 1100 & $<0.010$ & 0.010 & $<0.008$ & -- & -- & $<0.008$ & $<0.005$ & - & $<0.012$ & $<0.008$ & $<0.011$ & $<0.039$ \\
\hline 08 & 1200 & $<0.010$ & 0.011 & $<0.008$ & -- & -- & $<0.008$ & $<0.005$ & - & $<0.012$ & $<0.008$ & $<0.011$ & $<0.039$ \\
\hline 11 & 1130 & $<0.010$ & $<0.008$ & $<0.008$ & -- & -- & $<0.008$ & $<0.005$ & -- & $<0.012$ & $<0.008$ & $<0.011$ & $<0.039$ \\
\hline 15 & 1200 & $<0.010$ & 0.007 & $<0.008$ & -- & - & $<0.008$ & $<0.005$ & -- & $<0.012$ & 0.003 & $<0.011$ & $<0.039$ \\
\hline 18 & 1300 & $<0.010$ & 0.013 & $<0.008$ & -- & -- & $<0.008$ & $<0.005$ & -- & $<0.012$ & $<0.008$ & $<0.011$ & $<0.039$ \\
\hline 22 & 1300 & $<0.010$ & 0.009 & $<0.008$ & -- & -- & $<0.008$ & $<0.005$ & -- & $<0.012$ & $<0.008$ & $<0.011$ & $<0.039$ \\
\hline 25 & 1200 & $<0.010$ & 0.007 & $<0.008$ & -- & -- & $<0.008$ & $<0.005$ & -- & $<0.012$ & $<0.008$ & $<0.011$ & $<0.039$ \\
\hline 30 & 1145 & $<0.010$ & 0.006 & $<0.008$ & -- & -- & $<0.008$ & $<0.005$ & -- & $<0.012$ & $<0.008$ & $<0.011$ & $<0.039$ \\
\hline \multicolumn{14}{|l|}{ AUGUST } \\
\hline 01 & 1145 & $<0.010$ & 0.007 & $<0.008$ & -- & -- & $<0.008$ & $<0.005$ & -- & $<0.012$ & $<0.008$ & $<0.011$ & $<0.039$ \\
\hline 06 & 1200 & $<0.010$ & $<0.008$ & $<0.008$ & -- & - & $<0.008$ & $<0.005$ & -- & $<0.012$ & $<0.008$ & $<0.011$ & $<0.039$ \\
\hline 08 & 1300 & $<0.010$ & 0.005 & $<0.008$ & -- & - & $<0.008$ & $<0.005$ & -- & $<0.012$ & $<0.008$ & $<0.011$ & $<0.039$ \\
\hline 12 & 1100 & $<0.010$ & $<0.008$ & $<0.008$ & -- & -- & $<0.008$ & $<0.005$ & -- & $<0.012$ & $<0.008$ & $<0.011$ & $<0.039$ \\
\hline 15 & 1200 & $<0.010$ & 0.010 & $<0.008$ & -- & -- & $<0.008$ & $<0.005$ & -- & $<0.012$ & $<0.008$ & $<0.011$ & $<0.039$ \\
\hline 19 & 1115 & $<0.010$ & 0.011 & $<0.008$ & -- & - & $<0.008$ & $<0.005$ & -- & $<0.012$ & $<0.008$ & $<0.011$ & $<0.039$ \\
\hline 22 & 1230 & $<0.010$ & 0.006 & $<0.008$ & -- & -. & $<0.008$ & $<0.005$ & -- & $<0.012$ & $<0.008$ & $<0.011$ & $<0.039$ \\
\hline 26 & 1245 & $<0.010$ & 0.007 & $<0.008$ & -- & -- & $<0.008$ & $<0.005$ & -- & $<0.012$ & $<0.008$ & $<0.011$ & $<0.039$ \\
\hline 29 & 1200 & $<0.010$ & 0.009 & $<0.008$ & -- & -- & $<0.008$ & $<0.005$ & -. & $<0.012$ & $<0.008$ & $<0.011$ & $<0.039$ \\
\hline \multicolumn{14}{|c|}{ SEPTEMBER 1991} \\
\hline 09 & 1100 & $<0.010$ & 0.006 & $<0.008$ & -- & -- & $<0.008$ & $<0.005$ & -. & $<0.012$ & $<0.008$ & $<0.011$ & $<0.039$ \\
\hline 12 & 1100 & $<0.010$ & 0.006 & $<0.008$ & -- & -- & $<0.008$ & $<0.005$ & -- & $<0.012$ & $<0.008$ & $<0.011$ & $<0.039$ \\
\hline 19 & 1130 & $<0.010$ & 0.007 & $<0.008$ & - & -- & $<0.008$ & $<0.005$ & -- & $<0.012$ & $<0.008$ & $<0.011$ & $<0.039$ \\
\hline 26 & 1200 & $<0.010$ & 0.019 & $<0.008$ & -- & -- & $<0.008$ & $<0.005$ & - & $<0.012$ & $<0.008$ & $<0.011$ & $<0.039$ \\
\hline \multicolumn{14}{|c|}{ OCTOBER } \\
\hline 03 & 1000 & $<0.010$ & 0.010 & $<0.008$ & -- & -- & $<0.008$ & $<0.005$ & - & $<0.012$ & $<0.008$ & $<0.011$ & $<0.039$ \\
\hline 08 & 1100 & $<0.010$ & 0.011 & $<0.008$ & -- & -- & $<0.008$ & $<0.005$ & -- & $<0.012$ & $<0.008$ & $<0.011$ & $<0.039$ \\
\hline 17 & 1100 & $<0.010$ & 0.017 & $<0.008$ & -- & -- & $<0.008$ & $<0.005$ & - & $<0.012$ & $<0.008$ & $<0.011$ & $<0.039$ \\
\hline 24 & 1100 & $<0.010$ & $<0.008$ & $<0.008$ & -- & -- & $<0.008$ & $<0.005$ & -- & $<0.012$ & $<0.008$ & $<0.011$ & $<0.039$ \\
\hline 28 & 1130 & $<0.010$ & 0.012 & $<0.008$ & - & -- & $<0.008$ & $<0.005$ & -- & $<0.012$ & $<0.008$ & $<0.011$ & $<0.039$ \\
\hline 30 & 1130 & $<0.010$ & 0.014 & $<0.008$ & - & -- & $<0.008$ & $<0.005$ & -- & $<0.012$ & $<0.008$ & $<0.011$ & $<0.039$ \\
\hline \multicolumn{14}{|c|}{ NOVEMBER } \\
\hline 04 & 1130 & $<0.010$ & $<0.008$ & $<0.008$ & - & -- & $<0.008$ & $<0.005$ & - & $<0.012$ & $<0.008$ & $<0.011$ & $<0.039$ \\
\hline 13 & 1025 & $<0.010$ & 0.030 & $<0.008$ & - & -- & $<0.008$ & $<0.005$ & -- & $<0.012$ & $<0.008$ & $<0.011$ & $<0.039$ \\
\hline 27 & 1200 & $<0.010$ & 0.024 & $<0.008$ & -- & -- & $<0.008$ & $<0.005$ & -- & $<0.012$ & $<0.008$ & $<0.011$ & $<0.039$ \\
\hline \multicolumn{14}{|c|}{ DECEMBER } \\
\hline 18 & 1200 & $<0.010$ & 0.015 & $<0.008$ & $\cdots$ & -- & $<0.008$ & $<0.005$ & - & $<0.012$ & $<0.008$ & $<0.011$ & $<0.039$ \\
\hline \multicolumn{14}{|c|}{ JANUARY 1992} \\
\hline 02 & 1200 & $<0.010$ & $<0.008$ & $<0.008$ & - & -- & $<0.008$ & $<0.005$ & -- & $<0.012$ & $<0.008$ & $<0.011$ & $<0.039$ \\
\hline 16 & 1235 & $<0.010$ & $<0.008$ & $<0.008$ & $<0.006$ & $<0.024$ & $<0.008$ & $<0.005$ & $<0.013$ & $<0.012$ & $<0.008$ & $<0.011$ & $<0.039$ \\
\hline 30 & 1243 & $<0.010$ & $<0.008$ & $<0.008$ & $<0.006$ & $<0.024$ & $<0.008$ & $<0.005$ & $<0.013$ & $<0.012$ & $<0.008$ & $<0.011$ & $<0.039$ \\
\hline
\end{tabular}


Tabie 8.--Pesticide results from solid-phase extraction 1-Ilter method--Continued

\begin{tabular}{ccccccccccccccc}
\hline Date & Time & $\begin{array}{c}\text { DDE } \\
\text { diss } \\
(\mu g / L)\end{array}$ & $\begin{array}{c}\text { Dia- } \\
\text { zinon } \\
\text { diss } \\
(\mu g / L)\end{array}$ & $\begin{array}{c}\text { Diel- } \\
\text { drin } \\
\text { diss } \\
(\mu g / L)\end{array}$ & $\begin{array}{c}\text { Dethyl- } \\
\text { analine } \\
\text { diss } \\
(\mu g / L)\end{array}$ & $\begin{array}{c}\text { Dim- } \\
\text { ethoat } \\
(\mu g / L)\end{array}$ & $\begin{array}{c}\text { Disui- } \\
\text { foton } \\
\text { diss } \\
(\mu g / L)\end{array}$ & $\begin{array}{c}\text { EPTC } \\
\text { diss } \\
(\mu g / L)\end{array}$ & $\begin{array}{c}\text { Ethar- } \\
\text { flurain } \\
\text { diss } \\
(\mu g / L)\end{array}$ & $\begin{array}{c}\text { Etho- } \\
\text { prop } \\
\text { diss } \\
(\mu g / L)\end{array}$ & $\begin{array}{c}\text { Fon- } \\
\text { ofos } \\
\text { diss } \\
(\mu g / L)\end{array}$ & $\begin{array}{c}\text { Lin- } \\
\text { dane } \\
\text { diss } \\
(\mu g / L)\end{array}$ & $\begin{array}{c}\text { Lin- } \\
\text { uron } \\
\text { diss } \\
(\mu g / L)\end{array}$ \\
\hline
\end{tabular}

FEBRUARY

$\begin{array}{cccc}13 & 1240 & <0.010 & <0.008 \\ 27 & 1200 & <0.010 & <0.008 \\ \text { MARCH } & & & \\ 05 & 1130 & <0.010 & <0.008 \\ 12 & 1115 & <0.010 & <0.008 \\ 19 & 1133 & <0.010 & <0.008 \\ 26 & 1128 & 0.003 & <0.008 \\ \text { APRIL } & & & \\ 02 & 1135 & <0.010 & <0.008 \\ 09 & 1102 & <0.010 & <0.008 \\ 16 & 1112 & <0.010 & <0.008 \\ 20 & 1030 & <0.010 & <0.008 \\ 24 & 1035 & <0.010 & <0.008 \\ 29 & 1100 & <0.010 & 0.011\end{array}$

APRIL 1991

\begin{tabular}{|c|c|c|c|c|}
\hline 10 & 1200 & - & - & - \\
\hline 18 & 1045 & -- & -- & -- \\
\hline 23 & 1110 & - & -- & -- \\
\hline \multicolumn{5}{|l|}{ MAY } \\
\hline 01 & 1040 & $<0.010$ & $<0.008$ & $<0.008$ \\
\hline 07 & 1022 & $<0.010$ & $<0.008$ & $<0.008$ \\
\hline 14 & 1045 & -- & -- & -- \\
\hline 21 & 1045 & $<0.010$ & $<0.008$ & $<0.008$ \\
\hline 29 & 1030 & $<0.010$ & $<0.008$ & $<0.008$ \\
\hline \multicolumn{5}{|l|}{ JUNE } \\
\hline 04 & 1135 & $<0.010$ & 0.006 & $<0.008$ \\
\hline 11 & 1045 & $<0.010$ & $<0.008$ & $<0.008$ \\
\hline 18 & 1040 & $<0.010$ & $<0.008$ & $<0.008$ \\
\hline 25 & 0946 & $<0.010$ & 0.006 & $<0.008$ \\
\hline \multicolumn{5}{|c|}{ JULY 1991} \\
\hline 01 & 0950 & $<0.010$ & $<0.008$ & $<0.008$ \\
\hline 09 & 1030 & $<0.010$ & $<0.008$ & $<0.008$ \\
\hline 15 & 1100 & $<0.010$ & 0.004 & $<0.008$ \\
\hline 22 & 1040 & -- & -- & - \\
\hline 29 & 1110 & -- & -- & -- \\
\hline \multicolumn{5}{|c|}{ AUGUST } \\
\hline 05 & 1050 & -- & - & -- \\
\hline 12 & 1030 & $<0.010$ & 0.005 & $<0.008$ \\
\hline 20 & 1030 & $<0.010$ & $<0.008$ & $<0.008$ \\
\hline 27 & 0945 & $<0.010$ & $<0.008$ & $<0.008$ \\
\hline \multicolumn{5}{|c|}{ SEPTEMBER } \\
\hline 03 & 1040 & $<0.010$ & $<0.008$ & $<0.008$ \\
\hline 10 & 1030 & $<0.010$ & $<0.008$ & $<0.008$ \\
\hline 17 & 0952 & 0.030 & $<0.008$ & $<0.008$ \\
\hline 23 & 1040 & $<0.010$ & 0.004 & $<0.008$ \\
\hline 30 & 1122 & $<0.010$ & $<0.008$ & $<0.008$ \\
\hline
\end{tabular}

$0.008<0.006<0.024<0.008<0$

$<0.008<0.006<0.024<0.008<0.005$

$<0.008<0.006<0.024<0.008<0.005$

$<0.008<0.006<0.024<0.008<0.005$

$<0.008<0.006<0.024<0.008<0.005$

$<0.008<0.006$

$<0.024$

$<0.008$

$<0.005$

$<0.008$

$<0.008$

$<0.008$

$<0.006$

$<0.024<0.008<0.005$

$<0.006$

$<0.024$

$<0.006$

$<0.024$

$<0.008$

$<0.005$

$<0.008<0.005$

$<0.006$

$<0.024$

$<0.008$

0.001

$<0.024$

$<0.008$

$<0.005$

$<0.024$

$<0.008$

$<0.005$

$<0.005$

03612500 - Ohio River at Dam 53 near Grand Chain, III.

\section{$<0.013$}

$<0.013$

$<0.012$

$<0.012<0.008$

$<0.011$

$<0.039$

$<0.013$

$<0.012<0.008$

$<0.011$

$<0.012<0.008<0.011$

$<0.011$

$<0.013$

$<0.012<0.008$

$<0.012<0.008$

$<0.011$

$<0.039$

$<0.039$

$<0.039$

$<0.013$

$<0.012<0.008$

$<0.011$

$<0.039$

$<0.013$

$<0.012<0.008$

$<0.011$

$<0.039$

$<0.012<0.008$

$<0.012<0.008$

011

$<0.012<0.008$

$<0.011$

$<0.012$

$<0.008$

$<0.011$

$<0.039$

$<0.039$

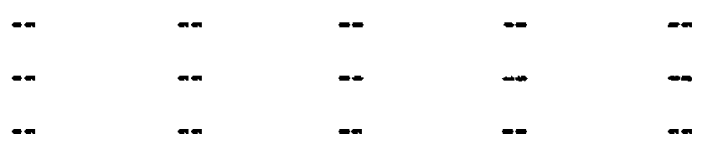

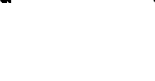

--
--
--
--
--
--
--
--
--
-
-
-
-
-
-

-
-
--
--
--
--
-
-
-

$\begin{array}{lccc}- & <0.008 & <0.005 & -- \\ -- & <0.008 & <0.005 & -- \\ -- & -- & -- & -- \\ -- & <0.008 & 0.009 & -- \\ -- & <0.008 & <0.005 & -- \\ & & & \\ -- & <0.008 & 0.015 & -- \\ -- & <0.008 & <0.005 & -- \\ -- & <0.008 & 0.003 & -- \\ -- & <0.008 & 0.003 & -- \\ & & & \\ - & <0.008 & <0.005 & - \\ -- & <0.008 & <0.005 & - \\ -- & <0.008 & <0.005 & - \\ -- & -- & -- & - \\ - & -- & -- & - \\ & & & - \\ -- & -- & -- & - \\ -- & <0.008 & <0.005 & -- \\ -- & <0.008 & <0.005 & - \\ -- & <0.008 & <0.005 & - \\ & & & - \\ - & <0.008 & <0.005 & - \\ -- & <0.008 & <0.005 & - \\ -- & <0.008 & <0.005 & - \\ -- & <0.008 & <0.005 & - \\ -- & <0.008 & <0.005 & - \\ & & & -\end{array}$

\begin{tabular}{|c|c|c|c|}
\hline$<0.012$ & $<0.008$ & $<0.011$ & $<0.039$ \\
\hline$<0.012$ & $<0.008$ & $<0.011$ & $<0.039$ \\
\hline -- & -- & -- & -- \\
\hline$<0.012$ & $<0.008$ & $<0.011$ & $<0.039$ \\
\hline$<0.012$ & $<0.008$ & $<0.011$ & $<0.039$ \\
\hline$<0.012$ & $<0.008$ & $<0.011$ & $<0.039$ \\
\hline$<0.012$ & 0.006 & $<0.011$ & $<0.039$ \\
\hline$<0.012$ & $<0.008$ & $<0.011$ & $<0.039$ \\
\hline$<0.012$ & $<0.008$ & $<0.011$ & $<0.039$ \\
\hline$<0.012$ & $<0.008$ & $<0.011$ & $<0.039$ \\
\hline$<0.012$ & $<0.008$ & $<0.011$ & $<0.039$ \\
\hline$<0.012$ & $<0.008$ & $<0.011$ & $<0.039$ \\
\hline-- & -- & -- & - \\
\hline-- & -- & -- & - \\
\hline-- & -- & -- & -- \\
\hline$<0.012$ & $<0.008$ & $<0.011$ & $<0.039$ \\
\hline$<0.012$ & $<0.008$ & $<0.011$ & $<0.039$ \\
\hline$<0.012$ & $<0.008$ & $<0.011$ & $<0.039$ \\
\hline$<0.012$ & $<0.008$ & $<0.011$ & $<0.039$ \\
\hline$<0.012$ & $<0.008$ & $<0.011$ & $<0.039$ \\
\hline$<0.012$ & $<0.008$ & $<0.011$ & $<0.039$ \\
\hline$<0.012$ & $<0.008$ & $<0.011$ & $<0.039$ \\
\hline$<0.012$ & $<0.008$ & $<0.011$ & $<0.039$ \\
\hline
\end{tabular}


Table 8.--Pesticide results from solid-phase extraction 1-liter method--Continued

\begin{tabular}{|c|c|c|c|c|c|c|c|c|c|c|c|c|c|}
\hline Date & Time & $\begin{array}{c}\text { DDE } \\
\text { diss } \\
(\mu g / L)\end{array}$ & $\begin{array}{l}\text { Dia- } \\
\text { zinon } \\
\text { diss } \\
(\mu g / L)\end{array}$ & $\begin{array}{l}\text { Diel- } \\
\text { drin } \\
\text { diss } \\
(\mu g / L)\end{array}$ & $\begin{array}{c}2,6- \\
\text { Dethyi- } \\
\text { analine } \\
\text { diss } \\
\text { ( } \mu \mathrm{g} / \text { ) }\end{array}$ & $\begin{array}{l}\text { Dim- } \\
\text { ethoat } \\
\text { e diss } \\
\text { ( } \mu g / \mathrm{L})\end{array}$ & $\begin{array}{l}\text { Disul- } \\
\text { foton } \\
\text { diss } \\
(\mu g / L)\end{array}$ & $\begin{array}{c}\text { EPTC } \\
\text { diss } \\
(\mu g / L)\end{array}$ & $\begin{array}{l}\text { Ethat- } \\
\text { fluralin } \\
\text { diss } \\
(\mu \mathrm{g} / \mathrm{L})\end{array}$ & $\begin{array}{l}\text { Etho- } \\
\text { prop } \\
\text { dlss } \\
(\mu g / L)\end{array}$ & $\begin{array}{l}\text { Fon- } \\
\text { ofos } \\
\text { diss } \\
(\mu g / L)\end{array}$ & $\begin{array}{l}\text { Lln- } \\
\text { dane } \\
\text { diss } \\
(\mu g / L)\end{array}$ & $\begin{array}{l}\text { Lin- } \\
\text { uron } \\
\text { diss } \\
(\mu g / L)\end{array}$ \\
\hline \multicolumn{14}{|c|}{03612500 - Ohio River at Dam 53 near Grand Chain, Ill.-Continned } \\
\hline \multicolumn{14}{|c|}{ OCTOBER 1991} \\
\hline 07 & 1125 & $<0.010$ & 0.004 & $<0.008$ & -- & -- & $<0.008$ & $<0.005$ & -- & $<0.012$ & $<0.008$ & $<0.011$ & $<0.039$ \\
\hline 15 & 0955 & $<0.010$ & 0.004 & $<0.008$ & - & - & $<0.008$ & $<0.005$ & -- & $<0.012$ & $<0.008$ & $<0.011$ & $<0.039$ \\
\hline 21 & 1020 & $<0.010$ & $<0.008$ & $<0.008$ & $-\cdot$ & -- & $<0.008$ & $<0.005$ & $-\cdot$ & $<0.012$ & $<0.008$ & $<0.011$ & $<0.039$ \\
\hline 29 & 0930 & $<0.010$ & $<0.008$ & $<0.008$ & -- & -. & $<0.008$ & $<0.005$ & -- & $<0.012$ & $<0.008$ & $<0.011$ & $<0.039$ \\
\hline \multicolumn{14}{|c|}{ NOVEMBER } \\
\hline 06 & 1040 & -- & -- & -- & -- & -- & -- & -- & -- & -. & -- & -- & -. \\
\hline 20 & 0920 & $<0.010$ & 0.004 & $<0.008$ & -- & -- & $<0.008$ & $<0.005$ & -- & $<0.012$ & $<0.008$ & $<0.011$ & $<0.039$ \\
\hline \multicolumn{14}{|c|}{ DECEMBER } \\
\hline 05 & 1045 & $<0.010$ & $<0.008$ & $<0.008$ & -- & -- & $<0.008$ & $<0.005$ & -- & $<0.012$ & $<0.008$ & $<0.011$ & $<0.039$ \\
\hline 17 & 1010 & $<0.010$ & $<0.008$ & $<0.008$ & -- & -- & $<0.008$ & $<0.005$ & -- & $<0.012$ & $<0.008$ & $<0.011$ & $<0.039$ \\
\hline \multicolumn{14}{|c|}{ JANUARY 1992} \\
\hline 02 & 1005 & $<0.010$ & $<0.008$ & $<0.008$ & -- & -- & $<0.008$ & $<0.005$ & - & $<0.012$ & $<0.008$ & $<0.011$ & $<0.039$ \\
\hline 21 & 1030 & $<0.010$ & $<0.008$ & $<0.008$ & 0.001 & $<0.024$ & $<0.008$ & $<0.005$ & $<0.013$ & $<0.012$ & $<0.008$ & $<0.011$ & $<0.039$ \\
\hline \multicolumn{14}{|c|}{ FEBRUARY } \\
\hline 04 & 1125 & 0.004 & $<0.008$ & $<0.008$ & $<0.006$ & $<0.024$ & $<0.008$ & $<0.005$ & $<0.013$ & $<0.012$ & 08 & 11 & $<0.039$ \\
\hline 18 & 1025 & $<0.010$ & $<0.008$ & $<0.008$ & $<0.006$ & $<0.024$ & $<0.008$ & $<0.005$ & $<0.013$ & $<0.012$ & $<0.008$ & $<0.011$ & $<0.039$ \\
\hline \multicolumn{14}{|c|}{ MARCH 1992} \\
\hline 03 & 1025 & $<0.010$ & $<0.008$ & $<0.008$ & $<0.006$ & $<0.024$ & $<0.008$ & $<0.005$ & $<0.013$ & $<0.012$ & $<0.008$ & 11 & $<0.039$ \\
\hline 11 & 1030 & $<0.010$ & $<0.008$ & $<0.008$ & $<0.006$ & $<0.024$ & $<0.008$ & $<0.005$ & $<0.013$ & $<0.012$ & $<0.008$ & $<0.011$ & $<0.039$ \\
\hline 16 & 1040 & $<0.010$ & $<0.008$ & $<0.008$ & $<0.006$ & $<0.024$ & $<0.008$ & $<0.005$ & $<0.013$ & 12 & & 11 & 0.039 \\
\hline 24 & 1020 & $<0.010$ & $<0.008$ & $<0.008$ & $<0.006$ & $<0.024$ & $<0.008$ & $<0.005$ & $<0.013$ & $<0.012$ & $<0.008$ & $<0.011$ & $<0.039$ \\
\hline 31 & 1030 & 0.003 & $<0.008$ & $<0.008$ & $<0.006$ & $<0.024$ & $<0.008$ & $<0.005$ & $<0.013$ & $<0.012$ & $<0.008$ & $<0.011$ & $<0.039$ \\
\hline \multicolumn{14}{|l|}{ APRIL } \\
\hline 07 & 1015 & $<0.010$ & $<0.008$ & $<0.008$ & $<0.006$ & $<0.024$ & $<0.008$ & $<0.005$ & $<0.013$ & $<0.012$ & $<0.008$ & $<0.011$ & $<0.039$ \\
\hline 14 & 1035 & $<0.010$ & $<0.008$ & $<0.008$ & $<0.006$ & $<0.024$ & $<0.008$ & $<0.005$ & $<0.013$ & $<0.012$ & $<0.008$ & $<0.011$ & $<0.039$ \\
\hline 22 & 1040 & $<0.010$ & $<0.008$ & $<0.008$ & $<0.006$ & $<0.024$ & $<0.008$ & $<0.005$ & $<0.013$ & $<0.012$ & $<0.008$ & $<0.011$ & $<0.039$ \\
\hline 28 & 1104 & 0.003 & 0.010 & $<0.008$ & $<0.006$ & $<0.024$ & $<0.008$ & 0.004 & $<0.013$ & $<0.012$ & $<0.008$ & $<0.011$ & $<0.039$ \\
\hline \multicolumn{14}{|l|}{ MAY } \\
\hline 06 & 0953 & 0.001 & 0.006 & $<0.008$ & $<0.006$ & $<0.024$ & $<0.008$ & $<0.005$ & $<0.013$ & $<0.012$ & $<0.008$ & $<0.011$ & $<0.039$ \\
\hline 12 & 0955 & 0.004 & 0.005 & $<0.008$ & $<0.006$ & $<0.024$ & $<0.008$ & 0.003 & $<0.013$ & $<0.012$ & $<0.008$ & $<0.011$ & $<0.039$ \\
\hline 18 & 1025 & $<0.010$ & $<0.008$ & $<0.008$ & $<0.006$ & $<0.024$ & $<0.008$ & 0.004 & $<0.013$ & $<0.012$ & $<0.008$ & $<0.011$ & $<0.039$ \\
\hline 27 & 1030 & 0.001 & 0.006 & $<0.008$ & $<0.006$ & $<0.024$ & $<0.008$ & 0.004 & $<0.013$ & $<0.012$ & $<0.008$ & $<0.011$ & $<0.039$ \\
\hline \multicolumn{14}{|c|}{ JUNE 1992} \\
\hline 02 & 1045 & $<0.010$ & 0.005 & $<0.008$ & $<0.006$ & $<0.024$ & $<0.008$ & 0.003 & $<0.013$ & $<0.012$ & 0.004 & $<0.011$ & $<0.039$ \\
\hline 09 & 1105 & $<0.010$ & 0.002 & $<0.008$ & $<0.006$ & $<0.024$ & $<0.008$ & 0.001 & $<0.013$ & $<0.012$ & $<0.008$ & $<0.011$ & $<0.039$ \\
\hline 16 & 1105 & 0.001 & 0.002 & $<0.008$ & $<0.006$ & $<0.024$ & $<0.008$ & 0.001 & $<0.013$ & $<0.012$ & $<0.008$ & $<0.011$ & $<0.039$ \\
\hline 23 & 1023 & $<0.010$ & 0.003 & $<0.008$ & $<0.006$ & $<0.024$ & $<0.008$ & $<0.005$ & $<0.013$ & $<0.012$ & 0.001 & $<0.011$ & $<0.039$ \\
\hline 29 & 1235 & $<0.010$ & 0.006 & $<0.008$ & $<0.006$ & $<0.024$ & $<0.008$ & $<0.005$ & $<0.013$ & $<0.012$ & 0.002 & $<0.011$ & $<0.039$ \\
\hline \multicolumn{14}{|l|}{ JULY } \\
\hline 08 & 1012 & 0.001 & 0.003 & $<0.008$ & $<0.006$ & $<0.024$ & $<0.008$ & $<0.005$ & $<0.013$ & $<0.012$ & 0.002 & $<0.011$ & $<0.039$ \\
\hline 14 & 1025 & 0.002 & 0.006 & $<0.008$ & $<0.006$ & $<0.024$ & $<0.008$ & $<0.005$ & $<0.013$ & $<0.012$ & 0.001 & $<0.011$ & $<0.039$ \\
\hline 23 & 1040 & $<0.010$ & 0.010 & $<0.008$ & $<0.006$ & $<0.024$ & $<0.008$ & $<0.005$ & $<0.013$ & $<0.012$ & $<0.008$ & $<0.011$ & $<0.039$ \\
\hline 28 & 1025 & 0.004 & 0.007 & $<0.008$ & 0.003 & $<0.024$ & $<0.008$ & $<0.005$ & $<0.013$ & $<0.012$ & $<0.008$ & $<0.011$ & $<0.039$ \\
\hline
\end{tabular}


Table 8.-Pesticide results from solid-phase extraction 1-liter method--Continued

\begin{tabular}{|c|c|c|c|c|c|c|c|c|c|c|c|c|c|}
\hline Date & Time & $\begin{array}{l}\text { Mala- } \\
\text { thion } \\
\text { dlss } \\
(\mu g / L)\end{array}$ & 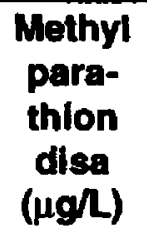 & $\begin{array}{l}\text { Meto- } \\
\text { lachlor } \\
\text { diss } \\
(\mu g / L)\end{array}$ & $\begin{array}{l}\text { Metri- } \\
\text { buzin } \\
\text { diss } \\
(\mu g / L)\end{array}$ & $\begin{array}{l}\text { Molin- } \\
\text { ate } \\
\text { diss } \\
(\mu \mathrm{g} / \mathrm{L})\end{array}$ & $\begin{array}{c}\text { Naprop- } \\
\text { amide } \\
\text { dlss } \\
(\mu g / L)\end{array}$ & $\begin{array}{l}\text { Para- } \\
\text { thlon } \\
\text { dlss } \\
(\mu g / 2)\end{array}$ & $\begin{array}{l}\text { Pebul- } \\
\text { ate } \\
\text { diss } \\
(\mu g / L)\end{array}$ & $\begin{array}{l}\text { Pendl- } \\
\text { meth- } \\
\text { alln } \\
\text { dlss } \\
\text { (ught) }\end{array}$ & $\begin{array}{l}\text { Perme- } \\
\text { thrin } \\
\text { dlss } \\
(\mu g / L)\end{array}$ & $\begin{array}{l}\text { Pho- } \\
\text { rate } \\
\text { diss } \\
(\mu g /))\end{array}$ & $\begin{array}{c}\text { Prome } \\
\text { ton } \\
\text { diss } \\
(\mu \mathrm{g} / \mathrm{L})\end{array}$ \\
\hline
\end{tabular}

APRIL 1991

$\begin{array}{rrrrrrr}02 & 1040 & -- & -- & - & -- & - \\ 11 & 1100 & -- & -- & - & - & - \\ 16 & 1150 & -- & -- & -- & -- & - \\ 23 & 1030 & -- & -- & -- & -- & -- \\ \text { MAY } & & & & & & \end{array}$

1

07

10

14

17

21

24

28

31

JUNE

04

02
11
16
23
MAY
01
07
10
14
17
21
24
28
31
JUNE
04
07
10
13
17
20
24
27

10

13

17

20

24

JULY

\section{0}

$1115<0.0$
1030

1030
1120

1045

1140

$1205<0.014<0.035$

$1210<0.014<0.035$

$1015<0.014<0.035$

$1300<0.014<0.035$

$1100<0.014<0.035$

$1130<0.014<0.035$

$1410<0.014<0.035$

$1040<0.014<0.035$

$1345<0.014<0.035$

$1055<0.014<0.035$

$0915<0.014<0.035$

$-\overline{0}$

JULY

01

05

11

15

18

22

29

AUGUST

06
12
19

\section{5}

$$
1205
$$

1130

1300

1025

1200

1050

1230

$$
124
$$

1125

1145

1205

SEPTEMBER

03

09

17

24

OCTOBER

$\begin{array}{cccccc}03 & 1200 & <0.014 & <0.035 & 0.073 & <0.012 \\ 09 & 1500 & <0.014 & <0.035 & 0.072 & <0.012 \\ 17 & 1215 & <0.014 & <0.035 & 0.072 & <0.012 \\ 24 & 1215 & <0.014 & <0.035 & 0.035 & <0.012 \\ \text { TOBER } & & & & & \\ 01 & 1200 & -- & -- & -- & -- \\ 07 & 1230 & <0.014 & <0.035 & 0.065 & <0.012 \\ 15 & 1300 & <0.014 & <0.035 & 0.065 & <0.012 \\ 22 & 1230 & <0.014 & <0.035 & 0.037 & <0.012 \\ 29 & 1330 & <0.014 & <0.035 & 0.032 & <0.012\end{array}$

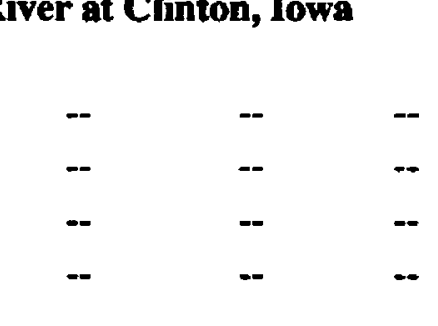


Table 8.--Pesticide results from solid-phase extraction 1-liter method--Continued

\begin{tabular}{|c|c|c|c|c|c|c|c|c|c|c|c|c|c|}
\hline Dale & Time & $\begin{array}{l}\text { Mala- } \\
\text { thion } \\
\text { djss } \\
(\mu g / L)\end{array}$ & $\begin{array}{l}\text { Mothyl } \\
\text { para- } \\
\text { thion } \\
\text { diss } \\
(\mu g / L)\end{array}$ & $\begin{array}{l}\text { Meto- } \\
\text { lachior } \\
\text { diss } \\
(\mu g /))\end{array}$ & $\begin{array}{l}\text { Metri- } \\
\text { buzin } \\
\text { diss } \\
(\mu g / L)\end{array}$ & $\begin{array}{l}\text { Molin- } \\
\text { ate } \\
\text { diss } \\
(\mu g / L)\end{array}$ & $\begin{array}{l}\text { Naprop- } \\
\text { amide } \\
\text { disa } \\
(\mu g / L)\end{array}$ & $\begin{array}{c}\text { Para- } \\
\text { thion } \\
\text { diss } \\
(\mu g / L)\end{array}$ & $\begin{array}{l}\text { Pebul- } \\
\text { ate } \\
\text { dlss } \\
(\mu \mathrm{g} / \mathrm{L})\end{array}$ & $\begin{array}{l}\text { Pendi- } \\
\text { meth- } \\
\text { allin } \\
\text { diss } \\
(\mu g / L)\end{array}$ & $\begin{array}{l}\text { Perme- } \\
\text { thrin } \\
\text { diss } \\
(\mu g / L)\end{array}$ & $\begin{array}{l}\text { Pho- } \\
\text { rate } \\
\text { diss } \\
(\mu g / L)\end{array}$ & $\begin{array}{c}\text { Prome } \\
\text { ton } \\
\text { diss } \\
(\mu g / L)\end{array}$ \\
\hline
\end{tabular}

NOVEMBER

$\begin{array}{ccccccccccccccc}05 & 1245 & <0.014 & <0.035 & 0.029 & <0.012 & -- & - & <0.022 & - & <0.018 & <0.016 & <0.011 & - \\ 12 & 1255 & <0.014 & <0.035 & 0.018 & <0.012 & -- & - & <0.022 & - & <0.018 & <0.016 & <0.011 & - \\ 26 & 1320 & <0.014 & <0.035 & 0.019 & <0.012 & -- & -- & <0.022 & -- & <0.018 & <0.016 & <0.011 & - \\ \begin{array}{c}\text { DECEMBER } \\ \quad 09\end{array} & 1200 & <0.014 & <0.035 & 0.027 & <0.012 & - & - & - & <0.022 & - & <0.018 & <0.016 & <0.011 & - \\ \begin{array}{c}22 \\ 22\end{array} & 1330 & <0.014 & <0.035 & 0.024 & 0.006 & -- & -- & <0.022 & -- & <0.018 & <0.016 & <0.011 & - \\ \begin{array}{c}\text { JANUARY 1992 } \\ 07\end{array} & 1210 & <0.014 & <0.035 & 0.012 & <0.012 & <0.007 & <0.010 & <0.022 & <0.009 & <0.018 & <0.016 & <0.011 & <0.008 \\ 22 & 1340 & <0.014 & <0.035 & 0.016 & <0.012 & <0.007 & <0.010 & <0.022 & <0.009 & <0.018 & <0.016 & <0.011 & <0.008 \\ \begin{array}{c}\text { FEBRUARY } \\ \text { 03 }\end{array} & 1245 & <0.014 & <0.035 & 0.010 & <0.012 & <0.007 & <0.010 & <0.022 & <0.009 & <0.018 & <0.016 & <0.011 & <0.008 \\ 20 & 1230 & <0.014 & <0.035 & 0.024 & <0.012 & <0.007 & <0.010 & <0.022 & <0.009 & <0.018 & <0.016 & <0.011 & 0.005\end{array}$

MARCH

04

13

17

24

$\begin{array}{rrrrr}30 & 1155 & <0.014 & <0.035 \\ \text { APRIL } & & & \\ 06 & 1215 & <0.014 & <0.035 & 0.02 \\ 14 & 1130 & <0.014 & <0.035 & 0.02 \\ 24 & 1145 & <0.014 & <0.035 & 0.05 \\ 28 & 1145 & <0.014 & <0.035 & 0.02 \\ \text { MAY } & & & & \\ 05 & 1115 & <0.014 & <0.035 \\ 12 & 1130 & <0.014 & <0.035 & 0.02 \\ 19 & 1130 & <0.014 & <0.035 & 0.04 \\ 26 & 1215 & <0.014 & <0.035 & 0.05\end{array}$

APRIL

and

APRIL 1991

$\begin{array}{cccc}\text { APRIL } 1991 & & & - \\ 26 & 1215 & - & - \\ 05 & 1230 & -- & - \\ 17 & 1330 & -- & - \\ 26 & 1000 & -- & -\end{array}$

$03 \quad 1545<0.014<0.035$

08

10

17

19

22

29

31

JUNE 1991

$\begin{array}{llllll}04 & 1215 & <0.014 & <0.035 & 2.70 & 0.057 \\ 06 & 1200 & <0.014 & <0.035 & 1.47 & 0.025 \\ 11 & 1345 & <0.014 & <0.035 & 1.91 & 0.035\end{array}$

$\begin{array}{llllllllllllll}1100 & -\overline{-} & -- & -- & -- & -- & -- & -- & -- & -- & - & -- & - \\ 1200 & <0.014 & <0.035 & 0.028 & <0.012 & <0.007 & <0.010 & <0.022 & <0.009 & <0.018 & <0.016 & <0.011 & <0.008 \\ 1400 & <0.014 & <0.035 & 0.024 & <0.012 & <0.007 & <0.010 & <0.022 & <0.009 & <0.018 & <0.016 & <0.011 & <0.008 \\ 1125 & <0.014 & <0.035 & 0.040 & <0.012 & <0.007 & <0.010 & <0.022 & <0.009 & <0.018 & <0.016 & <0.011 & <0.008 \\ 1155 & <0.014 & <0.035 & 0.031 & <0.012 & <0.007 & <0.010 & <0.022 & <0.009 & <0.018 & <0.016 & <0.011 & 0.007 \\ & & & & & & & & & & & & & \\ 1215 & <0.014 & <0.035 & 0.025 & <0.012 & <0.007 & <0.010 & <0.022 & <0.009 & <0.018 & <0.016 & <0.011 & 0.007 \\ 1130 & <0.014 & <0.035 & 0.023 & <0.012 & <0.007 & <0.010 & <0.022 & <0.009 & <0.018 & <0.016 & <0.011 & <0.008 \\ 1145 & <0.014 & <0.035 & 0.091 & <0.012 & <0.007 & <0.010 & <0.022 & <0.009 & <0.018 & <0.016 & <0.011 & <0.008 \\ 1145 & <0.014 & <0.035 & 0.025 & <0.012 & <0.007 & <0.010 & <0.022 & <0.009 & <0.018 & <0.016 & <0.011 & <0.008 \\ & & & & & & & & & & & & \\ 1115 & <0.014 & <0.035 & 0.021 & <0.012 & <0.007 & <0.010 & <0.022 & <0.009 & <0.018 & <0.016 & <0.011 & <0.008 \\ 1130 & <0.014 & <0.035 & 0.032 & <0.012 & <0.007 & <0.010 & <0.022 & <0.009 & <0.018 & <0.016 & <0.011 & <0.008 \\ 1130 & <0.014 & <0.035 & 0.043 & <0.012 & <0.007 & <0.010 & <0.022 & <0.009 & <0.018 & <0.016 & <0.011 & <0.008 \\ 1215 & <0.014 & <0.035 & 0.059 & 0.006 & <0.007 & <0.010 & <0.022 & <0.009 & <0.018 & <0.016 & <0.011 & 0.006\end{array}$


Table 8.--Pesticide results from solid-phase extraction 1-liter method-Continued

\begin{tabular}{|c|c|c|c|c|c|c|c|c|c|c|c|c|c|}
\hline Date & Tima & $\begin{array}{c}\text { Mala- } \\
\text { thion } \\
\text { diss } \\
(\mu g / L)\end{array}$ & $\begin{array}{l}\text { Methyl } \\
\text { para- } \\
\text { thion } \\
\text { diss } \\
(\mu \mathrm{g} / L)\end{array}$ & $\begin{array}{l}\text { Mato- } \\
\text { lachior } \\
\text { diss } \\
(\mu g / L)\end{array}$ & $\begin{array}{c}\text { Metri- } \\
\text { buzin } \\
\text { diss } \\
(\mu g / L)\end{array}$ & $\begin{array}{c}\text { Molin- } \\
\text { ate } \\
\text { diss } \\
(\mu g / L)\end{array}$ & $\begin{array}{c}\text { Naprop- } \\
\text { amide } \\
\text { diss } \\
(\mu g / L)\end{array}$ & $\begin{array}{l}\text { Para- } \\
\text { thlon } \\
\text { diss } \\
(\mu g / L)\end{array}$ & $\begin{array}{c}\text { Pebul- } \\
\text { ato } \\
\text { diss } \\
(\mu g / L)\end{array}$ & $\begin{array}{c}\text { Pendi- } \\
\text { meth- } \\
\text { alin } \\
\text { diss } \\
(\mu g / L)\end{array}$ & $\begin{array}{c}\text { Parme- } \\
\text { thrin } \\
\text { diss } \\
(\mu g / L)\end{array}$ & $\begin{array}{l}\text { Pho- } \\
\text { rate } \\
\text { diss } \\
(\mu g / L)\end{array}$ & $\begin{array}{c}\text { Prome- } \\
\text { ton } \\
\text { diss } \\
(\mu g / L)\end{array}$ \\
\hline \multicolumn{14}{|c|}{05586100 - Illinois River at Valley City-Continued } \\
\hline \multicolumn{14}{|c|}{ JUNE 1991 (cont.) } \\
\hline 14 & 1200 & $<0.014$ & $<0.035$ & 1.53 & 0.035 & -- & -- & $<0.022$ & -. & $<0.018$ & $<0.016$ & $<0.011$ & -- \\
\hline 18 & 1130 & $<0.014$ & $<0.035$ & 1.21 & 0.027 & -- & -- & $<0.022$ & -- & $<0.018$ & $<0.016$ & $<0.011$ & -- \\
\hline 20 & 1130 & $<0.014$ & $<0.035$ & 1.01 & 0.036 & -- & -- & $<0.022$ & -. & $<0.018$ & $<0.016$ & $<0.011$ & -- \\
\hline 24 & 1130 & $<0.014$ & $<0.035$ & 0.777 & 0.011 & -- & - & $<0.022$ & -- & $<0.018$ & $<0.016$ & $<0.011$ & - \\
\hline 27 & 1100 & $<0.014$ & $<0.035$ & 0.586 & 0.016 & -- & -- & $<0.022$ & -- & $<0.018$ & $<0.016$ & $<0.011$ & -- \\
\hline \multicolumn{14}{|l|}{ JULY } \\
\hline 01 & 0930 & $<0.014$ & $<0.035$ & 0.488 & 0.007 & -- & -- & $<0.022$ & -- & $<0.018$ & $<0.016$ & $<0.011$ & - \\
\hline 03 & 1100 & $<0.014$ & $<0.035$ & 0.350 & 0.008 & - & -- & $<0.022$ & - & $<0.018$ & $<0.016$ & $<0.011$ & -- \\
\hline 09 & 1000 & $<0.014$ & $<0.035$ & 0.216 & $<0.012$ & -- & - & $<0.022$ & -- & $<0.018$ & $<0.016$ & $<0.011$ & - \\
\hline 11 & 1045 & $<0.014$ & $<0.035$ & 0.168 & $<0.012$ & -- & -- & $<0.022$ & -- & $<0.018$ & $<0.016$ & $<0.011$ & -- \\
\hline 18 & 1115 & -- & -- & -- & -- & -- & -- & -- & -- & -- & -- & -- & -- \\
\hline 25 & 1340 & $<0.014$ & $<0.035$ & 0.079 & $<0.012$ & -- & -- & $<0.022$ & -- & $<0.018$ & $<0.016$ & $<0.011$ & -- \\
\hline 31 & 1100 & $<0.014$ & $<0.035$ & 0.068 & $<0.012$ & -- & -- & $<0.022$ & -- & $<0.018$ & $<0.016$ & $<0.011$ & - \\
\hline \multicolumn{14}{|c|}{ AUGUST 1991} \\
\hline 06 & 1015 & $<0.014$ & $<0.035$ & 0.087 & $<0.012$ & -- & -- & $<0.022$ & -- & $<0.018$ & $<0.016$ & $<0.011$ & -- \\
\hline 13 & 1200 & $<0.014$ & $<0.035$ & 0.091 & 0.005 & -- & -- & $<0.022$ & -- & $<0.018$ & $<0.016$ & $<0.011$ & -- \\
\hline 21 & 1230 & $<0.014$ & $<0.035$ & 0.037 & $<0.012$ & -. & -- & $<0.022$ & -- & $<0.018$ & $<0.016$ & $<0.011$ & -- \\
\hline 28 & 1015 & $<0.014$ & $<0.035$ & 0.024 & $<0.012$ & -- & -- & $<0.022$ & -- & $<0.018$ & $<0.016$ & $<0.011$ & -- \\
\hline \multicolumn{14}{|c|}{ SEPTEMBER } \\
\hline 03 & 1050 & $<0.014$ & $<0.035$ & 0.027 & $<0.012$ & -- & -- & $<0.022$ & -- & $<0.018$ & $<0.016$ & $<0.011$ & -- \\
\hline 10 & 1200 & $<0.014$ & $<0.035$ & 0.057 & $<0.012$ & -- & -- & $<0.022$ & -- & $<0.018$ & $<0.016$ & $<0.011$ & -- \\
\hline 17 & 1100 & $<0.014$ & $<0.035$ & 0.054 & $<0.012$ & -- & -- & $<0.022$ & -- & $<0.018$ & $<0.016$ & $<0.011$ & -. \\
\hline 24 & 1100 & $<0.014$ & $<0.035$ & 0.033 & $<0.012$ & -- & -- & $<0.022$ & -- & $<0.018$ & $<0.016$ & $<0.011$ & -- \\
\hline \multicolumn{14}{|l|}{ OCTOBER } \\
\hline 01 & 1130 & $<0.014$ & $<0.035$ & 0.041 & $<0.012$ & -- & -- & $<0.022$ & -- & $<0.018$ & $<0.016$ & $<0.011$ & -- \\
\hline 08 & 1330 & $<0.014$ & $<0.035$ & 0.409 & $<0.012$ & -- & -- & $<0.022$ & -- & $<0.018$ & $<0.016$ & $<0.011$ & -- \\
\hline 15 & 1215 & $<0.014$ & $<0.035$ & 0.069 & $<0.012$ & -- & -- & $<0.022$ & -- & $<0.018$ & $<0.016$ & $<0.011$ & -- \\
\hline 22 & 1130 & $<0.014$ & $<0.035$ & 0.040 & 0.005 & - & -- & $<0.022$ & -- & $<0.018$ & $<0.016$ & $<0.011$ & -- \\
\hline \multicolumn{14}{|c|}{ NOVEMBER } \\
\hline 05 & 1140 & $<0.014$ & $<0.035$ & 0.148 & $<0.012$ & -- & -- & $<0.022$ & -- & $<0.018$ & $<0.016$ & $<0.011$ & -- \\
\hline 15 & 1130 & $<0.014$ & $<0.035$ & 0.091 & $<0.012$ & - & -- & $<0.022$ & -- & $<0.018$ & $<0.016$ & $<0.011$ & -- \\
\hline \multicolumn{14}{|c|}{ DECEMBER 1991} \\
\hline 03 & 1030 & $<0.014$ & $<0.035$ & 0.205 & $<0.012$ & -- & -- & $<0.022$ & - & $<0.018$ & $<0.016$ & $<0.011$ & -- \\
\hline 18 & 1200 & $<0.014$ & $<0.035$ & 0.166 & 0.012 & -- & -- & $<0.022$ & -- & $<0.018$ & $<0.016$ & $<0.011$ & -- \\
\hline \multicolumn{14}{|c|}{ JANUARY 1992} \\
\hline 02 & 1330 & $<0.014$ & $<0.035$ & 0.109 & $<0.012$ & -- & -- & $<0.022$ & -- & $<0.018$ & $<0.016$ & $<0.011$ & -- \\
\hline 15 & 1645 & $<0.014$ & $<0.035$ & 0.110 & $<0.012$ & $<0.007$ & $<0.010$ & $<0.022$ & $<0.009$ & $<0.018$ & $<0.016$ & $<0.011$ & 0.020 \\
\hline 30 & 1345 & $<0.014$ & $<0.035$ & 0.130 & $<0.012$ & $<0.007$ & $<0.010$ & $<0.022$ & $<0.009$ & $<0.018$ & $<0.016$ & $<0.011$ & 0.019 \\
\hline \multicolumn{14}{|c|}{ FEBRUARY } \\
\hline 12 & 1200 & $<0.014$ & $<0.035$ & 0.100 & $<0.012$ & $<0.007$ & $<0.010$ & $<0.022$ & $<0.009$ & $<0.018$ & $<0.016$ & $<0.011$ & 0.019 \\
\hline 28 & 1245 & $<0.014$ & $<0.035$ & 0.140 & $<0.012$ & $<0.007$ & $<0.010$ & $<0.022$ & $<0.009$ & $<0.018$ & $<0.016$ & $<0.011$ & 0.027 \\
\hline \multicolumn{14}{|l|}{ MARCH } \\
\hline 03 & 1100 & $<0.014$ & $<0.035$ & 0.100 & $<0.012$ & $<0.007$ & $<0.010$ & $<0.022$ & $<0.009$ & $<0.018$ & $<0.016$ & $<0.011$ & 0.025 \\
\hline 10 & 1015 & $<0.014$ & $<0.035$ & 0.100 & $<0.012$ & $<0.007$ & $<0.010$ & $<0.022$ & $<0.009$ & $<0.018$ & $<0.016$ & $<0.011$ & 0.025 \\
\hline 17 & 1050 & $<0.014$ & $<0.035$ & 0.140 & $<0.012$ & $<0.007$ & $<0.010$ & $<0.022$ & $<0.009$ & $<0.018$ & $<0.016$ & $<0.011$ & 0.029 \\
\hline 25 & 1045 & $<0.014$ & .035 & 150 & .012 & 77 & 010 & $<0.022$ & 9 & 0.018 & $<0.016$ & $<0.011$ & 2 \\
\hline
\end{tabular}


Table 8.--Pesticide results from soiid-phase extraction 1-liter method--Continued

\begin{tabular}{|c|c|c|c|c|c|c|c|c|c|c|c|c|c|}
\hline Date & Time & $\begin{array}{l}\text { Mala- } \\
\text { thion } \\
\text { diss } \\
(\mu g / L)\end{array}$ & $\begin{array}{l}\text { Methyl } \\
\text { para- } \\
\text { thion } \\
\text { dlss } \\
\text { ( } \mathrm{gg} / \mathrm{L})\end{array}$ & $\begin{array}{l}\text { Meto- } \\
\text { lachior } \\
\text { diss } \\
(\mu g / L)\end{array}$ & $\begin{array}{c}\text { Metrl- } \\
\text { buzin } \\
\text { diss } \\
(\mu \mathrm{g} / \mathrm{L})\end{array}$ & $\begin{array}{l}\text { Molin- } \\
\text { ate } \\
\text { diss } \\
(\mu g / L)\end{array}$ & $\begin{array}{l}\text { Naprop- } \\
\text { amide } \\
\text { diss } \\
\text { ( } \mu \mathrm{g} / \mathrm{L})\end{array}$ & $\begin{array}{l}\text { Para- } \\
\text { thion } \\
\text { diss } \\
(\mu \mathrm{g} / \mathrm{L})\end{array}$ & $\begin{array}{l}\text { Pebul- } \\
\text { ate } \\
\text { diss } \\
(\mu g /)\end{array}$ & $\begin{array}{l}\text { Pendi- } \\
\text { meth- } \\
\text { alin } \\
\text { diss } \\
(\mu g / L)\end{array}$ & $\begin{array}{l}\text { Perme- } \\
\text { thrin } \\
\text { diss } \\
\text { ( } \mu \mathrm{g} / \mathrm{L} \text { ) }\end{array}$ & $\begin{array}{l}\text { Pho- } \\
\text { rate } \\
\text { diss } \\
(\mu g / L)\end{array}$ & $\begin{array}{c}\text { Prome- } \\
\text { ton } \\
\text { diss } \\
(\mu \mathrm{g} / \mathrm{L})\end{array}$ \\
\hline
\end{tabular}

APRIL 1991

\begin{tabular}{|c|c|c|c|c|c|c|c|c|c|c|c|c|c|}
\hline 09 & 1250 & -- & -- & -- & - & -- & -- & - & - & -- & - & - & - \\
\hline 16 & 1030 &.- & $\cdots$ & -- & -- & -- & -- & -- & -- & -- & -- & - & - \\
\hline 24 & 1030 & -- & - & -- & -- & -- & -- & -- & -- & -- & -- & - & - \\
\hline 29 & 1000 & $<0.014$ & $<0.035$ & 0.216 & $<0.012$ & $\cdots$ & -- & $<0.022$ & -- & $<0.018$ & 0.001 & $<0.011$ & -- \\
\hline \multicolumn{14}{|l|}{ MAY } \\
\hline 06 & 1115 & $<0.014$ & $<0.035$ & 0.562 & $<0.012$ & - & -- & $<0.022$ & -- & 0.004 & $<0.016$ & $<0.011$ & -- \\
\hline 09 & 0940 & $<0.014$ & $<0.035$ & 0.313 & $<0.012$ & -- & -- & $<0.022$ & -- & $<0.018$ & $<0.016$ & $<0.011$ & - \\
\hline 13 & 1110 & - & -- & -- & -- & -- & -- & -- & -- & -- & -- & -- & -- \\
\hline 16 & 1130 & -- & -- & -. & - & -- & $\cdots$ & $\cdots$ & $\cdots$ & -- & $\cdots$ & -- & -- \\
\hline 21 & 0945 & $<0.014$ & $<0.035$ & 5.17 & $<0.012$ & -- & -- & $<0.022$ & -- & $<0.018$ & $<0.016$ & $<0.011$ & -- \\
\hline 24 & 0740 & $<0.014$ & $<0.035$ & 0.902 & $<0.012$ & -- & -- & $<0.022$ & -- & $<0.018$ & $<0.016$ & $<0.011$ & - \\
\hline 29 & 1015 & $<0.014$ & $<0.035$ & 1.35 & 0.008 & $\cdots$ & $\cdots$ & $<0.022$ & - & $<0.018$ & $<0.016$ & $<0.011$ & - \\
\hline 31 & 0735 & $<0.014$ & $<0.035$ & 3.40 & 0.062 & -- & -- & $<0.022$ & -- & $<0.018$ & $<0.016$ & $<0.011$ & -- \\
\hline \multicolumn{14}{|c|}{ JUNE 1991} \\
\hline 04 & 0800 & $<0.014$ & $<0.035$ & 2.61 & 0.045 & -- & -- & $<0.022$ & -- & $<0.018$ & $<0.016$ & $<0.011$ & -. \\
\hline 07 & 1415 & $<0.014$ & $<0.035$ & 2.42 & 0.144 & -- & -- & $<0.022$ & -- & $<0.018$ & $<0.016$ & $<0.011$ & - \\
\hline 11 & 1045 & $<0.014$ & $<0.035$ & 1.81 & 0.126 & -- & -- & $<0.022$ & -- & $<0.018$ & $<0.016$ & $<0.011$ & -- \\
\hline 14 & 0750 & $<0.014$ & $<0.035$ & 1.52 & 0.268 & -- & -- & $<0.022$ & -- & $<0.018$ & $<0.016$ & $<0.011$ & - \\
\hline 18 & 0935 & $<0.014$ & $<0.035$ & 1.04 & 0.049 & -- & -- & $<0.022$ & -- & 0.017 & $<0.016$ & $<0.011$ & - \\
\hline 21 & 1020 & $<0.014$ & $<0.035$ & 0.460 & 0.020 & -- & -- & $<0.022$ & -- & 0.004 & $<0.016$ & $<0.011$ & -- \\
\hline 24 & 1110 & $<0.014$ & $<0.035$ & 0.474 & 0.028 & -- & - & $<0.022$ & - & 0.010 & $<0.016$ & $<0.011$ & -- \\
\hline 27 & 1020 & $<0.014$ & $<0.035$ & 0.249 & 0.013 & - & -- & $<0.022$ & -- & $<0.018$ & $<0.016$ & $<0,011$ & -- \\
\hline \multicolumn{14}{|l|}{ JULY } \\
\hline 01 & 0950 & $<0.014$ & $<0.035$ & 0.125 & 0.008 & -- &.- & $<0.022$ & -- & $<0.018$ & $<0.016$ & $<0.011$ & -. \\
\hline 03 & 0950 & $<0.014$ & $<0.035$ & 0.308 & 0.009 & -- & -- & $<0.022$ & -- & 0.014 & $<0.016$ & $<0.011$ & - \\
\hline 08 & 1100 & $<0.014$ & $<0.035$ & 0.157 & 0.005 & -- & -- & $<0.022$ & - & $<0.018$ & $<0.016$ & $<0.011$ & - \\
\hline 11 & 1020 & $<0.014$ & $<0.035$ & 0.307 & $<0.012$ & -- & -- & $<0.022$ & -- & $<0.018$ & $<0.016$ & $<0.011$ & -- \\
\hline 15 & 0950 & $<0.014$ & $<0.035$ & 0.297 & $<0.012$ & -- & -- & $<0.022$ & -- & $<0.018$ & $<0.016$ & $<0.011$ & -- \\
\hline 25 & 1000 & $<0.014$ & $<0.035$ & 0.104 & $<0.012$ & - & -- & $<0.022$ & -- & $<0.018$ & $<0.016$ & $<0.011$ & - \\
\hline 29 & 0940 & $<0.014$ & $<0.035$ & 0.064 & $<0.012$ & -- & -- & $<0.022$ & -- & $<0.018$ & $<0.016$ & $<0.011$ & -- \\
\hline \multicolumn{14}{|c|}{ AUGUST 1991} \\
\hline 06 & 1130 & $<0.014$ & $<0.035$ & 0.049 & $<0.012$ &.- & -- & $<0.022$ & -- & $<0.018$ & $<0.016$ & $<0.011$ & -- \\
\hline 12 & 1115 & $<0.014$ & $<0.035$ & 0.053 & $<0.012$ & -- & -- & $<0.022$ & - & $<0.018$ & $<0.016$ & $<0.011$ & -- \\
\hline 20 & 1000 & $<0.014$ & $<0.035$ & 0.059 & $<0.012$ & -- & -- & $<0.022$ & -- & $<0.018$ & $<0.016$ & $<0.011$ & -- \\
\hline 27 & 0940 & $<0.014$ & $<0.035$ & 0.025 & $<0.012$ & - & - & $<0.022$ & -- & $<0.018$ & $<0.016$ & $<0.011$ & -- \\
\hline \multicolumn{14}{|c|}{ SEPTEMBER } \\
\hline 03 & 0950 & $<0.014$ & $<0.035$ & 0.020 & $<0.012$ & -- & -- & $<0.022$ & -- & $<0.018$ & $<0.016$ & $<0.011$ & -- \\
\hline 10 & 1030 & $<0.014$ & $<0.035$ & 0.020 & $<0.012$ & -- & -- & $<0.022$ & -- & $<0.018$ & $<0.016$ & $<0.011$ & -- \\
\hline 17 & 1115 & $<0.014$ & $<0.035$ & 0.070 & $<0.012$ & - & -- & $<0.022$ & -- & $<0.018$ & $<0.016$ & $<0.011$ & -- \\
\hline 24 & 1135 & $<0.014$ & $<0.035$ & 0.018 & $<0.012$ & -- & -- & $<0.022$ & -- & $<0.018$ & $<0.016$ & $<0.011$ & -- \\
\hline \multicolumn{14}{|c|}{ OCTOBER } \\
\hline 01 & 1115 & $<0.014$ & $<0.035$ & 0.016 & $<0.012$ & -- & -- & $<0.022$ & -- & $<0.018$ & $<0.016$ & $<0.011$ & -- \\
\hline 08 & 1030 & $<0.014$ & $<0.035$ & 0.006 & $<0.012$ & -- & -- & $<0.022$ & -- & $<0.018$ & $<0.016$ & $<0.011$ & - \\
\hline 15 & 1130 & $<0.014$ & $<0.035$ & 0.012 & $<0.012$ & -- & -- & $<0.022$ & -- & $<0.018$ & $<0.016$ & $<0.011$ & -- \\
\hline 24 & 1200 & $<0.014$ & $<0.035$ & 0.008 & $<0.012$ & $-\cdot$ & - & $<0.022$ & -- & $<0.018$ & $<0.016$ & $<0.011$ & -- \\
\hline \multicolumn{14}{|c|}{ NOVEMBER } \\
\hline 05 & 1200 & $<0.014$ & $<0.035$ & 0.013 & $<0.012$ & -- & -- & $<0.022$ & -- & $<0.018$ & $<0.016$ & $<0.011$ & -- \\
\hline 19 & 1140 & $<0.014$ & $<0.035$ & 0.014 & $<0.012$ & -- & - & $<0.022$ & - & $<0.018$ & $<0.016$ & $<0.011$ & - \\
\hline
\end{tabular}


Table 8.-Pesticide results from solid-phase extraction 1-liter method-Continued

\begin{tabular}{|c|c|c|c|c|c|c|c|c|c|c|c|c|c|}
\hline Date & Time & $\begin{array}{c}\text { Mala- } \\
\text { thion } \\
\text { diss } \\
(\mu g / L)\end{array}$ & $\begin{array}{l}\text { Methyl } \\
\text { para- } \\
\text { thlon } \\
\text { diss } \\
(\mu g / L)\end{array}$ & $\begin{array}{l}\text { Meto- } \\
\text { lachior } \\
\text { diss } \\
(\mu g /))\end{array}$ & $\begin{array}{c}\text { Metri- } \\
\text { buzin } \\
\text { diss } \\
(\mu g / L)\end{array}$ & $\begin{array}{c}\text { Molin- } \\
\text { ate } \\
\text { dlss } \\
(\mu \mathrm{g} / L)\end{array}$ & $\begin{array}{l}\text { Naprop- } \\
\text { amide } \\
\text { diss } \\
(\mu g / L)\end{array}$ & $\begin{array}{l}\text { Para- } \\
\text { thlon } \\
\text { dls8 } \\
(\mu \mathrm{g} / L)\end{array}$ & $\begin{array}{l}\text { Pebul- } \\
\text { ate } \\
\text { diss } \\
(\mu g / L)\end{array}$ & $\begin{array}{l}\text { Pendi- } \\
\text { meth- } \\
\text { alin } \\
\text { diss } \\
(\mu g / L)\end{array}$ & $\begin{array}{l}\text { Permo- } \\
\text { thrin } \\
\text { diss } \\
(\mu g / L)\end{array}$ & $\begin{array}{l}\text { Pho- } \\
\text { rate } \\
\text { diss } \\
(\mu g /))\end{array}$ & $\begin{array}{c}\text { Prome- } \\
\text { Ion } \\
\text { diss } \\
(\mu \mathrm{g} / \mathrm{L})\end{array}$ \\
\hline
\end{tabular}

DECEMBER 1991

$\begin{array}{ccccccccccccccc}05 & 1130 & -- & -- & -- & -- & -- & - & - & - & - & -- & - & - \\ 20 & 1000 & <0.014 & <0.035 & 0.007 & <0.012 & <0.007 & <0.010 & <0.022 & <0.009 & <0.018 & <0.016 & <0.011 & 0.009 \\ 30 & 1020 & <0.014 & <0.035 & 0.025 & <0.012 & <0.007 & <0.010 & <0.022 & <0.009 & <0.018 & <0.016 & <0.011 & 0.011 \\ \text { JANUARY 1992 } & & & & & & & & & & & & & \\ 14 & 1000 & <0.014 & <0.035 & 0.014 & <0.012 & <0.007 & <0.010 & <0.022 & <0.009 & <0.018 & <0.016 & <0.011 & 0.008 \\ 28 & 1000 & <0.014 & <0.035 & 0.007 & <0.012 & <0.007 & <0.010 & <0.022 & <0.009 & <0.018 & <0.016 & <0.011 & 0.008 \\ \text { FEBRUARY } & & & & & & & & & & & & & \\ 11 & 1030 & <0.014 & <0.035 & 0.005 & <0.012 & <0.007 & <0.010 & <0.022 & <0.009 & <0.018 & <0.016 & <0.011 & 0.008 \\ 27 & 1000 & <0.014 & <0.035 & 0.009 & <0.012 & <0.007 & <0.010 & <0.022 & <0.009 & <0.018 & <0.016 & <0.011 & <0.008 \\ \text { MARCH } & & & & & & & & & & & & & \\ \text { 05 } & 1010 & <0.014 & <0.035 & 0.012 & <0.012 & <0.007 & <0.010 & <0.022 & <0.009 & <0.018 & <0.016 & <0.011 & 0.010 \\ 09 & 0945 & <0.014 & <0.035 & 0.014 & <0.012 & <0.007 & <0.010 & <0.022 & <0.009 & <0.018 & <0.016 & <0.011 & <0.008 \\ 17 & 1145 & <0.014 & <0.035 & 0.024 & <0.012 & <0.007 & <0.010 & <0.022 & <0.009 & <0.018 & <0.016 & <0.011 & 0.012 \\ 23 & 1040 & <0.014 & <0.035 & 0.013 & <0.012 & <0.007 & <0.010 & <0.022 & <0.009 & <0.018 & <0.016 & <0.011 & 0.012 \\ 31 & 1100 & <0.014 & <0.035 & 0.018 & <0.012 & <0.007 & <0.010 & <0.022 & <0.009 & <0.018 & <0.016 & <0.011 & <0.008 \\ \text { APRIL 1992 } & & & & & & & & & & & & & \\ 07 & 1100 & <0.014 & <0.035 & 0.019 & <0.012 & <0.007 & <0.010 & <0.022 & <0.009 & <0.018 & <0.016 & <0.011 & 0.012 \\ 14 & 1010 & <0.014 & <0.035 & 0.060 & <0.012 & <0.007 & <0.010 & <0.022 & <0.009 & <0.018 & <0.016 & <0.011 & <0.008 \\ 23 & 1020 & <0.014 & <0.035 & 0.050 & <0.012 & <0.007 & <0.010 & <0.022 & <0.009 & <0.018 & <0.016 & <0.011 & 0.015 \\ 29 & 1030 & <0.014 & <0.035 & 0.048 & <0.012 & <0.007 & <0.010 & <0.022 & <0.009 & <0.018 & <0.016 & <0.011 & 0.012 \\ \text { MAY } & & & & & & & & & & & & & & \end{array}$

APRIL 1991

\begin{tabular}{|c|c|c|c|c|c|c|c|c|c|c|c|c|c|}
\hline 09 & 1331 & .. & .. & -- & -- & -. & .- & -- & -- & .. & -- & -- & -. \\
\hline 16 & 1030 & -- & - & -- & -- & -. & -. & -- & .- & -- & -- & -- & -- \\
\hline 24 & 1200 & -- & -. & -- & - & -- & -- & -- & -- & -. & -. & -- & -- \\
\hline \multicolumn{14}{|l|}{ MAY } \\
\hline 01 & 1130 & -- & -- & .. & -- & -- & -- & -- & $-\infty$ & -- & - & -- & -- \\
\hline 06 & 1100 & $<0.014$ & $<0.035$ & 1.36 & $<0.012$ & .. & -. & $<0.022$ & - & $<0.018$ & $<0.016$ & $<0.011$ & - \\
\hline 09 & 0930 & -- & -- & -. & -- & -- & -- & - & -. & -- & -- & -. & -- \\
\hline 13 & 1000 & -- & - & -- & -- & -- & -- & - & -- & -- & -- & -- & -- \\
\hline 16 & 1020 & $<0.014$ & $<0.035$ & 0.656 & 0.020 & - & -- & $<0.022$ & -. & $<0.018$ & $<0.016$ & $<0.011$ & -. \\
\hline 20 & 1000 & $<0.014$ & $<0.035$ & 0.826 & 0.016 & -. & -- & $<0.022$ & -. & $<0.018$ & $<0.016$ & $<0.011$ & -- \\
\hline 24 & 1000 & $<0.014$ & $<0.035$ & 2.51 & 0.045 & -- & -- & $<0.022$ & -. & $<0.018$ & $<0.016$ & $<0.011$ & -- \\
\hline 28 & 1030 & $<0.014$ & $<0.035$ & 2.30 & 0.086 & -- & -- & $<0.022$ & -- & $<0.018$ & $<0.016$ & $<0.011$ & -- \\
\hline 31 & 1040 & $<0.014$ & $<0.035$ & 1.34 & 0.046 & -- & -- & $<0.022$ & -- & $<0.018$ & $<0.016$ & $<0.011$ & -- \\
\hline \multicolumn{14}{|c|}{ JUNE 1991} \\
\hline 03 & 1000 & $<0.014$ & $<0.035$ & 1.18 & 0.051 & -- & -- & $<0.022$ & -- & $<0.018$ & $<0.016$ & $<0.011$ & -- \\
\hline 06 & 1000 & $<0.014$ & $<0.035$ & 1.90 & 0.069 & -. & -- & $<0.022$ & -. & $<0.018$ & $<0.016$ & $<0.011$ & - \\
\hline 11 & 1000 & $<0.014$ & $<0.035$ & 1.28 & 0.063 & -- & -- & $<0.022$ & - & $<0.018$ & $<0.016$ & $<0.011$ & -- \\
\hline 13 & 1000 & $<0.014$ & $<0.035$ & 1.91 & 0.117 & - & -- & $<0.022$ & - & $<0.018$ & $<0.016$ & $<0.011$ & -- \\
\hline 21 & 1000 & 0.008 & $<0.035$ & 2.77 & 0.158 & - & -- & $<0.022$ & -- & 0.020 & $<0.016$ & $<0.011$ & -. \\
\hline 25 & 1130 & $<0.014$ & $<0.035$ & 1.84 & 0.057 & - & -- & $<0.022$ & - & 0.010 & $<0.016$ & $<0.011$ & - \\
\hline 27 & 1045 & $<0.014$ & $<0.035$ & 0.832 & 0.041 & - & -- & $<0.022$ & -- & 0.010 & $<0.016$ & $<0.011$ & .. \\
\hline \multicolumn{14}{|l|}{ JULY } \\
\hline 01 & 1100 & $<0.014$ & $<0.035$ & 0.740 & 0.036 & .. & -- & $<0.022$ & .. & $<0.018$ & $<0.016$ & $<0.011$ & -- \\
\hline 03 & 1000 & $<0.014$ & $<0.035$ & 0.384 & 0.013 & -- & -- & $<0.022$ & -- & $<0.018$ & $<0.016$ & $<0.011$ & -- \\
\hline
\end{tabular}


Table 8.--Pesticide results from soild-phase extraction 1-liter method-Continued

\begin{tabular}{|c|c|c|c|c|c|c|c|c|c|c|c|c|c|}
\hline Dete & Time & $\begin{array}{c}\text { Mala- } \\
\text { thion } \\
\text { diss } \\
(\mu g / L)\end{array}$ & $\begin{array}{l}\text { Methyl } \\
\text { para- } \\
\text { thion } \\
\text { diss } \\
(\mu g /)\end{array}$ & $\begin{array}{c}\text { Meto- } \\
\text { lachior } \\
\text { diss } \\
(\mu g / L)\end{array}$ & $\begin{array}{c}\text { Metri- } \\
\text { buzin } \\
\text { diss } \\
\left(\mu g^{\prime} L\right)\end{array}$ & $\begin{array}{l}\text { Molin- } \\
\text { ate } \\
\text { diss } \\
(\mu g / L)\end{array}$ & $\begin{array}{l}\text { Neprop- } \\
\text { emide } \\
\text { diss } \\
(\mu g /)\end{array}$ & $\begin{array}{c}\text { Para- } \\
\text { thion } \\
\text { diss } \\
(\mu g / L)\end{array}$ & $\begin{array}{l}\text { Pebul- } \\
\text { ate } \\
\text { diss } \\
(\mu g / L)\end{array}$ & $\begin{array}{l}\text { Pendi- } \\
\text { meth- } \\
\text { alin } \\
\text { diss } \\
(\mu g / L)\end{array}$ & $\begin{array}{l}\text { Perme- } \\
\text { thrin } \\
\text { diss } \\
(\mu g / L)\end{array}$ & $\begin{array}{l}\text { Pho- } \\
\text { rate } \\
\text { disa } \\
(\mu \mathrm{g} / \mathrm{L})\end{array}$ & $\begin{array}{c}\text { Prome- } \\
\text { ton } \\
\text { disa } \\
(\mu g / L)\end{array}$ \\
\hline \multicolumn{14}{|c|}{06934500 - Missouri River at Hermann, Mo.-Continued } \\
\hline JULY (cont.) & & & & & & & & & & & & & \\
\hline 08 & 1030 & $<0.014$ & $<0.035$ & 0.154 & 0.012 & -- & - & $<0.022$ & - & $<0.018$ & $<0.016$ & $<0.011$ & -- \\
\hline 11 & 1000 & $<0.014$ & $<0.035$ & 0.202 & $<0.015$ & -- & -- & $<0.022$ & -- & $<0.018$ & $<0.016$ & $<0.011$ & -- \\
\hline 15 & 1030 & $<0.014$ & $<0.035$ & 0.294 & 0.038 & -- & -. & $<0.022$ & -. & 0.010 & $<0.016$ & $<0.011$ & -. \\
\hline 22 & 1015 & $<0.014$ & $<0.035$ & 0.194 & 0.013 & -- & -- & $<0.022$ & -- & $<0.018$ & $<0.016$ & $<0.011$ & -- \\
\hline 29 & 1100 & $<0.014$ & $<0.035$ & 0.166 & 0.006 & -- & -- & $<0.022$ & -- & $<0.018$ & $<0.016$ & $<0.011$ & -- \\
\hline \multicolumn{14}{|c|}{ AUGUST 1991} \\
\hline 05 & 1000 & $<0.014$ & $<0.035$ & 0.107 & $<0.012$ & -- & -. & $<0.022$ & -. & $<0.018$ & $<0.016$ & $<0.011$ & -- \\
\hline 13 & 1100 & $<0.014$ & $<0.035$ & 0.188 & $<0.012$ & -- & -- & $<0.022$ & -. & $<0.018$ & $<0.016$ & $<0.011$ & -- \\
\hline 19 & 1000 & $<0.014$ & $<0.035$ & 0.190 & $<0.012$ & - & -. & $<0.022$ & -. & $<0.018$ & $<0.016$ & $<0.011$ & -- \\
\hline 27 & 1100 & $<0.014$ & $<0.035$ & 0.169 & $<0.012$ & - & -- & $<0.022$ & -- & $<0.018$ & $<0.016$ & $<0.011$ & -- \\
\hline \multicolumn{14}{|l|}{ SEPTEMBER } \\
\hline 05 & 1030 & $<0.014$ & $<0.035$ & 0.143 & $<0.012$ & - & - & $<0.022$ & - & $<0.018$ & $<0.016$ & $<0.011$ & -- \\
\hline 09 & 1000 & $<0.014$ & $<0.035$ & 0.123 & $<0.012$ & -- & -- & $<0.022$ & -- & $<0.018$ & $<0.016$ & $<0.011$ & -- \\
\hline 16 & 1000 & $<0.014$ & $<0.035$ & 0.079 & $<0.012$ & -- & -- & $<0.022$ & -- & $<0.018$ & $<0.016$ & $<0.011$ & - \\
\hline \multicolumn{14}{|l|}{ OCTOBER } \\
\hline 01 & 1030 & $<0.014$ & $<0.035$ & 0.055 & $<0.012$ & -- & -- & $<0.022$ & -- & $<0.018$ & $<0.016$ & $<0.011$ & - \\
\hline 07 & 1000 & $<0.014$ & $<0.035$ & 0.053 & $<0.012$ & -. & -. & $<0.022$ & -. & $<0.018$ & $<0.016$ & $<0.011$ & -. \\
\hline 15 & 1100 & $<0.014$ & $<0.035$ & 0.066 & $<0.012$ & -. & -- & $<0.022$ & -- & $<0.018$ & $<0.016$ & $<0.011$ & -- \\
\hline 21 & 1000 & $<0.014$ & $<0.035$ & 0.080 & $<0.012$ & -- & -- & $<0.022$ & -- & $<0.018$ & $<0.016$ & $<0.011$ & -- \\
\hline 29 & 1000 & $<0.014$ & $<0.035$ & 0.078 & $<0.012$ & -. &.- & $<0.022$ &.- & $<0.018$ & $<0.016$ & $<0.011$ & -- \\
\hline \multicolumn{14}{|l|}{ NOVEMBER } \\
\hline 04 & 1030 & $<0.014$ & $<0.035$ & 0.088 & $<0.012$ & -- & -- & $<0.022$ & -- & $<0.018$ & $<0.016$ & $<0.011$ & -- \\
\hline 21 & 1030 & $<0.014$ & $<0.035$ & 0.280 & $<0.012$ & -- & -- & $<0.022$ & -- & $<0.018$ & $<0.016$ & $<0.011$ & -- \\
\hline \multicolumn{14}{|c|}{ DECEMBER 1991} \\
\hline 06 & 1240 & $<0.014$ & $<0.035$ & 0.117 & $<0.012$ & -- & -- & $<0.022$ & -- & $<0.018$ & $<0.016$ & $<0.011$ & -- \\
\hline 16 & 1130 & $<0.014$ & $<0.035$ & 0.460 & $<0.012$ & -- & -- & $<0.022$ & -- & $<0.018$ & $<0.016$ & $<0.011$ & -- \\
\hline \multicolumn{14}{|c|}{ JANUARY 1992} \\
\hline 02 & 1030 & $<0.014$ & $<0.035$ & 0.102 & 0.005 & -- & -- & $<0.022$ & -- & $<0.018$ & $<0.016$ & $<0.011$ & -- \\
\hline 16 & 1200 & $<0.014$ & $<0.035$ & 0.430 & $<0.012$ & $<0.007$ & $<0.010$ & $<0.022$ & $<0.009$ & $<0.018$ & $<0.016$ & $<0.011$ & $<0.008$ \\
\hline 28 & 1300 & $<0.014$ & $<0.035$ & 0.320 & $<0.012$ & $<0.007$ & $<0.010$ & $<0.022$ & $<0.009$ & $<0.018$ & $<0.016$ & $<0.011$ & $<0.008$ \\
\hline \multicolumn{14}{|l|}{ FEBRUARY } \\
\hline 10 & 1000 & $<0.014$ & $<0.035$ & 0.170 & $<0.012$ & $<0.007$ & $<0.010$ & $<0.022$ & $<0.009$ & $<0.018$ & $<0.016$ & $<0.011$ & $<0.008$ \\
\hline 24 & 1200 & $<0.014$ & $<0.035$ & 0.130 & $<0.012$ & $<0.007$ & $<0.010$ & $<0.022$ & $<0.009$ & $<0.018$ & $<0.016$ & $<0.011$ & 0.007 \\
\hline \multicolumn{14}{|l|}{ MARCH } \\
\hline 03 & 1100 & $<0.014$ & $<0.035$ & 0.110 & $<0.012$ & $<0.007$ & $<0.010$ & $<0.022$ & $<0.009$ & 0.004 & $<0.016$ & $<0.011$ & $<0.008$ \\
\hline 12 & 1200 & $<0.014$ & $<0.035$ & 0.120 & $<0.012$ & $<0.007$ & $<0.010$ & $<0.022$ & $<0.009$ & $<0.018$ & $<0.016$ & $<0.011$ & $<0.008$ \\
\hline 20 & 0830 & $<0.014$ & $<0.035$ & 0.120 & $<0.012$ & $<0.007$ & $<0.010$ & $<0.022$ & $<0.009$ & $<0.018$ & $<0.016$ & $<0.011$ & $<0.008$ \\
\hline 26 & 1130 & $<0.014$ & $<0.035$ & 0.140 & $<0.012$ & $<0.007$ & $<0.010$ & $<0.022$ & $<0.009$ & $<0.018$ & $<0.016$ & $<0.011$ & $<0.008$ \\
\hline \multicolumn{14}{|l|}{ APRIL 1992} \\
\hline 01 & 1000 & $<0.014$ & $<0.035$ & 0.100 & $<0.012$ & $<0.007$ & $<0.010$ & $<0.022$ & $<0.009$ & $<0.018$ & $<0.016$ & $<0.011$ & 0.010 \\
\hline 06 & 1230 & $<0.014$ & 0.012 & 0.140 & $<0.012$ & $<0.007$ & $<0.010$ & $<0.022$ & $<0.009$ & $<0.018$ & $<0.016$ & $<0.011$ & $<0.008$ \\
\hline 14 & 1030 & $<0.014$ & $<0.035$ & 0.290 & $<0.012$ & $<0.007$ & $<0.010$ & $<0.022$ & $<0.009$ & $<0.018$ & $<0.016$ & $<0.011$ & $<0.008$ \\
\hline 21 & 1300 & $<0.014$ & $<0.035$ & 0.370 & 0.008 & $<0.007$ & $<0.010$ & $<0.022$ & $<0.009$ & $<0.018$ & $<0.016$ & $<0.011$ & $<0.008$ \\
\hline 29 & 1015 & $<0.014$ & $<0.035$ & 0.700 & 0.012 & $<0.007$ & $<0.010$ & $<0.022$ & $<0.009$ & $<0.018$ & $<0.016$ & $<0.011$ & 0.012 \\
\hline \multicolumn{14}{|l|}{ MAY } \\
\hline 12 & 1000 & $<0.014$ & $<0.035$ & 0.260 & $<0.012$ & $<0.007$ & $<0.010$ & $<0.022$ & $<0.009$ & $<0.018$ & $<0.016$ & $<0.011$ & $<0.008$ \\
\hline 21 & 1200 & $<0.014$ & $<0.035$ & 0.200 & $<0.012$ & $<0.007$ & $<0.010$ & $<0.022$ & $<0.009$ & $<0.018$ & $<0.016$ & $<0.011$ & 0.007 \\
\hline 26 & 1130 & $<0.014$ & $<0.035$ & 2.80 & 0.044 & $<0.007$ & $<0.010$ & $<0.022$ & $<0.009$ & 0.010 & $<0.016$ & $<0.011$ & 0.020 \\
\hline
\end{tabular}


Tabie 8.--Pesticide results from solid-phase extraction 1-iiter method--Continued

\begin{tabular}{|c|c|c|c|c|c|c|c|c|c|c|c|c|c|}
\hline Date & Time & $\begin{array}{l}\text { Maia- } \\
\text { thion } \\
\text { diss } \\
(\mu g / L)\end{array}$ & $\begin{array}{l}\text { Methyl } \\
\text { para- } \\
\text { thlon } \\
\text { diss } \\
\text { ( } \mu \mathrm{g} / \mathrm{L})\end{array}$ & $\begin{array}{l}\text { Meto- } \\
\text { lachlor } \\
\text { diss } \\
(\mu \mathrm{g} / \mathrm{L})\end{array}$ & $\begin{array}{c}\text { Metri- } \\
\text { buzin } \\
\text { diss } \\
(\mu g / L)\end{array}$ & $\begin{array}{l}\text { Molin- } \\
\text { ate } \\
\text { diss } \\
(\mu g / L)\end{array}$ & $\begin{array}{c}\text { Naprop- } \\
\text { amide } \\
\text { diss } \\
(\mu g / L)\end{array}$ & $\begin{array}{l}\text { Para- } \\
\text { thion } \\
\text { diss } \\
(\mu g / L)\end{array}$ & $\begin{array}{c}\text { Pebul- } \\
\text { ata } \\
\text { diss } \\
(\mu g / L)\end{array}$ & $\begin{array}{l}\text { Pendl- } \\
\text { meth- } \\
\text { alin } \\
\text { diss } \\
\text { ( } \mu \mathrm{g} / \mathrm{L} \text { ) }\end{array}$ & $\begin{array}{l}\text { Perme- } \\
\text { thrin } \\
\text { diss } \\
\text { ( } \mu \mathrm{g} / \mathrm{L})\end{array}$ & $\begin{array}{l}\text { Pho- } \\
\text { rate } \\
\text { diss } \\
(\mu g /))\end{array}$ & $\begin{array}{l}\text { Promo- } \\
\text { ton } \\
\text { diss } \\
(\mu \mathrm{g} / \mathrm{L})\end{array}$ \\
\hline
\end{tabular}

JUNE

06934500 - Missouri River at Hermann, Mo.-Continued

\begin{tabular}{|c|c|c|c|c|c|c|c|c|c|c|c|c|c|}
\hline 01 & 1330 & $<0.014$ & $<0.035$ & 1.50 & 0.017 & $<0.007$ & $<0.010$ & $<0.022$ & $<0.009$ & $<0.018$ & $<0.016$ & $<0.011$ & 0.014 \\
\hline 09 & 1030 & $<0.014$ & $<0.035$ & 0.250 & 0.027 & $<0.007$ & $<0.010$ & $<0.022$ & $<0.009$ & $<0.018$ & $<0.016$ & $<0.011$ & 0.010 \\
\hline 15 & 1230 & $<0.014$ & $<0.035$ & 0.500 & 0.021 & $<0.007$ & $<0.010$ & $<0.022$ & $<0.009$ & 0.006 & $<0.016$ & $<0.011$ & 0.019 \\
\hline 22 & 0945 & $<0.014$ & $<0.035$ & 0.410 & 0.015 & $<0.007$ & $<0.010$ & $<0.022$ & $<0.009$ & $<0.018$ & $<0.016$ & $<0.011$ & 0.018 \\
\hline 30 & 1130 & $<0.014$ & $<0.035$ & 0.560 & 0.011 & $<0.007$ & $<0.010$ & $<0.022$ & $<0.009$ & 0.006 & $<0.016$ & $<0.011$ & 0.020 \\
\hline \multicolumn{14}{|l|}{ JULY } \\
\hline 07 & 0930 & $<0.014$ & $<0.035$ & 0.170 & 0.004 & $<0.007$ & $<0.010$ & $<0.022$ & $<0.009$ & $<0.018$ & $<0.016$ & $<0.011$ & 0.01 \\
\hline 14 & 1200 & $<0.014$ & $<0.035$ & 0.420 & 0.023 & $<0.007$ & $<0.010$ & $<0.022$ & $<0.009$ & $<0.018$ & $<0.016$ & $<0.011$ & 0.030 \\
\hline 21 & 1030 & $<0.014$ & $<0.035$ & 0.820 & 0.037 & $<0.007$ & $<0.010$ & $<0.022$ & $<0.009$ & $<0.018$ & $<0.016$ & $<0.011$ & 0.033 \\
\hline 28 & 1230 & $<0.014$ & $<0.035$ & 0.270 & 0.013 & $<0.007$ & $<0.010$ & $<0.022$ & $<0.009$ & $<0.018$ & $<0.016$ & $<0.011$ & 0.01 \\
\hline
\end{tabular}

APRIL 1991

\begin{tabular}{|c|c|c|c|c|c|c|c|c|c|c|c|c|}
\hline 11 & 1500 & -- & - & -- & -- & -- & -- & -- & -- & -- & -- & -- \\
\hline 18 & 1130 & -- & -- & -- & -- & -- & -- & -- & -- & -- & -- & -- \\
\hline 24 & 0930 & -- & -- & -- & -- & - & -- & -- & -- & -. & - & $-\cdot$ \\
\hline 29 & 1230 & -- & -- & -- & -. & -- & -- & .- & .. & -. & -- & -- \\
\hline \multicolumn{13}{|l|}{ MAY } \\
\hline 07 & 1345 & $<0.014$ & $<0.035$ & 1.09 & 0.043 & - & -- & $<0.022$ & -. & 0.008 & $<0.016$ & $<0.011$ \\
\hline 09 & 0900 & $<0.014$ & $<0.035$ & 0.607 & 0.040 & -- & -- & $<0.022$ & -- & $<0.018$ & $<0.016$ & $<0.011$ \\
\hline 13 & 1330 & -- & -- & - & -- & -- & -- & -. & -- & -- & -- & -- \\
\hline 16 & 1200 & $<0.014$ & $<0.035$ & 1.15 & 0.020 & -- & -- & $<0.022$ & -. & $<0.018$ & $<0.016$ & $<0.011$ \\
\hline 20 & 1430 & $<0.014$ & $<0.035$ & 0.714 & $<0.012$ & -- & -- & $<0.022$ & -- & $<0.018$ & $<0.016$ & $<0.011$ \\
\hline 23 & 0916 & $<0.014$ & $<0.035$ & 0.758 & 0.035 & -- & -- & $<0.022$ & -- & $<0.018$ & $<0.016$ & $<0.011$ \\
\hline 28 & 1300 & $<0.014$ & $<0.035$ & 2.01 & 0.017 & -- & - & $<0.022$ & -- & $<0.018$ & $<0.016$ & $<0.011$ \\
\hline 30 & 1330 & $<0.014$ & $<0.035$ & 1.93 & 0.059 & -- & -- & $<0.022$ & -- & $<0.018$ & $<0.016$ & $<0.011$ \\
\hline \multicolumn{13}{|c|}{ JUNE 1991} \\
\hline 03 & 1345 & $<0.014$ & $<0.035$ & 1.74 & 0.037 & -- & -- & $<0.022$ & - & $<0.018$ & $<0.016$ & $<0.011$ \\
\hline 06 & 1045 & $<0.014$ & $<0.035$ & 1.45 & 0.044 & -- & -- & $<0.022$ & -- & $<0.018$ & $<0.016$ & $<0.011$ \\
\hline 10 & 1400 & $<0.014$ & $<0.035$ & 1.61 & 0.033 & -- & -- & $<0.022$ & -- & $<0.018$ & $<0.016$ & $<0.011$ \\
\hline 13 & 1500 & $<0.014$ & $<0.035$ & 1.38 & $<0.012$ & -- & -- & $<0.022$ & -- & $<0.018$ & $<0.016$ & $<0.011$ \\
\hline 18 & 1430 & $<0.014$ & $<0.035$ & 1.79 & 0.036 & -- & -- & $<0.022$ & - & $<0.018$ & $<0.016$ & $<0.011$ \\
\hline 20 & 1100 & $<0.014$ & $<0.035$ & 1.56 & 0.057 & - & -- & $<0.022$ & -- & $<0.018$ & $<0.016$ & $<0.011$ \\
\hline 24 & 1300 & $<0.014$ & $<0.035$ & 2.31 & 0.133 & -- & -- & $<0.022$ & -- & 0.010 & $<0.016$ & $<0.011$ \\
\hline 27 & 1400 & $<0.014$ & $<0.035$ & 1.97 & 0.047 & -- & -- & $<0.022$ & -- & 0.010 & $<0.016$ & $<0.011$ \\
\hline \multirow{2}{*}{\multicolumn{13}{|c|}{$1320<0.014<0.035$}} \\
\hline & & & & & & & & & & & & \\
\hline 03 & 0950 & $<0.014$ & $<0.035$ & 1.45 & 0.033 & -- & - & $<0.022$ & -- & $<0.018$ & $<0.016$ & $<0.011$ \\
\hline 08 & 1330 & $<0.014$ & $<0.035$ & 1.16 & 0.016 & -- & - & $<0.022$ & - & $<0.018$ & $<0.016$ & $<0.011$ \\
\hline 11 & 1400 & $<0.014$ & $<0.035$ & 1.09 & $<0.012$ & -- & -- & $<0.022$ & -- & $<0.018$ & $<0.016$ & $<0.011$ \\
\hline 18 & 0930 & -- & -- & -- & -- & -- & -- & -- & -- & -- & - & -- \\
\hline 23 & 1300 & $<0.014$ & $<0.035$ & 0.657 & 0.024 & -- & -. & $<0.022$ & -- & $<0.018$ & $<0.016$ & $<0.011$ \\
\hline 30 & 1000 & $<0.014$ & $<0.035$ & 0.539 & 0.020 &.- & -- & $<0.022$ & -- & $<0.018$ & $<0.016$ & $<0.011$ \\
\hline \multicolumn{13}{|c|}{ AUGUST 19} \\
\hline 06 & 1300 & $<0.014$ & $<0.035$ & 0.319 & $<0.012$ & $\cdots$ & -- & $<0.022$ & -- & $<0.018$ & $<0.016$ & $<0.011$ \\
\hline 13 & 0930 & $<0.014$ & $<0.035$ & 0.313 & $<0.012$ & $-\cdot$ & - & $<0.022$ & - & $<0.018$ & $<0.016$ & $<0.011$ \\
\hline 20 & 1330 & $<0.014$ & $<0.035$ & 0.247 & $<0.012$ & -- & -- & $<0.022$ & -- & $<0.018$ & $<0.016$ & $<0.011$ \\
\hline 29 & 1100 & $<0.014$ & $<0.035$ & 0.182 & 0.030 & -- & -- & $<0.022$ & -- & $<0.018$ & $<0.016$ & $<0.011$ \\
\hline
\end{tabular}


Table 8.-Pesticide results from solid-phase extraction 1-liter method--Continued

\begin{tabular}{|c|c|c|c|c|c|c|c|c|c|c|c|c|c|}
\hline Date & Time & $\begin{array}{l}\text { Mala- } \\
\text { thlon } \\
\text { diss } \\
(\mu g / L)\end{array}$ & $\begin{array}{c}\text { Mothyl } \\
\text { para- } \\
\text { thion } \\
\text { diss } \\
(\mu g / L)\end{array}$ & $\begin{array}{l}\text { Mato- } \\
\text { lachior } \\
\text { diss } \\
(\mu g / L)\end{array}$ & $\begin{array}{l}\text { Metri- } \\
\text { buzin } \\
\text { diss } \\
(\mu g / L)\end{array}$ & $\begin{array}{l}\text { Molin- } \\
\text { ate } \\
\text { diss } \\
(\mu g / L)\end{array}$ & $\begin{array}{l}\text { Naprop- } \\
\text { amida } \\
\text { dlss } \\
(\mu g / L)\end{array}$ & $\begin{array}{l}\text { Pare- } \\
\text { thion } \\
\text { diss } \\
(\mu g / 2)\end{array}$ & $\begin{array}{l}\text { Pabul- } \\
\text { ale } \\
\text { diss } \\
(\mu g / L)\end{array}$ & $\begin{array}{l}\text { Pendl- } \\
\text { math- } \\
\text { alln } \\
\text { diss } \\
(\mu \mathrm{g} / \mathrm{L})\end{array}$ & $\begin{array}{l}\text { Perme- } \\
\text { thrin } \\
\text { diss } \\
(\mu g / L)\end{array}$ & $\begin{array}{l}\text { Pho- } \\
\text { rata } \\
\text { dias } \\
(\mu g / L)\end{array}$ & $\begin{array}{l}\text { Prome- } \\
\text { ton } \\
\text { dlas } \\
(\mu g / L)\end{array}$ \\
\hline
\end{tabular}

SEPTEMBER

\begin{tabular}{|c|c|c|c|c|c|c|c|c|c|c|c|c|c|}
\hline 03 & 1500 & $<0.014$ & $<0.035$ & 0.157 & $<0.012$ & - & -- & $<0.022$ & -- & $<0.018$ & $<0.016$ & $<0.011$ & - \\
\hline 11 & 1000 & $<0.014$ & $<0.035$ & 0.106 & $<0.012$ & - & - & $<0.022$ & - & $<0.018$ & $<0.016$ & $<0.011$ & -- \\
\hline 17 & 1400 & $<0.014$ & $<0.035$ & 0.082 & $<0.012$ & -- & -- & $<0.022$ & -- & $<0.018$ & $<0.016$ & $<0.011$ & -- \\
\hline 26 & 0930 & $<0.014$ & $<0.035$ & 0.063 & $<0.012$ & -- & - & $<0.022$ & - & $<0.018$ & $<0.016$ & $<0.011$ & - \\
\hline \multicolumn{14}{|c|}{ OCTOBER } \\
\hline 02 & 1300 & $<0.014$ & $<0.035$ & 0.050 & $<0.012$ & -- & -- & $<0.022$ & -- & $<0.018$ & $<0.016$ & $<0.011$ & -- \\
\hline 09 & 1030 & $<0.014$ & $<0.035$ & 0.047 & $<0.012$ & -- & -- & $<0.022$ & -- & $<0.018$ & $<0.016$ & $<0.011$ & $\ldots$ \\
\hline 16 & 1430 & $<0.014$ & $<0.035$ & 0.072 & $<0.012$ & - & -- & $<0.022$ & -- & $<0.018$ & $<0.016$ & $<0.011$ & - \\
\hline 23 & 0945 & $<0.014$ & $<0.035$ & 0.065 & $<0.012$ & -- & -- & $<0.022$ & - & $<0.018$ & $<0.016$ & $<0.011$ & -- \\
\hline 30 & 1400 & $<0.014$ & $<0.035$ & 0.040 & $<0.012$ & - & -- & $<0.022$ & -- & $<0.018$ & $<0.016$ & $<0.011$ & -- \\
\hline \multicolumn{14}{|c|}{ NOVEMBER } \\
\hline 07 & 0800 & $<0.014$ & $<0.035$ & 0.068 & $<0.012$ & - & $\cdots$ & $<0.022$ & -- & $<0.018$ & $<0.016$ & $<0.011$ & -- \\
\hline 20 & 1030 & $<0.014$ & $<0.035$ & 0.100 & $<0.012$ & -- & - & $<0.022$ & -- & $<0.018$ & $<0.016$ & $<0.011$ & -- \\
\hline \multicolumn{14}{|c|}{ DECEMBER 1991} \\
\hline 05 & 1045 & $<0.014$ & $<0.035$ & 0.076 & $<0.012$ & -- & - & $<0.022$ & -- & $<0.018$ & $<0.016$ & $<0.011$ & - \\
\hline 18 & 1100 & $<0.014$ & $<0.035$ & 0.092 & $<0.012$ & -- & -- & $<0.022$ & -- & $<0.018$ & $<0.016$ & $<0.011$ & - \\
\hline 30 & 1400 & $<0.014$ & $<0.035$ & 0.125 & $<0.012$ & -- & -- & $<0.022$ & -- & $<0.018$ & $<0.016$ & $<0.011$ & -- \\
\hline \multicolumn{14}{|c|}{ JANUARY 1992} \\
\hline 24 & 0930 & $<0.014$ & $<0.035$ & 0.130 & $<0.012$ & $<0.007$ & $<0.010$ & $<0.022$ & $<0.009$ & $<0.018$ & $<0.016$ & $<0.011$ & $<0.008$ \\
\hline \multicolumn{14}{|c|}{ FEBRUARY } \\
\hline 04 & 1030 & $<0.014$ & $<0.035$ & 0.086 & $<0.012$ & $<0.007$ & $<0.010$ & $<0.022$ & $<0.009$ & $<0.018$ & $<0.016$ & $<0.011$ & 0.008 \\
\hline 19 & 0930 & $<0.014$ & $<0.035$ & 0.048 & $<0.012$ & $<0.007$ & $<0.010$ & $<0.022$ & $<0.009$ & $<0.018$ & $<0.016$ & $<0.011$ & $<0.008$ \\
\hline \multicolumn{14}{|l|}{ MARCH } \\
\hline 04 & 0945 & $<0.014$ & $<0.035$ & 0.076 & $<0.012$ & $<0.007$ & $<0.010$ & $<0.022$ & $<0.009$ & $<0.018$ & $<0.016$ & $<0.011$ & $<0.008$ \\
\hline 09 & 1300 & $<0.014$ & $<0.035$ & 0.085 & $<0.012$ & $<0.007$ & $<0.010$ & $<0.022$ & $<0.009$ & $<0.018$ & $<0.016$ & $<0.011$ & $<0.008$ \\
\hline 18 & 0945 & $<0.014$ & $<0.035$ & 0.068 & $<0.012$ & $<0.007$ & $<0.010$ & $<0.022$ & $<0.009$ & $<0.018$ & $<0.016$ & $<0.011$ & $<0.008$ \\
\hline 24 & 1330 & $<0.014$ & $<0.035$ & 0.140 & 0.007 & $<0.007$ & $<0.010$ & $<0.022$ & $<0.009$ & $<0.018$ & $<0.016$ & $<0.011$ & $<0.008$ \\
\hline \multicolumn{14}{|l|}{ APRIL } \\
\hline 02 & 0930 & $<0.014$ & $<0.035$ & 0.073 & 0.006 & $<0.007$ & $<0.010$ & $<0.022$ & $<0.009$ & $<0.018$ & $<0.016$ & $<0.011$ & 0.012 \\
\hline 07 & 1330 & $<0.014$ & $<0.035$ & 0.067 & $<0.012$ & $<0.007$ & $<0.010$ & $<0.022$ & $<0.009$ & $<0.018$ & $<0.016$ & $<0.011$ & $<0.008$ \\
\hline 15 & 1030 & $<0.014$ & $<0.035$ & 0.086 & $<0.012$ & $<0.007$ & $<0.010$ & $<0.022$ & $<0.009$ & 0.004 & $<0.016$ & $<0.011$ & $<0.008$ \\
\hline 23 & 1330 & $<0.014$ & $<0.035$ & 0.590 & 0.025 & $<0.007$ & $<0.010$ & $<0.022$ & $<0.009$ & $<0.018$ & $<0.016$ & $<0.011$ & 0.017 \\
\hline 29 & 1100 & $<0.014$ & $<0.035$ & 0.650 & 0.016 & $<0.007$ & $<0.010$ & $<0.022$ & $<0.009$ & $<0.018$ & $<0.016$ & $<0.011$ & $<0.008$ \\
\hline \multicolumn{14}{|l|}{ MAY 1992} \\
\hline 06 & 1300 & $<0.014$ & $<0.035$ & 0.300 & $<0.012$ & $<0.007$ & $<0.010$ & $<0.022$ & $<0.009$ & $<0.018$ & $<0.016$ & $<0.011$ & $<0.008$ \\
\hline 14 & 0930 & $<0.014$ & $<0.035$ & 0.180 & $<0.012$ & $<0.007$ & $<0.010$ & $<0.022$ & $<0.009$ & $<0.018$ & $<0.016$ & $<0.011$ & $<0.008$ \\
\hline 19 & 1300 & $<0.014$ & $<0.035$ & 0.130 & $<0.012$ & $<0.007$ & $<0.010$ & $<0.022$ & $<0.009$ & $<0.018$ & $<0.016$ & $<0.011$ & $<0.008$ \\
\hline 28 & 0900 & $<0.014$ & $<0.035$ & 0.150 & 0.014 & $<0.007$ & $<0.010$ & $<0.022$ & $<0.009$ & $<0.018$ & $<0.016$ & $<0.011$ & 0.009 \\
\hline \multicolumn{14}{|l|}{ JUNE } \\
\hline 02 & 1300 & $<0.014$ & $<0.035$ & 1.10 & 0.020 & $<0.007$ & $<0.010$ & $<0.022$ & $<0.009$ & $<0.018$ & $<0.016$ & $<0.011$ & 0.013 \\
\hline 09 & 0930 & $<0.014$ & $<0.035$ & 0.390 & 0.021 & $<0.007$ & $<0.010$ & $<0.022$ & $<0.009$ & $<0.018$ & $<0.016$ & $<0.011$ & 0.019 \\
\hline 16 & 1330 & $<0.014$ & $<0.035$ & 0.190 & 0.008 & $<0.007$ & $<0.010$ & $<0.022$ & $<0.009$ & $<0.018$ & $<0.016$ & $<0.011$ & 0.006 \\
\hline 25 & 1000 & $<0.014$ & $<0.035$ & 0.230 & 0.020 & $<0.007$ & $<0.010$ & $<0.022$ & $<0.009$ & $<0.018$ & $<0.016$ & $<0.011$ & 0.016 \\
\hline \multicolumn{14}{|l|}{ JULY } \\
\hline 01 & 1300 & $<0.014$ & $<0.035$ & 0.430 & 0.014 & $<0.007$ & $<0.010$ & $<0.022$ & $<0.009$ & $<0.018$ & $<0.016$ & $<0.011$ & 0.014 \\
\hline 09 & 0915 & $<0.014$ & $<0.035$ & 0.190 & 0.011 & $<0.007$ & $<0.010$ & $<0.022$ & $<0.009$ & $<0.018$ & $<0.016$ & $<0.011$ & 0.020 \\
\hline 15 & 1230 & $<0.014$ & $<0.035$ & 0.180 & 0.012 & $<0.007$ & $<0.010$ & $<0.022$ & $<0.009$ & $<0.018$ & $<0.016$ & $<0.011$ & 0.021 \\
\hline
\end{tabular}


Table 8.-Pesticide results from solid-phase extraction 1-liter method--Continued

\begin{tabular}{|c|c|c|c|c|c|c|c|c|c|c|c|c|c|}
\hline Date & Time & $\begin{array}{c}\text { Mala- } \\
\text { thion } \\
\text { diss } \\
(\mu g / L)\end{array}$ & $\begin{array}{l}\text { Methyl } \\
\text { para- } \\
\text { thion } \\
\text { diss } \\
(\mu g / L)\end{array}$ & $\begin{array}{l}\text { Meto- } \\
\text { lachlor } \\
\text { diss } \\
(\mu g / L)\end{array}$ & $\begin{array}{c}\text { Metri- } \\
\text { buzin } \\
\text { diss } \\
(\mu g / L)\end{array}$ & $\begin{array}{l}\text { Molin- } \\
\text { ate } \\
\text { diss } \\
(\mu g / L)\end{array}$ & $\begin{array}{c}\text { Naprop- } \\
\text { amlde } \\
\text { diss } \\
(\mu g / L)\end{array}$ & $\begin{array}{c}\text { Para- } \\
\text { thion } \\
\text { diss } \\
(\mu g /)\end{array}$ & $\begin{array}{c}\text { Pebul- } \\
\text { ate } \\
\text { diss } \\
(\mu g / L)\end{array}$ & $\begin{array}{l}\text { Pendi- } \\
\text { meth- } \\
\text { alin } \\
\text { diss } \\
\text { ( } \mu \mathrm{g} / \mathrm{L})\end{array}$ & $\begin{array}{l}\text { Perme- } \\
\text { thrin } \\
\text { diss } \\
(\mu g / L)\end{array}$ & $\begin{array}{l}\text { Pho- } \\
\text { rate } \\
\text { diss } \\
(\mu g / L)\end{array}$ & $\begin{array}{c}\text { Prome } \\
\text { ton } \\
\text { diss } \\
(\mu g / 2)\end{array}$ \\
\hline \multicolumn{14}{|c|}{07022000 - Mississippi River at Thebes, Ill.-Continned } \\
\hline \multicolumn{14}{|l|}{ JULY (cont.) } \\
\hline 23 & 1000 & $<0.014$ & $<0.035$ & 0.650 & 0.033 & $<0.007$ & $<0.010$ & $<0.022$ & 0.004 & 0.007 & $<0.016$ & $<0.011$ & 0.020 \\
\hline 28 & 1300 & $<0.014$ & $<0.035$ & 0.540 & 0.014 & $<0.007$ & $<0.010$ & $<0.022$ & $<0.009$ & $<0.018$ & $<0.016$ & $<0.011$ & 0.024 \\
\hline \multicolumn{14}{|c|}{03374100 - White River at Hazleton, Ind. } \\
\hline \multicolumn{14}{|l|}{ MAY 1991} \\
\hline 01 & 1000 & $<0.014$ & $<0.035$ & 0.190 & $<0.012$ & -- & -- & $<0.022$ & . & $<0.018$ & $<0.016$ & $<0.011$ & -- \\
\hline 06 & 1115 & 0.005 & $<0.035$ & 0.146 & 0.002 & - & -- & $<0.022$ & -- & $<0.018$ & $<0.016$ & $<0.011$ & -- \\
\hline 09 & 1215 & $<0.014$ & $<0.035$ & 0.223 & $<0.012$ & - & - & $<0.022$ & -- & $<0.018$ & $<0.016$ & $<0.011$ & 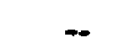 \\
\hline 13 & 1300 & -- & -- & -. & .- & -. & -. & -- & -- & -. & -- & -- & -. \\
\hline 16 & 1130 & -- & -- & -- & -- & -- & -- & -- & -- & - & -- & -- & -- \\
\hline 20 & 1045 & $<0.014$ & $<0.035$ & 0.834 & 0.020 & -. & - & $<0.022$ & -- & $<0.018$ & $<0.016$ & $<0.011$ & - \\
\hline 23 & 1100 & 0.006 & $<0.035$ & 1.92 & 0.090 & -- & -- & $<0.022$ & .- & $<0.018$ & $<0.016$ & $<0.011$ & .- \\
\hline 28 & 1100 & $<0.014$ & $<0.035$ & 3.50 & 0.341 & -- & -. & $<0.022$ & -- & $<0.018$ & $<0.016$ & $<0.011$ & -. \\
\hline 30 & 1200 & $<0.014$ & $<0.035$ & 5.10 & 0.328 & -. & -- & $<0.022$ & -- & $<0.018$ & $<0.016$ & $<0.011$ & -. \\
\hline \multicolumn{14}{|l|}{ JUNE } \\
\hline 03 & 1200 & $<0.014$ & $<0.035$ & 3.06 & 0.146 & -. & .. & $<0.022$ & - & $<0.018$ & $<0.016$ & $<0.011$ & -. \\
\hline 06 & 1145 & $<0,014$ & $<0.035$ & 2.39 & 0.154 & -- & -- & $<0.022$ & - & $<0.018$ & $<0.016$ & $<0.011$ & -- \\
\hline 10 & 1200 & $<0.014$ & $<0.035$ & 2.50 & 0.141 & -- & -- & $<0.022$ & -- & $<0.018$ & $<0.016$ & $<0.011$ & .- \\
\hline 13 & 1100 & $<0.014$ & $<0.035$ & 2.00 & 0.008 & -- & -- & $<0.022$ & -- & $<0.018$ & $<0.016$ & $<0.011$ & -- \\
\hline 17 & 1200 & $<0.014$ & $<0.035$ & 1.18 & 0.034 & -- & -- & $<0.022$ & .. & $<0.018$ & $<0.016$ & $<0.011$ & -- \\
\hline 20 & 1130 & $<0.014$ & $<0.035$ & 1.19 & 0.017 & -- & -- & $<0.022$ & -- & $<0.018$ & $<0.016$ & $<0.011$ & .. \\
\hline 24 & 1215 & $<0.014$ & $<0.035$ & 1.43 & 0.037 & -- & -- & $<0.022$ & - & $<0.018$ & $<0.016$ & $<0.011$ & .. \\
\hline 27 & 1130 & $<0.014$ & $<0.035$ & 0.857 & 0.017 & -- & .- & $<0.022$ & -- & $<0.018$ & $<0.016$ & $<0.011$ & - \\
\hline \multicolumn{14}{|l|}{ JULY 1991} \\
\hline 01 & 1145 & $<0.014$ & $<0.035$ & 0.434 & 0.009 & -. & -- & $<0.022$ & -- & $<0.018$ & $<0.016$ & $<0.011$ & -- \\
\hline 03 & 1100 & $<0.014$ & $<0.035$ & 0.526 & 0.009 & -- & -- & $<0.022$ & -- & $<0.018$ & $<0.016$ & $<0.011$ & -- \\
\hline 08 & 1200 & $<0.014$ & $<0.035$ & 0.461 & $<0.012$ & -- & -- & $<0.022$ & -. & $<0.018$ & $<0.016$ & $<0.011$ & -- \\
\hline 11 & 1130 & $<0.014$ & $<0.035$ & 0.316 & $<0.012$ & -- & - & $<0.022$ & -- & $<0.018$ & $<0.016$ & $<0.011$ & - \\
\hline 15 & 1200 & $<0.014$ & $<0.035$ & 1.51 & 0.014 & -- & -. & $<0.022$ & -- & $<0.018$ & $<0.016$ & $<0.011$ & .- \\
\hline 18 & 1300 & $<0.014$ & $<0.035$ & 0.797 & 0.011 & -- & -- & $<0.022$ & -. & $<0.018$ & $<0.016$ & $<0.011$ & .- \\
\hline 22 & 1300 & $<0.014$ & $<0.035$ & 0.386 & 0.006 & -- & .- & $<0.022$ & .- & $<0.018$ & $<0.016$ & $<0.011$ & -- \\
\hline 25 & 1200 & $<0.014$ & $<0.035$ & 0.264 & 0.123 & -- & -- & $<0.022$ & -. & $<0.018$ & $<0.016$ & $<0.011$ & -- \\
\hline 30 & 1145 & $<0.014$ & $<0.035$ & 0.248 & 0.086 & -- & -- & $<0.022$ & .. & $<0.018$ & $<0.016$ & $<0.011$ & -- \\
\hline \multicolumn{14}{|l|}{ AUGUST } \\
\hline 01 & 1145 & $<0.014$ & $<0.035$ & 0.191 & 0.066 & - & -- & $<0.022$ & .. & $<0.018$ & $<0.016$ & $<0.011$ & -- \\
\hline 06 & 1200 & $<0.014$ & $<0.035$ & 0.134 & 0.072 & -- & - & $<0.022$ & -- & $<0.018$ & $<0.016$ & $<0.011$ & -- \\
\hline 08 & 1300 & $<0.014$ & $<0.035$ & 0.106 & 0.052 & -- & -- & $<0.022$ & -. & $<0.018$ & $<0.016$ & $<0.011$ & -. \\
\hline 12 & 1100 & $<0.014$ & $<0.035$ & 0.228 & 0.062 & -- & -- & $<0.022$ & -. & $<0.018$ & $<0.016$ & $<0.011$ & -- \\
\hline 15 & 1200 & $<0.014$ & $<0.035$ & 0.111 & 0.060 & -- & .- & $<0.022$ & .- & $<0.018$ & $<0.016$ & $<0.011$ & - \\
\hline 19 & 1115 & $<0.014$ & $<0.035$ & 0.109 & $<0.012$ & -- & -- & $<0.022$ & -- & $<0.018$ & $<0.016$ & $<0.011$ & -- \\
\hline 22 & 1230 & $<0.014$ & $<0.035$ & 0.097 & 0.024 & -- & -. & $<0.022$ & -- & $<0.018$ & $<0.016$ & $<0.011$ & .- \\
\hline 26 & 1245 & $<0.014$ & $<0.035$ & 0.092 & 0.043 & -- & -- & $<0.022$ & -. & $<0.018$ & $<0.016$ & $<0.011$ & -. \\
\hline 29 & 1200 & $<0.014$ & $<0.035$ & 0.102 & 0.036 & -- & -- & $<0.022$ & -. & $<0.018$ & $<0.016$ & $<0.011$ & -- \\
\hline \multicolumn{14}{|c|}{ SEPTEMBER 1991} \\
\hline 09 & 1100 & $<0.014$ & $<0.035$ & 0.154 & $<0.012$ & -- & -. & $<0.022$ & -. & $<0.018$ & $<0.016$ & $<0.011$ & -- \\
\hline 12 & 1100 & $<0.014$ & $<0.035$ & 0.123 & $<0.012$ & -- & -- & $<0.022$ & -- & $<0.018$ & $<0.016$ & $<0.011$ & -- \\
\hline 19 & 1130 & $<0.014$ & $<0.035$ & 0.148 & $<0.012$ & -- & -. & $<0.022$ & -- & $<0.018$ & $<0.016$ & $<0.011$ & -- \\
\hline 26 & 1200 & $<0.014$ & $<0.035$ & 0.151 & $<0.012$ & -- & -. & $<0.022$ & .. & $<0.018$ & $<0.016$ & $<0.011$ & -. \\
\hline
\end{tabular}




\begin{tabular}{|c|c|c|c|c|c|c|c|c|c|c|c|c|c|}
\hline Date & TIme & $\begin{array}{l}\text { Mala- } \\
\text { thion } \\
\text { diss } \\
(\mu g / L)\end{array}$ & $\begin{array}{c}\text { Methyl } \\
\text { para- } \\
\text { thion } \\
\text { diss } \\
(\mu g / L)\end{array}$ & $\begin{array}{l}\text { Meto- } \\
\text { lachlor } \\
\text { diss } \\
(\mu g / L)\end{array}$ & $\begin{array}{c}\text { Metri- } \\
\text { buzin } \\
\text { dise } \\
(\mu g /)\end{array}$ & $\begin{array}{l}\text { Molin- } \\
\text { ate } \\
\text { diss } \\
(\mu g /)\end{array}$ & $\begin{array}{l}\text { Naprop- } \\
\text { amide } \\
\text { diss } \\
(\mu g / L)\end{array}$ & $\begin{array}{l}\text { Para- } \\
\text { thion } \\
\text { diss } \\
(\mu g / L)\end{array}$ & $\begin{array}{c}\text { Pebul- } \\
\text { ate } \\
\text { dieo } \\
(\mu g / L)\end{array}$ & $\begin{array}{l}\text { Pendi- } \\
\text { meth- } \\
\text { alin } \\
\text { diss } \\
(\mu \mathrm{g} / \mathrm{L})\end{array}$ & $\begin{array}{l}\text { Perme- } \\
\text { thrin } \\
\text { disa } \\
(\mu g / L)\end{array}$ & $\begin{array}{l}\text { Pho- } \\
\text { rate } \\
\text { disa } \\
(\mu g /)\end{array}$ & $\begin{array}{c}\text { Prome- } \\
\text { ton } \\
\text { diea } \\
(\mu g / L)\end{array}$ \\
\hline
\end{tabular}

OCTOBER

\begin{tabular}{|c|c|c|c|c|c|c|c|c|c|c|c|c|c|}
\hline & & & & & & & & & & & & & \\
\hline 03 & 1000 & $<0.014$ & $<0.035$ & 0.065 & $<0.012$ & -- & -- & $<0.022$ & -- & $<0.018$ & $<0.016$ & $<0.011$ & -- \\
\hline 08 & 1100 & $<0.014$ & $<0.035$ & 0.090 & $<0.012$ & -- & -- & $<0.022$ & -- & $<0.018$ & $<0.016$ & $<0.011$ & -- \\
\hline 17 & 1100 & $<0.014$ & $<0.035$ & 0.067 & $<0.012$ & -- & -- & $<0.022$ & -- & $<0.018$ & $<0.016$ & $<0.011$ & -- \\
\hline 24 & 1100 & $<0.014$ & $<0.035$ & 0.012 & $<0.012$ & -- & -. & $<0.022$ & -- & $<0.018$ & $<0.016$ & $<0.011$ & -- \\
\hline 28 & 1130 & $<0.014$ & $<0.035$ & 0.047 & $<0.012$ & -- & -- & $<0,022$ & -- & $<0.018$ & $<0.016$ & $<0.011$ & -. \\
\hline 30 & 1130 & $<0.014$ & $<0.035$ & 0.265 & $<0.012$ & -- & -- & $<0.022$ & - & $<0.018$ & $<0.016$ & $<0.011$ & - \\
\hline NOVEME & & & & & & & & & & & & & \\
\hline 04 & 1130 & $<0.014$ & $<0.035$ & 0.129 & $<0.012$ & -- & -- & $<0.022$ & - & $<0.018$ & $<0.016$ & $<0.011$ & - \\
\hline 13 & 1025 & $<0.014$ & $<0.035$ & 0.091 & $<0.012$ & 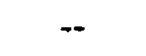 & -- & $<0.022$ & -- & $<0.018$ & $<0.016$ & $<0.011$ & -. \\
\hline 27 & 1200 & $<0.014$ & $<0.035$ & 0.154 & 0.009 & -. & -- & $<0.022$ & -- & $<0.018$ & $<0.016$ & $<0.011$ & -- \\
\hline DECEMB & & & & & & & & & & & & & \\
\hline 18 & 1200 & $<0.014$ & $<0.035$ & 0.130 & 0.004 & -- & -- & $<0.022$ & -- & $<0.018$ & $<0.016$ & $<0.011$ & - \\
\hline JANUAR & & & & & & & & & & & & & \\
\hline 02 & 1200 & $<0.014$ & $<0.035$ & 0.130 & 0.005 & - & -- & $<0.022$ & -- & $<0.018$ & $<0.016$ & $<0.011$ & - \\
\hline 16 & 1235 & $<0.014$ & $<0.035$ & 0.110 & $<0.012$ & $<0.007$ & $<0.010$ & $<0.022$ & $<0.009$ & $<0.018$ & $<0.016$ & $<0.011$ & 0.023 \\
\hline 30 & 1243 & $<0.014$ & $<0.035$ & 0.080 & $<0.012$ & $<0.007$ & $<0.010$ & $<0.022$ & $<0.009$ & $<0.018$ & $<0.016$ & $<0.011$ & 0.031 \\
\hline FEBRU, & & & & & & & & & & & & & \\
\hline 13 & 1240 & $<0.014$ & $<0.035$ & 0.062 & $<0.012$ & $<0.007$ & $<0.010$ & $<0.022$ & $<0.009$ & $<0.018$ & $<0.016$ & $<0.011$ & 0.023 \\
\hline 27 & 1200 & $<0.014$ & $<0.035$ & 0.070 & $<0.012$ & $<0.007$ & $<0.010$ & $<0.022$ & $<0.009$ & $<0.018$ & $<0.016$ & $<0.011$ & 0.024 \\
\hline MARCH & & & & & & & & & & & & & \\
\hline 05 & 1130 & $<0.014$ & $<0.035$ & 0.099 & $<0.012$ & $<0.007$ & $<0.010$ & $<0.022$ & $<0.009$ & $<0.018$ & $<0.016$ & $<0.011$ & 0.025 \\
\hline 12 & 1115 & $<0.014$ & $<0.035$ & 0.085 & $<0.012$ & $<0.007$ & $<0.010$ & $<0.022$ & $<0.009$ & $<0.018$ & $<0.016$ & $<0.011$ & 0.023 \\
\hline 19 & 1133 & $<0.014$ & $<0.035$ & 0.049 & $<0.012$ & $<0.007$ & $<0.010$ & $<0.022$ & $<0.009$ & $<0.018$ & $<0.016$ & $<0.011$ & 0.027 \\
\hline 26 & 1128 & $<0.014$ & $<0.035$ & 0.110 & $<0.012$ & $<0.007$ & $<0.010$ & $<0.022$ & $<0.009$ & $<0.018$ & $<0.016$ & $<0.011$ & 0.023 \\
\hline APRIL & & & & & & & & & & & & & \\
\hline 02 & 1135 & $<0.014$ & $<0.035$ & 0.170 & $<0.012$ & $<0.007$ & $<0.010$ & $<0.010$ & $<0.009$ & $<0.018$ & $<0.016$ & $<0.011$ & 0.023 \\
\hline 09 & 1102 & $<0.014$ & $<0.035$ & 0.130 & $<0.012$ & $<0.007$ & $<0.010$ & $<0.010$ & $<0.009$ & $<0.018$ & $<0.016$ & $<0.011$ & 0.026 \\
\hline 16 & 1112 & $<0.014$ & $<0.035$ & 0.300 & 0.024 & $<0.007$ & $<0.010$ & $<0.010$ & $<0.009$ & $<0.018$ & $<0.016$ & $<0.011$ & 0.034 \\
\hline 20 & 1030 & $<0.014$ & $<0.035$ & 0.620 & 0.015 & $<0.007$ & $<0.010$ & $<0.010$ & $<0.009$ & $<0.018$ & $<0.016$ & $<0.011$ & 0.026 \\
\hline 24 & 1035 & $<0.014$ & $<0.035$ & 2.20 & 0.140 & $<0.007$ & $<0.010$ & $<0.010$ & $<0.009$ & $<0.018$ & $<0.016$ & $<0.011$ & 0.035 \\
\hline 29 & 1100 & $<0.014$ & $<0.035$ & 1.00 & 0.074 & $<0.007$ & $<0.010$ & $<0.010$ & $<0.009$ & $<0.018$ & $<0.016$ & $<0.011$ & 0.028 \\
\hline
\end{tabular}

APRIL 1991

\begin{tabular}{|c|c|c|c|c|c|c|c|c|c|c|c|c|c|}
\hline 10 & 1200 & - & -- & -- & -. & -- & -- & -. & -- & -- & -. & .- & - \\
\hline 18 & 1045 & -. & - & -- & -. & -- & -- & -- & -- & -- & -- & -- & - \\
\hline 23 & 1110 & -- & -- & -- & - & -- & -- & -- & -- & -- & -- & -- & - \\
\hline \multicolumn{14}{|l|}{ MAY } \\
\hline 01 & 1040 & $<0.014$ & $<0.035$ & 0.098 & $<0.012$ & -- & -. & $<0.022$ & -- & $<0.018$ & 0.025 & $<0.011$ & - \\
\hline 07 & 1022 & $<0.014$ & $<0.035$ & 0.088 & $<0.012$ & -. & .. & $<0.022$ & -- & $<0.018$ & $<0.016$ & $<0.011$ & -- \\
\hline 14 & 1045 & -- & -- & -- & -- & -- & - & -- & -- & -- & -- & -- & - \\
\hline 21 & 1045 & $<0.014$ & $<0.035$ & 0.721 & 0.010 & -- & -- & $<0.022$ & - & 0.002 & 0.003 & $<0.011$ & .- \\
\hline 29 & 1030 & $<0.014$ & $<0.035$ & 0.168 & $<0.012$ & -- & $\ldots$ & $<0.022$ & -. & $<0.018$ & $<0.016$ & $<0.011$ & -. \\
\hline \multicolumn{14}{|l|}{ JUNE } \\
\hline 04 & 1135 & $<0.014$ & $<0.035$ & 0.495 & 0.018 & -- & -. & $<0.022$ & - & $<0.018$ & $<0.016$ & $<0.011$ & -- \\
\hline 11 & 1045 & $<0.014$ & $<0.035$ & 0.540 & 0.023 & -- & -- & $<0.022$ & -- & $<0.018$ & $<0.016$ & $<0.011$ & -. \\
\hline 18 & 1040 & $<0.014$ & $<0.035$ & 0.827 & 0.067 & -- & -- & $<0.022$ & -- & $<0.018$ & $<0.016$ & $<0.011$ & -- \\
\hline 25 & 0946 & $<0.014$ & $<0.035$ & 0.464 & 0.018 & -- & -. & $<0.022$ & -- & $<0.018$ & $<0.016$ & $<0.011$ & -- \\
\hline
\end{tabular}


Table 8.-Pesticide results from solid-phase extraction 1-liter method-Contlnued

\begin{tabular}{|c|c|c|c|c|c|c|c|c|c|c|c|c|c|}
\hline Date & Time & $\begin{array}{c}\text { Mala- } \\
\text { thion } \\
\text { diss } \\
(\mu g / L)\end{array}$ & $\begin{array}{l}\text { Methyl } \\
\text { pere- } \\
\text { thlon } \\
\text { diss } \\
(\mu g / L)\end{array}$ & $\begin{array}{l}\text { Meto- } \\
\text { lachlor } \\
\text { diss } \\
(\mu g / L)\end{array}$ & $\begin{array}{l}\text { Metri- } \\
\text { buzin } \\
\text { diss } \\
(\mu g / L)\end{array}$ & $\begin{array}{c}\text { Molin- } \\
\text { ele } \\
\text { diss } \\
(\mu g / L)\end{array}$ & $\begin{array}{l}\text { Neprop- } \\
\text { amide } \\
\text { diss } \\
(\mu g / L)\end{array}$ & $\begin{array}{l}\text { Para- } \\
\text { thion } \\
\text { diss } \\
(\mu g / 2)\end{array}$ & $\begin{array}{c}\text { Pebui- } \\
\text { ate } \\
\text { diss } \\
(\mu g / L)\end{array}$ & $\begin{array}{c}\text { Pendl- } \\
\text { moth- } \\
\text { elin } \\
\text { diss } \\
(\mu \mathrm{g} / \mathrm{L})\end{array}$ & $\begin{array}{c}\text { Perme- } \\
\text { thrin } \\
\text { diss } \\
(\mu g / L)\end{array}$ & $\begin{array}{l}\text { Pho- } \\
\text { rate } \\
\text { diss } \\
(\mu g / L)\end{array}$ & $\begin{array}{c}\text { Promo- } \\
\text { ton } \\
\text { diss } \\
(\mu g / L)\end{array}$ \\
\hline \multicolumn{14}{|c|}{ 03612500 - Ohio River at Dam 53 near Grand Chsin, Ill.-Continued } \\
\hline \multicolumn{14}{|c|}{ sets } \\
\hline 01 & 0950 & $<0.014$ & $<0.035$ & 0.226 & $<0.012$ & -- & -- & $<0.022$ & -- & $<0.018$ & $<0.016$ & $<0.011$ & -- \\
\hline 09 & 1030 & $<0.014$ & $<0.035$ & 0.201 & $<0.012$ & - & -- & $<0.022$ & -- & $<0.018$ & $<0.016$ & $<0.011$ & -- \\
\hline 15 & 1100 & $<0.014$ & $<0.035$ & 0.173 & $<0.012$ & -- & -- & $<0.022$ & -- & $<0.018$ & $<0.016$ & $<0.011$ & -- \\
\hline 22 & 1040 & -- & -- & -- & -- & -- & -- & - & -- & -- & - & - & -- \\
\hline 29 & 1110 & -- & -- & -- & -- & - & -- & - & -- & -- & -- & -- & -. \\
\hline \multicolumn{14}{|l|}{ AUGUST } \\
\hline 05 & 1050 & -- & -- & -- & -- & -- & -- & - & -- & -- & -- & - & -- \\
\hline 12 & 1030 & $<0.014$ & $<0.035$ & 0.099 & $<0.012$ & -- & -- & $<0.022$ & -- & $<0.018$ & $<0.016$ & $<0.011$ & -- \\
\hline 20 & 1030 & $<0.014$ & $<0.035$ & 0.062 & $<0.012$ & -- & - & $<0.022$ & -- & $<0.018$ & $<0.016$ & $<0.011$ & - \\
\hline 27 & 0945 & $<0.014$ & $<0.035$ & 0.032 & $<0.012$ & -- & -- & $<0.022$ & -- & $<0.018$ & $<0.016$ & $<0.011$ & - \\
\hline \multicolumn{14}{|c|}{ SEPTEMBER } \\
\hline 03 & 1040 & $<0.014$ & $<0.035$ & 0.036 & $<0.012$ & -- & - & $<0.022$ & -- & $<0.018$ & $<0.016$ & $<0.011$ & -- \\
\hline 10 & 1030 & $<0.014$ & $<0.035$ & 0.051 & $<0.012$ & -- & -- & $<0.022$ & -- & $<0.018$ & $<0.016$ & $<0.011$ & -- \\
\hline 17 & 0952 & $<0.014$ & $<0.035$ & 0.048 & $<0.012$ & -- & -- & $<0.022$ & -- & $<0.018$ & $<0.016$ & $<0.011$ & -- \\
\hline 23 & 1040 & $<0.014$ & $<0.035$ & 0.062 & $<0.012$ & -- & -- & $<0.022$ & -- & $<0.018$ & $<0.016$ & $<0.011$ & - \\
\hline 30 & 1122 & $<0.014$ & $<0.035$ & 0.038 & $<0.012$ & -- & -- & $<0.022$ & -- & $<0.018$ & $<0.016$ & $<0.011$ & -- \\
\hline \multicolumn{14}{|c|}{ OCTOBER 1991} \\
\hline 07 & 1125 & $<0.014$ & $<0.035$ & 0.020 & $<0.012$ & -- & -- & $<0.022$ & -- & $<0.018$ & $<0.016$ & $<0.011$ & -- \\
\hline 15 & 0955 & $<0.014$ & $<0.035$ & 0.026 & $<0.012$ & -- & -- & $<0.022$ & -- & $<0.018$ & $<0.016$ & $<0.011$ & - \\
\hline 21 & 1020 & $<0.014$ & $<0.035$ & 0.027 & $<0.012$ & -- & -- & $<0.022$ & -- & $<0.018$ & $<0.016$ & $<0.011$ & -- \\
\hline 29 & 0930 & $<0.014$ & $<0.035$ & 0.057 & $<0.012$ & -- & -- & $<0.022$ & - & $<0.018$ & $<0.016$ & $<0.011$ & - \\
\hline \multicolumn{14}{|c|}{ NOVEMBER } \\
\hline 06 & 1040 & -- & -- & -- & -- & -- & -- & -- & -- & -- & - & - & -- \\
\hline 20 & 0920 & $<0.014$ & $<0.035$ & 0.058 & $<0.012$ & -- & -- & $<0.022$ & -- & $<0.018$ & $<0.016$ & $<0.011$ & - \\
\hline \multicolumn{14}{|l|}{ DECEMBER } \\
\hline 05 & 1045 & $<0.014$ & $<0.035$ & 0.054 & $<0.012$ & -- & -- & $<0.022$ & -- & $<0.018$ & $<0.016$ & $<0.011$ & -- \\
\hline 17 & 1010 & $<0.014$ & $<0.035$ & 0.019 & $<0.012$ & -- & -- & $<0.022$ & -. & $<0.018$ & $<0.016$ & $<0.011$ & -- \\
\hline \multicolumn{14}{|c|}{ JANUARY 1992} \\
\hline 02 & 1005 & $<0.014$ & $<0.035$ & 0.029 & $<0.012$ & -- & -- & $<0.022$ & -- & $<0.018$ & $<0.016$ & $<0.011$ & -- \\
\hline 21 & 1030 & $<0.014$ & $<0.035$ & 0.038 & $<0.012$ & $<0.007$ & $<0.010$ & $<0.022$ & $<0.009$ & $<0.018$ & $<0.016$ & $<0.011$ & 0.011 \\
\hline \multicolumn{14}{|l|}{ FEBRUARY } \\
\hline 04 & 1125 & $<0.014$ & $<0.035$ & 0.022 & $<0.012$ & $<0.007$ & $<0.010$ & $<0.022$ & $<0.009$ & $<0.018$ & $<0.016$ & $<0.011$ & 0,007 \\
\hline 18 & 1025 & $<0.014$ & $<0.035$ & 0.023 & $<0.012$ & $<0.007$ & $<0.010$ & $<0.022$ & $<0.009$ & $<0.018$ & $<0.016$ & $<0.011$ & $<0.002$ \\
\hline \multicolumn{14}{|c|}{ MARCH 1992} \\
\hline 03 & 1025 & $<0.014$ & $<0.035$ & 0.015 & $<0.012$ & $<0.007$ & $<0.010$ & $<0.022$ & $<0.009$ & $<0.018$ & $<0.016$ & $<0.011$ & 0.012 \\
\hline 11 & 1030 & $<0.014$ & $<0.035$ & 0.012 & $<0.012$ & $<0.007$ & $<0.010$ & $<0.022$ & $<0.009$ & $<0.018$ & $<0.016$ & $<0.011$ & 0.010 \\
\hline 16 & 1040 & $<0.014$ & $<0.035$ & 0.016 & $<0.012$ & $<0.007$ & $<0.010$ & $<0.022$ & $<0.009$ & $<0.018$ & $<0.016$ & $<0.011$ & 0.012 \\
\hline 24 & 1020 & $<0.014$ & $<0.035$ & 0.032 & $<0.012$ & $<0.007$ & $<0.010$ & $<0.022$ & $<0.009$ & $<0.018$ & $<0.016$ & $<0.011$ & 0.012 \\
\hline 31 & 1030 & $<0.014$ & $<0.035$ & 0.030 & $<0.012$ & $<0.007$ & $<0.010$ & $<0.022$ & $<0.009$ & $<0.018$ & $<0.016$ & $<0.011$ & 0.010 \\
\hline \multicolumn{14}{|l|}{ APRIL } \\
\hline 07 & 1015 & $<0.014$ & $<0.035$ & 0.043 & $<0.012$ & $<0.007$ & $<0.010$ & $<0.022$ & $<0.009$ & $<0.018$ & $<0.016$ & $<0.011$ & 0.011 \\
\hline 14 & 1035 & $<0.014$ & $<0.035$ & 0.034 & $<0.012$ & $<0.007$ & $<0.010$ & $<0.022$ & $<0.009$ & $<0.018$ & $<0.016$ & $<0.011$ & 0.010 \\
\hline 22 & 1040 & $<0.014$ & $<0.035$ & 0.430 & 0.009 & $<0.007$ & $<0.010$ & $<0.022$ & $<0.009$ & $<0.018$ & $<0.016$ & $<0.011$ & 0.015 \\
\hline 28 & 1104 & $<0.014$ & $<0.035$ & 0.690 & 0.030 & $<0.007$ & $<0.010$ & $<0.022$ & $<0.009$ & $<0.018$ & $<0.016$ & $<0.011$ & 0.019 \\
\hline \multicolumn{14}{|l|}{ MAY } \\
\hline 06 & 0953 & $<0.014$ & $<0.035$ & 0.230 & 0.010 & $<0.007$ & $<0.010$ & $<0.022$ & $<0.009$ & $<0.018$ & $<0.016$ & $<0.011$ & 0.012 \\
\hline 12 & 0955 & $<0.014$ & $<0.035$ & 0.170 & 0.008 & $<0.007$ & $<0.010$ & $<0.022$ & $<0.009$ & $<0.018$ & $<0.016$ & $<0.011$ & 0.012 \\
\hline
\end{tabular}


Table 8.-Pesticide results from solid-phase extraction 1-liter method--Continued

\begin{tabular}{|c|c|c|c|c|c|c|c|c|c|c|c|c|c|}
\hline Date & Time & $\begin{array}{l}\text { Maia- } \\
\text { Ihion } \\
\text { diss } \\
(\mu g / L)\end{array}$ & $\begin{array}{c}\text { Methyi } \\
\text { para- } \\
\text { thion } \\
\text { disa } \\
\text { (ugh) }\end{array}$ & $\begin{array}{l}\text { Meto- } \\
\text { lachlor } \\
\text { diss } \\
(\mu g /)\end{array}$ & $\begin{array}{c}\text { Metri- } \\
\text { buzin } \\
\text { diss } \\
(\mu g / L)\end{array}$ & $\begin{array}{l}\text { Molin- } \\
\text { ate } \\
\text { diss } \\
\text { ( } \mu g / L)\end{array}$ & $\begin{array}{l}\text { Naprop- } \\
\text { emide } \\
\text { diss } \\
\text { ( } \mu \mathrm{g} / \mathrm{L})\end{array}$ & $\begin{array}{l}\text { Para- } \\
\text { thion } \\
\text { diss } \\
(\mu g / L)\end{array}$ & $\begin{array}{l}\text { Pebul- } \\
\text { ate } \\
\text { diss } \\
\text { ( } \mu \mathrm{g} / \text { ) }\end{array}$ & $\begin{array}{l}\text { Pendi- } \\
\text { meth- } \\
\text { alin } \\
\text { diss } \\
\text { ( } \mu g / L)\end{array}$ & $\begin{array}{l}\text { Perme- } \\
\text { thrin } \\
\text { diss } \\
(\mu g / L)\end{array}$ & $\begin{array}{l}\text { Pho- } \\
\text { rate } \\
\text { disa } \\
\text { (ugh) }\end{array}$ & 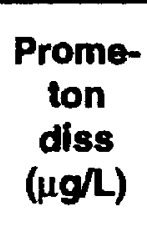 \\
\hline \multicolumn{14}{|c|}{03612500 - Ohio River at Dam 53 near Grand Chain, III.-Continued } \\
\hline \multicolumn{14}{|l|}{ MAY (cont.) } \\
\hline 18 & 1025 & $<0.014$ & $<0.035$ & 0.100 & $<0.012$ & $<0.007$ & $<0.010$ & $<0.022$ & $<0.009$ & $<0.018$ & $<0.016$ & $<0,011$ & 0.019 \\
\hline 27 & 1030 & $<0.014$ & $<0.035$ & 0.260 & 0.005 & $<0.007$ & $<0.010$ & $<0.022$ & $<0.009$ & $<0.018$ & $<0.016$ & $<0.011$ & 0.016 \\
\hline \multicolumn{14}{|l|}{ JUNE 1992} \\
\hline 02 & 1045 & $<0.014$ & $<0.035$ & 0.690 & 0.012 & $<0.007$ & $<0.010$ & $<0.022$ & $<0.009$ & $<0.018$ & $<0.016$ & $<0.011$ & 0.028 \\
\hline 09 & 1105 & $<0.014$ & $<0.035$ & 0.190 & $<0.012$ & $<0.007$ & $<0.010$ & $<0.022$ & $<0.009$ & $<0.018$ & $<0.016$ & $<0.011$ & 0.015 \\
\hline 16 & 1105 & $<0.014$ & $<0.035$ & 0.082 & 0.005 & $<0.007$ & $<0.010$ & $<0.022$ & $<0.009$ & $<0.018$ & $<0.016$ & $<0.011$ & 0.020 \\
\hline 23 & 1023 & $<0.014$ & $<0.035$ & 0.170 & 0.005 & $<0.007$ & $<0.010$ & $<0.022$ & $<0.009$ & $<0.018$ & $<0.016$ & $<0.011$ & 0.021 \\
\hline 29 & 1235 & $<0.014$ & $<0.035$ & 0.240 & 0.007 & $<0.007$ & $<0.010$ & $<0.022$ & $<0.009$ & 0.006 & $<0.016$ & $<0.011$ & 0.027 \\
\hline \multicolumn{14}{|l|}{ JULY } \\
\hline 08 & 1012 & $<0.014$ & $<0.035$ & 0.210 & 0.009 & $<0.007$ & $<0.010$ & $<0.022$ & $<0.009$ & 0.007 & $<0.016$ & $<0.011$ & 0.028 \\
\hline 14 & 1025 & $<0.014$ & $<0.035$ & 0.310 & 0.008 & $<0.007$ & $<0.010$ & $<0.022$ & $<0.009$ & $<0.018$ & $<0.016$ & $<0.011$ & 0.038 \\
\hline 23 & 1040 & $<0.014$ & $<0.035$ & 0.450 & 0.018 & $<0.007$ & $<0.010$ & $<0.022$ & $<0.009$ & $<0.018$ & $<0.016$ & $<0.011$ & 0.033 \\
\hline 28 & 1025 & $<0.014$ & $<0.035$ & 0.550 & 0.033 & $<0.007$ & $<0.010$ & $<0.022$ & $<0.009$ & 0.006 & $<0.016$ & $<0.011$ & 0.033 \\
\hline
\end{tabular}

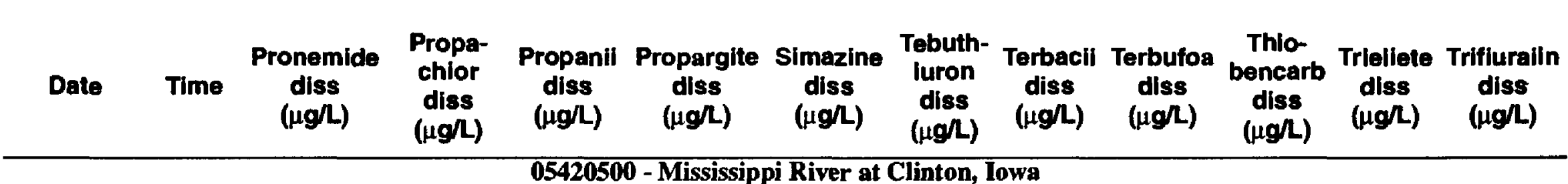

APRIL 1991

\begin{tabular}{|c|c|c|c|c|c|c|c|c|c|c|c|c|}
\hline 02 & 1040 & -- & -- & - & -- & -- & -- & -- & -- & - & - & - \\
\hline 11 & 1100 & -- & -- & -- & -- & -- & - & -- & - & -- & -- & -- \\
\hline 16 & 1150 & - & - & -- & - & - & -- & -- & -- & -- &.- & - \\
\hline 23 & 1030 & -- & -- & - & -- & -- & .. & -- & -- & -- & -- & - \\
\hline \multicolumn{13}{|l|}{ MAY } \\
\hline 01 & 1150 & -- & - & - & -- & $\cdots$ & -- & -- & -- &.- & $\cdots$ & $\cdots$ \\
\hline 07 & 1115 & -- & $<0.015$ & - & 0.041 & 0.008 & -- & -- & $<0.012$ & -- & -- & $<0.012$ \\
\hline 10 & 1030 & -- & - & -- & - & - & -- & -- & -- & -- & - & -- \\
\hline 14 & 1120 & -- & -- & - & - & - & -- & -- & -- & -- & - & -- \\
\hline 17 & 1045 & -- & $<0.015$ & $\sim$ & $<0.006$ & $<0.008$ & -- & -- & $<0.012$ & -- & - & $<0.012$ \\
\hline 21 & 1140 & -- & -- & -- & -- & - & -- & -- & -- & -- & -- & - \\
\hline 24 & 1205 & -- & 0.005 & -- & $<0.006$ & 0.013 & -- & -- & $<0.012$ & -- & -- & $<0.012$ \\
\hline 28 & 1210 & - & $<0.015$ & $\cdots$ & $<0.006$ & 0.015 & -- & -- & $<0.012$ & $-\cdot$ & -- & $<0.012$ \\
\hline 31 & 1015 & -- & $<0.015$ & -- & $<0.006$ & 0.017 & -- & -- & $<0.012$ & - & -- & $<0.012$ \\
\hline \multicolumn{13}{|l|}{ JUNE 1991} \\
\hline 04 & 1300 & -- & $<0.015$ & -- & $<0.006$ & 0.013 & -- & -- & $<0.012$ & -- & -- & $<0.012$ \\
\hline 07 & 1100 & - & $<0.015$ & -- & $<0.006$ & 0.010 & -- & -- & $<0.012$ & -- & -- & $<0.012$ \\
\hline 10 & 1130 & -- & $<0.015$ & -- & $<0.006$ & 0.015 & -- & -- & $<0.012$ & -- & -- & $<0.012$ \\
\hline 13 & 1410 & - & 0.003 & -- & $<0.006$ & 0.023 & -- & -- & $<0.012$ & -- & - & $<0.012$ \\
\hline 17 & 1040 & - & 0.006 & -- & $<0.006$ & 0.036 & -- & - & $<0.012$ & -- & -- & $<0.012$ \\
\hline 20 & 1345 & - & 0.005 & -- & $<0.006$ & 0.035 & - & -- & $<0.012$ & -- & -- & $<0.012$ \\
\hline 24 & 1055 & -- & 0.008 & -- & $<0.006$ & 0.025 & -- & -- & $<0.012$ & - & -- & $<0.012$ \\
\hline 27 & 0915 & - & 0.006 & - & $<0.006$ & 0.020 & -- & $\cdots$ & $<0.012$ & -- & -- & $<0.012$ \\
\hline \multicolumn{13}{|l|}{ JULY } \\
\hline 01 & 1025 & - & 0.002 & -- & $<0.006$ & 0.022 & -- & -- & $<0.012$ & -- & -- & $<0.012$ \\
\hline 05 & 1205 & - & $<0.015$ & -- & $<0.006$ & 0.017 & - & -- & $<0.012$ & -. & -- & $<0.012$ \\
\hline 08 & 1130 & -- & $<0.015$ & -- & $<0.006$ & 0.011 & -- & -- & $<0.012$ & -- & -- & $<0.012$ \\
\hline 11 & 1300 & -- & -- & -- & -- & -- & -- & -- & -- & $-\bullet$ & -- & -- \\
\hline 15 & 1025 & -- & $<0.015$ & -- & $<0.006$ & 0.019 & -- & -- & $<0.012$ & - & -- & $<0.012$ \\
\hline 18 & 1200 & -- & $-\cdot$ & - & -- & $\cdots$ & $\cdots$ & -- & -- & -- & - & -- \\
\hline
\end{tabular}


Table 8.-Pesticide results from solid-phase extraction 1-liter method-Continued

\begin{tabular}{|c|c|c|c|c|c|c|c|c|c|c|c|c|}
\hline Date & Time & $\begin{array}{c}\text { Pronamide } \\
\text { diss } \\
\text { ( } \mu g / \text { L) }\end{array}$ & $\begin{array}{l}\text { Propa- } \\
\text { chlor } \\
\text { diss } \\
(\mu g / L)\end{array}$ & $\begin{array}{c}\text { Propanii } \\
\text { diss } \\
(\mu g / L)\end{array}$ & $\begin{array}{c}\text { Propargite } \\
\text { dlse } \\
\text { ( } \mu g / \mathrm{L})\end{array}$ & $\begin{array}{c}\text { Simazina } \\
\text { diss } \\
(\mu g / L)\end{array}$ & $\begin{array}{l}\text { Tebuth- } \\
\text { Iuron } \\
\text { diss } \\
\text { (ugh) }\end{array}$ & $\begin{array}{c}\text { Terbacil } \\
\text { diss } \\
\text { (ught) }\end{array}$ & $\begin{array}{c}\text { Terbutos } \\
\text { diss } \\
(\mu g / L)\end{array}$ & $\begin{array}{l}\text { Thio- } \\
\text { bencerb } \\
\text { dlss } \\
(\mu g / \text { ) }\end{array}$ & $\begin{array}{c}\text { Triallata } \\
\text { diss } \\
(\mu \mathrm{g} / \mathrm{L})\end{array}$ & $\begin{array}{c}\text { Trifiuralin } \\
\text { diss } \\
(\mu g / L)\end{array}$ \\
\hline
\end{tabular}

05420500 - Mississippi River at Clinton, Iowa-Continued

JULY (cont.)

\begin{tabular}{|c|c|c|c|c|c|c|c|c|c|c|c|c|}
\hline 22 & 1050 & -- & -- & -- & -- & -. & $\cdots$ & - & -- & -- & - & - \\
\hline 29 & 1230 & -- & $<0.015$ & -- & $<0.006$ & 0.025 & -- & -- & $<0.012$ & - & - & $<0.012$ \\
\hline \multicolumn{13}{|c|}{ AUGUST 1991} \\
\hline 06 & 1240 & -- & $<0.015$ & -- & $<0.006$ & 0.018 & -- & - & $<0.012$ & -- & - & $<0.012$ \\
\hline 12 & 1125 & -- & $<0.015$ & -- & $<0.006$ & 0.018 & -- & -- & $<0.012$ & $\cdots$ & -- & $<0.012$ \\
\hline 19 & 1145 & -- & $<0.015$ & -- & $<0.006$ & 0.016 & -- & -- & $<0.012$ & -- & - & $<0.012$ \\
\hline 26 & 1205 & -- & $<0.015$ & -. & $<0.006$ & 0.012 & -- & -- & $<0.012$ & - & -- & $<0.012$ \\
\hline \multicolumn{13}{|c|}{ SEPTEMBER } \\
\hline 03 & 1200 & -- & $<0.015$ & - & $<0.006$ & 0.011 & -- & -- & $<0.012$ & - & - & $<0.012$ \\
\hline 09 & 1500 & -- & $<0.015$ & - & $<0.006$ & 0.011 & - & -- & $<0.012$ & $\cdots$ & -- & $<0.012$ \\
\hline 17 & 1215 & -- & $<0.015$ & -- & $<0.006$ & 0.009 & -- & -- & $<0.012$ & -- & - & $<0.012$ \\
\hline 24 & 1215 & -- & $<0.015$ & -- & $<0.006$ & 0.011 & -- & -- & $<0.012$ & -- & -- & $<0.012$ \\
\hline \multicolumn{13}{|c|}{ OCTOBER } \\
\hline 01 & 1200 & -- & -- & - & -- & - & -- & -- & -- & -- & -- & - \\
\hline 07 & 1230 & -- & $<0.015$ & -- & $<0.006$ & 0.006 & - & -- & $<0.012$ & -- & -- & $<0.012$ \\
\hline 15 & 1300 & -- & $<0.015$ & -- & $<0.006$ & 0.009 & -- & -- & $<0.012$ & -- & -- & $<0.012$ \\
\hline 22 & 1230 & -- & $<0.015$ & -- & $<0.006$ & 0.006 & - & -- & $<0.012$ & -- & - & $<0.012$ \\
\hline 29 & 1330 & - & $<0.015$ & -- & $<0.006$ & 0.007 & - & -- & $<0.012$ & $\cdots$ & $\cdots$ & $<0.012$ \\
\hline \multicolumn{13}{|c|}{ NOVEMBER 1991} \\
\hline 05 & 1245 & -- & $<0.015$ & -- & $<0.006$ & 0.007 & - & $\cdots$ & $<0.012$ & -- & -- & $<0.012$ \\
\hline 12 & 1255 & -- & $<0.015$ & -- & $<0.006$ & 0.009 & - & -- & $<0.012$ & -- & -- & $<0.012$ \\
\hline 26 & 1320 & -- & $<0.015$ & -- & $<0.006$ & $<0.008$ & - & - & $<0.012$ & $\cdots$ & $\cdots$ & $<0.012$ \\
\hline \multicolumn{13}{|c|}{ DECEMBER } \\
\hline 09 & 1200 & $-\cdots$ & $<0.015$ & -- & $<0.006$ & 0.006 & -- & -- & $<0.012$ & -- & -- & $<0.012$ \\
\hline 22 & 1330 & -- & $<0.015$ & -- & $<0.006$ & 0.004 & - & -- & $<0.012$ & -- & -- & $<0.012$ \\
\hline \multicolumn{13}{|c|}{ JANUARY 1992} \\
\hline 07 & 1210 & $<0.009$ & $<0.015$ & $<0.016$ & $<0.006$ & 0.005 & $<0.012$ & $<0.030$ & $<0.012$ & $<0.008$ & $<0.008$ & $<0.012$ \\
\hline 22 & 1340 & $<0.009$ & $<0.015$ & $<0.016$ & $<0.006$ & 0.004 & $<0.012$ & $<0.030$ & $<0.012$ & $<0.008$ & $<0.008$ & $<0.012$ \\
\hline \multicolumn{13}{|c|}{ FEBRUARY } \\
\hline 03 & 1245 & $<0.009$ & $<0.015$ & $<0.016$ & $<0.006$ & 0.004 & $<0.012$ & $<0.030$ & $<0.012$ & $<0.008$ & $<0.008$ & $<0.012$ \\
\hline 20 & 1230 & $<0.009$ & $<0.015$ & $<0.016$ & $<0.006$ & 0.005 & $<0.012$ & $<0.030$ & $<0.012$ & $<0.008$ & $<0.008$ & $<0.012$ \\
\hline \multicolumn{13}{|c|}{ MARCH 1992} \\
\hline 04 & 1100 & -- & - & -- & - & - & -- & -- & -- & - & - & -- \\
\hline 13 & 1200 & $<0.009$ & $<0.015$ & $<0.016$ & $<0.006$ & $<0.008$ & $<0.012$ & $<0.030$ & $<0.012$ & $<0.008$ & $<0.008$ & $<0.012$ \\
\hline 17 & 1400 & $<0.009$ & 0.002 & $<0.016$ & $<0.006$ & 0.006 & $<0.012$ & $<0.030$ & $<0.012$ & $<0.008$ & $<0.008$ & 0.003 \\
\hline 24 & 1125 & $<0.009$ & $<0.015$ & $<0.016$ & $<0.006$ & $<0.008$ & $<0.012$ & $<0.030$ & $<0.012$ & $<0.008$ & $<0.008$ & 0.003 \\
\hline 30 & 1155 & $<0.009$ & $<0.015$ & $<0.016$ & $<0.006$ & $<0.008$ & $<0.012$ & $<0.030$ & $<0.012$ & $<0.008$ & $<0.008$ & 0.003 \\
\hline \multicolumn{13}{|l|}{ APRIL } \\
\hline 06 & 1215 & $<0.009$ & $<0.015$ & $<0.016$ & $<0.006$ & 0.005 & 0.006 & $<0.030$ & $<0.012$ & $<0.008$ & $<0.008$ & $<0.012$ \\
\hline 14 & 1130 & $<0.009$ & $<0.015$ & $<0.016$ & $<0.006$ & 0.008 & 0.005 & $<0.030$ & $<0.012$ & $<0.008$ & $<0.008$ & $<0.012$ \\
\hline 24 & 1145 & $<0.009$ & $<0.015$ & $<0.016$ & $<0.006$ & 0.006 & $<0.012$ & $<0.030$ & $<0.012$ & $<0.008$ & $<0.008$ & $<0.012$ \\
\hline 28 & 1145 & $<0.009$ & $<0.015$ & $<0.016$ & $<0.006$ & 0.006 & $<0.012$ & $<0.030$ & $<0.012$ & $<0.008$ & $<0.008$ & $<0.012$ \\
\hline \multicolumn{13}{|l|}{ MAY } \\
\hline 05 & 1115 & $<0.009$ & $<0.015$ & $<0.016$ & $<0.006$ & 0.007 & $<0.012$ & $<0.030$ & $<0.012$ & $<0.008$ & $<0.008$ & $<0.012$ \\
\hline 12 & 1130 & $<0.009$ & $<0.015$ & $<0.016$ & $<0.006$ & 0.009 & $<0.012$ & $<0.030$ & $<0.012$ & $<0.008$ & $<0.008$ & $<0.012$ \\
\hline 19 & 1130 & $<0.009$ & $<0.015$ & $<0.016$ & $<0.006$ & 0.010 & $<0.012$ & $<0.030$ & $<0.012$ & $<0.008$ & $<0.008$ & 0.003 \\
\hline 26 & 1215 & $<0.009$ & $<0.015$ & $<0.016$ & $<0.006$ & 0.019 & $<0.012$ & $<0.030$ & $<0.012$ & $<0.008$ & $<0.008$ & 0.005 \\
\hline
\end{tabular}


Table 8.--Pesticide results from solid-phase extraction 1-liter method-Continued

\begin{tabular}{|c|c|c|c|c|c|c|c|c|c|c|c|c|}
\hline Date & Time & $\begin{array}{c}\text { Pronamide } \\
\text { diss } \\
(\mu g / L)\end{array}$ & $\begin{array}{l}\text { Propa- } \\
\text { chior } \\
\text { diss } \\
(\mu g / L)\end{array}$ & $\begin{array}{c}\text { Propanii } \\
\text { diss } \\
(\mu g / L)\end{array}$ & $\begin{array}{c}\text { Propargite } \\
\text { diss } \\
(\mu g / L)\end{array}$ & $\begin{array}{c}\text { Simazine } \\
\text { diss } \\
(\mu g / L)\end{array}$ & $\begin{array}{l}\text { Tebuth- } \\
\text { iuron } \\
\text { diss } \\
(\mu g / L)\end{array}$ & $\begin{array}{c}\text { Terbacii } \\
\text { diss } \\
(\mu g / L)\end{array}$ & $\begin{array}{c}\text { Terbufos } \\
\text { diss } \\
(\mu g / L)\end{array}$ & $\begin{array}{l}\text { Thio- } \\
\text { bencarb } \\
\text { diss } \\
(\mu g / L)\end{array}$ & $\begin{array}{c}\text { Trialiate } \\
\text { diss } \\
(\mu g / L)\end{array}$ & $\begin{array}{l}\text { Trifiuralin } \\
\text { diss } \\
(\mu g / L)\end{array}$ \\
\hline
\end{tabular}

APRIL 1991

\begin{tabular}{|c|c|c|c|c|c|c|c|c|c|c|c|c|}
\hline 05 & 1230 & -- & -- & 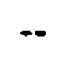 & -- & -- & - & -- & - & -- & -- & -. \\
\hline 17 & 1330 & -- & -- & -- & -- & -- & - & -- & - & -- & -- & -- \\
\hline 26 & 1000 & - & -- & -- & -- & - & - & -- & -- & -. & -. & -- \\
\hline \multicolumn{13}{|l|}{ MAY } \\
\hline 03 & 1545 & -- & $<0.016$ & -- & $<0.006$ & 0.033 & -. & $\therefore$ & $<0.015$ & -- & - & 0.002 \\
\hline 08 & 1245 & -- & 0.010 & -- & $<0.006$ & 0.115 & - & - & $<0.015$ & - & -- & 0.017 \\
\hline 10 & 1100 & -- & -- & -- & -- & - & -- & -- & -- & - & -- & -- \\
\hline 17 & 1145 & -- & $<0.016$ & -- & $<0.006$ & 0.026 & -- & -- & $<0.015$ & - & - & 0.005 \\
\hline 19 & 1115 & -- & 0.006 & -- & $<0.006$ & 0.088 & -- & -- & $<0.015$ &.- & -- & 0.010 \\
\hline 22 & 1715 & -- & 0.009 & -- & $<0.006$ & 0.131 & -- & -- & 0.012 & -- & -. & 0.009 \\
\hline 29 & 1130 & $\ldots$ & 0.011 & -- & $<0.006$ & 0.075 & -- & -- & $<0.015$ & -- & -- & 0.005 \\
\hline 31 & 1045 & -. & 0.010 & - & $<0.006$ & 0.084 & -- & -- & $<0.015$ & - & - & 0.003 \\
\hline \multicolumn{13}{|c|}{ JUNE 1991} \\
\hline 04 & 1215 & -- & 0.004 & -. & $<0.006$ & 0.102 & .- & -- & $<0.015$ & -- & -- & 0.003 \\
\hline 06 & 1200 & -- & $<0.016$ & -- & $<0.006$ & 0.081 & .. & -- & $<0.015$ & -- & -- & 0.004 \\
\hline 11 & 1345 & -. & $<0.016$ & -- & $<0.006$ & 0.119 & -- & -- & $<0.015$ & - & -- & $<0.012$ \\
\hline 14 & 1200 & -- & $<0.016$ & -- & $<0.006$ & 0.125 & - & -- & $<0.015$ & -- & - & $<0.012$ \\
\hline 18 & 1130 & -- & $<0.016$ & -- & $<0.006$ & 0.138 & -- & -- & $<0.015$ & $\ldots$ & -- & $<0.012$ \\
\hline 20 & 1130 & - & $<0.016$ & -- & $<0.006$ & 0.120 & -- & -- & $<0.015$ & -- & -- & $<0.012$ \\
\hline 24 & 1130 & -- & $<0.016$ & -- & $<0.006$ & 0.086 & -- & -- & $<0.015$ & .. & -- & $<0.012$ \\
\hline 27 & 1100 & -. & 0.004 & - & $<0.006$ & 0.070 & -- & -- & $<0.015$ & -- & -- & $<0.012$ \\
\hline \multicolumn{13}{|l|}{ JULY } \\
\hline 01 & 0930 & -- & $<0.016$ & -- & $<0.006$ & 0.084 & -- & -- & $<0.015$ & -- & -- & $<0.012$ \\
\hline 03 & 1100 & -- & $<0.016$ & -- & $<0.006$ & 0.079 & -- & -- & $<0.015$ & -- & -- & $<0.012$ \\
\hline 09 & 1000 & -- & $<0.016$ & -- & $<0.006$ & 0.067 & -- & -- & $<0.015$ & -- & -- & $<0.012$ \\
\hline 11 & 1045 & -- & $<0.016$ & -- & $<0.006$ & 0.068 & -- & -- & $<0.015$ & - & .. & $<0.012$ \\
\hline 18 & 1115 & -- & -- & - & -. & -- & -- & -- & .. & -. & .. & -- \\
\hline 25 & 1340 & - & $<0.016$ & -- & $<0.006$ & 0.043 & - & - & $<0.015$ & - & -- & $<0.012$ \\
\hline 31 & 1100 & -- & $<0.016$ & -- & $<0.006$ & 0.038 & -- & - & $<0.015$ & -- & -- & $<0.012$ \\
\hline \multicolumn{13}{|c|}{ AUGUST 1991} \\
\hline 06 & 1015 & -- & $<0.016$ & -- & $<0.006$ & 0.034 & -- & -- & $<0.015$ & -- & -- & $<0.012$ \\
\hline 13 & 1200 & - & $<0.016$ & - & $<0.006$ & 0.030 & -- & -- & $<0.015$ & -- & -- & $<0.012$ \\
\hline 21 & 1230 & -- & $<0.016$ & -- & $<0.006$ & 0.025 & -- & -- & $<0.015$ & -- & -- & $<0.012$ \\
\hline 28 & 1015 & -- & $<0.016$ & -- & $<0.006$ & 0.022 & -- & $\cdots$ & $<0.015$ & -- & -- & $<0.012$ \\
\hline \multicolumn{13}{|c|}{ SEPTEMBER } \\
\hline 03 & 1050 & - & $<0.016$ & - & $<0.006$ & 0.039 & -- & -- & $<0.015$ & -- & -- & $<0.012$ \\
\hline 10 & 1200 & -- & $<0.016$ & -- & $<0.006$ & 0.039 & -- & -- & $<0.015$ & -- & - & $<0.012$ \\
\hline 17 & 1100 & -- & $<0.016$ & -- & $<0.006$ & 0.049 & - & -- & $<0.015$ & -- & -- & $<0.012$ \\
\hline 24 & 1100 & -- & $<0.016$ & -- & $<0.006$ & $<0.015$ & - & - & $<0.015$ & - & -- & $<0.012$ \\
\hline \multicolumn{13}{|c|}{ OCTOBER } \\
\hline 01 & 1130 & -- & $<0.016$ & -- & $<0.006$ & 0.041 & - & -- & $<0.015$ & -- & -- & $<0.012$ \\
\hline 08 & 1330 & -- & $<0.016$ & -- & $<0.006$ & 0.041 & -- & - & $<0.015$ & -- & - & $<0.012$ \\
\hline 15 & 1215 & - & $<0.016$ &.- & $<0.006$ & 0.030 & -- & -- & $<0.015$ & -- & -- & $<0.012$ \\
\hline 22 & 1130 & -- & $<0.016$ & -- & $<0.006$ & 0.029 & -- & -- & $<0.015$ & -- & -- & $<0.012$ \\
\hline \multicolumn{13}{|c|}{ NOVEMBER } \\
\hline 05 & 1140 & -- & $<0.016$ & -- & $<0.006$ & 0.028 & -- & -- & $<0.015$ & -- & -- & $<0.012$ \\
\hline 15 & 1130 & -- & $<0.016$ & -- & $<0.006$ & $<0.015$ &.- & -- & $<0.015$ & -- & -- & $<0.012$ \\
\hline
\end{tabular}


Table 8.--Pesticide resuits from solid-phase extraction 1-liter method--Contlnued

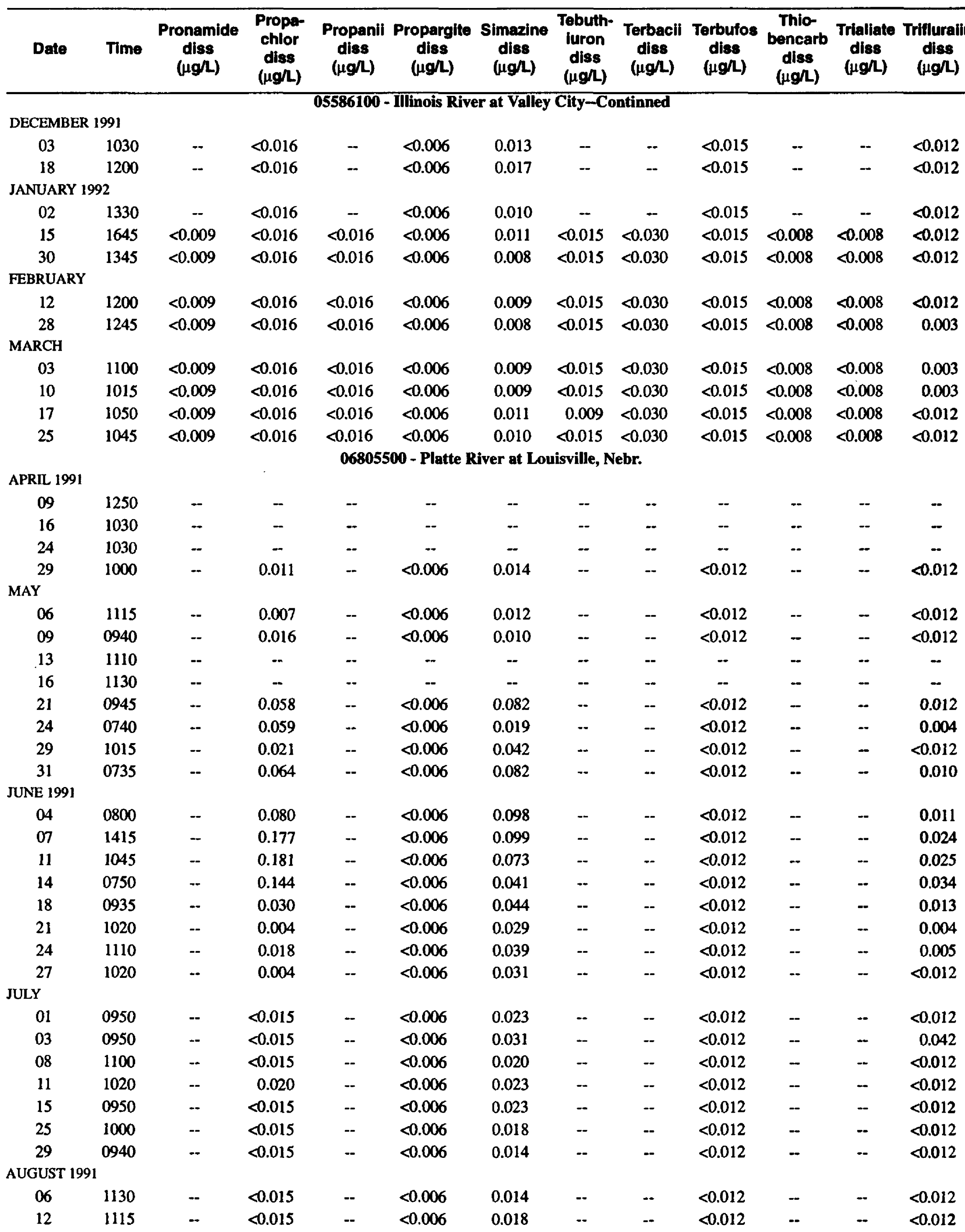


Table 8.-Pesticide resuits from solid-phase extraction 1-liter method--Continued

\begin{tabular}{|c|c|c|c|c|c|c|c|c|c|c|c|c|}
\hline Date & Time & $\begin{array}{c}\text { Pronamide } \\
\text { diss } \\
(\mu g / \mathrm{L})\end{array}$ & $\begin{array}{l}\text { Propa- } \\
\text { chlor } \\
\text { diss } \\
(\mu g / L)\end{array}$ & $\begin{array}{c}\text { Propanil } \\
\text { dlss } \\
(\mu g /))\end{array}$ & $\begin{array}{c}\text { Proparglte } \\
\text { diss } \\
(\mu g / L)\end{array}$ & $\begin{array}{c}\text { Simazina } \\
\text { diss } \\
(\mu g / L)\end{array}$ & $\begin{array}{c}\text { Tebuth- } \\
\text { iuron } \\
\text { diss } \\
(\mu g / L)\end{array}$ & $\begin{array}{c}\text { Terbacil } \\
\text { diss } \\
(\mu g / L)\end{array}$ & $\begin{array}{c}\text { Terbufos } \\
\text { diss } \\
(\mu g / L)\end{array}$ & $\begin{array}{l}\text { Thio- } \\
\text { bencarb } \\
\text { dlss } \\
(\mu g / L)\end{array}$ & $\begin{array}{c}\text { Triallate } \\
\text { diss } \\
(\mu g / L)\end{array}$ & $\begin{array}{c}\text { Trifluralin } \\
\text { diss } \\
(\mu g / L)\end{array}$ \\
\hline \multicolumn{13}{|c|}{ 06805500 - Plstte River at Louisville, Nebr.-Continued } \\
\hline \multicolumn{13}{|c|}{ AUGUST 1991 (cont.) } \\
\hline 20 & 1000 & - & $<0.015$ & -- & $<0.006$ & 0.021 & - & - & $<0.012$ & -- & -- & $<0.012$ \\
\hline 27 & 0940 & -- & $<0.015$ & -- & $<0.006$ & 0.009 & -- & -- & $<0.012$ & -- & -- & $<0.012$ \\
\hline \multicolumn{13}{|c|}{ SEPTEMBER } \\
\hline 03 & 0950 & -. & $<0.015$ & .. & $<0.006$ & 0.007 & .. & -- & $<0.012$ & -- & - & $<0.012$ \\
\hline 10 & 1030 & _. & $<0.015$ & .- & $<0.006$ & 0.017 & -- & -- & $<0.012$ & -- & -. & $<0.012$ \\
\hline 17 & 1115 & -- & $<0.015$ & -- & $<0.006$ & 0.007 & - & -- & $<0.012$ & -- & - & $<0.012$ \\
\hline 24 & 1135 & - & $<0.015$ & .- & $<0.006$ & 0.008 & - & -- & $<0.012$ & -- & -- & $<0.012$ \\
\hline \multicolumn{13}{|c|}{ OCTOBER } \\
\hline 01 & 1115 & -- & $<0.015$ & -- & $<0.006$ & 0.010 & -- & -- & $<0.012$ & -- & -- & $<0.012$ \\
\hline 08 & 1030 & -- & $<0.015$ & -- & $<0.006$ & 0.004 & -- & - & $<0.012$ & -- & -- & $<0.012$ \\
\hline 15 & 1130 & -- & $<0.015$ & -- & $<0.006$ & 0.019 & -- & - & $<0.012$ & -- & -- & $<0.012$ \\
\hline 24 & 1200 & -- & $<0.015$ & -- & $<0.006$ & 0.022 & -- & -- & $<0.012$ & -- & -- & $<0.012$ \\
\hline \multicolumn{13}{|c|}{ NOVEMBER } \\
\hline 05 & 1200 & -- & $<0.015$ & -- & $<0.006$ & 0.009 & -- & -- & $<0.012$ & - & -- & $<0.012$ \\
\hline 19 & 1140 & -. & $<0.015$ & -- & $<0.006$ & 0.011 & -. & -- & $<0.012$ & -- & -. & $<0.012$ \\
\hline \multicolumn{13}{|c|}{ DECEMBER 1991} \\
\hline 05 & 1130 & -- & -- & -- & -- & -- & -- & -- & -- & -- & -- & -- \\
\hline 20 & 1000 & $<0.009$ & $<0.015$ & $<0.016$ & $<0.006$ & 0.004 & $<0.015$ & $<0.030$ & $<0.012$ & $<0.008$ & $<0.008$ & $<0.012$ \\
\hline 30 & 1020 & $<0.009$ & $<0.015$ & $<0.016$ & $<0.006$ & 0.005 & $<0.015$ & $<0.030$ & $<0.012$ & $<0.008$ & $<0.008$ & 0.003 \\
\hline \multicolumn{13}{|c|}{ JANUARY 1992} \\
\hline 14 & 1000 & $<0.009$ & $<0.015$ & $<0.016$ & $<0.006$ & 0.005 & $<0.015$ & $<0.030$ & 0.070 & $<0.008$ & $<0.008$ & $<0.012$ \\
\hline 28 & 1000 & $<0.009$ & $<0.015$ & $<0.016$ & $<0.006$ & 0.004 & $<0.015$ & $<0.030$ & $<0.012$ & $<0.008$ & $<0.008$ & 0.004 \\
\hline \multicolumn{13}{|c|}{ FEBRUARY } \\
\hline 11 & 1030 & $<0.009$ & $<0.015$ & $<0.016$ & $<0.006$ & 0.004 & $<0.015$ & $<0.030$ & $<0.012$ & $<0.008$ & $<0.008$ & $<0.012$ \\
\hline 27 & 1000 & $<0.009$ & $<0.015$ & $<0.016$ & $<0.006$ & 0.004 & $<0.015$ & $<0.030$ & $<0.012$ & $<0.008$ & $<0.008$ & 0.003 \\
\hline \multicolumn{13}{|l|}{ MARCH } \\
\hline 05 & 1010 & $<0.009$ & $<0.015$ & $<0.016$ & $<0.006$ & 0.004 & $<0.015$ & $<0.030$ & $<0.012$ & $<0.008$ & $<0.008$ & $<0.012$ \\
\hline 09 & 0945 & $<0.009$ & 0.007 & $<0.016$ & $<0.006$ & 0.004 & $<0.015$ & $<0.030$ & $<0.012$ & $<0.008$ & $<0.008$ & $<0.012$ \\
\hline 17 & 1145 & $<0.009$ & $<0.015$ & $<0.016$ & $<0.006$ & 0.006 & $<0.015$ & $<0.030$ & $<0.012$ & $<0.008$ & $<0.008$ & $<0.012$ \\
\hline 23 & 1040 & $<0.009$ & $<0.015$ & $<0.016$ & $<0.006$ & 0.005 & $<0.015$ & $<0.030$ & $<0.012$ & $<0.008$ & $<0.008$ & $<0.012$ \\
\hline 31 & 1100 & $<0.009$ & $<0.015$ & $<0.016$ & $<0.006$ & 0.005 & $<0.015$ & $<0.030$ & $<0.012$ & $<0.008$ & $<0.008$ & 0.003 \\
\hline \multicolumn{13}{|c|}{ APRIL 1992} \\
\hline 07 & 1100 & $<0.009$ & $<0.015$ & $<0.016$ & $<0.006$ & 0.005 & 0.010 & $<0.030$ & 0.012 & $<0.008$ & $<0.008$ & 0.004 \\
\hline 14 & 1010 & $<0.009$ & $<0.015$ & $<0.016$ & $<0.006$ & 0.006 & 0.004 & 0.015 & $<0.012$ & $<0.008$ & $<0.008$ & 0.004 \\
\hline 23 & 1020 & $<0.009$ & $<0.015$ & $<0.016$ & $<0.006$ & 0.006 & $<0.015$ & 0.020 & $<0.012$ & $<0.008$ & $<0.008$ & 0.004 \\
\hline 29 & 1030 & $<0.009$ & 0.004 & $<0.016$ & $<0.006$ & 0.210 & $<0.015$ & 0.032 & $<0.012$ & $<0.008$ & $<0.008$ & $<0.012$ \\
\hline \multicolumn{13}{|l|}{ MAY } \\
\hline \multirow[t]{2}{*}{07} & 1100 & $<0.009$ & 0.003 & $<0.016$ & $<0.006$ & 0.210 & $<0.015$ & 0.032 & $<0.012$ & $<0.008$ & $<0.008$ & $<0.012$ \\
\hline & \multicolumn{12}{|c|}{ O6934500 - Missouri River at Hermsnn, Mo. } \\
\hline \multicolumn{13}{|c|}{ APRIL 1991} \\
\hline 09 & 1331 & -- & -. & -- & -. & -- & -- & -- & -- & -- & -- & -- \\
\hline 16 & 1030 & - & -- & -- & -- & -- & -- & -- & -- & -- & -- & -- \\
\hline 24 & 1200 & -- & -- & -- & -- & -- & -- & -- & -- & -- & -- & -- \\
\hline \multicolumn{13}{|l|}{ MAY } \\
\hline 01 & 1130 & -- & -- & -- & -- & -- & -- & -- & -- & - & -- & -- \\
\hline 06 & 1100 & -- & $<0.015$ & -- & 0.067 & 0.035 & -- & -- & $<0.012$ & -- & -- & 0.011 \\
\hline 09 & 0930 & -- & -- & -- & -- & -- & -- & -- & -- & -- & -- & -- \\
\hline 13 & 1000 & -- & -- & -- & -- & -- & -- & -- & -- & -- & -- & -- \\
\hline 16 & 1020 & -- & 0.005 & -- & $<0.006$ & 0.053 & -- & -- & $<0.012$ & -- & -- & 0.008 \\
\hline
\end{tabular}


Tabie 8.--Pesticide resuits from solid-phase extraction 1-iiter method--Continued

\begin{tabular}{|c|c|c|c|c|c|c|c|c|c|c|c|c|}
\hline Date & Time & $\begin{array}{c}\text { Pronamide } \\
\text { diss } \\
(\mu g / L)\end{array}$ & 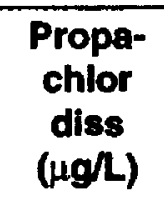 & $\begin{array}{c}\text { Propanil } \\
\text { dlss } \\
(\mu g / L)\end{array}$ & $\begin{array}{l}\text { Propargite } \\
\text { diss } \\
(\mu g / L)\end{array}$ & $\begin{array}{c}\text { Simazine } \\
\text { diss } \\
(\mu \mathrm{g} / \mathrm{L})\end{array}$ & $\begin{array}{l}\text { Tebuth- } \\
\text { iuron } \\
\text { diss } \\
(\mu g / L)\end{array}$ & $\begin{array}{c}\text { Terbacil } \\
\text { disss } \\
(\mu g / L)\end{array}$ & $\begin{array}{c}\text { Terbufos } \\
\text { diss } \\
(\mu g / L)\end{array}$ & $\begin{array}{c}\text { Thio- } \\
\text { bancarb } \\
\text { diss } \\
(\mu g / L)\end{array}$ & $\begin{array}{c}\text { Triailate } \\
\text { diss } \\
(\mu g / 2)\end{array}$ & $\begin{array}{c}\text { Trifluralin } \\
\text { diss } \\
(\mu g / L)\end{array}$ \\
\hline \multicolumn{13}{|c|}{06934500 - Missouri River at Hermann, Mo.-Continned } \\
\hline \multicolumn{13}{|c|}{ MAY (cont.) } \\
\hline 20 & 1000 & -. & $<0.015$ & -- & $<0.006$ & 0.027 & - & -- & $<0.012$ & -- & -- & 0.005 \\
\hline 24 & 1000 & -- & 0.085 & -- & $<0.006$ & 0.049 & - & -- & $<0.012$ & -- & -- & 0.014 \\
\hline 28 & 1030 & -- & $<0.015$ & - & $<0.006$ & 0.058 & -- & ـ & $<0.012$ & -- & -- & 0.011 \\
\hline 31 & 1040 & -- & 0.006 & -- & 0.026 & 0.044 & -- & -- & $<0.012$ & -- & -- & 0.005 \\
\hline \multicolumn{13}{|l|}{ JUNE } \\
\hline 03 & 1000 &.- & 0.007 & -- & $<0.006$ & 0.042 & - & -- & $<0.012$ & - & -- & 0.012 \\
\hline 06 & 1000 & -- & 0.010 & -- & $<0.006$ & 0.047 & -- & -- & $<0.012$ & -- & - & 0.006 \\
\hline 11 & 1000 & -- & $<0.015$ & -- & $<0.006$ & 0.047 & - & $\cdots$ & $<0.012$ & - & - & $<0.012$ \\
\hline 13 & 1000 & -- & 0.005 & -- & $<0.006$ & 0.056 & -- & -- & $<0.012$ & -- & -- & 0.010 \\
\hline 21 & 1000 & -- & $<0.015$ & -- & $<0.006$ & 0.057 & -- & -- & $<0.012$ & -- & - & 0.019 \\
\hline 25 & 1130 & -- & $<0.015$ & -- & $<0.006$ & 0.054 & -- & -- & $<0.012$ & -- & -- & 0.010 \\
\hline 27 & 1045 & -- & $<0.015$ & -- & $<0.006$ & 0.024 & -- & -- & $<0.012$ & -- & - & 0.005 \\
\hline \multicolumn{13}{|l|}{ JULY } \\
\hline 01 & 1100. & -- & $<0.015$ & -- & $<0.006$ & 0.032 & -- & -- & $<0.012$ & -- & -- & 0.002 \\
\hline 03 & 1000 & -. & $<0.015$ & -- & $<0.006$ & 0.014 & -. & -- & $<0.012$ & -- & -- & $<0.012$ \\
\hline 08 & 1030 & -- & $<0.015$ & -- & $<0.006$ & 0.010 & -- & -- & $<0.012$ & -- & - & $<0.012$ \\
\hline 11 & 1000 & -- & 0.010 & -- & $<0.006$ & 0.012 & - & -. & $<0.012$ & - & -- & $<0.012$ \\
\hline 15 & 1030 & -- & $<0.015$ & -- & $<0.006$ & 0.012 & -- & -- & $<0.012$ & -- & -- & 0.002 \\
\hline 22 & 1015 & - & $<0.015$ & -- & $<0.006$ & 0.013 & -- & -- & $<0.012$ & -- & -- & $<0.012$ \\
\hline 29 & 1100 & -- & $<0.015$ & -- & $<0.006$ & 0.013 & -- & -- & $<0.012$ & - & -- & $<0.012$ \\
\hline \multicolumn{13}{|l|}{ AUGUST } \\
\hline 05 & 1000 & -- & $<0.015$ & -- & $<0.006$ & 0.010 & -- & -- & $<0.012$ & - & -- & $<0.012$ \\
\hline 13 & 1100 & -- & $<0.015$ & -- & $<0.006$ & 0.008 & - & -- & $<0.012$ & - & -- & $<0.012$ \\
\hline 19 & 1000 & -- & $<0.015$ & -- & $<0.006$ & 0.010 & -- & -- & $<0.012$ & - & -- & $<0.012$ \\
\hline 27 & 1100 & -- & $<0.015$ & -- & $<0.006$ & 0.008 & -- & -- & $<0.012$ & - & -- & $<0.012$ \\
\hline \multicolumn{13}{|c|}{ SEPTEMBER 1991} \\
\hline 05 & 1030 & -- & $<0.015$ & -- & $<0.006$ & 0.007 & -- & -- & $<0.012$ & -- & -. & $<0.012$ \\
\hline 09 & 1000 & -- & $<0.015$ & -- & $<0.006$ & 0.005 & -- & -- & $<0.012$ & - & .- & $<0.012$ \\
\hline 16 & 1000 & -- & $<0.015$ & -- & $<0.006$ & 0.008 & -- & -- & $<0.012$ & -- & -- & $<0.012$ \\
\hline 23 & 0945 & -. & $<0.015$ & - & $<0.006$ & 0.010 & -- & - & $<0.012$ & - & -- & $<0.012$ \\
\hline \multicolumn{13}{|l|}{ OCTOBER } \\
\hline 01 & 1030 & -. & $<0.015$ & -. & $<0.006$ & $<0.008$ & -. & -- & $<0.012$ & - & -- & $<0: 012$ \\
\hline 07 & 1000 & -- & $<0.015$ & - & $<0.006$ & 0.006 & -- & - & $<0.012$ & -- & -- & $<0.012$ \\
\hline 15 & 1100 & -- & $<0.015$ & -- & $<0.006$ & 0.008 & -- & -- & $<0.012$ & - & -- & $<0.012$ \\
\hline 21 & 1000 & -- & $<0.015$ & -- & $<0.006$ & 0.005 & -- & -- & $<0.012$ & -- & -- & $<0.012$ \\
\hline 29 & 1000 & -- & $<0.015$ & -- & $<0.006$ & 0.005 & - & - & $<0.012$ & -- & -- & $<0.012$ \\
\hline \multicolumn{13}{|c|}{ NOVEMBER } \\
\hline 04 & 1030 & - & $<0.015$ & -- & $<0.006$ & 0.007 & -. & $-\cdot$ & $<0.012$ & - & -- & $<0.012$ \\
\hline 21 & 1030 & -- & $<0.015$ & - & $<0.006$ & 0.012 & -- & - & $<0.012$ & - & - & $<0.012$ \\
\hline \multicolumn{13}{|c|}{ DECEMBER } \\
\hline 06 & 1240 & -- & $<0.015$ & - & $<0.006$ & 0.011 & -- & -. & $<0.012$ & - & -- & $<0.012$ \\
\hline 16 & 1130 & -- & $<0.015$ & -- & $<0.006$ & 0.006 & -- & -- & $<0.012$ & - & -. & $<0.012$ \\
\hline \multicolumn{13}{|c|}{ JANUARY 1992} \\
\hline 02 & 1030 & -- & $<0.015$ & -- & $<0.006$ & 0.022 & -- & -. & $<0.012$ & -- & -- & $<0.012$ \\
\hline 16 & 1200 & $<0.009$ & $<0.015$ & $<0.016$ & $<0.006$ & 0.011 & $<0.012$ & $<0.030$ & $<0.012$ & $<0.008$ & $<0.008$ & 0.003 \\
\hline 28 & 1300 & $<0.009$ & $<0.015$ & 0.001 & $<0.006$ & $<0.008$ & $<0.012$ & $<0.030$ & $<0.012$ & $<0.008$ & $<0.008$ & $<0.012$ \\
\hline \multicolumn{13}{|c|}{ FEBRUARY } \\
\hline 10 & 1000 & $<0.009$ & $<0.015$ & $<0.016$ & $<0.006$ & 0.006 & $<0.012$ & $<0.030$ & $<0.012$ & $<0.008$ & $<0.008$ & 0.003 \\
\hline 24 & 1200 & $<0.009$ & $<0.015$ & $<0.016$ & $<0.006$ & 0.007 & $<0.012$ & $<0.030$ & $<0.012$ & $<0.008$ & $<0.008$ & 0.004 \\
\hline
\end{tabular}


Table 8.-Pesticide results from solid-phase extraction 1-liter method-Continued

\begin{tabular}{|c|c|c|c|c|c|c|c|c|c|c|c|c|}
\hline Date & Time & $\begin{array}{c}\text { Pronamide } \\
\text { diss } \\
(\mu g / L)\end{array}$ & $\begin{array}{l}\text { Propa- } \\
\text { chior } \\
\text { diss } \\
(\mu g / L)\end{array}$ & $\begin{array}{c}\text { Propanil } \\
\text { diss } \\
(\mu g / L)\end{array}$ & $\begin{array}{c}\text { Propargite } \\
\text { diss } \\
(\mu g / L)\end{array}$ & $\begin{array}{c}\text { Simazine } \\
\text { diss } \\
(\mu g / L)\end{array}$ & $\begin{array}{c}\text { Tebuth- } \\
\text { iuron } \\
\text { diss } \\
(\mu g / L)\end{array}$ & $\begin{array}{c}\text { Terbacil } \\
\text { dlss } \\
(\mu g / L)\end{array}$ & $\begin{array}{c}\text { Terbufos } \\
\text { diss } \\
(\mu g / L)\end{array}$ & $\begin{array}{c}\text { Thio- } \\
\text { bencarb } \\
\text { diss } \\
(\mu g / L)\end{array}$ & $\begin{array}{l}\text { Triailate } \\
\text { diss } \\
(\mu g / L)\end{array}$ & $\begin{array}{c}\text { Triflurailn } \\
\text { diss } \\
(\mu g / L)\end{array}$ \\
\hline
\end{tabular}

\begin{tabular}{|c|c|c|c|c|c|c|c|c|c|c|c|c|}
\hline \multirow{2}{*}{\multicolumn{13}{|c|}{ MARCH }} \\
\hline & & & & & & & & & & & & \\
\hline & 1100 & $<0.009$ & $<0.015$ & $<0.016$ & $<0.006$ & 0.007 & $<0.012$ & $<0.030$ & $<0.012$ & $<0.008$ & $<0.008$ & 0.003 \\
\hline 12 & 1200 & $<0.009$ & 0.006 & $<0.016$ & $<0.006$ & 0.009 & $<0.012$ & $<0.030$ & $<0.012$ & $<0.008$ & $<0.008$ & 0.004 \\
\hline 20 & 0830 & $<0.009$ & 0.013 & $<0.016$ & $<0.006$ & 0.006 & $<0.012$ & $<0.030$ & $<0.012$ & $<0.008$ & $<0.008$ & 0.004 \\
\hline 26 & 1130 & $<0.009$ & 0.017 & $<0.016$ & $<0.006$ & 0.011 & 0.014 & $<0.030$ & $<0.012$ & $<0.008$ & $<0.008$ & 0.004 \\
\hline \multicolumn{13}{|l|}{ APRIL } \\
\hline 01 & 1000 & $<0.009$ & 0.009 & $<0.016$ & $<0.006$ & 0.009 & 0.250 & $<0.030$ & $<0.012$ & $<0.008$ & $<0.008$ & 0.007 \\
\hline 06 & 1230 & $<0.009$ & $<0.015$ & $<0.016$ & $<0.006$ & 0.008 & $<0.012$ & $<0.030$ & $<0.012$ & $<0.008$ & $<0.008$ & 0.004 \\
\hline 14 & 1030 & $<0.009$ & $<0.015$ & $<0.016$ & $<0.006$ & 0.012 & 0.039 & $<0.030$ & $<0.012$ & $<0.008$ & $<0.008$ & 0.004 \\
\hline 21 & 1300 & $<0.009$ & $<0.015$ & $<0.016$ & $<0.006$ & 0.016 & 0.012 & $<0.030$ & $<0.012$ & $<0.008$ & $<0.008$ & 0.004 \\
\hline 29 & 1015 & $<0.009$ & 0.004 & $<0.016$ & $<0.006$ & 0.025 & 0.014 & $<0.030$ & $<0.012$ & $<0.008$ & $<0.008$ & 0.005 \\
\hline \multicolumn{13}{|c|}{ MAY 1992} \\
\hline 12 & 1000 & $<0.009$ & $<0.015$ & 0.005 & $<0.006$ & 0.018 & $<0.012$ & $<0.030$ & $<0.012$ & $<0.008$ & $<0.008$ & 0.004 \\
\hline 21 & 1200 & $<0.009$ & 0.001 & $<0.016$ & $<0.006$ & 0.010 & $<0.012$ & $<0.030$ & $<0.012$ & $<0.008$ & $<0.008$ & 0.003 \\
\hline 26 & 1130 & $<0.009$ & 0.019 & $<0.016$ & $<0.006$ & 0.044 & $<0.012$ & $<0.030$ & $<0.012$ & $<0.008$ & $<0.008$ & 0.005 \\
\hline \multicolumn{13}{|l|}{ JUNE } \\
\hline 01 & 1330 & $<0.009$ & 0.013 & $<0.016$ & $<0.006$ & 0.030 & $<0.012$ & $<0.030$ & $<0.012$ & $<0.008$ & $<0.008$ & 0.003 \\
\hline 09 & 1030 & $<0.009$ & 0.002 & $<0.016$ & $<0.006$ & 0.018 & $<0.012$ & $<0.030$ & $<0.012$ & $<0.008$ & $<0.008$ & 0.003 \\
\hline 15 & 1230 & $<0.009$ & 0.014 & $<0.016$ & $<0.006$ & 0.014 & 0.006 & $<0.030$ & 0.020 & $<0.008$ & $<0.008$ & 0.003 \\
\hline 22 & 0945 & $<0.009$ & $<0.015$ & $<0.016$ & $<0.006$ & 0.021 & $<0.012$ & $<0.030$ & $<0.012$ & $<0.008$ & $<0.008$ & 0.003 \\
\hline 30 & 1130 & $<0.009$ & $<0.015$ & $<0.016$ & $<0.006$ & 0.021 & $<0.012$ & $<0.030$ & $<0.012$ & $<0.008$ & $<0.008$ & 0.003 \\
\hline \multicolumn{13}{|l|}{ JULY } \\
\hline 07 & 0930 & $<0.009$ & $<0.015$ & $<0.016$ & $<0.006$ & 0.013 & $<0.012$ & $<0.030$ & $<0.012$ & $<0.008$ & $<0.008$ & $<0.012$ \\
\hline 14 & 1200 & $<0.009$ & $<0.015$ & $<0.016$ & $<0.006$ & 0.037 & $<0.012$ & $<0.030$ & $<0.012$ & $<0.008$ & $<0.008$ & 0.004 \\
\hline 21 & 1030 & $<0.009$ & $<0.015$ & $<0.016$ & $<0.006$ & 0.028 & $<0.012$ & $<0.030$ & $<0.012$ & $<0.008$ & $<0.008$ & 0.005 \\
\hline 28 & 1230 & $<0.009$ & $<0.015$ & $<0.016$ & $<0.006$ & 0.015 & $<0.012$ & $<0.030$ & $<0.012$ & $<0.008$ & $<0.008$ & 0.003 \\
\hline \multicolumn{13}{|c|}{07022000 - Mississippi River at Thebes, IIl. } \\
\hline \multicolumn{13}{|c|}{ APRIL 199} \\
\hline 11 & 1500 & -- & -- & -- & -- & -- & -- & -- & -- & - & -- & - \\
\hline 18 & 1130 & -- & -- & -- & -- & -- & -- & - & - & - & -. & -- \\
\hline 24 & 0930 & - & -- & -- & -- & -- & -- & -- & - & - & -- & -- \\
\hline 29 & 1230 & -- & -- & -- & -- & -- & -- & -- & -- & - & -- & - \\
\hline \multicolumn{13}{|l|}{ MAY } \\
\hline 07 & 1345 & -- & $<0.015$ & -- & $<0.006$ & 0.042 & - & - & $<0.012$ & -- & -- & 0.005 \\
\hline 09 & 0900 & -- & $<0.015$ & -- & $<0.006$ & 0.026 & -- & -- & $<0.012$ & -- & -- & 0.002 \\
\hline 13 & 1330 & - & -- & -- & -- & -- & - & - & - & -- & - & -. \\
\hline 16 & 1200 & -- & $<0.015$ & -- & $<0.006$ & 0.028 & - & - & $<0.012$ & -- & -- & $<0.012$ \\
\hline 20 & 1430 & -. & $<0.015$ & -- & $<0.006$ & 0.058 & -- & -- & $<0.012$ & -- & -- & 0.004 \\
\hline 23 & 0916 & -- & $<0.015$ & -- & $<0.006$ & 0.047 & - & -- & $<0.012$ & - & -- & 0.002 \\
\hline 28 & 1300 & -- & 0.004 & -- & $<0.006$ & 0.016 & -- & -- & $<0.012$ & -- & -- & 0.004 \\
\hline 30 & 1330 & -- & 0.006 & -- & $<0.006$ & 0.041 & -- & -- & $<0.012$ & -- & -- & 0.004 \\
\hline \multicolumn{13}{|c|}{ JUNE 1991} \\
\hline 03 & 1345 & -- & $<0.015$ & -- & $<0.006$ & 0.053 & -- & - & $<0.012$ & -- & -- & 0.003 \\
\hline 06 & 1045 & -- & $<0.015$ & -- & $<0.006$ & 0.053 & - & -- & $<0.012$ & -- & -- & 0.004 \\
\hline 10 & 1400 & -- & $<0.015$ & -- & $<0.006$ & 0.045 & - & -. & $<0.012$ & - & -- & 0.003 \\
\hline 13 & 1500 & -- & $<0.015$ & -- & $<0.006$ & 0.033 & -- & -- & $<0.012$ & - & - & $<0.012$ \\
\hline 18 & 1430 & -- & $<0.015$ & -- & $<0.006$ & 0.058 & -- & -- & $<0.012$ & - & - & 0.004 \\
\hline 20 & 1100 & -- & 0.003 & -- & $<0.006$ & 0.060 & -- & -- & $<0.012$ & -- & -- & 0.004 \\
\hline 24 & 1300 & -- & $<0.015$ & - & $<0.006$ & 0.042 & -. & $\bar{i}$ & $<0.012$ & -- & -- & 0.010 \\
\hline 27 & 1400 & -- & $<0.015$ & -- & $<0.006$ & 0.048 & - & 1 & $<0.012$ & - & -- & 0.004 \\
\hline
\end{tabular}


Table 8.-Pesticide results from solid-phase extraction 1-Ilter method-Continued

\begin{tabular}{|c|c|c|c|c|c|c|c|c|c|c|c|c|}
\hline Date & Time & $\begin{array}{c}\text { Pronamide } \\
\text { diss } \\
(\mu g / L)\end{array}$ & $\begin{array}{l}\text { Propa- } \\
\text { chior } \\
\text { diss } \\
\text { (ug/) }\end{array}$ & $\begin{array}{c}\text { Propanll } \\
\text { diss } \\
(\mu g / 2)\end{array}$ & $\begin{array}{c}\text { Propargite } \\
\text { dlss } \\
(\mu g / 4)\end{array}$ & $\begin{array}{c}\text { Simazine } \\
\text { dlss } \\
(\mu g / 4)\end{array}$ & $\begin{array}{l}\text { Tobuth- } \\
\text { luron } \\
\text { diss } \\
(\mu \mathrm{g} / \mathrm{L})\end{array}$ & $\begin{array}{c}\text { Terbacil } \\
\text { diss } \\
(\mu g / L)\end{array}$ & $\begin{array}{c}\text { Terbufos } \\
\text { diss } \\
(\mu g / L)\end{array}$ & $\begin{array}{l}\text { Thlo- } \\
\text { bencarb } \\
\text { dlss } \\
\text { (ugh) }\end{array}$ & $\begin{array}{l}\text { Triallate } \\
\text { diss } \\
\text { (ug/L) }\end{array}$ & $\begin{array}{l}\text { Trifluralin } \\
\text { dles } \\
(\mu g / L)\end{array}$ \\
\hline
\end{tabular}

JULY

\begin{tabular}{|c|c|c|c|c|c|c|c|c|c|c|c|c|}
\hline 01 & 1320 & -- & $<0.015$ & -- & $<0.006$ & 0.036 & -- & - & $<0.012$ & - & -- & 0.002 \\
\hline 03 & 0950 & -- & $<0.015$ & - & $<0.006$ & 0.032 & -- & -- & $<0.012$ & - & - & $<0.012$ \\
\hline 08 & 1330 & -- & $<0.015$ & -- & $<0.006$ & 0.028 & -- & -- & $<0.012$ & - & - & $<0.012$ \\
\hline 11 & 1400 & -- & $<0.015$ & -- & $<0.006$ & 0.027 & -- & -- & $<0.012$ & - & - & $<0.012$ \\
\hline 18 & 0930 & -- & $\ldots$ & - & -- & .- & -- & -- & - & - & -- & -- \\
\hline 23 & 1300 & -- & $<0.015$ & - & $<0.006$ & 0.024 & -- & -- & $<0.012$ & - & - & $<0.012$ \\
\hline 30 & 1000 & -- & $<0.015$ & -- & $<0.006$ & 0.021 & -- & -- & $<0.012$ & -- & -- & $<0.012$ \\
\hline \multicolumn{13}{|c|}{ AUGUST 1991} \\
\hline 06 & 1300 & -- & $<0.015$ & - & $<0.006$ & 0.023 & -- & -- & $<0.012$ & -- & - & $<0.012$ \\
\hline 13 & 0930 & - & $<0.015$ & -- & $<0.006$ & 0.022 & -- & -- & $<0.012$ & - & -- & $<0.012$ \\
\hline 20 & 1330 & -- & $<0.015$ & -- & $<0.006$ & 0.018 & -- & -- & $<0.012$ & -- & - & $<0.012$ \\
\hline 29 & 1100 & - & $<0.015$ & - & $<0.006$ & 0.018 & -- & -- & $<0.012$ & - & -- & $<0.012$ \\
\hline \multicolumn{13}{|c|}{ EEPTEMBER } \\
\hline 03 & 1500 & - & $<0.015$ & -- & $<0.006$ & 0.014 & -- & -- & $<0.012$ & -- & - & $<0.012$ \\
\hline 11 & 1000 & - & $<0.015$ & - & $<0.006$ & 0.015 & -- & -- & $<0.012$ & - & -- & $<0.012$ \\
\hline 17 & 1400 & -- & $<0.015$ & -- & $<0.006$ & 0.011 & -- & -- & $<0.012$ & -- & -- & $<0.012$ \\
\hline 26 & 0930 & -- & $<0.015$ & -- & $<0.006$ & 0.014 & - & - & $<0.012$ & -- & -- & $<0.012$ \\
\hline \multicolumn{13}{|c|}{ CTOBER } \\
\hline 02 & 1300 & - & $<0.015$ & $\cdots$ & $<0.006$ & 0.011 & -- & -- & $<0.012$ & -- & - & $<0.012$ \\
\hline 09 & 1030 & -- & $<0.015$ & -- & $<0.006$ & 0.015 & -- & - & $<0.012$ & -- & - & $<0.012$ \\
\hline 16 & 1430 & -- & $<0.015$ & $\cdots$ & $<0.006$ & 0.012 & -- & $\cdots$ & $<0.012$ & -- & -- & $<0.012$ \\
\hline 23 & 0945 & -- & $<0.015$ & - & $<0.006$ & 0.012 & -- & -- & $<0.012$ & -- & - & $<0.012$ \\
\hline 30 & 1400 & -- & $<0.015$ & - & $<0.006$ & $<0.008$ & -- & -- & $<0.012$ & -- & -- & $<0.012$ \\
\hline \multicolumn{13}{|c|}{ NOVEMBER } \\
\hline 07 & 0800 & -- & $<0.015$ & - & $<0.006$ & 0.010 & -- & -- & $<0.012$ & - & - & $<0.012$ \\
\hline 20 & 1030 & -- & $<0.015$ & - & $<0.006$ & 0.010 & -- & -- & $<0.012$ & -- & - & $<0.012$ \\
\hline \multicolumn{13}{|c|}{ DECEMBER 1991} \\
\hline 05 & 1045 & -- & $<0.015$ & - & $<0.006$ & 0.011 & -- & -- & $<0.012$ & -- & $m$ & $<0.012$ \\
\hline 18 & 1100 & -- & 0.004 & - & $<0.006$ & 0.011 & -- & - & $<0.012$ & - & - & $<0.012$ \\
\hline 30 & 1400 & - & $<0.015$ & -- & $<0.006$ & 0.014 & -- & -- & $<0.012$ & - & -- & $<0.012$ \\
\hline
\end{tabular}

JANUARY 1992

(1)

$24 \quad 0930$

FEBUARY

$04 \quad 1030$

$19 \quad 0930$

MARCH

$04 \quad 0945$

$0945<0.009$

$18 \quad 0945 \quad<0.009$

$24 \quad 1330<0.009$

APRIL

$02 \quad 0930<0.009$

$07 \quad 1330<0.009$

$15 \quad 1030<0.009$

$23 \quad 1330<0.009$

$29 \quad 1100<0.009$

MAY 1992

$\begin{array}{llllll}06 & 1300 & <0.009 & <0.015 & <0.016 & <0.006 \\ 14 & 0930 & <0.009 & <0.015 & <0.016 & <0.006\end{array}$

$\begin{array}{rrrrrrrr}0.007 & <0.010 & <0.030 & <0.012 & <0.008 & <0.008 & 0.003 \\ 0.006 & <0.010 & <0.030 & <0.012 & <0.008 & <0.008 & <0.012 \\ 0.011 & 0.041 & <0.030 & <0.012 & <0.008 & <0.008 & 0.004 \\ & & & & & & \\ <0.008 & <0.015 & <0.030 & <0.012 & <0.008 & <0.008 & 0.003 \\ 0.008 & <0.015 & <0.030 & <0.012 & <0.008 & <0.008 & <0.012 \\ 0.008 & <0.015 & <0.030 & <0.012 & <0.008 & <0.008 & 0.004 \\ 0.009 & 0.010 & <0.030 & <0.012 & <0.008 & <0.008 & 0.003 \\ & & & & & & \\ 0.017 & 0.014 & <0.030 & <0.012 & <0.008 & <0.008 & 0.005 \\ 0.012 & 0.024 & <0.030 & <0.012 & <0.008 & <0.008 & <0.012 \\ 0.012 & 0.019 & <0.030 & <0.012 & <0.008 & <0.008 & 0.004 \\ 0.060 & 0.018 & <0.030 & <0.012 & <0.008 & <0.008 & 0.004 \\ 0.040 & 0.013 & <0.030 & <0.012 & <0.008 & <0.008 & 0.004 \\ & & & & & & \\ 0.022 & <0.015 & <0.030 & <0.012 & <0.008 & <0.008 & <0.012 \\ 0.018 & <0.015 & <0.030 & <0.012 & <0.008 & <0.008 & 0.004\end{array}$


Table 8.-Pesticide results from solid-phase extraction 1-liter method-Continued

\begin{tabular}{|c|c|c|c|c|c|c|c|c|c|c|c|c|}
\hline Date & Time & $\begin{array}{c}\text { Pronamide } \\
\text { diss } \\
(\mu g / L)\end{array}$ & $\begin{array}{l}\text { Propa- } \\
\text { chlor } \\
\text { diss } \\
(\mu g / L)\end{array}$ & $\begin{array}{c}\text { Propanil } \\
\text { dias } \\
(\mu g / L)\end{array}$ & $\begin{array}{c}\text { Propargite } \\
\text { diss } \\
(\mu g / L)\end{array}$ & $\begin{array}{c}\text { Simazine } \\
\text { diss } \\
(\mu g / L)\end{array}$ & $\begin{array}{c}\text { Tebuth- } \\
\text { luron } \\
\text { diss } \\
(\mu g /)\end{array}$ & $\begin{array}{c}\text { Terbacil } \\
\text { diss } \\
(\mu g / L)\end{array}$ & $\begin{array}{c}\text { Terbufos } \\
\text { diss } \\
(\mu g / L)\end{array}$ & $\begin{array}{c}\text { Thlo- } \\
\text { bencarb } \\
\text { diss } \\
(\mu g / L)\end{array}$ & $\begin{array}{c}\text { Triallate } \\
\text { diss } \\
(\mu g /))\end{array}$ & $\begin{array}{c}\text { Trifluralin } \\
\text { diss } \\
(\mu g / L)\end{array}$ \\
\hline
\end{tabular}

MAY 1992 (cont.)

$\begin{array}{ccccccccccccc}19 & 1300 & <0.009 & <0.015 & <0.016 & <0.006 & 0.023 & <0.015 & <0.030 & <0.012 & <0.008 & <0.008 & 0.003 \\ 28 & 0900 & <0.009 & <0.015 & <0.016 & <0.006 & 0.052 & <0.015 & <0.030 & <0.012 & <0.008 & <0.008 & 0.003 \\ \text { JUNE } & & & & & & & & & & & & \\ 02 & 1300 & <0.009 & 0.002 & <0.016 & <0.006 & 0.042 & <0.015 & <0.030 & <0.012 & <0.008 & <0.008 & 0.003 \\ 09 & 0930 & <0.009 & <0.015 & <0.016 & <0.006 & 0.040 & <0.015 & <0.030 & <0.012 & <0.008 & <0.008 & 0.003 \\ 16 & 1330 & <0.009 & <0.015 & <0.016 & <0.006 & 0.024 & 0.005 & <0.030 & 0.027 & <0.008 & <0.008 & 0.003 \\ 25 & 1000 & <0.009 & <0.015 & <0.016 & <0.006 & 0.028 & 0.006 & <0.030 & <0.012 & <0.008 & <0.008 & 0.003 \\ \text { JULY } & & & & & & & & & & & & \\ 01 & 1300 & <0.009 & <0.015 & <0.016 & <0.006 & 0.036 & <0.015 & <0.030 & <0.012 & <0.008 & <0.008 & 0.003 \\ 09 & 0915 & <0.009 & <0.015 & <0.016 & <0.006 & 0.033 & <0.015 & <0.030 & <0.012 & <0.008 & <0.008 & <0.012 \\ 15 & 1230 & <0.009 & <0.015 & <0.016 & <0.006 & 0.032 & <0.015 & <0.030 & <0.012 & <0.008 & <0.008 & <0.012 \\ 23 & 1000 & <0.009 & <0.015 & <0.016 & <0.006 & 0.034 & <0.015 & <0.030 & <0.012 & <0.008 & <0.008 & 0.004 \\ 28 & 1300 & <0.009 & <0.015 & <0.016 & <0.006 & 0.036 & <0.015 & <0.030 & <0.012 & <0.008 & <0.008 & 0.003\end{array}$

MAY 1991

\begin{tabular}{|c|c|c|c|c|c|c|c|c|c|c|c|c|}
\hline 01 & 1000 & $\cdots$ & $<0.015$ & $\cdots$ & 0.062 & 0.045 & -. & - & $<0.012$ & -- & -- & $<0.005$ \\
\hline 06 & 1115 & - & $<0.015$ & -- & $<0.006$ & 0.080 & -. & - & $<0.012$ & -- & - & $<0.005$ \\
\hline 09 & 1215 & - & $<0.015$ & -- & $<0.006$ & 0.073 & -- & -- & $<0.012$ & -- & -- & $<0.005$ \\
\hline 13 & 1300 & -- & -- & - & - & -- & -- & - & - & - & - & - \\
\hline 16 & 1130 & -- & -- & -- & - & -- & -- & -- & - & -- & - & -- \\
\hline 20 & 1045 & -- & $<0.015$ & -- & $<0.006$ & 0.412 & -- & -- & $<0.012$ & -- & -- & $<0.005$ \\
\hline 23 & 1100 & -- & 0.042 & -- & $<0.006$ & 0.355 & -- & -- & $<0.012$ & - & - & 0.002 \\
\hline 28 & 1100 & - & $<0.015$ & - & $<0.006$ & 0.927 & - & - & $<0.012$ & - & - & $<0.005$ \\
\hline 30 & 1200 & - & $<0.015$ & -- & $<0.006$ & 1.04 & - & - & $<0.012$ & - & - & 0.003 \\
\hline \multicolumn{13}{|l|}{ JUNE } \\
\hline 03 & 1200 & -- & $<0.015$ & -- & $<0.006$ & 0.506 & - & -- & $<0.012$ & -- & -- & 0.004 \\
\hline 06 & 1145 & -- & $<0.015$ & -- & $<0.006$ & 0.437 & - & -- & $<0.012$ & -- & - & $<0.005$ \\
\hline 10 & 1200 & $\ldots$ & $<0.015$ & -- & $<0.006$ & 0.701 & - & -- & $<0.012$ & $\cdots$ & -- & $<0.005$ \\
\hline 13 & 1100 & - & 0.021 & - & $<0.006$ & 0.492 & - & - & $<0.012$ & -- & - & $<0.005$ \\
\hline 17 & 1200 & -- & $<0.015$ & -- & $<0.006$ & 0.184 & - & - & $<0.012$ & - & -- & $<0.005$ \\
\hline 20 & 1130 & -. & $<0.015$ & -. & $<0.006$ & 0.165 & -- & -- & $<0.012$ & -- & -- & $<0.005$ \\
\hline 24 & 1215 & -- & $<0.015$ & - & $<0.006$ & 0.277 & -- & - & $<0.012$ & -- & -- & $<0.005$ \\
\hline 27 & 1130 & -- & $<0.015$ & -- & $<0.006$ & 0.162 & $-\cdot$ & -. & $<0.012$ & -- & - & $<0.005$ \\
\hline \multicolumn{13}{|c|}{ JULY 1991} \\
\hline 01 & 1145 & -- & $<0.015$ & -- & $<0.006$ & 0.101 & - & -- & $<0.012$ & -- & -- & $<0.005$ \\
\hline 03 & 1100 & -- & $<0.015$ & -- & $<0.006$ & 0.110 & - & -- & $<0.012$ & -- & -- & $<0.005$ \\
\hline 08 & 1200 & -- & $<0.015$ & -- & $<0.006$ & 0.093 & -- & -- & $<0.012$ & -- & -- & $<0.005$ \\
\hline 11 & 1130 & -- & $<0.015$ & - & $<0.006$ & 0.073 & - & - & $<0.012$ & - & -- & $<0.005$ \\
\hline 15 & 1200 & $\cdots$ & $<0.015$ & $\cdots$ & $<0.006$ & 0.152 & -- & -. & $<0.012$ & -- & -- & $<0.005$ \\
\hline 18 & 1300 & -- & $<0.015$ & .. & $<0.006$ & 0.122 & - & -- & $<0.012$ & -- & -- & $<0.005$ \\
\hline 22 & 1300 &.- & $<0.015$ & - & $<0.006$ & 0.097 & - & -- & $<0.012$ & -- & -- & $<0.005$ \\
\hline 25 & 1200 & - & $<0.015$ & -- & $<0.006$ & 0.081 & -- & -- & $<0.012$ & -- & -- & $<0.005$ \\
\hline 30 & 1145 & - & $<0.015$ & - & $<0.006$ & 0.088 & - & - & $<0.012$ & -- & -- & $<0.005$ \\
\hline \multicolumn{13}{|c|}{ AUGUST } \\
\hline 01 & 1145 & - & $<0.015$ & -- & $<0.006$ & 0.069 & -- & -- & $<0.012$ & - & -- & $<0.005$ \\
\hline 06 & 1200 & - & $<0.015$ & -- & $<0.006$ & 0.066 & - & -- & $<0.012$ & - & $\cdots$ & $<0.005$ \\
\hline 08 & 1300 & - & $<0.015$ & -- & $<0.006$ & 0.054 & - & -- & $<0.012$ & -- & -- & $<0.005$ \\
\hline 12 & 1100 & -- & $<0.015$ & -- & $<0.006$ & 0.051 & - & -- & $<0.012$ & -- & -- & $<0.005$ \\
\hline
\end{tabular}


Table 8.--Pesticide results from solid-phase extraction 1-liter method-Continued

\begin{tabular}{|c|c|c|c|c|c|c|c|c|c|c|c|c|}
\hline Date & Time & 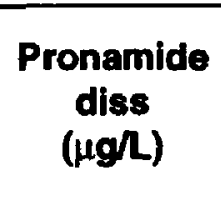 & $\begin{array}{l}\text { Propa- } \\
\text { chior } \\
\text { diss } \\
(\mu g / L)\end{array}$ & $\begin{array}{c}\text { Propanil } \\
\text { diss } \\
(\mu g / L)\end{array}$ & $\begin{array}{c}\text { Propargite } \\
\text { diss } \\
(\mu g / L)\end{array}$ & $\begin{array}{c}\text { Simazine } \\
\text { diss } \\
(\mu g / L)\end{array}$ & $\begin{array}{l}\text { Tebuth- } \\
\text { iuron } \\
\text { dlss } \\
(\mu g / L)\end{array}$ & $\begin{array}{c}\text { Terbacil } \\
\text { diss } \\
(\mu g / L)\end{array}$ & $\begin{array}{c}\text { Terbufos } \\
\text { diss } \\
(\mu g / L)\end{array}$ & $\begin{array}{l}\text { Thlo- } \\
\text { bencarb } \\
\text { diss } \\
(\mu g / L)\end{array}$ & 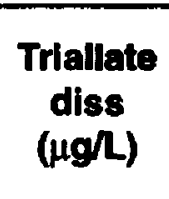 & $\begin{array}{c}\text { Trifluralin } \\
\text { diss } \\
(\mu g h)\end{array}$ \\
\hline \multicolumn{13}{|c|}{03374100 - White River at Hazleton, Ind.-Continued } \\
\hline \multicolumn{13}{|c|}{ AUGUST (cont.) } \\
\hline 15 & 1200 & -- & $<0.015$ & -- & $<0.006$ & 0.074 & $\ldots$ & -- & $<0.012$ & -- & - & $<0.005$ \\
\hline 19 & 1115 & -- & $<0.015$ & -- & $<0.006$ & 0.057 & -- & -- & $<0.012$ & - & - & $<0.005$ \\
\hline 22 & 1230 & -- & $<0.015$ & - & $<0.006$ & 0.038 & -- & -- & $<0.012$ & 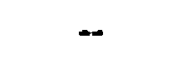 & - & $<0.005$ \\
\hline 26 & 1245 & - & $<0.015$ & -- & $<0.006$ & 0.037 &.- & $\cdots$ & $<0.012$ & - & -- & $<0.005$ \\
\hline 29 & 1200 & -- & $<0.015$ & -- & $<0.006$ & 0.044 & - & $\cdots$ & $<0.012$ & $\cdots$ & - & $<0.005$ \\
\hline \multicolumn{13}{|c|}{ SEPTEMBER 1991} \\
\hline 09 & 1100 & -- & $<0.015$ & -- & $<0.006$ & 0.046 & -- & -- & $<0.012$ & -- & - & $<0.005$ \\
\hline 12 & 1100 & - & $<0.015$ & -- & $<0.006$ & 0.047 & -- & -- & $<0.012$ & -- & -- & $<0.005$ \\
\hline 19 & 1130 & - & $<0.015$ & $\cdots$ & $<0.006$ & 0.070 & -- & -- & $<0.012$ & -- & - & $<0.005$ \\
\hline 26 & 1200 & -- & $<0.015$ & - & $<0.006$ & 0.059 & -- & -- & $<0.012$ & -- & - & $<0.005$ \\
\hline \multicolumn{13}{|c|}{ OCTOBER } \\
\hline 03 & 1000 & -- & $<0.015$ & -- & $<0.006$ & 0.042 & -- & -- & $<0.012$ & -- & - & $<0.012$ \\
\hline 08 & 1100 & - & $<0.015$ & -- & $<0.006$ & 0.033 & -- & - & $<0.012$ & -- & - & $<0.012$ \\
\hline 17 & 1100 & - & $<0.015$ & - & $<0.006$ & 0.035 & -- & $-\cdot$ & $<0.012$ & -- & - & $<0.012$ \\
\hline 24 & 1100 & - & $<0.015$ & -- & $<0.006$ & 0.034 & - & - & $<0.012$ & -- & - & $<0.012$ \\
\hline 28 & 1130 & -- & $<0.015$ & -- & $<0.006$ & 0.033 & - & - & $<0.012$ & - & - & $<0.012$ \\
\hline 30 & 1130 & -- & $<0.015$ & - & $<0.006$ & 0.058 & $\cdots$ & - & $<0.012$ & -- & -- & $<0.012$ \\
\hline \multicolumn{13}{|c|}{ NOVEMBER } \\
\hline 04 & 1130 & - & $<0.015$ & - & $<0.006$ & 0.052 & $\cdots$ & -- & $<0.012$ & -- & - & $<0.012$ \\
\hline 13 & 1025 & -- & $<0.015$ & -- & $<0.006$ & 0.050 & -- & - & $<0.012$ & -- & -- & $<0.012$ \\
\hline 27 & 1200 & -- & $<0.015$ & -- & $<0.006$ & 0.063 & -- & -- & $<0.012$ & -- &. & $<0.012$ \\
\hline \multicolumn{13}{|c|}{ DECEMBER } \\
\hline 18 & 1200 & -- & $<0.015$ & -- & $<0.006$ & 0.031 & -- & -- & $<0.012$ & $\cdots$ & -- & $<0.012$ \\
\hline \multicolumn{13}{|c|}{ JANUARY 1992} \\
\hline 02 & 1200 & -- & $<0.015$ & -- & $<0.006$ & $<0.008$ & -- & - & $<0.012$ & -- & -- & $<0.012$ \\
\hline 16 & 1235 & $<0.009$ & $<0.015$ & $<0.016$ & $<0.006$ & 0.019 & $<0.015$ & $<0.030$ & $<0,012$ & $<0.008$ & $<0.008$ & $<0.012$ \\
\hline 30 & 1243 & $<0.009$ & $<0.015$ & $<0.016$ & $<0.006$ & 0.020 & $<0.015$ & $<0.030$ & $<0.012$ & $<0.008$ & $<0.008$ & $<0.012$ \\
\hline \multicolumn{13}{|c|}{ FEBRUARY } \\
\hline 13 & 1240 & $<0.009$ & $<0.015$ & $<0.016$ & $<0.006$ & 0.017 & $<0.015$ & $<0.030$ & $<0.012$ & $<0.008$ & $<0.008$ & $<0.012$ \\
\hline 27 & 1200 & $<0.009$ & 0.001 & $<0.016$ & $<0.006$ & 0.036 & 0.009 & $<0.030$ & $<0.012$ & $<0.008$ & $<0.008$ & $<0.012$ \\
\hline \multicolumn{13}{|l|}{ MARCH } \\
\hline 05 & 1130 & $<0.009$ & $<0.015$ & $<0.016$ & $<0.006$ & 0.042 & $<0.015$ & $<0.030$ & $<0.012$ & $<0.008$ & $<0.008$ & $<0.012$ \\
\hline 12 & 1115 & $<0.009$ & $<0.015$ & $<0.016$ & $<0.006$ & 0.034 & $<0.015$ & $<0.030$ & $<0.012$ & $<0.008$ & $<0.008$ & $<0.012$ \\
\hline 19 & 1133 & $<0.009$ & $<0.015$ & $<0.016$ & $<0.006$ & 0.088 & $<0.015$ & $<0.030$ & $<0.012$ & $<0.008$ & $<0.008$ & 0.004 \\
\hline 26 & 1128 & $<0.009$ & $<0.015$ & $<0.016$ & $<0.006$ & 0.048 & 0.015 & $<0.030$ & $<0.012$ & $<0.008$ & $<0.008$ & $<0.012$ \\
\hline \multicolumn{13}{|l|}{ APRIL } \\
\hline 02 & 1135 & $<0.009$ & $<0.015$ & $<0.016$ & $<0.006$ & 0.055 & 0.017 & $<0.030$ & $<0.012$ & $<0.008$ & $<0.008$ & 0.008 \\
\hline 09 & 1102 & $<0.009$ & $<0.015$ & $<0.016$ & $<0.006$ & 0.082 & 0.037 & $<0.030$ & $<0.012$ & $<0.008$ & $<0.008$ & 0.004 \\
\hline 16 & 1112 & $<0.009$ & $<0.015$ & $<0.016$ & $<0.006$ & 0.330 & 0.041 & $<0.030$ & $<0.012$ & $<0.008$ & $<0.008$ & 0.003 \\
\hline 20 & 1030 & $<0.009$ & $<0.015$ & $<0.016$ & $<0.006$ & 0.170 & 0.021 & $<0.030$ & $<0.012$ & $<0.008$ & $<0.008$ & 0.004 \\
\hline 24 & 1035 & $<0.009$ & $<0.015$ & $<0.016$ & $<0.006$ & 0.130 & 0.022 & $<0.030$ & $<0.012$ & 0.002 & $<0.008$ & 0.006 \\
\hline 29 & 1100 & $<0.009$ & $<0.015$ & $<0.016$ & $<0.006$ & 0.140 & 0.019 & $<0.030$ & $<0.012$ & $<0.008$ & $<0.008$ & 0.004 \\
\hline
\end{tabular}

APRIL 1991

$\begin{array}{llllll}10 & 1200 & -- & - & - & - \\ 18 & 1045 & -- & -- & -- & - \\ 23 & 1110 & -- & -- & -- & -\end{array}$


Table 8.--Pesticide results from solid-phase extraction 1-liter method--Continued

\begin{tabular}{|c|c|c|c|c|c|c|c|c|c|c|c|c|}
\hline Date & Time & $\begin{array}{c}\text { Pronamide } \\
\text { diss } \\
\text { ( } \mu g / L)\end{array}$ & $\begin{array}{l}\text { Propa- } \\
\text { chlor } \\
\text { diss } \\
(\mu \mathrm{g} / \mathrm{L})\end{array}$ & $\begin{array}{c}\text { Propanii } \\
\text { diss } \\
(\mu g / L)\end{array}$ & $\begin{array}{c}\text { Propargite } \\
\text { diss } \\
(\mu g / L)\end{array}$ & $\begin{array}{c}\text { Simazine } \\
\text { diss } \\
(\mu g / L)\end{array}$ & $\begin{array}{l}\text { Tebuth- } \\
\text { luron } \\
\text { diss } \\
(\mu g / L)\end{array}$ & $\begin{array}{c}\text { Terbacii } \\
\text { diss } \\
\text { (ug/L) }\end{array}$ & $\begin{array}{c}\text { Terbufos } \\
\text { diss } \\
(\mu g / L)\end{array}$ & 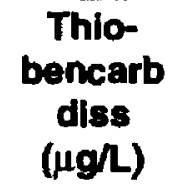 & $\begin{array}{c}\text { Trlaliate } \\
\text { diss } \\
\text { ( } \mu \mathrm{g} / \mathrm{L})\end{array}$ & $\begin{array}{c}\text { Trifluralin } \\
\text { diss } \\
(\mu g / L)\end{array}$ \\
\hline \multicolumn{13}{|c|}{ 03612500 - Ohio River at Dam 53 near Grand Chain, Ill.-Continued } \\
\hline \multicolumn{13}{|l|}{ MAY } \\
\hline 01 & 1040 & -- & $<0.015$ & -- & $<0.006$ & 0.035 & -- & -- & $<0.012$ & - & -- & $<0.012$ \\
\hline 07 & 1022 & -- & $<0.015$ & -- & $<0.006$ & 0.030 & -- & - & $<0.012$ & -- & - & $<0.012$ \\
\hline 14 & 1045 & -. & -. & -- & -- & -- & -- & -- & -- & - & -- & - \\
\hline 21 & 1045 & -- & $<0.015$ & -- & $<0.006$ & 0.763 & - & -- & $<0.012$ & - & -- & 0.002 \\
\hline 29 & 1030 & -- & $<0.015$ & -- & $<0.006$ & 0.071 & -- & -- & $<0.012$ & - & - & $<0.012$ \\
\hline \multicolumn{13}{|l|}{ JUNE } \\
\hline 04 & 1135 & -- & $<0.015$ & -- & $<0.006$ & 0.122 & -- & -- & $<0.012$ & - & - & 0.004 \\
\hline 11 & 1045 & -- & $<0.015$ & -- & $<0.006$ & 0.089 & -- & -- & $<0.012$ & -- & -- & $<0.012$ \\
\hline 18 & 1040 & -- & $<0.015$ & -- & $<0.006$ & 0.103 & -- & -- & $<0.012$ & -- & - & $<0.012$ \\
\hline 25 & 0946 & - & $<0.015$ & -- & $<0.006$ & 0.120 & - & -- & $<0.012$ & -- & - & 0.002 \\
\hline \multicolumn{13}{|c|}{ JULY 1991} \\
\hline 01 & 0950 & -- & $<0.015$ & - & $<0.006$ & 0.058 & - & - & $<0.012$ & -- & -- & $<0.012$ \\
\hline 09 & 1030 & -- & $<0.015$ & -- & $<0.006$ & 0.081 & -- & -- & $<0.012$ & -- & -- & $<0.012$ \\
\hline 15 & 1100 & -- & $<0.015$ & -- & $<0.006$ & 0.083 & -- & -- & $<0.012$ & .. &.- & $<0.012$ \\
\hline 22 & 1040 &.- & -. & -- & -- & -- & -- & -- & -- & -- & -- & $-\infty$ \\
\hline 29 & 1110 & -- & -- & -- & - & - & -- & - & -- & -- & - & -- \\
\hline \multicolumn{13}{|l|}{ AUGUST } \\
\hline 05 & 1050 & -- & -- & -- & -- & -- & -- & -- & -- & - & -- & - \\
\hline 12 & 1030 & -- & $<0.015$ & -- & $<0.006$ & 0.049 & -- & -- & $<0.012$ & -- & -- & $<0.012$ \\
\hline 20 & 1030 & $\cdots$ & $<0.015$ & -- & $<0.006$ & 0.047 & -- & -- & $<0.012$ & -- & -- & $<0.012$ \\
\hline 27 & 0945 & -- & $<0.015$ & -- & $<0.006$ & 0.035 & -- & -- & $<0.012$ & -- & - & $<0.012$ \\
\hline \multicolumn{13}{|c|}{ SEPTEMBER } \\
\hline 03 & 1040 & -- & $<0.015$ & -- & $<0.006$ & 0.036 & -- & - & $<0.012$ & -- & $\cdots$ & $<0.012$ \\
\hline 10 & 1030 & -- & $<0.015$ & -- & $<0.006$ & 0.036 & -- & -- & $<0.012$ & -- & - & $<0.012$ \\
\hline 17 & 0952 & -- & $<0.015$ & -- & $<0.006$ & 0.049 & -- & - & $<0.012$ & - & -- & $<0.012$ \\
\hline 23 & 1040 & -- & $<0.015$ & $\cdots$ & $<0.006$ & 0.040 & - & - & $<0.012$ & -- & -- & $<0.012$ \\
\hline 30 & 1122 & -- & $<0.015$ & - & $<0.006$ & 0.032 & - & - & $<0.012$ & -- & -- & $<0.012$ \\
\hline \multicolumn{13}{|c|}{ OCTOBER 1991} \\
\hline 07 & 1125 & -- & $<0.015$ & - & $<0.006$ & 0.019 & - & -- & $<0.012$ & -- & -- & $<0.012$ \\
\hline 15 & 0955 & -- & $<0.015$ & -- & $<0.006$ & 0.023 & - & - & $<0.012$ & -- & -- & $<0.012$ \\
\hline 21 & 1020 & -- & $<0.015$ & -- & $<0.006$ & 0.028 & -- & -- & $<0.012$ & -- & -- & $<0,012$ \\
\hline 29 & 0930 & $-\cdot$ & $<0.015$ & -- & $<0.006$ & 0.030 & -- & -- & $<0.012$ & -- & -- & $<0.012$ \\
\hline \multicolumn{13}{|c|}{ NOVEMBER } \\
\hline 06 & 1040 & -- & -- & -- & -- & -- & - & -- & -- & -- & - & -- \\
\hline 20 & 0920 &.- & $<0.015$ & -- & $<0.006$ & 0.027 & -- & -- & $<0.012$ & - & - & $<0.012$ \\
\hline \multicolumn{13}{|c|}{ DECEMBER } \\
\hline 05 & 1045 & -- & $<0.015$ & - & $<0.006$ & 0.028 & -- & -- & $<0.012$ & -- & - & $<0.012$ \\
\hline 17 & 1010 & - & $<0.015$ & - & $<0.006$ & 0.017 & -- & - & $<0.012$ & - & - & $<0.012$ \\
\hline \multicolumn{13}{|c|}{ JANUARY 1992} \\
\hline 02 & 1005 & -- & $<0.015$ & -- & $<0.006$ & 0.016 & -- & -- & $<0.012$ & -- & - & $<0.012$ \\
\hline 21 & 1030 & $<0.009$ & $<0.015$ & $<0.016$ & $<0.006$ & 0.016 & $<0.015$ & $<0.030$ & $<0.012$ & $<0.008$ & $<0.008$ & $<0.012$ \\
\hline \multicolumn{13}{|c|}{ FEBRUARY } \\
\hline 04 & 1125 & $<0.009$ & $<0.015$ & $<0.016$ & $<0.006$ & 0.010 & $<0.015$ & $<0.030$ & $<0.012$ & $<0.008$ & $<0.008$ & 0.003 \\
\hline 18 & 1025 & $<0.009$ & $<0.015$ & $<0.016$ & $<0.006$ & 0.013 & $<0.015$ & $<0.030$ & $<0.012$ & $<0.008$ & $<0.008$ & $<0.012$ \\
\hline \multicolumn{13}{|c|}{ MARCH 1992} \\
\hline 03 & 1025 & $<0.009$ & $<0.015$ & $<0.016$ & $<0.006$ & 0.010 & 0.016 & $<0.030$ & $<0.012$ & $<0.008$ & $<0.008$ & $<0.012$ \\
\hline 11 & 1030 & $<0.009$ & $<0.015$ & $<0.016$ & $<0.006$ & 0.009 & $<0.015$ & $<0.030$ & $<0.012$ & $<0.008$ & $<0.008$ & $<0.012$ \\
\hline 16 & 1040 & $<0.009$ & $<0.015$ & $<0.016$ & $<0.006$ & 0.012 & $<0.015$ & $<0.030$ & $<0.012$ & $<0.008$ & $<0.008$ & 0.003 \\
\hline
\end{tabular}


Table 8.-Pesticide results from solid-phase extraction 1-liter method-Continued

\begin{tabular}{|c|c|c|c|c|c|c|c|c|c|c|c|c|}
\hline Date & Time & $\begin{array}{c}\text { Pronamide } \\
\text { diss } \\
(\mu g / L)\end{array}$ & $\begin{array}{l}\text { Prope- } \\
\text { chlor } \\
\text { diss } \\
(\mu g / L)\end{array}$ & $\begin{array}{c}\text { Propanil } \\
\text { diss } \\
(\mu g / L)\end{array}$ & $\begin{array}{c}\text { Proparglte } \\
\text { diss } \\
(\mu g / L)\end{array}$ & $\begin{array}{c}\text { Simazine } \\
\text { diss } \\
(\mu g / L)\end{array}$ & $\begin{array}{l}\text { Tebuth- } \\
\text { luron } \\
\text { diss } \\
(\mu g / L)\end{array}$ & $\begin{array}{c}\text { Terbacll } \\
\text { diss } \\
(\mu \mathrm{g} / \mathrm{L})\end{array}$ & $\begin{array}{c}\text { Terbufos } \\
\text { diss } \\
(\mu g / L)\end{array}$ & $\begin{array}{c}\text { Thlo- } \\
\text { bencarb } \\
\text { diss } \\
(\mu \mathrm{g} / \mathrm{L})\end{array}$ & $\begin{array}{c}\text { Triallate } \\
\text { diss } \\
(\mu g / 2)\end{array}$ & $\begin{array}{c}\text { Trifiurailin } \\
\text { dises } \\
(\mu g / L)\end{array}$ \\
\hline \multicolumn{13}{|c|}{ 03612500 - Ohio River at Dam 53 near Grand Chain, Iil.-Continued } \\
\hline \multicolumn{13}{|c|}{ MARCH 1992 (cont.) } \\
\hline 24 & 1020 & $<0.009$ & $<0.015$ & $<0.016$ & $<0.006$ & 0.019 & 0.018 & $<0.030$ & $<0.012$ & $<0.008$ & $<0.008$ & 0.003 \\
\hline 31 & 1030 & $<0.009$ & $<0.015$ & $<0.016$ & $<0.006$ & 0.017 & 0.014 & $<0.030$ & $<0.012$ & $<0.008$ & $<0.008$ & $<0.012$ \\
\hline \multicolumn{13}{|l|}{ APRIL } \\
\hline 07 & 1015 & $<0.009$ & $<0.015$ & $<0.016$ & $<0.006$ & 0.029 & 0.030 & $<0.030$ & $<0.012$ & $<0.008$ & $<0.008$ & 0.004 \\
\hline 14 & 1035 & $<0.009$ & $<0.015$ & $<0.016$ & $<0.006$ & 0.026 & 0.035 & $<0.030$ & $<0.012$ & $<0.008$ & $<0.008$ & $<0.012$ \\
\hline 22 & 1040 & $<0.009$ & $<0.015$ & $<0.016$ & $<0.006$ & 0.180 & 0.012 & $<0.030$ & $<0.012$ & $<0.008$ & $<0.008$ & 0.003 \\
\hline 28 & 1104 & $<0.009$ & $<0.015$ & $<0.016$ & $<0.006$ & 0.150 & 0.016 & $<0.030$ & $<0.012$ & $<0.008$ & $<0.008$ & 0.004 \\
\hline \multicolumn{13}{|l|}{ MAY } \\
\hline 06 & 0953 & $<0.009$ & $<0.015$ & $<0.016$ & $<0.006$ & 0.076 & 0.012 & $<0.030$ & $<0.012$ & $<0.008$ & $<0.008$ & 0.003 \\
\hline 12 & 0955 & $<0.009$ & $<0.015$ & $<0.016$ & $<0.006$ & 0.046 & 0.011 & $<0.030$ & $<0.012$ & $<0.008$ & $<0.008$ & 0.004 \\
\hline 18 & 1025 & $<0.009$ & $<0.015$ & $<0.016$ & $<0.006$ & 0.047 & 0.012 & $<0.030$ & $<0.012$ & $<0.008$ & $<0.008$ & $<0.012$ \\
\hline 27 & 1030 & $<0.009$ & $<0.015$ & $<0.016$ & $<0.006$ & 0.160 & 0.015 & $<0.030$ & $<0.012$ & $<0.008$ & $<0.008$ & 0.003 \\
\hline \multicolumn{13}{|l|}{ JUNE 1992} \\
\hline 02 & 1045 & $<0.009$ & $<0.015$ & $<0.016$ & $<0.006$ & 0.480 & 0.020 & $<0.030$ & $<0.012$ & $<0.008$ & $<0.008$ & 0.004 \\
\hline 09 & 1105 & $<0.009$ & $<0.015$ & $<0.016$ & $<0.006$ & 0.090 & 0.016 & $<0.030$ & $<0.012$ & $<0.008$ & $<0.008$ & 0.002 \\
\hline 16 & 1105 & $<0.009$ & $<0.015$ & $<0.016$ & $<0.006$ & 0.045 & 0.023 & $<0.030$ & $<0.012$ & $<0.008$ & $<0.008$ & 0.003 \\
\hline 23 & 1023 & $<0.009$ & $<0.015$ & $<0.016$ & $<0.006$ & 0.063 & 0.023 & $<0.030$ & $<0.012$ & $<0.008$ & $<0.008$ & 0.002 \\
\hline 29 & 1235 & $<0.009$ & $<0.015$ & $<0.016$ & $<0.006$ & 0.100 & 0.013 & $<0.030$ & $<0.012$ & $<0.008$ & $<0.008$ & 0.003 \\
\hline \multicolumn{13}{|l|}{ JULY } \\
\hline 08 & 1012 & $<0.009$ & $<0.015$ & $<0.016$ & $<0.006$ & 0.072 & 0.029 & $<0.030$ & $<0.012$ & $<0.008$ & $<0.008$ & 0.004 \\
\hline 14 & 1025 & $<0.009$ & $<0.015$ & $<0.016$ & $<0.006$ & 0.093 & 0.015 & $<0.030$ & $<0.012$ & $<0.008$ & $<0.008$ & 0.003 \\
\hline 23 & 1040 & $<0.009$ & $<0.015$ & $<0.016$ & $<0.006$ & 0.088 & 0.030 & $<0.030$ & $<0.012$ & $<0.008$ & $<0.008$ & 0.003 \\
\hline 28 & 1025 & $<0.009$ & $<0.015$ & $<0.016$ & $<0.006$ & 0.086 & 0.026 & $<0.030$ & $<0.012$ & $<0.008$ & $<0.008$ & 0.003 \\
\hline
\end{tabular}


$\left[\mathrm{m}^{3} / \mathrm{s}\right.$, cubic meter per second; ${ }^{\circ} \mathrm{C}$, degree Celsius; $\mu \mathrm{S} / \mathrm{cm}$, microsiemens per centimeter at

25 degrees Celsius; $\mathrm{mg} / \mathrm{L}$, milligrams per liter; --, no data]

\begin{tabular}{|c|c|c|c|c|c|c|c|c|c|c|c|c|}
\hline Date & Time & $\begin{array}{c}\text { Water } \\
\text { dischsrge } \\
\left(\mathrm{m}^{3} / \mathrm{s}\right)\end{array}$ & $\begin{array}{c}\text { Temper- } \\
\text { ature } \\
\left({ }^{\circ} \mathrm{C}\right)\end{array}$ & pH & $\begin{array}{c}\text { Specific } \\
\text { conduct- } \\
\text { ance } \\
(\mu \mathrm{S} / \mathrm{cm})\end{array}$ & $\begin{array}{c}\text { Nitrogen, } \\
\text { ammonia } \\
\text { diss } \\
\text { (mg/L) }\end{array}$ & $\begin{array}{l}\text { Nitrogen, } \\
\text { nitrite } \\
\text { diss } \\
\text { (mg/L) }\end{array}$ & $\begin{array}{l}\text { Nitrogen, } \\
\text { nitrete } \\
\text { diss } \\
\text { (mg/l) }\end{array}$ & $\begin{array}{c}\text { Nitrogen } \\
\text { emmonia } \\
+ \\
\text { organic, } \\
\text { totai } \\
\text { (mg/l) }\end{array}$ & $\begin{array}{c}\text { Phos- } \\
\text { phorus, } \\
\text { total } \\
\text { (mg/l) }\end{array}$ & $\begin{array}{l}\text { Ortho- } \\
\text { phos- } \\
\text { phorus } \\
\text { diss } \\
\text { (mg/) }\end{array}$ & $\begin{array}{l}\text { Sus- } \\
\text { pended } \\
\text { sedl- } \\
\text { ment } \\
\text { (mg/h) }\end{array}$ \\
\hline \multicolumn{13}{|c|}{05420500 - Mississippi River at Clinton, Iowa } \\
\hline \multicolumn{13}{|c|}{ 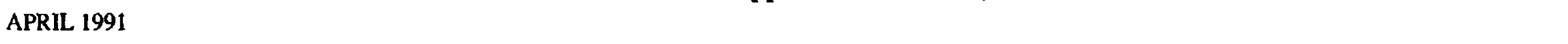 } \\
\hline 02 & 1040 & 3,140 & 8.0 & 7.5 & 305 & 0.13 & $<0.01$ & 0.550 & -- & -- & 0.02 & -- \\
\hline 11 & 1100 & 2,700 & 11.5 & 8.2 & 332 & 0.07 & 0.02 & 1.78 & -- & -- & 0.04 & -- \\
\hline 16 & 1150 & 3,200 & 9.0 & 8.2 & 335 & 0.13 & 0.03 & 3.57 & -- & - & 0.03 & -- \\
\hline 23 & 1030 & 3,170 & 11.5 & 8.6 & 295 & 0.01 & 0.02 & 2.48 & -- & - & 0.02 & - \\
\hline \multicolumn{13}{|c|}{ MAY 1991} \\
\hline 01 & 1150 & 2,820 & 14.0 & 8.5 & 366 & 0.05 & 0.02 & 2.58 & -- & -- & 0.03 & -- \\
\hline 07 & 1115 & 2,660 & 11.0 & 8.6 & 372 & 0.04 & 0.04 & 3.56 & -- & -- & 0.03 & - \\
\hline 10 & 1030 & 2,660 & 14.0 & 8.5 & 375 & 0.02 & 0.04 & 3.36 & -- & - & 0.08 & - \\
\hline 14 & 1120 & 2,970 & 19.0 & 8.4 & 355 & 0.02 & 0.02 & 2.88 & -- & -- & 0.02 & -- \\
\hline 17 & 1045 & 3,310 & 20.5 & 8.7 & 355 & 0.04 & 0.03 & 2.67 & - & - & 0.05 & -- \\
\hline 21 & 1140 & 3,570 & 18.5 & 8.3 & 326 & 0.07 & 0.05 & 3.05 & -- & - & 0.04 & - \\
\hline 24 & 1205 & 3,480 & 20.0 & 8.3 & 364 & 0.05 & 0.04 & 3.56 & -- & -- & 0.04 & - \\
\hline 28 & 1210 & 2,970 & 24.0 & 8.4 & 370 & 0.03 & 0.06 & 3.74 & - & - & 0.04 & - \\
\hline 31 & 1015 & 2,680 & 24.5 & 8.6 & 361 & 0.04 & 0.07 & 3.63 & - & - & 0.03 & - \\
\hline \multicolumn{13}{|c|}{ JUNE 1991} \\
\hline 04 & 1300 & 2,660 & 23.5 & 8.4 & 338 & 0.03 & 0.13 & 3.47 & 1.5 & 0.26 & 0.06 & 179 \\
\hline 07 & 1100 & 3,260 & 23.0 & 8.2 & 303 & 0.06 & 0.10 & 2.90 & 1.2 & 0.15 & 0.06 & - \\
\hline 10 & 1130 & 3,680 & 23.5 & 8.8 & 273 & 0.07 & 0.06 & 2.24 & 1.1 & 0.20 & 0.05 & 142 \\
\hline 13 & 1410 & 3,710 & 25.5 & 8.3 & 279 & 0.05 & 0.07 & 2.23 & 1.1 & 0.13 & 0.06 & 130 \\
\hline 17 & 1040 & 3,820 & 23.5 & 8.3 & 328 & 0.06 & 0.12 & 2.48 & 2.1 & 0.28 & 0.09 & 454 \\
\hline 20 & 1345 & 3,120 & 26.0 & 8.2 & 362 & 0.08 & 0.12 & 3.18 & 1.1 & 0.10 & 0.08 & 148 \\
\hline 24 & 1055 & 2,480 & 24.0 & 8.1 & 396 & 0.07 & 0.12 & 3.28 & 1.1 & 0.10 & 0.09 & 105 \\
\hline 27 & 0915 & 2,320 & 25.0 & 8.3 & 425 & 0.02 & 0.11 & 3.69 & -- & - & 0.08 & 103 \\
\hline \multicolumn{13}{|l|}{ JULY } \\
\hline 01 & 1025 & 2,260 & 27.5 & 8.5 & 368 & 0.02 & 0.05 & 2.85 & 1.4 & 0.21 & 0.08 & 79 \\
\hline 05 & 1205 & 1,970 & 27.0 & 8.3 & 439 & 0.01 & 0.04 & 2.76 & 1.3 & 0.21 & 0.08 & 58 \\
\hline 08 & 1130 & 1,860 & 25.5 & 8.3 & 457 & $<0.01$ & 0.05 & 2.75 & 1.8 & 0.17 & 0.08 & 71 \\
\hline 11 & 1300 & 1,910 & 26.0 & 8.4 & 467 & 0.02 & 0.04 & 2.76 & 1.4 & 0.17 & 0.07 & 54 \\
\hline 15 & 1025 & 2,050 & 24.5 & 8.2 & 474 & 0.02 & 0.05 & 1.65 & 1.3 & 0.18 & 0.10 & 70 \\
\hline 18 & 1200 & 1,840 & 26.5 & - & 486 & 0.10 & 0.02 & 2.28 & - & -- & 0.06 & -- \\
\hline 22 & 1050 & 1,770 & 27.0 & 8.0 & 465 & 0.05 & 0.02 & 1.78 & 1.3 & 0.23 & 0.08 & 55 \\
\hline 29 & 1230 & 1,770 & 22.5 & 8.2 & 412 & 0.05 & 0.01 & 1.19 & 1.7 & 0.23 & 0.07 & 82 \\
\hline \multicolumn{13}{|c|}{ AUGUST 1991} \\
\hline 06 & 1240 & 1,310 & 23.0 & 8.4 & 417 & 0.04 & 0.02 & 1.18 & 1.3 & 0.20 & 0.05 & 52 \\
\hline 12 & 1125 & 1,640 & 23.0 & 7.7 & 417 & 0.03 & 0.04 & 1.46 & 1.1 & 0.24 & 0.10 & 64 \\
\hline 19 & 1145 & 1,280 & 22.0 & 8.4 & 409 & 0.03 & 0.03 & 1.37 & 1.1 & 0.25 & 0.10 & 85 \\
\hline 26 & 1205 & 1,010 & 25.5 & 8.4 & 443 & 0.02 & 0.02 & 1.18 & 1.3 & 0.20 & 0.04 & 54 \\
\hline \multicolumn{13}{|c|}{ SEPTEMBER } \\
\hline 03 & 1200 & 657 & 25.5 & 8.6 & 433 & 0.03 & 0.02 & 0.94 & 1.2 & 0.20 & 0.05 & 46 \\
\hline 09 & 1500 & 643 & 25.0 & 8.6 & 422 & 0.02 & 0.01 & 0.77 & 1.4 & 0.25 & 0.05 & 59 \\
\hline 17 & 1215 & 1,950 & 20.5 & 8.4 & 456 & 0.01 & 0.01 & 1.29 & 0.90 & 0.24 & 0.09 & 118 \\
\hline 24 & 1215 & 1,960 & 14.0 & 8.2 & 441 & 0.03 & 0.02 & 1.08 & 1.3 & 0.27 & 0.09 & 103 \\
\hline
\end{tabular}




\begin{tabular}{|c|c|c|c|c|c|c|c|c|c|c|c|c|}
\hline Date & Time & $\begin{array}{c}\text { Water } \\
\text { discherge } \\
\left(\mathrm{m}^{3} / \mathrm{s}\right)\end{array}$ & $\begin{array}{c}\text { Temper- } \\
\text { ature } \\
\left({ }^{\circ} \mathrm{C}\right)\end{array}$ & pH & $\begin{array}{c}\text { Specific } \\
\text { conduct- } \\
\text { ance } \\
(\mu \mathrm{S} / \mathrm{cm})\end{array}$ & $\begin{array}{l}\text { Nitrogen, } \\
\text { ammonia } \\
\text { diss } \\
\text { (mg/L) }\end{array}$ & $\begin{array}{l}\text { Nitrogen, } \\
\text { nitrite } \\
\text { dlsa } \\
\text { (mg/t) }\end{array}$ & $\begin{array}{l}\text { Nitrogen, } \\
\text { nitrate } \\
\text { disa } \\
\text { (mg/) }\end{array}$ & $\begin{array}{c}\text { Nitrogen } \\
\text { ammonia } \\
+ \\
\text { organic, } \\
\text { total } \\
\text { (mg/L) }\end{array}$ & $\begin{array}{c}\text { Phos- } \\
\text { phorus, } \\
\text { total } \\
\text { (mg/L) }\end{array}$ & $\begin{array}{l}\text { Ortho- } \\
\text { phos- } \\
\text { phorus } \\
\text { diss } \\
\text { (mg/h) }\end{array}$ & $\begin{array}{l}\text { Sus- } \\
\text { pended } \\
\text { sedi- } \\
\text { ment } \\
\text { (mgl) }\end{array}$ \\
\hline \multicolumn{13}{|c|}{05420500 - Mississippi River st Clinton, Iowa-Continued } \\
\hline \multicolumn{13}{|c|}{ OCTOBER } \\
\hline 01 & 1200 & 1,530 & 14.5 & 8.1 & 393 & 0.03 & 0.01 & 0.94 & -- & - & 0.08 & - \\
\hline 07 & 1230 & 1,240 & 11.0 & 8.4 & 395 & 0.05 & 0.02 & 1.48 & 0.70 & 0.18 & 0.09 & 43 \\
\hline 15 & 1300 & 821 & 10.0 & 8.8 & 411 & 0.01 & 0.02 & 1.88 & 0.80 & 0.16 & 0.08 & 39 \\
\hline 22 & 1230 & 818 & 11.5 & 8.3 & 424 & 0.02 & $<0.01$ & 1.50 & 0.80 & 0.14 & 0.07 & 21 \\
\hline 29 & 1330 & 1,010 & 13.0 & 8.4 & 413 & 0.08 & 0.02 & 1.28 & 0.70 & 0.13 & 0.04 & 21 \\
\hline \multicolumn{13}{|c|}{ NOVEMBER 1991} \\
\hline 05 & 1245 & 1,710 & 2.0 & 8.0 & 405 & 0.10 & 0.01 & 1.59 & 1.0 & 0.17 & 0.07 & 89 \\
\hline 12 & 1255 & 2,390 & 0.5 & 7.8 & 325 & 0.09 & 0.02 & 1.48 & 0.90 & 0.18 & 0.06 & 49 \\
\hline 26 & 1320 & 2,500 & 0.0 & - & 362 & 0.08 & 0.02 & 1.98 & 0.80 & 0.17 & 0.06 & 51 \\
\hline \multicolumn{13}{|c|}{ DECEMBER } \\
\hline 09 & 1200 & 1,580 & 0.0 & 8.2 & 398 & 0.11 & 0.02 & 2.58 & 0.70 & 0.90 & 0.05 & 7 \\
\hline 22 & 1330 & 1,880 & 0.0 & 7.8 & 401 & 0.13 & 0.02 & 3.58 & 0.80 & 0.10 & 0.06 & 13 \\
\hline \multicolumn{13}{|c|}{ JANUARY 1992} \\
\hline 07 & 1210 & 1,610 & 0.5 & 7.7 & 391 & 0.11 & 0.02 & 2.98 & 0.60 & 0.10 & 0.06 & 4 \\
\hline 22 & 1340 & 1,190 & 0.5 & 7.8 & 398 & 0.11 & 0.021 & 2.88 & 0.70 & 0.11 & 0.04 & 4 \\
\hline \multicolumn{13}{|c|}{ FEBRUARY } \\
\hline 03 & 1245 & 1,160 & 1.0 & 7.8 & 415 & 0.08 & 0.02 & 2.78 & 0.70 & 0.08 & 0.06 & 4 \\
\hline 20 & 1230 & 1,630 & 0.5 & 7.9 & 400 & 0.18 & 0.02 & 2.68 & 0.90 & 0.15 & 0.10 & 49 \\
\hline \multicolumn{13}{|c|}{ MARCH 1992} \\
\hline 04 & 1100 & 1,750 & 4.5 & 8.2 & 390 & 0.06 & 0.03 & 2.27 & 0.80 & 0.14 & 0.06 & 17 \\
\hline 13 & 1200 & 2,890 & 2.0 & 8.1 & 390 & 0.12 & 0.03 & 2.77 & 1.0 & 0.18 & 0.07 & 56 \\
\hline 17 & 1400 & 3,600 & 3.0 & 8.2 & 356 & 0.11 & 0.03 & 2.27 & 1.0 & 0.18 & 0.05 & 88 \\
\hline 24 & 1125 & 3,460 & 4.0 & 7.8 & 385 & 0.10 & 0.03 & 3.67 & 0.70 & 0.08 & 0.05 & 47 \\
\hline 30 & 1155 & 2,890 & 5.0 & 8.0 & 408 & 0.15 & 0.03 & 4.17 & 0.60 & 0.08 & 0.04 & 40 \\
\hline \multicolumn{13}{|l|}{ APRIL } \\
\hline 06 & 1215 & 2,760 & 9.0 & 8.1 & 406 & 0.02 & 0.02 & 3.48 & 0.50 & 0.05 & 0.02 & 31 \\
\hline 14 & 1130 & 2,270 & 8.5 & 8.0 & 400 & $<0.01$ & 0.02 & 2.98 & 0.80 & 0.12 & 0.02 & 34 \\
\hline 24 & 1145 & 3,510 & 9.0 & 8.1 & 355 & 0.06 & 0.02 & 2.98 & 0.50 & 0.10 & 0.04 & 135 \\
\hline 28 & 1145 & 4,050 & 9.0 & 8.1 & 308 & $<0.01$ & 0.01 & 2.09 & 0.50 & 0.06 & 0.02 & 101 \\
\hline \multicolumn{13}{|l|}{ MAY } \\
\hline 05 & 1115 & 3,480 & 14.0 & 8.4 & 345 & 0.02 & 0.01 & 1.89 & 0.80 & 0.12 & 0.02 & 115 \\
\hline 12 & 1130 & 2,330 & 19.0 & 8.4 & 370 & 0.05 & 0.02 & 1.88 & 0.80 & 0.12 & 0.02 & 66 \\
\hline 19 & 1130 & 1,560 & 19.0 & 8.8 & 385 & 0.02 & 0.02 & 1.78 & 0.60 & 0.08 & 0.03 & 47 \\
\hline 26 & 1215 & 1,690 & 15.5 & 8.6 & 374 & 0.02 & 0.02 & 1.28 & 0.60 & 0.09 & 0.03 & 86 \\
\hline \multicolumn{13}{|c|}{ JUNE 1992} \\
\hline 01 & 1200 & 1,350 & 19.5 & 8.8 & 332 & 0.03 & 0.02 & 0.81 & 0.80 & 0.11 & 0.03 & 38 \\
\hline 08 & 1235 & 929 & 22.0 & 8.4 & 378 & 0.09 & 0.02 & 0.81 & 0.70 & 0.10 & 0.07 & 27 \\
\hline 16 & 1130 & 886 & 23.0 & 8.5 & 371 & 0.08 & 0.02 & 0.40 & 1.0 & 0.08 & 0.08 & 30 \\
\hline 22 & 1230 & 1,190 & 21.0 & 8.3 & 385 & 0.12 & 0.05 & 0.76 & 0.60 & 0.12 & 0.10 & 41 \\
\hline 29 & 1300 & 1,180 & 23.0 & 8.2 & 405 & 0.04 & 0.01 & 0.88 & 0.80 & 0.19 & 0.07 & 27 \\
\hline \multicolumn{13}{|l|}{ JULY } \\
\hline 08 & 0815 & 1,350 & 23.5 & 8.3 & 450 & 0.05 & 0.02 & 1.38 & 0.80 & 0.18 & 0.09 & 43 \\
\hline 13 & 1225 & 1,710 & 24.5 & 8.4 & 458 & 0.06 & 0.03 & 2.47 & 0.90 & 0.20 & 0.10 & 54 \\
\hline 20 & 1145 & 1,990 & 23.5 & 8.4 & 431 & 0.05 & 0.03 & 2.17 & 1.0 & 0.20 & 0.10 & 59 \\
\hline 27 & 1215 & 1,530 & 22.0 & 8.4 & 430 & .0 .05 & 0.04 & 1.86 & 0.90 & 0.15 & 0.10 & 36 \\
\hline
\end{tabular}




\begin{tabular}{|c|c|c|c|c|c|c|c|c|c|c|c|c|}
\hline Date & Time & $\begin{array}{c}\text { Water } \\
\text { discharge } \\
\left(\mathrm{m}^{3} / \mathrm{s}\right)\end{array}$ & $\begin{array}{l}\text { Temper- } \\
\text { ature } \\
\left.{ }^{\circ} \mathrm{C}\right)\end{array}$ & pH & $\begin{array}{c}\text { Specific } \\
\text { conduct- } \\
\text { ance } \\
(\mu S / \mathrm{cm})\end{array}$ & $\begin{array}{c}\text { Nitrogen, } \\
\text { ammonia } \\
\text { diss } \\
(\mathrm{mg} / \mathrm{L})\end{array}$ & $\begin{array}{l}\text { Nitrogen, } \\
\text { nitrite } \\
\text { diss } \\
\text { (mg/L) }\end{array}$ & $\begin{array}{l}\text { Nitrogen, } \\
\text { nitrate } \\
\text { diss } \\
\text { (mg/L) }\end{array}$ & $\begin{array}{c}\text { Nitrogen } \\
\text { ammonia } \\
+ \\
\text { orgsnic, } \\
\text { totai } \\
(m g / L)\end{array}$ & $\begin{array}{c}\text { Phos: } \\
\text { phorus, } \\
\text { totai } \\
\text { (mg/L) }\end{array}$ & $\begin{array}{l}\text { Ortho- } \\
\text { phos- } \\
\text { phorus } \\
\text { diss } \\
\text { (mg/l) }\end{array}$ & $\begin{array}{l}\text { Sus- } \\
\text { pended } \\
\text { sedl- } \\
\text { ment } \\
\text { (mgll) }\end{array}$ \\
\hline
\end{tabular}

APRIL 1991

\begin{tabular}{|c|c|c|c|c|c|c|c|c|c|c|c|c|}
\hline 05 & 1230 & 1,510 & -- & 8.2 & 657 & 0.06 & 0.06 & 1.34 & -- & -- & 0.11 & -- \\
\hline 17 & 1330 & 1,230 & -- & 7.0 & 670 & 0.06 & 0.07 & 5.33 & - & -- & 0.07 & - \\
\hline 26 & 1000 & 1,510 & 14.5 & 6.9 & 631 & 0.02 & 0.07 & 6.23 & - & -- & 0.07 & - \\
\hline \multicolumn{13}{|l|}{ MAY } \\
\hline 03 & 1545 & 1,240 & 15.0 & 7.4 & 642 & 0.01 & 0.08 & 5.72 & - & -- & 0.07 & - \\
\hline 08 & 1245 & 1,650 & 16.0 & 7.0 & 529 & 0.07 & 0.06 & 4.84 & - & -- & 0.08 & -- \\
\hline 10 & 1100 & 1,650 & 17.0 & 6.8 & 516 & 0.06 & 0.07 & 5.23 & - & -- & 0.10 & - \\
\hline 17 & 1145 & 1,260 & 22.5 & 7.8 & 642 & 0.09 & 0.08 & 5.72 & -- & -- & 0.06 & -- \\
\hline 19 & 1115 & 1,460 & 21.0 & 8.0 & 499 & 0.12 & 0.10 & 4.70 & -- & -. & 0.06 & -- \\
\hline 22 & 1715 & 1,420 & 21.0 & 7.5 & 495 & 0.07 & 0.12 & 6.38 & -- & -- & 0.11 & -- \\
\hline 29 & 1130 & 1,510 & 25.5 & 8.1 & 544 & 0.04 & 0.11 & 5.59 & -- & -- & 0.09 & -- \\
\hline 31 & 1045 & 1,820 & 25.0 & 8.1 & 468 & 0.06 & 0.10 & 4.60 & -. & -- & 0.07 & -- \\
\hline \multicolumn{13}{|c|}{ JUNE 1991} \\
\hline 04 & 1215 & 1,550 & 27.0 & 7.9 & 585 & 0.05 & 0.13 & 5.67 & -- & -- & 0.10 & -- \\
\hline 06 & 1200 & 1,450 & 25.0 & 7.9 & 581 & 0.08 & 0.14 & 6.06 & -- & -- & 0.11 & - \\
\hline 11 & 1345 & 1,140 & 25.0 & 7.9 & 627 & 0.04 & 0.11 & 5.69 & 0.90 & 0.28 & 0.14 & -- \\
\hline 14 & 1200 & 980 & 27.0 & 8.0 & 641 & 0.09 & 0.08 & 5.32 & 1.9 & 0.32 & 0.11 & - \\
\hline 18 & 1130 & 855 & 27.5 & 8.1 & 651 & 0.05 & 0.10 & 4.60 & 1.9 & 0.35 & 0.11 & -. \\
\hline 20 & 1130 & 784 & 28.0 & 8.2 & 664 & 0.04 & 0.09 & 4.31 & 1.7 & 0.34 & 0.10 & - \\
\hline 24 & 1130 & 493 & 28.0 & 7.9 & 661 & 0.12 & 0.16 & 3.34 & 1.6 & 0.33 & 0.11 & -- \\
\hline 27 & 1100 & 445 & 28.0 & 7.9 & 680 & 0.05 & 0.13 & 2.97 & 1.3 & 0.30 & 0.11 & 124 \\
\hline \multicolumn{13}{|l|}{ JULY } \\
\hline 01 & 0930 & 377 & 29.5 & 8.1 & 682 & 0.04 & 0.22 & 3.68 & 1.1 & 0.35 & 0.13 & 83 \\
\hline 03 & 1100 & 346 & 30.0 & 7.6 & 666 & 0.05 & 0.13 & 2.57 & 0.90 & 0.24 & 0.11 & 86 \\
\hline 09 & 1000 & 300 & 29.0 & 7.8 & 680 & 0.10 & 0.13 & 1.97 & 1.7 & 0.20 & 0.11 & 59 \\
\hline 11 & 1045 & 303 & 29.0 & 8.2 & 740 & 0.16 & 0.17 & 1.53 & 1.0 & 0.30 & 0.15 & 66 \\
\hline 18 & 1115 & 262 & 29.5 & 8.0 & 680 & 0.06 & 0.09 & 2.01 & 1.3 & 0.20 & 0.08 & 124 \\
\hline 25 & 1340 & 253 & 29.5 & 8.0 & 690 & 0.14 & 0.09 & 1.31 & 0.80 & 0.26 & 0.12 & 38 \\
\hline 31 & 1100 & 201 & 30.0 & 7.7 & 695 & 0.08 & 0.13 & 0.97 & 1.2 & 0.36 & 0.14 & -. \\
\hline \multicolumn{13}{|c|}{ AUGUST 1991} \\
\hline 06 & 1015 & 275 & 26.5 & 7.9 & 690 & 0.07 & 0.22 & 1.08 & 1.1 & 0.33 & 0.14 & 68 \\
\hline 13 & 1200 & 266 & 26.0 & 8.3 & 733 & $<0.01$ & 0.20 & 1.50 & 0.90 & 0.42 & 0.27 & 60 \\
\hline 21 & 1230 & 243 & 27.5 & 7.6 & 670 & 0.03 & 0.13 & 1.57 & 1.2 & 0.32 & 0.13 & 59 \\
\hline 28 & 1015 & 193 & 29.5 & 7.7 & 685 & 0.01 & 0.21 & 1.79 & 1.3 & 0.34 & 0.13 & 45 \\
\hline \multicolumn{13}{|c|}{ SEPTEMBER } \\
\hline 03 & 1050 & 208 & -- & 7.8 & 685 & 0.04 & 0.25 & 1.45 & 1.0 & 0.33 & 0.16 & 41 \\
\hline 10 & 1200 & 360 & 27.0 & 7.7 & 630 & $<0.01$ & 0.29 & 1.81 & 0.60 & 0.33 & 0.16 & 88 \\
\hline 17 & 1100 & 263 & -- & 7.9 & 650 & $<0.01$ & 0.16 & 1.84 & 1.2 & 0.34 & 0.16 & 102 \\
\hline 24 & 1100 & 175 & 19.5 & 7.7 & 640 & 0.04 & 0.13 & 1.97 & 0.80 & 0.29 & 0.16 & 53 \\
\hline \multicolumn{13}{|c|}{ OCTOBER } \\
\hline 01 & 1130 & 103 & 19.5 & 8.1 & 630 & 0.02 & 0.04 & 2.16 & 0.80 & 0.28 & 0.15 & 76 \\
\hline 08 & 1330 & 728 & 14.5 & 7.4 & 484 & 0.10 & 0.04 & 1.76 & 1.3 & 0.58 & 0.14 & 476 \\
\hline 15 & 1215 & 248 & 16.0 & 8.0 & 680 & 0.19 & 0.06 & 2.74 & 0.80 & 0.43 & 0.25 & 86 \\
\hline 22 & 1130 & 170 & 14.0 & 8.0 & 696 & 0.18 & 0.08 & 2.82 & 0.80 & 0.32 & 0.24 & 38 \\
\hline \multicolumn{13}{|c|}{ NOVEMBER 1991} \\
\hline 05 & 1140 & 680 & 5.0 & 8.0 & 635 & 0.28 & 0.05 & 3.35 & 1.0 & 0.39 & 0.24 & -- \\
\hline 15 & 1130 & 385 & 5.5 & 7.8 & 724 & 0.18 & 0.07 & 4.13 & 1.1 & 0.40 & 0.18 & 90 \\
\hline
\end{tabular}




\begin{tabular}{|c|c|c|c|c|c|c|c|c|c|c|c|c|}
\hline Date & Time & $\begin{array}{c}\text { Water } \\
\text { discharge } \\
\left(\mathrm{m}^{3} / \mathrm{s}\right)\end{array}$ & $\begin{array}{c}\text { Temper- } \\
\text { ature } \\
\left({ }^{\circ} \mathrm{C}\right)\end{array}$ & PH & $\begin{array}{c}\text { Specific } \\
\text { conduct- } \\
\text { ence } \\
(\mu S / c m)\end{array}$ & $\begin{array}{c}\text { Nitrogen, } \\
\text { ammonia } \\
\text { diss } \\
\text { (mgll) }\end{array}$ & $\begin{array}{l}\text { Nitrogen, } \\
\text { nitrite } \\
\text { diss } \\
\text { (mg/L) }\end{array}$ & $\begin{array}{l}\text { Nitrogen, } \\
\text { nitrate } \\
\text { diss } \\
\text { (mg/L) }\end{array}$ & $\begin{array}{c}\text { Nitrogen } \\
\text { ammonie } \\
+ \\
\text { organic, } \\
\text { total } \\
\text { (mg/L) }\end{array}$ & $\begin{array}{l}\text { Phoa- } \\
\text { phorus, } \\
\text { total } \\
\text { (mg/L) }\end{array}$ & $\begin{array}{l}\text { Ortho- } \\
\text { phos- } \\
\text { phorus } \\
\text { diss } \\
\text { (mg/L) }\end{array}$ & $\begin{array}{c}\text { Sus- } \\
\text { pended } \\
\text { sedl- } \\
\text { ment } \\
\text { (mght) }\end{array}$ \\
\hline \multicolumn{13}{|c|}{05586100 - Illinois River at Valley City-Continned } \\
\hline \multicolumn{13}{|c|}{ DECEMBER } \\
\hline 03 & 1030 & 725 & 4.5 & 8.2 & 700 & 0.09 & 0.05 & 5.95 & 1.3 & 0.53 & 0.14 & 344 \\
\hline 18 & 1200 & 864 & 3.0 & 7.2 & 747 & 0.11 & 0.06 & 7.74 & 1.2 & 0.37 & 0.14 & 147 \\
\hline \multicolumn{13}{|c|}{ JANUARY 1992} \\
\hline 02 & 1330 & 564 & 3.5 & 7.9 & 749 & 0.17 & 0.06 & 5.94 & 1.1 & 0.32 & 0.13 & 101 \\
\hline 15 & 1645 & 507 & -- & 8.0 & 813 & -- & -- & -- & -- & - & -- & -- \\
\hline 30 & 1345 & 493 & 2.5 & 8.1 & 828 & 0.24 & 0.10 & 6.70 & 1.2 & 0.43 & 0.20 & 92 \\
\hline \multicolumn{13}{|c|}{ FEBRUARY } \\
\hline 12 & 1200 & 413 & 4.0 & 8.1 & 862 & 0.26 & 0.05 & 5.55 & 1.1 & 0.39 & 0.17 & 100 \\
\hline 28 & 1245 & 742 & 8.0 & 7.8 & 740 & 0.21 & 0.06 & 9.24 & 1.1 & 0.32 & 0.15 & 108 \\
\hline \multicolumn{13}{|l|}{ MARCH } \\
\hline 03 & 1100 & 716 & 8.5 & 7.8 & 773 & 0.07 & 0.05 & 8.95 & 1.0 & 0.31 & 0.10 & 111 \\
\hline 10 & 1015 & 575 & 11.0 & 8.3 & 747 & 0.05 & 0.05 & 7.15 & -- & -- & 0.12 & -- \\
\hline 17 & 1050 & 649 & 7.0 & 8.0 & 785 & 0.09 & 0.06 & 6.94 & 1.0 & 0.35 & 0.16 & 92 \\
\hline 25 & 1045 & 708 & 8.5 & 8.2 & 813 & 0.03 & 0.04 & 6.76 & 1.1 & 0.34 & 0.12 & 115 \\
\hline \multicolumn{13}{|c|}{ O6805500 - Platte River at Louisville, Nebr. } \\
\hline \multicolumn{13}{|c|}{ APRIL 1991} \\
\hline 09 & 1250 & 144 & -- & 8.7 & 635 & 0.03 & 0.01 & 0.16 & - & -- & 0.14 & -- \\
\hline 16 & 1030 & 215 & 11.5 & 8.2 & 533 & 0.24 & 0.05 & 1.35 & -- & -- & 0.24 & -- \\
\hline 24 & 1030 & 162 & 13.0 & 8.0 & 546 & 0.02 & 0.07 & 0.73 & -- & -- & 0.18 & -- \\
\hline 29 & 1000 & 149 & 11.0 & 8.8 & 595 & $<0.01$ & 0.01 & 0.29 & - & -- & 0.10 & -- \\
\hline \multicolumn{13}{|l|}{ MAY } \\
\hline 06 & 1115 & 244 & 10.0 & 8.4 & 470 & 0.04 & 0.02 & 0.68 & -- & -- & 0.14 & .- \\
\hline 09 & 0940 & 206 & 18.0 & 8.3 & 588 & 0.03 & 0.03 & 0.97 & -- & -- & 0.18 & -- \\
\hline 13 & 1110 & 183 & - & 8.7 & 544 & 0.01 & 0.02 & 0.29 & -- & -- & 0.10 & -- \\
\hline 16 & 1130 & 161 & 23.0 & 8.9 & 483 & 0.04 & $<0.01$ & $<0.05$ & -- & -- & 0.10 & -- \\
\hline 21 & 0945 & 255 & 19.0 & 8.2 & 445 & 0.09 & 0.05 & 1.25 & -- & -- & 0.19 & - \\
\hline 24 & 0740 & 179 & 22.0 & 8.0 & 480 & 0.04 & 0.03 & 0.74 & -- & -- & 0.18 & -. \\
\hline 29 & 1015 & 306 & 27.0 & 8.5 & 269 & 0.05 & 0.01 & 0.78 & -- & $-\cdot$ & 0.14 & -- \\
\hline 31 & 0735 & 292 & 24.0 & 7.5 & 555 & 0.07 & 0.03 & 1.27 & -- & -- & 0.42 & -. \\
\hline \multicolumn{13}{|c|}{ JUNE 1991} \\
\hline 04 & 0800 & 572 & 24.0 & 6.3 & 437 & 0.03 & 0.03 & 1.27 & -- & -- & 0.25 & -- \\
\hline 07 & 1415 & 1,000 & -- & 6.4 & 347 & 0.24 & 0.08 & 1.72 & -. & -- & 0.15 & -- \\
\hline 11 & 1045 & 374 & 23.0 & 6.1 & 491 & 0.08 & 0.05 & 1.25 & -- & - & 0.26 & -- \\
\hline 14 & 0750 & 714 & 22.0 & 6.4 & 360 & 0.03 & 0.04 & 1.26 & -. & -- & 0.15 & -- \\
\hline 18 & 0935 & 295 & -- & 7.4 & 540 & 0.02 & 0.04 & 1.16 & -- & -- & 0.22 & -. \\
\hline 21 & 1020 & 206 & 23.0 & 6.3 & 521 & 0.04 & $<0.01$ & 0.86 & -. & -- & 0.18 & -- \\
\hline 24 & 1110 & 217 & 22.0 & 7.4 & 540 & 0.05 & $<0.01$ & 1.20 & -- & -- & 0.22 & -- \\
\hline 27 & 1020 & 153 & 26.0 & 6.3 & 678 & $<0.01$ & -- & -- & -- & -- & - & -. \\
\hline \multicolumn{13}{|l|}{ JULY } \\
\hline 01 & 0950 & 102 & 28.0 & 6.4 & 611 & 0.02 & $<0.01$ & $<0.05$ & -- & -- & 0.10 & -- \\
\hline 03 & 0950 & 144 & 26.0 & 6.1 & 600 & $<0.01$ & $<0.01$ & 1.20 & -- & -- & 0.18 & -- \\
\hline 08 & 1100 & 65.7 & 25.0 & 6.3 & 714 & 0.02 & 0.02 & 0.17 & -- & -- & 0.15 & -- \\
\hline 11 & 1020 & 125 & 22.5 & 6.8 & 432 & 0.04 & 0.03 & 0.53 & -. & -- & 0.13 & -- \\
\hline 15 & 0950 & 121 & 25.5 & 9.0 & 558 & -. & -. & -- & -- & -- & $-\cdot$ & -- \\
\hline 25 & 1000 & 49.3 & 24.0 & 8.4 & 746 & 0.05 & $<0.01$ & $<0.05$ & -- & -. & 0.06 & -- \\
\hline 29 & 0940 & 48.4 & 21.0 & 8.8 & 741 & 0.05 & $<0.01$ & $<0.05$ & -- & -- & 0.08 & -- \\
\hline
\end{tabular}




\begin{tabular}{|c|c|c|c|c|c|c|c|c|c|c|c|c|}
\hline Date & Time & $\begin{array}{c}\text { Water } \\
\text { discharge } \\
\left(\mathrm{m}^{3} / \mathrm{s}\right)\end{array}$ & $\begin{array}{l}\text { Temper- } \\
\text { ature } \\
\left.\text { ( }^{\circ} \mathrm{C}\right)\end{array}$ & pH & $\begin{array}{c}\text { Specific } \\
\text { conduct- } \\
\text { ance } \\
(\mu \mathrm{S} / \mathrm{cm})\end{array}$ & $\begin{array}{c}\text { Nitrogen, } \\
\text { ammonia } \\
\text { disa } \\
\text { (mg/l) }\end{array}$ & $\begin{array}{l}\text { Nitrogen, } \\
\text { nitrite } \\
\text { diss } \\
\text { (mg/l) }\end{array}$ & $\begin{array}{l}\text { Nitrogen, } \\
\text { nitrate } \\
\text { diss } \\
\text { (mg/l) }\end{array}$ & $\begin{array}{c}\text { Nitrogen } \\
\text { ammonia } \\
+ \\
\text { organic, } \\
\text { total } \\
\text { (mg/L) }\end{array}$ & $\begin{array}{c}\text { Phos- } \\
\text { phorus, } \\
\text { total } \\
\text { (mg/L) }\end{array}$ & $\begin{array}{c}\text { Ortho- } \\
\text { phos- } \\
\text { phorus } \\
\text { diss } \\
\text { (mg/L) }\end{array}$ & $\begin{array}{c}\text { Sus- } \\
\text { pended } \\
\text { sedf- } \\
\text { ment } \\
\text { (mg/L) }\end{array}$ \\
\hline
\end{tabular}

AUGUST 1991

\begin{tabular}{|c|c|c|c|c|c|c|c|c|c|c|c|c|}
\hline 06 & 1130 & 47.9 & 20.0 & 8.4 & 1,190 & 0.02 & 0.06 & 0.13 & _- & -- & 0.30 & -- \\
\hline 12 & 1115 & 45.3 & 22.5 & 8.8 & 818 & $<0.01$ & $<0.01$ & $<0.05$ & -- & -- & 0.16 & -. \\
\hline 20 & 1000 & 50.4 & 21.0 & 8.8 & 769 & $<0.01$ & $<0.01$ & $<0.05$ & -- & - & 0.17 & - \\
\hline 27 & 0940 & 34.6 & 24.0 & 8.6 & 1,080 & $<0.01$ & $<0.01$ & $<0.05$ & -- & -- & 0.23 & -- \\
\hline \multicolumn{13}{|c|}{ SEPTEMBER } \\
\hline 03 & 0950 & 28.6 & 25.0 & 8.6 & 1,430 & 0.06 & 0.01 & 0.055 & -- & -- & 0.43 & -- \\
\hline 10 & 1030 & 34.0 & 24.0 & 8.5 & 734 & $<0.01$ & $<0.01$ & $<0.05$ & 2.1 & 0.56 & 0.19 & 179 \\
\hline 17 & 1115 & 71.1 & 24.0 & 8.6 & 595 & 0.02 & 0.01 & $<0.05$ & 1.9 & 0.67 & 0.15 & 462 \\
\hline 24 & 1135 & 53.2 & 13.0 & 8.8 & 781 & 0.02 & 0.04 & 0.09 & 1.0 & 0.52 & 0.23 & 133 \\
\hline \multicolumn{13}{|c|}{ OCTOBER } \\
\hline 01 & 1115 & 53.0 & 14.0 & 8.5 & 887 & 0.01 & $<0.01$ & $<0.05$ & 1.1 & 0.44 & 0.17 & 109 \\
\hline 08 & 1030 & 58.1 & 13.0 & 8.4 & 845 & 0.03 & 0.02 & 0.16 & 1.3 & 0.44 & 0.23 & 108 \\
\hline 15 & 1130 & 67.7 & 9.0 & 8.3 & 879 & $<0.01$ & 0.02 & 0.14 & 1.1 & 0.40 & 0.20 & 74 \\
\hline 24 & 1200 & 79.0 & 12.0 & 8.4 & 757 & 0.02 & 0.03 & 0.43 & -- & -- & 0.25 & 107 \\
\hline \multicolumn{13}{|c|}{ NOVEMBER } \\
\hline 05 & 1200 & 68.0 & 0.5 & 8.1 & 1,140 & 0.37 & 0.04 & 0.96 & 0.90 & 0.51 & 0.37 & 68 \\
\hline 19 & 1140 & 170 & 6.5 & 8.0 & 590 & 0.28 & 0.03 & 1.17 & 1.6 & 0.52 & 0.22 & -- \\
\hline \multicolumn{13}{|c|}{ DECEMBER 1991} \\
\hline 05 & 1130 & 62.6 & 1.0 & 7.9 & 755 & 0.28 & 0.03 & 1.37 & 0.80 & 0.36 & 0.27 & 119 \\
\hline 20 & 1000 & 135 & 0.5 & 8.1 & 669 & 0.47 & 0.03 & 1.67 & 1.4 & 0.46 & 0.27 & 84 \\
\hline 30 & 1020 & 169 & 2.0 & 8.0 & 647 & 0.16 & 0.05 & 1.45 & 0.30 & 0.24 & 0.26 & 402 \\
\hline \multicolumn{13}{|c|}{ IANUARY 1992} \\
\hline 14 & 1000 & 186 & 0.0 & 8.1 & 615 & 0.17 & 0.01 & 1.49 & 1.2 & 0.45 & 0.19 & 234 \\
\hline 28 & 1000 & 188 & 0.0 & 8.0 & 590 & 0.12 & $<0.01$ & 1.40 & 0.60 & 0.30 & 0.20 & 268 \\
\hline \multicolumn{13}{|c|}{ FBRUARY } \\
\hline 11 & 1030 & $175^{\circ}$ & 0.5 & 8.1 & 724 & 0.11 & 0.02 & 1.28 & 0.90 & 0.37 & 0.19 & 168 \\
\hline 27 & 1000 & 198 & 6.0 & 8.1 & 660 & 0.06 & 0.01 & 1.49 & 0.90 & 0.36 & 0.15 & 153 \\
\hline \multicolumn{13}{|c|}{ MARCH } \\
\hline 05 & 1010 & 197 & 12.0 & 8.1 & 562 & 0.05 & $<0.01$ & 1.40 & 1.1 & 0.37 & 0.16 & 181 \\
\hline 09 & 0945 & 281 & 11.0 & 8.0 & 643 & 0.07 & 0.03 & 1.47 & 1.1 & 0.34 & 0.17 & 599 \\
\hline 17 & 1145 & 297 & 11.0 & 8.5 & 705 & 0.05 & 0.02 & 1.38 & 0.80 & 0.30 & 0.17 & 446 \\
\hline 23 & 1040 & 249 & 7.5 & 8.1 & 723 & 0.03 & 0.01 & 1.19 & 0.50 & 0.21 & 0.15 & 130 \\
\hline 31 & 1100 & 297 & 8.0 & 8.1 & 721 & 0.04 & 0.01 & 1.49 & 1.4 & 0.46 & 0.13 & 334 \\
\hline \multicolumn{13}{|c|}{ APRIL 1992} \\
\hline 07 & 1100 & 201 & 15.0 & 8.3 & 706 & 0.06 & $<0.01$ & 1.10 & 1,0 & 0.34 & 0.11 & 72 \\
\hline 14 & 1010 & 182 & 9.0 & 8.4 & 710 & 0.04 & 0.01 & 0.62 & 1.0 & 0.24 & 0.09 & 193 \\
\hline 23 & 1020 & 206 & 7.0 & 8.1 & 659 & 0.04 & $<0.01$ & 0.95 & $<0.20$ & 0.18 & 0.12 & 122 \\
\hline 29 & 1030 & 172 & 14.0 & 7.9 & 615 & 0.01 & 0.02 & 1.88 & 0.30 & 0.23 & 0.14 & 186 \\
\hline \multicolumn{13}{|l|}{ MAY } \\
\hline 07 & 1100 & 134 & 16.0 & 8.0 & 488 & 0.04 & $<0.01$ & $<0.05$ & $<0.20$ & 0.08 & $<0.01$ & 240 \\
\hline \multicolumn{13}{|c|}{06934500 - Missouri River at Hermann, Mo. } \\
\hline \multicolumn{13}{|c|}{ APRIL 1991} \\
\hline 09 & 1331 & 1,180 & -- & 8.2 & 570 & 0.04 & 0.02 & 1.68 & -- & -- & 0.11 & - \\
\hline 16 & 1030 & 3,260 & 16.5 & 8.8 & 429 & 0.05 & 0.02 & 0.87 & -- & -- & 0.06 & -- \\
\hline 24 & 1200 & 3,460 & 14.0 & 7.9 & 365 & 0.07 & 0.17 & 2.13 & -- & -- & 0.06 & -- \\
\hline
\end{tabular}




\begin{tabular}{|c|c|c|c|c|c|c|c|c|c|c|c|c|}
\hline Dete & Time & $\begin{array}{c}\text { Water } \\
\text { discharge } \\
\left(\mathrm{m}^{3} / \mathrm{s}\right)\end{array}$ & $\begin{array}{c}\text { Temper- } \\
\text { ature } \\
\left.\text { ( }{ }^{\circ} \mathrm{C}\right)\end{array}$ & pH & $\begin{array}{c}\text { Specific } \\
\text { conduct- } \\
\text { ance } \\
(\mu \mathrm{S} / \mathrm{cm})\end{array}$ & $\begin{array}{c}\text { Nitrogen, } \\
\text { ammonio } \\
\text { disa } \\
\text { (mg/L) }\end{array}$ & $\begin{array}{l}\text { Nitrogen, } \\
\text { nitrite } \\
\text { diss } \\
\text { (mg/L) }\end{array}$ & $\begin{array}{l}\text { Nitrogen, } \\
\text { nitrate } \\
\text { diaa } \\
\text { (mg/L) }\end{array}$ & $\begin{array}{c}\text { Nitrogen } \\
\text { ammonia } \\
+ \\
\text { organic, } \\
\text { total } \\
(\mathrm{mg} / \mathrm{L})\end{array}$ & $\begin{array}{c}\text { Phos- } \\
\text { phorua, } \\
\text { total } \\
\text { (mg/) }\end{array}$ & $\begin{array}{l}\text { Ortho- } \\
\text { phos- } \\
\text { phorus } \\
\text { dlss } \\
(\mathrm{mgh})\end{array}$ & $\begin{array}{l}\text { Sus- } \\
\text { pended } \\
\text { sodl- } \\
\text { ment } \\
\text { (mg/L) }\end{array}$ \\
\hline \multicolumn{13}{|c|}{06934500 - Missouri River at Hermann, Mo.-Continned } \\
\hline \multicolumn{13}{|l|}{ MAY } \\
\hline 01 & 1130 & 3,460 & 17.5 & 8.0 & 502 & 0.02 & 0.05 & 2.45 & 0.60 & - & 0.07 & - \\
\hline 06 & 1100 & 4,220 & 16.5 & 7.6 & 361 & 0.08 & 0.07 & 1.63 & 2.7 & 0.43 & 0.13 & 3,400 \\
\hline 09 & 0930 & 4,050 & 16.5 & 7.9 & 375 & 0.03 & 0.05 & 1.55 & -- & -- & 0.06 & -- \\
\hline 13 & 1000 & 2,920 & 18.0 & 8.1 & 429 & 0.03 & 0.04 & 1.76 & -. & - & 0.07 & -- \\
\hline 16 & 1020 & 3,620 & -- & 8.1 & 370 & 0.02 & 0.04 & 1.36 & -- & -. & 0.06 & - \\
\hline 20 & 1000 & 2,460 & 22.0 & 7.9 & 515 & 0.04 & 0.04 & 2.06 & - & -- & 0.07 & - \\
\hline 24 & 1000 & 2,450 & 23.0 & 8.1 & 549 & 0.01 & $<0.01$ & 2.20 & -- & -- & 0.08 & -- \\
\hline 28 & 1030 & 3,230 & 24.0 & 7.9 & 406 & 0.02 & $<0.01$ & 1.80 & -- & - & 0.08 & -. \\
\hline 31 & 1040 & 3,060 & 25.5 & 8.2 & 430 & $<0.01$ & $<0.01$ & 1.80 & - & - & 0.07 & -- \\
\hline \multicolumn{13}{|c|}{ JUNE 1991} \\
\hline 03 & 1000 & 3,510 & 26.5 & 8.1 & 538 & $<0.01$ & $<0.01$ & 2.20 & -- & - & 0.09 & -- \\
\hline 06 & 1000 & 3,290 & 25.5 & 8.0 & 433 & 0.02 & 0.01 & 2.39 & -- & -- & 0.10 & -- \\
\hline 11 & 1000 & 2,830 & 25.0 & 8.1 & 539 & 0.01 & $<0.01$ & 2.90 & 1.8 & 0.47 & 0.13 & 2,760 \\
\hline 13 & 1000 & 2,410 & 26.0 & 8.2 & 458 & 0.04 & 0.01 & 2.59 & 3.4 & 0.15 & 0.14 & 2,510 \\
\hline 21 & 1000 & 2,630 & 27.5 & 7.9 & 470 & 0.02 & 0.02 & 2.48 & 4.2 & 0.67 & 0.12 & -- \\
\hline 25 & 1130 & 2,070 & 28.0 & 8.2 & 486 & 0.06 & 0.02 & 2.98 & 1.4 & 0.46 & 0.15 & 1,470 \\
\hline 27 & 1045 & 2,020 & 29.0 & 8.1 & 551 & 0.01 & $<0.01$ & 2.90 & 0.70 & 0.61 & 0.13 & 1,220 \\
\hline \multicolumn{13}{|l|}{ JULY } \\
\hline 01 & 1100 & 1,580 & 30.0 & 8.2 & 593 & 0.02 & $<0.01$ & 2.50 & 1.0 & 0.33 & 0.13 & 382 \\
\hline 03 & 1000 & 1,640 & 28.5 & 8.2 & 613 & 0.01 & $<0.01$ & 2.40 & 0.80 & 0.28 & 0.15 & - \\
\hline 08 & 1030 & 1,370 & 30.0 & 8.3 & 662 & $<0.01$ & $<0.01$ & 2.10 & 0.40 & 0.26 & 0.14 & 136 \\
\hline 11 & 1000 & 1,630 & 27.5 & 8.1 & 563 & 0.03 & 0.02 & 1.68 & 0.70 & 0.32 & 0.13 & 201 \\
\hline 15 & 1030 & 2,390 & 28.0 & 7.6 & 463 & $<0.01$ & $<0.01$ & 1.60 & 2.3 & 0.49 & 0.09 & 1,650 \\
\hline 22 & 1015 & 1,250 & 31.0 & 8.2 & 642 & 0.02 & $<0.01$ & 1.30 & 0.70 & 0.18 & 0.13 & 161 \\
\hline 29 & 1100 & 1,160 & 29.0 & 8.2 & 678 & 0.03 & 0.02 & 0.98 & 0.80 & 0.20 & 0.11 & 109 \\
\hline \multicolumn{13}{|c|}{ AUGUST } \\
\hline 05 & 1000 & 1,130 & 28.0 & 7.8 & 690 & 0.05 & 0.01 & 0.34 & 0.40 & 0.19 & 0.09 & 75 \\
\hline 13 & 1100 & 1,130 & 28.5 & 8.3 & 667 & 0.02 & $<0.01$ & 0.49 & 0.70 & 0.20 & 0.09 & 81 \\
\hline 19 & 1000 & 1,150 & 27.5 & 8.3 & 694 & $<0.01$ & $<0.01$ & 0.60 & 0.70 & 0.17 & 0.09 & -- \\
\hline 27 & 1100 & 1,060 & 28.0 & 8.3 & 681 & $<0.01$ & $<0.01$ & 0.57 & 0.70 & 0.19 & 0.09 & 70 \\
\hline \multicolumn{13}{|c|}{ SEPTEMBER 1991} \\
\hline 05 & 1030 & 1,120 & 27.0 & 8.3 & 712 & $<0.01$ & $<0.01$ & 0.17 & 0.60 & 0.23 & 0.05 & 79 \\
\hline 09 & 1000 & 1,080 & 27.0 & 8.0 & 750 & 0.01 & $<0.01$ & 0.33 & 0.50 & 0.21 & 0.05 & 66 \\
\hline 16 & 1000 & 1,190 & 27.0 & 8.0 & 662 & 0.02 & 0.02 & 0.48 & 0.70 & 0.23 & 0.10 & 115 \\
\hline 23 & 0945 & 1,260 & 17.5 & 7.8 & 646 & 0.07 & $<0.01$ & 0.47 & 0.90 & 0.19 & 0.10 & 228 \\
\hline \multicolumn{13}{|c|}{ OCTOBER } \\
\hline 01 & 1030 & 1,100 & 19.0 & 8.1 & 709 & 0.01 & $<0.01$ & 0.21 & 0.60 & 0.21 & 0.08 & 104 \\
\hline 07 & 1000 & 1,400 & 16.0 & 7.5 & 556 & 0.03 & $<0.01$ & 0.28 & 0.60 & 0.50 & 0.08 & 375 \\
\hline 15 & 1100 & 1,130 & 16.0 & 8.1 & 649 & 0.02 & $<0.01$ & 0.19 & 0.40 & 0.16 & 0.07 & 127 \\
\hline 21 & 1000 & 1,110 & 14.0 & 8.4 & 706 & $<0.01$ & $<0.01$ & 0.21 & 0.40 & 0.15 & 0.07 & 121 \\
\hline 29 & 1000 & 1,140 & 17.5 & 8.1 & 728 & 0.02 & $<0.01$ & 0.39 & 0.60 & 0.21 & 0.08 & 124 \\
\hline \multicolumn{13}{|c|}{ NOVEMBER } \\
\hline 04 & 1030 & 1,310 & 6.5 & 8.3 & 614 & 0.04 & $<0.01$ & 0.48 & 0.60 & 0.22 & 0.07 & 119 \\
\hline 21 & 1030 & 1,290 & 11.0 & 7.8 & 560 & 0.18 & 0.02 & 0.97 & 0.70 & 0.22 & 0.09 & 380 \\
\hline \multicolumn{13}{|c|}{ DECEMBER } \\
\hline 06 & 1240 & 1,310 & 5.0 & 7.6 & 530 & 0.10 & 0.01 & 0.97 & 0.70 & 0.25 & 0.08 & 172 \\
\hline 16 & 1130 & 923 & 5.5 & 7.7 & 652 & 0.11 & 0.03 & 1.57 & 0.70 & 0.21 & 0.10 & 117 \\
\hline
\end{tabular}




\begin{tabular}{|c|c|c|c|c|c|c|c|c|c|c|c|c|}
\hline Date & Time & $\begin{array}{c}\text { Water } \\
\text { discharge } \\
\left(\mathrm{m}^{3} / \mathrm{s}\right)\end{array}$ & $\begin{array}{l}\text { Tomper- } \\
\text { ature } \\
\text { ('C) }\end{array}$ & pH & $\begin{array}{c}\text { Specific } \\
\text { conduct- } \\
\text { ance } \\
(\mu \mathrm{S} / \mathrm{cm})\end{array}$ & $\begin{array}{c}\text { Nitrogen, } \\
\text { ammonie } \\
\text { diss } \\
\text { (mg/l) }\end{array}$ & $\begin{array}{l}\text { Nitrogen, } \\
\text { nitrite } \\
\text { diss } \\
\text { (mg/L) }\end{array}$ & $\begin{array}{c}\text { Nitrogen, } \\
\text { nitrate } \\
\text { diss } \\
\text { (mg/L) }\end{array}$ & $\begin{array}{c}\text { Nitrogen } \\
\text { ammonie } \\
+ \\
\text { organic, } \\
\text { lotal } \\
\text { (mg/L) }\end{array}$ & $\begin{array}{c}\text { Phos- } \\
\text { phorus, } \\
\text { iotal } \\
\text { (mg/) }\end{array}$ & $\begin{array}{l}\text { Ortho- } \\
\text { phos- } \\
\text { phorus } \\
\text { diss } \\
\text { (mg/L) }\end{array}$ & $\begin{array}{c}\text { Sus- } \\
\text { pended } \\
\text { sodt- } \\
\text { ment } \\
\text { (mg/L) }\end{array}$ \\
\hline
\end{tabular}
JANUARY 1992

\begin{tabular}{|c|c|c|c|c|c|c|c|c|c|c|c|c|}
\hline 02 & 1030 & 1,080 & 5.5 & 7.5 & 592 & 0.10 & 0.03 & 1.47 & 0.90 & 0.30 & 0.08 & 157 \\
\hline 16 & 1200 & 1,210 & 2.5 & 8.0 & 528 & 0.04 & 0.02 & 1.38 & 0.40 & 0.23 & 0.23 & 93 \\
\hline 28 & 1300 & 991 & 3.0 & 8.1 & 668 & 0.07 & 0.02 & 1.58 & 0.40 & 0.16 & 0.10 & 62 \\
\hline \multicolumn{13}{|c|}{ FEBRUARY } \\
\hline 10 & 1000 & 864 & 3.0 & 8.0 & 702 & 0.04 & 0.02 & 1.48 & 0.40 & 0.19 & 0.11 & - \\
\hline 24 & 1200 & 1,630 & 7.5 & 7.6 & 525 & 0.05 & 0.02 & 1.78 & 1.0 & 0.37 & 0.07 & 346 \\
\hline \multicolumn{13}{|c|}{ MARCH } \\
\hline 03 & 1100 & 1,160 & 8.5 & 8.2 & 532 & 0.03 & 0.02 & 1.98 & 0.80 & 0.36 & 0.09 & 762 \\
\hline 12 & 1200 & 1,870 & 10.0 & 7.6 & 525 & 0.02 & 0.03 & 1.57 & 0.80 & 0.32 & 0.09 & 401 \\
\hline 20 & 0830 & 1,800 & 8.0 & 7.5 & 550 & 0.04 & 0.02 & 2.28 & 0.40 & 0.15 & 0.12 & 436 \\
\hline 26 & 1130 & 2,070 & 9.0 & 7.8 & 437 & 0.04 & 0.04 & 2.26 & 0.50 & 0.14 & 0.08 & 661 \\
\hline \multicolumn{13}{|l|}{ APRIL } \\
\hline 01 & 1000 & 2,490 & 10.0 & 7.9 & 539 & 0.02 & 0.01 & 1.59 & 0.50 & 0.15 & 0.07 & 224 \\
\hline 06 & 1230 & 1,610 & 11.0 & 7.8 & 572 & 0.03 & 0.01 & 1.89 & 0.60 & 0.25 & 0.09 & 337 \\
\hline 14 & 1030 & 1,960 & 15.0 & 7.5 & 638 & 0.04 & 0.01 & 1.59 & 0.30 & 0.14 & 0.09 & 282 \\
\hline 21 & 1300 & 3,290 & 15.5 & 7.4 & 535 & 0.03 & 0.02 & 1.18 & 0.60 & 0.19 & 0.07 & 1,070 \\
\hline 29 & 1015 & 3,740 & 14.0 & 7.4 & 422 & 0.07 & 0.09 & 1.81 & 0.40 & 0.06 & 0.06 & 1,190 \\
\hline \multicolumn{13}{|c|}{ MAY 1992} \\
\hline 12 & 1000 & 1,500 & - & 8.1 & 656 & 0.02 & $<0.01$ & 2.20 & $<0.20$ & 0.10 & 0.09 & -- \\
\hline 21 & 1200 & 1,440 & 22.5 & 8.3 & 586 & 0.05 & $<0.01$ & 1.20 & 0.50 & 0.17 & 0.08 & 70 \\
\hline 26 & 1130 & 1,750 & 19.5 & 7.5 & 592 & 0.02 & $<0.01$ & 2.20 & 2.5 & 1.0 & 0.12 & 872 \\
\hline \multicolumn{13}{|l|}{ JUNE } \\
\hline 01 & 1330 & 1,510 & 22.0 & 8.0 & 704 & 0.03 & 0.02 & 2.78 & 1.4 & 0.44 & 0.12 & 369 \\
\hline 09 & 1030 & 1,420 & 23.0 & 8.2 & 681 & 0.04 & $<0.01$ & 1.50 & 0.20 & 0.12 & 0.09 & 103 \\
\hline 15 & 1230 & 1,780 & 23.5 & 7.8 & 617 & 0.03 & 0.02 & 1.38 & 1.1 & 0.32 & 0.09 & 354 \\
\hline 22 & 0945 & 2,160 & 25.0 & 8.0 & 619 & 0.03 & 0.01 & 1.19 & 0.40 & 0.21 & 0.10 & 321 \\
\hline 30 & 1130 & 1,460 & 26.0 & 7.7 & 599 & 0.04 & 0.01 & 1.29 & 1.1 & 0.46 & 0.11 & 381 \\
\hline \multicolumn{13}{|l|}{ JULY } \\
\hline 07 & 0930 & 1,310 & 28.0 & 8.0 & 688 & 0.03 & $<0.01$ & 1.30 & 0.60 & 0.34 & 0.12 & \\
\hline 14 & 1200 & 2,110 & 27.0 & 7.6 & 598 & 0.02 & $<0.01$ & 1.20 & 0.80 & 0.33 & 0.09 & \\
\hline 21 & 1030 & 3,770 & 26.5 & 7.8 & 560 & 0.06 & 0.01 & 1.29 & 0.30 & 0.15 & 0.07 & \\
\hline 28 & 1230 & 6,830 & 26.5 & 8.2 & 396 & 0.03 & 0.01 & 1.39 & 1.8 & 0.72 & 0.08 & \\
\hline
\end{tabular}

APRIL 1991

\begin{tabular}{|c|c|c|c|c|c|c|c|c|c|c|c|c|}
\hline 11 & 1500 & 8,070 & 14.5 & 7.8 & 489 & 0.02 & 0.08 & 5.02 & - & -- & 0.09 & - \\
\hline 18 & 1130 & 10,900 & 17.0 & 7.8 & 425 & 0.04 & 0.07 & 2.63 & -- & - & 0.07 & -- \\
\hline 24 & 0930 & 12,900 & 13.5 & 7.8 & 408 & 0.02 & 0.05 & 3.75 & -- & -- & 0.06 & - \\
\hline 29 & 1230 & 10,600 & 16.5 & 7.9 & 454 & 0.02 & 0.05 & 4.75 & 0.70 & - & 0.06 & 713 \\
\hline \multicolumn{13}{|c|}{ MAY } \\
\hline 07 & 1345 & 11,100 & 17.0 & 7.8 & 407 & 0.03 & 0.06 & 3.94 & -- & -- & 0.08 & -- \\
\hline 09 & 0900 & 12,300 & 17.0 & 7.8 & 377 & 0.02 & 0.06 & 3.44 & -- & -- & 0.08 & - \\
\hline 13 & 1330 & 11,600 & - & 7.9 & 393 & 0.04 & 0.07 & 3.73 & - & -- & 0.07 & 557 \\
\hline 16 & 1200 & 10,800 & -- & 7.9 & 482 & $<0.01$ & 0.07 & 4.33 & - & -- & 0.08 & - \\
\hline 20 & 1430 & 10,800 & 23.5 & 7.8 & 434 & 0.03 & 0.07 & 4.13 & -- & - & 0.06 & 582 \\
\hline 23 & 0916 & 9,770 & 22.0 & 7.7 & 433 & 0.04 & 0.04 & 3.96 & -- & -- & 0.07 & -- \\
\hline 28 & 1300 & 10,800 & 25.5 & 8.2 & 468 & $<0.01$ & 0.02 & 4.08 & -- & -- & 0.02 & -- \\
\hline 30 & 1330 & 11,800 & 25.0 & 8.0 & 422 & $<0.01$ & $<0.01$ & 4.10 & 0.80 & 0.24 & 0.08 & -- \\
\hline
\end{tabular}




\begin{tabular}{|c|c|c|c|c|c|c|c|c|c|c|c|c|}
\hline Date & Time & $\begin{array}{c}\text { Water } \\
\text { discharge } \\
\left(\mathrm{m}^{3} / \mathrm{s}\right)\end{array}$ & $\begin{array}{c}\text { Temper- } \\
\text { ature } \\
\left({ }^{\circ} \mathrm{C}\right)\end{array}$ & pH & $\begin{array}{c}\text { Specific } \\
\text { conduct- } \\
\text { ance } \\
(\mu S / c m)\end{array}$ & $\begin{array}{c}\text { Nitrogen, } \\
\text { ammonia } \\
\text { diss } \\
\text { (mg/l) }\end{array}$ & $\begin{array}{l}\text { Nitrogen, } \\
\text { nitrite } \\
\text { diss } \\
\text { (mg/L) }\end{array}$ & $\begin{array}{l}\text { Nitrogen, } \\
\text { nitrate } \\
\text { diss } \\
\text { (mg/L) }\end{array}$ & $\begin{array}{c}\text { Nitrogen } \\
\text { ammonia } \\
+ \\
\text { organic, } \\
\text { totai } \\
\text { (mgl) }\end{array}$ & $\begin{array}{c}\text { Phos- } \\
\text { phorus, } \\
\text { totai } \\
\text { (mg/L) }\end{array}$ & $\begin{array}{l}\text { Ortho- } \\
\text { phos- } \\
\text { phorus } \\
\text { diss } \\
\text { (mgh) }\end{array}$ & $\begin{array}{c}\text { Sus- } \\
\text { pendec } \\
\text { sedi- } \\
\text { ment } \\
\text { (mg/L) }\end{array}$ \\
\hline \multicolumn{13}{|c|}{07022000 - Mississippi River at Thebes, Ill.--Continued } \\
\hline \multicolumn{13}{|c|}{ JUNE 1991} \\
\hline 03 & 1345 & 11,600 & 25.5 & 7.9 & 392 & 0.03 & 0.02 & 4.88 & .- & -- & 0.09 & - \\
\hline 06 & 1045 & 11,200 & 26.0 & 8.2 & 478 & 0.01 & 0.01 & 4.89 & -- & - & 0.10 & - \\
\hline 10 & 1400 & 9,910 & 26.5 & 7.9 & 471 & 0.04 & 0.01 & 4.99 & 1.0 & 0.13 & 0.12 & 585 \\
\hline 13 & 1500 & 9,600 & 26.0 & 8.2 & 489 & 0.03 & $<0.01$ & 5.10 & 2.1 & 0.15 & 0.13 & 802 \\
\hline 18 & 1430 & 9,120 & 26.0 & 7.7 & 464 & 0.02 & $<0.01$ & 4.60 & 1.8 & 0.18 & 0.10 & 462 \\
\hline 20 & 1100 & 9,400 & 26.5 & 8.1 & 468 & 0.02 & $<0.01$ & 4.40 & 1.3 & 0.17 & 0.10 & 313 \\
\hline 24 & 1300 & 8,920 & 29.0 & 8.1 & 451 & 0.04 & 0.02 & 4.38 & 1.9 & 0.73 & 0.12 & 1210 \\
\hline 27 & 1400 & 8,100 & 27.0 & 8.2 & 434 & $<0.01$ & $<0.01$ & 4.30 & 0.30 & 0.69 & 0.12 & 627 \\
\hline \multicolumn{13}{|l|}{ JULY } \\
\hline 01 & 1320 & 6,940 & 28.0 & 8.2 & 495 & 0.10 & 0.01 & 4.79 & 1.6 & 0.44 & 0.13 & 347 \\
\hline 03 & 0950 & 6,660 & 28.0 & 8.2 & 492 & 0.03 & 0.01 & 4.69 & 1.3 & 0.38 & 0.14 & 250 \\
\hline 08 & 1330 & 5,580 & 29.5 & 8.2 & 507 & $<0.01$ & 0.02 & 4.38 & 1.0 & 0.30 & 0.13 & - \\
\hline 11 & 1400 & 5,720 & 29.0 & 8.3 & 515 & 0.02 & 0.04 & 3.76 & 0.90 & 0.24 & 0.13 & 93 \\
\hline 18 & 0930 & 5,980 & 28.0 & 8.1 & 472 & 0.03 & 0.01 & 2.89 & 1.0 & 0.47 & 0.12 & 544 \\
\hline 23 & 1300 & 4,760 & 30.5 & 8.2 & 480 & 0.02 & 0.03 & 2.47 & 1.4 & 0.30 & 0.07 & 226 \\
\hline 30 & 1000 & 4,470 & 26.5 & 8.6 & 539 & 0.03 & 0.01 & 2.59 & 0.60 & 0.18 & 0.06 & 74 \\
\hline \multicolumn{13}{|c|}{ AUGUST 1991} \\
\hline 06 & 1300 & 3,460 & 29.0 & 8.5 & 568 & 0.04 & 0.02 & 1.18 & 1.2 & 0.12 & 0.05 & 106 \\
\hline 13 & 0930 & 3,740 & 25.0 & 8.7 & 538 & 0.02 & 0.01 & 1.39 & 1.2 & 0.19 & 0.05 & 76 \\
\hline 20 & 1330 & 3,790 & 29.0 & 8.0 & 556 & $<0.01$ & 0.01 & 1.49 & 1.1 & 0.18 & 0.07 & 152 \\
\hline 29 & 1100 & 2,920 & 28.5 & 8.6 & 571 & 0.13 & 0.03 & 1.47 & 1.2 & 0.22 & 0.05 & 64 \\
\hline \multicolumn{13}{|c|}{ SEPTEMBER } \\
\hline 03 & 1500 & 2,730 & 28.5 & 8.1 & 565 & 0.02 & 0.07 & 1.23 & 0.90 & 0.24 & 0.07 & 70 \\
\hline 11 & 1000 & 2,300 & 26.5 & 8.2 & 622 & 0.03 & 0.07 & 0.84 & 0.60 & 0.24 & 0.07 & 69 \\
\hline 17 & 1400 & 3,260 & 27.5 & 8.7 & 577 & 0.02 & 0.03 & 0.85 & 0.30 & 0.245 & 0.11 & -- \\
\hline 26 & 0930 & 3,790 & 18.5 & 8.0 & 545 & 0.03 & 0.03 & 0.68 & 0.70 & 0.21 & 0.12 & 105 \\
\hline \multicolumn{13}{|c|}{ OCTOBER } \\
\hline 02 & 1300 & 3,310 & 19.0 & 8.2 & 540 & 0.03 & 0.01 & 1.09 & 0.80 & 0.26 & 0.12 & 76 \\
\hline 09 & 1030 & 4,050 & 17.0 & 8.0 & 561 & 0.04 & 0.03 & 1.07 & 0.70 & 0.29 & 0.12 & 102 \\
\hline 16 & 1430 & 2,760 & 16.0 & 8.1 & 553 & 0.06 & 0.02 & 0.77 & 0.50 & 0.20 & 0.09 & 106 \\
\hline 23 & 0945 & 2,510 & 17.0 & 8.3 & 593 & 0.05 & 0.01 & 0.88 & 0.60 & 0.21 & 0.10 & 56 \\
\hline 30 & 1400 & 3,510 & 17.0 & 7.7 & 550 & 0.08 & 0.02 & 1.28 & 0.90 & 0.27 & 0.11 & 88 \\
\hline \multicolumn{13}{|c|}{ NOVEMBER } \\
\hline 07 & 0800 & 4,620 & 7.5 & 8.1 & 573 & 0.14 & 0.02 & 1.28 & 0.80 & 0.28 & 0.10 & 91 \\
\hline 20 & 1030 & 4,760 & 11.0 & 7.9 & 445 & 0.22 & 0.02 & 2.08 & 1.7 & 0.63 & 0.10 & 780 \\
\hline \multicolumn{13}{|c|}{ DECEMBER 1991} \\
\hline 05 & 1045 & 6,770 & 3.0 & 7.9 & 485 & 0.13 & 0.02 & 2.98 & 1.1 & 0.39 & 0.11 & 81 \\
\hline 18 & 1100 & 6,120 & 4.0 & 8.1 & 518 & 0.13 & 0.02 & 4.08 & 1.2 & 0.31 & 0.11 & 156 \\
\hline 30 & 1400 & 6,830 & 4.0 & 7.9 & 487 & 0.15 & 0.03 & 4.27 & 1.0 & 0.30 & 0.09 & 166 \\
\hline \multicolumn{13}{|c|}{ JANUARY 1992} \\
\hline 24 & 0930 & 3,310 & 2.5 & 8.1 & 570 & 0.16 & 0.03 & 3.67 & 0.70 & 0.21 & 0.10 & 177 \\
\hline \multicolumn{13}{|c|}{ FEBRUARY } \\
\hline 04 & 1030 & 3,910 & 4.0 & 8.2 & 583 & 0.10 & 0.03 & 3.77 & 0.70 & 0.18 & 0.10 & 114 \\
\hline 19 & 0930 & 5,180 & 4.5 & 8.4 & 510 & 0.10 & 0.02 & 2.78 & 1.2 & 0.31 & 0.08 & 250 \\
\hline \multicolumn{13}{|l|}{ MARCH } \\
\hline 04 & 0945 & 4,810 & 8.0 & 7.7 & 494 & 0.12 & 0.03 & 3.77 & 0.90 & 0.24 & 0.10 & 200 \\
\hline 09 & 1300 & 5,210 & 12.0 & 8.0 & 522 & 0.11 & 0.04 & 4.26 & 1.1 & 0.27 & 0.11 & 72 \\
\hline
\end{tabular}




\begin{tabular}{|c|c|c|c|c|c|c|c|c|c|c|c|c|}
\hline Date & Time & $\begin{array}{c}\text { Water } \\
\text { discharge } \\
\left(\mathrm{m}^{3} / \mathrm{s}\right)\end{array}$ & $\begin{array}{c}\text { Temper- } \\
\text { ature } \\
\left({ }^{\circ} \mathrm{C}\right)\end{array}$ & pH & $\begin{array}{c}\text { Specific } \\
\text { conduct- } \\
\text { ence } \\
(\mu \mathrm{S} / \mathrm{cm})\end{array}$ & $\begin{array}{c}\text { Nitrogen, } \\
\text { emmonie } \\
\text { dlsa } \\
\text { (mg/l) }\end{array}$ & $\begin{array}{l}\text { Nitrogen, } \\
\text { nitrite } \\
\text { diss } \\
\text { (mg/L) }\end{array}$ & $\begin{array}{c}\text { Nitrogen, } \\
\text { nitrate } \\
\text { diss } \\
\text { (mgh) }\end{array}$ & $\begin{array}{c}\text { Nitrogen } \\
\text { ammonie } \\
+ \\
\text { organic, } \\
\text { total } \\
\text { (mg/L) }\end{array}$ & $\begin{array}{c}\text { Phos- } \\
\text { phorua, } \\
\text { totai } \\
\text { (mg/h) }\end{array}$ & $\begin{array}{l}\text { Ortho- } \\
\text { phos- } \\
\text { phorus } \\
\text { diss } \\
\text { (mg/L) }\end{array}$ & $\begin{array}{c}\text { Sus- } \\
\text { pended } \\
\text { sedi- } \\
\text { ment } \\
\text { (mg/L) }\end{array}$ \\
\hline \multicolumn{13}{|c|}{07022000 - Mississippi River st Thebes, III.-Continued } \\
\hline \multicolumn{13}{|c|}{ MARCH (cont.) } \\
\hline 18 & 0945 & 7,000 & 8.0 & 7.6 & 502 & 0.05 & 0.03 & 3.57 & 0.60 & 0.24 & 0.09 & 215 \\
\hline 24 & 1330 & 9,630 & 6.0 & 7.5 & 472 & 0.06 & 0.03 & 2.97 & 0.90 & 0.27 & 0.08 & 568 \\
\hline \multicolumn{13}{|l|}{ APRIL } \\
\hline 02 & 0930 & 8,580 & 7.0 & 7.8 & 475 & 0.11 & 0.02 & 3.38 & 0.80 & 0.23 & 0.09 & 224 \\
\hline 07 & 1330 & 7,140 & 10.0 & 7.8 & 497 & 0.04 & 0.01 & 2.39 & 0.80 & 0.21 & 0.04 & 203 \\
\hline 15 & 1030 & 5,660 & 14.0 & 8.4 & 553 & 0.06 & 0.02 & 3.38 & 1.0 & 0.19 & 0.06 & 141 \\
\hline 23 & 1330 & 10,300 & 15.5 & 7.6 & 448 & 0.06 & 0.05 & 2.25 & 0.60 & 0.14 & 0.06 & 501 \\
\hline 29 & 1100 & 10,700 & 11.5 & 8.1 & 436 & 0.06 & 0.06 & 3.14 & 1.1 & 0.38 & 0.06 & - \\
\hline \multicolumn{13}{|c|}{ MAY 1992} \\
\hline 06 & 1300 & 8,840 & 16.0 & 7.4 & 466 & 0.03 & 0.03 & 4.07 & 0.30 & 0.10 & 0.07 & 294 \\
\hline 14 & 0930 & 6,200 & 18.0 & 8.1 & 497 & 0.06 & 0.03 & 3.07 & 0.30 & 0.08 & 0.07 & - \\
\hline 19 & 1300 & 4,500 & 24.0 & 8.5 & 519 & 0.06 & 0.04 & 2.66 & 0.40 & 0.14 & 0.07 & 77 \\
\hline 28 & 0900 & 4,980 & 20.0 & 8.3 & 544 & 0.05 & 0.03 & 2.27 & 0.50 & 0.09 & 0.07 & 84 \\
\hline \multicolumn{13}{|l|}{ JUNE } \\
\hline 02 & 1300 & 4,130 & 20.0 & 8.0 & 559 & 0.04 & 0.05 & 2.45 & 1.2 & 0.37 & 0.07 & 230 \\
\hline 09 & 0930 & 3,740 & 23.0 & 8.3 & 553 & 0.09 & 0.05 & 2.45 & 0.50 & 0.11 & 0.09 & 97 \\
\hline 16 & 1330 & 3,570 & 25.0 & 8.2 & 555 & 0.03 & 0.03 & 1.77 & 1.2 & 0.19 & 0.08 & 78 \\
\hline 25 & 1000 & 4,220 & 24.0 & 7.8 & 545 & 0.04 & 0.04 & 1.66 & 0.90 & 0.20 & 0.09 & -- \\
\hline \multicolumn{13}{|l|}{ JULY } \\
\hline 01 & 1300 & 3,710 & 25.0 & 7.9 & 493 & 0.02 & 0.03 & 1.97 & 1.2 & 0.48 & 0.10 & \\
\hline 09 & 0915 & 3,880 & 26.5 & 8.2 & 545 & 0.06 & 0.05 & 1.45 & 0.70 & 0.24 & 0.09 & \\
\hline 15 & 1230 & 5,470 & 27.0 & 8.3 & 504 & 0.02 & $<0.01$ & 1.20 & 1.0 & 0.34 & 0.08 & \\
\hline 23 & 1000 & 7,930 & 28.0 & 8.0 & 437 & 0.04 & $<0.01$ & 3.20 & 0.40 & 0.17 & 0.13 & \\
\hline 28 & 1300 & 8,160 & 26.5 & 7.9 & 525 & 0.04 & $<0.01$ & 3.10 & 0.80 & 0.30 & 0.12 & \\
\hline \multicolumn{13}{|c|}{03374100 - White River at Hazleton, Ind. } \\
\hline \multicolumn{13}{|c|}{ MAY 1991} \\
\hline 01 & 1000 & 399 & 17.5 & 7.9 & 436 & 0.01 & 0.01 & 2.09 & 0.50 & 0.11 & 0.03 & 51 \\
\hline 06 & 1115 & 337 & 17.5 & 8.0 & 444 & $<0.01$ & $<0.01$ & 1.60 & 0.80 & 0.08 & 0.02 & 20 \\
\hline 09 & 1215 & 329 & 17.5 & 8.2 & 481 & $<0.01$ & 0.01 & 1.59 & 0.80 & 0.11 & $<0.01$ & 67 \\
\hline 13 & 1300 & 269 & 20.5 & 8.0 & 494 & 0.01 & 0.02 & 1.58 & 0.80 & 0.16 & 0.02 & 116 \\
\hline 16 & 1130 & 228 & 23.5 & 8.2 & 568 & 0.01 & 0.02 & 1.18 & 1.4 & 0.05 & $<0.01$ & 87 \\
\hline 20 & 1045 & 246 & 24.0 & 8.1 & 572 & 0.02 & 0.02 & 1.48 & 0.90 & 0.19 & $<0.01$ & 102 \\
\hline 23 & 1100 & 282 & 24.5 & 8.0 & 537 & 0.03 & 0.04 & 2.16 & 0.90 & 0.03 & 0.02 & 148 \\
\hline 28 & 1100 & 269 & 26.0 & 7.8 & 523 & 0.02 & 0.12 & 3.98 & 1.7 & 0.24 & 0.09 & 161 \\
\hline 30 & 1200 & 240 & 27.5 & 7.9 & 540 & $<0.01$ & 0.03 & 3.07 & 0.80 & 0.23 & 0.07 & 130 \\
\hline \multicolumn{13}{|l|}{ JUNE } \\
\hline 03 & 1200 & 223 & 28.5 & 7.9 & 511 & 0.05 & 0.05 & 3.05 & 3.1 & 0.32 & 0.07 & 229 \\
\hline 06 & 1145 & 312 & 27.0 & 7.9 & 537 & $<0.01$ & 0.03 & 3.27 & 0.70 & 0.10 & 0.11 & 294 \\
\hline 10 & 1200 & 187 & 26.5 & -- & 522 & $<0.01$ & 0.01 & 2.89 & 0.80 & 0.25 & 0.08 & 133 \\
\hline 13 & 1100 & 153 & 27.0 & 8.3 & 609 & 0.02 & 0.02 & 2.48 & 1.2 & 0.20 & 0.06 & 84 \\
\hline 17 & 1200 & 140 & 27.5 & 8.3 & 581 & 0.04 & 0.02 & 1.28 & 1.5 & 0.21 & 0.02 & 377 \\
\hline 20 & 1130 & 138 & 28.5 & 8.2 & 583 & 0.01 & 0.02 & 0.740 & 1.4 & 0.14 & $<0.01$ & 101 \\
\hline 24 & 1215 & 121 & 27.5 & 8.3 & 577 & 0.01 & 0.02 & 0.860 & 1.5 & 0.17 & $<0.01$ & 86 \\
\hline 27 & 1130 & 105 & 29.0 & 8.4 & 564 & 0.02 & 0.02 & 0.180 & 1.1 & 0.15 & 0.06 & 84 \\
\hline \multicolumn{13}{|c|}{ JULY 1991} \\
\hline 01 & 1145 & 100 & 31.0 & 8.2 & 595 & 0.03 & $<0.01$ & $<0.050$ & 1.2 & 0.13 & $<0.01$ & 66 \\
\hline 03 & 1100 & 101 & 30.0 & 8.1 & 560 & 0.01 & $<0.01$ & $<0.050$ & 1.3 & 0.14 & 0.02 & 68 \\
\hline
\end{tabular}




\begin{tabular}{|c|c|c|c|c|c|c|c|c|c|c|c|c|}
\hline Date & Time & $\begin{array}{c}\text { Water } \\
\text { dlecharge } \\
\left(\mathrm{m}^{3} / \mathrm{s}\right)\end{array}$ & $\begin{array}{c}\text { Temper- } \\
\text { ature } \\
\left({ }^{\circ} \mathrm{C}\right)\end{array}$ & pH & $\begin{array}{c}\text { Specific } \\
\text { conduct- } \\
\text { ance } \\
(\mu \mathrm{S} / \mathrm{cm})\end{array}$ & $\begin{array}{c}\text { Nitrogen, } \\
\text { ammonia } \\
\text { diss } \\
\text { (mg/L) }\end{array}$ & $\begin{array}{l}\text { Nitrogen, } \\
\text { nitrite } \\
\text { dise } \\
\text { (mgll) }\end{array}$ & $\begin{array}{l}\text { Nitrogen, } \\
\text { nitrate } \\
\text { dies } \\
\text { (mgll) }\end{array}$ & $\begin{array}{c}\text { Nitrogen } \\
\text { ammonia } \\
+ \\
\text { organic, } \\
\text { lotal } \\
\text { (mg/L) }\end{array}$ & $\begin{array}{c}\text { Phos- } \\
\text { phorua, } \\
\text { total } \\
\text { (mgl) }\end{array}$ & $\begin{array}{l}\text { Ortho- } \\
\text { phos- } \\
\text { phorus } \\
\text { dlss } \\
\text { (mg/l) }\end{array}$ & $\begin{array}{l}\text { Sus- } \\
\text { pended } \\
\text { cedl- } \\
\text { ment } \\
\text { (mgll) }\end{array}$ \\
\hline
\end{tabular}

JULY 1991 (cont)

08

$08 \quad 1200$

$11 \quad 1130$

$15 \quad 1200$

$18 \quad 1300$

$22 \quad 1300$

$25 \quad 1200$

$30 \quad 1145$

AUGUST

$01 \quad 1145$

$06 \quad 1200$

$08 \quad 1300$

$12 \quad 1100$

$15 \quad 1200$

$19 \quad 1115$

$22 \quad 1230$

$26 \quad 1245$

$29 \quad 1200$

SEPTEMBER 1991

$\begin{array}{ll}09 & 1100 \\ 12 & 1100 \\ 19 & 1130 \\ 26 & 1200\end{array}$

OCTOBER

$03 \quad 1000$

$08 \quad 1100$

$17 \quad 1100$

$24 \quad 1100$

$28 \quad 1130$

$30 \quad 1130$

NOVEMBER

$04 \quad 1130$

$13 \quad 1025$

$27 \quad 1200$

DECEMBER

$\begin{array}{cc}18 & 120 \\ \text { JANUARY } & 1992\end{array}$

$\begin{array}{ll}02 & 1200 \\ 16 & 1235\end{array}$

$\begin{array}{ll}16 & 1235 \\ 30 & 1243\end{array}$

FEBRUARY

$27 \quad 1200$

MARCH

$\begin{array}{rrrrrr}0 & 1130 & 121 & 14.0 & 8.5 & 635 \\ 12 & 1115 & 120 & 9.0 & 8.6 & 651 \\ 19 & 1133 & 159 & 9.5 & 8.3 & 674 \\ 26 & 1128 & 262 & 9.5 & 7.9 & 478\end{array}$

$8.1 \quad 647$

$\begin{array}{cccc}86.4 & 31.0 & 8.2 & 545 \\ 82.7 & 29.5 & 8.1 & 561 \\ 147 & 27.5 & 7.7 & 386 \\ 100 & 30.0 & 8.8 & 467 \\ 74.5 & 32.0 & 8.4 & 510 \\ 65.4 & 29.0 & 8.3 & 573 \\ 55.5 & 27.5 & 8.2 & 600\end{array}$

$52.1 \quad 29.0$

$49.3 \quad 28.0$

$54.4 \quad 29.5$

$62.3 \quad 28.0$

$60.3 \quad 27.0$

$50.4 \quad 28.0$

$66.0 \quad 28.0$

$56.9 \quad 29.5$

$47.9 \quad 29.5$

$\begin{array}{ll}77.9 & 27.5\end{array}$

$52.7 \quad 28.5$

$41.3 \quad 23.0$

$36.2 \quad 18.5$

$32.9 \quad 21.5$

$32.3 \quad 16.0$

$31.4 \quad 14.0$

$28.9 \quad 17.5$

$100 \quad 20.5$

$8.2 \quad 625$

8.3664

$8.4 \quad 656$

$8.4 \quad 690$

$8.2 \quad 646$

$8.3 \quad 643$

$8.4 \quad 635$

8.1702

152

$83.8 \quad 9.5$

52.7

154

$8.2 \quad 614$

$8.5 \quad 597$

8.3

8.7

651

612

$8.4 \quad 701$

8.5

8.7

8.4

8.2

767

766

771

477

$8.3 \quad 572$

$8.4 \quad 715$

$8.2 \quad 582$

$\begin{array}{llll}128 & 5.5 & 8.2 & 600\end{array}$

130

170

163

$\begin{array}{lll}6.0 & 8.2 & 595 \\ 2.0 & 8.2 & 611 \\ 4.5 & 8.2 & 662\end{array}$

109

172

$\begin{array}{lll}5.5 & 8.5 & 690\end{array}$

$\begin{array}{lll}9.0 & 8.3 & 610\end{array}$

$<0.01$

$<0.01$

0.01

$<0.01$

.0 .01

0.06

$<0.01$

0.02

$\begin{array}{rc}<0.01 & <0.050 \\ <0.01 & <0.050 \\ 0.10 & 1.60 \\ 0.02 & 0.090 \\ <0.01 & <0.050 \\ <0.01 & <0.050 \\ 0.01 & --\end{array}$

1.2

1.3

2.3

1.1

1.3

1.4

1.8

$0.13 \quad 0.01$
0.13

$0.13<0.01$

$0.33 \quad 0.03$

$0.17 \quad 0.02$

$0.02<0.01$

$0.11<0.01$

$0.18<0.01$

68

48

336

47

$<0.01$
$<0.01$

$<0.01$
$<0.01$

$<0.01<0.050$

1.0

$0.14<0.01$

$0.12<0.01$

$0.11<0.01$

0.60

$0.13 \quad 0.02$

$0.14<0.01$

1.7

1.1

$0.15 \quad 0.02$

$0.13<0.01$

$1.4<0.09<0.01$

$<0.01<0.050$

0.70

$0.14<0.01$

56

54

222

\section{$<0.01<0.01<0.050$}

1.3

1.2

1.2

$0.17<0.01$

$0.11<0.01$

$0.12 \quad 0.01$

$0.14<0.01$

36

31

36

46

46

48

46

49

38

$<0.01<0.01<0.050$

1.4

$<0.01<0.050$

$0.16<0.01$

$<0.01<0.050$

60

31

35

30

$\begin{array}{lll}0.05 & 0.03 & 0.610 \\ 0.10 & 0.03 & 0.890\end{array}$

$0.22 \quad 0.04$

0.80
0.50

$0.19 \quad 0.05$
1.4

1.3

$0.14<0.01$

$0.20<0.01$

1.2

0.25

0.25
0.28

0.04

0.02

$0.52 \quad 0.05$

1.3
1.4

0.70

$0.19 \quad 0.06$

0.40

$0.10 \quad 0.10$

0.80

0.26

0.50

0.1

0.40

0.1

0.90

0.28

0.80

0.1

0.60

$\begin{array}{lll}0.12 & 0.07 & 21\end{array}$

0.60

0.1

0.90

0.90

$0.15 \quad 0.04$

$0.20 \quad 0.05$

33

29

77

73

98

279

66

26

89

32

21

63

25

21

$\begin{array}{ll}0.01 & 1.69\end{array}$

$\begin{array}{ll}0.02 & 1.88 \\ 0.03 & 2.47\end{array}$

72
41
63
115




\begin{tabular}{|c|c|c|c|c|c|c|c|c|c|c|c|c|}
\hline Date & Time & $\begin{array}{c}\text { Weter } \\
\text { discharge } \\
\left(\mathrm{m}^{3} / \mathrm{s}\right)\end{array}$ & $\begin{array}{l}\text { Temper- } \\
\text { oture } \\
\left.{ }^{\circ} \mathrm{C}\right)\end{array}$ & PH & $\begin{array}{c}\text { Specific } \\
\text { conduct- } \\
\text { ance } \\
(\mu \mathrm{S} / \mathrm{cm})\end{array}$ & $\begin{array}{c}\text { Nitrogen, } \\
\text { ammonis } \\
\text { diss } \\
\text { (mg/L) }\end{array}$ & $\begin{array}{l}\text { Nitrogen, } \\
\text { nitrite } \\
\text { diss } \\
\text { (mg/L) }\end{array}$ & $\begin{array}{l}\text { Nitrogen, } \\
\text { nitrate } \\
\text { diss } \\
\text { (mg/L) }\end{array}$ & $\begin{array}{c}\text { Nitrogen } \\
\text { ammonia } \\
+ \\
\text { organic, } \\
\text { total } \\
\text { (mgll) }\end{array}$ & $\begin{array}{c}\text { Phos- } \\
\text { phorus, } \\
\text { total } \\
\text { (mg/L) }\end{array}$ & $\begin{array}{c}\text { Ortho- } \\
\text { phos- } \\
\text { phorus } \\
\text { diss } \\
\text { (mg/L) }\end{array}$ & $\begin{array}{c}\text { Sus- } \\
\text { pended } \\
\text { sedl- } \\
\text { ment } \\
\text { (mg/L) }\end{array}$ \\
\hline
\end{tabular}

APRIL

02

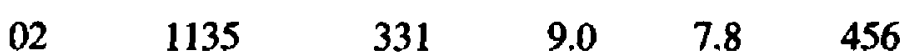

$\begin{array}{rrrrrr}09 & 1102 & 215 & 14.0 & 8.2 & 559\end{array}$

$\begin{array}{llllll}16 & 1112 & 247 & 18.0 & 8.1 & 552\end{array}$

$\begin{array}{llllll}20 & 1030 & 606 & 17.5 & 7.6 & 340\end{array}$

$\begin{array}{llllll}24 & 1035 & 1,140 & 17.5 & 7.6 & 385\end{array}$

$\begin{array}{llllll}29 & 1100 & 447 & 14.0 & 7.8 & 512\end{array}$

$\begin{array}{rrr}0.04 & 0.02 & 2.58 \\ 0.01 & 0.02 & 2.48 \\ <0.01 & 0.02 & 2.38 \\ 0.06 & 0.03 & 1.67 \\ 0.05 & 0.06 & 3.24 \\ 0.06 & 0.04 & 4.26\end{array}$

$\begin{array}{lllr}0.90 & 0.28 & 0.08 & 142 \\ 0.70 & 0.20 & 0.09 & 68 \\ 0.90 & 0.25 & 0.07 & 95 \\ 1.0 & 0.32 & 0.06 & 368 \\ 1.6 & 0.26 & 0.05 & 155 \\ 0.40 & 0.09 & 0.04 & 176\end{array}$

MAY 1992

$06 \quad 1115$

$12 \quad 1130$

$\begin{array}{llll}257 & 15.5 & 8.1 & 570\end{array}$

$\begin{array}{lll}<0.01 & 0.01 \quad 3.09\end{array}$

$\begin{array}{cccc}0.20 & 0.10 & 0.08 & 103 \\ <0.20 & 0.07 & 0.04 & 82 \\ 1.0 & 0.19 & 0.02 & 82 \\ 1.1 & 0.23 & 0.02 & -\end{array}$

JUNE

$20 \quad 1130$

$201 \quad 19.5$

$\begin{array}{ll}8.1 & 570 \\ 8.3 & 561\end{array}$

$$
0.02
$$

$0.01 \quad 2.19$

$0.02 \quad 1.38$

$\begin{array}{ll}150 & 22.5 \\ 153 & 19.5\end{array}$

$8.4 \quad 609$

$<0.01$

$0.02 \quad 1.38$

$120 \quad 21.0$

$8.5 \quad 596$

$<0.01$

$0.01 \quad 1.49$

$\begin{array}{llll}0.40 & 0.07 & 0.02 & 109\end{array}$

$10 \quad 1115$

$149 \quad 25.0$

8.3

640

$<0.01$

$0.02 \quad 0.840$

$\begin{array}{llll}0.40 & 0.06 & <0.01 \quad 201\end{array}$

$19 \quad 1100$

86.927 .5

1130

$\begin{array}{llll}439 & 23.0 & 7.8 & 450\end{array}$

$\begin{array}{lll}0.02 & 0.01 & 0.610\end{array}$

$\begin{array}{llll}1.2 & 0.25 & 0.03 & 238\end{array}$

$25 \quad 1115$

$\begin{array}{llll}547 & 22.5 & 7.7 & 345\end{array}$

0.05

$0.07 \quad 2.83$

1.8

$\begin{array}{lll}0.70 & 0.07 & 527\end{array}$

$\begin{array}{lll}0.10 & 0.12 & 4.28\end{array}$

1.6

0.40

$\begin{array}{lll}0.58 & 0.18 & 383\end{array}$

JULY

$\begin{array}{llllll}02 & 1215 & 142 & 27.0 & 8.2 & 538\end{array}$

$\begin{array}{llllll}10 & 1130 & 234 & 27.0 & 7.8 & 396\end{array}$

16

$23 \quad 114$

$31 \quad 1045$

AUGUST 1992

$\begin{array}{llllllrll}06 & 1100 & 532 & 24.0 & 7.8 & 416 & 0.03 & 0.02 & 2.48 \\ 12 & 1100 & 558 & 25.0 & 7.8 & 314 & <0.01 & 0.01 & 1.29 \\ 20 & 1145 & 260 & 24.0 & 7.8 & 373 & 0.04 & 0.01 & 0.420 \\ 26 & 1130 & 116 & 27.0 & 8.2 & 534 & 0.04 & 0.01 & 0.500\end{array}$

03612500 - Ohio River at Dam 53 near Grand Chain, Ill.

APRIL 1991

$\begin{array}{ccccccccccccc}10 & 1200 & 11,400 & 15.5 & 8.5 & 150 & 0.05 & 0.02 & 0.980 & - & - & 0.05 & -- \\ 18 & 1045 & 16,900 & 16.5 & 7.6 & 231 & 0.01 & 0.02 & 0.850 & -- & - & 0.02 & - \\ 23 & 1110 & 16,000 & 16.0 & 7.3 & 236 & 0.02 & 0.01 & 1.09 & -- & -- & 0.02 & - \\ \text { MAY } & & & & & & & & & & & & \\ 01 & 1040 & 10,200 & 18.0 & 8.3 & 202 & 0.02 & <0.01 & 0.970 & - & -- & 0.02 & - \\ 07 & 1022 & 8,270 & 19.5 & 8.1 & 179 & 0.02 & 0.02 & 0.630 & - & -- & 0.01 & -- \\ 14 & 1045 & 9,860 & 20.5 & 8.5 & 170 & 0.02 & 0.03 & 0.700 & - & -- & 0.02 & - \\ 21 & 1045 & 6,740 & 23.0 & 8.3 & 230 & 0.04 & 0.03 & 1.07 & - & - & <0.01 & - \\ 29 & 1030 & 14,300 & 24.0 & 7.8 & 170 & 0.03 & 0.03 & 0.610 & 0.50 & 0.16 & 0.02 & 82 \\ \text { JUNE } & & & & & & & & & & & & \\ 04 & 1135 & 5,950 & 26.5 & 7.6 & 290 & 0.02 & 0.03 & 0.970 & - & - & 0.01 & 29 \\ 11 & 1045 & 2,970 & 26.0 & 6.4 & 246 & 0.03 & 0.03 & 1.47 & 0.70 & 0.07 & 0.02 & 23 \\ 18 & 1040 & 3,770 & 27.0 & 5.6 & 197 & <0.01 & 0.03 & 0.960 & 0.50 & 0.12 & 0.03 & 23 \\ 25 & 0946 & 3,850 & 27.5 & 6.2 & 272 & 0.06 & 0.02 & 0.790 & 0.90 & 0.08 & 0.02 & 20\end{array}$




\begin{tabular}{|c|c|c|c|c|c|c|c|c|c|c|c|c|}
\hline Date & Time & $\begin{array}{c}\text { Water } \\
\text { discharge } \\
\left(\mathrm{m}^{3} / \mathrm{s}\right)\end{array}$ & $\begin{array}{l}\text { Temper- } \\
\text { ature } \\
\left.\text { ( }{ }^{\circ} \mathrm{C}\right)\end{array}$ & PH & $\begin{array}{c}\text { Specific } \\
\text { conduct- } \\
\text { ance } \\
(\mu S / c m)\end{array}$ & $\begin{array}{c}\text { Nitrogen, } \\
\text { ammonla } \\
\text { dlss } \\
\text { (mg/L) }\end{array}$ & $\begin{array}{l}\text { Nitrogen, } \\
\text { nitrite } \\
\text { dlss } \\
\text { (mg/L) }\end{array}$ & $\begin{array}{c}\text { Nitrogen, } \\
\text { nitrate } \\
\text { diss } \\
\text { (mg/L) }\end{array}$ & $\begin{array}{c}\text { Nitrogen } \\
\text { ammonia } \\
+ \\
\text { organic, } \\
\text { total } \\
\text { (mg/L) }\end{array}$ & $\begin{array}{c}\text { Phos- } \\
\text { phorus, } \\
\text { total } \\
\text { (mg/L) }\end{array}$ & $\begin{array}{c}\text { Ortho- } \\
\text { phos- } \\
\text { phorus } \\
\text { diss } \\
\text { (mg/) }\end{array}$ & $\begin{array}{l}\text { Sus- } \\
\text { pendoc } \\
\text { sodf- } \\
\text { ment } \\
\text { (mg/L) }\end{array}$ \\
\hline \multicolumn{13}{|c|}{ 03612500 - Ohio River at Dam 53 near Grand Chain, Ill.--Continued } \\
\hline \multicolumn{13}{|c|}{ JULY 1991} \\
\hline 01 & 0950 & 3,000 & 28.5 & 7.6 & 264 & 0.02 & 0.02 & 0.500 & 0.50 & 0.06 & $<0.01$ & 17 \\
\hline 09 & 1030 & 3,310 & 29.5 & 7.2 & 240 & 0.06 & 0.02 & 0.270 & 0.50 & 0.06 & $<0.01$ & 7 \\
\hline 15 & 1100 & 3,290 & 29.5 & 6.1 & 224 & 0.01 & 0.02 & 0.380 & 0.90 & 0.06 & $<0.01$ & 18 \\
\hline 22 & 1040 & 1,980 & 31.0 & 8.0 & 233 & 0.01 & 0.03 & 0.290 & 0.40 & 0.05 & $<0.01$ & 29 \\
\hline 29 & 1110 & 2,190 & 29.0 & 7.6 & 244 & $<0.01$ & 0.09 & 0.260 & 0.40 & 0.10 & $<0.01$ & 16 \\
\hline \multicolumn{13}{|c|}{ AUGUST } \\
\hline 05 & 1050 & 2,340 & 29.0 & 7.6 & 222 & 0.01 & 0.08 & 0.200 & 0.60 & 0.05 & $<0.01$ & 1 \\
\hline 12 & 1030 & 2,770 & 29.0 & 7.0 & 298 & 0.01 & 0.09 & 0.260 & 0.50 & 0.08 & $<0.01$ & 18 \\
\hline 20 & 1030 & 2,380 & 27.5 & 7.7 & 310 & $<0.01$ & 0.05 & 0.160 & 0.50 & 0.05 & 0.02 & 450 \\
\hline 27 & 0945 & 2,550 & 28.0 & 6.5 & 249 & 0.03 & 0.06 & 0.160 & 0.60 & 0.06 & $<0.01$ & 28 \\
\hline \multicolumn{13}{|c|}{ SEPTEMBER } \\
\hline 03 & 1040 & 2,310 & 28.0 & 6.3 & 207 & 0.03 & 0.04 & 0.200 & 0.40 & 0.05 & $<0.01$ & 33 \\
\hline 10 & 1030 & 2,440 & 28.0 & 6.3 & 233 & $<0.01$ & 0.04 & 0.210 & 0.50 & 0.07 & 0.02 & 12 \\
\hline 17 & 0952 & 2,470 & 28.0 & 6.3 & 236 & 0.02 & 0.03 & 0.270 & 0.50 & 0.06 & 0.03 & 12 \\
\hline 23 & 1040 & 1,920 & 24.0 & 6.4 & 230 & 0.03 & 0.04 & 0.330 & 0.40 & 0.07 & 0.03 & 10 \\
\hline 30 & 1122 & 1,760 & 22.5 & 6.7 & 228 & -- & -. & -- & 0.30 & 0.06 & -- & 9 \\
\hline \multicolumn{13}{|c|}{ OCTOBER 1991} \\
\hline 07 & 1125 & 1,810 & 19.5 & 6.5 & 225 & 0.04 & $<0.01$ & 0.190 & 0.30 & 0.10 & 0.02 & 9 \\
\hline 15 & 0955 & 1,550 & 19.5 & 6.3 & 264 & 0.06 & $<0.01$ & 0.210 & 0.30 & 0.05 & 0.03 & 8 \\
\hline 21 & 1020 & 1,520 & 17.0 & 6.4 & 258 & 0.04 & 0.01 & 0.310 & 0.40 & 0.06 & 0.02 & 5 \\
\hline 29 & 0930 & 2,200 & 18.5 & 6.7 & 378 & 0.08 & 0.02 & 0.410 & 0.60 & 0.10 & 0.03 & 9 \\
\hline \multicolumn{13}{|c|}{ NOVEMBER } \\
\hline 06 & 1040 & 2,700 & 14.5 & 6.4 & 286 & 0.09 & 0.01 & 0.410 & 0.40 & 0.07 & 0.03 & 19 \\
\hline 20 & 0920 & 2,540 & 12.5 & 6.5 & 237 & 0.05 & $<0.01$ & 0.600 & 0.30 & 0.07 & 0.22 & 10 \\
\hline \multicolumn{13}{|c|}{ DECEMBER } \\
\hline 05 & 1045 & 18,900 & 9.0 & 7.6 & 284 & 0.09 & 0.01 & 0.920 & 0.60 & 0.22 & 0.05 & 134 \\
\hline 17 & 1010 & 15,600 & 9.5 & 5.7 & 191 & 0.04 & 0.02 & 0.800 & 0.50 & 0.16 & 0.05 & 71 \\
\hline \multicolumn{13}{|c|}{ JANUARY 1992} \\
\hline 02 & 1005 & 8,780 & 7.5 & 5.5 & 209 & 0.07 & 0.02 & 1.08 & 0.30 & 0.08 & 0.04 & 17 \\
\hline 21 & 1030 & 6,970 & 3.5 & 7.1 & 204 & 0.04 & 0.02 & 0.970 & 0.30 & 0.10 & 0.05 & 19 \\
\hline \multicolumn{13}{|c|}{ FEBRUARY } \\
\hline 04 & 1125 & 4,420 & 5.5 & 7.2 & 244 & 0.06 & 0.02 & 1.08 & 0.30 & 0.06 & 0.03 & 13 \\
\hline 18 & 1025 & 5,860 & 8.0 & 7.8 & 350 & 0.05 & 0.02 & 1.18 & 0.40 & 0.08 & $<0.01$ & 24 \\
\hline \multicolumn{13}{|c|}{ MARCH 1992} \\
\hline 03 & 1025 & 11,800 & 10.0 & 7.7 & 234 & 0.07 & 0.02 & 1.08 & 0.40 & 0.04 & 0.04 & 73 \\
\hline 11 & 1030 & 11,100 & 10.5 & 7.6 & 240 & 0.06 & 0.02 & 0.930 & 0.40 & 0.13 & 0.02 & 62 \\
\hline 16 & 1040 & 14,800 & 9.5 & 7.7 & 197 & 0.04 & 0.02 & 0.930 & 0.20 & 0.07 & 0.04 & 160 \\
\hline 24 & 1020 & 14,000 & 9.5 & 5.8 & 263 & 0.03 & 0.02 & 1.28 & 0.60 & 0.10 & 0.03 & 248 \\
\hline 31 & 1030 & 14,800 & 10.5 & 7.7 & 243 & 0.02 & 0.02 & 1.38 & 0.40 & 0.15 & 0.02 & 101 \\
\hline \multicolumn{13}{|l|}{ APRIL } \\
\hline 07 & 1015 & 8,270 & 9.0 & 7.0 & 260 & 0.07 & 0.02 & 1.48 & 0.40 & 0.08 & 0.02 & 99 \\
\hline 14 & 1035 & 4,080 & 14.0 & -- & 321 & 0.03 & 0.02 & 1.48 & 0.20 & 0.06 & 0.03 & 35 \\
\hline 22 & 1040 & 7,000 & 16.0 & 7.4 & 316 & -- & -- & - & 0.50 & 0.12 & -- & 71 \\
\hline 28 & 1104 & 9,660 & 15.0 & 7.4 & 311 & 0.04 & 0.05 & 2.35 & 0.40 & 0.17 & 0.05 & 112 \\
\hline \multicolumn{13}{|l|}{ MAY } \\
\hline 06 & 0953 & 4,590 & 16.5 & 6.9 & 368 & 0.03 & 0.01 & 2.19 & 0.30 & 0.05 & 0.04 & 29 \\
\hline 12 & 0955 & 5,300 & 18.5 & 6.4 & 344 & 0.02 & $<0.01$ & 1.80 & $<0.20$ & 0.03 & 0.03 & 25 \\
\hline
\end{tabular}




\begin{tabular}{|c|c|c|c|c|c|c|c|c|c|c|c|c|}
\hline Date & Time & $\begin{array}{c}\text { Water } \\
\text { discharge } \\
\left(\mathbf{m}^{3} / \mathbf{s}\right)\end{array}$ & $\begin{array}{l}\text { Temper- } \\
\text { ature } \\
\left({ }^{\circ} \mathrm{C}\right)\end{array}$ & pH & $\begin{array}{c}\text { Specific } \\
\text { conduct- } \\
\text { ance } \\
(\mu \mathrm{S} / \mathrm{cm})\end{array}$ & $\begin{array}{c}\text { Nitrogen, } \\
\text { ammonia } \\
\text { diss } \\
\text { (mg/L) }\end{array}$ & $\begin{array}{l}\text { Nitrogen, } \\
\text { nitrite } \\
\text { diss } \\
\text { (mg/l) }\end{array}$ & $\begin{array}{l}\text { Nitrogen, } \\
\text { nitrate } \\
\text { diss } \\
\text { (mg/L) }\end{array}$ & $\begin{array}{c}\text { Nitrogen } \\
\text { ammonia } \\
+ \\
\text { organic, } \\
\text { totai } \\
\text { (mgll) }\end{array}$ & $\begin{array}{c}\text { Phos- } \\
\text { phorus, } \\
\text { totai } \\
\text { (mg/l) }\end{array}$ & $\begin{array}{l}\text { Ortho- } \\
\text { phos- } \\
\text { phorus } \\
\text { diss } \\
\text { (mgh) }\end{array}$ & $\begin{array}{c}\text { Sus- } \\
\text { pended } \\
\text { sedi- } \\
\text { ment } \\
\text { (mg/L) }\end{array}$ \\
\hline \multicolumn{13}{|c|}{03612500 - Ohio River at Dam 53 near Grand Chain, III.-Continned } \\
\hline \multicolumn{13}{|c|}{ MAY (cont.) } \\
\hline 18 & 1025 & 4,250 & 20.5 & 7.8 & 260 & 0.02 & $<0.01$ & 0.950 & $<0.20$ & 0.02 & 0.02 & 24 \\
\hline 27 & 1030 & 3,170 & 21.0 & 6.8 & 292 & 0.03 & 0.01 & 0.860 & 0.30 & 0.03 & 0.02 & 18 \\
\hline \multicolumn{13}{|c|}{ JUNE 1992} \\
\hline 02 & 1045 & 4,670 & 20.0 & 6.9 & 258 & 0.03 & 0.03 & 1.17 & 0.50 & 0.11 & 0.05 & 24 \\
\hline 09 & 1105 & 6,400 & 22.0 & 6.1 & 269 & - & -- & -- & 0.20 & 0.07 & - & 36 \\
\hline 16 & 1105 & 4,130 & 24.5 & 6.6 & 243 & 0.02 & $<0.01$ & 0.530 & 0.20 & 0.05 & 0.01 & 27 \\
\hline 23 & 1023 & 6,400 & 24.5 & 6.8 & 297 & 0.06 & 0.02 & 0.940 & 0.30 & 0.06 & 0.04 & 45 \\
\hline 29 & 1235 & 3,790 & 25.5 & 7.5 & 289 & 0.04 & 0.01 & 0.990 & 0.30 & 0.05 & 0.04 & 17 \\
\hline \multicolumn{13}{|l|}{ JULY } \\
\hline 08 & 1012 & 5,470 & 27.0 & 7.0 & 213 & 0.03 & 0.01 & 0.770 & 0.40 & 0.12 & 0.04 & 43 \\
\hline 14 & 1025 & 5,010 & 28.0 & 6.1 & 264 & 0.05 & $<0.01$ & 0.960 & 0.50 & 0.10 & 0.04 & 10 \\
\hline 23 & 1040 & 6,880 & 28.0 & 6.5 & 276 & 0.08 & 0.02 & 1.68 & 0.30 & 0.08 & 0.06 & 66 \\
\hline 28 & 1025 & 7,870 & 28.0 & 6.7 & 324 & 0.01 & 0.01 & 2.09 & 0.50 & 0.21 & 0.06 & 86 \\
\hline \multicolumn{13}{|c|}{07373420 - Mississippi River near St. Francisville, La. } \\
\hline \multicolumn{13}{|c|}{ APRIL 1991} \\
\hline 02 & 0945 & 22,800 & 22.0 & 7.6 & 322 & 0.01 & 0.03 & 1.47 & 0.70 & 0.21 & 0.04 & 238 \\
\hline \multicolumn{13}{|l|}{ MAY } \\
\hline 06 & 1130 & 32,900 & 19.0 & 8.1 & 270 & -- & -- & -. & 0.46 & 0.22 & -. & 198 \\
\hline \multicolumn{13}{|l|}{ JUNE } \\
\hline 17 & 1115 & 20,500 & 27.5 & 7.8 & 389 & 0.02 & $<0.01$ & 2.60 & 0.50 & 0.13 & 0.08 & 272 \\
\hline \multicolumn{13}{|l|}{ JULY } \\
\hline 23 & 1030 & 6,850 & 30.0 & 8.1 & 425 & -- & -- & -- & 0.85 & 0.20 & -- & 118 \\
\hline \multicolumn{13}{|c|}{ SEPTEMBER } \\
\hline 23 & 1245 & 6,030 & 27.0 & 7.6 & 433 & 0.03 & $<0.01$ & 0.55 & 0.50 & 0.13 & 0.06 & 90 \\
\hline \multicolumn{13}{|c|}{ NOVEMBER } \\
\hline 05 & 1000 & 9,460 & 16.0 & 7.4 & 560 & 0.04 & $<0.01$ & 0.64 & 0.30 & 0.16 & 0.06 & 360 \\
\hline 19 & 1045 & 9,180 & 12.0 & 7.6 & 418 & -. & -- & -- & 0.50 & 0.15 & -- & -. \\
\hline \multicolumn{13}{|c|}{ DECEMBER } \\
\hline 10 & 1030 & 18,700 & 8.0 & 7.5 & 321 & 0.05 & 0.03 & 1.27 & 0.40 & 0.41 & 0.06 & 414 \\
\hline \multicolumn{13}{|c|}{ JANUARY 1992} \\
\hline 28 & 1115 & 14,700 & 5.0 & 7.5 & 348 & 0.04 & 0.03 & 1.67 & 0.40 & 0.22 & 0.06 & 187 \\
\hline \multicolumn{13}{|c|}{ FEBRUARY } \\
\hline 26 & 1015 & 13,100 & 9.5 & 7.5 & 411 & 0.05 & 0.02 & 1.88 & 0.30 & 0.20 & 0.05 & 172 \\
\hline \multicolumn{13}{|l|}{ MARCH } \\
\hline 25 & 1000 & 21,400 & 12.5 & 7.4 & 306 & -. & -- & -- & 0.33 & 0.28 & .. & 225 \\
\hline \multicolumn{13}{|l|}{ MAY } \\
\hline 14 & 1200 & 12,200 & 18.5 & 7.8 & 410 & 0.03 & 0.01 & 2.99 & 0.50 & 0.44 & 0.07 & 228 \\
\hline 26 & 1145 & 9,630 & 24.0 & 7.7 & 404 & 0.02 & 0.01 & 1.89 & 0.60 & 0.25 & 0.06 & 177 \\
\hline \multicolumn{13}{|l|}{ JUNE } \\
\hline 17 & 1000 & 13,400 & 25.5 & 7.4 & 392 & 0.03 & $<0.01$ & 1.40 & 0.30 & 0.25 & 0.07 & 255 \\
\hline \multicolumn{13}{|l|}{ JULY } \\
\hline \multicolumn{13}{|c|}{ AUGUST } \\
\hline 18 & 1015 & 14,500 & 26.0 & 7.9 & 360 & 0.04 & $<0.01$ & 1.80 & 0.50 & 0.15 & 0.09 & 368 \\
\hline SEPTEMI & & & & & & & & & & & & \\
\hline 15 & 0900 & 7,390 & 26.5 & 7.5 & 424 & -- & -- & -- & 0.88 & 0.20 & -- & 115 \\
\hline
\end{tabular}




\begin{tabular}{|c|c|c|c|c|c|c|c|c|c|c|c|c|}
\hline Date & Time & $\begin{array}{c}\text { Water } \\
\text { discharge } \\
\left(\mathrm{m}^{3} / \mathrm{s}\right)\end{array}$ & $\begin{array}{l}\text { Temper- } \\
\text { ature } \\
\left(^{\circ} \mathrm{C}\right)\end{array}$ & pH & $\begin{array}{c}\text { Specific } \\
\text { conduct- } \\
\text { ance } \\
(\mu S / \mathrm{cm})\end{array}$ & $\begin{array}{c}\text { Nitrogen, } \\
\text { ammonia } \\
\text { diss } \\
\text { (mg/L) }\end{array}$ & $\begin{array}{l}\text { Nitrogen, } \\
\text { nitrite } \\
\text { diss } \\
\text { (mg/L) }\end{array}$ & $\begin{array}{c}\text { Nitrogen, } \\
\text { nitrale } \\
\text { diss } \\
\text { (mg/L) }\end{array}$ & $\begin{array}{c}\text { Nitrogen } \\
\text { ammonia } \\
+ \\
\text { organic, } \\
\text { total } \\
\text { (mg/L) }\end{array}$ & $\begin{array}{c}\text { Phos- } \\
\text { phorus, } \\
\text { total } \\
\text { (mg/L) }\end{array}$ & $\begin{array}{l}\text { Ortho- } \\
\text { phos- } \\
\text { phorus } \\
\text { dias } \\
\text { (mgh) }\end{array}$ & $\begin{array}{c}\text { Sus- } \\
\text { pended } \\
\text { sedi- } \\
\text { ment } \\
\text { (mg/L) }\end{array}$ \\
\hline \multicolumn{13}{|c|}{ 07374000 - Mississippi River at Baton Ronge, La. } \\
\hline \multicolumn{13}{|c|}{ APRIL 1991} \\
\hline 11 & 1430 & 25,000 & 16.5 & 7.9 & 366 & 0.02 & 0.01 & 1.99 & -- & -- & 0.03 & \\
\hline 17 & 1415 & 27,500 & 18.0 & 7.8 & 306 & 0.02 & 0.02 & 1.88 & - & .- & 0.04 & \\
\hline 24 & 1045 & 28,800 & 18.0 & 7.8 & 293 & 0.02 & 0.01 & 1.69 & -- & -- & 0.05 & \\
\hline \multicolumn{13}{|l|}{ MAY } \\
\hline 01 & 1030 & 32,200 & 18.5 & 7.6 & 300 & 0.02 & $<0.01$ & 1.50 & -- & - & 0.04 & \\
\hline 06 & 0800 & 32,700 & 19.0 & 7.6 & 280 & 0.02 & 0.01 & 1.59 & -- & -- & 0.04 & \\
\hline 09 & 1030 & 33,000 & 19.0 & 7.6 & 277 & 0.02 & $<0.01$ & 1.70 & -- & -- & 0.05 & \\
\hline 13 & 0930 & 32,600 & 21.0 & 7.6 & 281 & 0.02 & $<0.01$ & 1.80 & -- & -- & 0.05 & \\
\hline 16 & 1130 & 31,700 & 22.0 & 7.5 & 295 & 0.04 & 0.01 & 1.89 & & & 0.04 & - \\
\hline 20 & 1330 & 29,800 & 22.5 & -- & 291 & 0.04 & 0.01 & 1.89 & & & 0.04 & -- \\
\hline 24 & 1315 & 28,900 & 23.0 & 7.7 & 294 & 0.07 & 0.01 & 1.89 & & & 0.07 & -- \\
\hline 28 & 1255 & 27,300 & 24.5 & 7.8 & 311 & 0.05 & $<0.01$ & 2.00 & & & 0.06 & -- \\
\hline 30 & 1130 & 26,500 & 25.0 & 7.7 & 316 & 0.03 & $<0.01$ & 1.90 & & & 0.07 & -- \\
\hline \multicolumn{13}{|c|}{ JUNE 1991} \\
\hline 03 & 1045 & 24,600 & 26.0 & 7.7 & 336 & 0.02 & $<0.01$ & 2.00 & & & 0.06 & - \\
\hline 06 & 1300 & 24,600 & 27.0 & 7.7 & 353 & 0.02 & $<0.01$ & 2.40 & & & 0.07 & - \\
\hline 10 & 1315 & 24,200 & 26.5 & 7.7 & 307 & 0.03 & $<0.01$ & 2.00 & & & 0.07 & .- \\
\hline 13 & 1400 & 22,900 & 27.0 & 7.8 & 338 & 0.02 & $<0.01$ & 2.30 & & & 0.07 & -- \\
\hline 17 & 1315 & 20,500 & 27.5 & 7.8 & 382 & 0.04 & 0.01 & 2.59 & & & 0.09 & - \\
\hline 20 & 1415 & 18,200 & 28.0 & 8.0 & 403 & 0.05 & $<0.01$ & 2.60 & & & 0.07 & -- \\
\hline 24 & 1345 & 16,500 & 28.0 & 8.0 & 410 & 0.04 & $<0.01$ & 3.30 & & & 0.09 & -. \\
\hline 27 & 1400 & 16,300 & 28.0 & 8.0 & 409 & -- & -- & -- & & & -- & -- \\
\hline \multicolumn{13}{|l|}{ JULY } \\
\hline 03 & 1345 & 13,800 & 29.5 & 7.9 & 426 & 0.02 & $<0.01$ & 2.90 & & & 0.10 & -- \\
\hline 08 & 1500 & 12,000 & 29.0 & 7.9 & 413 & 0.01 & $<0.01$ & 2.60 & & & 0.08 & -. \\
\hline 11 & 1500 & 11,000 & 30.0 & 7.9 & 390 & 0.03 & $<0.01$ & 2.60 & & & 0.10 & -- \\
\hline 17 & 1330 & 9,490 & 30.5 & 8.0 & 426 & 0.04 & $<0.01$ & 2.70 & & & 0.10 & -- \\
\hline 23 & 1315 & 9,460 & 30.5 & 8.1 & 440 & 0.02 & $<0.01$ & 2.10 & & & 0.08 & -- \\
\hline 23 & 1316 & 9,460 & -- & -- & -- & 0.02 & $<0.01$ & 2.10 & & & 0.07 & -. \\
\hline 23 & 1317 & 9,460 & -- & -- & -. & 0.01 & $<0.01$ & 2.00 & & & 0.07 & -- \\
\hline 23 & 1318 & 9,460 & -- & -- & -- & 0.01 & $<0.01$ & 2.00 & -- & - & 0.07 & -- \\
\hline 23 & 1319 & 9,460 & -- & -- & -- & $<0.01$ & $<0.01$ & 2.00 & -- & -- & 0.07 & -- \\
\hline 23 & 1320 & 9,460 & -- & -- & -- & $<0.01$ & $<0.01$ & 2.00 & -. & -- & 0.07 & -- \\
\hline 30 & 0815 & 7,840 & 30.0 & 8.0 & 413 & 0.04 & $<0.01$ & 1.80 & -- & -- & 0.08 & -- \\
\hline \multicolumn{13}{|c|}{ AUGUST 1991} \\
\hline 06 & 0900 & 7,080 & 30.5 & 8.1 & 450 & 0.03 & $<0.01$ & 1.70 & -- & -- & 0.06 & -- \\
\hline 12 & 1030 & 6,830 & 30.5 & 8.4 & 456 & 0.03 & $<0.01$ & 1.30 & -- & -- & 0.05 & -- \\
\hline 20 & 0900 & 6,600 & 29.5 & 8.0 & 464 & 0.01 & 0.01 & 0.880 & -- & -. & 0.05 & .- \\
\hline 26 & 0900 & 6,290 & 29.0 & 8.2 & 451 & 0.02 & $<0.01$ & 0.750 & -- & -- & 0.03 & -. \\
\hline \multicolumn{13}{|c|}{ SEPTEMBER } \\
\hline 03 & 1350 & 6,340 & 29.0 & 8.5 & 468 & $<0.01$ & $<0.01$ & 0.740 & -- & - & 0.04 & -- \\
\hline 09 & 1341 & 6,200 & 29.0 & 8.1 & 466 & 0.03 & $<0.01$ & 0.820 & -- & - & 0.04 & -- \\
\hline 18 & 1000 & 5,890 & 30.5 & 8.1 & 425 & 0.08 & $<0.01$ & 0.750 & -- & -- & 0.07 & -- \\
\hline 23 & 0915 & 6,030 & 28.0 & 8.2 & 457 & 0.04 & 0.01 & 0.610 & -- & -. & 0.07 & -- \\
\hline 30 & 0845 & 6,680 & 25.5 & 8.2 & 449 & .- & -- & -- & -- & -- & -- & -- \\
\hline \multicolumn{13}{|c|}{ OCTOBER } \\
\hline 07 & 1415 & 6,200 & 23.0 & 8.1 & 447 & 0.02 & $<0.01$ & 0.640 & -- & -- & 0.07 & -- \\
\hline 16 & 1400 & 5,810 & 22.0 & 8.1 & 479 & 0.05 & $<0.01$ & 0.630 & -- & -- & 0.05 & _- \\
\hline
\end{tabular}




\begin{tabular}{|c|c|c|c|c|c|c|c|c|c|c|c|c|}
\hline Date & Time & $\begin{array}{c}\text { Water } \\
\text { discharge } \\
\left(\mathrm{m}^{3} / \mathrm{s}\right)\end{array}$ & $\begin{array}{l}\text { Temper- } \\
\text { ature } \\
\left.\text { ( }{ }^{\circ} \mathrm{C}\right)\end{array}$ & pH & $\begin{array}{c}\text { Specific } \\
\text { conduct- } \\
\text { ance } \\
(\mu \mathrm{S} / \mathrm{cm})\end{array}$ & $\begin{array}{l}\text { Nitrogen, } \\
\text { ammonia } \\
\text { diss } \\
\text { (mg/L) }\end{array}$ & $\begin{array}{l}\text { Nitrogen, } \\
\text { nitrite } \\
\text { diss } \\
\text { (mg/L) }\end{array}$ & $\begin{array}{l}\text { Nitrogen, } \\
\text { nitrate } \\
\text { diss } \\
\text { (mg/l) }\end{array}$ & $\begin{array}{c}\text { Nitrogen } \\
\text { ammonia } \\
+ \\
\text { organic, } \\
\text { totai } \\
\text { (mg/l) }\end{array}$ & $\begin{array}{c}\text { Phos- } \\
\text { phorus, } \\
\text { total } \\
\text { (mgll) }\end{array}$ & $\begin{array}{l}\text { Ortho- } \\
\text { phos- } \\
\text { phorus } \\
\text { diss } \\
\text { (mg/L) }\end{array}$ & $\begin{array}{l}\text { Sus- } \\
\text { pended } \\
\text { sodl- } \\
\text { ment } \\
\text { (mg/L) }\end{array}$ \\
\hline
\end{tabular}

OCTOBER (cont.)

07374000 - Mississippi River at Baton Rouge, La.-Continued

\begin{tabular}{|c|c|c|c|c|c|c|c|c|c|c|c|c|}
\hline 23 & 1315 & 5,270 & 20.5 & 8.1 & 489 & 0.06 & $<0.01$ & 0.780 & -. & - & 0.09 & - \\
\hline 31 & 1100 & 6,150 & 21.0 & 8.0 & 453 & 0.05 & 0.02 & 0.810 & -- & -- & 0.03 & - \\
\hline \multicolumn{13}{|c|}{ NOVEMBER 1991} \\
\hline 07 & 1600 & 10,600 & 17.0 & 8.1 & 596 & $<0.01$ & $<0.01$ & 0.630 & - & -- & 0.06 & - \\
\hline 14 & 0900 & 9,910 & 12.0 & 8.1 & 462 & 0.05 & 0.03 & 0.740 & - & - & 0.07 & - \\
\hline 19 & 1330 & 9,180 & 16.0 & 7.9 & 455 & -. & -- & -- & -- & $\cdots$ & - & -- \\
\hline 25 & 1700 & 11,800 & 13.0 & 7.8 & 407 & 0.07 & 0.02 & 0.980 & - & -- & 0.06 & - \\
\hline \multicolumn{13}{|c|}{ DECEMBER } \\
\hline 02 & 1415 & 12,500 & 14.5 & 7.6 & 353 & 0.05 & 0.04 & 1.26 & - & - & 0.06 & - \\
\hline 10 & 1015 & 18,700 & 10.0 & 7.8 & 312 & 0.07 & 0.03 & 1.17 & - & - & 0.06 & -- \\
\hline 17 & 1330 & 23,800 & 10.5 & 7.8 & 318 & 0.03 & 0.03 & 1.07 & - & -- & 0.05 & -- \\
\hline 24 & 1130 & 23,400 & 10.0 & 7.6 & 280 & 0.05 & 0.03 & 1.07 & - & - & 0.06 & -. \\
\hline 31 & 1100 & 20,900 & 9.0 & 8.3 & 316 & 0.04 & 0.03 & 1.37 & - & -- & 0.06 & -- \\
\hline \multicolumn{13}{|c|}{ JANUARY 1992} \\
\hline 07 & 1100 & 18,500 & 8.5 & 7.9 & 341 & 0.05 & 0.02 & 1.78 & -- & -- & 0.06 & m \\
\hline 15 & 1430 & 18,700 & 8.0 & 7.9 & 353 & 0.05 & 0.03 & 1.67 & -- & -- & 0.06 & - \\
\hline 20 & 1645 & 17,700 & 7.0 & 8.0 & 313 & -- & -- & -- & -- & -- & - & -- \\
\hline 29 & 1600 & 14,300 & 6.5 & 8.0 & 358 & 0.05 & 0.03 & 1.57 & - & -- & 0.06 & -- \\
\hline \multicolumn{13}{|c|}{ FEBRUARY 1992} \\
\hline 06 & 1500 & 13,300 & 8.5 & 7.9 & 379 & 0.06 & 0.02 & 1.58 & -. & -- & 0.05 & - \\
\hline 13 & 1500 & 10,500 & 11.0 & 8.1 & 416 & 0.04 & 0.01 & 1.89 & -- & .. & 0.05 & -- \\
\hline 20 & 1745 & 11,800 & 12.0 & 7.9 & 407 & 0.06 & 0.02 & 1.78 & -- & -- & 0.06 & - \\
\hline 26 & 1015 & 13,100 & 10.5 & 7.9 & 434 & 0.07 & 0.02 & 2.08 & - & -- & 0.06 & -- \\
\hline \multicolumn{13}{|c|}{ MARCH } \\
\hline 05 & 1100 & 15,900 & 11.0 & 8.2 & 420 & 0.10 & 0.02 & 1.68 & -- & -- & 0.05 & -- \\
\hline 11 & 1300 & 17,600 & 12.5 & 8.0 & 378 & 0.04 & 0.03 & 1.97 & -. & -. & 0.05 & -- \\
\hline 19 & 0900 & 19,500 & 12.0 & 8.0 & 378 & 0.06 & 0.03 & 1.97 & -- & -- & 0.06 & -- \\
\hline 26 & 1030 & 21,400 & 12.5 & 7.9 & 328 & 0.03 & 0.01 & 1.59 & - & -. & 0.06 & -. \\
\hline 30 & 1630 & 21,800 & 12.5 & 7.4 & 335 & 0.03 & 0.02 & 1.88 & - & -. & 0.05 & -- \\
\hline \multicolumn{13}{|l|}{ APRIL } \\
\hline 10 & 1500 & 18,200 & 13.0 & 7.6 & 370 & 0.03 & 0.02 & 2.28 & -- & -- & 0.06 & -- \\
\hline 15 & 0930 & 14,500 & 15.0 & -- & 384 & 0.04 & 0.03 & 2.07 & - & -. & 0.06 & -. \\
\hline 23 & 1030 & 11,200 & 18.5 & 8.0 & 430 & 0.04 & 0.02 & 2.28 & - & -- & 0.06 & - \\
\hline 30 & 0900 & 15,800 & 19.0 & 8.2 & 462 & 0.03 & $<0.01$ & 1.20 & -- & -- & 0.04 & - \\
\hline \multicolumn{13}{|c|}{ MAY 1992} \\
\hline 06 & 0915 & 17,800 & 18.0 & 7.9 & 396 & 0.02 & $<0.01$ & 2.10 & -- & -- & 0.06 & -- \\
\hline 14 & 1330 & 12,200 & 23.0 & 8.2 & 434 & 0.03 & $<0.01$ & 2.90 & $\ddot{-}$ & -- & 0.07 & -- \\
\hline 20 & 1110 & 11,000 & 23.0 & 7.7 & 469 & 0.03 & 0.01 & 1.79 & -. & -. & 0.05 & -- \\
\hline 28 & 1100 & 9,690 & 25.0 & 7.8 & 456 & 0.04 & 0.02 & 1.78 & -- & -- & 0.06 & -- \\
\hline \multicolumn{13}{|l|}{ JUNE } \\
\hline 03 & 1325 & 9,200 & 24.0 & 7.6 & 470 & 0.02 & $<0.01$ & 1.60 & -- & -- & 0.06 & -- \\
\hline 12 & 1440 & 11,800 & 25.0 & 7.7 & 443 & 0.02 & $<0.01$ & 1.60 & -- & -. & 0.06 & -. \\
\hline 19 & 0925 & 13,100 & 27.0 & 7.7 & 418 & 0.03 & $<0.01$ & 1.30 & -- & -. & 0.06 & - \\
\hline 25 & 1415 & 11,800 & 29.0 & -- & -- & 0.03 & $<0.01$ & 1.00 & -- & -. & 0.06 & -- \\
\hline \multicolumn{13}{|l|}{ JULY } \\
\hline 02 & 1145 & 11,600 & 27.0 & 7.7 & 425 & 0.03 & $<0.01$ & 1.10 & -- & -. & 0.06 & -- \\
\hline 09 & 1500 & 10,300 & 28.0 & 7.8 & 450 & 0.04 & 0.01 & 1.09 & -- & -- & 0.07 & -- \\
\hline
\end{tabular}


Table 9.--Water-discharge, physical-property, nutrient, and suspended-sediment data--Continued

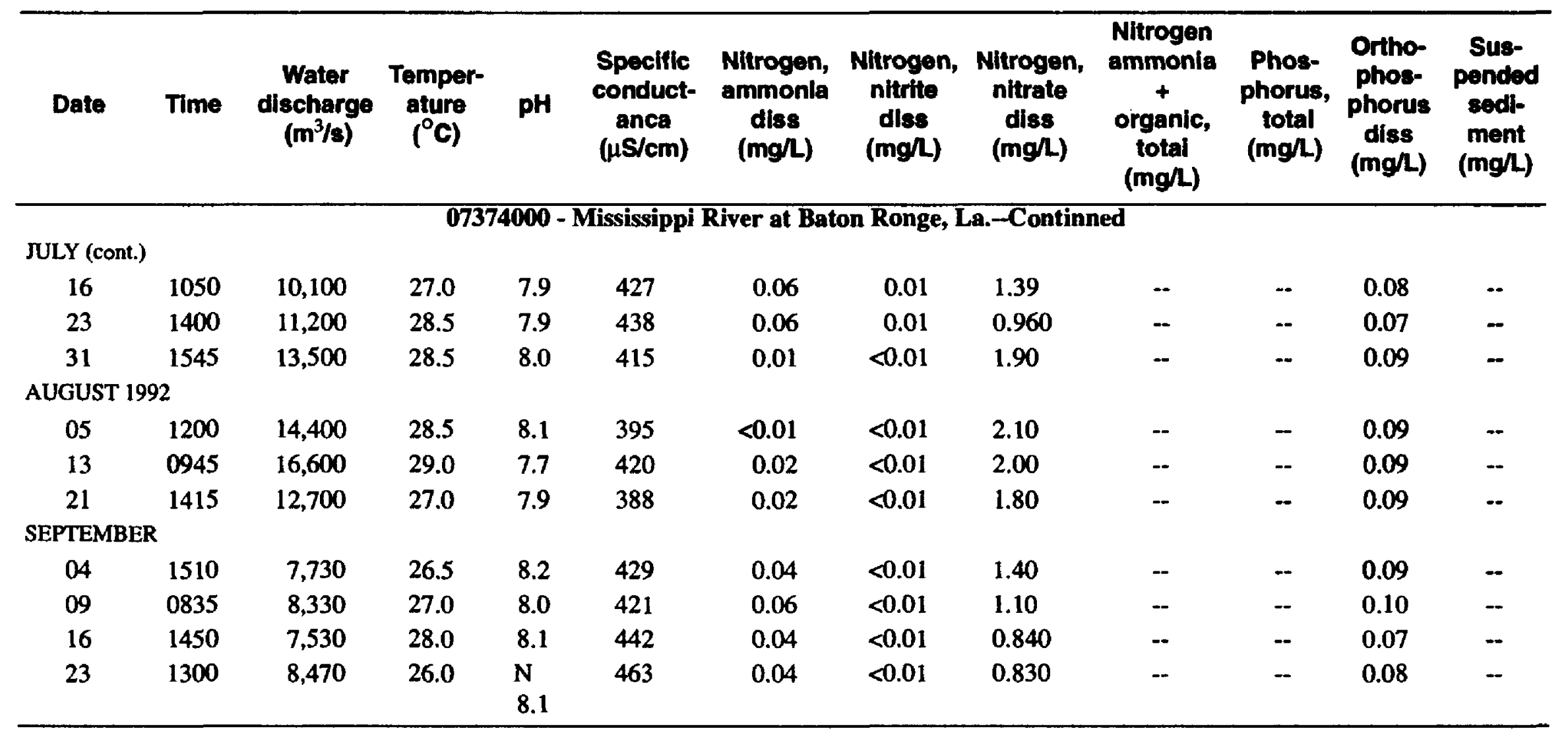

